\title{
THE INTEGRATION OF SEISMIC ANISOTROPY AND RESERVOIR PERFORMANCE DATA FOR CHARACTERIZATION OF NATURALLY FRACTURED RESERVOIRS USING DISCRETE FEATURE NETWORK MODELS
}

\author{
A Dissertation \\ by \\ ROBERT WILL \\ Submitted to the Office of Graduate Studies of \\ Texas A\&M University \\ in partial fulfillment of the requirements for the degree of \\ DOCTOR OF PHILOSOPHY
}

May 2004

Major Subject: Petroleum Engineering 


\title{
THE INTEGRATION OF SEISMIC ANISOTROPY AND RESERVOIR PERFORMANCE DATA FOR CHARACTERIZATION OF NATURALLY FRACTURED RESERVOIRS USING DISCRETE FEATURE NETWORK MODELS
}

\author{
A Dissertation \\ by \\ ROBERT WILL \\ Submitted to Texas A\&M University \\ in partial fulfillment of the requirements \\ for the degree of \\ DOCTOR OF PHILOSOPHY
}

Approved as to style and content by:

Rosalind Archer

(Chair of Committee)

W. John Lee

(Member)

Jerry Jensen

(Member)

Richard Gibson

(Member)

Stephen Holditch

(Head of Department)

May 2004

Major Subject: Petroleum Engineering 


\author{
ABSTRACT \\ The Integration of Seismic Anisotropy and Reservoir Performance Data for \\ Characterization of Naturally Fractured Reservoirs Using Discrete Feature Network \\ Models. (May 2004) \\ Robert Will, B.A., Hope College; \\ M.S., New Mexico Institute of Mining and Technology \\ Chair of Advisory Committee: Dr. Rosalind Archer
}

This dissertation presents the development of a method for quantitative integration of seismic (elastic) anisotropy attributes with reservoir performance data as an aid in characterization of systems of natural fractures in hydrocarbon reservoirs. This new method incorporates stochastic Discrete Feature Network (DFN) fracture modeling techniques, DFN model based fracture system hydraulic property and elastic anisotropy modeling, and non-linear inversion techniques, to achieve numerical integration of production data and seismic attributes for iterative refinement of initial trend and fracture intensity estimates. Although DFN modeling, flow simulation, and elastic anisotropy modeling are in themselves not new technologies, this dissertation represents the first known attempt to integrate advanced models for production performance and elastic anisotropy in fractured reservoirs using a rigorous mathematical inversion. The following new developments are presented:

- Forward modeling and sensitivity analysis of the upscaled hydraulic properties of realistic DFN fracture models through use of effective permeability modeling techniques. 
- Forward modeling and sensitivity analysis of azimuthally variant seismic attributes based on the same DFN models.

- Development of a combined production and seismic data objective function and computation of sensitivity coefficients.

- Iterative model-based non-linear inversion of DFN fracture model trend and intensity through minimization of the combined objective function.

This new technique is demonstrated on synthetic models with single and multiple fracture sets as well as differing background (host) reservoir hydraulic and elastic properties. Results on these synthetic control models show that, given a well conditioned initial DFN model and good quality field production and seismic observations, the integration procedure results in convergence of both fracture trend and intensity in models with both single and multiple fracture sets. Tests show that for a single fracture set convergence is accelerated when the combined objective function is used as compared to a similar technique using only production data in the objective function. Tests performed on multiple fracture sets show that, without the addition of seismic anisotropy, the model fails to converge. These tests validate the importance of the new process for use in more realistic reservoir models. 


\section{DEDICATION}

This dissertation is dedicated:

To my father for teaching me that I should,

To a few very special people for believing that I could,

To my mother who said I was brave to try,

To my wife for a reason why. 


\section{ACKNOWLEDGMENTS}

I wish to express my sincere appreciation to Dr. John Lee, Dr. Jerry Jensen and Dr. Rick Gibson for serving on my graduate advisory committee, and for their contributions toward the success of this dissertation. Special thanks goes to the chair of my graduate

advisory committee, Dr. Rosalind Archer, for her support, guidance, encouragement, and help in completing this dissertation.

I would like to thank express my appreciation to Dr. John Lee, Dr. Thomas Blasingame, Dr. Rosalind Archer, Dr. Maria Barrufet, Darla Jean Weatherford, and many others for their dedication to the Distance Learning program at Texas A\&M University. I would also like to thank my employer Schlumberger for making software and computing facilities available for this work, and Bill Dershowitz of Golder Associates for providing software support and technical advice.

Finally, I wish to thank Eleanor Schuler for her kind assistance throughout these years, as well as the rest of the faculty and staff at the Harold Vance Department of Petroleum Engineering for their sharing of knowledge, support, and encouragement during my studies at Texas A\&M University. 


\section{TABLE OF CONTENTS}

Page

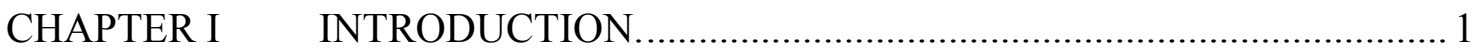

$1.1 \quad$ Background of This Research............................................................... 1

1.2 Objectives of This Research .............................................................. 4

1.3 Application of the Method ..................................................................

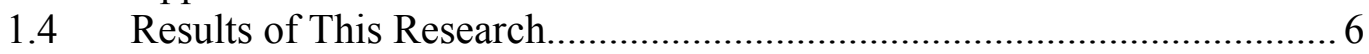

1.5 Organization of This Dissertation.......................................................... 6

CHAPTER II LITERATURE REVIEW ................................................................ 9

2.1 Flow Through Fractured Media ...........................................................

2.2 Fracture Related Elastic Anisotropy ………………………………….... 17

$2.3 \quad$ Discrete Feature Network Modeling ........................................................2 24

$2.4 \quad$ History Matching and Numerical Optimization Methods ……………..... 26

CHAPTER III INVERSION PROCEDURE........................................................ 29

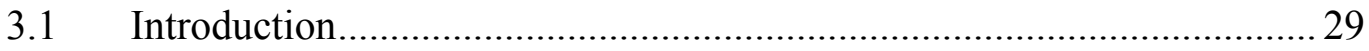

3.2 Least Squares Inversion Fundamentals...………………………………....30

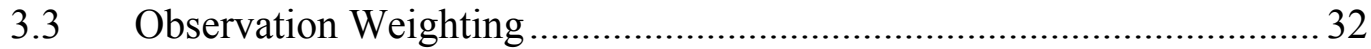

3.4 Addition of Random Observation Noise .................................................. 33

3.5 Computation of the Jacobian Matrix …………………………………........ 32

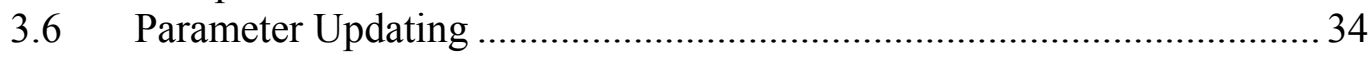

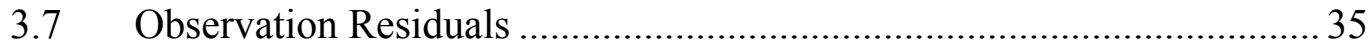

3.8 Application to the New Method................................................................ 35

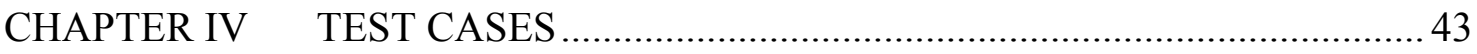

4.1 Base Case Reservoir Model and Production Configuration ...................... 44

4.2 Test T2 - Single Fracture Set in a Tight Sandstone Matrix …………..... 45

4.3 Test T3 - Two Fracture Sets in a Tight Sandstone Matrix........................58

4.4 Test T4 - Two Fracture Sets in a Dolomite Matrix................................... 76

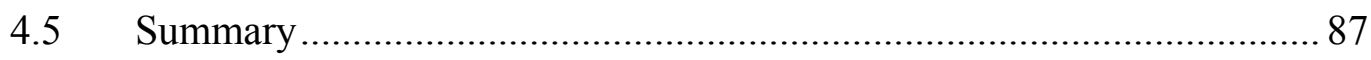




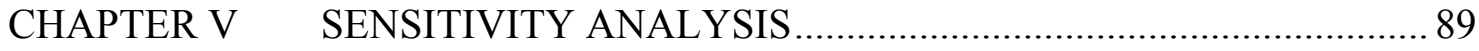

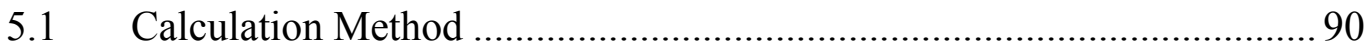

5.2 Sensitivity to Trend and $\mathrm{P}_{32}$ Intensity ............................................... 92

5.3 Sensitivity to Hydraulic Parameters …................................................. 93

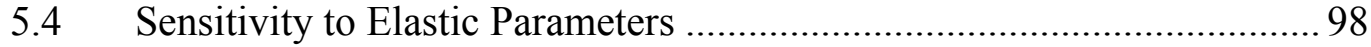

5.5 Sensitivity to Reservoir Thickness .................................................... 101

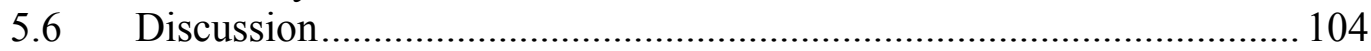

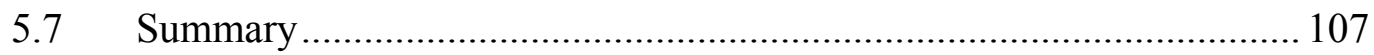

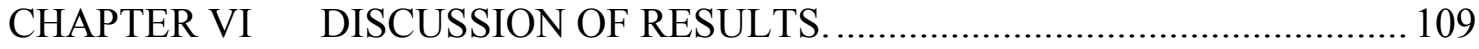

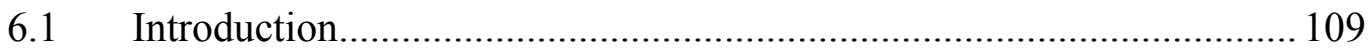

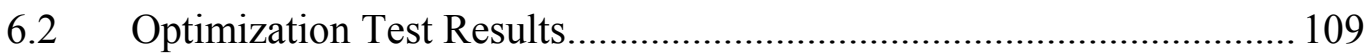

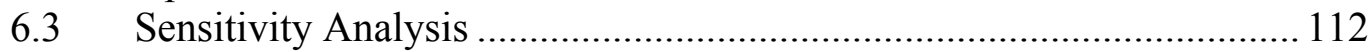

6.4 Integrated Modeling Workflow .................................................. 112

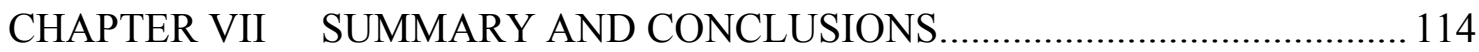

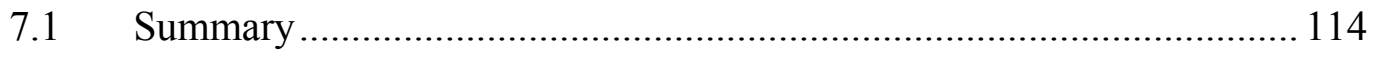

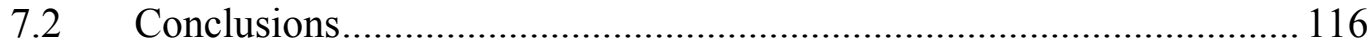

7.3 Recommendations for Future Work ................................................ 119

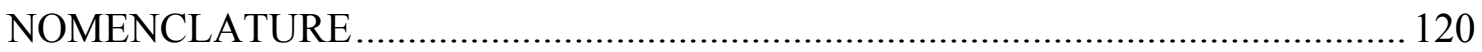

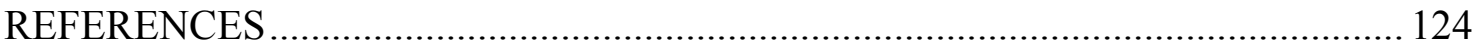

APPENDIX A OVERVIEW OF THE METHOD............................................. 130

APPENDIX B SUMMARY OF TEST T2 RESULTS ........................................ 142

APPENDIX C SUMMARY OF TEST T3 RESULTS ..................................... 180

APPENDIX D SUMMARY OF TEST T4 RESULTS ....................................239

APPENDIX E SUMMARY OF SENSITIVITY TEST RESULTS ..................... 276

APPENDIX F MATLAB SCRIPT STIFF (EFFECTIVE ELASTIC MEDIA

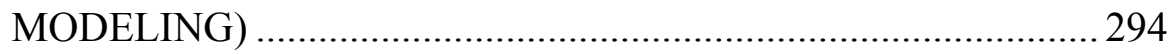

APPENDIX G MATLAB SCRIPT CREATE_ATTR (SEISMIC ATTRIBUTE

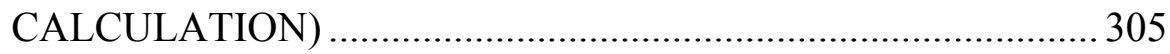

APPENDIX H MATLAB SCRIPT SENS (SENSITIVITY COEFFICIENTS AND PARAMETER UPDATES) .......................................... 311

APPENDIX I SAMPLE ECLIPSE DATA FILE .............................................. 325

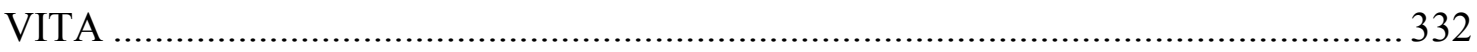




\section{LIST OF FIGURES}

FIGURE

Page

2.1 The conventional "sugar cube" dual porosity model (after Warren and $\left.\operatorname{Root}^{24}\right)$. 10

2.2 The representative elemental volume from a fractured reservoir (after Kazemi et al. ${ }^{25}$ ). 12

2.3 The relationship between fracture scale and upscaling gridblock size (after Dershowitz et al. ${ }^{5}$ ).

2.4 Boundary element nodal grid used by Lough et al. ${ }^{4}$ for upscaling of fracture permeability. 16

4.1a Top view of production configuration for optimization test cases................ 45

4.1b Side view of production configuration for optimization test cases................ 45

4.2 Graph of absolute error in fracture set $\mathrm{P}_{32}$ intensity versus iteration for test $\mathrm{T} 2$. Iteration 0 is the initial estimate.

4.3 Graph of absolute error in fracture set trend versus iteration for test T2.

Iteration 0 is the initial estimate. 48

4.4 Graph of objective function and normalized residual errors by observation type for fracture set trend and $\mathrm{P}_{32}$ intensity at each iteration using the combined objective function. Iteration 0 is the initial estimate.

4.5 Parameter sensitivity coefficients by observation number for the single fracture set initial model case T2.

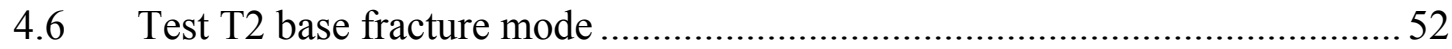

4.7 Test T2 initial fracture system estimate .................................................. 53

4.8 Final model using the combined data objective function.............................53

4.9 Final model using the production data only objective function .....................54 
4.10 Fracture water saturation at report step 20 (600 days) for test T2 base model.

4.11 Fracture water saturation at report step 20 (600 days) for test T2 initial estimate.

4.12 Fracture water saturation at report step 20 (600 days) for test T2 final model, combined data 56

4.13 Base model quasi-P wave velocity modulation amplitude and maximum quasi-P wave velocity direction.

4.14 Initial estimate quasi-P wave velocity modulation amplitude and maximum quasi-P wave velocity direction

4.15 Final quasi-P wave velocity modulation amplitude and maximum quasi$\mathrm{P}$ wave velocity direction. 58

4.16 Graph of absolute error in fracture set trend versus iteration for test T3 using the combined objective function. Iteration 0 is the initial estimate 60

4.17 Graph of absolute error in fracture set $\mathrm{P}_{32}$ intensity versus iteration for test T3 using the combined objective function. Iteration 0 is the initial estimate.

4.18 Graph of absolute error in fracture set $1 \mathrm{P}_{32}$ intensity versus iteration for test $\mathrm{T} 3$ for both combined and production data only objective function. Iteration 0 is the initial estimate

4.19 Graph of absolute error in fracture set $2 \mathrm{P}_{32}$ intensity versus iteration for test $\mathrm{T} 3$ for both combined and production data only objective function. Iteration 0 is the initial estimate

4.20 Graph of absolute error in fracture set 1 trend versus iteration for test $\mathrm{T} 3$ for both combined and production data only objective function. Iteration 0 is the initial estimate

4.21 Graph of absolute error in fracture set 2 trend versus iteration for test T3 for both combined and production data only objective function. Iteration 0 is the initial estimate. 
4.22 Graph of objective function and normalized residual errors by observation type for fracture set trend and $\mathrm{P}_{32}$ intensity using the combined objective function. Iteration 0 is the initial estimate

4.23 Graph of objective function and normalized oil production rate residual errors for the combined objective function and production data only objective function. Iteration 0 is the initial estimate 66

4.24 Graph of normalized bottom hole pressure residual errors for the combined objective function and production data only objective function. Iteration 0 is the initial estimate

4.25 Parameter sensitivity coefficients by observation number for the multiple fracture set initial model combined objective function case T3 ...... 68

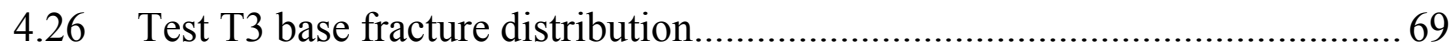

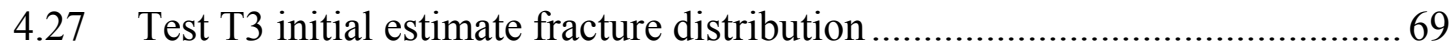

4.28 Test T3 final model fracture distribution using the combined objective function.

4.29 Test T3 final model fracture distribution using the production data only objective function

4.30 Fracture water saturation at report step 20 (600 days) for test T3 base model

4.31 Fracture water saturation at report step 20 (600 days) for test T3 initial estimate

4.32 Fracture water saturation at report step 20 (600 days) for test T3 final model

4.33 Test T3 base model quasi-P wave velocity modulation amplitude and maximum quasi-P wave velocity direction

4.34 Test T3 initial estimate quasi-P wave velocity modulation amplitude and maximum quasi-P wave velocity direction

4.35 Test T3 final quasi-P wave velocity modulation amplitude and maximum quasi-P wave velocity direction 
4.36 Graph of absolute error in fracture set trend versus iteration for test T4. Iteration 0 is the initial estimate..... 78

4.37 Graph of absolute error in fracture set $\mathrm{P}_{32}$ intensity versus iteration for test $\mathrm{T} 4$. Iteration 0 is the initial estimate

4.38 Graph of objective function and normalized residual errors by observation type for fracture set trend and $\mathrm{P}_{32}$ intensity at each iteration. Iteration 0 is the initial estimate 80

4.39 Parameter sensitivity coefficients by observation number for the multiple fracture set initial model combined objective function case $\mathrm{T} 4$

4.40 Test T4 base model fracture distribution............................................... 82

4.41 Test T4 initial estimate fracture distribution fracture distribution ................. 82

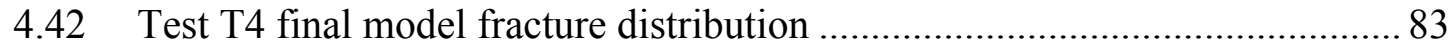

4.43 Fracture water saturation at report step 20 (600 days) for test T4 base model

4.44 Fracture water saturation at report step 20 (600 days) for test T4 initial estimate.

4.45 Fracture water saturation at report step 20 (600 days)for test T4 final model

4.46 Base model quasi-P wave velocity modulation amplitude and maximum quasi-P wave velocity direction

4.47 Initial estimate quasi-P wave velocity modulation amplitude and maximum quasi-P wave velocity direction 86

4.48 Final quasi-P wave velocity modulation amplitude and maximum quasi$\mathrm{P}$ wave velocity direction

5.1 Objective function sensitivity to fracture set 2 trend and $\mathrm{P}_{32}$ intensity. 93

5.2 Sensitivity of objective function to hydraulic model parameters and fracture $\mathrm{P}_{32}$ intensity. 
FIGURE Page

5.3 Sensitivity of objective function to hydraulic model parameters and

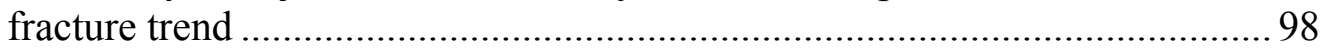

5.4 Sensitivity of objective function to elastic model parameters and fracture $\mathrm{P}_{32}$ intensity

5.5 Sensitivity of objective function to elastic model parameters and fracture trend

5.6 Sensitivity of objective function to reservoir thickness and fracture $\mathrm{P}_{32}$ intensity

5.7 Sensitivity of objective function to reservoir thickness and fracture trend. 


\section{LIST OF TABLES}

TABLE

Page

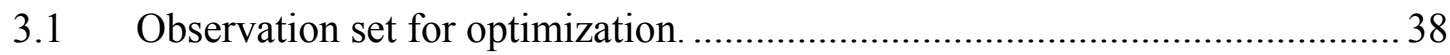

4.1 Test T2 base model fracture distribution parameters. ................................ 46

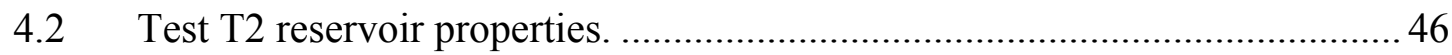

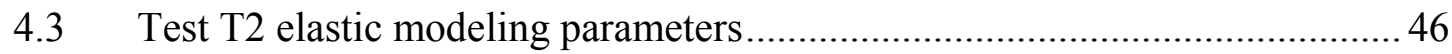

4.4 Summary of absolute errors for fracture set trend and $\mathrm{P}_{32}$ intensity at each update step for both production data only and combined data tests. Step 0 is the initial estimate

4.5 Summary of objective function and normalized residual errors for fracture set trend and $\mathrm{P}_{32}$ intensity at each update step using the combined objective function. Step 0 is the initial estimate.

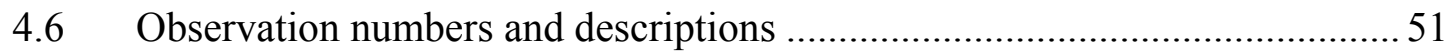

4.7 Test T3 base model fracture distribution parameters ................................... 58

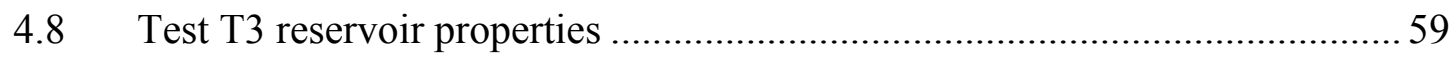

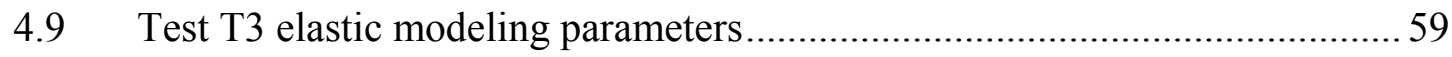

4.10 Summary of absolute errors for fracture set trend and $\mathrm{P}_{32}$ intensity at each update step using the combined objective function. Step 0 is the initial estimate

4.11 Summary of absolute errors for fracture set trend and $\mathrm{P}_{32}$ intensity at each update step using the production data only objective function. Step 0 is the initial estimate.

4.12 Summary of objective function and normalized residual errors by observation type for fracture using the combined objective function. Iteration 0 is the initial estimate..... 65

4.13 Summary of normalized residuals by observation type for fracture set trend and $\mathrm{P}_{32}$ intensity at each iteration using the production data only objective function. Iteration 0 is the initial estimate 
TABLE

4.14 Test T4 base model fracture distribution parameters ................................ 76

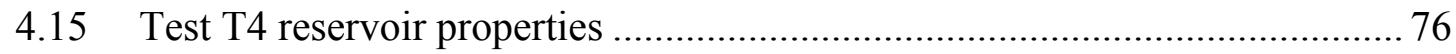

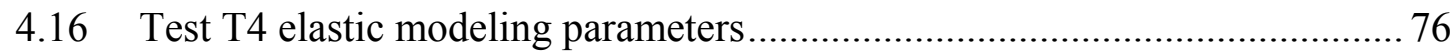

4.17 Summary of absolute errors for fracture set trend and $\mathrm{P}_{32}$ intensity at each update step. Step 0 is the initial estimate ......................................... 77

4.18 Summary of objective function and normalized residual errors by observation type for fracture set trend and $\mathrm{P}_{32}$ intensity at each iteration. Iteration 0 is the initial estimate

5.1 Reference model parameters and sensitivity test parameter perturbation limits.

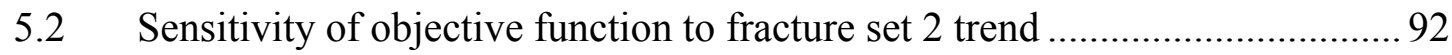

5.3 Sensitivity of objective function to fracture set $2 \mathrm{P}_{32}$ intensity ...................... 92

5.4 Sensitivity of objective function to hydraulic REV block size ..................... 94

5.5 Matrix block size for sensitivity test dual porosity shape factor values assuming cubic matrix blocks ............................................................... 95

5.6 Sensitivity of objective function to dual porosity shape factor ..................... 96

5.7 Sensitivity of objective function to matrix permeability ............................. 97

5.8 Sensitivity of objective function to elastic REV radius ............................ 100

5.9 Sensitivity of objective function to elastic incidence angle ....................... 100

5.10 Sensitivity of objective function to fracture compliance ........................... 101

5.11 Sensitivity of objective function to reservoir thickness ............................. 102 


\section{CHAPTER I}

\section{INTRODUCTION}

In this chapter we introduce the general scope of this dissertation, in particular:

- The background and motivation for the research.

- The specific objectives of the research.

- The application of the method developed.

- A brief summary of the results of the research.

To aid the reader, the last section of this chapter provides the organizational sequence for this dissertation.

\subsection{Background of This Research}

Anisotropy and heterogeneity in reservoir permeability present unique challenges during the development of hydrocarbon reserves in naturally fractured reservoirs. Predicting primary reservoir performance, planning development drilling or EOR programs, completion design, and facilities design all require accurate estimates of reservoir properties and the predictions of future reservoir behavior computed from such estimates. Over the history of naturally fractured reservoir development many methods have been employed for characterization of fracture systems and their effect on fluid flow in the reservoir. These include the use of geologic surface outcrop analogues, core, single and multi-well pressure transient analysis, full-field simulation history matching, borehole imaging logs, and surface and borehole seismic observations. This dissertation focuses on integration of production data and surface seismic observations for improved prediction of fractured reservoir performance.

This dissertation follows the style and format of the SPEJ. 
The foundation for computation of single phase flow through fractured media was laid out by Barenblatt et al. ${ }^{1}$, who concluded that existing methods for description of 'seepage' through porous media using single porosity models were suitable only for 'qualitative investigations'. Barenblatt went on to develop the 'dual porosity' continuum model. Kazemi ${ }^{2}$ later developed the first practical finite difference implementation of the dual porosity model for reservoir simulation.

Although stable technologies exist for continuum modeling of fractured reservoirs, determination of hydraulic properties for fracture systems, and the interaction between fractures and their host medium remains a subject of intense study. Oda ${ }^{3}$ developed the first method for computing continuum model properties from discrete fracture networks. More recently, Lough et al. ${ }^{4}$ developed boundary element computational methods for computing the effective permeability of fractured reservoirs. Dershowitz et $a l^{5}$ developed permeability upscaling methods for discrete fracture networks using finiteelement computational techniques.

Linear elastic theory provides the framework for computation of elastic behavior of a generalized medium. Oda's original continuum modeling techniques were later developed into effective media models for both elasticity and permeability ${ }^{6,7}$ using discrete models for discontinuities (fractures). Schoenberg ${ }^{8}$ introduced the linear slip model for fractured media which, further elaborated on the elastic characteristics of the discrete discontinuities. This theory was further developed by Schoenberg and Sayers ${ }^{9}$ and Schoenberg and Douma ${ }^{10}$ to represent elastic media with arbitrary fracture systems represented by elastic discontinuities.

Brown et al. ${ }^{11}$, Pickup et al. ${ }^{12}$, and $\mathrm{King}^{13,14}$ made laboratory investigations into the relationship between seismic anisotropy and permeability anisotropy. These authors 
present basic effective media theories for both flow and elastic behavior in fractured media and contemplate possible models for direct relationships between elastic and hydraulic anisotropy. Brown et al. ${ }^{11}$ discussed the need for calibration of both the hydraulic parameters (primarily transmissivity) and elastic parameters (compliance) using laboratory and field observations. Pyrak-Nolte ${ }^{15}$ reported consistent interrelationships between elastic attenuation and fluid flow laboratory studies conducted on three rock samples from the same tectonic setting.

To date, efforts to integrate seismic data into the workflow for characterization of naturally fractured reservoirs have been focused on the use of post-stack data. Seismic data are typically used to define main structural elements of the reservoir. Fracture density has been successfully correlated with horizon curvature determined from seismic horizons ${ }^{16}$. Seismic attributes can frequently be correlated with reservoir properties such as shale fraction, which often correlates with fracture population statistics. Acoustic impedance computed from seismic data frequently exhibits dim spots in the presence of fractures. Parney and LaPointe ${ }^{17,18}$ were the first to correlate seismic anisotropy with DFN realizations for a field case.

Precedents exist for rigorous numerical history matching in non-fractured reservoirs. Datta-Gupta et al. ${ }^{19}$ used sensitivity coefficients computed from a streamline simulator for refinement of reservoir models with multiple data types. Gradient optimization methods have been used successfully by Landa, ${ }^{20}$ and Landa and Horne ${ }^{21}$ for integration of conventional seismic attributes with well test data. Many authors including Huang et $a l .{ }^{22,23}$ used linear gradient techniques for integration of field production data and timelapse seismic data. No such rigorous, model based inversion approach to the fractured reservoir problem has been documented prior to this research program. 


\subsection{Objectives of This Research}

The objective of this research is to build on prior work in the areas of discrete feature network modeling, dual porosity simulation, and seismic anisotropy in order to develop an improved method for history matching of naturally fractured reservoir models which takes advantage of the combined resolving power of both production data and specialized elastic anisotropy attributes computed from 3-dimensional seismic data. The new method involves simultaneous inversion of production and seismic data through an iterative, linear gradient optimization technique. The known relationships between the direction of maximum quasi-P wave velocity and fracture trend, and between the degree of systematic quasi-P wave velocity variation and fracture intensity will be exploited through combination of these observations with well bottom hole pressures and oil production rates in a single optimization objective function. This research program involved the following specific objectives:

- Forward modeling and sensitivity analysis of upscaled hydraulic properties from realistic DFN fracture models through use of effective permeability modeling techniques. DFN modeling is an established method for developing field-wide stochastic realizations of fracture networks in petroleum reservoirs. Hydraulic properties are computed from these realizations through effective media modeling. This dissertation includes a systematic analysis of these hydraulic properties for a realistic field production configuration as observed through oil production rate and flowing bottom hole pressures for a realistic five spot injector-producer pattern. The effects of fracture population parameters, host reservoir properties, upscaling parameters, and certain flow simulation parameters were studied.

- Forward modeling and sensitivity analysis of azimuthally variant seismic observations computed from the same DFN models. Elastic effective media modeling techniques were used to investigate the sensitivity of the modeled anisotropic elastic characteristics of the fractured reservoir as observed through 
azimuthally variant quasi-P wave velocities. This analysis includes the effects of fracture elastic properties, host rock properties, and upscaling parameters.

- Development of a combined production and seismic data objective function and computation of sensitivity coefficients. This objective function includes oil production rate, flowing bottom hole pressure, direction of maximum quasi-P wave seismic velocity, and seismic interval P-wave velocity anisotropy. Noise is added to simulated data. Sensitivity coefficients with respect to fracture system trend and intensity were computed through incremental perturbation of model parameters.

- Iterative model-based non-linear inversion of DFN fracture model trend and intensity through minimization of the combined objective function. New sensitivity coefficients are computed for each iteration and model update. Estimated observation standard deviations are applied and normalized residuals reported for all update steps.

\subsection{Application of the Method}

The new method was demonstrated on three synthetic cases. In each case a base (truth) case fracture realization was established. The common measure of fracture intensity " $\mathrm{P}_{32}$ " was adopted for this research. $\mathrm{P}_{32}$ intensity is defined as the ratio of fracture surface area to reservoir volume. Initial erroneous estimates of fracture set trend and $\mathrm{P}_{32}$ intensity were made as a starting point for the refinement process. Success of the method was judged in terms of the rate and final accuracy of convergence of trend and $\mathrm{P}_{32}$ intensity to the base case. The three cases analyzed explore the applicability of the method in the presence of single and multiple fracture sets as well as different host medium porosity, permeability, densities, and seismic wave propagation velocities. In all cases the reservoir was of constant thickness with homogeneous host medium reservoir properties. Stochastic fracture realizations were spatially stationary with respect to all relevant hydraulic and mechanical parameters. The production 
configuration used was a five spot pattern with a center injector. The objective function was made up of well oil production rates, well bottom hole pressures, and the amplitude and phase angle of the sinusoidal azimuth dependence of seismic p-wave propagation velocity.

\section{$1.4 \quad$ Results of This Research}

The results of this research support the conclusion that use of the combined production and seismic data objective function is a viable process that improves characterization of naturally fractured reservoirs. In all three cases studied the new method both sped up and stabilized convergence of fracture trend and intensity to the known base parameters when compared with similar optimization method using only production data. The method was successful in tests involving single and multiple fracture sets and was also successful in cases using various host medium properties. Some sensitivity to base model parameters and host medium properties was observed. The results of extensive sensitivity analyses are presented in Chapter V.

\subsection{Organization of This Dissertation}

The outline of this dissertation is as follows:

- Chapter I - Introduction

- Background of this research

- Objectives of this research

- Application of results

- Chapter II - Literature Review

- Flow through fractured media

- Fracture related elastic anisotropy

○ Discrete Feature Network (DFN) modeling

- History matching and numerical optimization methods

- Chapter III - Inversion Procedure

- Introduction 
- Least squares inversion fundamentals

- Observation weighting

- Addition of observation noise

- Computing the Jacobian matrix

- Parameter updating

- Observation residuals

- Application to the new method

- Chapter IV - Test Cases

- Base reservoir model and production configuration

- Single fracture sets in a tight sandstone matrix

- Two fracture sets in a tight sandstone matrix

- Two fracture sets in a dolomite matrix

○ Summary

- Chapter V - Sensitivity Analysis

- Calculation method

- Sensitivity to trend and $\mathrm{P}_{32}$ intensity

- Sensitivity to hydraulic parameters

- Sensitivity to elastic parameters

- Sensitivity to reservoir thickness

$\circ$ Discussion

- Chapter VI - Discussion of Results

○ Optimization test results

- Sensitivity analysis

- Integration workflow

- Chapter VII - Summary and Conclusions

○ Summary

○ Conclusions

○ Recommendations for future work 
- Appendix A - Overview of the Workflow

- Appendix B - $\quad$ Detailed Summary of Test T2 Results

- Appendix C - Detailed Summary of Test T3 Results

- Appendix D - $\quad$ Detailed Summary of Test T4 Results

- Appendix E - Detailed Summary of Sensitivity Tests

- Appendix F - $\quad$ Matlab Script STIFF (elastic effective media modeling)

- Appendix G - Matlab Script ATTR (attribute calculation)

- Appendix H - Matlab Script SENS (sensitivity coefficients and parameter updates)

- Appendix I - $\quad$ Sample Eclipse DATA file 


\section{CHAPTER II}

\section{LITERATURE REVIEW}

In this chapter we present a review of the literature used as a basis for the development of the new technique. The following sections cover;

- Flow through fractured media.

- Fracture related elastic anisotropy.

- Discrete Feature Network modeling.

- History matching and numerical methods in reservoir engineering.

These sections briefly cover the mathematical and conceptual foundations of each of these fundamental components of the new method.

\subsection{Flow Through Fractured Media}

\subsubsection{The Dual Porosity Continuum Model}

The foundation for computation of single phase flow through fractured media was laid out by Barenblatt et al. ${ }^{1}$. These authors observed the lack of detailed information regarding the fracture and matrix properties, and thus concluded that existing methods for description of 'seepage' through porous media using single porosity models were suitable only for 'qualitative investigations'. Based on a conceptual model of a fractured reservoir, Barenblatt developed the 'dual porosity' continuum model in which the fracture system and matrix blocks are represented as distinct but interacting continuous flow systems, each represented by average or effective media parameters of a region much larger than the individual matrix block dimension. Barenblatt also first introduced use of the characteristic 'specific fracture surface', relating the amount of fracture 
surface area per area of rock volume, in formulating solutions to problems involving matrix-fracture flow. This work also included the first solutions for the problem of radial flow into an infinitesimally small wellbore.

The dual porosity model pioneered by Barrenblat was adopted and used as a basis for further development by Warren and $\operatorname{Root}^{24}$ who developed analytical solutions to the single phase radial flow problem. In this work the authors developed the well known $\lambda, \omega$ parameterization of fracture storativity and fracture-matrix transfer to describe the hydraulic behavior of a regularized model (Fig. 2.1) of a fracture media.

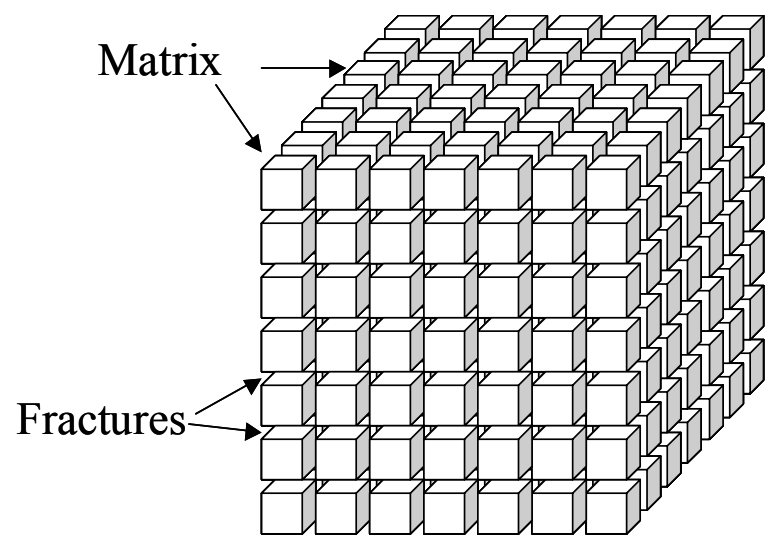

Fig 2.1 - The conventional "sugar cube" dual porosity model (after Warren and $\operatorname{Root}^{24}$ ).

Kazemi et $a l .{ }^{25}$ developed the first practical implementation of a finite difference multiphase, dual porosity simulator which included gravity and imbibition effects. Kazemi considered the mass balance on an elemental volume of the grid including both matrix and fractures; 


$$
\begin{aligned}
& \nabla\left[\lambda_{\alpha f} \rho_{\alpha}\left(\nabla \Psi_{f}-\frac{1}{144} \nabla D_{f}\right)\right]-\left[T_{\alpha m a}\left(S_{\alpha m a}\right)\left(\Psi_{\alpha f}-\Psi_{\alpha m a}\right)\right] \\
& +q_{\alpha m a} \delta\left(X-X_{0}\right)=\frac{1}{5.6146} \frac{\partial}{\partial t}\left(\phi_{f} S_{\alpha f} / B_{\alpha f}\right) \\
& T_{\alpha m a}\left(S_{\alpha m a}\right) \rho_{\alpha}\left(\Psi_{\alpha f}-\Psi_{\alpha m a}\right)=\frac{1}{5.6146} \frac{\partial}{\partial t}\left(\phi_{m a} S_{\alpha m a} / B_{\alpha m a}\right) \ldots
\end{aligned}
$$

where;

$$
\begin{aligned}
& k=\text { matrix permeability } \\
& k_{\alpha \rho}=\text { relative permeability with respect to phase } \alpha \\
& \mu_{\mathrm{o}} \quad=\quad \text { viscosity } \\
& B_{\alpha m a}=\text { formation volume factor of phase in matrix } \\
& N=\text { number of normal sets of fractures } \\
& L=\text { characteristic dimension of the matrix block } \\
& \Sigma=\frac{4 N(N+2)}{L^{2}} \\
& \lambda_{\alpha f}=0.0011271\left(\frac{k k_{\alpha r}}{\mu_{\alpha} B_{\alpha}}\right)_{f} \\
& T_{\alpha m a}\left(S_{\alpha m a}\right)=0.0011271\left(\frac{k k_{\alpha r}}{\mu_{\alpha} B_{\alpha}}\right)_{m} \Sigma \\
& \rho \quad=\quad \text { density } \\
& p_{\alpha} \quad=\quad \text { pressure of phase } \alpha \\
& \Psi_{\alpha}=\int_{o_{o}}^{p} \frac{d p_{\alpha}}{\rho\left(p_{\alpha}\right)}
\end{aligned}
$$

$\delta\left(X-X_{o}\right)=$ Dirac delta function $=1$ for $\mathrm{X}=\mathrm{X}_{0}, 0$ otherwise

$S_{\alpha m a}=\quad$ phase saturation in matrix

$S_{w f}+S_{n w f}=1$

$S_{w m a}+S_{n w m a}=1$ 
$p_{c}=$ capillary pressure

$p_{n w m a}, p_{w m a}=$ non-wetting and wetting phase capillary pressures

$$
\begin{aligned}
& p_{n w f}-p_{w f}=p_{c f}\left(S_{w f}\right) \\
& p_{n w m a}-p_{w m a}=p_{c m a}\left(S_{w m a}\right)
\end{aligned}
$$

Eq. 2.1 represents the mass balance within the fracture system including production terms and mass transfer to and from the matrix. Eq. 2.2 represents the mass transfer between the matrix and fracture system. Eq. $2.3(\Sigma)$ is the shape factor, which describes the effect of the relative fracture surface area per matrix block volume on the matrix-fracture mass transfer. In this formulation it is assumed that production (flow into the wellbore) is from the fracture system only. Fig. 2.2 illustrates Kazemi's representative elemental volume and modeled pressure distribution in a dual porosity grid.

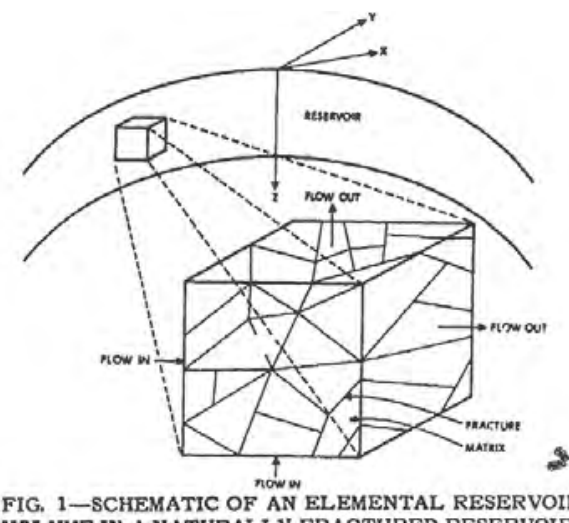

FO. 1-SCHEMATTRAFY FRACTURED RESERVOIR
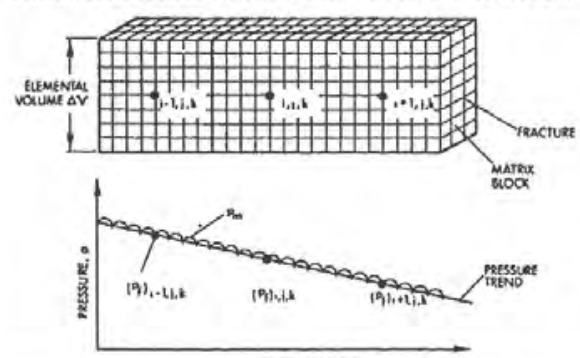

FIG, 2 - IDEALIZATION OF FLOW AND ELEMENTAL RESERVOIR VOLUMES CONTAINING MATRIX BLOCKS IN A NATURALLY FRACTURED RESERVOIR.

Fig 2.2 - $\quad$ The representative elemental volume from a fractured reservoir (after Kazemi et $_{\text {al. }}{ }^{25}$ ). 
Kazemi used a discrete difference formulation of Eqs. 2.1 and 2.2 to perform finite difference simulation of fractured reservoir flow. Flow between the fracture system and matrix is typically modeled as pseudosteady state flow, which assumes pressure equilibration between fractures and matrix within the duration of each time step. This approximation is generally accurate for small matrix blocks and when matrix permeability is high. The pseudosteady state assumption will begin to fail in situations where equilibration is slow with respect to the time step. This may happen, for example, with large matrix blocks, lower matrix permeability permeability, or in the event of silicification of fracture surfaces.

The characteristic shape factor $\Sigma$ should be calibrated through history matching of dynamic data such as pressure transient data. Alternate methods have been developed, each of which provide some enhancement to portions of the overall problem of representing flow through fractured media. However, none of these yet provide as robust a solution to the generalized multi-phase problem as the finite difference dual porosity formulation. Finite element methods implemented by Dershowitz et al. ${ }^{5}$ allow use of Discrete Feature Network (DFN) models of fracture distributions for more 'realistic' representations of the reservoir. However, finite element simulators do not currently provide adequate solutions to the problem of multi-phase flow in fractured reservoirs. Bourbiaux et $a l .{ }^{26}$ demonstrate a new method of modeling the matrix-fracture system interaction for use in dual porosity simulation. This method uses individual functions to describe the different flow mechanisms such as capillarity and gravity forces, thus eliminating the need for the dual porosity shape factor and implicitly representing the actual matrix block size. However, calibration of these functions is non-trivial. More recently, Basquet et al. ${ }^{27}$ developed a technique for more representative modeling flow of single phase compressible fluids (gas) in fractured reservoirs through an extension of the original formulation by Barrenblat to include non-Darcy flow terms and development of an appropriate dual porosity simulation scheme. 


\subsubsection{Permeability Upscaling}

Upscaling of permeability is even more problematic in fractured reservoirs than in conventional, single porosity reservoirs. Permeability of the fracture system is a function of fracture parameters (length, aperture, density), which are largely unknown. Permeability of the fracture system exhibits extreme scale sensitivity. Depending upon the simulation gridblock size a fracture of length $L$ may or may not contribute to interblock connectivity. That is, depending upon fracture length, the permeability of the individual fracture may contribute to the permeability of the fracture system, or may be attributed to the matrix (Fig. 2.3). Therefore, in dual porosity finite difference simulation it is critical to evaluate the relationship between simulator block size and fracture length population statistics. $\mathrm{Oda}^{3}$ developed analytical relations for computing the permeability tensor of an arbitrary fracture distribution in which the fracture population is above the percolation point. The Oda fracture tensor, representing the volume weighted average product of fracture areas and transmissivity, is

$F_{i j}=\frac{1}{V} \sum_{k=1}^{N} A_{c k} T_{k} n_{i k} n_{j k}$

where;

$$
\begin{array}{lll}
\mathrm{T} & = & \text { fracture transmissivity }\left(\mathrm{m}^{2} / \mathrm{s}\right) \\
\mathrm{A}_{\mathrm{c}} & = & \text { fracture cross-sectional area }\left(\mathrm{m}^{2}\right) \\
\mathrm{n}_{\mathrm{ij}} & = & \text { unit normal vectors }
\end{array}
$$

$$
T=\frac{\rho g b^{3}}{12 \mu}
$$

where;

$$
\begin{array}{lll}
\rho & = & \text { fluid density }\left(\mathrm{g} / \mathrm{cm}^{3}\right) \\
\mu & = & \text { fluid viscosity }(\mathrm{cp}) \\
\mathrm{b} & = & \text { fracture aperture }(\mathrm{cm}) \\
\mathrm{g} & = & \text { acceleration due to gravity }\left(\mathrm{m} / \mathrm{s}^{2}\right)
\end{array}
$$


And the Oda fracture hydraulic conductivity tensor is;

$k_{i j}=\frac{1}{12}\left(F_{k k} \delta_{i j}-F_{i j}\right)$

where;

$$
\mathrm{k}_{\mathrm{ij}}=\text { fracture hydraulic conductivity tensor }(\mathrm{m} / \mathrm{s})
$$

In this study the off-diagonal terms of the hydraulic conductivity tensor in Eq. 2.10 were ignored. In order to minimize the impact of this simplification the simulator grid lines were aligned as closely as possible to the direction of fracture strike.
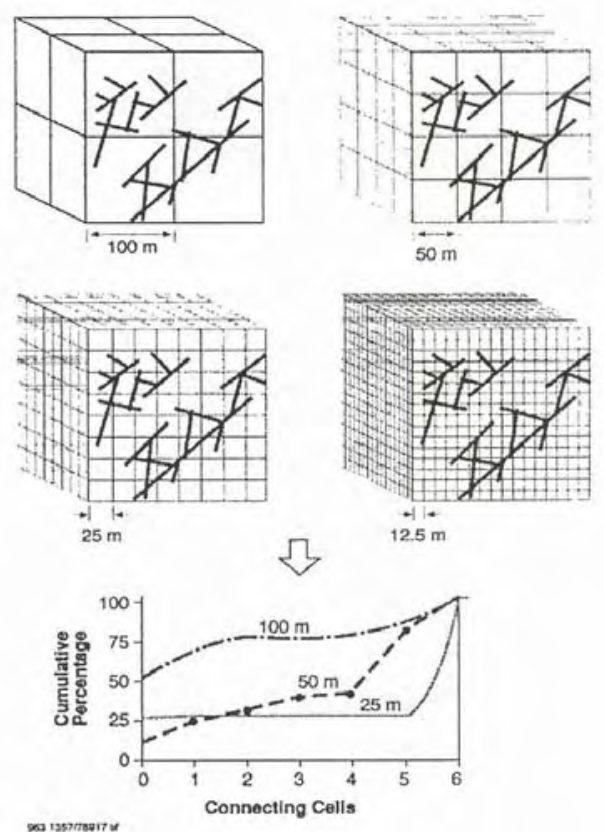

Fig. 2.3 - $\quad$ The relationship between fracture scale and upscaling gridblock size (after Dershowitz et al. ${ }^{5}$ ).

Various authors ${ }^{4,5}$ have developed techniques for upscaling of permeabilities given a generalized discrete fracture distribution. Dershowitz et al. ${ }^{5}$ used finite element 
simulation to construct permeability tensors for each individual simulator block. This technique involves gridding of a discrete characterization of the fracture-matrix system, and successively simulating the flow through each block under appropriate boundary conditions. This technique suffers from the drawback that the finite element simulator is single phase and does not include matrix-fracture flow. Lough et al. ${ }^{4}$ implemented the boundary element method (BEM) for permeability upscaling from a discrete fracture model. In this method, flow through each individual simulator gridblock is modeled using a BEM formulation with periodic boundary conditions. Fig. 2.4 shows fractures defined by systems of nodes for use in the BEM by Lough. The advantages of this method are that the matrix-fracture interaction is accurately modeled, and the dimensionality of the scale-up problem is reduced. Later Lee and Lough ${ }^{28}$ addressed computational inefficiency in their method by introducing a scale-dependent hierarchical upscaling technique. In this method, permeability attributed to fractures of length much smaller that the grid block are first modeled analytically. The resulting permeability is attributed to the matrix. Fractures with length scale on the order of the simulation block size are treated using their BEM technique.

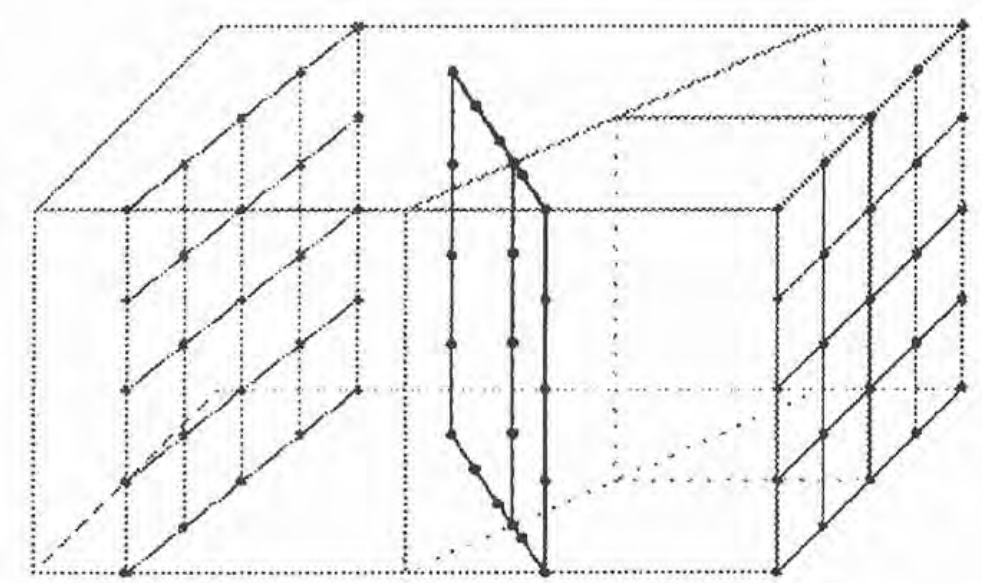

Fig. $2.4 \quad$ - $\quad$ Boundary element nodal grid used by Lough et al. ${ }^{4}$ for upscaling of fracture permeability.

He et al. ${ }^{29}$ avoided the high cost of boundary element permeability upscaling in large grids by using the boundary element method to create "pseudo" hard data points at 
selected locations, followed by the use of Sequential Gaussian Simulation to populate the entire grid with fracture permeability based upon the known correlation between DFN intensity and upscaled permeability. Gurpinar and Kossack ${ }^{30}$ developed techniques for calibrating dual porosity media parameters through history matching of fine scale single porosity models.

\subsection{Fracture Related Elastic Anisotropy}

\subsubsection{Elastic Anisotropy}

Current applications for the use of seismic anisotropy in fractured reservoir characterization rely upon the existence of spatially systematic variations (anisotropy) in elastic rock properties caused by fractures. These variations in rock elastic properties are detectable in certain special seismic attributes such as azimuthal variation of amplitude with azimuth (AVAZ), shear wave bi-refringence, and azimuthal variation of propagation velocity for the fractured interval. In the simple case of a single set of vertical aligned fractures the anisotropy has a single vertical plane of symmetry aligned with the mean trend of the fractures. In this case, the elasticity and related seismic attributes exhibit systematic and symmetrical azimuthal variations, which may be approximated with a sinusoidal function. It can be shown through rigorous anisotropic elastic theory that the amplitude and phase angle of the sinusoidal function are related to the intensity and trend of the fracture set. This phenomenon forms the basis for the use of seismic anisotropy in the new method. The following sections outline the relevant anisotropic elastic theory which have been implemented as part of the development of the new method.

Seismic anisotropy resulting from elongated voids in the host media can be described through various models including Oda ${ }^{5}$, Kuster-Tokzos ${ }^{31}$, Hudson ${ }^{32}$, and Schoenberg's linear slip discontinuity model ${ }^{8}$. The latter model was selected for this study because it allows computation using discrete fracture descriptions rather than fracture population statistics. Further, this model was used by Schoenberg and Sayers ${ }^{9}$ as the basis for 
seismic anisotropy studies as referenced earlier in this disserttion. The linear slip discontinuity model is derived from the anisotropic form of Hooke's law;

$\sigma_{i j}=c_{i j k l} \varepsilon_{k l}$

where;

$$
\begin{array}{lll}
\sigma_{\mathrm{ij}} & = & \text { stress }(\mathrm{Pa}) \\
\varepsilon_{\mathrm{k} 1} & = & \text { strain } \\
\mathrm{c}_{\mathrm{ijkl}} & = & \text { elastic stiffness }(\mathrm{Pa}) .
\end{array}
$$

Equivalently, it can be expressed as

$$
\varepsilon_{k l}=s_{i j k l} \sigma_{i j}=c_{i j k l}^{-1} \sigma_{i j}
$$

where;

$$
\mathrm{s}_{\mathrm{ijkl}}=\quad \text { elastic compliance }\left(\mathrm{Pa}^{-1}\right)
$$

In fractured media, the compliance tensor $s$ can be expressed as

$$
s_{i j k l}=s_{i j k l}^{h}+\Delta s_{i j k l}
$$

where;

$$
\begin{array}{lll}
\mathrm{s}_{\mathrm{ijkl}}^{\mathrm{h}}= & \text { elastic compliance of host media } \\
\Delta \mathrm{s} & = & \text { fracture compliance }
\end{array}
$$


Schoenberg and Sayers have shown that the additional compliance of the medium resulting from the presence of fractures can be expressed as

$$
\begin{aligned}
& \Delta s_{i j k l}=\frac{1}{4}\left(\delta_{i k} \alpha_{j l}+\delta_{i l} \alpha_{j k}+\delta_{j k} \alpha_{i l}+\delta_{j l} \alpha_{i k}\right)+\beta_{i j k l} \\
& \alpha_{i j}=\frac{1}{V} \sum_{r} B_{T}^{*(r)} n_{i}^{(r)} n_{j}^{(r)} A_{s}^{(r)} \ldots \ldots \ldots \ldots \ldots \ldots \ldots \ldots \ldots \ldots \ldots \ldots \\
& \beta_{i j k l}=\frac{1}{V} \sum\left(B_{N}^{*(r)}-B_{T}^{*(r)}\right) n_{i}^{(r)} n_{j}^{(r)} n_{k}^{(r)} n_{l}^{(r)} A_{s}^{(r)} \ldots \ldots
\end{aligned}
$$

where;

$$
\begin{array}{lll}
\mathrm{B}^{*}{ }_{\mathrm{N}} & = & \text { fracture normal compliance }\left(\mathrm{Pa}^{-1}-\mathrm{m}\right) \\
& \text { compliance } \\
\mathrm{B}^{*}{ }_{\mathrm{T}}= & \text { fracture shear compliance }\left(\mathrm{Pa}^{-1}-\mathrm{m}\right) \\
\mathrm{A}_{\mathrm{fs}}= & \text { fracture surface area }\left(\mathrm{m}^{2}\right) \\
\mathrm{n} & = & \text { unit normal vectors }
\end{array}
$$

Given a discrete description of the fracture distribution and knowledge of $\mathrm{B}_{\mathrm{N}}^{*}$ and $\mathrm{B}_{\mathrm{T}}{ }_{\mathrm{T}}$ in Eqs. 2.23 and 2.24, it is possible model the anisotropic elastic behavior of the fractured medium. Although no method exists for directly measuring $\mathrm{B}_{\mathrm{N}}{ }_{\mathrm{N}}$ and $\mathrm{B}_{\mathrm{T}}{ }_{\mathrm{T}}$, Pyrak-Nolte ${ }^{17}$ presented experimental results of a method for estimating fracture shear stiffness from seismic interference. Sayers ${ }^{33}$ provides analytical expressions for $\mathrm{B}^{*}{ }_{\mathrm{N}}$ and $\mathrm{B}_{\mathrm{T}}{ }_{\mathrm{T}}$ as functions of fracture aspect ratio, host medium, and fluid prioperties. Values selected for $\mathrm{B}^{*}{ }_{\mathrm{N}}$ and $\mathrm{B}_{\mathrm{T}}{ }_{\mathrm{T}}$ in this research program generally agree with the results of computations using the Sayers analytical expression. These yield velocity anisotropy of approximately $90 \%$ for the studied DFN. A library of generic fracture stiffnesses $\left(\mathrm{Yip}^{34}\right)$ is available to support this analysis in future studies. 
The compliance tensor computed above is used in solution of the momentum balance equation via the Cristofel equation. The result is the vector of ray parameters for the three modes of elastic wave propagation, quasi-P wave compressional, fast shear, and slow shear. From this solution the directional dependent velocity of propagation of all modes in any direction may be computed. Typical fracture anisotropy analyses rely on qualitative or semi quantitative interpretation of seismic derivatives or "attributes" which are equal to or directly related to one or more of these propagation velocities.

\subsubsection{P-wave Anisotropy}

Directional quasi-P wave elastic moduli and propagation velocities may be computed for for any arbitrary fractured medium can be computed from the compliance tensor given by Eqs. 2.14 through 2.16. This azimuthal variation in velocity results in a systematic variation in reflection amplitude at the interface of the anisotropic layer. Perez et al. ${ }^{35}$ used the expression in Eq. 2.17 for constant incidence $\mathrm{P}$ wave reflectivity to parameterize the $\mathrm{P}$-wave response in fractured reservoirs.

$R_{p p}(\theta)=A+B \sin ^{2}(\theta)$

where;

$$
\begin{array}{lll}
\theta & = & \text { incidence angle } \\
A & = & \text { offset term independent of } \theta
\end{array}
$$

Ikelle ${ }^{36}$ derived expressions for P-wave reflection coefficient as a function of angle and azimuth as a Fourier series in azimuth. For fracture distributions with weak anisotropy and monoclinic symmetry with horizontal plane of mirror symmetry, P-wave reflectivity can be written as the series expansion;

$$
\begin{aligned}
& R_{p p}\left(\theta_{i}, \phi\right)=D+G_{2}\left(\theta_{i}\right) \cos 2\left(\phi-\phi_{2}\right)+ \\
& G_{4}\left(\theta_{i}\right) \cos 4\left(\phi-\phi_{4}\right)+G_{6}\left(\theta_{i}\right) \cos 6\left(\phi-\phi_{6}\right)+\ldots
\end{aligned}
$$


where;

$$
\begin{array}{lll}
D & = & \text { offset term independent of } \theta \text { and } \phi \\
G_{n}\left(\theta_{l}\right) & = & \text { modulation amplitude of nth Fourier component } \\
\phi & = & \text { raypath azimuth } \\
\phi_{\mathrm{n}} & = & \text { azimuth of nth Fourier component } \\
p p & = & \text { compressional wave mode } \\
\theta_{i} & = & \text { constant incidence angle }
\end{array}
$$

The Fourier components $\mathrm{D}$ and $\mathrm{G}\left(\theta_{\mathrm{i}}\right)$ in Eq. 2.18 are independent of azimuth angle but are functions of the elastic moduli $c_{i j k l}$ (Eq. 2.12) of the fractured medium. Eg. 2.18 is frequently terminated to obtain the well known expression expression for P-wave reflection amplitude versus azimuth AVAZ used by Mallick et al. ${ }^{37}$, and Lynn et al. ${ }^{38}$

$$
R_{p p}\left(\phi, \theta_{i}\right)=A^{\prime}+B\left(\theta_{i}\right) \cos 2\left(\phi-\phi_{q p p}\right)
$$

where;

$\begin{array}{lll}\mathrm{R}_{\mathrm{pp}}\left(\phi, \theta_{\mathrm{i}}\right) & = & \text { compressional wave reflection coefficient } \\ A^{\prime} & = & \text { amplitude offset } \\ B\left(\theta_{i}\right) & = & \text { amplitude modulation constant } \\ \phi & = & \text { raypath azimuth } \\ \phi_{\mathrm{qpp}} & = & \text { direction of maximum quasi-P wave amplitude }\end{array}$

The offset $\mathrm{A}^{\prime}$, modulation amplitude $\mathrm{B}\left(\theta_{\mathrm{i}}\right)$, and direction of maximum quasi-P wave amplitude $\phi_{\mathrm{qpp}}$ are dependent on fracture system trend and $\mathrm{P}_{32}$ intensity through the elastic moduli $\mathrm{c}_{\mathrm{ijkl}}$ of the fractured medium. Therefore, for a constant angle of incidence $\theta_{\mathrm{i}} \mathrm{Eq}$. 2.19 is a function of the independent variable $\phi$ with the dependency on the fracture 
system characteristics carried in the $\mathrm{B}\left(\theta_{\mathrm{i}}\right)$, and $\phi_{\mathrm{qp}}$ terms. Amplitude versus azimuth (AVAZ) anisotropy analysis is performed by sorting seismic data into incidence angle and azimuth limited gathers and plotting reflection amplitude $\mathrm{R}(\phi)$ versus azimuth $\phi$ for a set of gathers at a selected incidence angle. A best fit sinusoidal function (Eg. 2.19) is computed to determine $\mathrm{B}\left(\theta_{\mathrm{i}}\right)$ and $\phi_{\text {qpp }}$ for qualitative estimation of fracture system trend and intensity. For a single set of fractures with constant compliance, modulation amplitude $\mathrm{B}\left(\theta_{\mathrm{i}}\right)$ is proportional to the intensity of the fracture system; the direction maximum quasi-P wave amplitude $\phi_{\mathrm{qpp}}$ is aligned parallel with the fracture system orientation.

A silimar analysis technique may be applied to the seismic velocities of the fractured interval. For development of the new method the quasi-P wave velocity of the fractured interval was used as the primary seismic observation. From Eq. 2.19 can be shown ${ }^{39}$ that;

$V_{q p}\left(\theta_{p}, \phi\right)=A^{\prime}\left(\theta_{p}\right)+B^{\prime}\left(\theta_{p}\right) \cos 2\left(\phi-\phi_{q p v}\right)$

Where;

$\begin{array}{lll}\mathrm{V}_{\mathrm{qp}}\left(\theta_{\mathrm{p}}, \phi\right) & = & \text { quasi-P wave velocity } \\ \theta_{\mathrm{p}} & = & \text { angle of velocity propagation measured from vertical } \\ A^{\prime}\left(\theta_{\mathrm{p}}\right) & = & \text { velocity offset } \\ B^{\prime}\left(\theta_{p}\right) & = & \text { velocity modulation constant } \\ \phi & = & \text { raypath azimuth } \\ \phi_{\mathrm{qpv}} & = & \text { direction of maximum quasi-P wave velocity }\end{array}$

Here the offset $\mathrm{A}^{\prime}$, modulation amplitude $\mathrm{B}^{\prime}\left(\theta_{\mathrm{i}}\right)$, and direction of maximum quasi-P wave propagation velocity $\phi_{\mathrm{qpv}}$ are dependent on fracture system trend and $\mathrm{P}_{32}$ intensity through the elastic moduli $c_{\mathrm{ijkl}}$ of the fractured medium. Therefore, for a constant angle of angle of propagation through the fractured medium $\theta_{p}$, Eq. 2.20 is a function of the 
independent variable $\phi$ with the dependency on the fracture system characteristics carried in the $\mathrm{A}^{\prime}, \mathrm{B}^{\prime}\left(\theta_{\mathrm{p}}\right)$, and $\phi_{\mathrm{qpv}}$ terms. A best fit sinusoidal function (Eg. 2.19) is computed determine $\mathrm{B}^{\prime}\left(\theta_{\mathrm{p}}\right)$ and $\phi_{\mathrm{qpv}}$ for qualitative estimation of fracture system trend and intensity.

Modulation amplitude B', and direction of maximum quasi-P wave velocity $\phi_{\mathrm{qpv}}$ may be derived from appropriately acquired and processed 3D seismic data using the following procedure;

1. The 3D data is sorted into common image point gathers on the pre-determined $\mathrm{x}$ y grid spacing.

2. The gathers are sorted into a minimum of eight azimuth sectors (i.e. $0^{\circ}-45^{\circ}, 45^{\circ}$ $\left.90^{\circ}, 90^{\circ}-135^{\circ}, 135^{\circ}-180^{\circ}, 180^{\circ}-225^{\circ}, 225^{\circ}-270^{\circ}, 270^{\circ}-315^{\circ}, 315^{\circ}-360^{\circ}\right)$.

3. RMS velocity versus depth is computed for all azimuth sector gathers.

4. RMS velocities are converted to interval velocities for each azimuth sector gather.

5. For each CDP gather (grid block), and at the target reservoir depth, interval velocity is plotted as a function of azimuth.

6. For each grid block a best fit sinusoidal function in the form of Eq. 2.20 is computed for the velocity versus azimuth data.

7. For each grid block modulation amplitude $\mathrm{B}^{\prime}$ is determined from the peak-to peak amplitude of the simusoidal function. Direction of maximum quasi-P wave velocity $\phi_{\mathrm{qpv}}$ is computed from the phase angle of the sine function.

In conventional seismic velocity analysis the quasi-P wave propagation velocity is assumed to be independent of incidence angle. In cases where anisotropy is strong the data may be further sorted into incidence angle limited gathers or an anisotropic velocity model may be used for the velocity analysis. 
For a single set of fractures with constant compliance, modulation amplitude B' is proportional to the intensity of the fracture system; the direction maximum quasi-P wave velocity $\phi_{\mathrm{qpv}}$ is aligned parallel with the fracture system orientation. In this general case it is also true that $\phi_{\mathrm{qpv}}=\phi_{\mathrm{qpp}}$.

\subsection{Discrete Feature Network Modeling}

Discrete Feature Network (DFN) modeling (Dershowitz et al. ${ }^{40}$ ) is a powerful tool for developing field-wide stochastic realizations of fracture networks in petroleum reservoirs. Given a comprehensive set of geologic, geophysical, borehole, and engineering observations it is possible to create a conditioned stochastic estimate of the fracture system in the reservoir. Creating such a model involves several data analysis and integration steps, requiring specialized software and training. Such comprehensive characterization work is outside the scope of this approach. However, the assumption of a well conditioned initial DFN model, and the use of stochastic modeling tools for forward modeling are central to the proposed research program. Such models are typically well conditioned in the vicinity of the wellbore through incorporation of core data, borehole imagery, and pressure transient data. Model uncertainty generally increases with distance from the borehole.

The following is a brief discussion of DFN methods and some of the capabilities of the FracMan software package provided for this research under academic license terms by Golder Associates.

Individual fractures and systems of natural fractures can be parameterized as follows ${ }^{41}$;

Individual Fractures

Orientation

Size

Location

Termination style

\section{Systems of Fractures}

Sets

Chronology

Heirarchy

Termination $\%$ 
Aperture

Roughness

Planarity
Intensity

Connectivity

Many of the above parameters may be determined directly or inferred through integrated analysis of various field observations including;

Core

Outcrop analogs

Borehole imaging (FMI)

Conventional well logs

Borehole seismic

Packer tests

Spinners

Flow meters

The above parameterization may be used to describe several geologic conceptual models, nine of which have been implemented in the FracMan stochastic modeling software package from Golder.

Simplistic fracture models assume stationarity of the fracture sytsem using a Poisson distribution. Various methods gave been developed to create non-stationary fracture populations. Elementary seismic attributes such as horizon curvature and impedance anomalies have been used to guide estimates of fracture trend and intensity $\left(\mathrm{P}_{32}\right)$ (Dershowitz and $\mathrm{Herda}^{42}$ ) in DFN modeling through geostatistical calibration with borehole and other data. However, these attributes often provide only weak statistical correlation with fracture system characteristics. Bushara ${ }^{43}$ imposed variations in fracture intensity through integration with stress the field derived from horizon flexure. Ouenes et al. $^{44}$ used artificial intelligence to develop non-linear multi-variate correlations for 
mapping non-stationary fracture intensity. Tran et $a l .{ }^{45}$ identified a hierarchy in the importance of fracture parameters and used a nested neural network technique to develop the relationships between primary and secondary fracture parameters through training. The resulting neural network was used to create a multi-scale scheme for characterizing fracture related heterogeneity scheme.

\subsection{History Matching and Numerical Optimization Methods}

Due to the strongly anisotropic effects of fractured media, and the uncertainty in fracture distribution parameters, history matching of a fractured reservoir simulator model is an extremely complex task. Gurpinar et al. ${ }^{46}$ use a scale hierarchical approach in which seven key reservoir parameters were systematically calibrated using well tests and production performance:

- Fracture permeability and direction.

- Clay layer transmissibility.

- Aquifer strength and location.

- Rock type.

- Block height.

- Dual porosity shape factor and matrix permeability.

Agarwal et al. ${ }^{47}$, describe simulator model building and history matching for the Ekofisk field. In this study where a DFN model was not used, key history matching parameters were:

- Permeability anisotropy.

- Fault transmissibilities.

- Non-neighbor connections across faults.

- Vertical permeability.

- Relative permeability pseudo functions.

- Endpoint $\mathrm{k}_{\mathrm{rw}}$.

- Permeability and skin in wells 
- Well productivity index.

- Bubble point pressure.

- Rock compressibility.

Key observations used by the author for history matching were GOR and water-cut.

The objective of this study is to build on previous work in order to further improve the characterization of fracture systems. The method presented here is involves simultaneous inversion of production and seismic data through an iterative, linear gradient optimization technique. A preconditioned discrete fracture model is used as the basis for forward modeling of elastic and hydraulic properties of the fracture system. Several examples of optimized data integration techniques exist in the literature. Datta-Gupta et al. ${ }^{19}$ used sensitivity coefficients computed from a streamline simulator for refinement of reservoir models with multiple data types. These researchers used linearization of the diffusivity equation in order to perform a direct inversion of pressure and saturation data for reservoir static properties. Gradient optimization methods have been used successfully by Landa, ${ }^{20}$ and Landa and Horne ${ }^{21}$ for integration of static data, including permeability fields interpreted from seismic data, with well test data. These works involved modification of a conventional simulator to compute gradients of "static" reservoir model variables. These sensitivity coefficients were passed to a linear parameter update model which used the Gauss-Newton method. In all of the above works the authors did not solve the elastic portion of the inversion problem. All of these methods used estimates of reservoir lithology or fluid saturations which had been derived (inverted) from seismic data. Avoiding the seismic inversion problem significantly simplifies the problems associated with the use of seismic data. The seismic inversion is equally as difficult as the production data inversion.

Other authors including Huang $e t$ al. $^{22,23}$ used linear gradient techniques for integration of field production data and time-lapse seismic data. Due to the integration of seismic 
data, the workflow for the new method closely resembles these time-lapse seismic integration workflows. These workflows involve parallel modeling of both hydraulic and elastic observations from tan initial model realization. As a result, these methods also solve the seismic inversion problem. The new method differs from the time-lapse seismic optimization problem in the following way. In the time-lapse seismic problem the seismic response is expected to change significantly in response to changes in reservoir pressure and saturation state. Multiple observations of these time variant seismic attributes are combined as quasi-static observations with dynamic (production) data in a numerical optimization scheme for refining the reservoir petrophysical properties. In the new method, specially designed for fractured reservoirs, the assumption is made that the anisotropic seismic attributes are functions of only the fracture system.

The new method is a scheme for integrating static anisotropic seismic attributes with production data in a numerical optimization scheme for refining fracture system trend and stochastic intensity $\mathrm{P}_{32}$. The new method starts with "raw" seismic observations as input and, hence, also solves the seismic inversion problem. Effective media models for anisotropic elasticity and directional permeability are used to forward model the hydraulic and elastic properties of discrete feature network models. Production data are generated using a dual porosity formulation. The reservoir is modeled as an undersaturated single layer oil reservoir with constant matrix properties, constant dual porosity shape factor, and zero fracture capillary pressure. For elastic modeling the matrix elastic properties are constant and normal and shear components of fracture compliance are equal and constant. 


\section{CHAPTER III}

\section{INVERSION PROCEDURE}

In this chapter we describe the inversion procedure which were used in this study to resolve fracture system trend and $\mathrm{P}_{32}$ intensity. Detailed descriptions of the following elements are provided:

- Introduction

- Least squares inversion fundamentals.

- Observation weighting.

- Observation noise.

- Computation of the Jacobian matrix of sensitivity coefficients.

- Parameter updating.

- Observation residuals.

- Application to the new method.

\subsection{Introduction}

Although least squares inversion is by construction a linear process, iterative application of the least squares method is commonly utilized to invert non-linear models. This method is generally only feasible when a small number of unknown parameters are considered, as is the case here. The iterative least squares process involves the following steps;

- Making an initial estimate or educated guess of the unknown model parameters

- Computing the value of the objective function (model) using the estimated parameter set.

- Comparing the model prediction to observed data to form a residual vector. 
- Calculating the gradients or sensitivities of the residuals with respect the unknown parameters.

- Computing linear updates to the parameter estimates which are proportional to the magnitude and sign of both the residuals and the gradients.

- Computing a new estimate of the model based on the updated parameters and repeating the process until the magnitude of the residual vector falls below an acceptable value, signifying that the model predictions fit the data.

The following sections contain a brief review of the mathematical development of the least squares method and it's application to this research topic. This method was adapted from techniques commonly used in geophysical model inversion problems ${ }^{48}$.

\subsection{Least Squares Inversion Fundamentals}

Any function can be represented by its Taylor expansion about a specified point. If a function has more than one variable then the Taylor expansion contains partial derivatives and takes the form;

$y_{i}=f_{i}\left(x_{j}^{o}\right)+\frac{1}{1 !} \sum_{j=1}^{m} \frac{\partial f_{i}}{\partial x_{j}}\left(x_{j}-x_{j}^{0}\right)+\frac{1}{2 !} \sum_{j=1}^{m} \frac{\partial f_{i}^{2}}{\partial x_{j}^{2}}\left(x_{j}-x_{j}^{0}\right)^{2}+\frac{1}{3 !} \sum_{j=1}^{m} \frac{\partial f_{i}^{3}}{\partial x_{j}^{3}}\left(x_{j}-x_{j}^{0}\right)^{3}$

where;

$$
\begin{array}{lll}
\mathrm{y}_{\mathrm{i}} & = & \text { observed data } \\
\mathrm{x}_{\mathrm{j}} & = & \text { unknown parameters } \\
\mathrm{x}_{\mathrm{j}}{ }^{\mathrm{O}} & = & \text { initial estimates of unknown parameters } \\
\mathrm{f}_{\mathrm{i}}\left(\mathrm{x}^{\mathrm{o}}\right) & = & \text { forward model of } \mathrm{y}_{\mathrm{i}} \text { using parameter set } \mathrm{x}_{\mathrm{j}}{ }^{\mathrm{O}} \\
\mathrm{n} & = & \text { number of observations } \\
\mathrm{m} & = & \text { number of unknown parameters }
\end{array}
$$

In this research program the left hand side of Eq. 3.1 represents the field observations of bottom hole pressure, oil production rate, seismic velocity modulation parameter, and 
maximum quasi-P wave velocity direction. The unknown parameter vector $\mathrm{x}_{\mathrm{j}}$ in Eq. 3.1 is made up of trend and $\mathrm{P}_{32}$ intensity of one or more fracture sets.

Truncating to first order terms and rearranging, Eq 3.1 may be written;

$$
\left(y_{i}-f_{i}\left(x_{j}^{o}\right)\right)=\left(y_{i}-y_{i}^{0}\right)=\sum_{j=1}^{m} \frac{\partial f_{i}}{\partial x_{j}}\left(x_{j}-x_{j}^{0}\right)
$$

The left hand side of Eq. 3.2 represents the vector of residual errors.

Eq. 3.2 can be written as;

$$
\Delta y=J \Delta x
$$

where;

$$
\begin{aligned}
& \mathrm{J}=\text { Jacobian or sensitivity coefficient matrix } \\
& \Delta \mathrm{x}=\text { vector of model parameter errors }
\end{aligned}
$$

When there are more independent data observations than there are unknown parameters then it is expected that the model will not fit the data and we will have a residual error such that;

$$
\Delta y_{i}-J_{i j} \Delta x_{j}=\varepsilon_{i}^{\prime}
$$

We seek to minimize the errors in a least squares sense and start by forming the squared residual function;

$$
\xi^{2}=\varepsilon_{i}^{T} \varepsilon_{i}^{\prime}=(y-J \Delta x)^{T}(y-J \Delta x)
$$


In order to impose the least squares condition on the solution to Eq. 3.5 it is necessary that;

$$
\frac{\partial \xi^{2}}{\partial x_{l}}=0
$$

It can be shown ${ }^{48}$ that for the overdetermined case where $i>j$, by substitution of Eq. 3.5 into Eq. 3.6 and expanding the derivative the following result is obtained;

$$
\Delta x=\left(J^{T} J\right)^{-1} J^{T} \Delta y
$$

\subsection{Observation Weighting}

In order to account for differences in observation type and data quality it is necessary to weight the components of vector $\Delta y$. This is typically done by dividing each term in $\Delta y$ by an estimate of the observation standard deviation, resulting in a normalized vector $\Delta y$ '. Thus, Eq. 3.3 becomes;

$$
\Delta y^{\prime}=\frac{\Delta y}{S T D_{o b s}}=\frac{J}{S T D_{o b s}} \Delta x
$$

where;

$$
\mathrm{STD}_{\mathrm{obs}}=\quad \text { the vector of observation standard deviations. }
$$

and;

$$
\Delta y^{\prime}=J^{\prime} \Delta x
$$


where;

$$
J^{\prime}=\frac{J}{S T D_{o b s}}
$$

And for the underdetermined case Eq. 3.7 becomes;

$$
\Delta x=\left(J^{\prime^{T}} J^{\prime}\right)^{-1} J^{T^{T}} \Delta y
$$

\subsection{Addition of Random Observation Noise}

In order to make the synthetic base case more representative of actual data random noise was added to each observation.

If;

$$
\mathrm{z}_{\mathrm{i}}=\text { synthetic observation }
$$

and;

$$
\begin{gathered}
\eta_{i}=N\left[0, N O I S E_{i}\right]= \\
\text { type }
\end{gathered}
$$

then in Eq. 3.2;

$$
y_{i}=z_{i}+\eta_{i}
$$

\subsection{Computation of the Jacobian Matrix}

For a single fracture set the Jacobian matrix in Eq. 3.7 takes the form; 


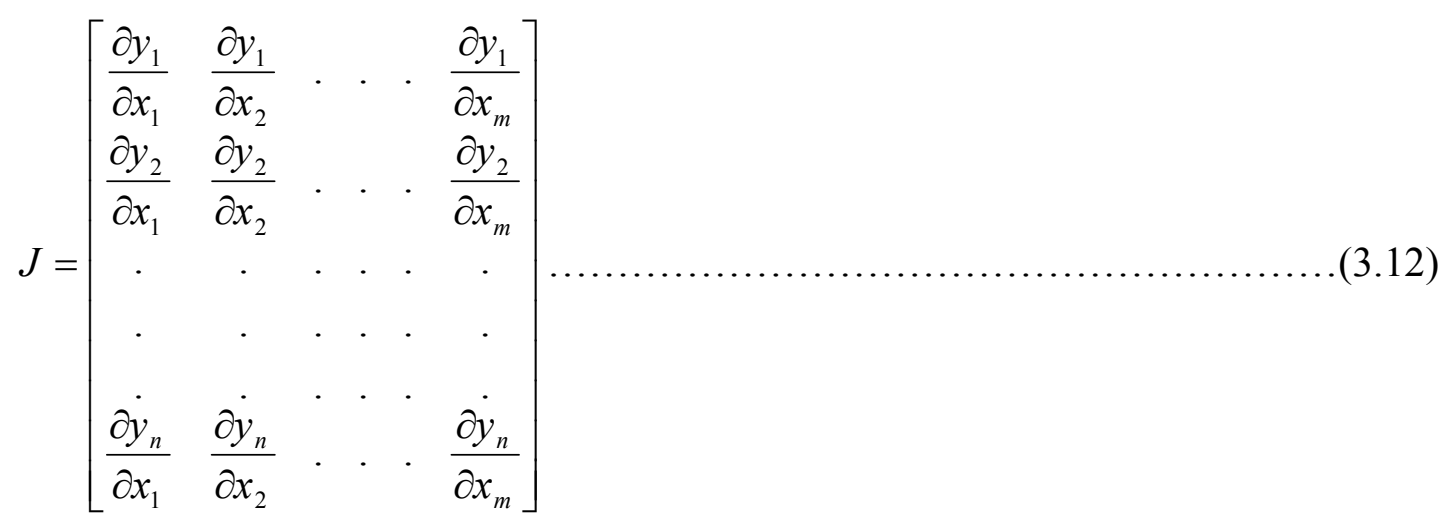

If an analytical derivative is not available it is necessary to compute the Jacobian matrix components through forward modeling. Derivatives for each observation with respect to unknown model parameters can be computed at each iteration in the inversion by systematically perturbing each unknown parameter one at a time and computing the entire set of observations with the current model parameter set and the perturbed parameter set.

Then the Jacobian terms are computed as;

$J_{i j}=\frac{\partial\left(y_{i}\right)}{\partial\left(x_{j}\right)} \approx \frac{y_{i}\left(x_{j}+\Delta x_{j}\right)-y_{i}\left(x_{j}\right)}{\Delta x_{j}}$

where;

$$
\begin{array}{lll}
\mathrm{y}_{\mathrm{i}}\left(\mathrm{x}_{\mathrm{j}}\right) & = & \text { modeled } \mathrm{i}^{\text {th }} \text { observation at current parameter set } \\
\mathrm{y}_{\mathrm{i}}\left(\mathrm{x}_{\mathrm{j}}+\Delta \mathrm{x}_{\mathrm{j}}\right) & = & \text { modeled } \mathrm{i}^{\text {th }} \text { observation at perturbed parameter set } \\
\Delta \mathrm{x}_{\mathrm{j}} & = & \mathrm{j}^{\text {th }} \text { parameter perturbation }
\end{array}
$$

\subsection{Parameter Updating}

The parameter error vector takes the form;

$$
\Delta x=\left[x_{1}^{n e w}-x_{1}^{i n i t}, x_{2}^{n e w}-x_{2}^{i n i t}, \ldots . ., x_{m}^{n e w}-x_{m}^{i n i t}\right]
$$


where;

$$
\begin{array}{lll}
\Delta \mathrm{x} & = & \text { vector of parameter differences } \\
\mathrm{x}_{\mathrm{m}}{ }^{\text {init }} & = & \text { initial estimate for the } \mathrm{i}^{\text {th }} \text { parameter } \\
\mathrm{x}_{\mathrm{m}}{ }^{\text {new }} & = & \text { adjusted estimate for the } \mathrm{m}^{\text {th }} \text { parameter }
\end{array}
$$

Thus, parameter updates are computed as;

$$
x_{m}^{\text {new }}=\Delta x_{1}+x_{m}^{\text {init }}
$$

\subsection{Observation Residuals}

Convergence of the solution for unknown model parameters may be quantified after each iteration by means of evaluating the root mean residual error terms. These root mean residuals were computed as;

$$
R^{\prime}=S Q R T\left\{\sum_{i=f o b s}^{l o b s}\left(y_{i}^{o}-y_{i}^{*}\right)^{2}\right\}
$$

where;

$$
\begin{array}{lll}
\mathrm{y}_{\mathrm{i}}{ }^{*} & = & \text { Computed value at current model parameter state. } \\
\text { fobs } & = & \text { first observation type } \\
\text { lobs } & = & \text { last observation type }
\end{array}
$$

\subsection{Application to the New Method}

\subsubsection{Forward Modeling of Observations}

The observation set chosen for optimization includes both production performance (bottom hole pressures and oil production rates) and quasi-P wave velocity parameters (quasi-P wave velocity modulation amplitude and azimuth of maximum quasi-P wave velocity). Each DFN model generated using the new method was discretized into 2dimensional $\mathrm{x}-\mathrm{y}$ grids for computation of both production observations and seismic 
velocities. For this research program it was assumed that all fractures contribute to both flow and the elastic properties according to the models described in Chapter II. Fracture permeability for each grid block was computed using Oda's method. Grid corner point geometry, matrix and fracture porosity and permeability were generated using Eclipse FloGrid. Fluid properties and relative permeabilities were taken from Kazemi ${ }^{25}$. Production observations were computed using a finite difference dual porosity simulator with the upscaled fracture permeability. The following assumptions and simplifications were made in performing all flow simulations;

- constant matrix porosity and permeability.

- constant fracture porosity.

- constant reservoir thickness.

- constant dual porosity shape factor.

- zero fracture capillary pressure.

All simulations were run for a 600 day duration with twenty 30 day report steps. Pressure, injection, and production summary vectors were also written to summary files. Fracture and matrix water saturation maps were created using Eclipse FloViz at selected report steps for visualization and inclusion in Appendices B, C, and D of this dissertation. Bottom hole pressure and oil production rate vectors were extracted from summary files. Bottom hole pressures and oil production rates were averaged over the duration of simulation time for each individual well.

Elastic moduli were computed for each grid block using the linear slip discontinuity formulation. For the purpose of computing quasi-P wave phase velocity a two layer elastic model was defined with an isotropic layer over the anisotropic fractured layer. Quasi-P wave phase velocities for the anisotropic medium were computed for each grid cell using Matlab and a FORTRAN executable. Phase velocities for the anisotropic layer were computed for all azimuths and all angles of incidence at the interface between the 
two layers. The overburden layer was used to allow selection of quasi-P wave velocity gathers based on angle of incidence as would be the case for the process of sorting angle gathers in seismic processing. For the selected angle of incidence the quasi-P wave velocity versus azimuth data were fitted to determine velocity modulation amplitude B, and azimuth of maximum quasi-P wave velocity $\phi_{\mathrm{qpv}}$ for each block. These values were averaged over all blocks for the objective function (Eq. 3.17). Seismic velocity displays in this chapter show quasi-P wave phase velocity versus azimuth for all blocks before averaging. Since the fracture trend and $\mathrm{P}_{32}$ distributions are stationary and have infinite spatial correlation length it is felt that this averaging process does not significantly degrade the information content in the quasi-P wave velocity observations. For fracture distributions which have correlation length on the order of the reservoir model dimensions or smaller this assumption may not be sufficiently accurate. More detailed descriptions of the methods used for forward modeling of these observations are provided in Appendix A of this dissertation.

\subsubsection{Objective Function}

The observations listed in Table 3.8.1 were combined to form an objective function for minimization (Eq. 3.17) which is equivalent to the total residual in Eq. 3.5. The production and injection time series were averaged over the duration of the simulation for each well. Seismic observations were averaged over the entire active 2D map extent.

$$
\begin{aligned}
& Q=\sum_{i=1}^{n w}\left(\operatorname{avg}\left(B H P^{o}\right)-\operatorname{avg}\left(B H P_{i}^{c}\right)\right)^{2}+\sum_{i=1}^{n w}\left(\operatorname{avg}\left(O P R_{i}^{o}\right)-\operatorname{avg}\left(O P R_{i}^{c}\right)\right)^{2} \\
& +\left(\operatorname{avg}\left(B^{\prime o}\right)-\operatorname{avg}\left(B^{\prime c}\right)\right)^{2}+\left(\operatorname{avg}\left(\varphi_{q p v}^{o}\right)-\operatorname{avg}\left(\varphi_{q p v}^{c}\right)\right)^{2}
\end{aligned}
$$

where;

$$
\begin{array}{lll}
\mathrm{BHP}= & \text { bottom-hole pressure }(\mathrm{psi}) \\
\mathrm{OPR}= & \text { oil production rate }(\mathrm{STB} / \mathrm{D}) \\
\mathrm{nw} & = & \text { number of wells }
\end{array}
$$




$$
\begin{array}{lll}
\phi_{\mathrm{qpv}} & = & \text { maximum quasi-P wave velocity azimuth } \\
\mathrm{B}^{\prime} & = & \text { velocity modulation amplitude } \\
\mathrm{c} & = & \text { computed value } \\
\mathrm{o} & = & \text { observed value }
\end{array}
$$

Table 3.8.1 - Observation set for optimization.

\begin{tabular}{|c|l|}
\hline $\begin{array}{c}\text { Observation } \\
\text { Number }\end{array}$ & \multicolumn{1}{c|}{$\begin{array}{c}\text { Observation } \\
\text { Name }\end{array}$} \\
\hline $\mathbf{1}$ & BHP - Injector \\
\hline $\mathbf{2}$ & $\mathrm{BHP}-\mathrm{P} 1$ \\
\hline $\mathbf{3}$ & $\mathrm{BHP}-\mathrm{P} 2$ \\
\hline $\mathbf{4}$ & $\mathrm{BHP}-\mathrm{P} 3$ \\
\hline $\mathbf{5}$ & $\mathrm{BHP}-\mathrm{P} 4$ \\
\hline $\mathbf{6}$ & $\mathrm{OPR}-\mathrm{P} 1$ \\
\hline $\mathbf{7}$ & OPR $-\mathrm{P} 2$ \\
\hline $\mathbf{8}$ & OPR $-\mathrm{P} 3$ \\
\hline $\mathbf{9}$ & OPR $-\mathrm{P} 4$ \\
\hline $\mathbf{1 0}$ & $\mathrm{B}$ \\
\hline $\mathbf{1 1}$ & $\phi_{\mathrm{apv}}$ \\
\hline
\end{tabular}

Referring to table 3.8.1, in this inversion problem the vector $\Delta y$ takes the form;

$$
\Delta y=\left[\begin{array}{c}
\operatorname{avg}\left(B H P_{P 1}^{o}\right)-\operatorname{avg}\left(B H P_{P 1}^{c}\right) \\
\operatorname{avg}\left(B H P_{P 2}^{o}\right)-\operatorname{avg}\left(B H P_{P 2}^{c}\right) \\
\operatorname{avg}\left(B H P_{P 3}^{o}\right)-\operatorname{avg}\left(B H P_{P 3}^{c}\right) \\
\operatorname{avg}\left(B H P_{P 4}^{o}\right)-\operatorname{avg}\left(B H P_{P 4}^{c}\right) \\
\operatorname{avg}\left(B H P_{I}^{o}\right)-\operatorname{avg}\left(B H P_{I}^{c}\right) \\
\operatorname{avg}\left(O P R_{P 2}^{o}\right)-\operatorname{avg}\left(O P R_{P 2}^{c}\right) \\
\operatorname{avg}\left(O P R_{P 2}^{o}\right)-\operatorname{avg}\left(O P R_{P 2}^{c}\right) \\
\operatorname{avg}\left(O P R_{P 3}^{o}\right)-\operatorname{avg}\left(O P R_{P 3}^{c}\right) \\
\operatorname{avg}\left(O P R_{P 4}^{o}\right)-\operatorname{avg}\left(O P R_{P 4}^{c}\right) \\
\operatorname{avg}\left(B^{\prime o}\right)-\operatorname{avg} B\left(B^{\prime c}\right) \\
\operatorname{avg}\left(\varphi_{q p v}^{o}\right)-\operatorname{avg}\left(\varphi_{q p v}^{c}\right)
\end{array}\right]
$$

where;

$$
\mathrm{BHP}=\text { bottom-hole pressure }(\mathrm{psi})
$$




$$
\begin{array}{lll}
\mathrm{OPR} & = & \text { oil production rate }(\mathrm{STB} / \mathrm{D}) \\
\phi_{\mathrm{qpv}} & = & \text { maximum quasi-P wave velocity azimuth } \\
\mathrm{B} & = & \text { velocity modulation amplitude } \\
\mathrm{c} & = & \text { computed value } \\
\mathrm{o} & = & \text { observed value }
\end{array}
$$

\subsubsection{Random Noise}

Noise estimates for the types of observations used in the new method are highly subjective. Values used in this research were determined from various sources. Estimates of noise for production observations, oil production rate and bottom hole pressure, were obtained through personal communication with engineers and are highly dependent on the type and condition of the gauges and measurement techniques used. The noise in maximum quasi-P wave velocity direction is related to the number of azimuth groups available for the velocity analysis to define the sinusoidal velocity relationship. The estimate used in this research was obtained by personal communication with practitioners. The noise estimate for the amplitude modulation factor was calculated as a small percentage $(5-10 \%)$ of the mean of the predicted data. These noise components were sampled from a normal distribution with zero mean and standard deviation equal to the individual observation noise estimates.

\subsubsection{Computation of the Jacobian Matrix - Single Fracture Set}

The Jacobian matrix in Eq. 3.7 takes the form; 
$J=\left[\begin{array}{cc}\frac{\partial\left(o b s_{1}\right)}{\partial P_{32}} & \frac{\partial\left(\text { obs }_{1}\right)}{\partial \text { Trend }} \\ \frac{\partial\left(o b s_{2}\right)}{\partial P_{32}} & \frac{\partial\left(\text { obs }_{2}\right)}{\partial \text { Trend }} \\ \cdot & \cdot \\ \cdot & \cdot \\ \frac{\partial\left(o b s_{11}\right)}{\partial P_{32}} & \frac{\partial\left(o \dot{b}_{11}\right)}{\partial \text { Trend }}\end{array}\right]$

where;

$$
\mathrm{obs}_{\mathrm{i}}=\quad \mathrm{i}^{\text {th }} \text { observed data type }
$$

\subsubsection{Parameter Updates}

The parameter error vector takes the form;

$$
\Delta x=\left[P_{32}^{n e w}-P_{32}^{\text {init }}, \text { Trend }^{\text {new }}-\text { Trend }^{\text {init }}\right]
$$

where;

$$
\begin{array}{lll}
\Delta \mathrm{x} & = & \text { vector of parameter differences } \\
\text { init } & = & \text { initial parameter estimate } \\
\text { new } & = & \text { adjusted parameter }
\end{array}
$$

Thus, parameter updates are computed as;

$$
\begin{aligned}
& P_{32}^{\text {new }}=\Delta x_{1}+P_{32}^{\text {init }} \\
& \text { Trend }^{\text {new }}=\Delta x_{2}+\text { Trend }^{\text {init }}
\end{aligned}
$$

\subsubsection{Observation Residuals}


Convergence of the solution for model parameters $x_{j}$ was quantified after each iteration by means of evaluating the root mean residual errors (Eq. 3.16). This error estimate was computed observation over all observations, and also separated by observation type for bottom hole pressure (all wells), oil production rate (all wells), and seismic (both observations). These root mean residuals were computed as;

$$
\begin{aligned}
& R_{\text {TOTAL }}^{\prime}=\operatorname{SQRT}\left\{\sum_{i=1}^{5}\left(y_{i}^{o}-y_{i}^{*}\right)^{2}\right\} \\
& R_{\text {BHP }}^{\prime}=\operatorname{SQRT}\left\{\sum_{i=1}^{5}\left(y_{i}^{o}-y_{i}^{*}\right)^{2}\right\} \ldots \\
& R_{\text {OPR }}^{\prime}=\operatorname{SQRT}\left\{\sum_{i=6}^{9}\left(y_{i}^{o}-y_{i}^{*}\right)^{2}\right\} \ldots \\
& R_{\text {SEIS }}^{{ }^{2}}=\operatorname{SQRT}\left\{\sum_{i=10}^{11}\left(y_{i}^{o}-y_{i}^{*}\right)^{2}\right\} .
\end{aligned}
$$

where;

$$
\mathrm{y}_{\mathrm{i}}^{*}=\text { Computed value at current model parameter state. }
$$

\subsubsection{Application to Multiple Fracture Sets}

Application of this solution method to three synthetic test cases is presented in Chapter IV. Two of the synthetic test cases are systems with multiple fracture sets. Extension of this formulation for multiple fracture sets is easily accomplished by increasing number of parameters $m$ in Eq. 3.1 to include terms for the additional fracture system trend and $\mathrm{P}_{32}$ parameters. This results in the extension of the sensitivity matrix in Eq. 3.19 with additional columns of sensitivity coefficients the second fracture set parameters, and extending vector $\Delta \mathrm{x}$ in Eq. 3.20 to include initial parameter estimates for the second fracture set as follows;

$$
\Delta x=\left(P_{32}^{1}-P_{32}^{\text {init } 1}, \text { Trend }^{1}-\text { Trend }^{\text {init } 1}, P_{32}^{2}-P_{32}^{\text {init } 2}, \text { Trend }^{2}-\text { Trend }^{\text {init } 2}\right)
$$


Fracture set parameter updates are computed through the following revision of Eqs. 3.21.

$$
\begin{aligned}
& P_{32}^{\text {new } 1}=\Delta x_{1}+P_{32}^{\text {init } 1} \\
& \text { Trend }^{\text {new } 1}=\Delta x_{2}+\text { Trend }^{\text {init } 1} \\
& P_{32}^{\text {new } 2}=\Delta x_{3}+P_{32}^{\text {init } 2} \\
& \text { Trend }^{\text {new } 2}=\Delta x_{4}+\text { Trend }^{\text {init } 2}
\end{aligned}
$$

\subsubsection{Starting Models}

In practice initial estimates for the trend and $\mathrm{P}_{32}$ intensity of the fracture system(s) will be well constrained at the boreholes through analysis of wireline, core, and other data. Given the assumptions of stationarity and long spatial correlation lengths in trend and $\mathrm{P}_{32}$, the error in these initial estimates should be limited to the error in these assumptions and the effect of finite sampling of the populations for these parameters. Errors in the initial estimates used in the test cases conducted using the new method are felt to be sufficiently large to represent realistic scenarios, and in part, compensate for the simplicity of the models tested. 


\section{CHAPTER IV}

\section{TEST CASES}

In this chapter we present the results of application of the new method to three cases representing different fracture population characteristics and host medium (reservoir rock) lithological conditions. These are:

- Test T2 - Single fracture set, moderate permeability, "tight sandstone" host medium elastic properties.

- Test T3 - Two fracture sets, moderate permeability, "tight sandstone" host medium elastic properties.

- Test T4 - Two fracture sets, low permeability, dolomite host medium elastic properties.

Variations in reservoir types are defined through the seismic compressional wave and shear wave velocities, bulk density, porosity, and permeability of the host rock (matrix) in elastic modeling and reservoir simulation. A summary of the results of test cases T2, T3, and T4 will be presented in this chapter. These results will be presented in the following form:

- Tables and graphs of trend and $\mathrm{P}_{32}$ intensity history.

- Tables and graphs of objective function and residual error values.

- Graphs of sensitivity coefficients.

- Maps of the fracture system geometry for the base model, initial estimate, and final optimized model.

- Maps of water saturation in the fracture system at selected simulator report steps for the base model, initial estimate, and final optimized model. 
- Plots of azimuthally dependent seismic velocity for the base model, initial estimate, and final optimized model.

In order to demonstrate the value of the new method, as part of tests T2 and T3 a similar optimization technique was performed without elastic observations in the objective function. These results are presented and discussed within the discussion of tests T2 and T3. Test T1 was a preliminary trial using single porosity simulation and is not reported here.

\subsection{Base Case Reservoir Model and Production Configuration}

Reservoir Model; The reservoir model used as a base case for testing the new procedure consisted of a single $2560 \mathrm{ft} \times 2560 \mathrm{ft} \times 100 \mathrm{ft}$ thick interval with homogeneous host matrix properties and either 1 or 2 fracture sets. Petrophysical and elastic properties of the host reservoir rock were selected to conform roughly with various rock types for typical fractured reservoirs ${ }^{49}$. Fracture systems used in these tests were spatially stationary with respect to both geometry and hydrologic properties (i.e. the population statistics did not vary with location in the reservoir). The specific host matrix and fracture system properties used in each test will be presented along with test results in the following sections.

Simulator Grid and Production Configuration; For flow simulation a single layer corner point grid was constructed with block size $10 \mathrm{ft}$ x $10 \mathrm{ft}$ x $100 \mathrm{ft}$. The reservoir was undersaturated, with fluid properties, relative permeabilities, and capillary data taken from Kazemi ${ }^{25}$. A single production configuration was used for all three test cases. This configuration consisted of a five spot pattern on a 20 acre spacing with a center water injector (Figs. 4.1a and 4.1b). In order to overcome instabilities associated with lateral variations in fracture system permeability at the small block size chosen, wells were completed in six consecutive reservoir blocks in a direction perpendicular to fracture trend. In an effort to maintain constant reservoir pressure, all wells were set on liquid 
rate control, with injection and total field production rates selected to maintain close to zero net voidage. No aquifer support was provided.

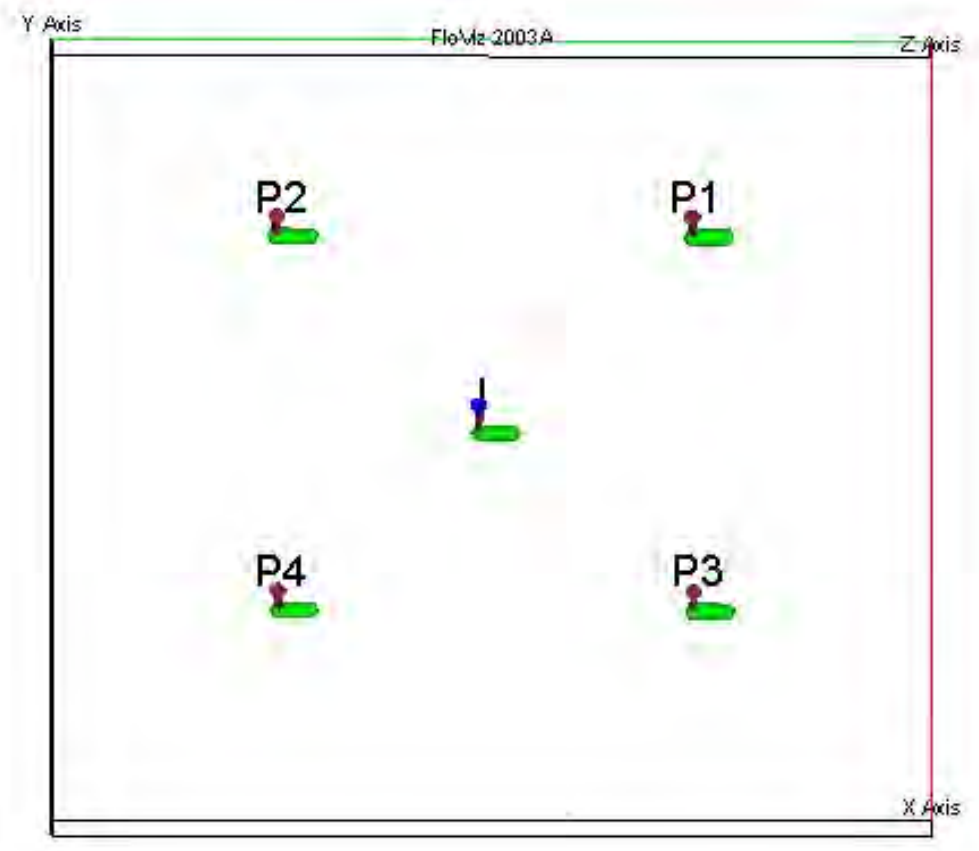

Fig. 4.1a - Top view of production configuration for optimization test cases.

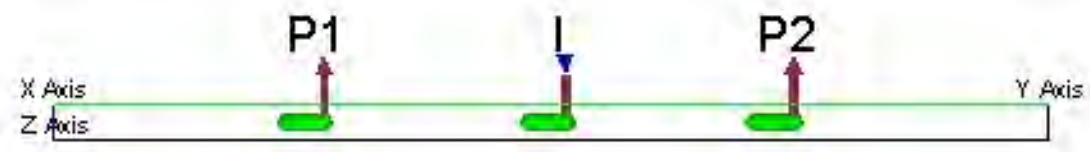

Fig. 4.1b - Side view of production configuration for optimization test cases.

\subsection{Test $\mathbf{T} 2$ - Single Fracture Set in a Tight Sandstone Reservoir}

The first test of the new method was conducted on a reservoir model with a single fracture set having the population statistics listed in Table 4.1. Petrophysical and elastic reservoir properties are listed in Tables 4.2 and 4.3. In order to test the effect of including seismic anisotropy in the objective function a parallel test was performed 
using only production data in the objective function. These tests with and without seismic anisotropy are referred to as "combined" and "production only" respectively in the following summary Tables and Figures.

Table 4.1 - Test T2 base model fracture distribution parameters.

\begin{tabular}{|l|c|}
\hline \multicolumn{1}{|c|}{ Parameter } & Set 1 \\
\hline Trend & Normal $[0,5]$ \\
\hline $\mathbf{P}_{\mathbf{3 2}}$ Intensity (1/m) & 0.1 \\
\hline Length (m) & Lognormal[50,3] \\
\hline Height (m) & 60 \\
\hline Transmissivity $\left(\mathbf{m}^{\mathbf{2}} / \mathbf{s}\right)$ & $8 \mathrm{e}-04$ \\
\hline
\end{tabular}

Table 4.2 - Test $\mathrm{T} 2$ reservoir properties.

\begin{tabular}{|l|c|}
\hline Matrix Porosity & 0.10 \\
\hline Matrix Permeability & $10 \mathrm{mD}$ \\
\hline Fracture Porosity & 0.015 \\
\hline
\end{tabular}

Table 4.3 - Test T2 elastic modeling parameters.

\begin{tabular}{|l|c|}
\hline Matrix P-wave Velocity & $4670 \mathrm{ft} / \mathrm{sec}$ \\
\hline Matrix Shear-wave Velocity & $3060 \mathrm{ft} / \mathrm{sec}$ \\
\hline Matrix Density & $2510 \mathrm{~kg} / \mathrm{m}^{3}$ \\
\hline Overburden P wave Velocity & $4670 \mathrm{ft} / \mathrm{sec}$ \\
\hline Overburden S wave Velocity & $3060 \mathrm{ft} / \mathrm{sec}$ \\
\hline Overburden Density & $2510 \mathrm{~kg} / \mathrm{m}^{3}$ \\
\hline
\end{tabular}

\subsubsection{Parameter Updates}

Parameter error history for this optimization test is listed in Table 4.4 and shown in Figs. 4.2 and 4.3. Parameter perturbations and a priori model weights for each iteration are listed in Tables B.5 and B.6 in Appendix B. The initial estimate of fracture trend was 30 
degrees away from the base model trend of 0 degrees. The initial estimate of $\mathrm{P}_{32}$ intensity was 0.15 , which was 0.05 away from the base model value of 0.2 . Fig. 4.2 depicting the $\mathrm{P}_{32}$ intensity error shows a monotonic, almost linear convergence from the initial error of 0.05 toward 0.002 using the combined objective function. The production only $\mathrm{P}_{32}$ error also converges linearly, but less rapidly. Fig. 4.3 depicting the trend error also shows monotonic convergence from the initial estimate of 30 degrees to approximately 3 degrees for the combined objective function. This convergence is rapid during the first few iteration steps and then slows during later iterations. The convergence of trend for the production only objective function follows the same trend but slightly lags the combined objective function result.

Table 4.4 - $\quad$ Summary of absolute errors for fracture set trend and $\mathrm{P}_{32}$ intensity at each update step for both production data only and combined data tests. Step 0 is the initial estimate.

\begin{tabular}{|r|r|r|r|r|}
\cline { 2 - 5 } \multicolumn{1}{c|}{} & \multicolumn{3}{c|}{ Parameter Error } \\
\cline { 2 - 5 } \multicolumn{1}{c|}{ Production Only } & \multicolumn{2}{c|}{ Combined } \\
\hline Iteration & Trend & \multicolumn{1}{c|}{ P32 } & Trend & \multicolumn{1}{c|}{ P32 } \\
\hline $\mathbf{0}$ & 30.0 & 0.050 & 30.0 & 0.050 \\
\hline $\mathbf{1}$ & 23.1 & 0.038 & 19.8 & 0.034 \\
\hline $\mathbf{2}$ & 9.3 & 0.032 & 8.7 & 0.022 \\
\hline $\mathbf{3}$ & 7.4 & 0.022 & 5.9 & 0.009 \\
\hline $\mathbf{4}$ & 5.9 & 0.016 & 4.0 & 0.001 \\
\hline $\mathbf{5}$ & 5.0 & 0.008 & 2.7 & 0.002 \\
\hline
\end{tabular}




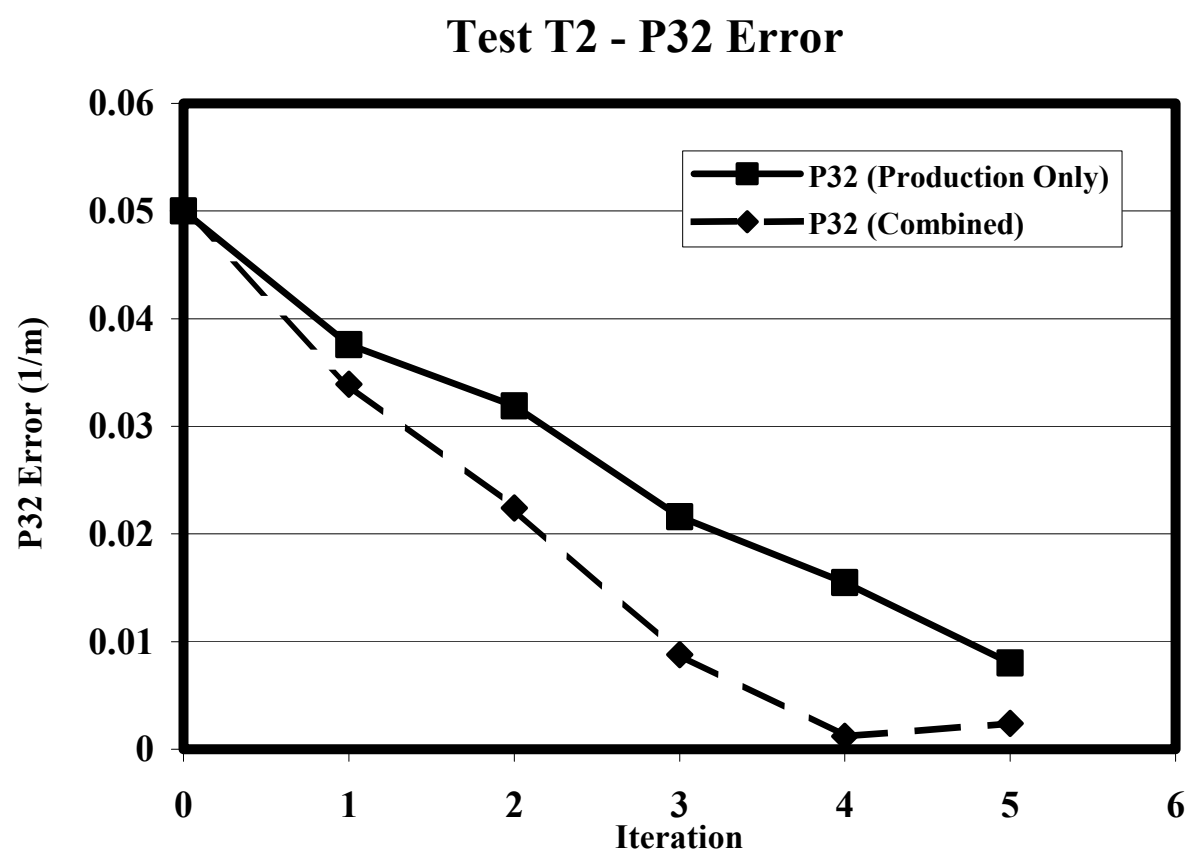

Fig. 4.2 - Graph of absolute error in fracture set $\mathrm{P}_{32}$ intensity versus iteration for test $\mathrm{T} 2$. Iteration 0 is the initial estimate.

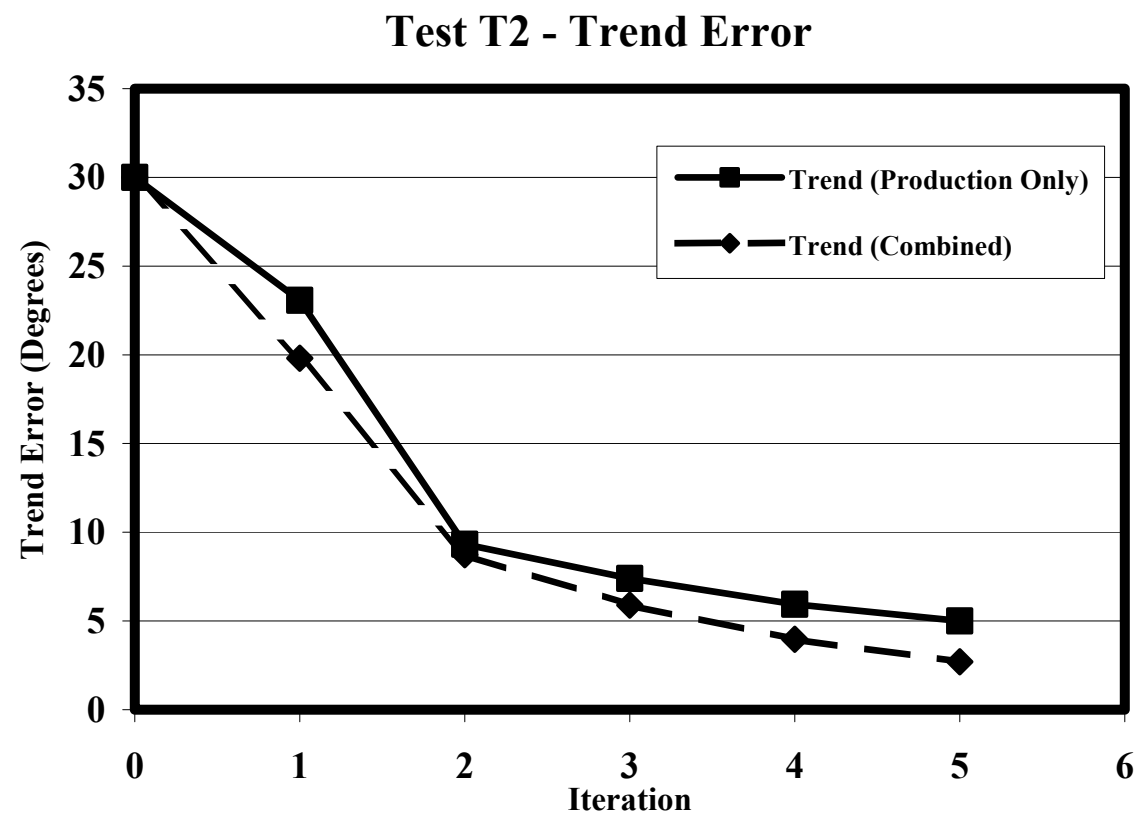

Fig. 4.3 - Graph of absolute error in fracture set trend versus iteration for test T2. Iteration 0 is the initial estimate. 


\subsubsection{Objective Function and Observation Residuals}

In order to stabilize parameter updates in the presence of noisy data, ten parameter updates were computed at each iteration step. Each of these parameter updates used independent samples of random noise. The results of these 10 updates at each iteration step were averaged. The average objective function and observation type residuals computed using the combined objective function are listed in Table 4.5 and illustrated in Fig. 4.4. The objective function (Eq. 3.17) and partial residuals for bottom hole pressure, oil production rate, and seismic observations (Eq. 3.23 through 3.25) are shown separately. All residuals are listed in Table B.8 in Appendix B. It can be seen that error in the initial model estimate of bottom hole pressure is much higher than that for oil production rate or seismic observations. The behavior of the bottom hole pressure residual follows the same trend as the convergence of fracture system trend, suggesting a strong inter-dependence. The seismic residual is between bottom hole pressure and oil production rate, and exhibits a monotonic decrease throughout the optimization procedure. The model is less sensitive to oil production rate because the simulations are controlled by liquid production rate. As a result, oil production rate is assured to be constant until the time of water breakthrough. Therefore, the only part of the oil production observation contributing information to the inversion is that occurring after breakthrough.

Table 4.5 $\quad-\quad$ Summary of objective function and normalized residual errors for fracture set trend and $\mathrm{P}_{32}$ intensity at each update step using the combined objective function. Step 0 is initial the estimate.

\begin{tabular}{|r|r|r|r|r|}
\cline { 3 - 5 } \multicolumn{2}{c|}{} & \multicolumn{3}{c|}{ RMS Residuals } \\
\hline Iteration & Q & \multicolumn{1}{c|}{ BHP } & \multicolumn{1}{c|}{ OPR } & \multicolumn{1}{c|}{ SEIS } \\
\hline $\mathbf{0}$ & 778.4 & 26.410 & 5.2 & 7.250 \\
\hline $\mathbf{1}$ & 432.6 & 20.240 & 2.5 & 4.750 \\
\hline $\mathbf{2}$ & 37.2 & 5.390 & 1.2 & 2.490 \\
\hline $\mathbf{3}$ & 11.6 & 2.656 & 1.9 & 1.400 \\
\hline $\mathbf{4}$ & 11.0 & 2.630 & 1.8 & 0.820 \\
\hline
\end{tabular}




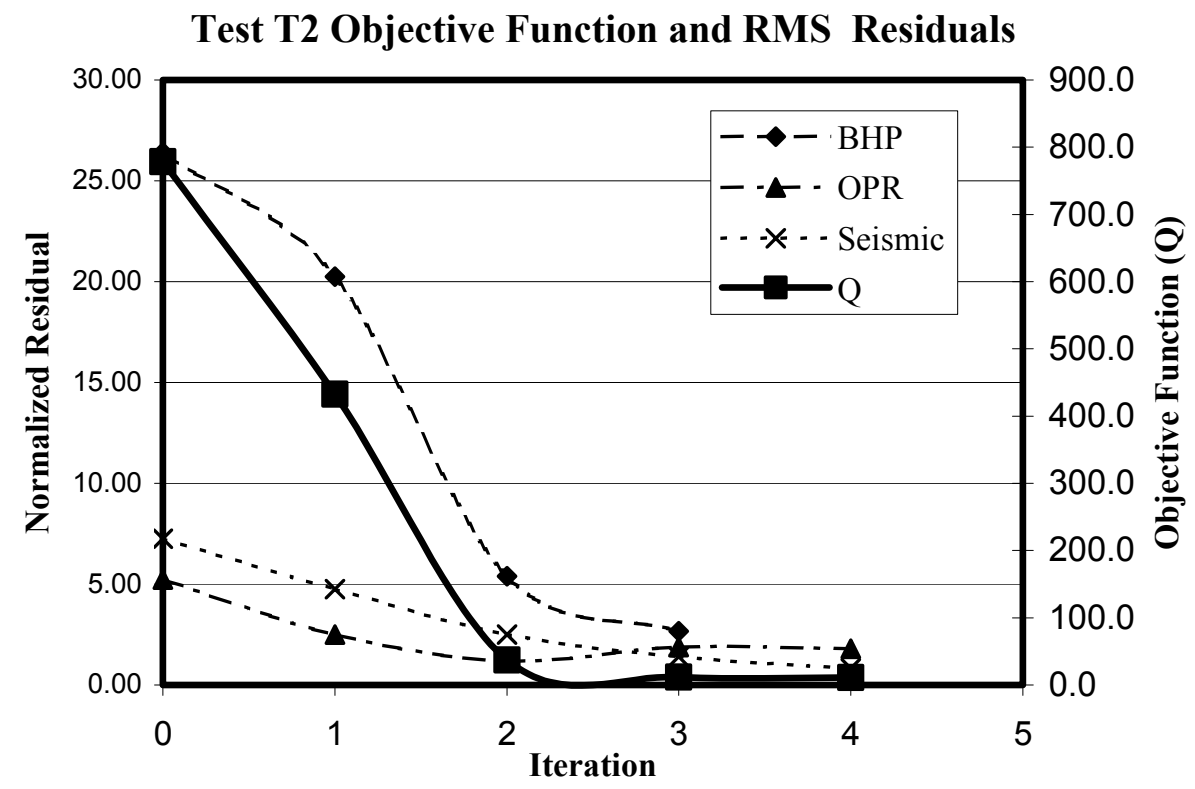

Fig. 4.4 - Graph of objective function and normalized residual errors by observation type for fracture set trend and $\mathrm{P}_{32}$ intensity at each iteration using the combined objective function. Iteration 0 is the initial estimate.

\subsubsection{Sensitivity Coefficients}

Fig. 4.5 shows a sample of Jacobian matrix sensitivity coefficients computed using Eq. 3.14 for the initial estimate model state of the single fracture set optimization test. Table 4.6 contains a list of observation numbers and descriptions. These sensitivity coefficients are the gradients of the observations with respect to the unknown parameters and carry information regarding the relative contribution of each observation to the parameter update. Observations with large positive or negative sensitivity coefficients will have a larger impact on the parameter update than those with lower sensitivity coefficients. The sample sensitivity coefficients in Fig. 4.5 show the sensitivity of both trend and $\mathrm{P}_{32}$ intensity to the bottom hole pressure observations for all wells. All sensitivity coefficients for the combined objective function test are listed shown in Figs. B.15a to B.15e in Appendix B. These data also confirm the sensitivity of $\mathrm{P}_{32}$ intensity to the velocity modulation amplitude and sensitivity of trend to maximum quasi-P wave velocity direction. 
Table 4.6 $\quad-\quad$ Observation numbers and descriptions.

\begin{tabular}{|c|l|}
\hline $\begin{array}{c}\text { Observation } \\
\text { Number }\end{array}$ & \multicolumn{1}{c|}{$\begin{array}{c}\text { Observation } \\
\text { Name }\end{array}$} \\
\hline $\mathbf{1}$ & $\mathrm{BHP}-$ Injector \\
\hline $\mathbf{2}$ & $\mathrm{BHP}-\mathrm{P} 1$ \\
\hline $\mathbf{3}$ & $\mathrm{BHP}-\mathrm{P} 2$ \\
\hline $\mathbf{4}$ & $\mathrm{BHP}-\mathrm{P} 3$ \\
\hline $\mathbf{5}$ & $\mathrm{BHP}-\mathrm{P} 4$ \\
\hline $\mathbf{6}$ & $\mathrm{OPR}-\mathrm{P} 1$ \\
\hline $\mathbf{7}$ & $\mathrm{OPR}-\mathrm{P} 2$ \\
\hline $\mathbf{8}$ & $\mathrm{OPR}-\mathrm{P} 3$ \\
\hline $\mathbf{9}$ & $\mathrm{OPR}-\mathrm{P} 4$ \\
\hline $\mathbf{1 0}$ & $\mathrm{B}$ \\
\hline $\mathbf{1 1}$ & $\phi_{\mathrm{qpv}}$ \\
\hline
\end{tabular}
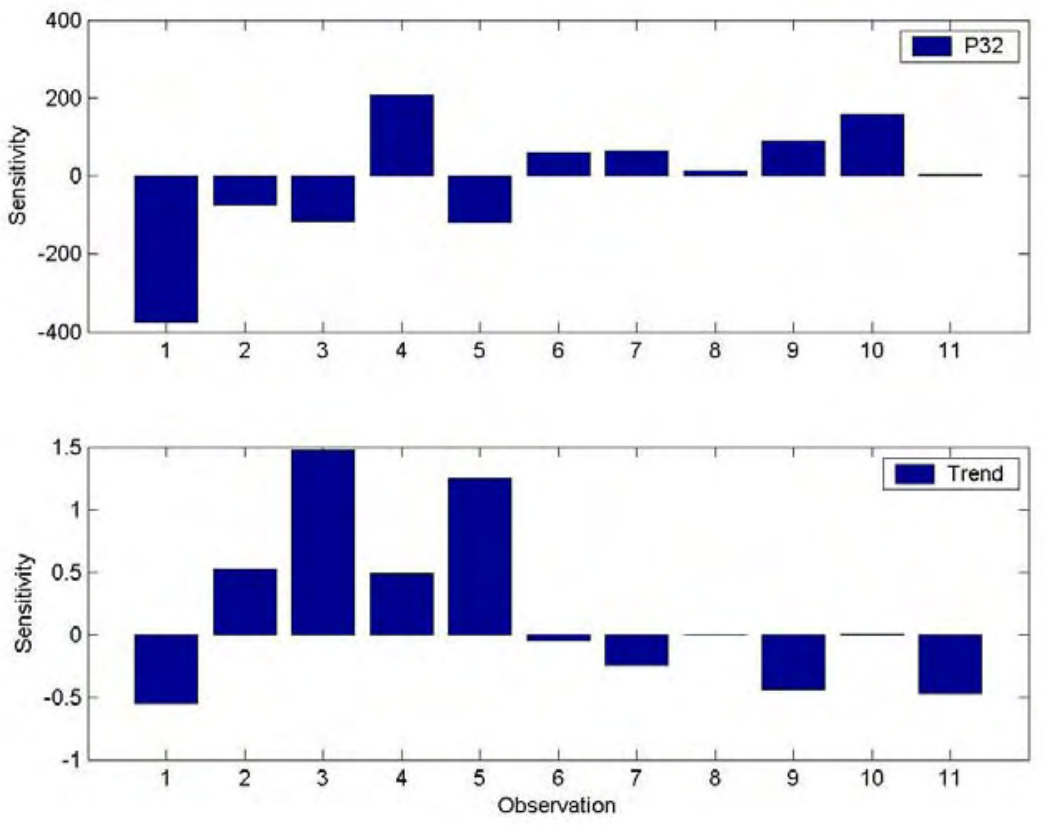

Fig. 4.5 - Parameter sensitivity coefficients by observation number for the single fracture set initial model case $\mathrm{T} 2$. 


\subsubsection{Fracture Distribution Maps}

Maps of the fracture system and selected observation sets taken from the base case, initial estimate, and optimized model are shown in Figs. 4.6 to 4.9. These figures show aerial views of 3-dimensional fracture models with fracture faces shaded in blue. Fracture density is aerially uniform and all fractures are vertical. The apparent systematic variation in fracture dip and density in these figures are caused by the viewing perspective. Figs. 4.6 to 4.9 illustrate the fracture distribution for the base model (Fig. 4.6), the initial model estimate (Fig. 4.7), optimized model using the combined data objective function (Fig. 4.8), and the optimized model using the production data only objective function (Fig. 4.9). Comparison of Figs. 4.7 and 4.8 clearly illustrates the success of the new method in optimizing fracture trend and $\mathrm{P}_{32}$ intensity for this model using the combined data objective function. The improvement in resolution of fracture trend using the combined data objective function as opposed to the production data only objective function may also detected in the slight difference in fracture trend between the distributions in Figs. 4.8 and 4.9.

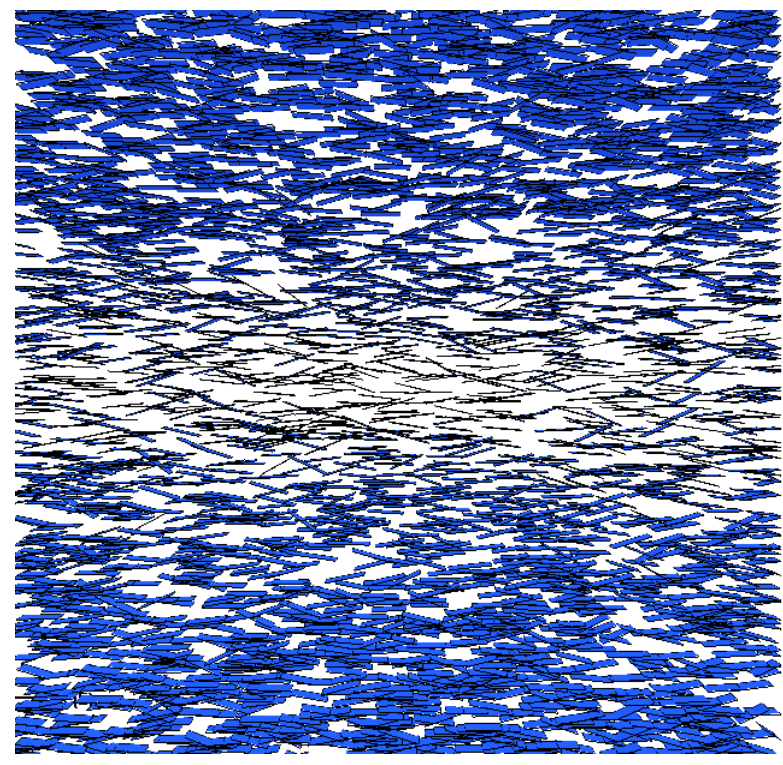

Fig. 4.6 - $\quad$ Test T2 base fracture model 


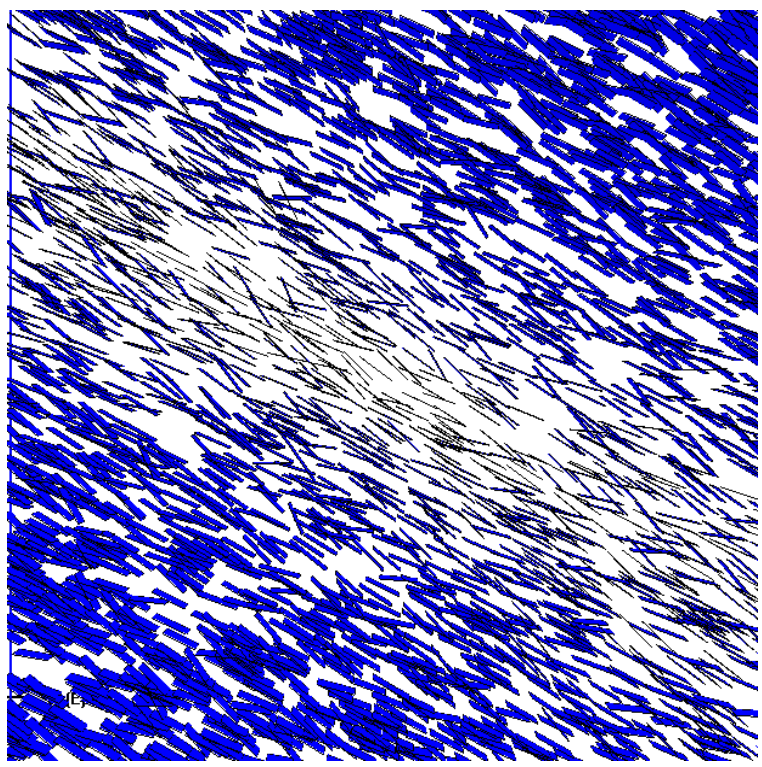

Fig. 4.7 - Test T2 initial fracture system estimate.

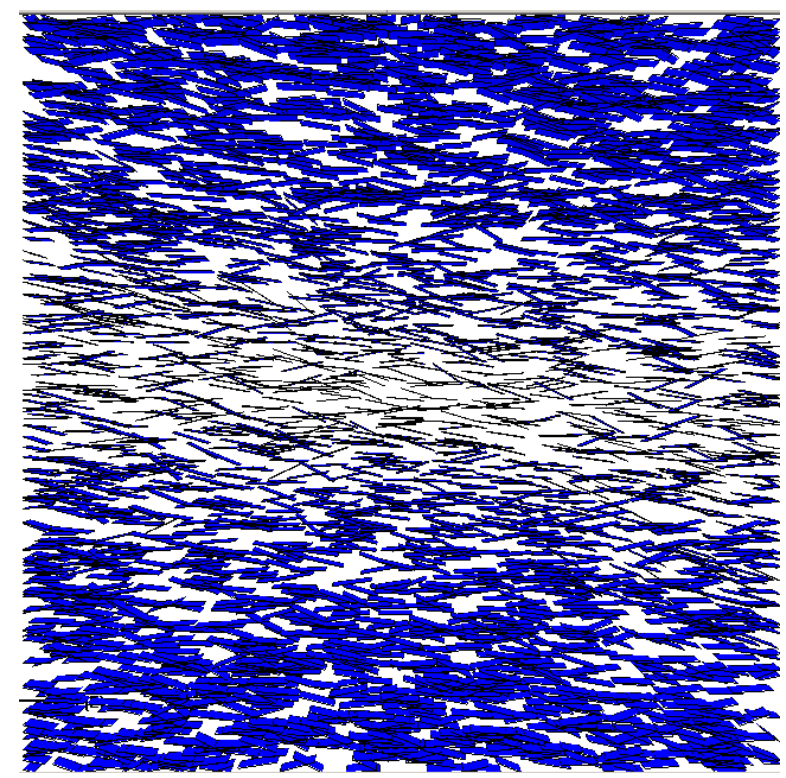

Fig. 4.8 - Final model using the combined data objective function. 


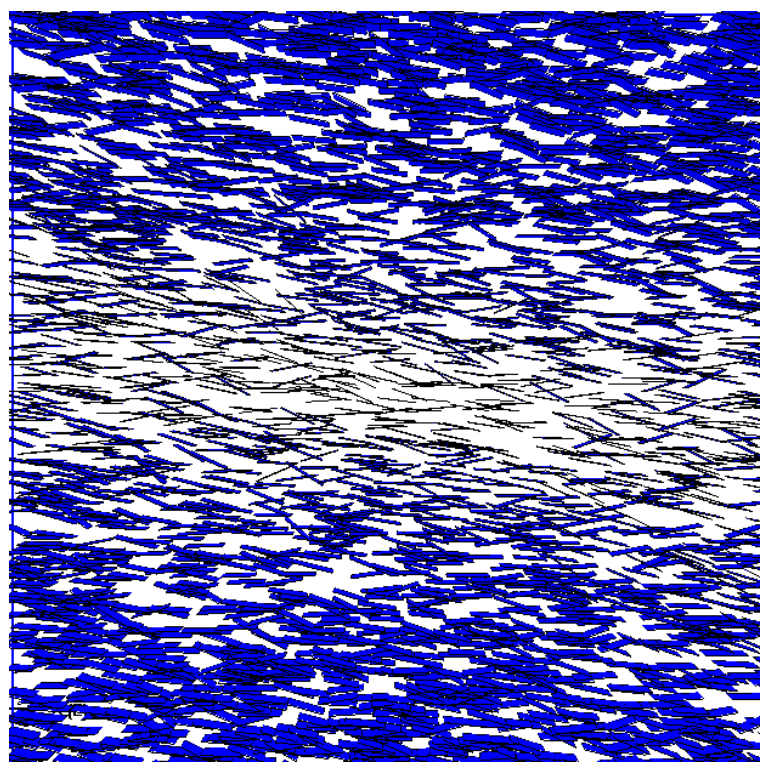

Fig. 4.9 - Final model using the production data only objective function.

\subsubsection{Water Saturation Maps}

Figs. 4.10 to 4.12 show water saturation in the fracture system at the final report step for the base model (Fig. 4.10), the initial model estimate (Fig. 4.11), and the optimized model (Fig. 4.12). Fig. 4.10 exhibits preferential water flow through the fracture system resulting in minor and more or less equal breakthrough at each producing well for the base case. Fig. 4.11, showing the same data for the initial model estimate, also clearly exhibits preferential flow through the fracture system. However, it can be seen that the combination of well placement and permeability anisotropy of the fracture system results in strong breakthrough in producing wells P1 and P4, which are aligned with the injector and the fracture system. Breakthrough is delayed in producing wells P2 and P3 due to their position with respect to the injector perpendicular to the direction of fracture trend and maximum permeability. Fig. 4.12 for the optimized model clearly shows that the production performance has returned to very close to the base case. Fracture and matrix water saturation maps for report steps 5 (150 days), 10 (300 days), 15 (450 days), and 20 (600 days) are depicted in Figs. B.5 to B.12 located in Appendix B. 


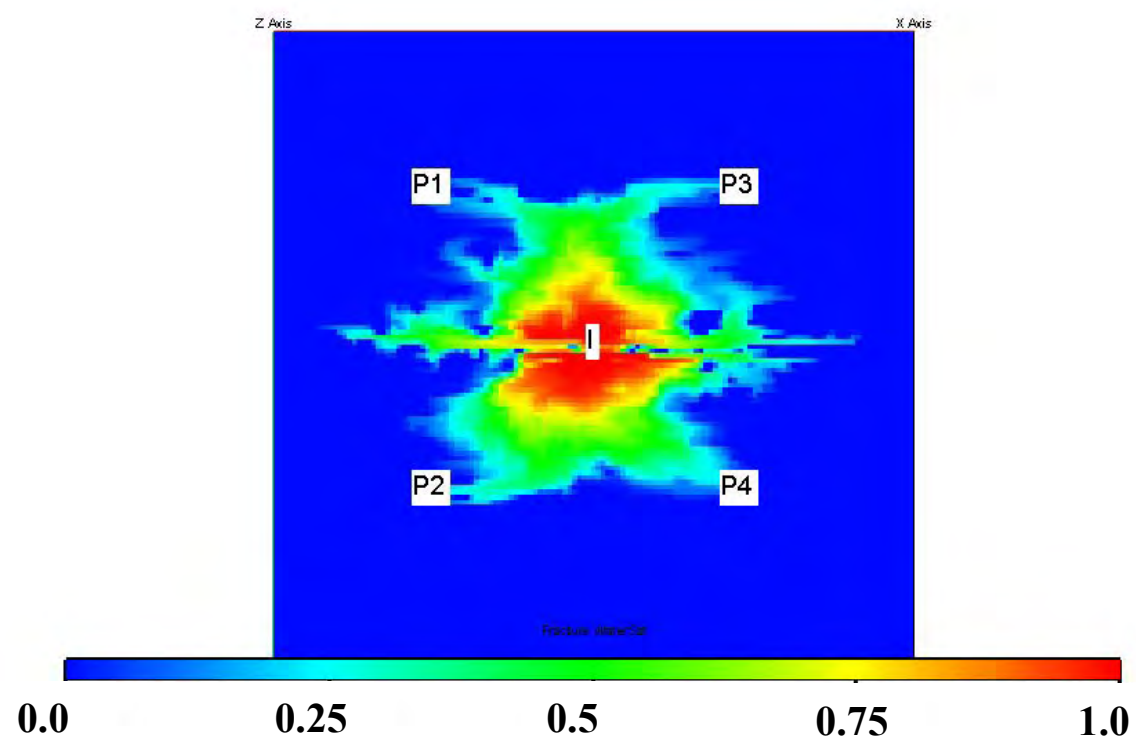

Fig. 4.10 - Fracture water saturation at report step 20 (600 days) for test T2 base model.

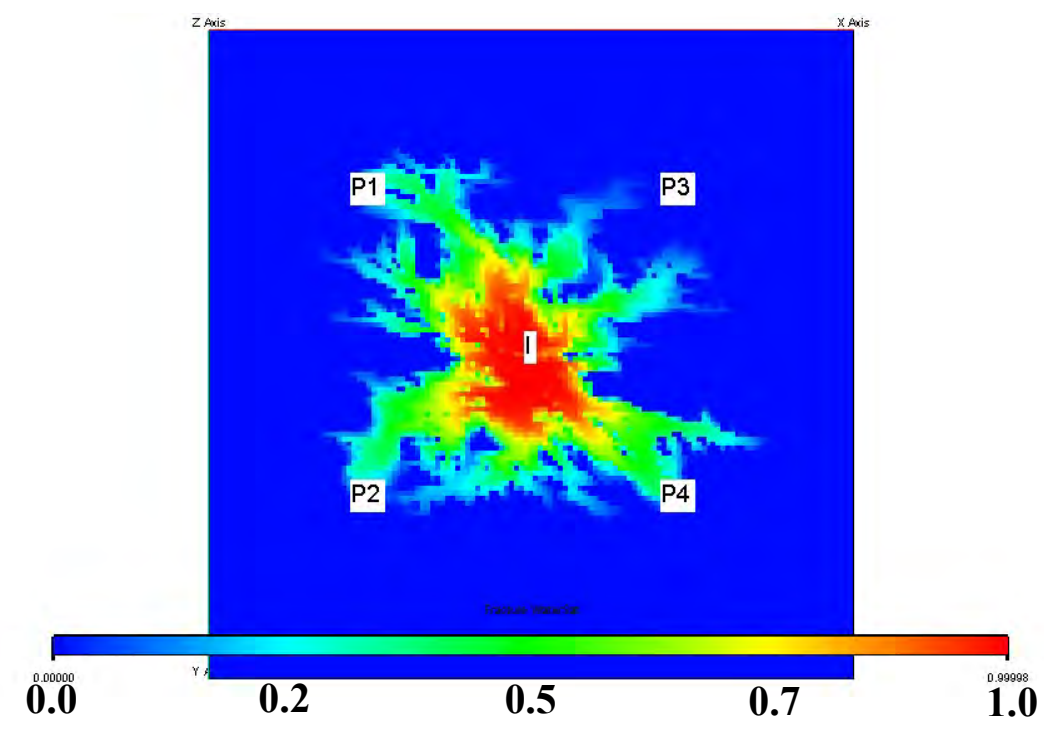

Fig. 4.11 - Fracture water saturation at report step 20 (600 days) for test T2 initial estimate. 


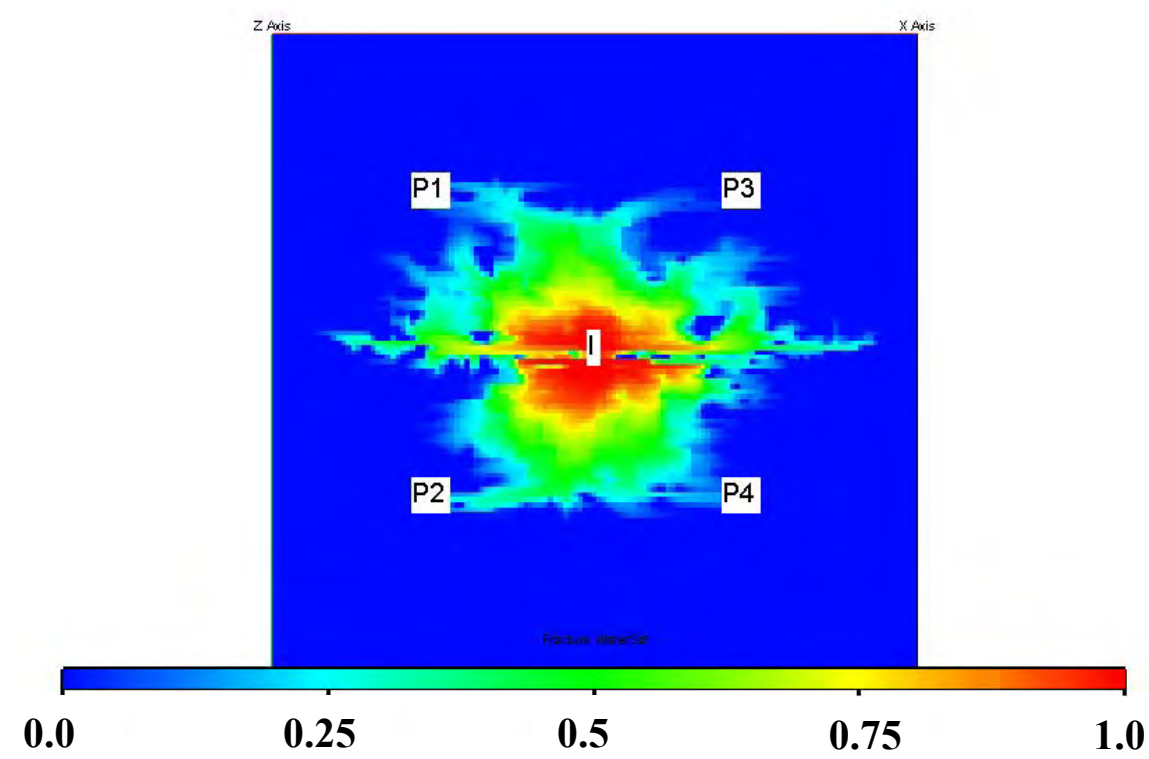

Fig. 4.12 - Fracture water saturation at report step 20 (600 days) for test T2 final model, combined data.

\subsubsection{Seismic Attribute Plots}

Figs. 4.13 to 4.15 show seismic velocity plots for the base model (Fig. 4.13), the initial model estimate (Fig. 4.14), and the optimized model (Fig. 4.15). The vertical axis is the quasi-p wave velocity of propagation. The horizontal axis in the azimuth of energy propagation. Quasi-P wave velocity versus azimuth is plotted for all cell blocks prior to averaging for computation of the objective function. Average values for peak-to-peak quasi-P wave modulation amplitude and direction of maximum quasi-P wave velocity are indicated on each plot. Fig. 4.13 for the base model clearly shows the maximum quasi-P wave velocity direction with its modulation phase aligned with fracture trend with average peak-to-peak velocity modulation amplitude is approximately 0.34 . Fig. 4.14 for the initial estimate shows a 30 degree shift on the maximum quasi-P wave velocity direction phase and reduced average peak-to-peak modulation amplitude of approximately 0.26 , as would be expected due to the 30 degree error in trend estimate and low estimate of $\mathrm{P}_{32}$ intensity. Fig. 4.15 for the optimized model shows the return of maximum quasi-P wave velocity direction and average peak-to-peak velocity 
modulation to the base case values. Seismic attributes for all iterations are depicted in Figs. B.16 and B.17 located in Appendix B.

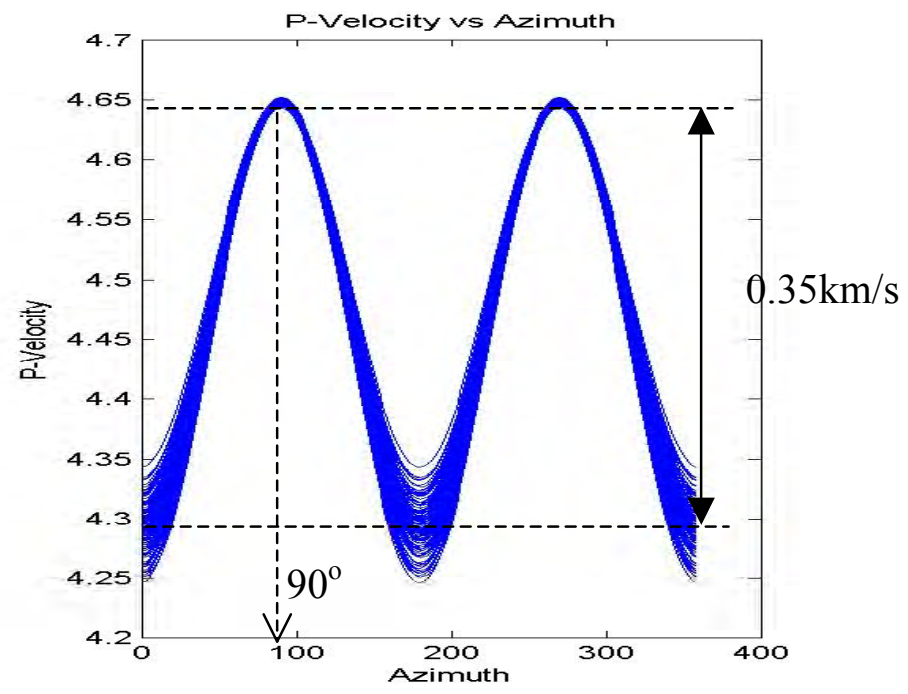

Fig. 4.13 - Base model quasi-P wave velocity modulation amplitude and maximum quasi-P wave velocity direction.

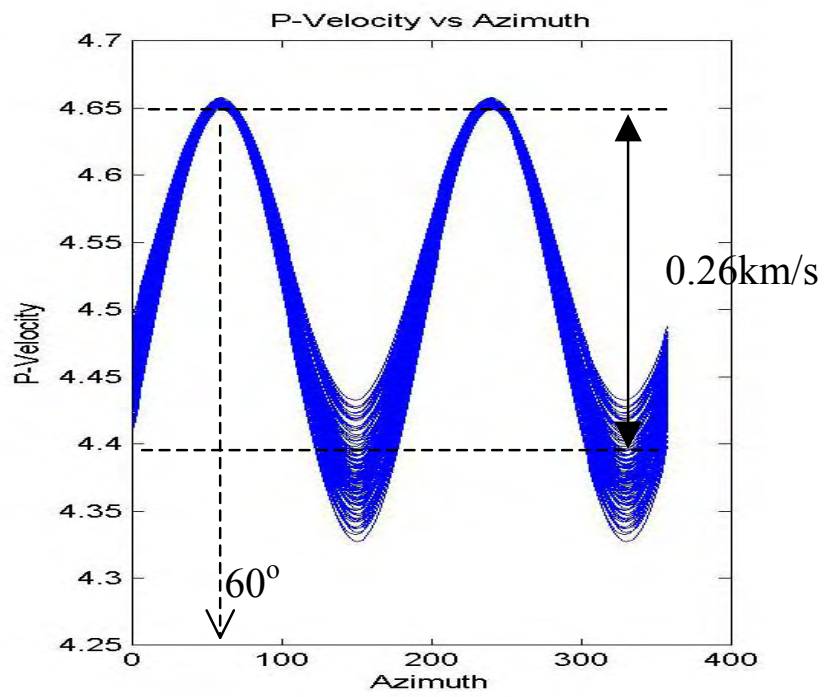

Fig. 4.14 - Initial estimate quasi-P wave velocity modulation amplitude and maximum quasi-P wave velocity direction. 


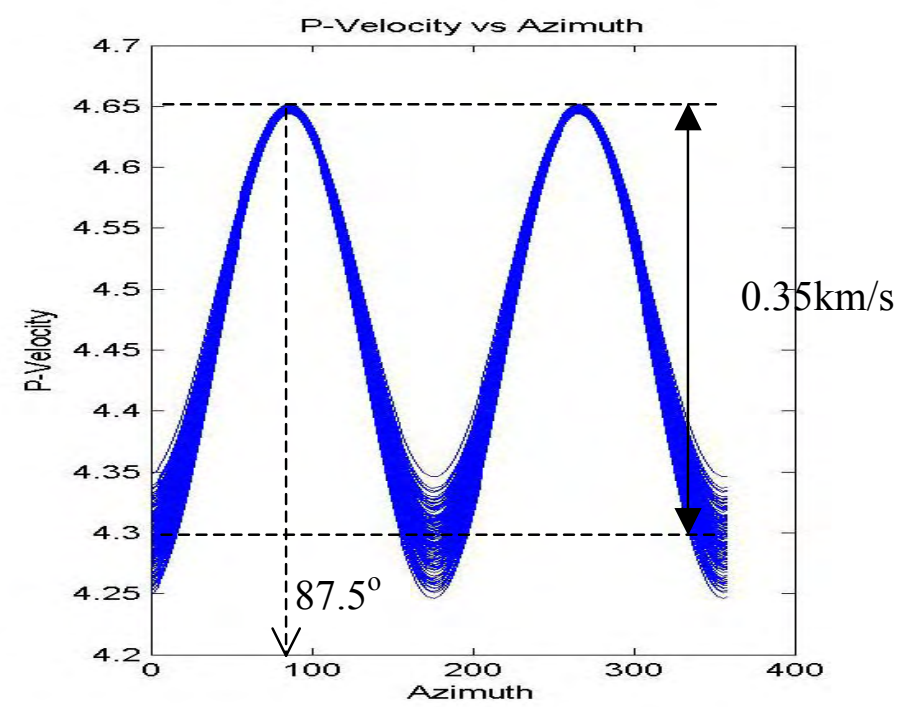

Fig. 4.15 - Final quasi-P wave velocity modulation amplitude and maximum quasi-wave velocity direction.

\subsection{Test T3 - Two Fracture Sets in a Tight Sandstone Matrix}

The next test of the new method was conducted on a reservoir model with a two nonparallel fracture sets having the population statistics listed in Table 4.7 and elastic and petrophysical properties representative of a fractured tight sandstone reservoir. Reservoir matrix properties are listed in Tables 4.8 and 4.9. In order to test the effect of including seismic anisotropy in the objective function a parallel test was performed using only production data in the objective function. These tests with and without seismic anisotropy are referred to as "combined" and "production only" respectively in the following summary Tables and Figures.

Table 4.7 - Test T3 base model fracture distribution parameters.

\begin{tabular}{|c|c|c|}
\hline Parameter & Set 1 & Set 2 \\
\hline Trend (Geographical) & $\mathrm{N}[335,10]$ & $\mathrm{N}[45,10]$ \\
\hline$P_{32}$ Intensity $(1 / \mathrm{m})$ & 0.1 & 0.15 \\
\hline Length (m) & $\mathrm{LN}[50,3]$ & $\mathrm{LN}[50,3]$ \\
\hline Height (m) & 60 & 60 \\
\hline Transmissivity $\left(\mathrm{m}^{2} / \mathrm{s}\right)$ & $\mathrm{LN}[8 \mathrm{e}-04,4.3 \mathrm{e}-05]$ & $\mathrm{LN}[8 \mathrm{e}-04,4.3 \mathrm{e}-05]$ \\
\hline
\end{tabular}


Table 4.8 - Test T3 reservoir properties.

\begin{tabular}{|l|c|}
\hline Matrix Porosity & 0.10 \\
\hline Matrix Permeability & $10 \mathrm{mD}$ \\
\hline Fracture Porosity & 0.015 \\
\hline DP shape factor & 0.08 \\
\hline
\end{tabular}

Table 4.9 - Test T3 elastic modeling parameters.

\begin{tabular}{|l|c|}
\hline Matrix P-wave Velocity & $4670 \mathrm{ft} / \mathrm{sec}$ \\
\hline Matrix Shear-wave Velocity & $3060 \mathrm{ft} / \mathrm{sec}$ \\
\hline Matrix Density & $2510 \mathrm{~kg} / \mathrm{m}^{3}$ \\
\hline Overburden P wave Velocity & $4670 \mathrm{ft} / \mathrm{sec}$ \\
\hline Overburden S wave Velocity & $3060 \mathrm{ft} / \mathrm{sec}$ \\
\hline Overburden Density & $2510 \mathrm{~kg} / \mathrm{m}^{3}$ \\
\hline Incidence angle & $30 \mathrm{degrees}$ \\
\hline
\end{tabular}

\subsubsection{Parameter Updates}

a. Combined Data Objective Function - Parameter error history for this optimization test are listed in Table 4.10 and shown in Figs. 4.16 and 4.17. Parameter perturbations and a priori model weights for each iteration are listed in Tables C.5 and C.6 in Appendix C. The initial estimates of fracture trend for both fracture sets were 20 degrees away from the base model values. The initial estimates of $\mathrm{P}_{32}$ intensity were 0.05 and 0.1 away from the base model values for fracture sets 1 and 2 respectively. Fig. 4.16 depicting trend error shows that trend for fracture set 2 experienced monotonic convergence from the initial error of 20 degrees toward approximately 1 degree. Convergence of fracture set 1 trend was not as smooth as that for set 2 and showed unexplained erratic behavior before converging to within 2 degrees of the base value. Fig. 4.17 depicting $\mathrm{P}_{32}$ intensity error shows smooth, almost linear decrease in error for fracture set 1, while trend error for fracture set 2 experienced erratic behavior before 
converging. Ultimately $\mathrm{P}_{32}$ intensity estimates for both fracture sets converged to within 0.005 of the base case value.

Table 4.10 - $\quad$ - Summary of absolute errors for fracture set trend and $\mathrm{P}_{32}$ intensity at each update step using the combined objective function. Step 0 is the initial estimate.

\begin{tabular}{|r|r|r|r|r|}
\cline { 2 - 5 } \multicolumn{1}{c|}{} & \multicolumn{4}{c|}{ Parameter Error } \\
\hline Iteration & Trend (1) & $\mathbf{P}_{\mathbf{3 2}}$ (1) & Trend (2) & $\mathbf{P}_{\mathbf{3 2}}$ (2) \\
\hline $\mathbf{0}$ & 20.0 & 0.100 & 20.0 & 0.050 \\
\hline $\mathbf{1}$ & 19.3 & 0.074 & 18.6 & 0.043 \\
\hline $\mathbf{2}$ & 16.4 & 0.050 & 14.6 & 0.056 \\
\hline $\mathbf{3}$ & 16.1 & 0.036 & 9.5 & 0.057 \\
\hline $\mathbf{4}$ & 8.2 & 0.024 & 4.5 & 0.035 \\
\hline $\mathbf{5}$ & 12.1 & 0.007 & 4.1 & 0.018 \\
\hline $\mathbf{6}$ & 1.8 & 0.003 & 0.6 & 0.005 \\
\hline
\end{tabular}

Test T3 - Trend Error

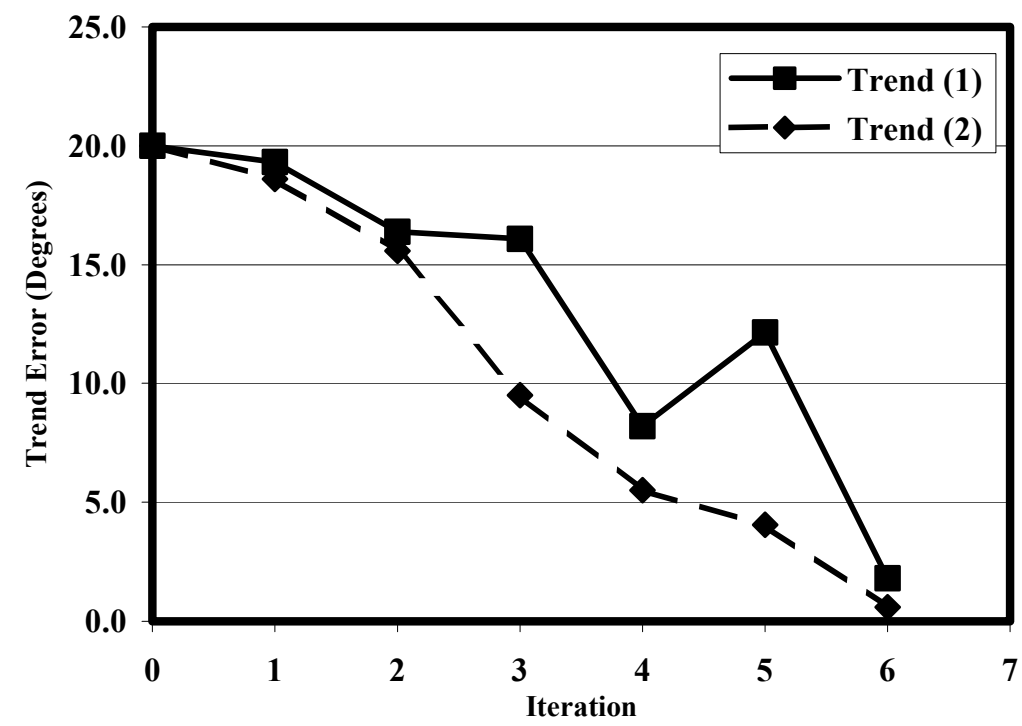

Fig. 4.16 - Graph of absolute error in fracture set trend versus iteration for test T3 using the combined objective function. Iteration 0 is the initial estimate. 


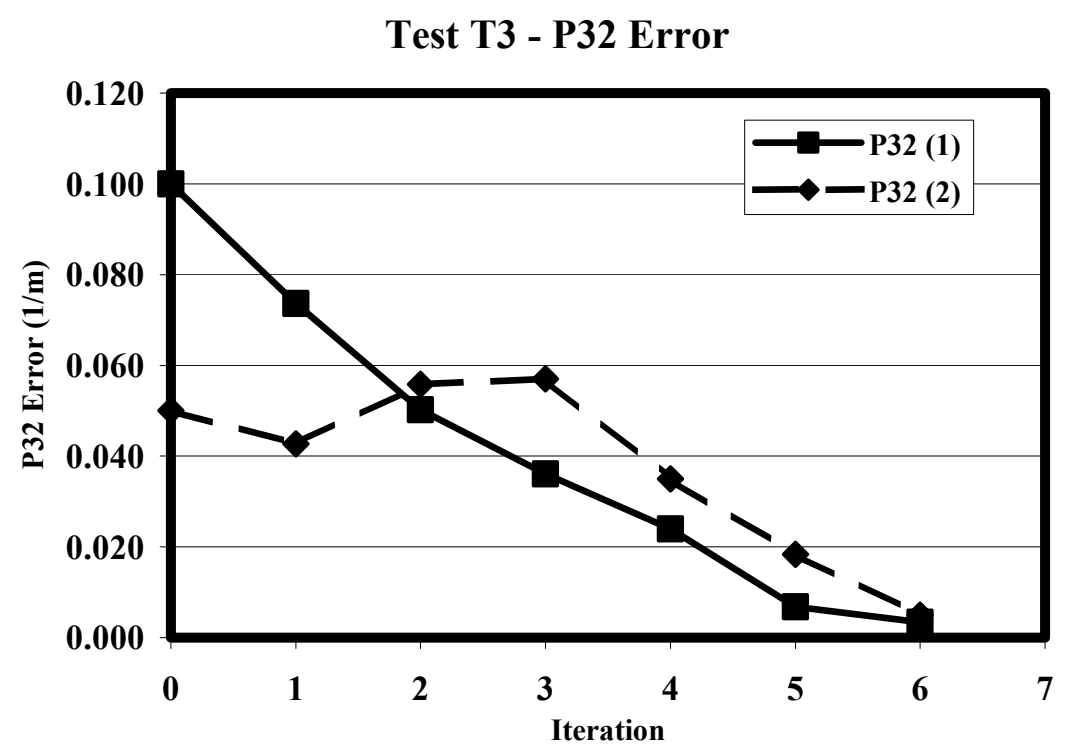

Fig. 4.17 $\quad-\quad$ Graph of absolute error in fracture set $\mathrm{P}_{32}$ intensity versus iteration for test $\mathrm{T} 3$ using the combined objective function. Iteration 0 is the initial estimate.

b. Production Data Only Objective Function - Parameter error history for the production only optimization test are listed in Table 4.11 and shown in Figs. 4.18 through 4.21. Parameter perturbations and a priori model weights for each iteration are listed in Tables C.10 and C.11 in Appendix C. For reference the result of the combined objective function test is plotted on the same figure. with the production data only results. The initial estimates of fracture trend and intensity for both fracture sets was the same as was used for the combined objective function test. These figures show that, unlike the successful convergence result achieved using the combined objective function, the production data only objective function failed to converge for either $\mathrm{P}_{32}$ intensity or trend of fracture sets 1 or 2 . All target inversion parameters experienced erratic random or divergent behavior. 
Table 4.11 - Summary of absolute errors for fracture set trend and $\mathrm{P}_{32}$ intensity at each update step using the production data only objective function. Step 0 is the initial estimate.

\begin{tabular}{|r|r|r|r|r|}
\cline { 2 - 5 } \multicolumn{1}{c|}{} & \multicolumn{4}{c|}{ Parameter Error } \\
\hline Iteration & Trend (1) & P32 (1) & Trend (2) & P32 (2) \\
\hline $\mathbf{0}$ & 20.0 & 0.100 & 20.0 & 0.050 \\
\hline $\mathbf{1}$ & 16.0 & 0.111 & 17.7 & 0.046 \\
\hline $\mathbf{2}$ & 15.2 & 0.116 & 12.8 & 0.065 \\
\hline $\mathbf{3}$ & 10.9 & 0.111 & 11.6 & 0.083 \\
\hline $\mathbf{4}$ & 12.2 & 0.118 & 16.8 & 0.068 \\
\hline $\mathbf{5}$ & 15.2 & 0.104 & 20.7 & 0.078 \\
\hline
\end{tabular}

Test T3 - P32 Error - Set 1

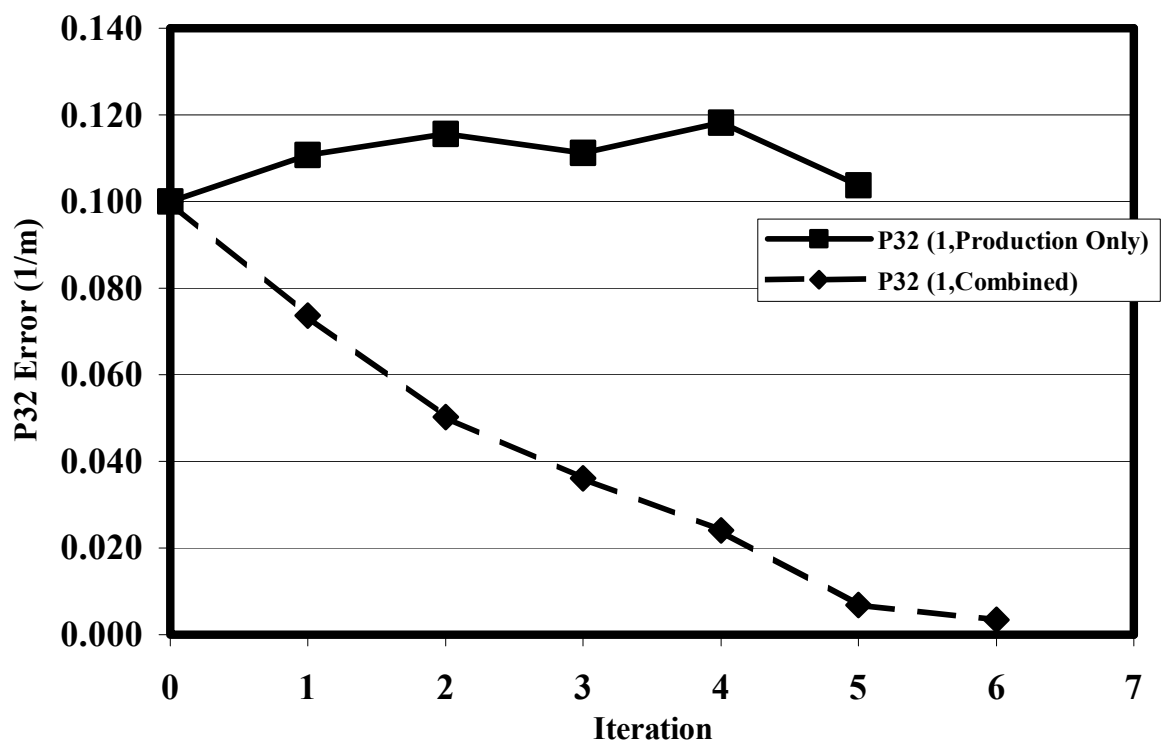

Fig. 4.18 - Graph of absolute error in fracture set $1 \mathrm{P}_{32}$ intensity versus iteration for test $\mathrm{T} 3$ for both combined and production data only objective function. Iteration 0 is the initial estimate. 


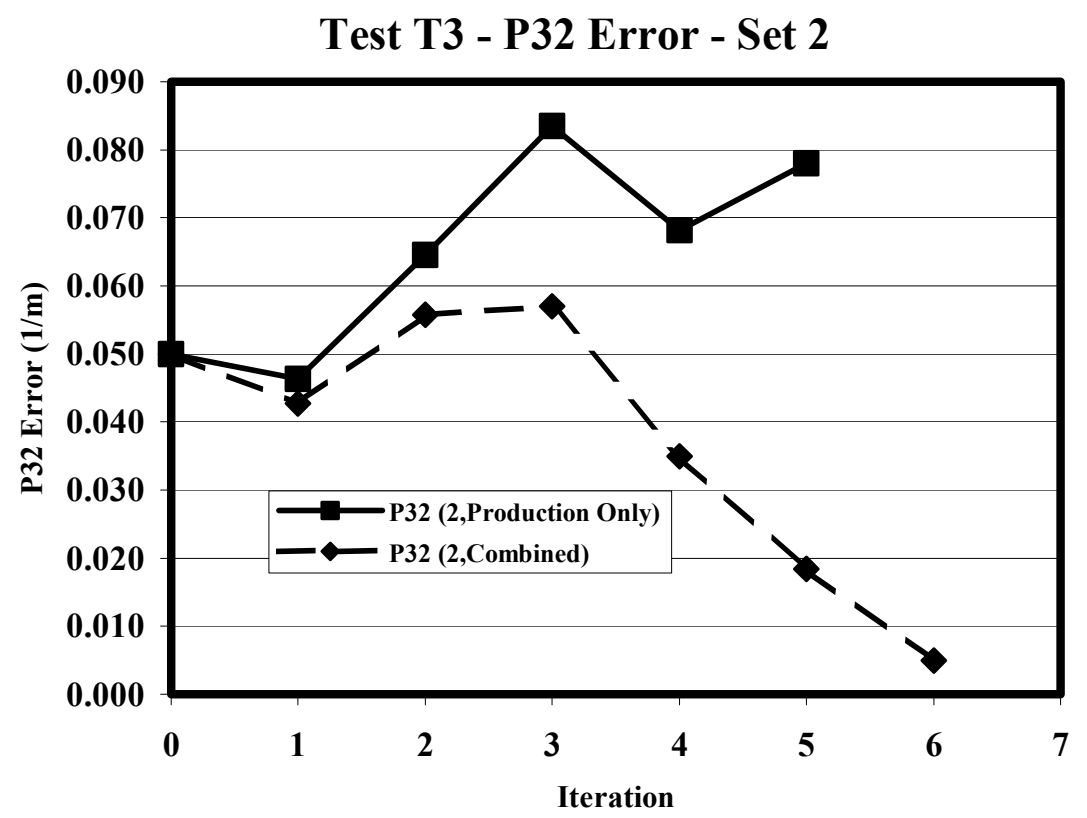

Fig. $4.19-$ Graph of absolute error in fracture set $2 \mathrm{P}_{32}$ intensity versus iteration for test $\mathrm{T} 3$ for both combined and production data only objective function. Iteration 0 is the initial estimate.

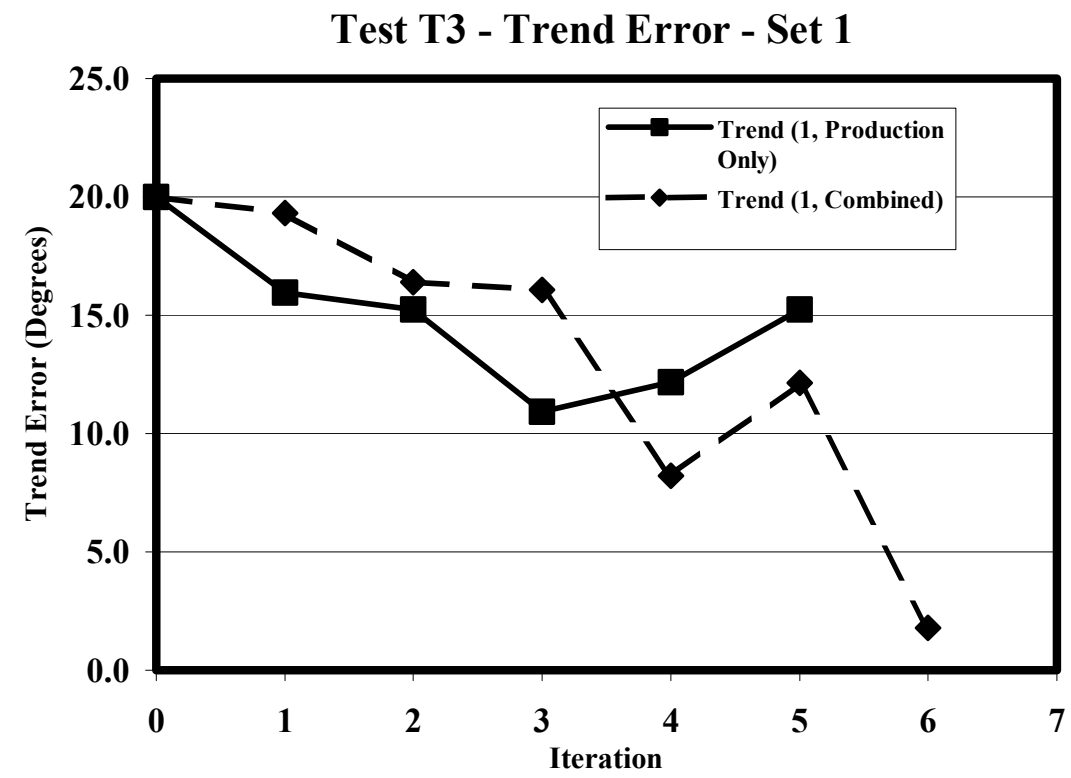

Fig. 4.20 - Graph of absolute error in fracture set 1 trend versus iteration for test $\mathrm{T} 3$ for both combined and production data only objective function. Iteration 0 is the initial estimate. 
Test T3 - Trend Error - Set 2

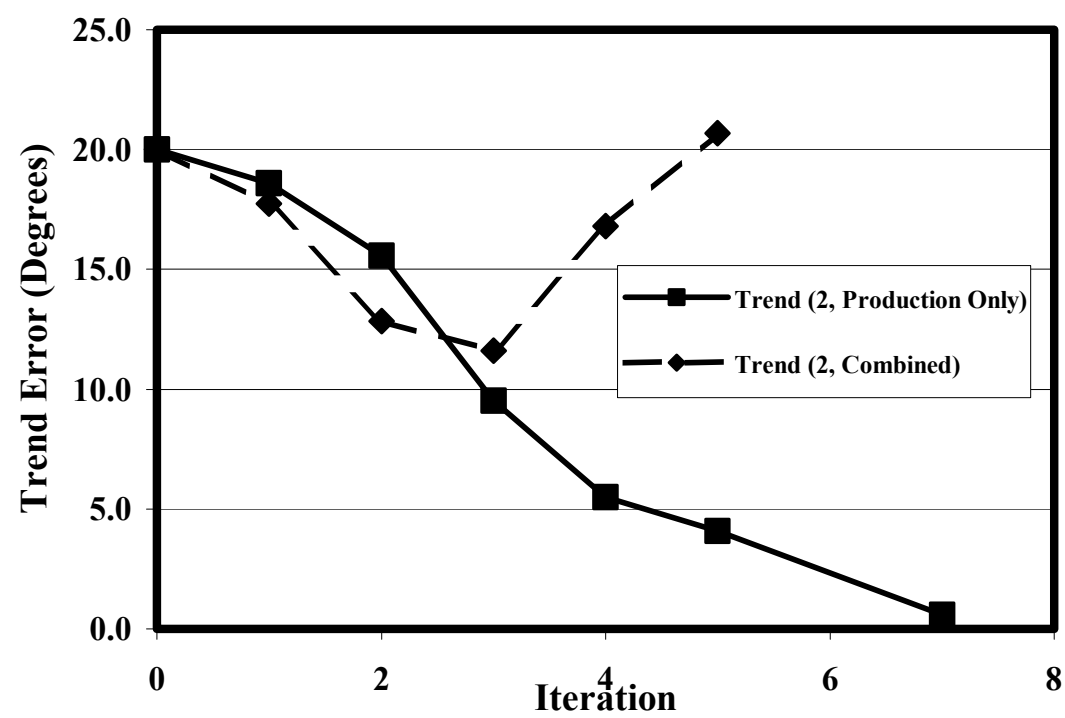

Fig. 4.21 - Graph of absolute error in fracture set 2 trend versus iteration for test $\mathrm{T} 3$ for both combined and production data only objective function. Iteration 0 is the initial estimate.

\subsubsection{Objective Function and Observation Residuals}

a. Combined Objective Function - The average normalized residuals for the 10 update trials of combined objective function optimization test are listed in Table 4.12 and illustrated in Fig. 4.22. The objective function and partial residuals for bottom hole pressure, oil production rate, and seismic attributes are shown separately. All test residuals are listed in Table C.9a to Table C.9f in Appendix C. Similar to test T2, the bottom hole pressure residual is seen to be larger than that for either seismic attributes or oil production rate. However, in this test the magnitude of the seismic residual compared to the bottom hole pressure residual is larger than in test $\mathrm{T} 2$. The initial seismic residual is approximately one half the magnitude of the bottom hole pressure residual. Also similar to observations in test $\mathrm{T} 2$, the oil production rate residual is the lowest. Bottom hole pressure and seismic attribute residuals show fairly steady convergence through the optimization process except for one iteration which is associated with the erratic 
parameter update behavior in Fig. 4.20. The oil production rate residual shows little convergent behavior, indicating that this observation is contributing little to the optimization process.

Table 4.12 - Summary of objective function and normalized residual errors by observation type for fracture using the combined objective function. Iteration 0 is the initial estimate.

\begin{tabular}{|r|r|r|r|r|}
\cline { 3 - 5 } \multicolumn{2}{c|}{} & \multicolumn{3}{c|}{ RMS Residuals } \\
\hline Iteration & \multicolumn{1}{c|}{$\mathbf{Q}$} & \multicolumn{1}{c|}{ BHP } & \multicolumn{1}{c|}{ OPR } & \multicolumn{1}{c|}{ SEIS } \\
\hline $\mathbf{0}$ & 353.52 & 8.31 & 3.93 & 16.40 \\
\hline $\mathbf{1}$ & 272.91 & 6.73 & 4.23 & 14.47 \\
\hline $\mathbf{2}$ & 139.95 & 3.93 & 3.29 & 10.68 \\
\hline $\mathbf{3}$ & 87.80 & 3.17 & 3.80 & 7.94 \\
\hline $\mathbf{4}$ & 25.81 & 1.34 & 1.18 & 4.74 \\
\hline $\mathbf{5}$ & 50.27 & 3.57 & 3.29 & 5.18 \\
\hline $\mathbf{6}$ & 20.43 & 3.26 & 3.06 & 0.77 \\
\hline
\end{tabular}

Test T3 Objective Function and RMS Residuals

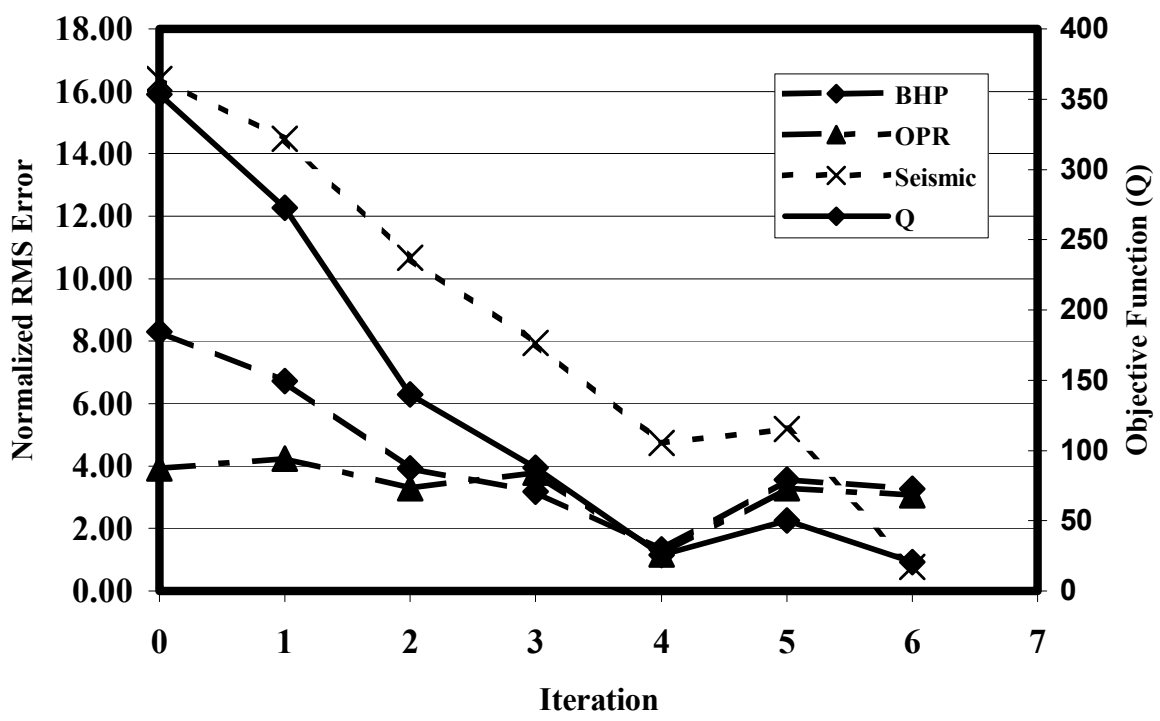

Fig. 4.22 - Graph of objective function and normalized residual errors by observation type for fracture set trend and $\mathrm{P}_{32}$ intensity using the combined objective function. Iteration 0 is the initial estimate. 
b. Production Data Only Objective Function - The normalized residuals during optimization using the production data only objective function are listed in Table 4.13 and illustrated in Figs. 4.23 and 4.24. Residuals for both oil production rate and bottom hole pressure show erratic behavior with the oil production rate residual reflecting the divergence of the objective function seen in Figs. 4.18 through 4.21. The bottom hole pressure residual shows a slight but erratic tendency toward convergence. All test residuals are listed in Tables C.14a to C.14e in Appendix C.

Test T3 - OPR Residuals

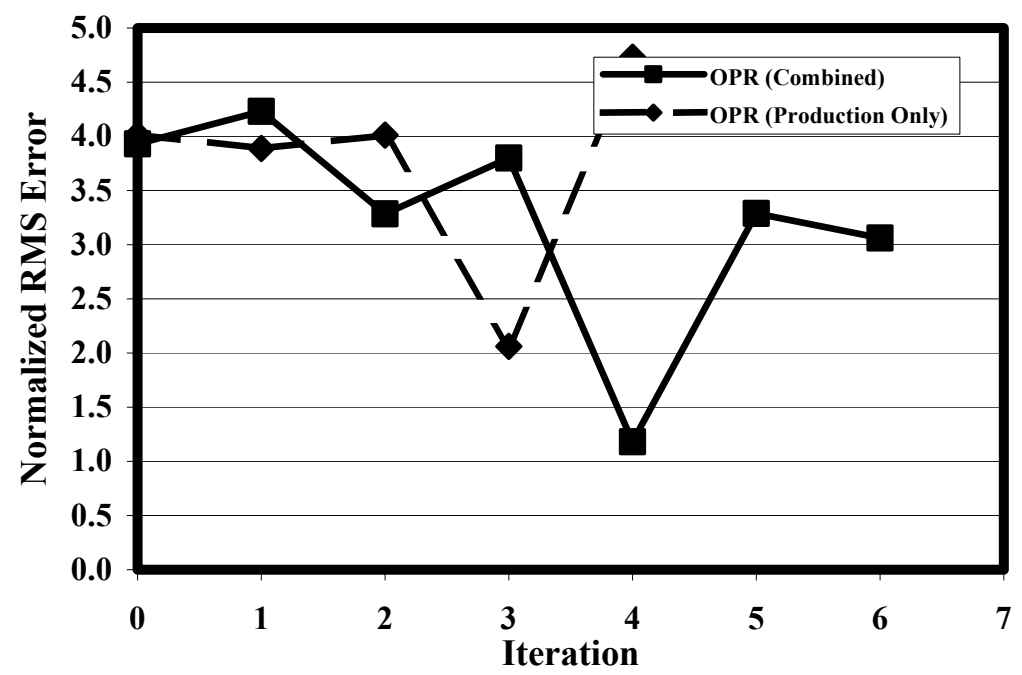

Fig. 4.23 - Graph of objective function and normalized oil production rate residual errors for the combined objective function and production data only objective function. Iteration 0 is the initial estimate.

Table 4.13 - Summary of normalized residuals by observation type for fracture set trend and $\mathrm{P}_{32}$ intensity at each iteration using the production data only objective function. Iteration 0 is the initial estimate.

\begin{tabular}{|r|r|r|r|}
\cline { 2 - 4 } \multicolumn{1}{c|}{} & \multicolumn{3}{c|}{ Residuals } \\
\hline Iteration & Total & \multicolumn{1}{c|}{ BHP } & \multicolumn{1}{c|}{ OPR } \\
\hline $\mathbf{0}$ & 9.24 & 8.33 & 4.01 \\
\hline $\mathbf{1}$ & 5.27 & 3.55 & 3.89 \\
\hline $\mathbf{2}$ & 8.40 & 7.38 & 4.01 \\
\hline $\mathbf{3}$ & 3.46 & 2.76 & 2.06 \\
\hline $\mathbf{4}$ & 6.32 & 4.18 & 4.73 \\
\hline
\end{tabular}




\section{Test T3 - BHP Residuals}

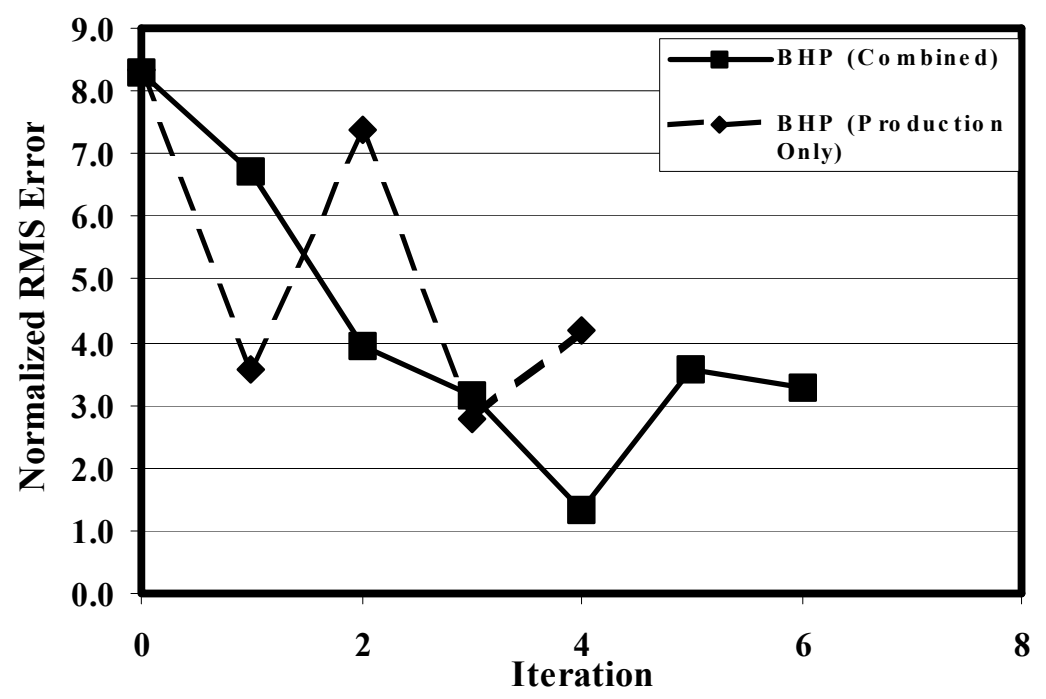

Fig. 4.24 - Graph of normalized bottom hole pressure residual errors for the combined objective function and production data only objective function. Iteration 0 is the initial estimate.

\subsubsection{Sensitivity Coefficients}

Fig. 4.25 shows a sample of Jacobian matrix sensitivity coefficients computed using Eq. 3.14 for the initial estimate model state of the multiple fracture set optimization test T3. Table 4.6 contains a list of observation numbers and descriptions. These sensitivity coefficients are the gradients of the observations with respect to the unknown parameters and carry information regarding the relative contribution of each observation to the parameter update. Observations with large positive or negative sensitivity coefficients will have a larger impact on the parameter update than those with lower sensitivity coefficients. The sensitivity coefficients in Fig. 4.25 show high sensitivity of trend and $\mathrm{P}_{32}$ intensity for both fracture sets to the bottom hole pressure observations. Fracture set 1 also shows some sensitivity to oil production rate. Interestingly $\mathrm{P}_{32}$ intensity and trend for both fracture sets show sensitivity to the velocity modulation amplitude while only the trend of fracture set 1 shows significant sensitivity to the maximum quasi-P wave velocity direction attribute. It is also notable that all sensitivity coefficients are opposite 
in sign between fracture sets 1 and 2. All sensitivity coefficients for the combined objective function test are shown in Figs. C.4a to C.4f in Appendix C.
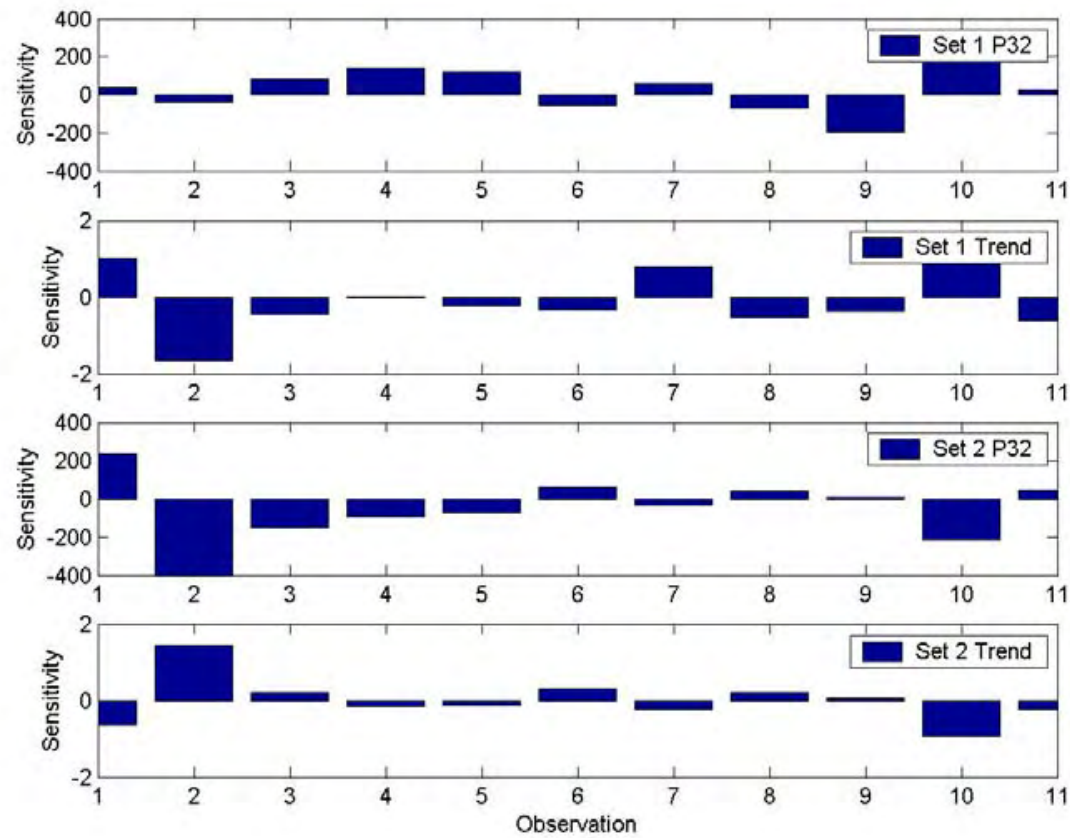

Fig. 4.25 - Parameter sensitivity coefficients by observation number for the multiple fracture set initial model combined objective function case T3.

\subsubsection{Fracture Distribution Maps}

Maps of the fracture system for the base case, initial estimate, and optimized model are shown in Figs. 4.26 to 4.29. Fig. 4.26 shows the fracture distribution for the base model. Fig. 4.27 shows the fracture distribution for the initial estimate. Fig. 4.28 shows the fracture distribution for the model optimized using the combined objective function. Fig. 4.29 shows the fracture distribution for the model optimized using the production data only objective function. Comparison of Figs. 4.26 and 4.28 illustrate the successful convergence of the model back to the base case when using the combined objective function. Failure to converge when using the production data only objective function is clearly seen by comparison of Figs. 4.26 and 4.29. 


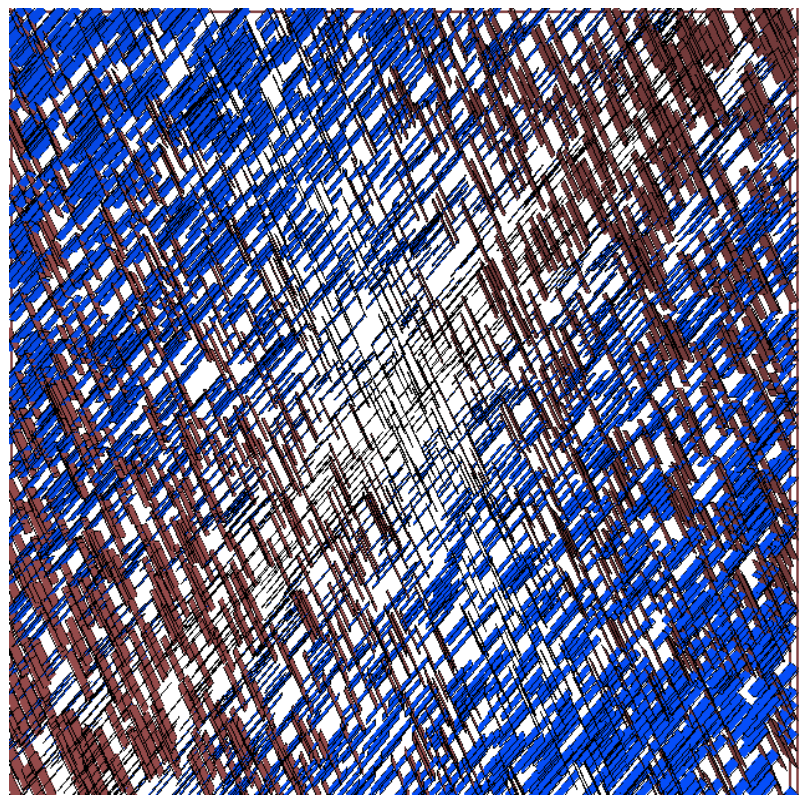

Fig. 4.26 - Test T3 base fracture distribution.

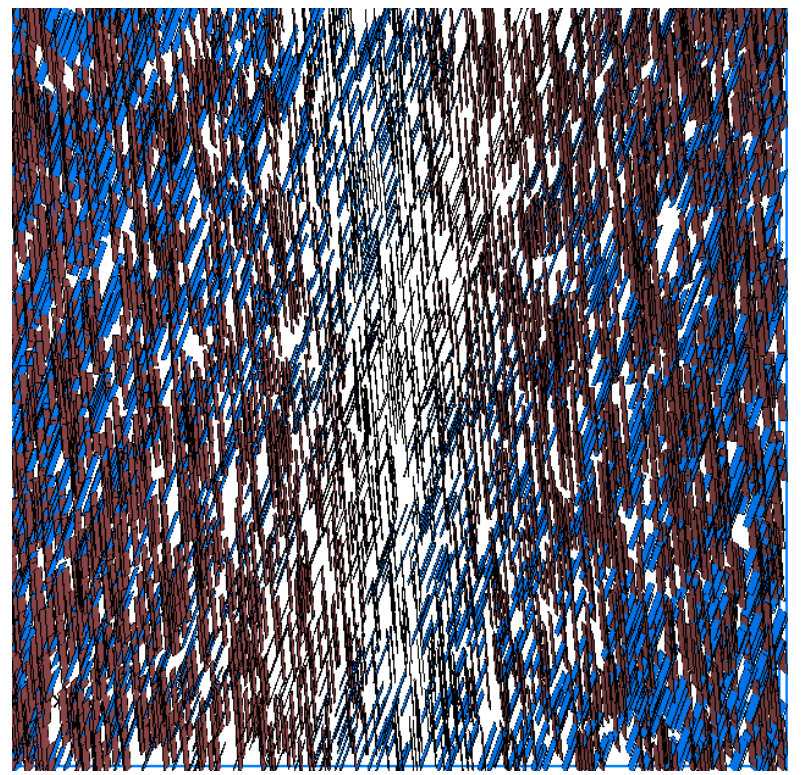

Fig. 4.27 - Test T3 initial estimate fracture distribution. 


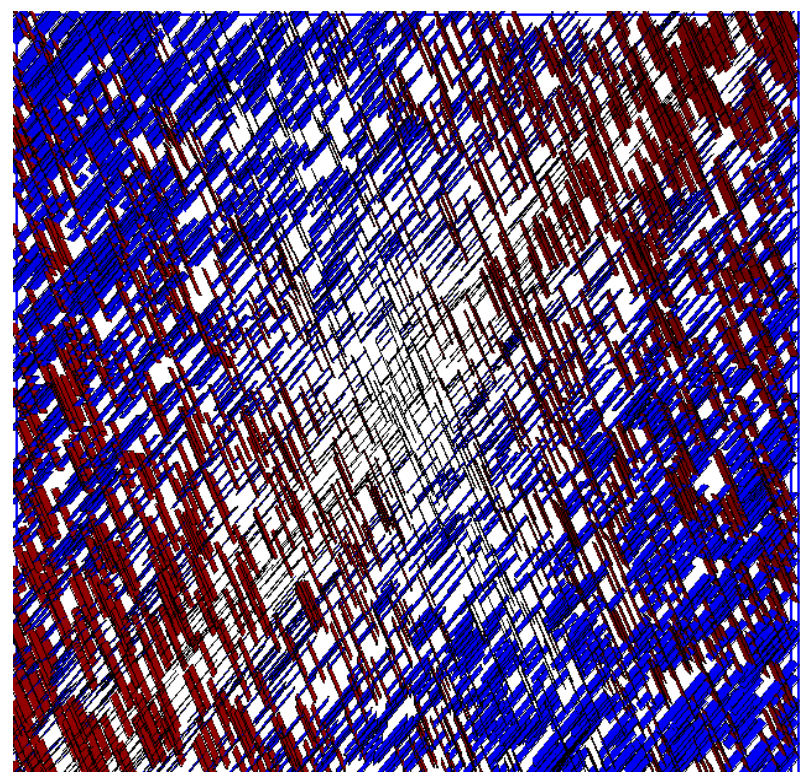

Fig. 4.28 - Test T3 final model fracture distribution using the combined objective function.

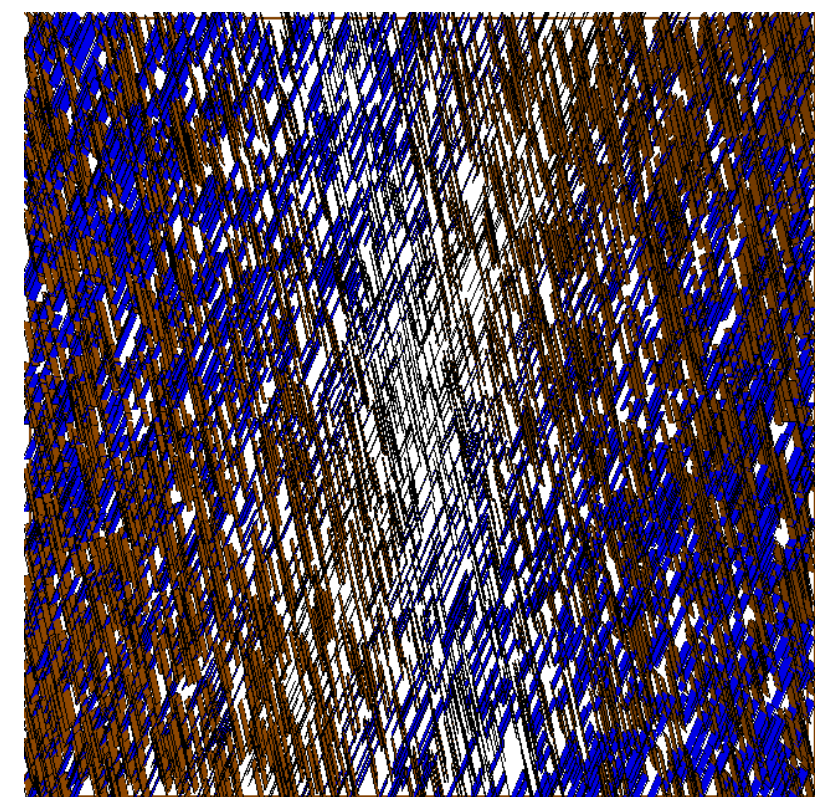

Fig. 4.29 - Test T3 final model fracture distribution using the production data only objective function. 


\subsubsection{Water Saturation Maps}

Figs. 4.30 to 4.32 show water saturation in the fracture system at the final report step for the base model (Fig. 4.30), the initial model estimate (Fig. 4.31), and the optimized model (Fig. 4.32). Fracture water saturation for the base model shows strong symmetry with less linear character than observed in the single fracture set case seen in test T2. There is a subtle preferential flow of water through fracture set 2 because of its alignment with the azimuth of the trajectory between the injector and producers P2 and P3. The water saturation distribution for the initial model estimate shown in Fig. 4.31 exhibits preferential flow aligned along the direction corresponding to the mean direction of the initial erroneous trend estimates. This effect was anticipated because the initial estimates for these parameters increased the apparent alignment of the fracture sets and, as a result, degree of anisotropy. A significant difference in water breakthrough to all producing wells can be seen between this initial estimate and the base case (Fig. 4.30). The water saturation distribution from the optimized model illustrated in Fig. 4.32 shows that the production performance has returned to very close to the base case. However, agreement with the base case is not as striking as seen in the case using only a single fracture set. This is because the optimization process was not able to resolve parameters as well for both fracture sets in this test as it was for the single fracture set. Fracture and matrix water saturation maps for report steps 5 (150 days), 10 (300 days), 15 (450 days), and 20 (600 days) are depicted in Appendix C Figs. C.6 to C.13 for the combined objective function test, and in Figs. C.19 to C.26 for the production data only objective function test. 


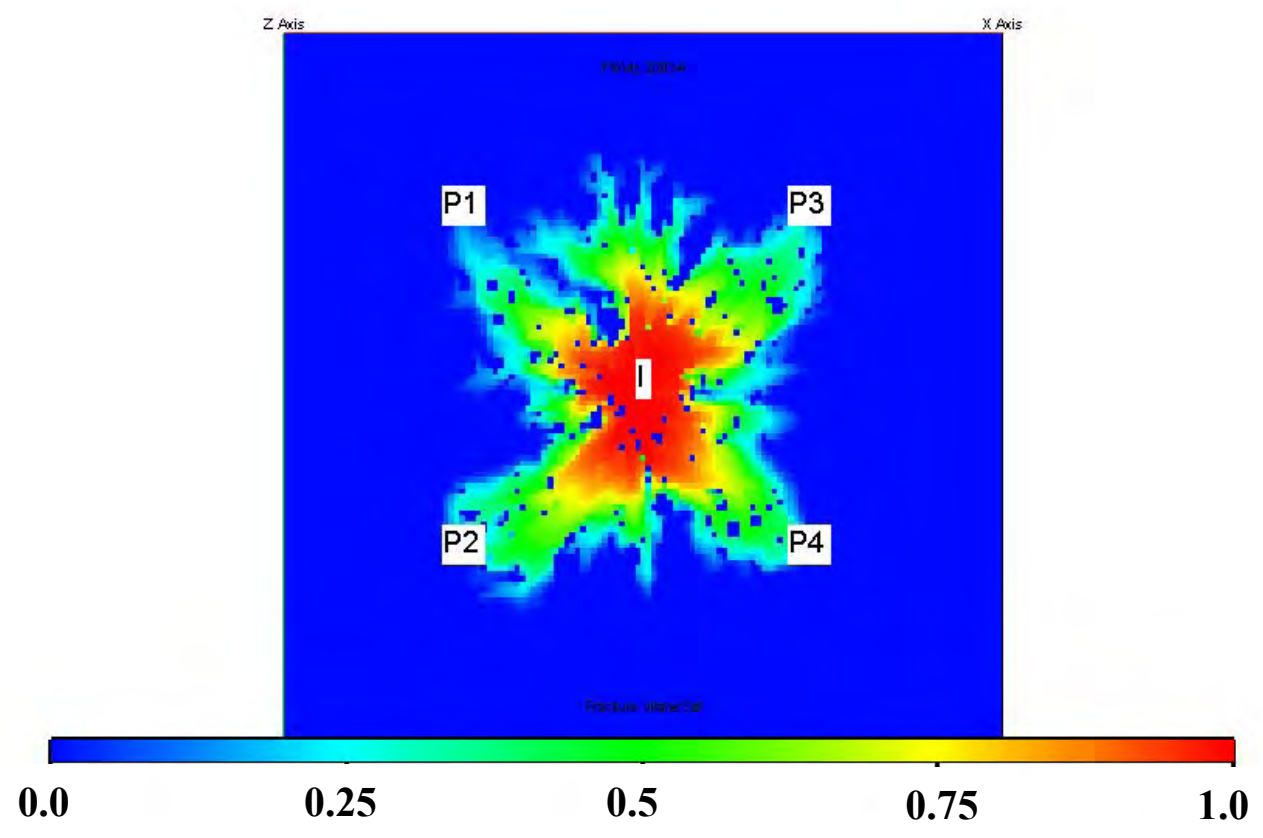

Fig. 4.30 - Fracture water saturation at report step 20 (600 days) for test T3 base model.

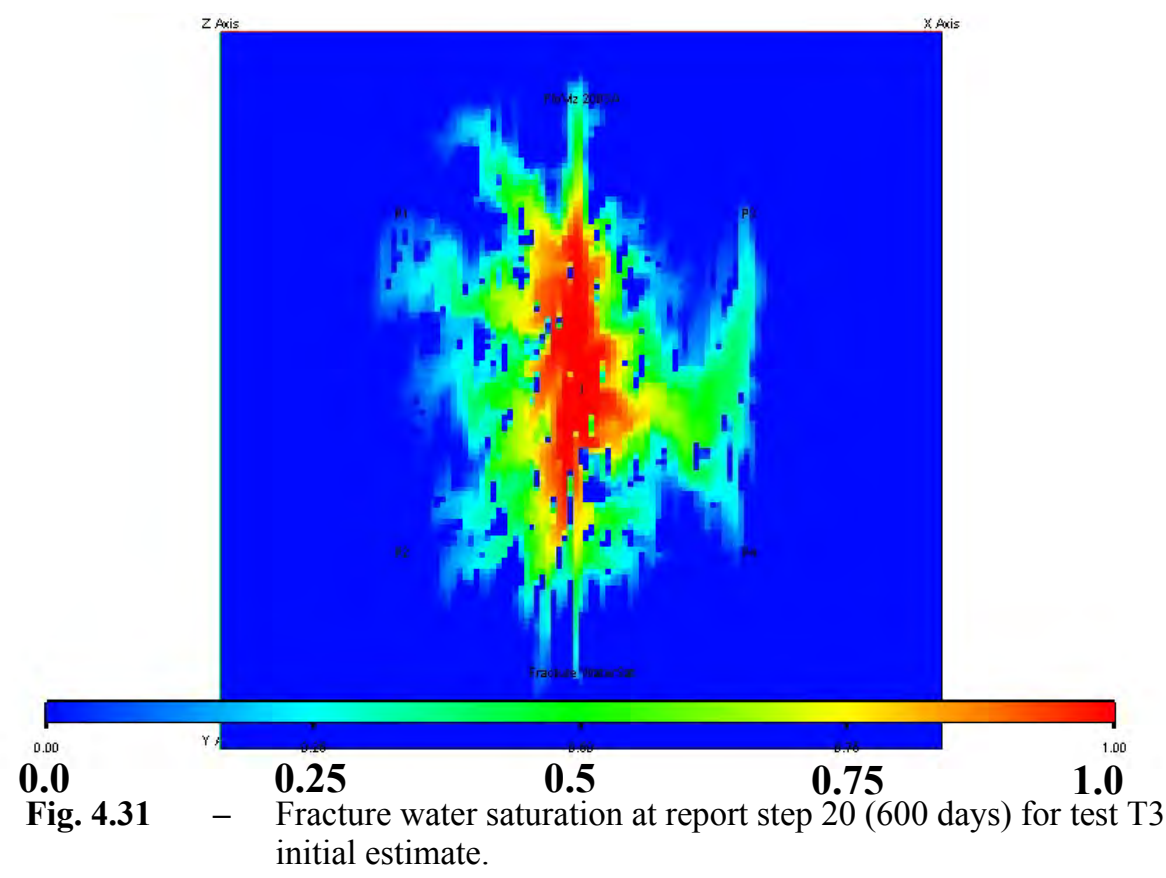




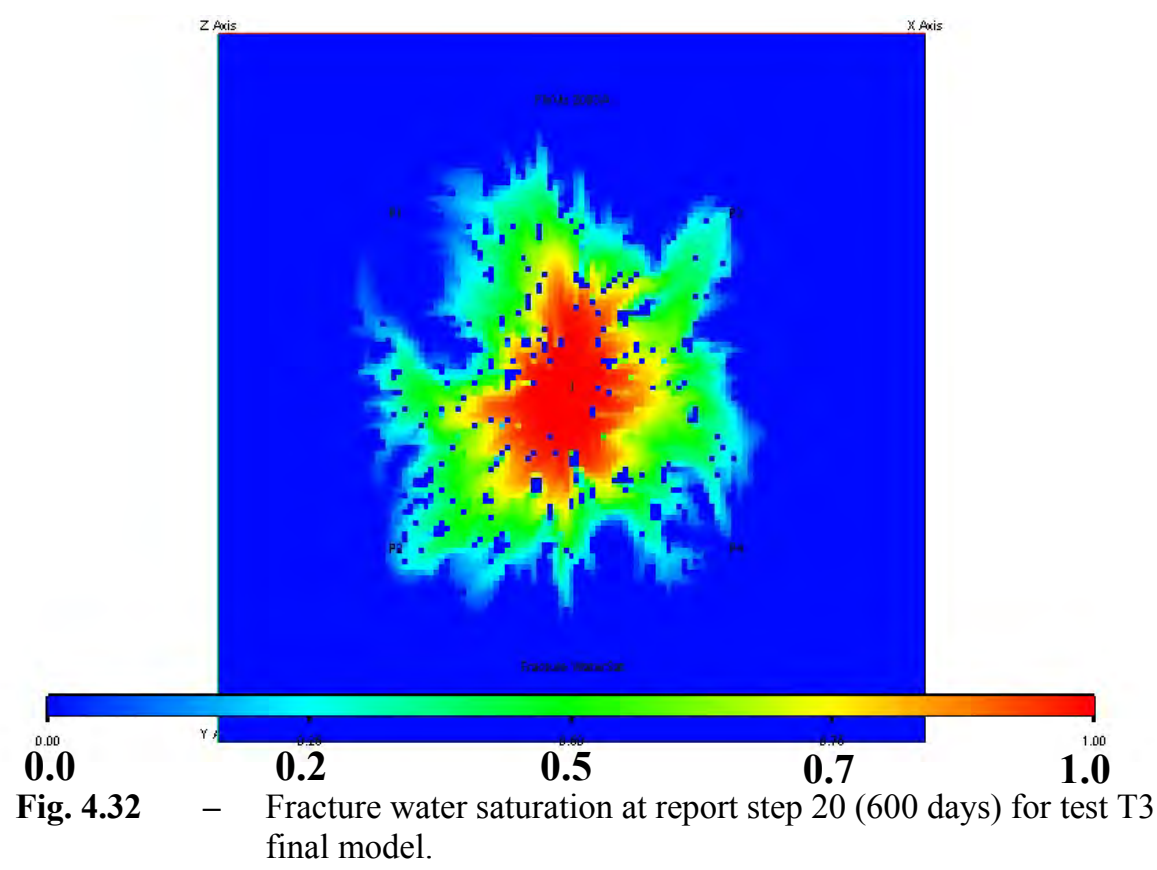

\subsubsection{Seismic Attribute Plots}

Figs. 4.33 to 4.35 show seismic velocity plots for the base model (Fig. 4.33), the initial model estimate (Fig. 4.34) and the optimized model (Fig. 4.35). The vertical axis is the quasi-p wave velocity of propagation. The horizontal axis in the azimuth of energy propagation. Quasi-P wave velocity versus azimuth is plotted for all cell blocks prior to averaging for computation of the objective function. Average values for peak-to-peak quasi-P wave modulation amplitude and direction of maximum quasi-P wave velocity are indicated on each plot. The seismic velocity anisotropy in Fig. 4.33 shows dispersion in both the peak-to-peak amplitude of quasi-P wave velocity modulation and direction of maximum quasi-P wave velocity as compared to the same attribute from the single fracture set model of T2 (Fig. 4.13). This is due to the existence of two separate fracture sets. Both the average velocity modulation amplitude and the maximum quasi-P wave velocity direction (phase angle) show the averaging effect of the effective media model process. This results in an apparent $\mathrm{P}_{32}$ fracture intensity that is lower than the sum of the actual $\mathrm{P}_{32}$ intensity values for the two fracture sets, and a maximum quasi-P wave 
velocity direction which is between the two fracture system trends. The maximum quasi$\mathrm{P}$ wave velocity direction in this medium is approximately 24 degrees true azimuth. Average velocity modulation amplitude is approximately 0.24 . Fig. 4.30 shows the seismic anisotropy attribute for the initial model estimate. This plot shows the effect of the erroneous initial estimates which incorrectly increase the alignment of the two fracture sets. This false alignment has the effect of adding the contribution of the two fracture sets more constructively in the elastic effective media modeling process, resulting in less dispersion in both the maximum quasi-P wave velocity direction and average velocity modulation amplitude. For this initial estimate the average peak-to-peak velocity modulation amplitude has increased to 0.57 and the maximum quasi-P wave velocity direction has shifted to approximately 0 degrees. Seismic attributes for the final model shown in Fig. 4.35 show convergence of the seismic attributes close to the base model parameters as a result of the optimization process.

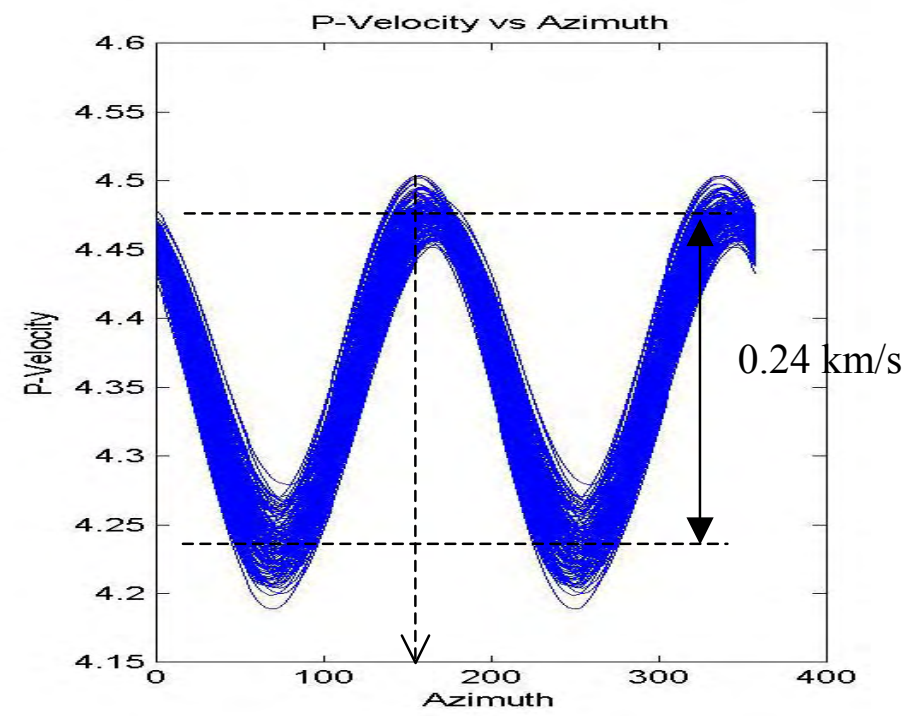

Fig. 4.33 - Test T3 base model quasi-P wave velocity modulation amplitude and maximum quasi-P wave velocity direction. 


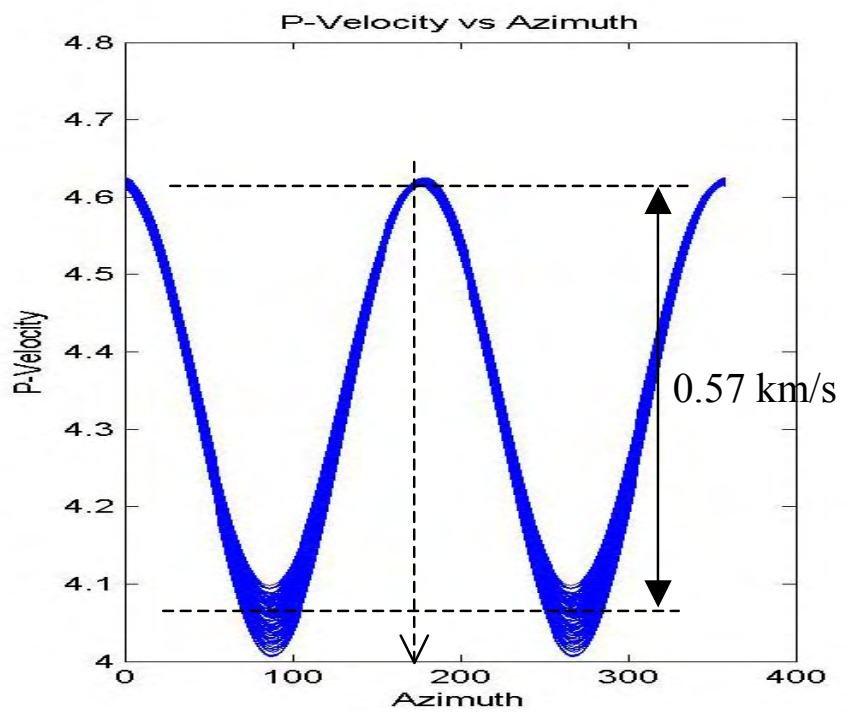

Fig. 4.34 - $\quad$ Test T3 initial estimate quasi-P wave velocity modulation amplitude and maximum quasi-P wave velocity direction.

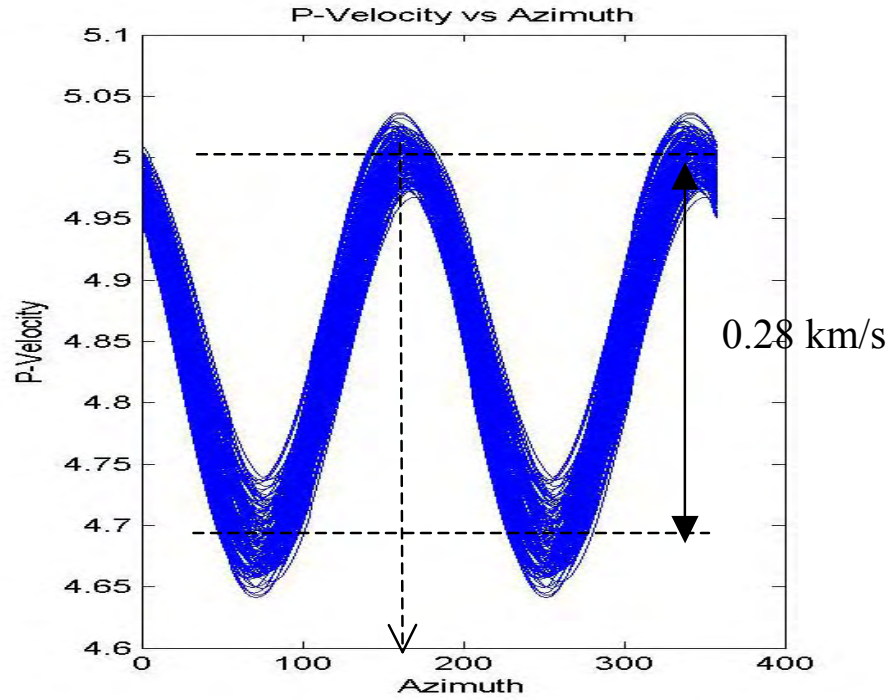

Fig. 4.35 - Test T3 final quasi-P wave velocity modulation amplitude and maximum quasi-P wave velocity direction. 


\subsection{Test T4 - Two Fracture Sets in a Dolomite Matrix}

The last test of the new method was conducted on a reservoir model with a two nonparallel fracture sets having the population statistics listed in Table 4.14 and elastic and petrophysical properties representative of a fractured dolomite reservoir. Reservoir matrix properties are listed in Tables 4.15 and 4.16. Only combined objective function optimization was performed on this model.

Table 4.14 - Test T4 base model fracture distribution parameters.

\begin{tabular}{|l|c|c|}
\hline \multicolumn{1}{|c|}{ Set } & Set 1 & Set 2 \\
\hline Trend (Geographical) & $\mathrm{N}[335,10]$ & $\mathrm{N}[45,10]$ \\
\hline $\mathbf{P}_{\mathbf{3 2}}$ Intensity (1/m) & 0.1 & 0.15 \\
\hline Length (m) & $\mathrm{LN}[50,3]$ & $\mathrm{LN}[50,3]$ \\
\hline Height (m) & 60 & 60 \\
\hline Transmissivity (m²) & $\mathrm{LN}[8 \mathrm{e}-04,4.3 \mathrm{e}-05]$ & $\mathrm{LN}[8 \mathrm{e}-04,4.3 \mathrm{e}-05]$ \\
\hline
\end{tabular}

Table 4.15 - Test T4 reservoir properties.

\begin{tabular}{|l|c|}
\hline Matrix Porosity & 0.13 \\
\hline Matrix Permeability & $0.1 \mathrm{mD}$ \\
\hline Fracture Porosity & 0.015 \\
\hline
\end{tabular}

Table 4.16 - Test T4 elastic modeling parameters.

\begin{tabular}{|l|c|}
\hline Matrix P-wave Velocity & $5390 \mathrm{ft} / \mathrm{sec}$ \\
\hline Matrix Shear-wave Velocity & $2970 \mathrm{ft} / \mathrm{sec}$ \\
\hline Matrix Density & $2950 \mathrm{~kg} / \mathrm{m}^{3}$ \\
\hline Overburden P wave Velocity & $4670 \mathrm{ft} / \mathrm{sec}$ \\
\hline Overburden S wave Velocity & $3060 \mathrm{ft} / \mathrm{sec}$ \\
\hline Overburden Density & $2510 \mathrm{~kg} / \mathrm{m}^{3}$ \\
\hline Incidence angle & $30 \mathrm{degrees}$ \\
\hline
\end{tabular}




\subsubsection{Parameter Updates}

Parameter error history for this optimization test is listed in Table 4.17 and shown in Figs. 4.36 and 4.37. Parameter perturbations and a priori model weights for each iteration are listed in Tables D.5 and D.6 in Appendix D. The initial estimates of fracture trend for both fracture sets were the same as used for optimization test T3. The initial estimates of $\mathrm{P}_{32}$ intensity were 0.05 and 0.1 away from the base model values. Fig. 4.36 depicting trend error shows that trend for fracture set 2 experienced a large convergent change at the first update step, followed by variation during intermediate update steps and further convergence to within 1 degree of the base case at later update steps. Trend error for fracture set 1 initially diverged. In order to achieve convergence of this parameter it was necessary to reduce the bottom hole pressure noise standard deviation and observation weighting to 1 psi. After this change was made fracture set 1 trend experienced dramatic convergence at the third update step to within 1 degree of the base case. $\mathrm{P}_{32}$ intensity error for fracture set 1 showed steady monotonic convergence to 0.001. $\mathrm{P}_{32}$ intensity error for fracture set 2 diverged at the first update, followed by steady convergence to 0.001 in following update steps.

Table 4.17 - Summary of absolute errors for fracture set trend and $\mathrm{P}_{32}$ intensity at each update step. Step 0 is the initial estimate.

\begin{tabular}{|r|r|r|r|r|}
\cline { 2 - 5 } \multicolumn{1}{c|}{} & \multicolumn{4}{c|}{ Parameter Error } \\
\hline Iteration & Trend (1) & \multicolumn{1}{|c|}{$\mathbf{P}_{\mathbf{3 2}} \mathbf{( 1 )}$} & \multicolumn{1}{c|}{ Trend (2) } & $\mathbf{P}_{\mathbf{3 2}} \mathbf{( 2 )}$ \\
\hline $\mathbf{0}$ & 20.0 & 0.100 & 20.0 & 0.050 \\
\hline $\mathbf{1}$ & 21.4 & 0.090 & 3.4 & 0.111 \\
\hline $\mathbf{2}$ & 22.0 & 0.061 & 2.5 & 0.080 \\
\hline $\mathbf{3}$ & 3.1 & 0.041 & 4.6 & 0.054 \\
\hline $\mathbf{4}$ & 0.4 & 0.029 & 5.4 & 0.043 \\
\hline $\mathbf{5}$ & 1.1 & 0.020 & 4.9 & 0.029 \\
\hline $\mathbf{6}$ & 1.5 & 0.001 & 0.6 & 0.019 \\
\hline $\mathbf{7}$ & 1.3 & 0.002 & 0.2 & 0.005 \\
\hline $\mathbf{8}$ & 0.7 & 0.001 & 0.8 & 0.001 \\
\hline
\end{tabular}




\section{Test T4 - Trend Error}

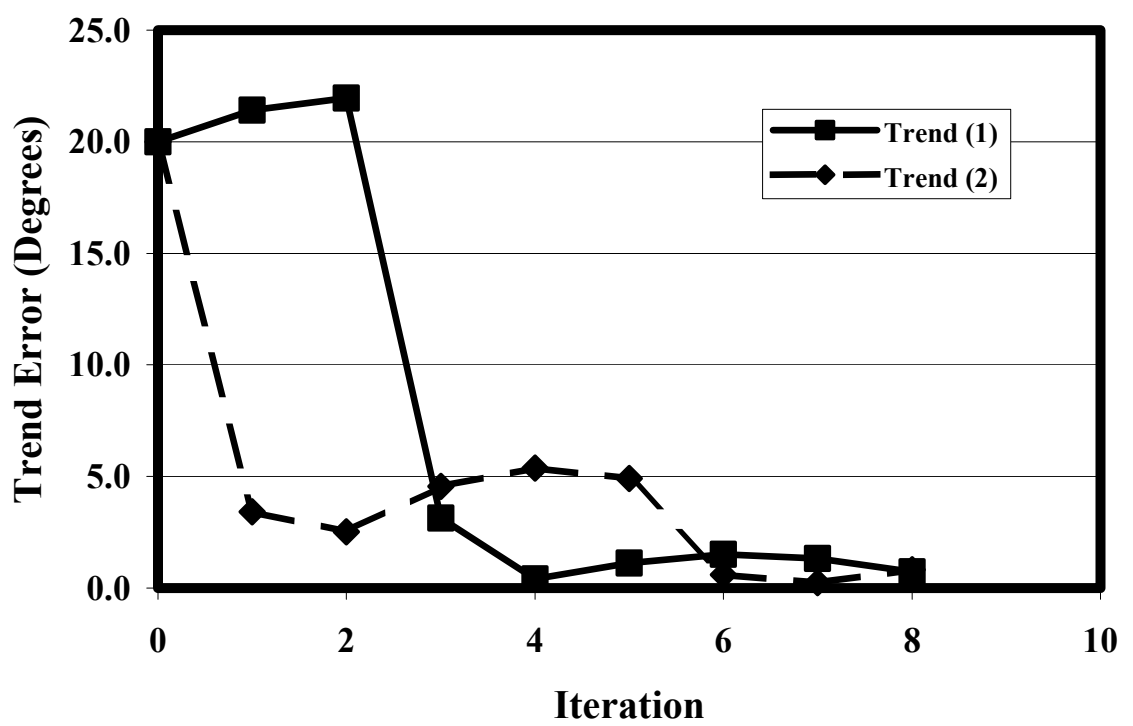

Fig. 4.36 - Graph of absolute error in fracture set trend versus iteration for test T4. Iteration 0 is the initial estimate.

\section{Test T4 - P32 Error}

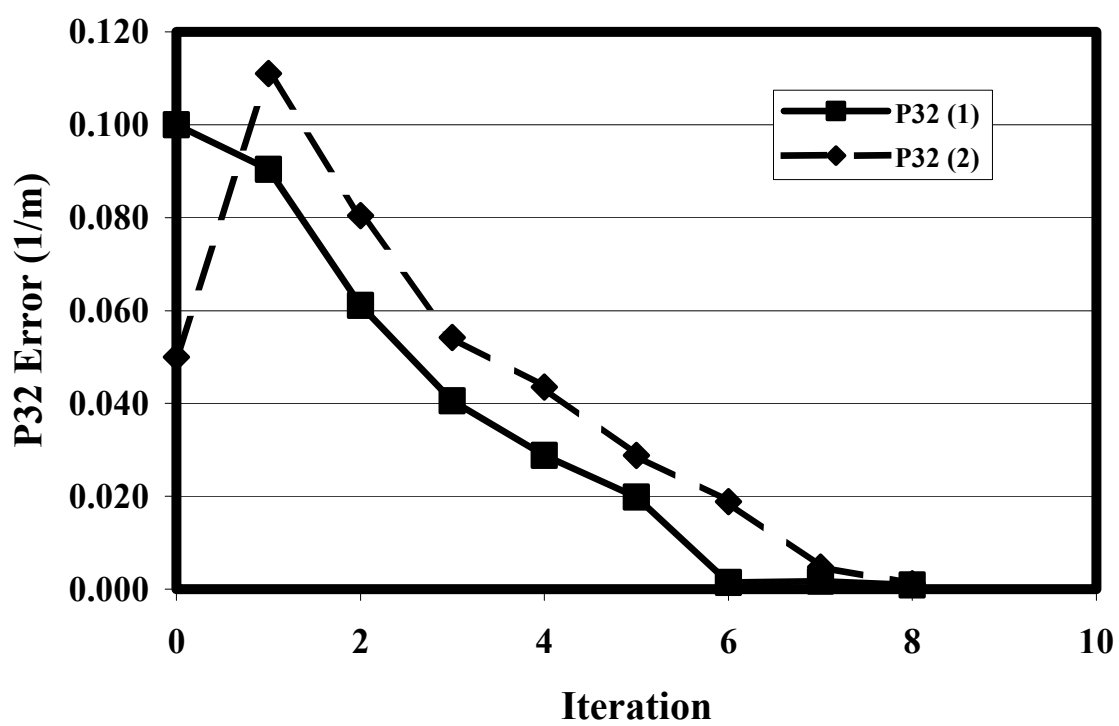

Fig. 4.37 - Graph of absolute error in fracture set $\mathrm{P}_{32}$ intensity versus iteration for test $\mathrm{T} 4$. Iteration 0 is the initial estimate. 


\subsubsection{Objective Function and Observation Residuals}

The average normalized residuals for the 10 update trials of the combined objective function optimization test are listed in Table 4.18 and illustrated in Fig. 4.38. The objective function and partial residuals for bottom hole pressure, oil production rate, and seismic attributes are shown separately. The dominant feature in Fig. 4.38 is the large spike in bottom hole pressure residual and total residual associated with the divergent behavior of fracture set 1 trend. After reduction of the observation uncertainty the significant improvement in convergence of fracture set 1 trend seen in Fig. 4.17 also results in marked reduction of the bottom hole pressure and total residuals in Fig. 4.18. As was the case in previous tests, the bottom hole pressure residual is seen to be larger than that for either seismic attributes or oil production rate. Further, in this test the magnitude of the seismic residual compared to the bottom hole pressure residual is smaller than in test T3. This difference in the seismic component for the same fracture model must be the result of the different elastic properties of the reservoir. The initial seismic residual is approximately one quarter the magnitude of the bottom hole pressure residual. Also, similar to observations in previous cases, the oil production rate residual is the lowest. Bottom hole pressure and seismic attribute residuals show fairly steady convergence through the optimization process. The oil production rate residual shows little convergent behavior and has an unexplained spurious value at the third update step. All residuals are listed in Tables D.9a through D.9h in Appendix D.

Table 4.18 - Summary of objective function and normalized residual errors by observation type for fracture set trend and $\mathrm{P}_{32}$ intensity at each iteration. Iteration 0 is the initial estimate.

\begin{tabular}{|r|r|r|r|r|}
\cline { 3 - 5 } \multicolumn{2}{c|}{} & \multicolumn{3}{c|}{ RMS Residuals } \\
\hline Iteration & \multicolumn{1}{c|}{$\mathbf{Q}$} & \multicolumn{1}{c|}{ BHP } & \multicolumn{1}{c|}{ OPR } & \multicolumn{1}{c|}{ SEIS } \\
\hline $\mathbf{0}$ & 453.26 & 5.39 & 1.58 & 20.58 \\
\hline $\mathbf{1}$ & 216.76 & 5.45 & 2.06 & 13.52 \\
\hline $\mathbf{2}$ & 141.28 & 4.27 & 1.54 & 10.99 \\
\hline $\mathbf{3}$ & 105.08 & 0.23 & 8.97 & 4.96 \\
\hline $\mathbf{4}$ & 18.13 & 1.00 & 0.61 & 4.09 \\
\hline $\mathbf{5}$ & 11.03 & 0.61 & 0.90 & 3.14 \\
\hline $\mathbf{6}$ & 1.10 & 0.32 & 0.55 & 0.83 \\
\hline $\mathbf{7}$ & 1.57 & 0.81 & 0.55 & 0.79 \\
\hline
\end{tabular}


Test T4 Objective Function and Residuals

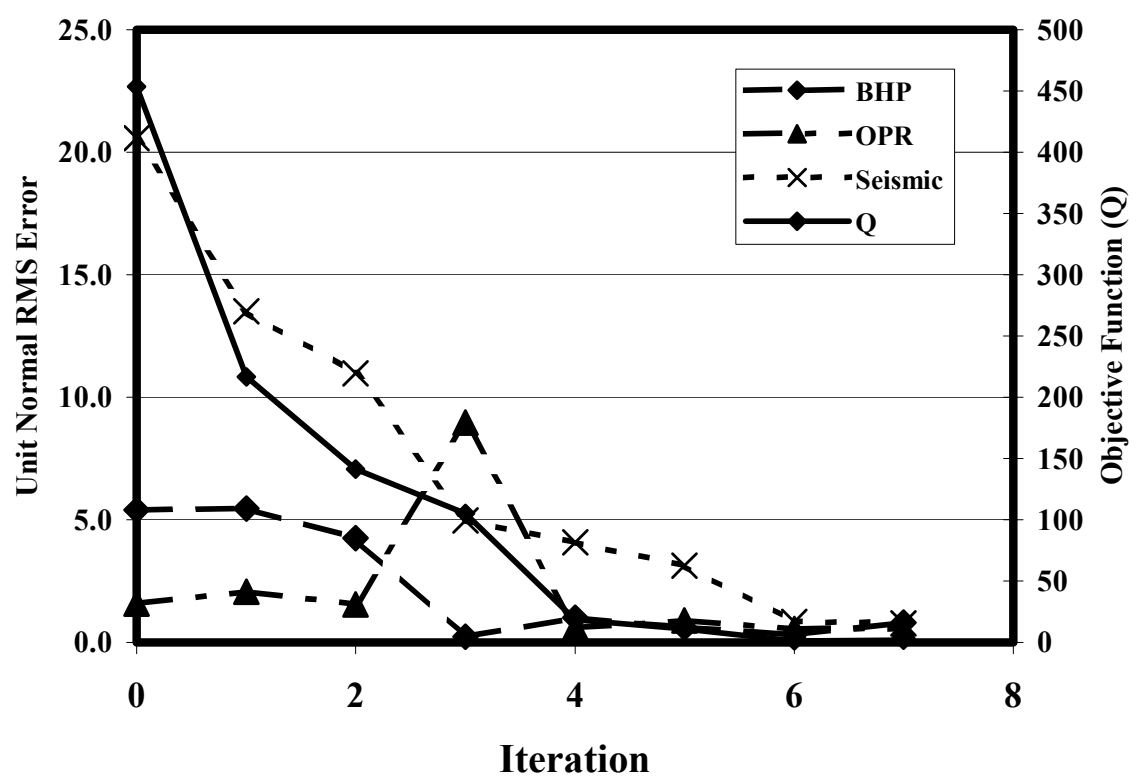

Fig. 4.38 $\quad-$ Graph of objective function and normalized residual errors by observation type for fracture set trend and $\mathrm{P}_{32}$ intensity at each iteration. Iteration 0 is the initial estimate.

\subsubsection{Sensitivity Coefficients}

Fig. 4.39 shows a sample of Jacobian matrix sensitivity coefficients computed using Eq. 3.14 for the initial estimate model state of the multiple fracture set optimization test T4. Table 4.6 contains a list of observation numbers and descriptions. These sensitivity coefficients are the gradients of the observations with respect to the unknown parameters and carry information regarding the relative contribution of each observation to the parameter update. Observations with large positive or negative sensitivity coefficients will have a larger impact on the parameter update than those with lower sensitivity coefficients. Except for the injector and producer P1 bottom hole pressure, these sensitivity coefficients show an overall reduced sensitivity of trend and $\mathrm{P}_{32}$ intensity to all production observations. Similar to test $\mathrm{T} 3, \mathrm{P}_{32}$ intensity and trend for both fracture sets show sensitivity to the velocity modulation amplitude while only the trend of 
fracture set 1 shows significant sensitivity to the maximum quasi-P wave velocity direction attribute. Unlike test $\mathrm{T} 3$, the sensitivity coefficients are not all of opposite in sign between fracture sets 1 and 2 . All sensitivity coefficients for the combined objective function test are listed shown in Figs. D.4a to D.4h in Appendix D.
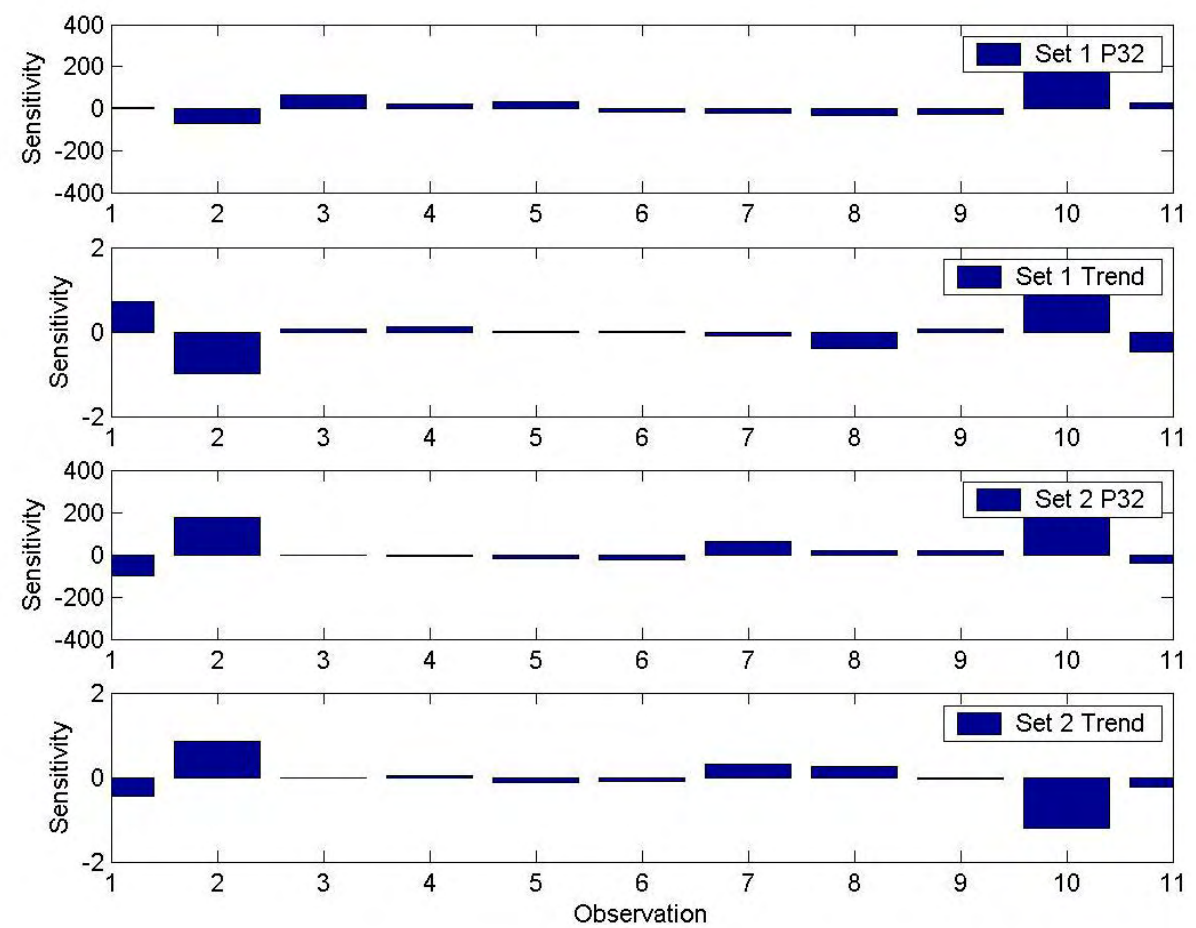

Fig. 4.39 - Parameter sensitivity coefficients by observation number for the multiple fracture set initial model combined objective function case T4.

\subsubsection{Fracture Distribution Maps}

Maps of the fracture system for the base case, initial estimate, and optimized model are shown in Figs. 4.40 to 4.42. Fig. 4.40 shows the fracture distribution for the base model. Fig. 4.41 shows the fracture distribution for the initial estimate. Fig. 4.42 shows the fracture distribution for the final model. Comparison of Figs. 4.40 and 4.42 illustrate the successful convergence of the model back to the base case. 


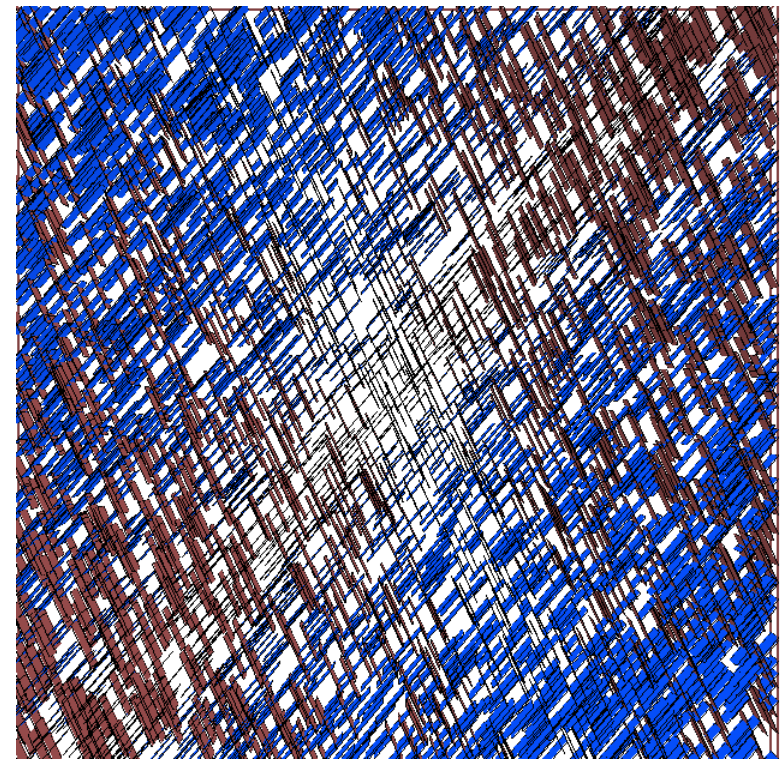

Fig. 4.40 - $\quad$ Test T4 base model fracture distribution.

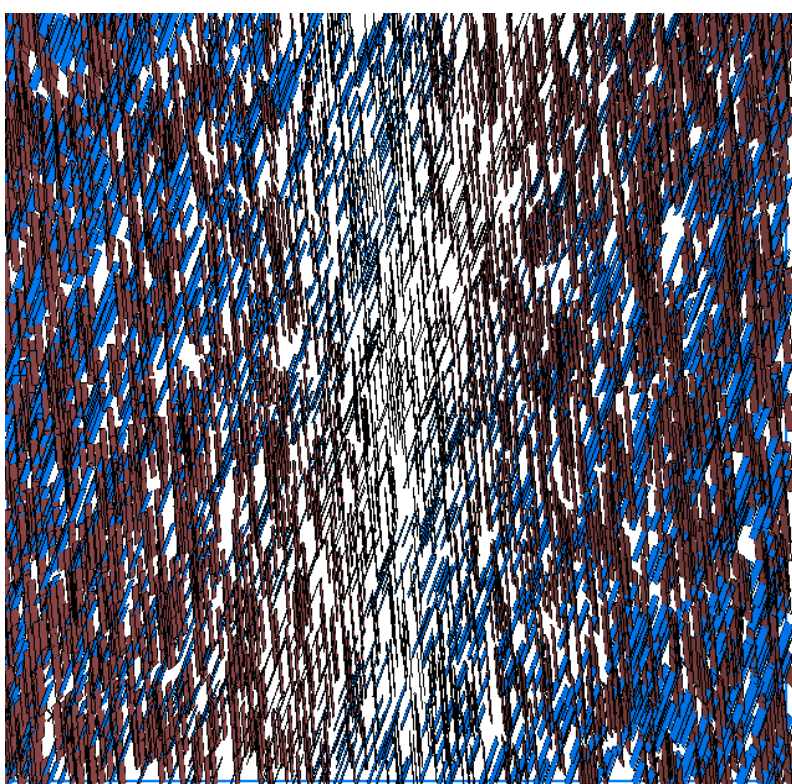

Fig. 4.41 $\quad-\quad$ Test T4 initial estimate fracture distribution fracture distribution. 


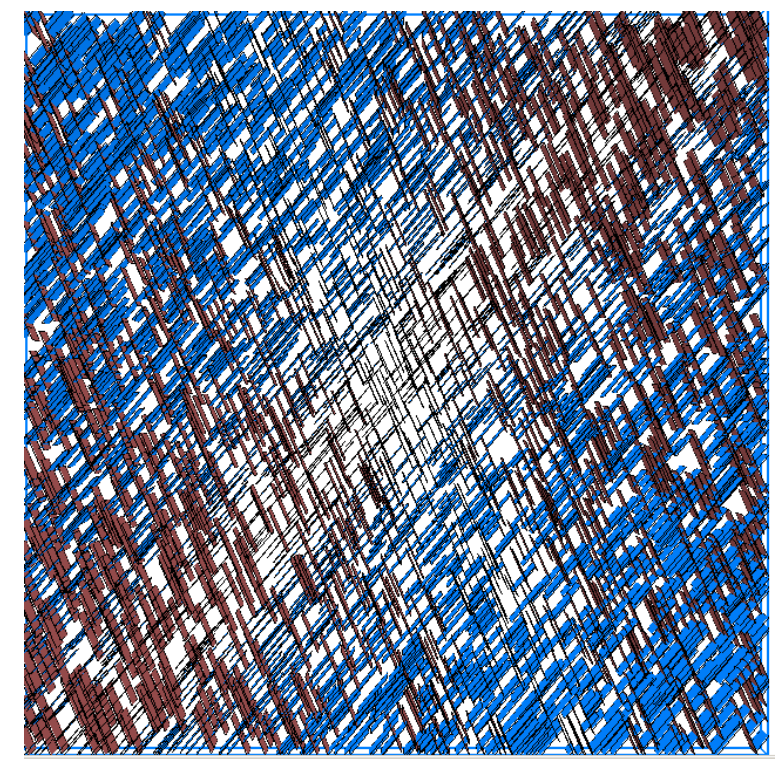

Fig. 4.42 - Test T4 final model fracture distribution.

\subsubsection{Water Saturation Maps}

Figs. 4.43 to 4.45 show water saturation in the fracture system at the final report step for the base model (Fig. 4.43), the initial model estimate (Fig. 4.44), and the optimized model (Fig. 4.45). Fracture water saturation for the base model shows strong symmetry with less linear character than observed in the single fracture set case seen in test $\mathrm{T} 2$. The water saturation front in this test shows a different character than the same report step in test T3. The fracture system water saturation distribution in test T4 is less concentrated and is more disperse than the equivalent display from test T3. This is possibly due to the difference in matrix permeability between the two models. The lower matrix permeability in test T4 would result in more water imbibition into the matrix, thus lowering fracture water saturation and causing more dispersion of water saturation in the fracture system. This also results in more general symmetry in the water distribution. This indirect effect of matrix permeability on fracture water saturation may be partially responsible for the less well behaved convergence of this model. Fracture and matrix water saturation maps for report steps 5 (150 days), 10 (300 days), 15 (450 days), and 20 (600 days) are depicted in Figs. D.6 to D.13 located in Appendix D. 


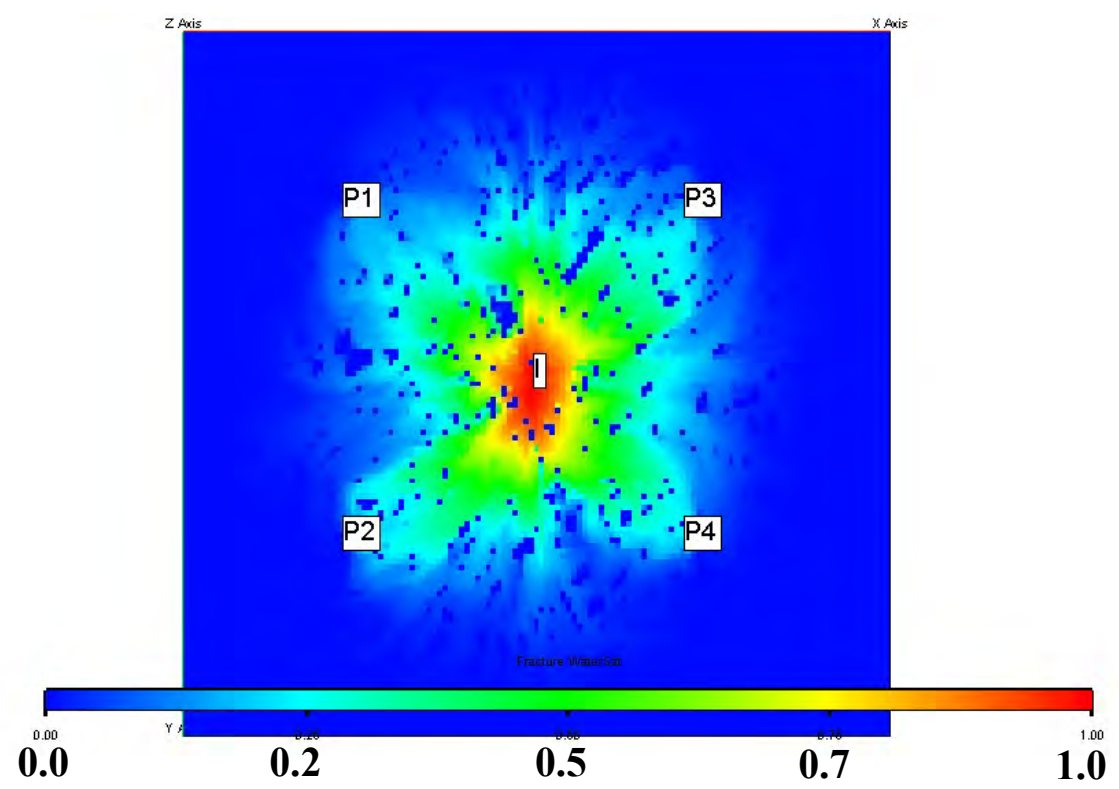

Fig. 4.43 - Fracture water saturation at report step 20 (600 days) for test T4 base model.

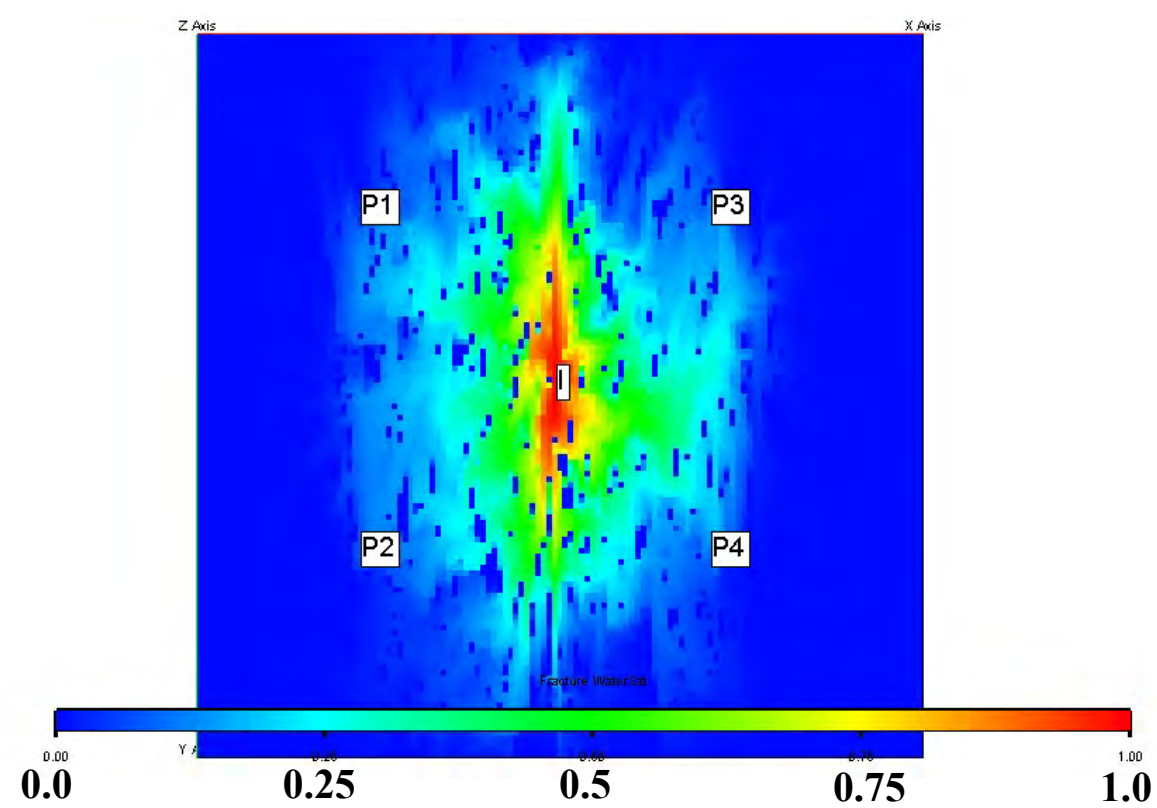

Fig. 4.44 - Fracture water saturation at report step 20 (600 days) for test T4 initial estimate. 


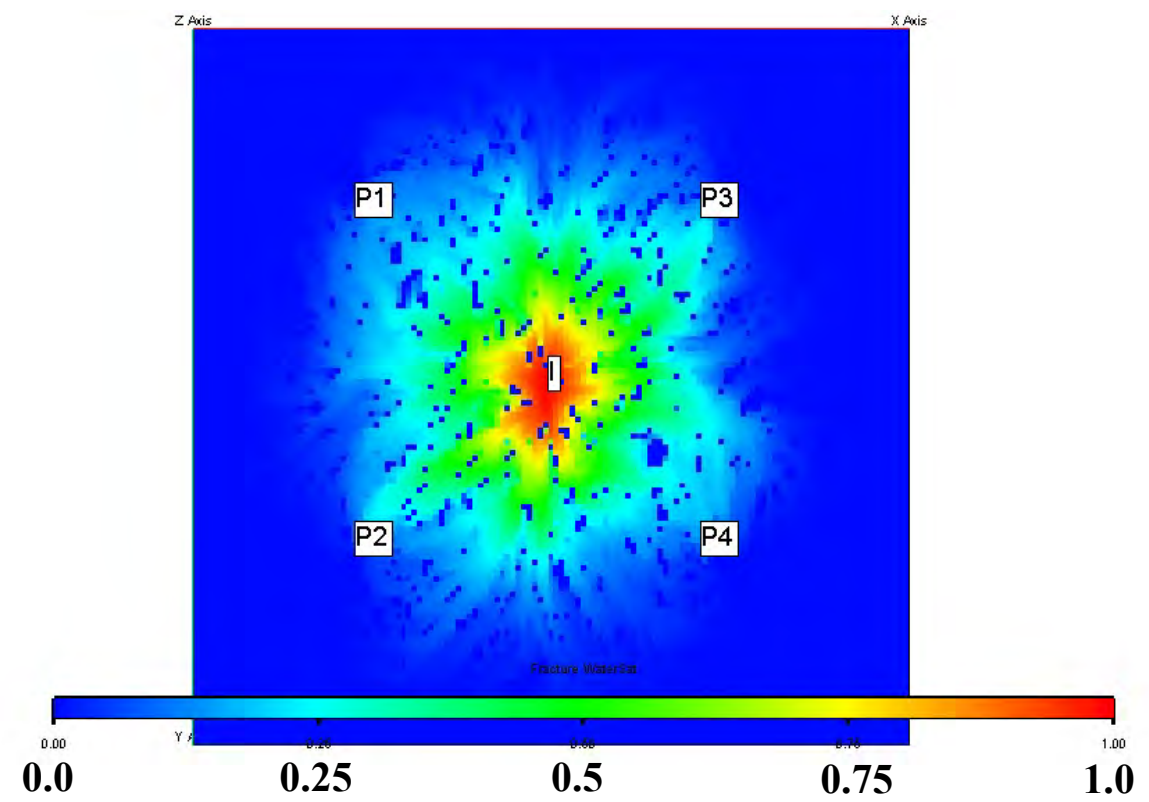

Fig. 4.45 - Fracture water saturation at report step 20 (600 days) for test T4 final model.

\subsubsection{Seismic Attribute Plots}

Figs. 4.46 to 4.48 show seismic velocity plots for the base model (Fig. 4.46), the initial model estimate (Fig. 4.47) and the optimized model (Fig. 4.48). The vertical axis is the quasi-p wave velocity of propagation. The horizontal axis in the azimuth of energy propagation. Quasi-P wave velocity versus azimuth is plotted for all cell blocks prior to averaging for computation of the objective function. Average values for peak-to-peak quasi-P wave modulation amplitude and direction of maximum quasi-P wave velocity are indicated on each plot. The seismic velocity anisotropy in Fig. 4.46 shows dispersion in both the peak-to-peak amplitude of quasi-P wave velocity modulation and the direction of maximum quasi-P wave velocity similar to test T3. The average velocity peak-to-peak modulation amplitude seen here is higher than observed in test T3 due to the difference in elastic properties of the medium. Average peak-to-peak velocity modulation amplitude for the base case is approximately $20 \%$ higher than that seen in test T3 for the sandstone matrix. The ratio of average peak-to-peak velocity modulation amplitude between the initial estimate and base case is $2.4: 1$ as opposed to $2: 1$ for test 
T3. These results indicate a higher degree of sensitivity of this observation to changes in geometrical parameters in the elastically stiffer dolomite matrix. Fig. 4.48 for the final model shows convergence of the seismic attribute to values close to the base case. Seismic attributes for all iterations are depicted in Figs. D.16 and D.17 located in Appendix D.

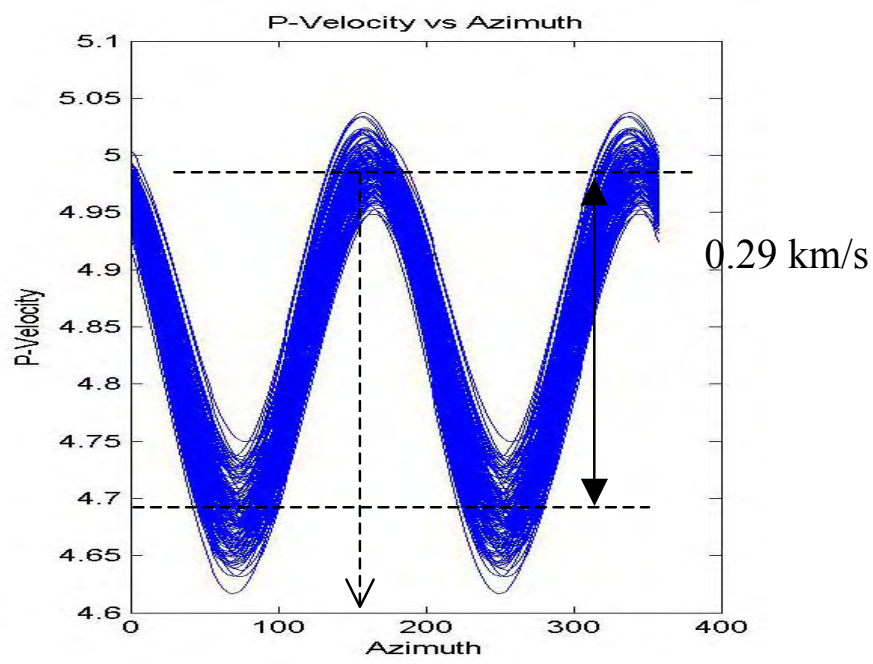

Fig. 4.46 - Base model quasi-P wave velocity modulation amplitude and maximum quasi-P wave velocity direction.

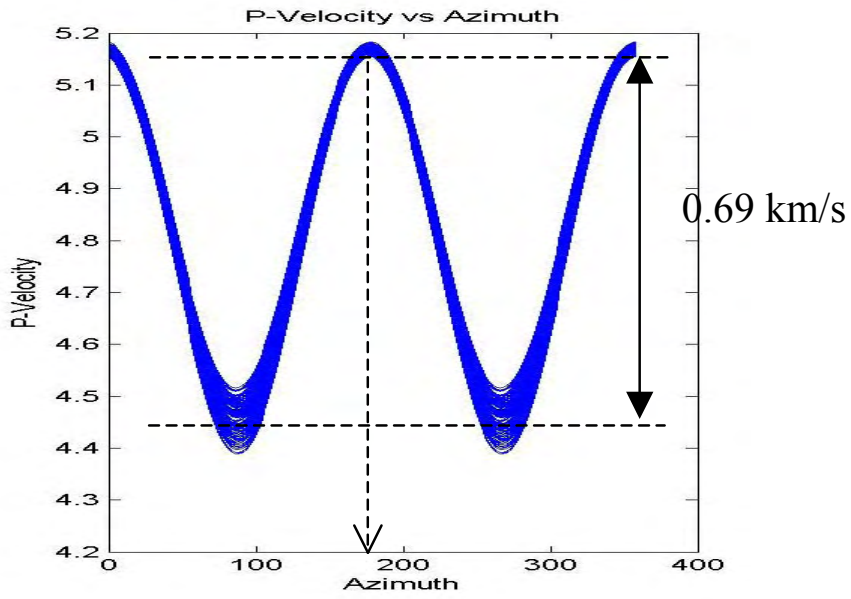

Fig. 4.47 - Initial estimate quasi-P wave velocity modulation amplitude and maximum quasi-P wave velocity direction. 


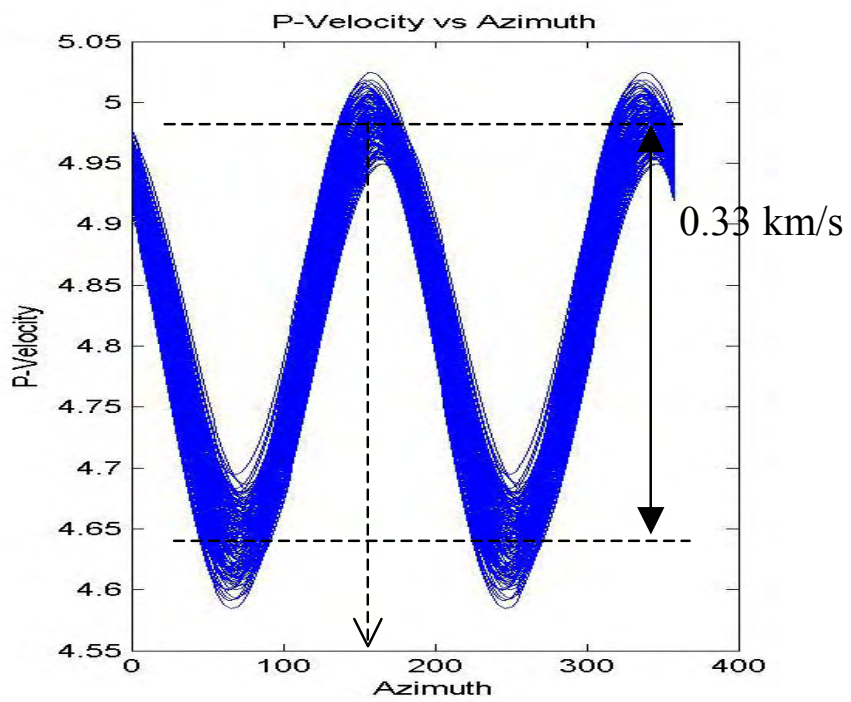

Fig. 4.48 - Final quasi-P wave velocity modulation amplitude and maximum quasi-P wave velocity direction.

\subsection{Summary}

In this chapter we presented the results of three optimization tests representing different fracture systems and reservoir lithologies. The results presented here include verification of the new method and a comparison of a parallel optimization procedure using production data only in the optimization objective function. The optimization performance for these tests were presented in terms of tabular and graphical representation of $\mathrm{P}_{32}$ intensity, trend, residual errors for each update step, and sensitivity coefficients for the initial estimate model state of each test case. The results of the new method were also illustrated through graphical display of fracture distributions and observations at selected steps in the optimization process representing the base case, the initial model estimate, and the final optimized model. These test results indicate that the new method of integrating seismic anisotropy attributes not only speeds and stabilizes convergence in models having a single fracture, but also provides the additional information required to resolve multiple fracture sets. These tests also demonstrate that the stability of the new process may be dependent upon the reservoir model parameters. 
One example of such instability seen in test T4 was resolved through reduction of the observation noise on the bottom hole observation, such as may be achieved through use of an improved measuring method or device. These test results will be further discussed in Chapter VI. The limitations on the process imposed by parameter sensitivity and the resulting requirements for model preconditioning will be investigated in Chapter V. 


\section{CHAPTER V}

\section{SENSITIVITY ANALYSIS}

In this chapter we present the results of sensitivity analysis of the objective function to various model parameters. This chapter will include:

- A discussion of the method used.

- Sensitivity analysis with respect to the inversion target parameters fracture set trend and $\mathrm{P}_{32}$ intensity.

- Sensitivity analysis with respect to three general categories of parameters which are assumed to be "known" during the inversion.

- A discussion of the results.

This sensitivity analysis is based on an investigation of the residual errors discussed in section 3.8 of Chapter III, computed at the initial model state (M1) of test case T3. The three categories of parameters investigated are;

- Hydraulic parameters - matrix permeability, shape factor, and the block size for effective permeability upscaling (REV volume).

- Elastic parameters - fracture compliance, radius for effective elastic media modeling (REV volume), and the angle of incidence for azimuthally varying quasi-P wave velocity computation.

- Geometrical parameters - reservoir thickness.

For direct comparison, the sensitivity of the objective function to the inversion target parameters of fracture set trend and $\mathrm{P}_{32}$ intensity are presented first. 


\subsection{Calculation Method}

This sensitivity analysis was performed by computing RMS observation residuals using Eqs. 3.22 through 3.25 with systematic perturbations in several model parameters. These perturbations are centered on a representative model state which will be referred to as the 'reference' model state. Sensitivities will be displayed in graphical form by plotting the normalized residual difference versus percent parameter perturbation. As such, these graphical present information about the gradient of the objective function with respect to the inversion parameters.

Residual differences were computed as;

$$
\Delta \mathrm{R}_{\mathrm{n}}(\mathrm{i})=\mathrm{R}\left(\mathrm{M} 1, \mathrm{x}_{\mathrm{n}}^{*}(\mathrm{i})\right)-\mathrm{R}(\mathrm{M} 1)
$$

where;

\begin{tabular}{|c|c|c|}
\hline$\Delta \mathrm{R}_{\mathrm{n}}^{\prime}(\mathrm{i})$ & $=$ & $\begin{array}{l}\text { Normalized residual difference for the } \mathrm{n}^{\text {th }} \text { parameter at the } \\
\mathrm{i}^{\text {th }} \text { perturbation of the } n \text {th parameter }\end{array}$ \\
\hline $\mathrm{R}^{\prime}(\mathrm{M} 1)$ & $=$ & $\begin{array}{l}\text { Total residual for iteration } \mathrm{M} 1 \mathrm{using} \text { the reference } \\
\text { parameter set }\end{array}$ \\
\hline$\Delta \mathrm{x}_{\mathrm{n}}(\mathrm{i})$ & $=$ & $i^{\text {th }}$ perturbation of parameter $x_{n}$ \\
\hline $\mathrm{R}^{\prime}\left(\mathrm{M} 1, \mathrm{x}_{\mathrm{n}}^{\mathrm{o}}+\Delta \mathrm{x}_{\mathrm{n}}(\mathrm{i})\right)$ & $=$ & $\begin{array}{l}\text { Total residual for iteration } \mathrm{M} 1 \mathrm{using} \text { the reference } \\
\text { parameter set } \mathrm{x}^{\mathrm{o}} \text { and the } \mathrm{i}^{\text {th }} \text { perturbation of the } \mathrm{n}^{\text {th }} \\
\text { parameter }\end{array}$ \\
\hline
\end{tabular}

Percent parameter perturbation $\mathrm{x}^{*}$ for the $\mathrm{i}^{\text {th }}$ perturbation of the $\left(\mathrm{n}^{\text {th }}\right)$ parameter was computed as;

$$
\mathrm{x}^{*}{ }_{\mathrm{n}}(\mathrm{i})=100 *\left(\mathrm{x}^{\mathrm{o}}{ }_{\mathrm{n}}+\Delta \mathrm{x}_{\mathrm{n}}(\mathrm{i})\right) / \mathrm{x}^{\mathrm{o}}{ }_{\mathrm{n}}
$$


In each case only the portion of the objective function affected by the parameter change was recomputed. For example, since variation in thickness effected both the elastic and hydraulic properties of the model both production and elastic data were recomputed. The resulting residual errors are then compared to those for the reference model state and presented in tabular and graphical format as differences from the reference model. Total residual error and observation type residual error components will be presented. The model state selected to be used as the reference for this analysis is the initial estimate (M1) in test T3. Reference model parameters and ranges of perturbations are listed in Table 5.1.

Table 5.1 - $\quad$ Reference model parameters and sensitivity test parameter perturbation limits.

\begin{tabular}{|l|c|c|c|}
\hline \multicolumn{1}{|c|}{ Parameter } & Reference & Min. & Max. \\
\hline Fracture Set 1 Trend (degrees) & 130 & 110 & 150 \\
\hline Fracture Set 1 P32 Intensity (1/m) & 0.15 & 0.1 & 0.2 \\
\hline Matrix Permeability (mD) & 10 & 0.1 & 100 \\
\hline Dual Porosity Shape Factor (ft $\left.{ }^{2}\right)$ & 0.08 & 0.01 & 0.5 \\
\hline Hydraulic REV Radius (m) & 3 & 10 & 50 \\
\hline Fracture Compliance (m/Pa) & $3.0 \mathrm{e}-11$ & $2.0 \mathrm{e}-11$ & $3.5 \mathrm{e}-11$ \\
\hline Incidence Angle (degrees) & 30 & 20 & 36 \\
\hline Elastic REV Radius (m) & 100 & 20 & 200 \\
\hline Reservoir Thickness (ft) & 100 & 60 & 262 \\
\hline
\end{tabular}

In the following sections model parameter perturbations are expressed in terms of percentages of the reference model parameter values. 


\subsection{Sensitivity to Trend and $\mathbf{P}_{32}$ Intensity}

In order to first establish the sensitivity of the objective function to the inversion target parameters sensitivity tests were first performed on trend and $\mathrm{P}_{32}$ intensity of one of the two fracture sets. The observations made with regard to sensitivity will be assumed to be representative of both fracture sets. The results of these analyses are summarized in Tables 5.2 and 5.3 and Fig. 5.1 below. The sign of the parameter perturbations indicate the magnitude of the perturbed parameter value with respect to reference value. Zero residual difference values indicate no perturbation from the reference parameter value. Negative residual difference values indicate a negative change in the value of the residual with the parameter perturbation.

Table 5.2 - Sensitivity of objective function to fracture set 2 trend.

\begin{tabular}{|r|r|r|r|r|}
\cline { 2 - 5 } \multicolumn{1}{c|}{} & \multicolumn{4}{c|}{ Unit Residual Difference from Ref. } \\
\hline $\begin{array}{r}\text { \% Parameter } \\
\text { Perturbation }\end{array}$ & \multicolumn{1}{c|}{ TOTAL } & \multicolumn{1}{c|}{ BHP } & \multicolumn{1}{c|}{ OPR } & \multicolumn{1}{c|}{ SEIS } \\
\hline $\mathbf{- 1 5 . 4}$ & -7.68 & -2.25 & -1.95 & -7.30 \\
\hline $\mathbf{- 7 . 7}$ & -4.87 & -2.20 & -2.95 & -3.91 \\
\hline $\mathbf{0 . 0}$ & 0.00 & 0.00 & 0.00 & 0.00 \\
\hline $\mathbf{7 . 7}$ & 3.47 & -0.31 & -1.38 & 4.24 \\
\hline $\mathbf{1 5 . 4}$ & 5.57 & -1.69 & -2.58 & 7.03 \\
\hline
\end{tabular}

Table 5.3 - Sensitivity of objective function to fracture set $2 \mathrm{P}_{32}$ intensity.

\begin{tabular}{|r|r|r|r|r|}
\cline { 2 - 5 } \multicolumn{1}{c|}{} & \multicolumn{4}{c|}{ Unit Residual Difference from Ref. } \\
\hline $\begin{array}{r}\text { \% Parameter } \\
\text { Perturbation }\end{array}$ & TOTAL & \multicolumn{1}{c|}{ BHP } & \multicolumn{1}{c|}{ OPR } & \multicolumn{1}{c|}{ SEIS } \\
\hline $\mathbf{- 3 3 . 3}$ & 2.64 & 1.22 & 1.11 & 2.14 \\
\hline $\mathbf{- 2 0 . 0}$ & 1.33 & -0.03 & 0.48 & 1.40 \\
\hline $\mathbf{0 . 0}$ & 0.00 & 0.00 & 0.00 & 0.00 \\
\hline $\mathbf{2 0 . 0}$ & -2.32 & -1.28 & -0.99 & -1.80 \\
\hline $\mathbf{3 3 . 3}$ & -3.39 & -1.41 & -1.10 & -2.93 \\
\hline
\end{tabular}




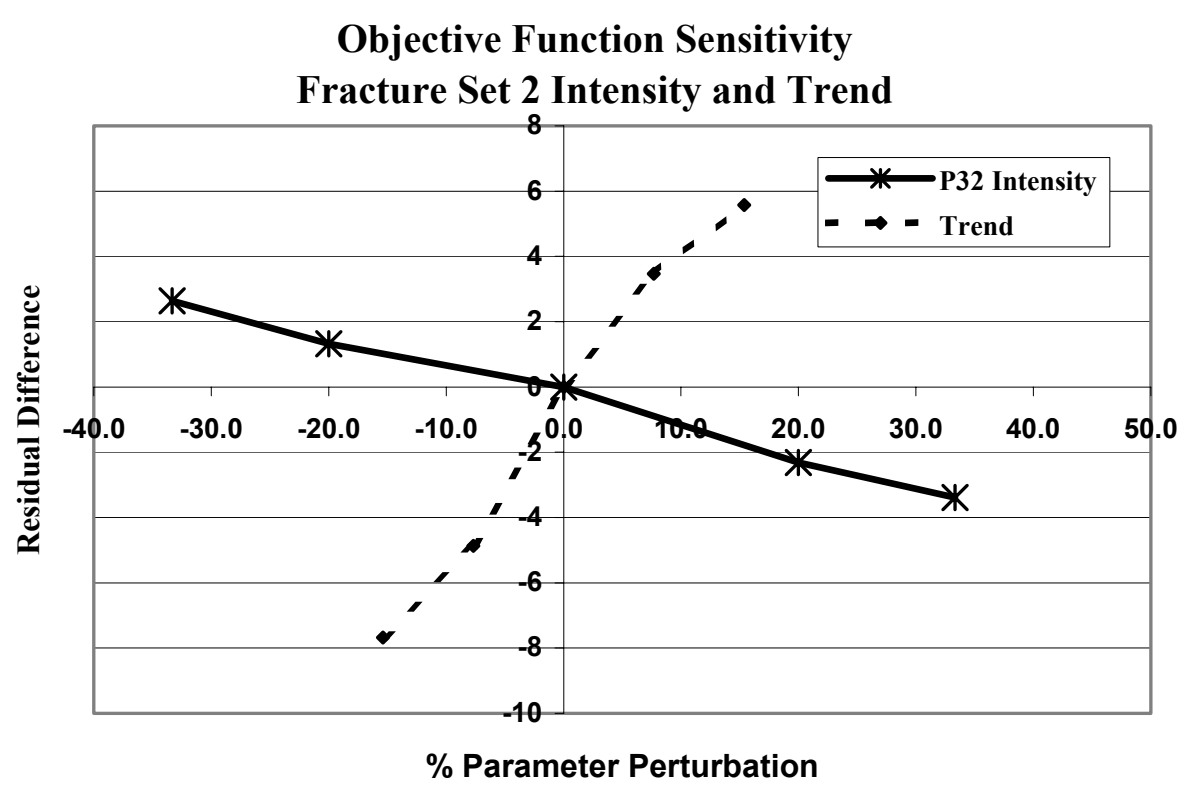

Fig. 5.1 - Objective function sensitivity to fracture set 2 trend and $\mathrm{P}_{32}$ intensity.

Sensitivity for both parameters is relatively linear about the reference point. However, the objective function is roughly three times more sensitive to variations in fracture trend than in fracture $\mathrm{P}_{32}$ intensity. In both cases the variation in the normalized residual difference is dominated by the seismic component as seen in Tables 5.2 and 5.3. Further, in the trend sensitivity data (Table 5.2), the dominant seismic component can be seen to change sign at the reference point, and is more or less symmetrical about this point. This is not the case for production residual components. In the $\mathrm{P}_{32}$ intensity sensitivity data (Table 5.3), it can be seen that all observation type residuals have the same sign on either side of the reference point.

\subsection{Sensitivity to Hydraulic Parameters}

The results of sensitivity tests for the hydraulic parameters dual porosity shape factor, matrix permeability, and the permeability upscaling representative elemental volume (REV) block size are listed in Tables 5.4 through 5.6 and shown in Figs. 5.2 and 5.3. 
Permeability upscaling and/or flow simulation were recomputed following each of these hydraulic parameter perturbations.

\subsubsection{REV Radius}

The results of sensitivity tests for perturbations in hydraulic REV radius from $0 \%$ to $+1600 \%$ of the reference value are listed in Table 5.4. Since an extremely small hydraulic representative elemental volume was chosen by design for the optimization tests it was not practical to test perturbations less than the reference value. As a result, the REV radius parameter perturbations presented here are one sided. It can be seen in Table 5.4 that, as expected, the sensitivity of the objective function is dominated by the production components, with the elastic component showing no sensitivity to this hydraulic parameter. Because of the use of the simplified $\mathrm{Oda}^{5}$ formulation for permeability upscaling the sensitivity to this parameter will depend on the degree of connectivity at the scales under consideration. The maximum REV radius tested here is roughly the same length as the average fracture. This lack of sensitivity to REV radius is somewhat surprising, and can possibly be attributed to the effect of averaging over long production time periods.

Table 5.4 - Sensitivity of objective function to hydraulic REV block size.

\begin{tabular}{|c|c|c|c|c|}
\hline \multirow[b]{2}{*}{$\begin{array}{l}\text { \% Parameter } \\
\text { Perturbation }\end{array}$} & \multicolumn{4}{|c|}{ Unit Residual Difference from Base } \\
\hline & TOTAL & BHP & OPR & SEIS \\
\hline 0.0 & 0.00 & 0.00 & 0.00 & 0.00 \\
\hline 233.3 & -0.94 & -1.62 & -1.94 & 0.05 \\
\hline 565.7 & -0.93 & -1.66 & -2.01 & 0.09 \\
\hline 900.0 & -0.95 & -1.56 & -2.14 & 0.04 \\
\hline 1233.3 & -0.89 & -1.58 & -1.91 & 0.08 \\
\hline 1565.7 & -0.96 & -1.63 & -2.08 & 0.05 \\
\hline
\end{tabular}




\subsubsection{Dual Porosity Shape Factor}

The results of sensitivity tests for perturbations in dual porosity shape factor from $-90 \%$ to $+525 \%$ of the reference value are listed in Table 5.6. Dual porosity shape factor is related to the assumed matrix block size by eqn. 5.3.1. Note that $\mathrm{L}$ is not the same as the computational grid block size.

$$
\Sigma=4 *\left(\frac{1}{L_{x}}+\frac{1}{L_{y}}+\frac{1}{L_{z}}\right)
$$

Where $\mathrm{L}$ is the matrix block size.

Table 5.5 shows the block dimension L computed from Eq. 5.1 for the dual porosity shape factor values tested based on the assumption that matrix blocks are cubic.

Table 5.5 - $\quad$ Matrix block size for sensitivity test dual porosity shape factor values assuming cubic matrix blocks.

\begin{tabular}{|r|r|}
\hline $\begin{array}{r}\text { SHAPE } \\
\text { FACTOR }\end{array}$ & \multicolumn{1}{c|}{$\mathbf{L}(\mathbf{f t})$} \\
\hline $\mathbf{0 . 0 1}$ & 34.6 \\
\hline $\mathbf{0 . 0 8}$ & 12.2 \\
\hline $\mathbf{0 . 1 5}$ & 8.9 \\
\hline $\mathbf{0 . 2}$ & 7.7 \\
\hline $\mathbf{0 . 2 5}$ & 5.9 \\
\hline
\end{tabular}

The results listed in Table 5.6 show that for dual porosity shape factor values corresponding to very small matrix block sizes the sensitivity is very low. The single point tested at dual porosity shape factor corresponding to a larger matrix block size (lower values of dual porosity shape factor) shows higher sensitivity. As expected, the sensitivity of the objective function is totally reflected in the production components, with the elastic component showing no sensitivity to this hydraulic parameter. Figs. 5.2 and 5.3 show that, relative to the inversion target parameters, the objective function 
sensitivity to dual porosity shape factor is higher than the sensitivity to $\mathrm{P}_{32}$ intensity and approximately equal to the sensitivity to trend.

Table 5.6 - Sensitivity of objective function to dual porosity shape factor.

\begin{tabular}{|r|r|r|r|r|}
\cline { 2 - 5 } \multicolumn{1}{c|}{} & \multicolumn{4}{c|}{ Unit Residual Difference from Ref. } \\
\hline $\begin{array}{r}\text { \% Parameter } \\
\text { Perturbation }\end{array}$ & \multicolumn{1}{|c|}{ TOTAL } & \multicolumn{1}{c|}{ BHP } & \multicolumn{1}{c|}{ OPR } & \multicolumn{1}{c|}{ SEIS } \\
\hline $\mathbf{8 7 . 5}$ & 3.70 & 5.88 & 1.85 & 0.07 \\
\hline $\mathbf{0 . 0}$ & 0.00 & 0.00 & 0.00 & 0.00 \\
\hline $\mathbf{8 7 . 5}$ & 0.09 & -0.02 & 0.23 & 0.06 \\
\hline $\mathbf{2 1 2 . 5}$ & 0.25 & 0.27 & 0.45 & 0.03 \\
\hline $\mathbf{5 2 5 . 0}$ & 0.25 & 0.27 & 0.45 & 0.03 \\
\hline
\end{tabular}

\subsubsection{Matrix Permeability}

The results of sensitivity tests for perturbations in matrix permeability from $-100 \%$ to $+900 \%$ of the reference value are listed in Table 5.7. It can be seen that the objective function is sensitive to variations in matrix permeability only at very low permeabilities. This sensitivity is highly dependent on dual porosity shape factor. Since the value of dual porosity shape factor used in the reference model corresponds to a very small matrix block size, the objective function is not sensitive to variations at high permeability levels. This is probably because variations in permeability at this level do not significantly effect the transient matrix to fracture flow response from such small matrix blocks. At very low permeabilities the effect on matrix to fracture flow may be affected, even on such small matrix blocks. As expected, the sensitivity of the objective function is dominated by the production components, with the elastic component showing no sensitivity to this hydraulic parameter. Figs. 5.2 and 5.3 show that, relative to the inversion target parameters, the objective function sensitivity to matrix permeability is higher than the sensitivity to $\mathrm{P}_{32}$ intensity and approximately equal to the sensitivity to trend. 
Table 5.7 - Sensitivity of objective function to matrix permeability.

\begin{tabular}{|r|r|r|r|r|}
\cline { 2 - 5 } \multicolumn{1}{c|}{} & \multicolumn{4}{c|}{ Unit Residual Difference from Base } \\
\hline $\begin{array}{r}\text { \% Parameter } \\
\text { Perturbation }\end{array}$ & \multicolumn{1}{c|}{ TOTAL } & \multicolumn{1}{c|}{ BHP } & \multicolumn{1}{c|}{ OPR } & \multicolumn{1}{c|}{ SEIS } \\
\hline $\mathbf{- 9 9 . 0}$ & 31.60 & 35.89 & 11.11 & -0.01 \\
\hline $\mathbf{- 9 0 . 0}$ & 4.66 & 7.30 & 2.22 & -0.02 \\
\hline $\mathbf{0 . 0}$ & 0.00 & 0.00 & 0.00 & 0.00 \\
\hline $\mathbf{4 0 0 . 0}$ & 0.35 & 0.39 & 0.57 & 0.05 \\
\hline $\mathbf{9 0 0 . 0}$ & 0.50 & 0.57 & 0.88 & 0.05 \\
\hline
\end{tabular}

Objective Function Sensitivity

Hydraulic Parameters and Fracture Set 2 Intensity

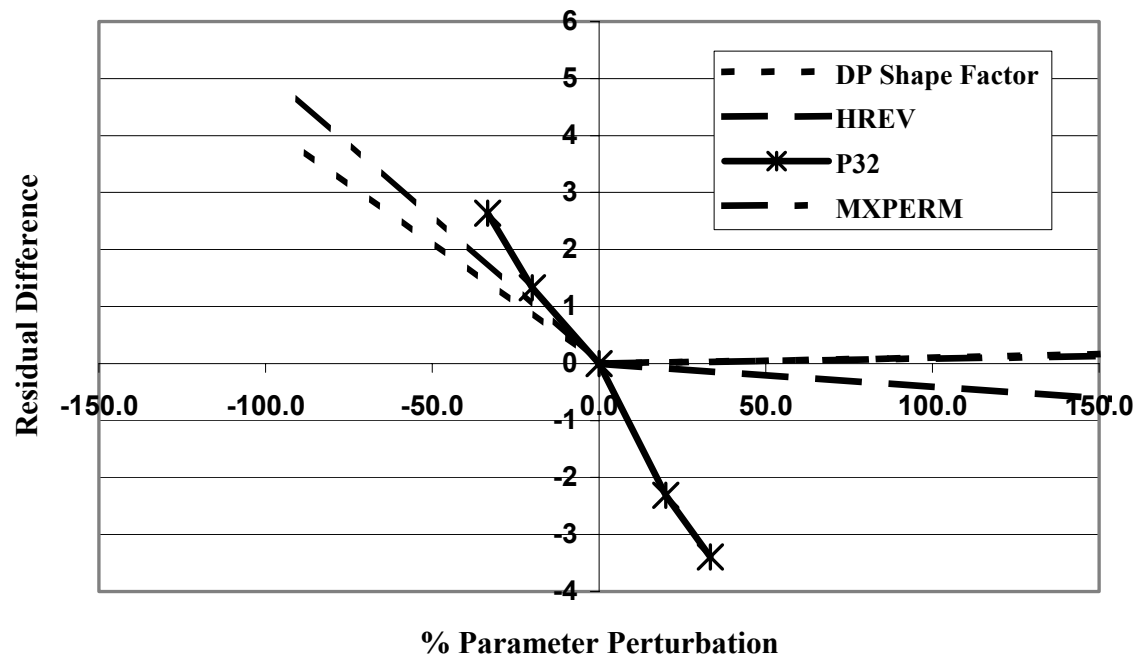

Fig. 5.2 - Sensitivity of objective function to hydraulic model parameters and fracture $\mathrm{P}_{32}$ intensity. 


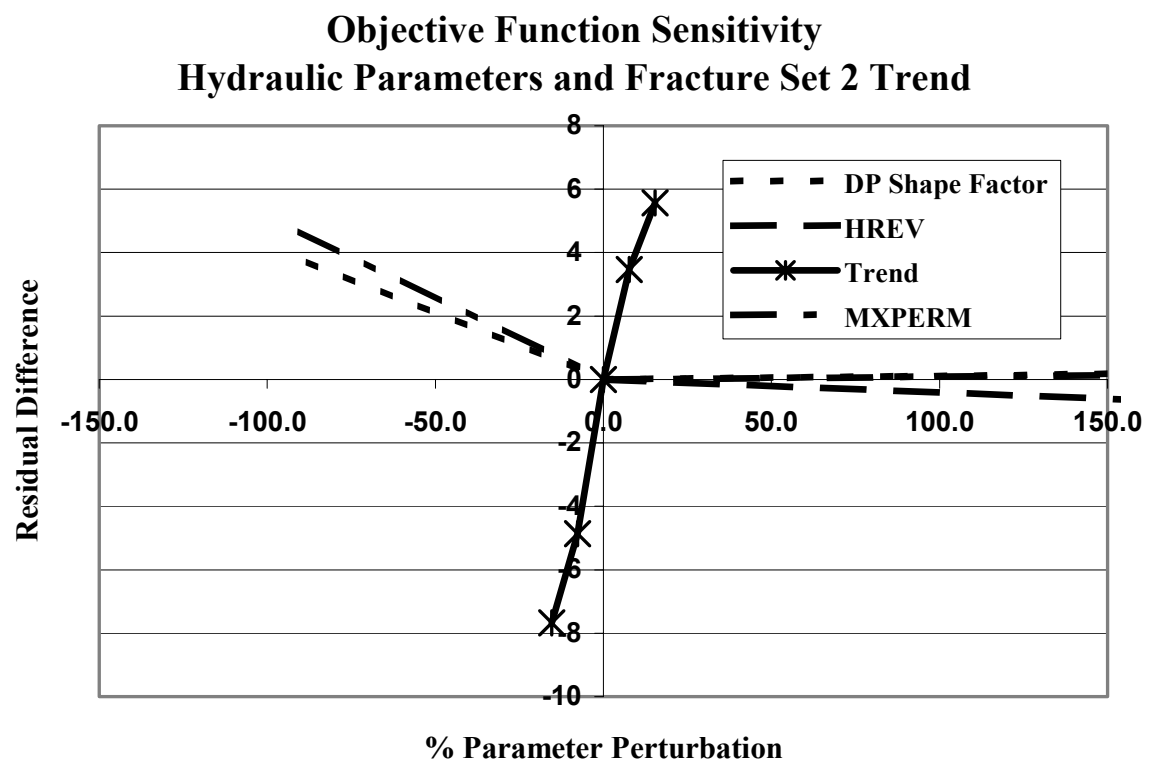

Fig. 5.3 - Sensitivity of objective function to hydraulic model parameters and fracture trend.

\subsection{Sensitivity to Elastic Parameters}

The results of sensitivity tests for the elastic parameters fracture compliance, elastic stiffness upscaling representative elemental volume (REV) radius, and the incidence angle used for calculation of quasi-P wave velocity are shown in Figs. 5.4 and 5.5. Elastic modeling and seismic attributes were recomputed following each of these elastic parameter perturbations.

\subsubsection{Elastic REV Radius}

The results of sensitivity tests for perturbations in elastic REV radius from $-80 \%$ to $+100 \%$ of the reference value are listed in Table 5.8. As expected, the sensitivity of the objective function is totally dominated by the seismic component, with the production components showing no sensitivity to this elastic parameter. Figs. 5.4 and 5.5 show that, relative to the inversion target parameters, the objective function sensitivity to elastic REV radius is lower than the sensitivity to both $\mathrm{P}_{32}$ intensity and trend. 


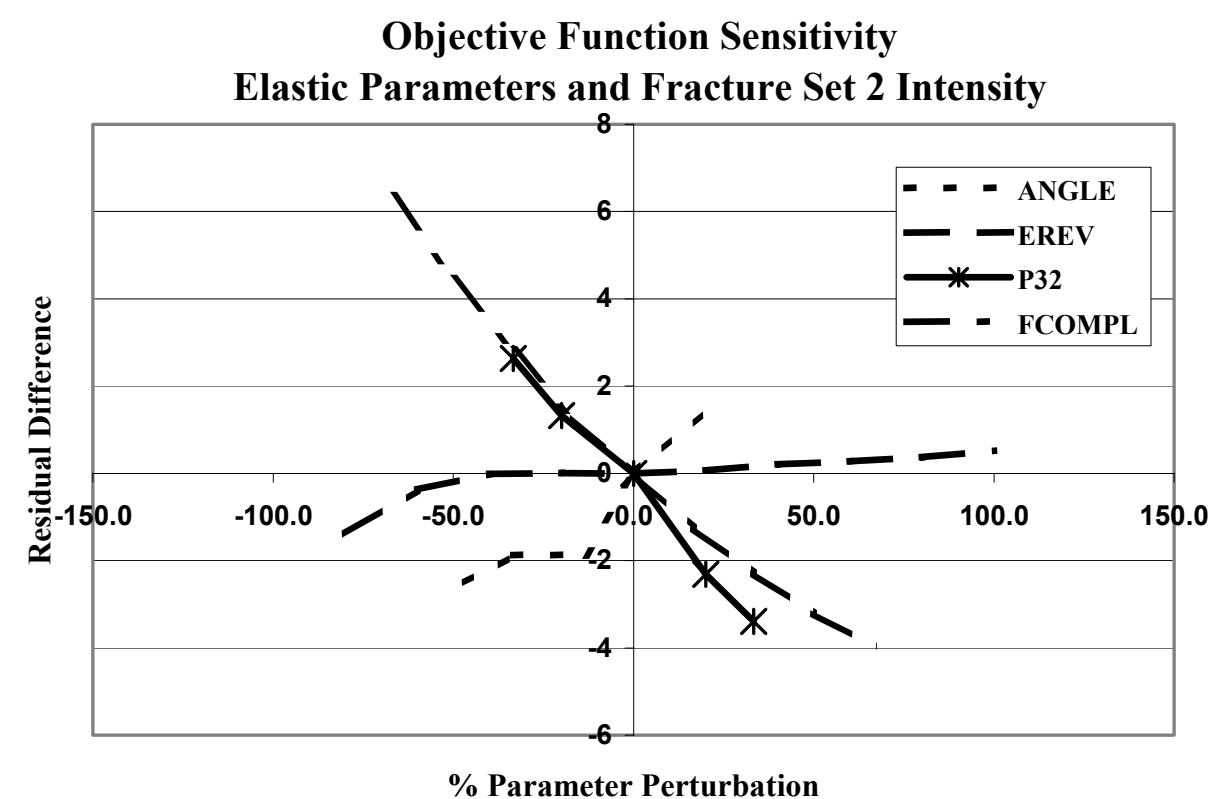

Fig. 5.4 - Sensitivity of objective function to elastic model parameters and fracture $\mathrm{P}_{32}$ intensity.

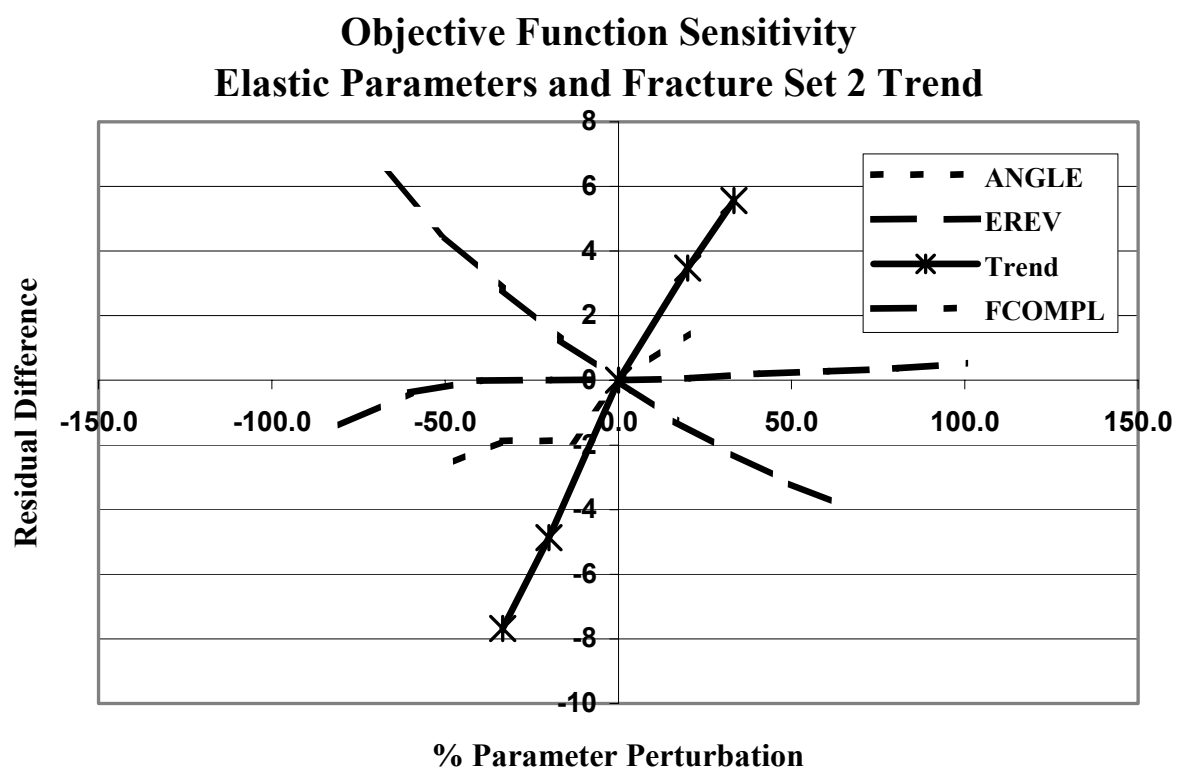

Fig. 5.5 - Sensitivity of objective function to elastic model parameters and fracture trend. 
Table 5.8 - Sensitivity of objective function to elastic REV radius.

\begin{tabular}{|rr|r|r|r|}
\cline { 2 - 6 } \multicolumn{1}{c|}{} & \multicolumn{4}{c|}{ Unit Residual Difference from Base } \\
\hline $\begin{array}{r}\text { \% Parameter } \\
\text { Perturbation }\end{array}$ & \multicolumn{1}{c|}{ TOTAL } & \multicolumn{1}{c|}{ BHP } & \multicolumn{1}{c|}{ OPR } & \multicolumn{1}{c|}{ SEIS } \\
\hline $\mathbf{- 8 0 . 0}$ & -1.39 & 0.10 & 0.04 & -1.68 \\
\hline$-\mathbf{6 0 . 0}$ & -0.37 & -0.01 & 0.12 & -0.46 \\
\hline$-\mathbf{4 0 . 0}$ & -0.01 & 0.04 & 0.12 & -0.06 \\
\hline $\mathbf{- 2 0 . 0}$ & 0.01 & -0.05 & 0.12 & 0.01 \\
\hline $\mathbf{0 . 0}$ & 0.00 & 0.00 & 0.00 & 0.00 \\
\hline $\mathbf{2 0 . 0}$ & 0.06 & 0.03 & 0.01 & 0.05 \\
\hline $\mathbf{4 0 . 0}$ & 0.20 & -0.01 & 0.24 & 0.18 \\
\hline $\mathbf{6 0 . 0}$ & 0.27 & -0.01 & 0.07 & 0.30 \\
\hline $\mathbf{8 0 . 0}$ & 0.37 & 0.00 & 0.10 & 0.40 \\
\hline $\mathbf{1 0 0 . 0}$ & 0.52 & 0.06 & 0.05 & 0.55 \\
\hline
\end{tabular}

\subsubsection{Incidence Angle}

The results of sensitivity tests for perturbations in incidence angle used for selecting quasi-P wave velocity versus azimuth gathers from $-50 \%$ to $+20 \%$ of the reference value are listed in Table 5.9. As expected, the sensitivity of the objective function is totally dominated by the seismic component, with the production components showing no sensitivity to this elastic parameter. Figs. 5.4 and 5.5 show that, relative to the inversion target parameters, the objective function sensitivity to incidence angle is lower than the sensitivity to both $\mathrm{P}_{32}$ intensity and trend. Sensitivity to incidence angle is non-linear about the reference point.

Table 5.9 - Sensitivity of objective function to elastic incidence angle.

\begin{tabular}{|rr|r|r|r|}
\cline { 2 - 6 } \multicolumn{1}{c|}{} & \multicolumn{4}{c|}{ Unit Residual Difference from Base } \\
\hline $\begin{array}{r}\text { \% Parameter } \\
\text { Perturbation }\end{array}$ & \multicolumn{1}{c|}{ TOTAL } & \multicolumn{1}{c|}{ BHP } & \multicolumn{1}{c|}{ OPR } & \multicolumn{1}{c|}{ SEIS } \\
\hline $\mathbf{- 4 5 . 7}$ & -2.51 & -0.02 & -0.02 & -2.94 \\
\hline $\mathbf{- 3 3 . 3}$ & -1.88 & 0.01 & -0.02 & -2.20 \\
\hline $\mathbf{- 1 3 . 3}$ & -1.88 & 0.01 & -0.02 & -2.20 \\
\hline $\mathbf{0 . 0}$ & 0.00 & 0.00 & 0.00 & 0.00 \\
\hline $\mathbf{2 0 . 0}$ & 1.45 & -0.02 & 0.03 & 1.65 \\
\hline
\end{tabular}




\subsubsection{Fracture Compliance}

The results of sensitivity tests for perturbations in fracture compliance from $-67 \%$ to $+67 \%$ of the reference value are listed in Table 5.10. As expected, the sensitivity of the objective function is totally reflected in the seismic component, with the production components showing no sensitivity to this elastic parameter. Figs. 5.4 and 5.5 show that, relative to the inversion target parameters, the objective function sensitivity to incidence angle is significantly higher than the sensitivity to $\mathrm{P}_{32}$ intensity and approximately equal to the sensitivity to trend. Sensitivity to fracture compliance is relatively linear about the reference point.

Table 5.10 - Sensitivity of objective function to fracture compliance.

\begin{tabular}{|r|r|r|r|r|}
\cline { 2 - 5 } \multicolumn{1}{c|}{} & \multicolumn{3}{c|}{ Unit Residual Difference from Base } \\
\hline $\begin{array}{r}\text { \% Parameter } \\
\text { Perturbation }\end{array}$ & \multicolumn{1}{c|}{ TOTAL } & \multicolumn{1}{c|}{ BHP } & \multicolumn{1}{c|}{ OPR } & \multicolumn{1}{c|}{ SEIS } \\
\hline $\mathbf{- 6 5 . 7}$ & 5.37 & -0.11 & -0.07 & 7.10 \\
\hline $\mathbf{- 5 0 . 0}$ & 4.46 & -0.03 & -0.10 & 5.01 \\
\hline $\mathbf{- 3 3 . 3}$ & 2.83 & -0.03 & -0.10 & 3.22 \\
\hline $\mathbf{- 1 5 . 7}$ & 1.27 & -0.04 & -0.03 & 1.48 \\
\hline $\mathbf{0 . 0}$ & 0.00 & 0.00 & 0.00 & 0.00 \\
\hline $\mathbf{1 5 . 7}$ & -1.25 & -0.10 & -0.10 & -1.38 \\
\hline $\mathbf{3 3 . 3}$ & -2.29 & -0.08 & 0.00 & -2.65 \\
\hline $\mathbf{5 0 . 0}$ & -3.21 & -0.14 & -0.05 & -3.71 \\
\hline $\mathbf{6 5 . 7}$ & -3.97 & 0.05 & -0.12 & -4.77 \\
\hline
\end{tabular}

\subsection{Sensitivity to Reservoir Thickness}

The results of sensitivity tests for perturbations in reservoir thickness from $-33 \%$ to $+100 \%$ of the reference value are listed in Table 5.11 and illustrated in Figs. 5.6 and 5.7. Both production data and seismic velocities are dependent upon reservoir thickness. Larger vertical dimensions of the fractures effects both the permeability and the elastic moduli of the fracture system. When all other fracture parameters are held constant, an increase in fracture height will result in an increase in the permeability parallel to the 
fracture and a decrease in elastic stiffness of the fractured media normal to the fracture trend, lowering the quasi-P wave velocity. Therefore, permeability scaling, flow simulation, elastic modeling, and seismic velocity modeling was recomputed after each thickness change. This data shows a very strong sensitivity in the production components of the objective function, but a surprisingly low sensitivity of the elastic components of the objective function to reservoir thickness. The sensitivity of production components is dominated by the bottom hole pressure observation. Results suggest that sensitivity increases as thickness decreases. Testing of the sensitivity for a reservoir thinner than $60 \mathrm{ft}$. was not possible because the simulation solution failed to converge. It is believed that this failure to converge for this reservoir was caused by a combination of the production controls (rates and pressures) used, the matrix and fracture permeability used, and the reduced pore volume. Sensitivity to reservoir thickness is very linear both near to and far from the reference point.

Table 5.11 - Sensitivity of objective function to reservoir thickness.

\begin{tabular}{|r|r|r|r|r|}
\cline { 2 - 5 } \multicolumn{1}{c|}{} & \multicolumn{4}{c|}{ Unit Residual Difference from Base } \\
\hline $\begin{array}{r}\text { \% Parameter } \\
\text { Perturbation }\end{array}$ & \multicolumn{1}{c|}{ TOTAL } & \multicolumn{1}{c|}{ BHP } & \multicolumn{1}{c|}{ OPR } & \multicolumn{1}{c|}{ SEIS } \\
\hline $\mathbf{3 3 . 3}$ & 27.93 & 34.57 & 4.42 & 0.07 \\
\hline $\mathbf{0 . 0}$ & 0.00 & 0.00 & 0.00 & 0.00 \\
\hline $\mathbf{3 3 . 3}$ & 0.58 & 1.37 & -0.48 & 0.04 \\
\hline $\mathbf{6 5 . 7}$ & 3.45 & 5.22 & -0.44 & 0.08 \\
\hline $\mathbf{1 0 0 . 0}$ & 5.61 & 9.34 & -0.43 & 0.07 \\
\hline $\mathbf{1 3 3 . 3}$ & 7.45 & 11.82 & -0.40 & 0.05 \\
\hline $\mathbf{1 6 5 . 7}$ & 8.75 & 13.45 & -0.41 & 0.11 \\
\hline
\end{tabular}




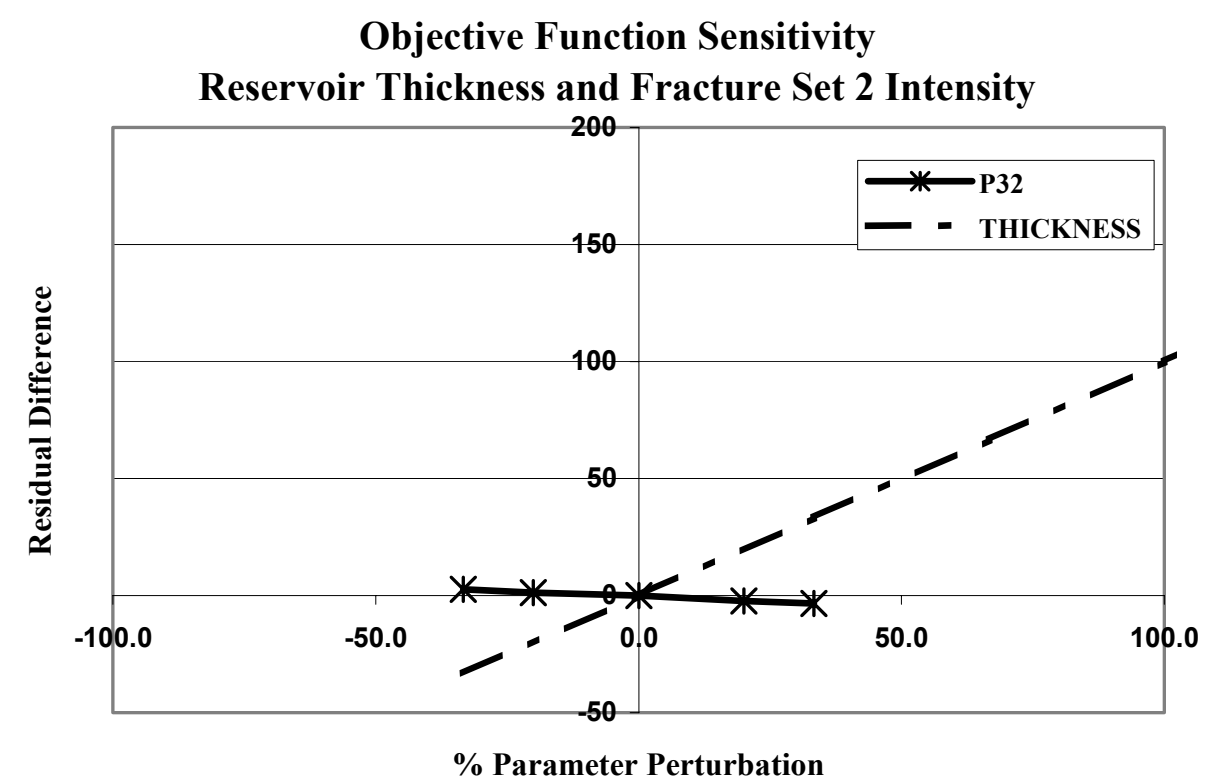

Fig. 5.6 - Sensitivity of objective function to reservoir thickness and fracture $\mathrm{P}_{32}$ intensity.

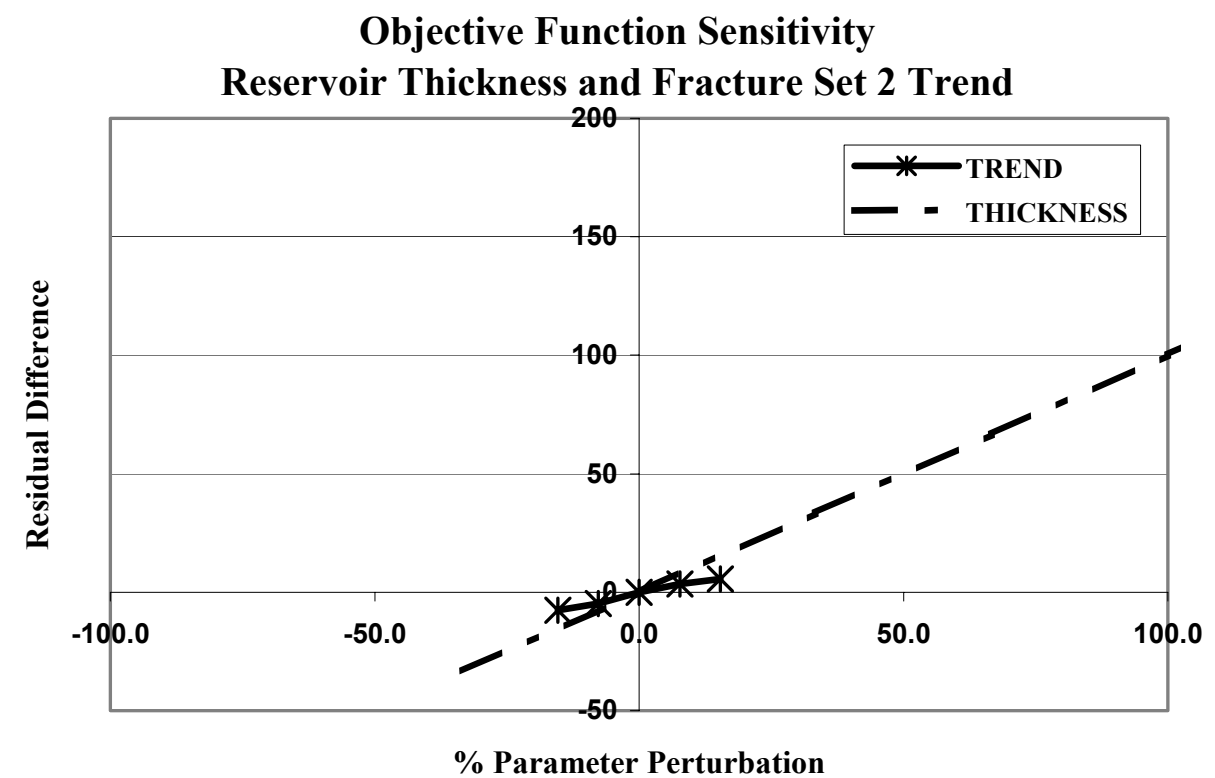

Fig. 5.7 - Sensitivity of objective function to reservoir thickness and fracture trend. 


\subsection{Discussion}

\subsubsection{Hydraulic REV Radius}

This effective media model parameter is scale dependent with respect to the fracture length and spatial distribution. Sensitivity of the objective function used in this study to hydraulic REV radius is relatively low. Selection of the hydraulic REV radius may be optimized by spatial analysis of the fracture system and by sensitivity testing through forward modeling. In theory, the hydraulic REV radius should be chosen such that the hydraulic effective media properties do not change with small variation in radius. However, it is not known whether, when using the new method in practice, a single optimal hydraulic REV radius may be selected which would be appropriate for all model parameter updates. Although more rigorous methodology could have been applied in selecting this parameter for actual optimization tests, the results of the new method presented in this research are not compromised by poor selection or uncertainty in this parameter.

\subsubsection{Dual Porosity Shape Factor}

Dual porosity shape factor (Eq. 5.1) is dependent on fracture system trend and $\mathrm{P}_{32}$ intensity. Local heterogeneities in fracture intensity and trend can result in spatial variation in shape factor as well as dependency on the size of the region considered in formulating this parameter. Techniques are available to estimate this parameter through spatial analysis of the fracture population ${ }^{5}$. These analytical tools were not available for this research. In most cases, particularly when using a fracture distribution that is spatially stationary, a single representative value of dual porosity shape factor is computed for the population and used in the dual porosity simulator. Although it is possible to compute spatially variant dual porosity shape factor for a fracture population, this is not common practice. The sensitivity tests indicate the possibility of significant sensitivity to dual porosity shape factor indicating that the results of the new method may be compromised by poor estimates of this parameter. Further work on this new technique should include computation and use of spatially varying dual porosity shape 
factor from the fracture population. The need to update this parameter at each iteration should also be investigated.

\subsubsection{Matrix Permeability}

Matrix permeability is a real petrophysical property which may be measured at the borehole through a variety of measurements and populated within the interwell space using deterministic or stochastic methods as appropriate. These tests indicate that the sensitivity of the objective function to matrix permeability may depend on matrix block size and may be significant for larger block sizes. Based on these results it is the authors opinion that sensitivity to matrix permeability should be considered as a first order effect. As such, it is strongly suggested that when using the new method, attention must be paid to characterization of both the magnitude and spatial distribution of matrix permeability. Wireline logs such as magnetic resonance should be used for point estimates in wells. Well test analysis may be used to extend permeability estimates deeper into the reservoir. 3D seismic attribute correlations for matrix porosity may be used along with porosity-permeability relations from wireline or core data in order to estimate the spatial distribution of permeability in the inter-well space.

\subsubsection{Elastic REV}

Like hydraulic REV radius, elastic REV radius is an effective media model parameter and is scale dependent with respect to the fracture length and spatial distribution. Sensitivity of the objective function used in this study to elastic REV radius is significant with respect to sensitivity to trend and $\mathrm{P}_{32}$ intensity. An optimal value for this parameter for any fracture distribution may be selected through forward modeling tests. In theory, the elastic REV radius should be chosen such that the elastic effective media properties do not change with small variation in radius. However, like hydraulic REV radius, it is not known whether, in practice, a single optimal elastic REV radius may be selected which would be appropriate for all model parameter updates. 


\subsubsection{Incidence Angle}

The anisotropic compressional wave response to fractures is known to be stronger at higher angles of incidence. This is seen in the sensitivity test data presented in section 5.4. As such, in order to derive the greatest benefit from anisotropic seismic attributes the incidence angle used should be as large as possible while still satisfying the constraints of the low angle approximation used to derive equation 5.12. In practice, there is some uncertainty in the actual value of incidence angle in the seismic gathers used for the ANMO analysis. This uncertainty is caused by the cumulative effect of errors in estimates of overburden seismic velocities on the process of sorting of the seismic data into gathers at the reservoir depth. However, an understanding of how these errors propagate to errors in incidence angle, and the effect of these errors on the objective function, is outside the scope of this research.

\subsubsection{Fracture Compliance}

The sensitivity of the objective function to fracture compliance is high. This parameter represents the largest component of potential error of all parameters studied in the sensitivity tests. An error component not investigated here is the use of equivalent normal and tangential fracture compliances, representative of gas filled rather than liquid filled fractures. At this time no well established methods exist for measuring fracture compliance. Sayers ${ }^{50}$ has developed a formulation of the linear slip discontinuity suitable

for inversion of laboratory experimental data for elastic stiffness moduli. Sayers ${ }^{33}$ also provides a derivation of fracture compliance from estimates of fracture geometry and host media elastic properties based upon the Hudson formulation for stiffness of an elliptical inclusion. Through personal communication Sayers has further suggested the possibility of deriving estimates of fracture compliance through joint inversion of acoustic data from a developmental Schlumberger wireline tool along with other colocated electrical image and petrophysical observations. 


\subsubsection{Reservoir Thickness}

As noted in section 5.5, both the permeability and seismic velocities of the fracture system are dependent on reservoir thickness (fracture height) with increasing height resulting in higher fracture permeability parallel to fracture trend and lower velocity in the direction normal to fracture trend. Since the seismic velocity of the fractured media parallel to the fracture trend will remain approximately constant the host medium velocity, the increase in fracture height will result in an increase in quasi-P wave modulation amplitude. The data presented in section 5.5 indicates significant sensitivity of the objective function to the estimate of reservoir thickness. Without proper treatment poor estimates of reservoir thickness will compromise the results achieved with the new method. Reservoir thickness may be estimated with fair accuracy using stratigraphic interpretation and integration of wireline logs and good quality seismic data using one of many available algorithms such as such as trend kriging. Further, in reservoirs where assuming that fracture height equaling reservoir thickness is not a good approximation it may be necessary to model the reservoir with multiple layers.

\subsection{Summary}

In this chapter we presented the results of sensitivity tests of the optimization objective function with respect to various hydraulic, elastic, and geometric model parameters. To enhance the validity of these tests, the trend and $\mathrm{P}_{32}$ intensity parameters used for the reference model were deliberately not equal to the known base case values for the model. As a result, the solution space explored in this sensitivity analysis was representative of an estimate of trend and $\mathrm{P}_{32}$ intensity with realistic errors.

The sensitivity tests presented here are not exhaustive. Although many of the primary model parameters have been tested, the fractured reservoir model contains many other parameters to which the sensitivity of the new method has not yet been explored. However, this work does represent a systematic investigation of model sensitivity and 
serves as a starting point for future studies concerning other model parameters. These results will be further discussed in Chapter VI. 


\section{CHAPTER VI}

\section{DISCUSSION OF RESULTS}

\subsection{Introduction}

In this chapter we discuss the results of the optimization and sensitivity testing presented in Chapters IV and V. The new method was applied to three synthetic test cases. The strength of the new process was validated in both the single and multiple fracture set scenarios through application of a parallel optimization workflow in which seismic anisotropy was absent from the objective function. Sensitivity testing provided valuable information regarding the need for pre-conditioning of the fracture model prior to application of the new process.

In this chapter we will also review and discuss the workflow for the new process which is presented in Appendix A. This workflow is a challenging multi-disciplinary effort involving stochastic fracture modeling, petrophysical and elastic modeling, reservoir simulation, and numerical optimization.

\subsection{Optimization Test Results}

Models for the test cases were developed using elastic and petrophsyical reservoir properties representative of the following reservoir types:

- A tight sandstone reservoir with a single fracture set.

- A tight sandstone reservoir with two fracture sets.

- A dolomite reservoir with two fracture sets. 


\subsubsection{Single Fracture Set}

The single fracture set test case showed stable convergence of both $\mathrm{P}_{32}$ intensity and trend of the fracture set, resulting in a very good history match of production data and prediction of seismic anisotropy attributes. The following specific observations have been made based upon to these test results;

- The new method provides well behaved systematic convergence of $\mathrm{P}_{32}$ intensity and trend in five iterations.

- Elimination of seismic attributes from the objective function results in slower convergence. However, this convergence is relatively stable.

- $\mathrm{P}_{32}$ intensity error after five iterations of the production data only method was eight times that achieved using the combined objective function while trend error was twice that achieved using the combined objective function.

- Bottom hole pressure was the dominant observation in the inversion, followed by the seismic attributes.

- Fracture trend was most influenced by bottom hole pressure and the maximum quasi-P wave velocity direction seismic attribute while $\mathrm{P}_{32}$ intensity was influenced by bottom-hole pressure and the seismic velocity modulation amplitude attribute.

\subsubsection{Multiple Fracture Sets}

Multiple fracture set optimization tests showed convergence of the objective function, but with less stability than that seen in the single fracture set case. Stability of the multiple fracture set optimization displayed sensitivity to matrix petrophysical properties. Initial update steps required adjustments of the prior model weighting. Further, the production only optimization test clearly showed the need for the seismic observation in the objective function when refining multiple fracture set models. The following specific observations are made from tests $\mathrm{T} 3$ and $\mathrm{T} 4$; 
- For multiple fracture sets the new method provides convergence of $\mathrm{P}_{32}$ intensity and trend. However, this convergence is not as stable as experienced with a single fracture set.

- Adjustment of prior model weights was required to assure convergence to the local solution.

- Sensitivity of the process to a priori model weights indicates the importance of model preconditioning in application of the new process.

- For multiple fracture sets the elimination of the seismic observation from the objective function results in non-convergence.

- In all cases bottom hole pressure is the most significant observation for the optimization process.

- In all cases oil production rate appears to contribute little to the optimization process.

- Decrease in matrix permeability appears to mask the hydraulic behavior of the fracture system.

- The sensitivity to all production observations was reduced in the test with reduced permeability as observed in the dolomite reservoir test.

- The reduced sensitivity to production observations when the matrix was less permeable caused instability in the inversion.

- Instability in fracture trend convergence seen in the dolomite reservoir test required reduction of noise and uncertainty estimates for bottom hole pressure observations.

- In multiple fracture set optimization tests, trend and $\mathrm{P}_{32}$ intensity did not exhibit unique sensitivity to maximum quasi-P wave velocity direction and velocity modulation amplitude as anticipated from elastic theory. 


\subsection{Sensitivity Analysis}

The results of objective function sensitivity testing were presented in Chapter V. These tests included investigation of sensitivity to trend and $\mathrm{P}_{32}$ intensity as well as three classes of model parameters. These were;

- Hydraulic parameters

- Elastic parameters

- Geometrical parameters

The following specific observations are made from these sensitivity tests;

- The new method has a very high level of sensitivity to fracture elastic compliance. Future developments in well logging, acoustic measurements, and data inversion techniques promise to provide more reliable estimates of these elastic properties.

- The new method has a high level of sensitivity to reservoir thickness, which can be controlled through use of a combination of borehole measurements and seismic imaging.

- The new method is sensitive to estimates of dual porosity shape factor, suggesting that any available method to calibrate this parameter from spatial analysis of fracture distribution estimates should be utilized.

- The new method can be sensitive to estimates of the matrix permeability, indicating that all available methods for accurate population of the $3 \mathrm{D}$ volume from well logs and from transient analysis, should be utilized.

\subsection{Integrated Modeling Workflow}

The new process workflow is documented in Appendix A. This workflow requires repetitive execution of a process, which includes the following sequence of tasks:

1. Stochastic fracture modeling 
2. Fracture permeability upscaling through application of an appropriate effective media model.

3. Dual porosity flow simulation.

4. Anisotropic elastic effective media modeling.

5. Elastic attribute modeling.

6. 3-dimensional earth model building and gridding.

7. Residual analysis.

8. Calculation of Jacobian matrix sensitivity coefficients.

9. Linear parameter updating.

Not only does each iteration of the new method requires execution of the above set of tasks, calculation of sensitivity coefficients requires execution of tasks 1-6 up to 5 times within each iteration loop for models having 2 fracture sets. Total CPU clock time for each iteration of the multiple fracture set case was approximately $10 \mathrm{hrs}$, with approximately 23 hours of intervention for data reformatting. Computation of sensitivity coefficients and linear parameter updates was very quick and efficient. 


\section{CHAPTER VII}

\section{SUMMARY AND CONCLUSIONS}

\subsection{Summary}

\subsubsection{Introduction}

In this chapter we summarize the work performed in this research program and provide conclusions concerning the applicability of the new method. At this point it is appropriate to recall the objectives of the research program as stated in Chapter I.

The objective of this research is to develop an improved method for history matching of naturally fractured reservoir models which takes advantage of the combined resolving power of both production data and specialized elastic anisotropy attributes computed from 3-dimensional seismic data.

\subsubsection{New Optimization Method}

We have developed the new approach for history matching of naturally fractured reservoir models with the aid of seismic anisotropic attributes. This new method involved a multi-disciplinary data integration workflow and included the following distinct tasks;

- Forward modeling and sensitivity analysis of hydraulic properties from realistic discrete feature network models.

- Forward modeling and sensitivity analysis of elastic anisotropy seismic attributes from the same realistic discrete feature network models.

- Formulation of an optimization objective function containing contributions of both production data and seismic anisotropy attributes.

- Computation of sensitivity coefficients with respect to fracture system trend and $\mathrm{P}_{32}$ intensity. 
- Refinement of an initial fracture model estimate through systematic linear gradient based updates to fracture system trend and $\mathrm{P}_{32}$ intensity.

\subsubsection{Data Analysis}

The new method has been applied to the following synthetic test cases:

- Single fracture set in a tight sandstone matrix.

- Multiple fracture sets in a tight sandstone matrix.

- Multiple fracture sets in a dolomite matrix.

In addition to the above applications of the new method, the following synthetic comparison tests were made;

- Comparison test for a single fracture set in a tight sandstone matrix without seismic attributes in the objective function.

- Comparison test for multiple fracture sets in a tight sandstone matrix without seismic attributes in the objective function.

These tests were performed using a typical five spot pattern production configuration. Test results are presented in Chapter V and discussed in Chapter VII.

\subsubsection{Sensitivity}

Extensive testing was performed to document the sensitivity of the optimization objective function with respect to the inversion target parameters fracture system trend and $\mathrm{P}_{32}$ intensity, as well as several key hydraulic, elastic, and geometrical model parameters.

The following parameters were tested:

- Hydraulic - Dual porosity shape factor, matrix permeability, and hydraulic effective media model representative elemental volume. 
- Elastic - Fracture compliance, seismic ANMO angle of incidence, and elastic effective media model representative elemental volume.

- Geometrical - Reservoir thickness.

This sensitivity analysis was performed using the behavior of normalized residuals in the neighborhood of the initial estimate for one of the multiple fracture test cases during systematic parameter perturbations. Total residual and residuals by observation type were analyzed. These results are presented in Chapter VI and discussed in Chapter VII. These results are also extensively documented in Appendix E.

\subsection{Conclusions}

The following conclusions were derived from this study. For a single fracture set;

- The new method successfully achieves stable refinement of fracture system trend and $\mathrm{P}_{32}$ intensity for a single fracture set in the representative tight sandstone matrix.

- Model convergence for the single fracture set case is stable with monotonic decrease in parameter errors and observation residuals.

- For a given production configuration, production observations such as bottom hole pressure and oil production rate can have dramatically different degrees of influence on the optimization process.

- For this production configuration, the seismic observation is more influential than some production observations.

For multiple fracture sets;

- The new method successfully achieves refinement in models with two fracture systems. 
- Model convergence for the multiple fracture set test cases is less stable than as observed for the single fracture set case, exhibiting convergent but somewhat erratic non-linear behavior of parameter errors and observation residuals.

- Model prior weighting was a critical factor. This illustrates the importance of model preconditioning in application of the new process.

- The need to reduce bottom hole pressure noise and uncertainty in test T4 demonstrates the sensitivity on the new method to the quality of field data observations.

- Variation of matrix hydraulic and elastic properties can have a significant effect on the convergence using the new method.

For production data only optimization;

- In a simple model such as that having a single fracture set, the seismic observations are not needed to achieve convergence. However, the inclusion of the seismic attribute in the objective function speeds convergence.

- In models having two fracture sets the seismic observation was required in order to achieve convergence.

For sensitivity;

- The models studied in this program exhibit first order sensitivity to the inversion target parameters fracture set trend and $\mathrm{P}_{32}$ intensity.

- These models also display high levels of sensitivity to some hydraulic, elastic, and geometric parameters, some of which may be adequately conditioned in the initial estimate through conventional data integration methods.

- The success of the new method will depend on the degree of model preconditioning with respect to parameters other than fracture system trend and $\mathrm{P}_{32}$ intensity.

- Preconditioning with respect to some parameter sensitivities, such as fracture compliance, may be problematic given the current state of technology. 
Uniqueness;

- The uniqueness of the solutions was not explored rigorously. However, some information about uniqueness may be inferred from test results.

- Since the inversion technique used is a local inversion and not a global inversion, non-convergence, such as seen in the multiple fracture set cases without the seismic velocity observations in the objective function, may be an indirect indication of non-uniqueness. Although not conclusive, non-convergence to the base case model may be the result of the inversion seeking an alternate solution minimum.

- Conversely, convergence of the multiple fracture set cases using the combined objective function suggests reduction of the non-uniqueness for these inversions.

- Plots of the azimuthally dependent quasi-P wave velocity for multiple fracture sets show that the seismic observations within one elastic computation block are averaged. As a result, the velocity observations alone are not sufficient to resolve the trend and $\mathrm{P}_{32}$ intensity of multiple fracture sets.

- Sensitivity test results reported in Chapter V suggest areas where model prior conditioning is critical to minimize non-uniqueness related to the assumptions imposed during application of the new method.

General;

- Despite the relative simplicity of this study, it is felt that the new method is potentially very powerful and holds the promise of serving as a template for other engineering and seismic data integration efforts.

- Instabilities in the inversion may be addressed using a more sophisticated optimization scheme.

- This new method is unique in its combined use of advanced stochastic fracture modeling, current anisotropic elastic theory, state of the art reservoir engineering methodology, and rigorous numerical optimization. 


\subsection{Recommendations for Future Work}

We recommend the following work for future development of this new method;

- Test the method on field data. Although engineering data requirements for the new process are minimal, the additional need for fracture characterization and initial DFN modeling, as well as appropriate seismic data with specialized processing and analysis, is problematic. The new method requires good quality 3D data acquired with wide source-receiver azimuthal distribution. In addition, anisotropy analysis involves some non-standard processing steps demanding additional time, cost, and special expertise over conventional seismic processing. One reservoir with adequate production data, seismic data, and anisotropic analysis is the Emilio field operated by AGIP in the Adriatic. However, AGIP could not be convinced to make the required data available for this study.

- Use P-wave reflectivity amplitude versus azimuth (AVAZ) instead of phase velocity.

- Implement the more sophisticated 'finite element permeameter' method for upscaling of fracture system permeability.

- Implement a global optimization scheme for initial updates prior to the gradient optimization.

- Use of fractal relationships for fracture geometry to allow more representative modeling of fracture compliances.

- Extend the process to comprehend spatial structures for application to nonstationary fracture systems.

- Develop a single well technique using VSP and well test data based on this new method. 


\section{NOMENCLATURE}

\section{Variables}

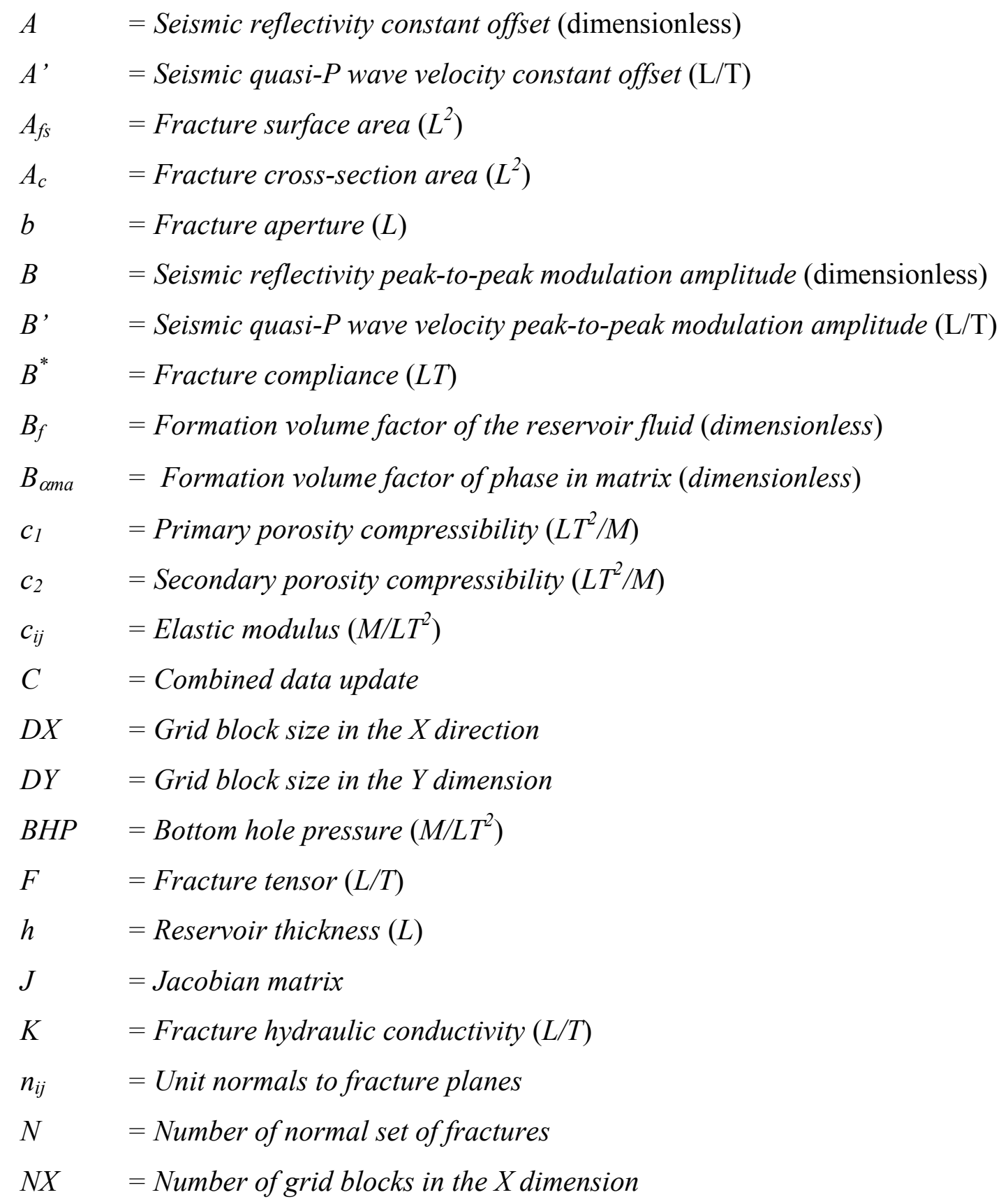




$$
\begin{aligned}
& \text { NY = Number of grid blocks in the Y dimension } \\
& O P R=\text { Oil production rate }\left(M^{3} / T\right) \\
& p \quad=\text { pressure }\left(M / L T^{2}\right) \\
& P \quad=\text { Initial pressure } \\
& p_{c} \quad=\text { Capillary pressure }\left(M / L T^{2}\right) \\
& P_{32}=\text { Stochastic fracture intensity }\left(L^{2} / L^{3}\right) \\
& q \quad=\text { Flow }\left(L^{3} / T\right) \\
& Q \quad=\text { Objective function (dimensionless) } \\
& r \quad=\text { Integration radius }(L) \\
& R \quad=\text { Reflection amplitude (dimensionless) } \\
& R^{\prime} \quad=R M S \text { residual errors (dimensionless) } \\
& s \quad=\text { Elastic compliance }\left(M / L T^{2}\right) \\
& S_{w c}=\text { Connate water saturation (fraction) } \\
& S_{\text {ama }} \quad=\text { Phase saturation in matrix (faction) } \\
& \text { STD = Observation standard deviation } \\
& T=\text { Transmissivity }\left(L^{2} / T\right) \\
& T^{\prime} \quad=\text { Fracture system trend (degrees) } \\
& V \quad=\text { Volume }\left(L^{3}\right) \\
& V \quad=\text { Seismic velocity }(L / T) \\
& x \quad=\text { Parameter vector } \\
& y \quad=\text { Weighted observation residual vector }
\end{aligned}
$$

\section{Greek Letters}

$$
\begin{array}{ll}
\varepsilon & =\text { Elastic strain (fraction) } \\
\varepsilon_{i}^{\prime} & =\text { Residual error component } \\
\varphi & =\text { Porosity (fraction) } \\
\phi & =\text { Aziumuth }(\text { degrees }) \\
\mu & =\text { Fluid viscosity }(c p) \\
\rho & =\text { Fluid density }\left(M / L^{3}\right)
\end{array}
$$




$$
\begin{array}{ll}
\sigma & =\text { Stress }\left(M / L T^{2}\right) \\
\xi & =\text { Total residua error } \\
\theta_{i} & =\text { Incidence angle (degrees) } \\
\Sigma & =\text { Dual porosity shape factor }\left(\mathrm{m}^{2}\right)
\end{array}
$$

\section{Subscript}

$$
\begin{array}{ll}
\text { BHP } & =\text { Bottom hole pressure } \\
c & =\text { Fracture opening cross section } \\
i, j & =\text { Principle indices of the arbitary reference frame } \\
I & =\text { Injector } \\
n & =\text { Normal } \\
n w & =\text { Non-wetting phase } \\
o & =\text { Host media property } \\
\text { OPR } & =\text { Oil production rate } \\
p p & =\text { Compressional wave } \\
P_{n} & =\text { Producer number } n \\
q P p & =\text { Quasi-P wave reflection } \\
q p r & =\text { Quasi-P wave velocity } \\
s_{1} & =\text { Fast Shear } \\
S & =\text { Fracture surface } \\
S S & =\text { Shear wave } \\
S E I S & =\text { Seismic observations } \\
T & =\text { Tangential } \\
w & =\text { Well } \\
w t & =\text { Wetting phase } \\
&
\end{array}
$$

\section{Superscripts}

$\begin{array}{ll}c & =\text { Computed } \\ h & =\text { Host media property } \\ o & =\text { Observed }\end{array}$




$$
\begin{array}{ll}
\text { init } & =\text { Initial estimate for update } \\
n e w & =\text { After linear update } \\
n w & =\text { Number of wells } \\
T & =\text { Transpose }
\end{array}
$$

\section{Operators}

$\nabla \quad=$ Divergence 


\section{REFERENCES}

1. Barenblatt, G.I., Zheltov, I.P., and Kochina, I.N.: "Basic Theory in the Theory of Seepage of Homogeneous Liquids in Fissured Rocks [Strata]," J. Applied Math (1960) 24, No. 5, 1286.

2. Kazemi, H, Merril, L.S., Porterfield, K.L., and Zeman, P.R.: "Numerical Simulation of Water-Oil Flow in Naturally Fractured Reservoirs", paper SPE 5719 presented at the 1976 SPE-AIME $4^{\text {th }}$ Symposium on Numerical Simulation of Reservoir Performance held in Los Angeles, California, 19-20 February

3. Oda, M.: "Permeability Tensor for Discontinuous Rock Masses", Geotechnique, (1985) 35, 483-495.

4. Lough, M.F., Lee, H., and Kamath, J.:“A New Method to Calculate the Effective Permeability of Grid Blocks Used in the Simulation of Naturally Fractured Reservoirs", paper SPE 36730 presented at the 1996 SPE Annual Technical Conference and Exhibition held in Denver, Colorado, 6-9 October.

5. Dershowitz, B., Lapointe, P., Eiben, T., and Wei, L.: "Integration of Discrete Feature Network Methods with Conventional Simulator Approaches," paper SPE paper 62498 presented at the 1998 SPE Annual Technical Conference and Exhibition held in New Orleans, Louisiana, 27-30 September.

6. Oda, M.: "Fabric Tensor for Discontinuous Geologic Materials," Soils Fdns (1982) 22, No. 4, 96.

7. Oda, M., Suzuki, K., and Maeshibu, T.: "Elastic Compliance for Rock-Like Materials," Soils Fdns (1984) 24, No. 3, 27.

8. Schoenberg, M.: "Reflection of Elastic Waves from Periodically Stratified Media with Interfacial Slip,” Geophys. Prosp. (1983) 31, 265.

9. Schoenberg, M. and Sayers, C.M.: "Seismic Anisotropy of Fractured Rock," Geophysics (1995) 60, No. 1, 204.

10. Schoenberg, M. and Douma, J.: "Elastic Wave Propagation in Media with Parallel Fractures and Aligned Fractures," Geophysical Prospecting (1988) 36, 571. 
11. Brown, R.L. and Gupta, A.: "Problems Calibrating Production and Seismic Data for Fractured Reservoirs," paper SPE 67317 presented at the 2001 SPE Production and Operations Symposium held in Oklahoma City, Oklahoma, 24-27 March.

12. Pickup, G.E. and MacBeth, C.: "Integrating Effective Flow and Seismic Properties," presented at the EAGE/SEG Technical Conference and Exhibition held in Zurich, Switzerland (May 2001).

13. King, M.S., Xu, S., and Shakeel, A.: "Elastic Wave Propagation and Fluid Permeability in Rocks with Systems of Natural Fractures," Int. J. Rock Mech. Min. Sci. (1998), 35, No. 4, 445.

14. King, M.S.: "Elastic Wave Propagation in and Permeability for Rocks with Multiple Parallel Fractures”, Int. J. Rock Mech. Min. Sci. (2002) 39, 1033.

15. Pyrak-Nolte, L.J.: "The Seismic Response of Fractures and the Interrelations among Fracture Properties," Int. J. Rock Mech. Min. Sci. \& Geomech. Abstr. (1996) 33, No. 8, 787.

16. Qui, Y., Holtz, M.H., and Yang, A.: “Applying Curvature Analysis to the Placement of Horizontal Wells: Example from the Mabee (San Andres) Reservoir, Texas," paper SPE 70010 presented at the 2001 SPE Permian Basin Oil and Gas Recovery Conference, Midland, Texas, 15-16 May.

17. Parney, B. and LaPointe, P.: "Fractures Can Come into Focus", AAPG Explorer (Oct. 2002), 31-32.

18. Parney, B. and LaPointe, P.: "Simple Seismic, Complex Fractures," AAPG Explorer (Nov. 2002), 31-32.

19. Datta-Gupta, A., Vasco, D.W., and Yoon, S.: "Integrating Dynamic Data into High-Resolution Reservoir Models Using Streamline-Based Analytic Sensitivity Coefficients," SPEJ (Dec. 1999) 389.

20. Landa, J.: "Technique to Integrate Production and Static Data in a SelfConsistent Way," paper SPE 71597 presented at the 2001 SPE Annual Technical Conference and Exhibition, New Orleans, Louisiana, 30 September - 3 October. 
21. Landa, J., Horne, R.N., Kamal, M.M., and Jenkins, C.D.: "Reservoir Characterization Constrained to Well-Test Data: A Field Example," SPE Reservoir Evaluation and Engineering (Aug. 2000) 3, No. 4.

22. Huang, X., Meister, L., and Workman, R.: "Improvement and Sensitivity of Reservoir Characterization Derived from Time-Lapse Seismic Data," accepted for presentation at the 1998 SPE Annual Technical Conference and Exhibition, New Orleans, Louisiana, 27-30 September.

23. Huang, X., Will, R., and Waggoner, J.: "Reconciliation of Time-lapse Seismic Data with Production Data for Reservoir Management: A Gulf of Mexico Reservoir," paper SPE 65155 presented at the 2000 SPE European Petroleum Conference, Paris, France, 24-25 October.

24. Warren, J.E. and Root, P.J.: "The Behavior of Naturally Fractured Reservoirs," The Society of Petroleum Engineering Journal, Trans. AIME (Sept 1963) 28, 245 .

25. Kazemi, H, Merrill, L.S., Porterfield, K.L., and Zeman, P.R.: "Numerical Simulation of Water-Oil Flow in Fractured Reservoirs," SPEJ (1976), 317.

26. Bourbiaux, B., Basquet, R., Cacas, M, and Daniel, J: “An Integrated Workflow to Account for Multi-Scale Fractures in Reservoir Simulation Models: Implementation and Benefits," paper SPE 78489 presented at the $10^{\text {th }}$ Abu Dhabi International Petroleum Exhibition and Conference, Adu Dhabi, 13-16 October, 2002.

27. Basquet, R., Jeannin, L., Lange, A., and Bourbiaux, B.: "Gas Flow Simulation in Discrete Fracture Network Models," paper SPE 79708 presented at the SPE Reservoir Simulation Symposium, Houston, Texas, 3-5 February, 2003.

28. Lee, S.H., Jensen, C.L., and Lough, M.F.: “An Efficient Finite Difference Model for Flow in a Reservoir with Multiple Length Fractures," paper SPE 56752 presented at the 1999 SPE Annual Technical Conference and Exhibition, Denver, Colorado, 6-9 October. 
29. He, N., Lee, S. and Jensen, C.: "Combination of Analytical, Numerical, and Geostatistical Methods to Model Naturally Fractured Reservoirs," paper SPE 68832 presented at the SPE Western Regional Meeting, Bakersfield, California, 26-30 March.

30. Gurpinar, O. and Kossack, C.: "Realistic Numerical Models for Fractured Reservoirs," paper SPE 68268 presented at the 2000 SPE International Petroleum Conference and Exhibition, Villahermosa, Mexico, 1-3 February.

31. Kuster, G.T. and Toksoz, M.N.: "Velocity and Attenuation of Seismic Waves in Two-Phase Media," Geophysics (1974), 587.

32. Hudson, J.A.: "Wave Speeds and Attenuation of Elastic Waves in Material Cracks," Geophys. J. Royal Astronom. Soc. 64, 133.

33. Sayers, C.M.: "Fluid Dependent Shear-Wave Splitting in Fractured Media," Geophys. Prospecting (2002) 50, 393.

34. Yip, C.K.: "Shear Strength and Deformability of Rock Joints," SM Thesis, Department of Civil Engineering, Massachusetts Institute of Technology, Cambridge, Massachsetts (1979).

35. Perez, M.A. and Gibson, R.L.: "Detection of Fracture Orientation Using Azimuthal Variation of P-wave AVO Response: Barinas Field (Venezuela)," Geophysics (1999) 64, No. 4, 1253.

36. Ikelle, L.T.: "Amplitude Variations with Azimuth (AVAZ) Inversion Based on Linearized Inversion of Common Azimuth Sections," Seismic Anisotropy, SEG, Tulsa, 601-644.

37. Mallick, S., Craft, K., Meister, L., and Chambers, R.: "Determination of the Principle Directions of Azimuthal Anisotropy from P-Wave Seismic Data," Geophysics (1998) 63, No. 2, 692.

38. Lynn, H.B., Simon, K.M., Layman, M., Schneider, R., Bates, C.R., and Jones, M.: "Use of Anisotropy in P-Wave and S-Wave Data for Fracture 
Characterization in a Naturally Fractured Gas Reservoir," The Leading Edge (1995), 887-893.

39. Sayers, C.M. and Ebrom, D.A.: "Seismic Traveltime Analysis for Azimuthally Anisotropic Media: Theory and Experiment," Geophysics (1997) 62, No. 5, 1570-1582.

40. Dershowitz, W., Hurley, N., and Been, K.: "Stochastic Discrete Fracture Modeling of Heterogeneous and Fractured Reservoirs," Proceedings of the 3rd European Conference on the Mathematics of Oil Recovery, Delft, The Netherlands, 22-28 October, 1992.

41. Dershowitz, W., Lee, G., Geier, J., Foxford, T., LaPointe, P., and Thomas, A.: "Fracman: Discrete Feature Data Analysis, Geometric Modeling, and Exploration Simulation. User Documentation, Ver. 2.6," Golder Associates, (2003).

42. Dershowitz, W. and Herda, H.H.: "Interpretation of Fracture Spacing and Intensity," Proceedings of the 33rd U.S. Symposium on Rock Mechanics, Santa Fe, New Mexico, 757, 1-3 September, 1992.

43. Bushara, M.N., Tawel, A., Borougha, H., Dabbouk, C., and Qotb, M.: "Effective Permeability Modeling: Geostatistical Integration of Permeability Indicators, Offshore Abu Dhabi," paper SPE 78583 presented at the $10^{\text {th }}$ Abu Dhabi International Petroleum Exhibition and Conference, Dubai, Abu-Dhabi, 13-16 October, 2002.

44. Ouenes, A. and Hartley, L.J.: "Integrated Fractured Reservoir Modeling Using Both Discrete and Continuum Approaches," paper SPE paper 62939 presented at the 2000 SPE Annual Conference and Exhibition, Dallas, Texas, 1-4 October.

45. Tran, N.H., Rahman M.K., and Rahman, S.S.: "A Nested Neural-FractalStochastic Technique for Modeling Naturally Fractured Reservoirs," paper SPE 77877, presented at the 2002 SPE Asia Oil and Gas Conference and Exhibition, Melbourne, Australia, 8-10 October. 
46. Gurpinar, O., Kalbus, J., and List, D.F.: "Numerical Modeling of a Large, Naturally Fractured Oil Complex", paper SPE 59061 presented at the 2000 SPE Petroleum Conference and Exhibition in Mexico held in Villahermosa, Mexico, 1-3 Feb.

47. Agarwal, B., Hermansen, H., Sylte, J.E., and Thomas, L.K.: "Reservoir Characterization of Ekofisk Field: A Giant, Fractured Chalk Reservoir in the Norwegian North Sea - History Match”, paper SPE 51893, presented at the 1999 SPE Reservoir Simulation Symposium held in Houston, Texas, 14-17 Feb.

48. Schlue, J.: "Geophysical Inverse Methods - Course Notes," New Mexico Institute of Mining and Technology (1980).

49. Mavko, G., Mukerji, T., and Dvorkin, J.: The Rock Physics Handbook , Cambridge University Press (1998).

50. Sayers, C.M.: "Stress-Dependent Elastic Anisotropy of Sandstones", Geophysical Prospecting (2002) 50, 85-95. 


\section{APPENDIX A}

\section{OVERVIEW OF THE METHOD}

In this appendix we present the overall workflow and software components used in the new method. This chapter will start with an overview of the process making up the new method. Each section will include comments regarding the limitations and approximations imposed.

\section{A.1 Process Overview}

The new process involves parallel workflows in which both the hydraulic and elastic effective media properties are computed from an initial estimate of the fracture distribution. These effective media models are used to predict the production and seismic responses from the model, such as rates, pressures, seismic amplitudes, and elastic moduli. The differences between predicted observations from the model estimate and the actual field data are used as input to an iterative, linear gradient based optimization technique designed to optimize estimates of fracture system trend and P32 intensity. The required workflow was synthesized from a combination of existing software and published algorithmic components. In some cases existing third party software was directly employed. In other cases, published algorithms were implemented using the Matlab programming language. The software packages used were;

- FracMan - Interactive Discrete Data Analysis, Geometric Modeling, and Exploration Simulation made available under academic license by Golder Associates.

- Eclipse Office - The complete Eclipse Office software suite made available by the author's employer Schlumberger.

- MATLAB - Mathworks MATLAB Version 6.5 made available by Schlumberger.

Table A.1 lists the discrete tasks in the workflow and the specific applications used. 
Table A.1 - Process tasks and software applications used

\begin{tabular}{|c|c|c|}
\hline Task & Description & Application \\
\hline 1 & Conditional stochastic fracture modeling & FracWorks \\
\hline 2 & Permeability upscaling & StrataFrac \\
\hline 3 & 3D simulator model building & Eclipse FloGrid \\
\hline 4 & Dual porosity flow simulation & Eclipse 300 \\
\hline 5 & Computation of elastic moduli & Matlab \\
\hline 6 & Seismic attribute modeling & Matlab \\
\hline 7 & $\begin{array}{l}\text { Computation of sensitivity coefficients for } \\
\text { fracture system trend and P32 intensity }\end{array}$ & Matlab \\
\hline 8 & Residual analysis & Matlab \\
\hline 9 & Parameter updating & Matlab \\
\hline & $\begin{array}{l}\text { Various format conversion, visualization, } \\
\text { and reporting functions. }\end{array}$ & Matlab, Eclipse Office \\
\hline
\end{tabular}

The process workflow is illustrated in figure A.1. Each iteration of the new method required execution of the tasks 1 through 9. Also, as will be discussed in Section A.8 below, the computation of sensitivity coefficients for sensitivity coefficients required additional execution of tasks 1-6 with a perturbation of each unknown at each iteration step. This means that for models with two fracture sets tasks 1-6 were repeated five times within each iteration step. 


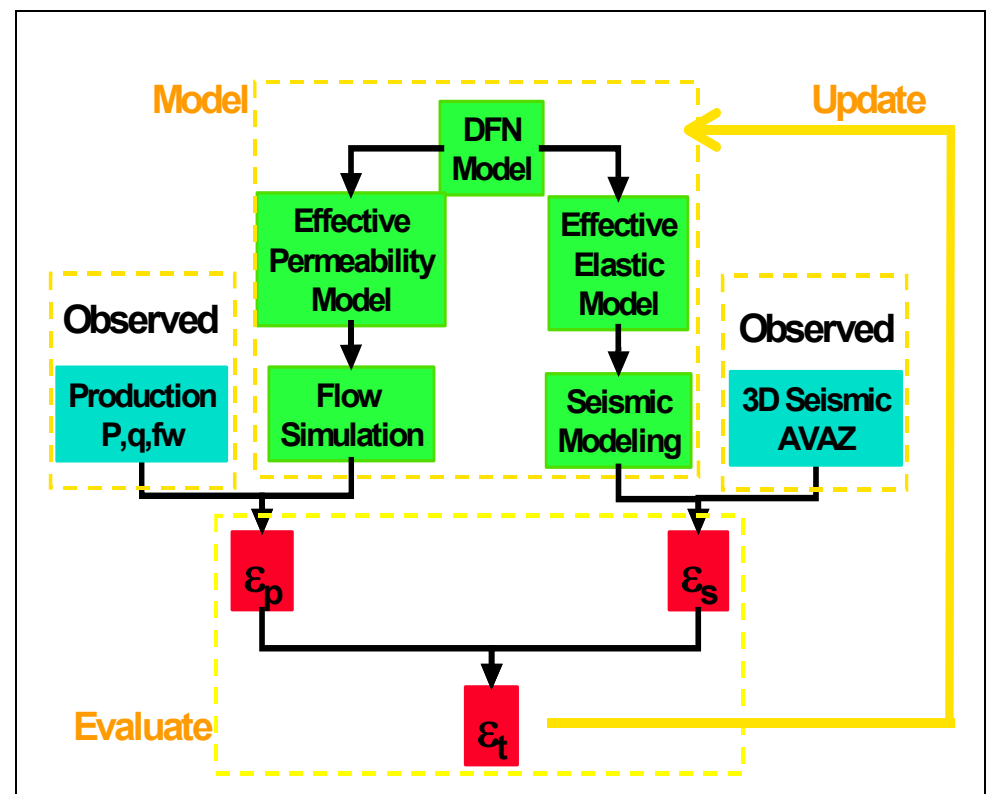

Fig. A.1 - Overview of the process for the new method.

\section{A.2 Description of Individual Tasks}

\section{Task 1 - Conditional Stochastic Fracture Modeling}

Stochastic fracture models used in this process were created using FracWorks from Golder. Table A.2 lists the input parameters required for fracture model generation. For the purpose of this study reasonable estimates for geometrical parameters and properties were from one of the following sources;

- Available literature.

- Dershowitz of Golder Associates through personal communication.

- Simplification of the above required due to process constraints. For example, all fractures were modeled as vertical and extending the entire thickness of the reservoir in order to simplify elastic modeling.

The fracture model geometry was determined by the production configuration. All geometric and rock/fluid property parameters were held fixed with the exception of fracture system trend and P32 intensity, which were the target parameters for the new process. 
Table A.2 - FracWorks Parameters

\begin{tabular}{|l|l|}
\hline Section & Parameters \\
\hline Region & Shape, dimensions, truncation \\
\hline Geometry & Orientation, size, shape, intensity \\
\hline Properties & Transmissivity, storativity, thickness \\
\hline
\end{tabular}

FracWorks allows recording of a macro file of all parameters and instructions used in a modeling session. For this new process a master macro file was recorded for the initial model. This master macro file was systematically modified and a version saved corresponding to each trend and P32 intensity parameter change either required for computation of sensitivity coefficients, or as dictated by data driven parameter updates. All macro files are saved, providing a process audit trail and facilitating reliable regeneration of any fracture models.

A DFN model was generated for the base case, the initial estimate, and each parameter update. In addition, DFN models were created for each trend and P32 intensity perturbation used in computation of sensitivity coefficients.

\section{Task 2 - Permeability Upscaling}

Use of the dual porosity finite difference simulation for this new method required the conversion, or upscaling, of the discrete fracture model to a representative continuum model for permeability of the fracture system. The FracMan StrataFrac application was used to upscale discrete fracture conductivity to effective continuum media conductivity on a regular grid system using Oda's ${ }^{3}$ method. Fracture model files generated by FracWorks are input to StrataFrac along with a grid definition file describing grid geometry. Typical upscaled x-y grid size for this study was $10 \mathrm{ft}$ x $10 \mathrm{ft}$. The StrataFrac upscaling process generates an ASCII file containing hydraulic conductivity tensor components for each grid block in the model. Using Matlab the diagonal tensor 
components were converted to $\mathrm{k}_{\mathrm{xx}}, \mathrm{k}_{\mathrm{yy}}$, and $\mathrm{k}_{\mathrm{zz}}$, and output in a format suitable for $3 \mathrm{D}$ simulator model building. Although it would have been desirable to use full a full permeability tensor for flow simulation, the added complexity and computational overhead in flow simulation for this feature was prohibitive. Therefore, off-diagonal tensor components were neglected. Permeability upscaling was performed on the DFN distributions for the base case, the initial estimate, and each parameter update. In addition, permeability upscaling was performed on all DFN models created using parameter perturbations for computation of sensitivity coefficients.

\section{Task 3 - Simulator Model Building}

Gridded models for dual porosity flow simulation were generated using Eclipse FloGrid using the following procedure;

- Reservoir Top and Base - Surfaces representing the reservoir top and base were generated using a simple text file editor and input to FloGrid as generic surfaces.

- Matrix Porosity and Permeability - Maps of constant matrix porosity and permeability were generated using a text editor and input to FloGrid as generic property maps and assigned to the matrix.

- Fracture Porosity and Permeability - A table constant matrix porosity vs. depth values was generated using a text editor. This data was input to FloGrid as a generic property map and assigned to the matrix system. The format converted output from the fracture permeability upscaling process described in the previous section was input as a generic property map and assigned to the fracture system.

- Grid - A structured grid was created with the desired x, y, and z dimensions and block sizes. All models used in this study were single layer models. The typical xy grid block dimension for this study was $20 \mathrm{ft}$ x $20 \mathrm{ft}$.

- Property Population - Fracture and matrix porosity and permeability were upscaled from property maps to grid blocks using the arithmetic-harmonic option in FloGrid.

- Export - Fracture and matrix porosity and permeability were exported to ASCII files in .GRDECL format. 
A simulator model was created for the base case, the initial estimate, and each updated model. In addition, the simulator model was created for all models created using parameter perturbations for computation of sensitivity coefficients.

\section{Task 4 - Dual Porosity Flow Simulation}

Production data used in the new optimization method were generated using Eclipse 300 in dual porosity mode. Dual porosity simulation was performed on the base case, the initial estimate, and each updated model. In addition, dual porosity simulation was performed on all models created using parameter perturbations for computation of sensitivity coefficients. Grid corner point geometry, matrix and fracture porosity and permeability generated using FloGrid were input using INCLUDE statements in the Eclipse data files. Fluid properties and relative permeabilities were taken from Kazemi ${ }^{25}$. All simulations were run for a 600 day duration with twenty 30 day report steps. Restart files containing matrix and fracture oil and water saturations were output at each report step. Pressure, injection, and production summary vectors were also written to summary files. Fracture and matrix water saturation maps were created at selected report steps for visualization and inclusion in Chapter IV and Appendices B to D of this dissertation using Eclipse FloViz. Bottom hole pressure and oil production rate vectors were extracted from summary files and converted to formatted text files using Eclipse Office Result.

\section{Task 5 - Computation of Elastic Moduli}

Elastic moduli for the reservoir were computed for each DFN models using a Matlab implementation of the linear slip discontinuity model described in section 2.2 of Chapter II. This computation was performed by first establishing a regular computational grid over the fracture model (Fig. A.2). The following process was repeated for each grid block using the Matlab script STIFF (Appendix E);

1. Computation of the circle representing the representative elemental volume $(\mathrm{REV})$ radius centered at the cell center. 
2. Calculating the unit normal vectors for each fracture.

3. Testing of each fracture in the system for intersection with the REV volume.

4. Calculation of the surface area lying within the REV for each fracture intersecting the REV radius.

5. Calculation and integration of the unit normal weighted fracture compliance components for each contributing fracture.

6. Calculation of the $\alpha$ and $\beta$ terms for linear slip discontinuity elastic effective media model .

7. Calculation of the elastic components (elastic moduli) from $\alpha$ and $\beta$.

The results of the computation in the form of the lower half of the symmetrical stiffness tensors for all grid cells are output to a single ASCII file for use in computation of seismic attributes. Elastic moduli were computed for the base case, the initial estimate, and each updated model. In addition, elastic moduli were computed for all models created using parameter perturbations for computation of sensitivity coefficients. 


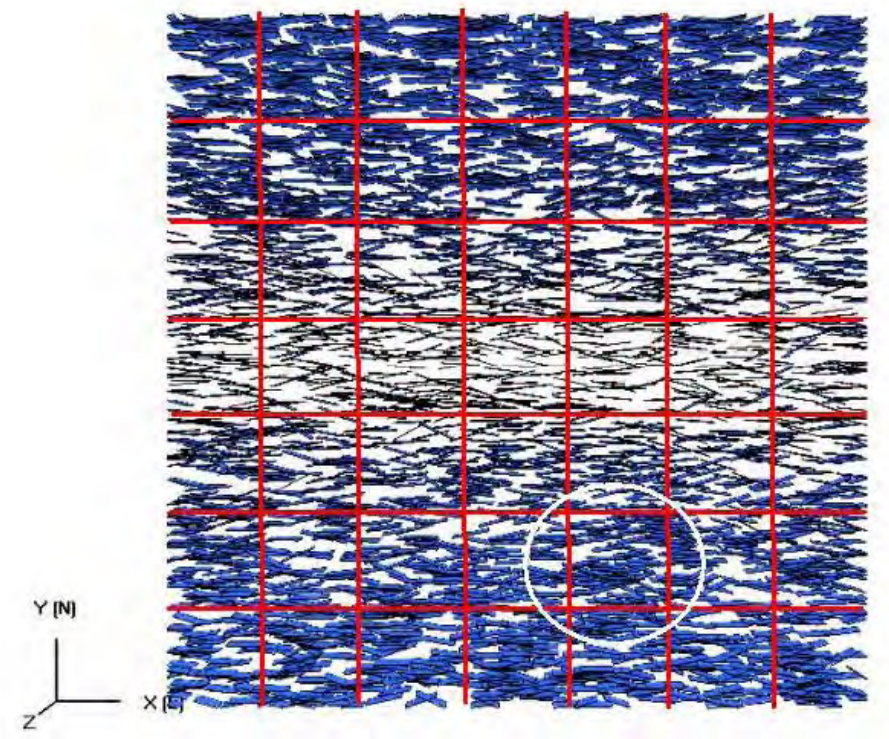

Fig. A.2 - Elastic effective media modeling computational grid and elastic REV radius (white circle). 


\section{Task 6 - Seismic Velocity Modeling}

For the purpose of computing quasi-P wave phase velocity a two layer elastic model was defined with an isotropic layer over the anisotropic fractured layer. Quasi-P wave phase velocities for the anisotropic medium were computed for each grid cell using Matlab and a FORTRAN executable. Phase velocities for the anisotropic layer were computed for all azimuths and all angles of incidence at the interface between the two layers. The overburden layer was used to allow selection of quasi-P wave velocity gathers based on angle of incidence as would be the case for the process of sorting angle gathers in seismic processing. For a selected angle of incidence the quasi-P wave velocity versus azimuth data were fitted to determine velocity modulation amplitude B, and azimuth of maximum quasi-P wave velocity $\phi_{\mathrm{qpv}}$.

The following tasks were performed for each grid cell using the Matlab script CREATE_ATTR (Appendix F);

1. The elastic stiffness tensor data is read in from the output of STIFF.

2. These data, along with overburden elastic properties, are passed to a FORTRAN program for calculation of azimuthally and incidence angle variant elastic phase velocities for azimuths of 0-360 degrees and incidence angles of 0-90 degrees..

3. Phase velocities for all azimuths are extracted for a user selected incidence angle.

4. The peak modulation amplitude of azimuthally variant seismic velocity and the fast shear direction (modulation phase angle) are computed.

The resulting attributes for all grid cells are written to a single ASCII file for later use in computation of sensitivity coefficients. Fig. A.3 shows an example of the quality control quiver/contour plot from the Matlab script CREATE_ATTR. Arrows indicate the fast shear direction. Contours indicate the velocity modulation peak amplitude. 


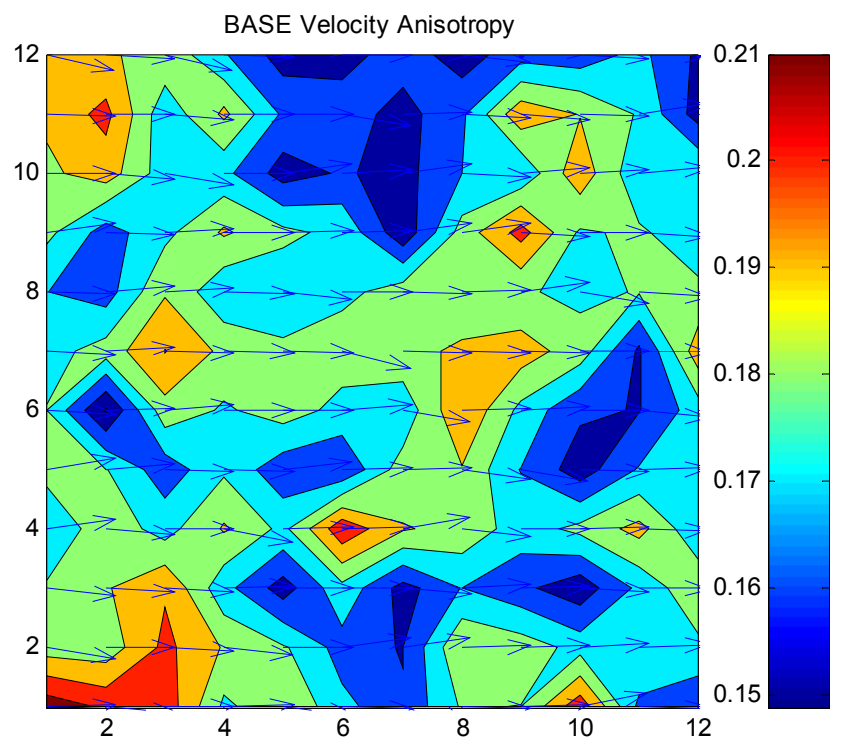

Fig. A.3 - Seismic anisotropy attribute map computed from elastic stiffness tensors.

Seismic attributes were computed for the base case, the initial estimate, and each updated model. In addition, seismic attributes were computed for all models created using parameter perturbations for computation of sensitivity coefficients.

\section{Task 7 - Computation of Sensitivity Coefficients}

Sensitivity coefficients were computed using a Matlab script script implementation of Eqs. 3.14 (script SENS located in Appendix G). This script read in all observation data files for the current model parameter state and the associated parameter perturbations. Production observations for each well were averaged over the entire simulation timespan. Seismic attributes were averaged over the entire 2D map area. Sensitivity coefficients were computed as the derivative of the observation averages. This process involved execution of tasks 1-6 in Table A.1 for the new model estimate as well as for each target inversion parameter (trend and P32 intensity) using the parameter perturbations. As a result, each iteration of the new method for a model with two fracture sets involved five executions of steps 1-6 of the workflow. Sensitivity coefficients for test cases are displayed in bar chart format in Appendices B to D. 


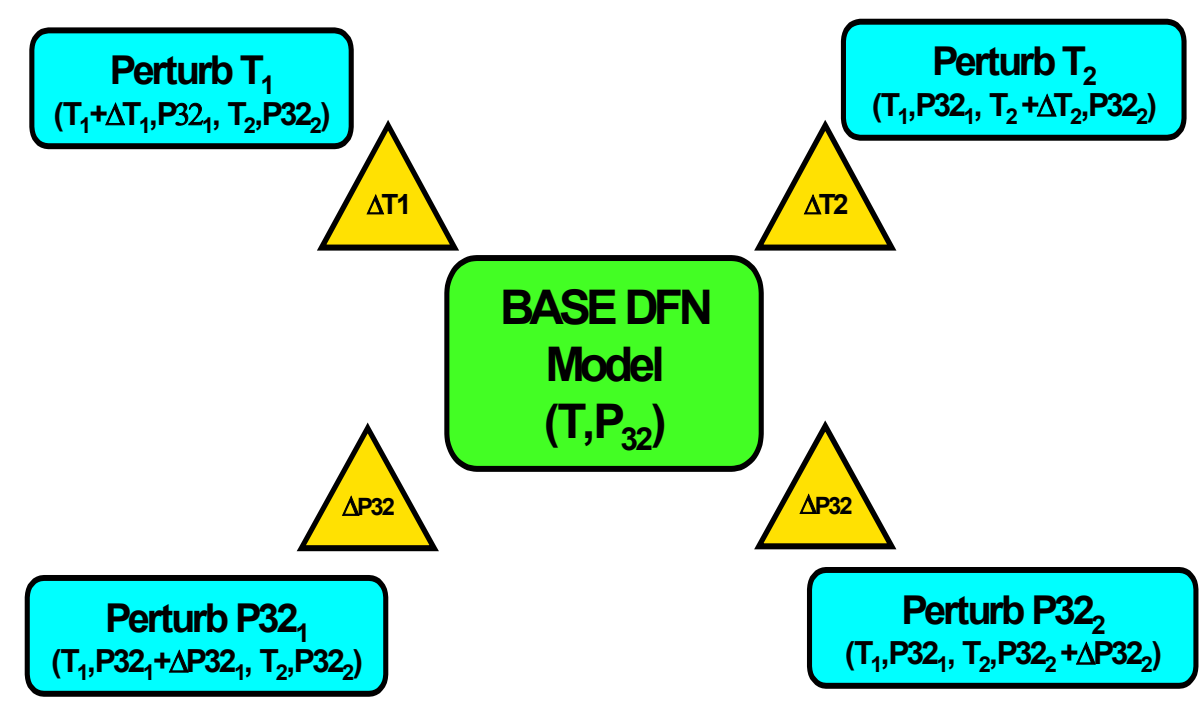

Fig. A.4 - Schematic diagram of sensitivity coefficient computation.

\section{Task 8 - Residual Analysis}

Residual errors were computed using a Matlab implementation of Eqs. 3.17 to 3.20. The residual errors were reported in four ways;

- The combined residual error of all observations.

- The combined residual error of the bottom hole pressure observations of all injection and producing wells.

- The combined residual error of oil production rate for all producing wells.

- The combined residual error of the seismic attributes B and $\phi_{\mathrm{s} 1}$.

All residual vectors were normalized using observation standard deviations. For each iteration of the new method ten trials were run, each with a new sample of random observation noise. Residual errors for these 10 trials were averaged and reported in the discussions of test results in Chapter IV as well as in Appendices B to D. 


\section{Task 9 - Parameter Updates}

Parameter updates were computed using a Matlab implementation of the inversion methodology described in Chapter III at each of the 10 trials during each iteration. The resulting 10 parameter updates were averaged and the result used as the initial estimate in the following iteration. A priori model weighting was applied independently for each parameter and at each iteration based on an ad hoc evaluation of the behavior of the residual errors and parameter updates. A priori weights for all tests are listed in Appendices B to D. 


\section{APPENDIX B}

\section{SUMMARY OF TEST T2 RESULTS}

In this Appendix we provide plots and tables detailing optimization test T2. As this dissertation is based upon the application of the new optimization process to the test cases we provide this Appendix as a comprehensive documentation set for this test case. Selected portions of this information may be found in Chapter IV with a concise description of the optimization test.

\section{General Information}

- Fracture system properties.

- Petrophysical properties.

- Simulation parameters.

- Elastic properties.

\section{Summarized Test Results}

- Parameter error summary table and plot.

- Residual summary tables.

\section{Data for Each Iteration}

- Table of derivative perturbations.

- Table of prior model weights.

- Tables of raw parameter updates.

- Tables of raw residuals.

- Plots of sensitivity coefficients. 
For base case, initial estimate, and optimized model;

- Plots of fracture distributions.

- Maps of fracture system water saturation report step 150 days, 300 days, 450 days, 600 days.

- Maps of matrix water saturation report step 150 days, 300 days, 450 days, 600 days.

- Bottom hole pressure plots.

- Oil production rate plots.

- Seismic P-wave velocity versus azimuth plots.

- Seismic anisotropy attribute maps. 
Table B.1 - $\quad$ Test T2 Base Model Fracture Distribution Parameters.

\begin{tabular}{|l|c|}
\hline \multicolumn{1}{|c|}{ Parameter } & Set 1 \\
\hline Trend & Normal $[0,5]$ \\
\hline $\mathbf{P}_{\mathbf{3 2}}$ Intensity (1/m) & 0.1 \\
\hline Length (m) & Lognormal[50,3] \\
\hline Height (m) & 60 \\
\hline Transmissivity $\left.\mathbf{( m}^{\mathbf{2}} / \mathbf{s}\right)$ & $8 \mathrm{e}-04$ \\
\hline
\end{tabular}

Table B.2 - Test T2 Reservoir Properties.

\begin{tabular}{|l|c|}
\hline Matrix Porosity & 0.10 \\
\hline Matrix Permeability & $10 \mathrm{mD}$ \\
\hline Fracture Porosity & 0.015 \\
\hline
\end{tabular}

Table B.3 - Test T2 Flow Simulation Parameters.

\begin{tabular}{|l|c|}
\hline Sigma & 0.08 \\
\hline Report step & 30 days \\
\hline Number cells (i) & 131 \\
\hline Number cells (j) & 131 \\
\hline Cell size (i) & $20 \mathrm{ft}$ \\
\hline Cell size (j) & $20 \mathrm{ft}$ \\
\hline
\end{tabular}

Table B.4 - Test T2 Elastic Modeling Parameters.

\begin{tabular}{|l|c|}
\hline Matrix P-wave Velocity & $4670 \mathrm{ft} / \mathrm{sec}$ \\
\hline Matrix Shear-wave Velocity & $3060 \mathrm{ft} / \mathrm{sec}$ \\
\hline Matrix Density & $2510 \mathrm{~kg} / \mathrm{m}^{3}$ \\
\hline Overburden P wave Velocity & $4670 / \mathrm{ft} / \mathrm{sec}$ \\
\hline Overburden S wave Velocity & $3060 \mathrm{ft} / \mathrm{sec}$ \\
\hline Overburden Density & $2510 \mathrm{~kg} / \mathrm{m}^{3}$ \\
\hline Incidence Angle & $30 \mathrm{degrees}$ \\
\hline
\end{tabular}


Table B.5a - Test T2 production data only derivative perturbations.

\begin{tabular}{|l|r|r|r|r|r|}
\hline Iterations & \multicolumn{1}{|c|}{ 1 } & \multicolumn{1}{c|}{ 2 } & \multicolumn{1}{c|}{ 3 } & \multicolumn{1}{c|}{ 4 } & \multicolumn{1}{c|}{5} \\
\hline Trend 1 (degrees) & -6 & -6 & -6 & -6 & -3 \\
\hline P32 1 (1/m) & 0.03 & 0.02 & 0.02 & 0.02 & 0.01 \\
\hline
\end{tabular}

Table B.5b - Test T2 combined data derivative perturbations.

\begin{tabular}{|l|r|r|r|r|r|}
\hline Iterations & \multicolumn{1}{|c|}{ 1 } & \multicolumn{1}{c|}{ 2 } & \multicolumn{1}{c|}{ 3 } & \multicolumn{1}{c|}{$\mathbf{4}$} & \multicolumn{1}{c|}{$\mathbf{5}$} \\
\hline Trend 1 (degrees) & -6 & -6 & -6 & -3 & -3 \\
\hline P32 1 (1/m) & 0.03 & 0.02 & 0.02 & 0.01 & -0.005 \\
\hline
\end{tabular}

Table B.6a - Test T2 production data only prior model weights.

\begin{tabular}{|l|c|c|c|c|c|}
\hline Iterations & $\mathbf{1}$ & $\mathbf{2}$ & $\mathbf{3}$ & $\mathbf{4}$ & $\mathbf{5}$ \\
\hline Trend (1) & 2 & 2 & 2 & 2 & 2 \\
\hline P32 (1) & 2 & 2 & 2 & 2 & 2 \\
\hline
\end{tabular}

Table B.6b - Test T2 combined data prior model weights.

\begin{tabular}{|l|c|c|c|c|c|}
\hline Iterations & $\mathbf{1}$ & $\mathbf{2}$ & $\mathbf{3}$ & $\mathbf{4}$ & $\mathbf{5}$ \\
\hline Trend (1) & 1 & 2 & 2 & 2 & 2 \\
\hline P32 (1) & 0.2 & 2 & 2 & 2 & 2 \\
\hline
\end{tabular}


Table B.7 - $\quad$ Summary of absolute errors for fracture set trend and P32 intensity at each update step for both production data only and combined data tests. Step 0 is the initial estimate.

\begin{tabular}{|r|r|r|r|r|}
\cline { 2 - 5 } \multicolumn{1}{c|}{} & \multicolumn{4}{c|}{ Parameter Error } \\
\cline { 2 - 5 } \multicolumn{1}{c|}{} & \multicolumn{1}{c|}{ Production Only } & \multicolumn{2}{c|}{ Combined } \\
\hline Iteration & Trend & \multicolumn{1}{c|}{ P32 } & Trend & \multicolumn{1}{c|}{ P32 } \\
\hline $\mathbf{0}$ & 30.0 & 0.050 & 30.0 & 0.050 \\
\hline $\mathbf{1}$ & 23.1 & 0.038 & 19.8 & 0.034 \\
\hline $\mathbf{2}$ & 9.3 & 0.032 & 8.7 & 0.022 \\
\hline $\mathbf{3}$ & 7.4 & 0.022 & 5.9 & 0.009 \\
\hline $\mathbf{4}$ & 5.9 & 0.016 & 4.0 & 0.001 \\
\hline $\mathbf{5}$ & 5.0 & 0.008 & 2.7 & 0.002 \\
\hline
\end{tabular}

Table B.8 - $\quad$ Summary of unit normalized residual errors for fracture set trend and P32 intensity at each update step for combined data tests. Step 0 is the initial estimate.

\begin{tabular}{|r|r|r|r|r|}
\cline { 2 - 5 } \multicolumn{1}{c|}{} & \multicolumn{4}{c|}{ Residuals } \\
\hline Iteration & Total & \multicolumn{1}{c|}{ BHP } & \multicolumn{1}{c|}{ OPR } & SEIS \\
\hline $\mathbf{0}$ & 27.9 & 26.410 & 5.2 & 7.250 \\
\hline $\mathbf{1}$ & 20.8 & 20.240 & 2.5 & 4.750 \\
\hline $\mathbf{2}$ & 6.1 & 5.390 & 1.2 & 2.490 \\
\hline $\mathbf{3}$ & 3.4 & 2.656 & 1.9 & 1.400 \\
\hline $\mathbf{4}$ & 3.3 & 2.630 & 1.8 & 0.820 \\
\hline
\end{tabular}




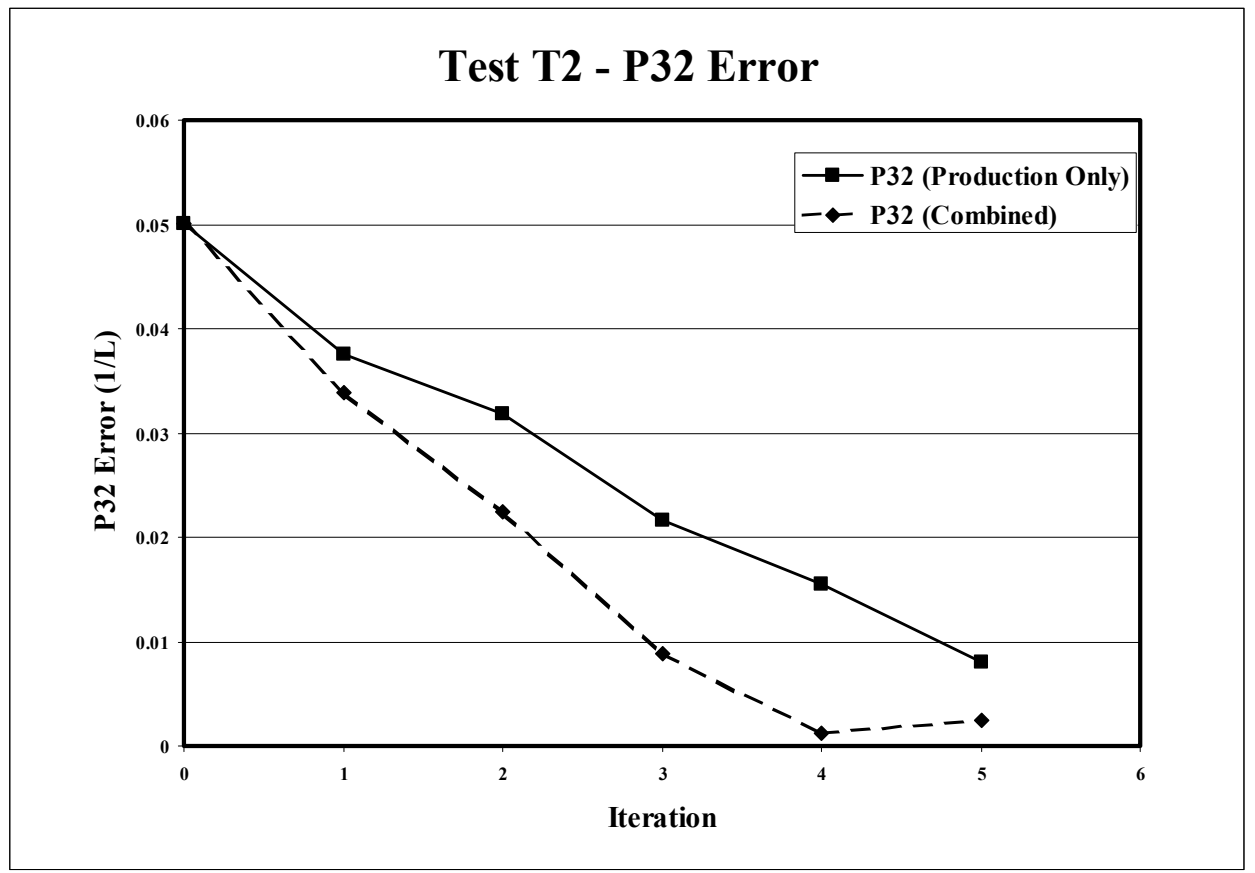

Fig. B.1 - Graph of absolute error in fracture set trend versus iteration for test $\mathrm{T} 2$. Iteration 0 is the initial estimate.

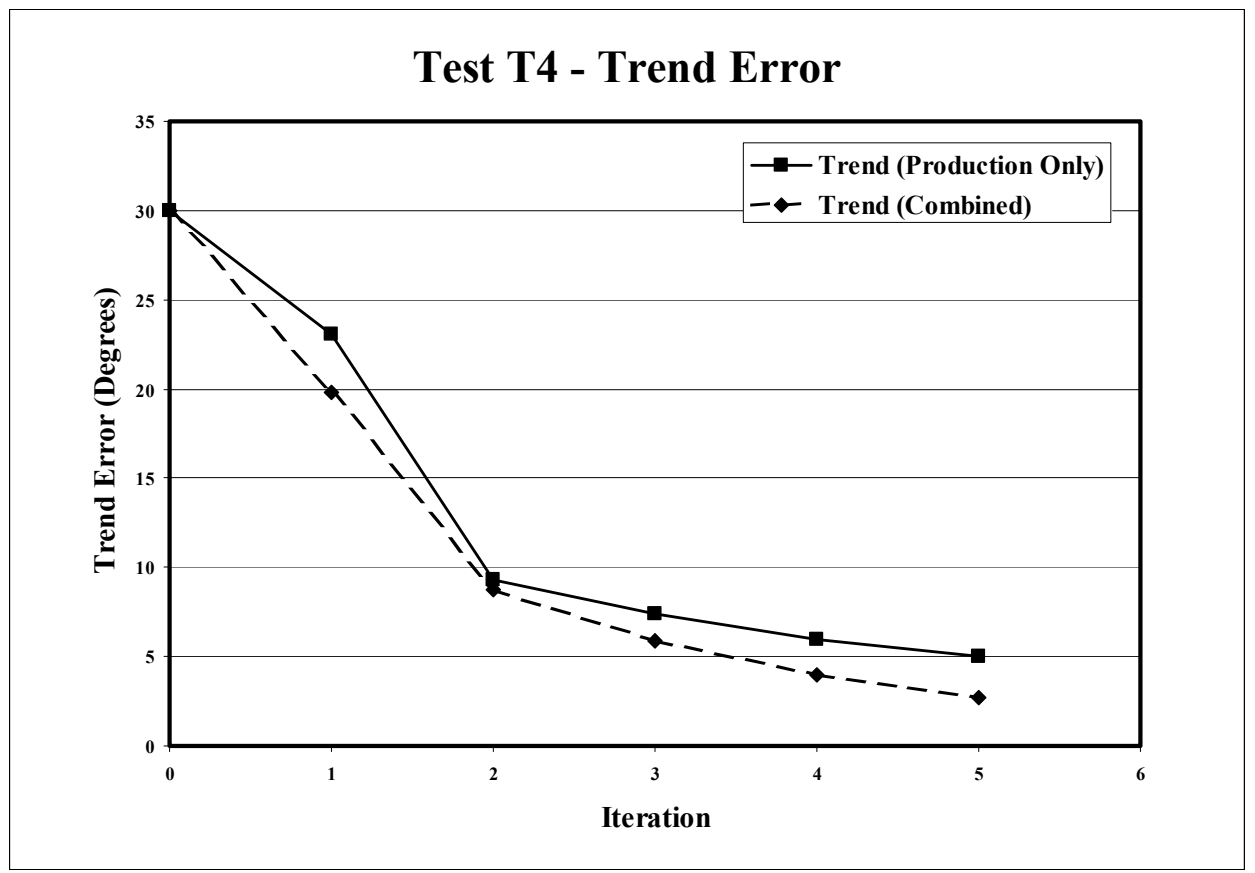

Fig. B.2 - Graph of absolute error in fracture set P32 intensity versus iteration for test $\mathrm{T} 2$. Iteration 0 is the initial estimate. 


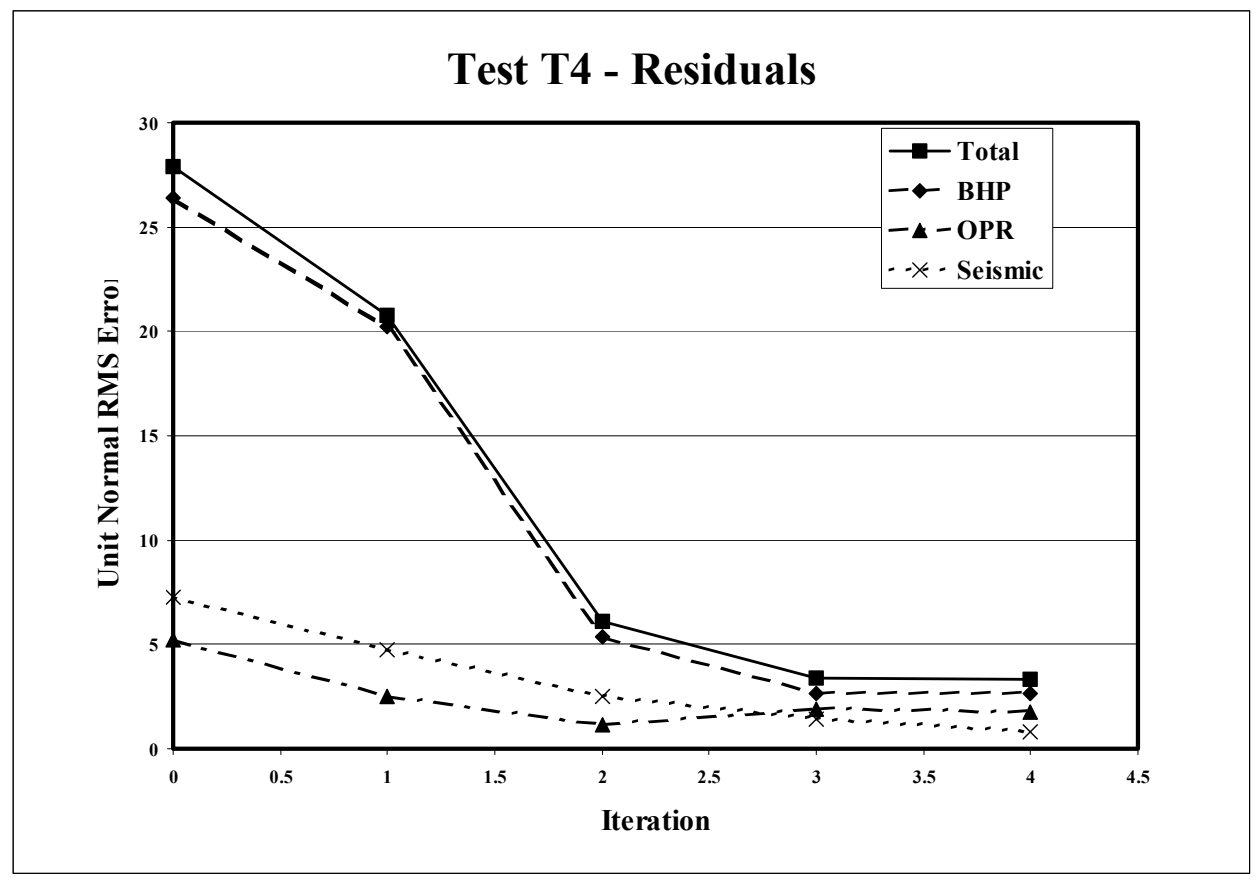

Fig. B.3 - Graph of unit normalized residual errors by observation type for fracture set trend and P32 intensity at each iteration, combined data test. Iteration 0 is the initial estimate.

Table B.9a - Summary of iteration 1 Trend and P32 intensity updates and residual errors for 10 samples.

\begin{tabular}{|c|c|c|c|c|c|c|c|c|}
\hline & \multicolumn{8}{|c|}{ Step M1 } \\
\hline & \multicolumn{4}{|c|}{ Updates } & \multirow{2}{*}{\multicolumn{4}{|c|}{ Combined Test Residuals }} \\
\hline & \multicolumn{2}{|c|}{ Production Only } & \multicolumn{2}{|c|}{ Combined } & & & & \\
\hline SAMPLE & Trend & P32 & Trend & P32 & TOTAL & $\overline{\text { BHP }}$ & OPR & SEIS \\
\hline 1 & 20.9 & 0.155 & 20.3 & 0.166 & 28.10 & 26.20 & 5.00 & 7.40 \\
\hline 2 & 18.0 & 0.142 & 21.3 & 0.166 & 27.50 & 26.60 & 5.30 & 7.30 \\
\hline 3 & 23.3 & 0.164 & 19.5 & 0.169 & 28.00 & 26.60 & 5.40 & 7.10 \\
\hline 4 & 17.0 & 0.143 & 20.0 & 0.163 & 27.80 & 26.40 & 5.20 & 7.20 \\
\hline 5 & 14.1 & 0.126 & 20.3 & 0.168 & 28.20 & 26.80 & 5.30 & 7.20 \\
\hline 6 & 25.7 & 0.176 & 20.5 & 0.166 & 27.70 & 26.20 & 5.30 & 7.30 \\
\hline 7 & 26.4 & 0.182 & 19.3 & 0.166 & 27.90 & 26.30 & 5.30 & 7.00 \\
\hline 8 & 32.4 & 0.195 & 18.6 & 0.167 & 27.80 & 26.10 & 5.00 & 7.50 \\
\hline 9 & 23.7 & 0.158 & 18.5 & 0.166 & 28.20 & 26.20 & 5.00 & 7.20 \\
\hline 10 & 29.2 & 0.183 & 19.7 & 0.164 & 27.90 & 26.70 & 5.40 & 7.30 \\
\hline Average & 23.1 & 0.162 & 19.8 & 0.166 & 27.91 & 26.41 & 5.22 & 7.25 \\
\hline
\end{tabular}


Table B.9b - Summary of iteration 2 Trend and P32 intensity updates and residual errors for 10 samples.

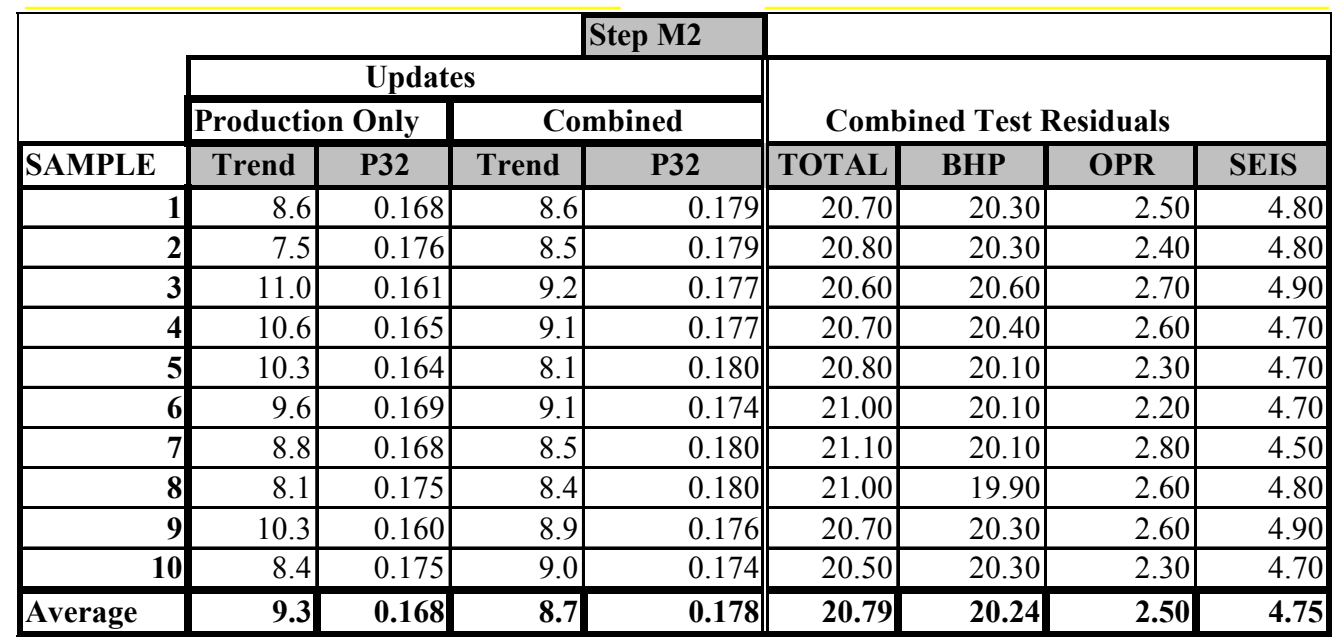

Table B.9c - Summary of iteration 3 Trend and P32 intensity updates and residual errors for 10 samples.

\begin{tabular}{|c|c|c|c|c|c|c|c|c|}
\hline & \multicolumn{4}{|c|}{ Step M3 } & & & & \\
\hline & \multicolumn{4}{|c|}{ Updates } & \multirow{2}{*}{\multicolumn{3}{|c|}{ Combined Test Residuals }} & \multirow[b]{3}{*}{ SEIS } \\
\hline & \multicolumn{2}{|c|}{ Production Only } & \multicolumn{2}{|c|}{ Combined } & & & & \\
\hline SAMPLE & Trend & P32 & Trend & P32 & TOTAL & $\overline{\text { BHP }}$ & $\overline{\text { OPR }}$ & \\
\hline 1 & 7.3 & 0.179 & 5.9 & 0.192 & 6.10 & 5.20 & 1.20 & 2.40 \\
\hline 2 & 7.4 & 0.178 & 5.6 & 0.193 & 6.20 & 5.20 & 1.40 & 2.60 \\
\hline 3 & 7.5 & 0.178 & 5.9 & 0.191 & 6.00 & 5.70 & 1.00 & 2.30 \\
\hline 4 & 7.4 & 0.179 & 6.0 & 0.192 & 6.10 & 5.50 & 1.20 & 2.60 \\
\hline 5 & 7.5 & 0.178 & 5.8 & 0.191 & 6.10 & 5.40 & 1.10 & 2.50 \\
\hline 6 & 7.5 & 0.178 & 6.0 & 0.190 & 6.50 & 5.40 & 1.00 & 2.40 \\
\hline 7 & 7.4 & 0.178 & 6.1 & 0.189 & 5.70 & 5.30 & 1.10 & 2.60 \\
\hline 8 & 7.2 & 0.179 & 6.0 & 0.190 & 5.80 & 5.40 & 1.20 & 2.40 \\
\hline 9 & 7.4 & 0.179 & 5.9 & 0.192 & 6.10 & 5.70 & 1.20 & 2.50 \\
\hline 10 & 7.4 & 0.178 & 5.8 & 0.192 & 6.00 & 5.10 & 1.40 & 2.60 \\
\hline Average & 7.4 & 0.178 & 5.9 & 0.191 & 6.06 & 5.39 & 1.18 & 2.49 \\
\hline
\end{tabular}


Table B.9d - Summary of iteration 4 Trend and P32 intensity updates and residual errors for 10 samples.

\begin{tabular}{|c|c|c|c|c|c|c|c|c|}
\hline & \multicolumn{8}{|c|}{ Step M4 } \\
\hline & \multicolumn{4}{|c|}{ Updates } & \multirow{2}{*}{\multicolumn{4}{|c|}{ Combined Test Residuals }} \\
\hline & \multicolumn{2}{|c|}{ Production Only } & \multicolumn{2}{|c|}{ Combined } & & & & \\
\hline SAMPLE & Trend & P32 & Trend & P32 & TOTAL & BHP & OPR & SEIS \\
\hline 1 & 5.8 & 0.184 & 3.9 & 0.199 & 3.30 & 2.60 & 1.80 & 1.30 \\
\hline 2 & 6.1 & 0.184 & 4.2 & 0.198 & 3.20 & 2.80 & 1.80 & 1.50 \\
\hline 3 & 5.9 & 0.185 & 4.3 & 0.196 & 3.50 & 2.70 & 1.80 & 1.30 \\
\hline 4 & 6.0 & 0.185 & 3.8 & 0.200 & 3.50 & 2.60 & 2.10 & 1.30 \\
\hline 5 & 5.9 & 0.184 & 3.9 & 0.200 & 3.50 & 2.90 & 1.70 & 1.50 \\
\hline 6 & 5.8 & 0.185 & 4.0 & 0.199 & 3.20 & 2.50 & 1.60 & 1.40 \\
\hline 7 & 5.9 & 0.185 & 3.7 & 0.201 & 3.10 & 2.60 & 2.20 & 1.40 \\
\hline 8 & 6.0 & 0.184 & 4.1 & 0.197 & 3.50 & 2.60 & 1.90 & 1.20 \\
\hline 9 & 6.1 & 0.185 & 3.9 & 0.199 & 3.60 & 2.60 & 2.10 & 1.70 \\
\hline 10 & 5.9 & 0.184 & 4.0 & 0.199 & 3.50 & & & \\
\hline Average & 5.9 & 0.185 & 4.0 & 0.199 & 3.39 & 2.66 & 1.89 & 1.40 \\
\hline
\end{tabular}

Table B.9e - Summary of iteration 5 Trend and P32 intensity updates and residual errors for 10 samples.

\begin{tabular}{|c|c|c|c|c|c|c|c|c|}
\hline & \multicolumn{4}{|c|}{ Step M5 } & & & & \\
\hline & \multicolumn{4}{|c|}{ Updates } & \multirow{2}{*}{\multicolumn{4}{|c|}{ Combined Test Residuals }} \\
\hline & \multicolumn{2}{|c|}{ Production Only } & \multicolumn{2}{|c|}{ Combined } & & & & \\
\hline SAMPLE & Trend & P32 & Trend & P32 & TOTAL & BHP & OPR & SEIS \\
\hline 1 & 5.3 & 0.191 & 2.8 & 0.198 & 3.10 & 2.50 & 1.70 & 0.80 \\
\hline 2 & 5.0 & 0.192 & 2.5 & 0.197 & 3.60 & 3.00 & 1.70 & 1.00 \\
\hline 3 & 4.9 & 0.193 & 2.4 & 0.197 & 3.70 & 2.90 & 2.10 & 0.70 \\
\hline 4 & 5.1 & 0.193 & 2.7 & 0.198 & 3.40 & 2.90 & 1.60 & 0.70 \\
\hline 5 & 4.9 & 0.193 & 2.9 & 0.198 & 3.20 & 2.50 & 1.70 & 0.90 \\
\hline 6 & 5.0 & 0.192 & 2.8 & 0.198 & 3.30 & 2.30 & 2.20 & 0.70 \\
\hline 7 & 5.1 & 0.193 & 2.7 & 0.197 & 3.20 & 2.40 & 1.60 & 0.90 \\
\hline$\overline{8}$ & 4.9 & 0.192 & 2.8 & 0.198 & 3.20 & 2.60 & 1.80 & 0.90 \\
\hline$\overline{9}$ & 5.0 & 0.193 & 2.6 & 0.197 & 3.50 & 2.80 & 1.80 & 0.90 \\
\hline 10 & 5.0 & 0.192 & 2.8 & 0.198 & 3.00 & 2.40 & 1.70 & 0.70 \\
\hline Average & $\mathbf{5 . 0}$ & 0.192 & 2.7 & 0.198 & 3.32 & 2.63 & 1.79 & 0.82 \\
\hline
\end{tabular}


Fracture Distribution Maps

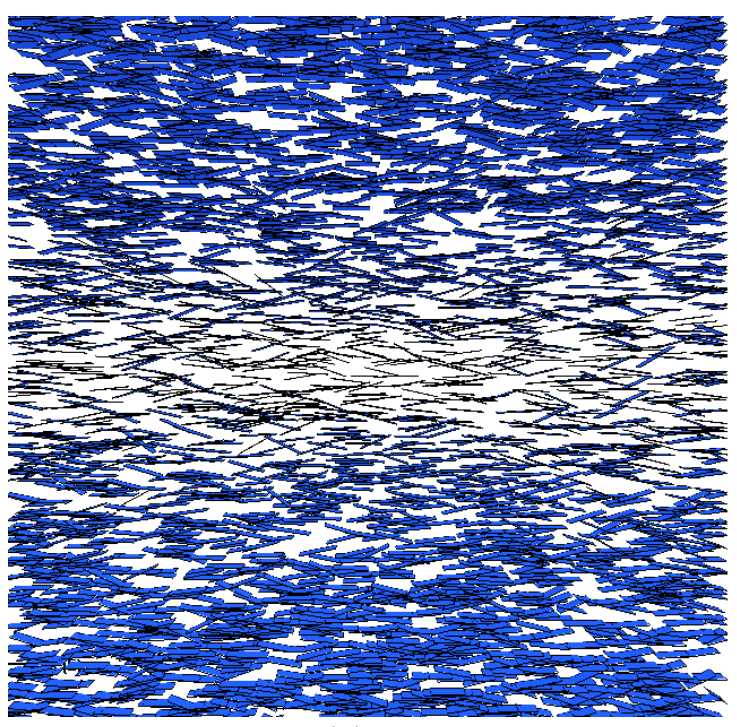

(a)

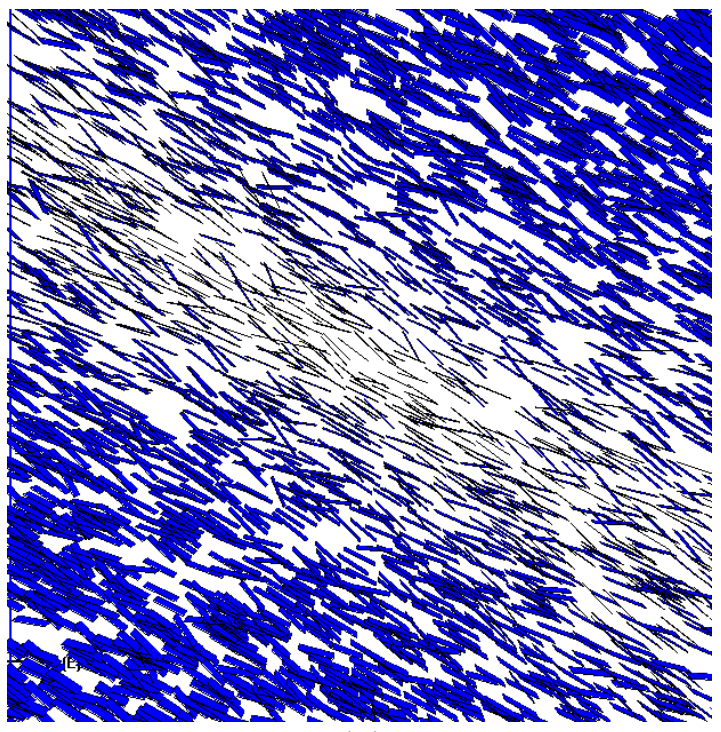

(b)

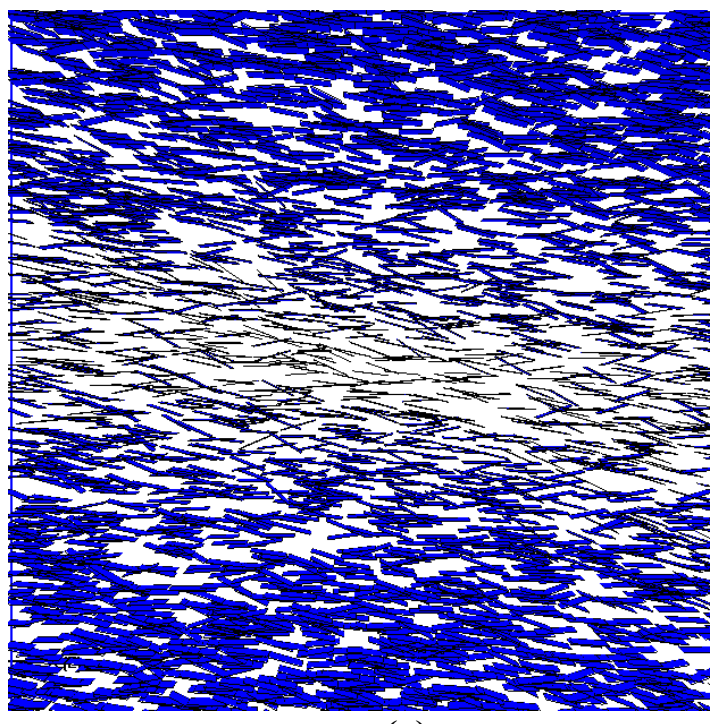

(c)

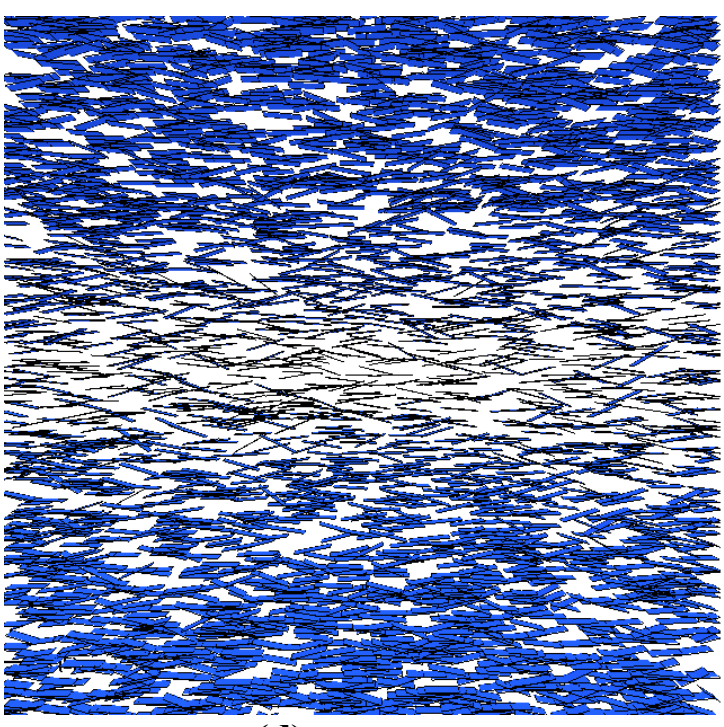

(d)

Fig. B.4 - Test T2 fracture models; (a) base model, (b) initial estimate, (c) final model using production data only, (d) final model using production and seismic data distribution. 


\section{Fracture Water Saturation Maps (Base, Initial, Final)}

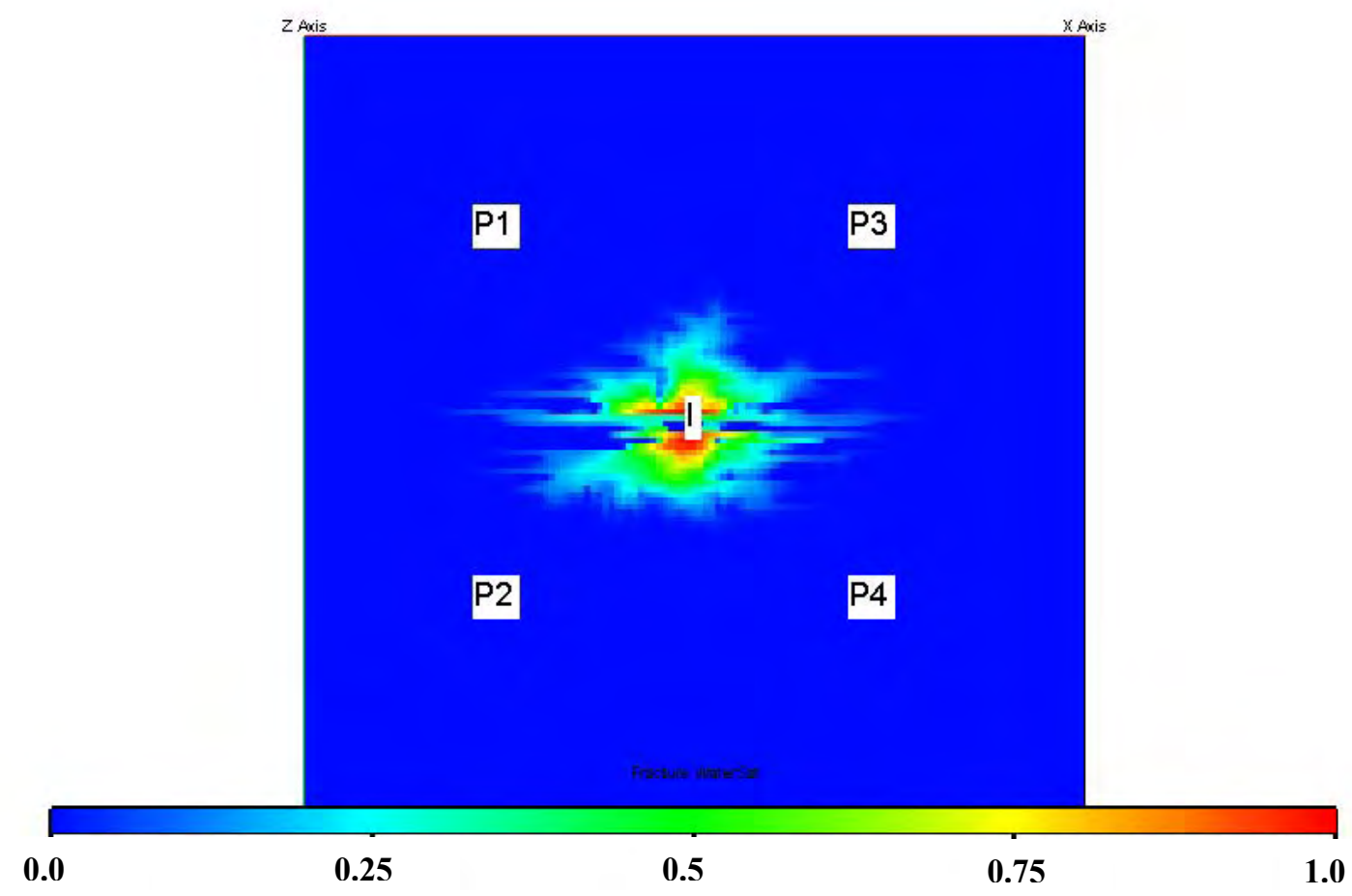

Fig. B.5a - Fracture water saturation at report step 5 (150 days) for test T2 base model.

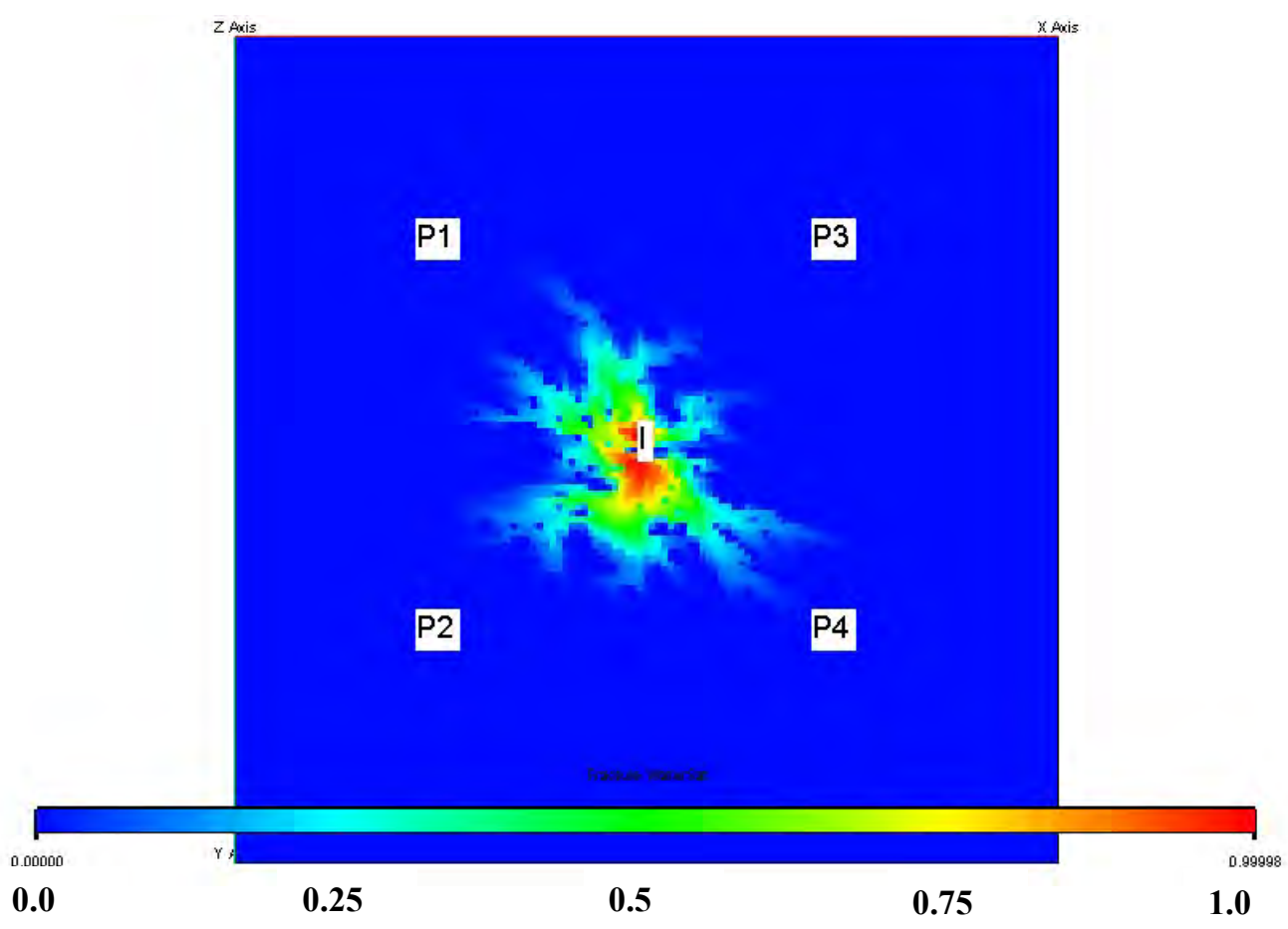

Fig. B.5b - Fracture water saturation at report step 5 (150 days) for test T2 initial estimate. 


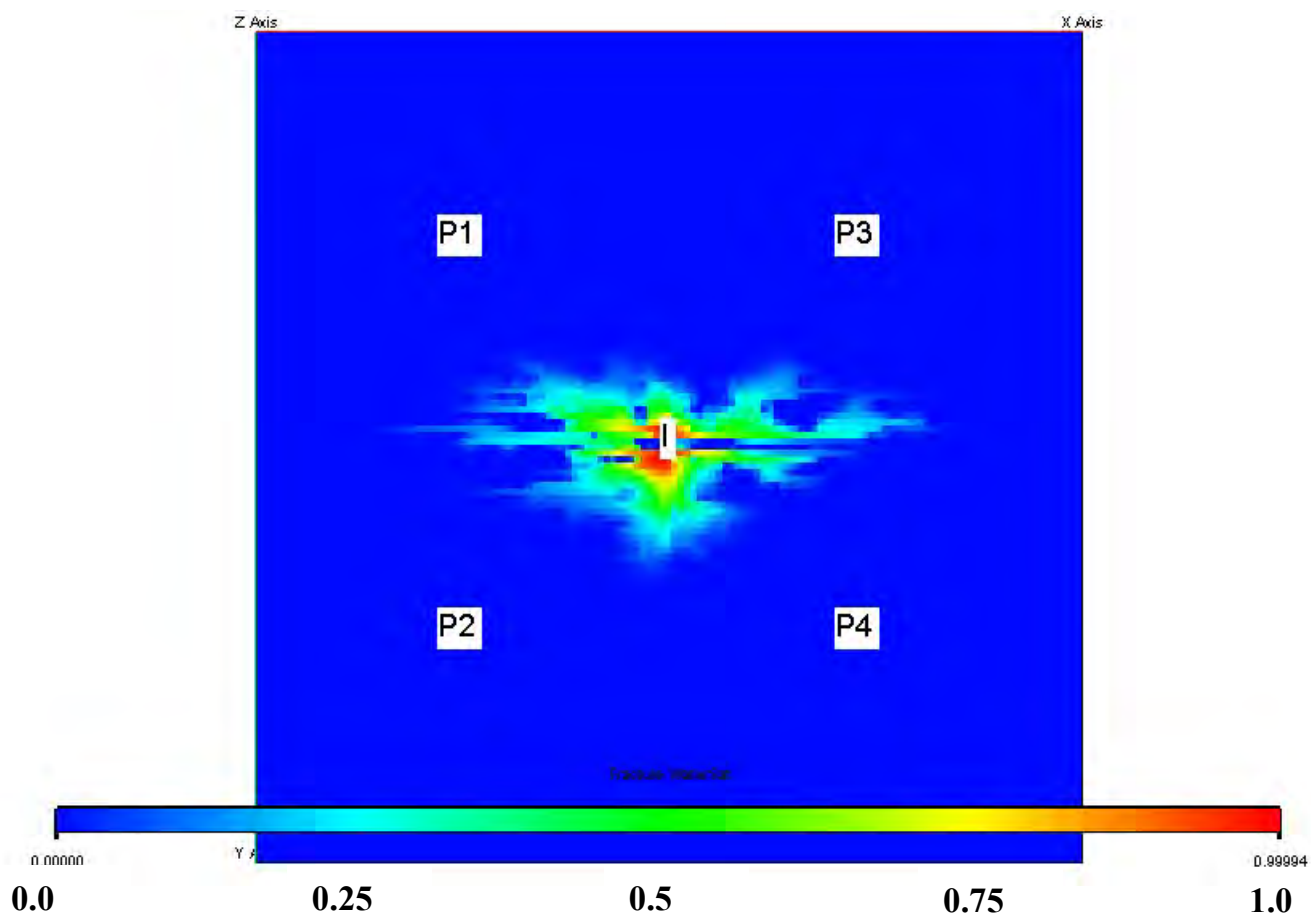

Fig. B.5c - Fracture water saturation at report step 5 (150 days) for test T2 final model using production data only.

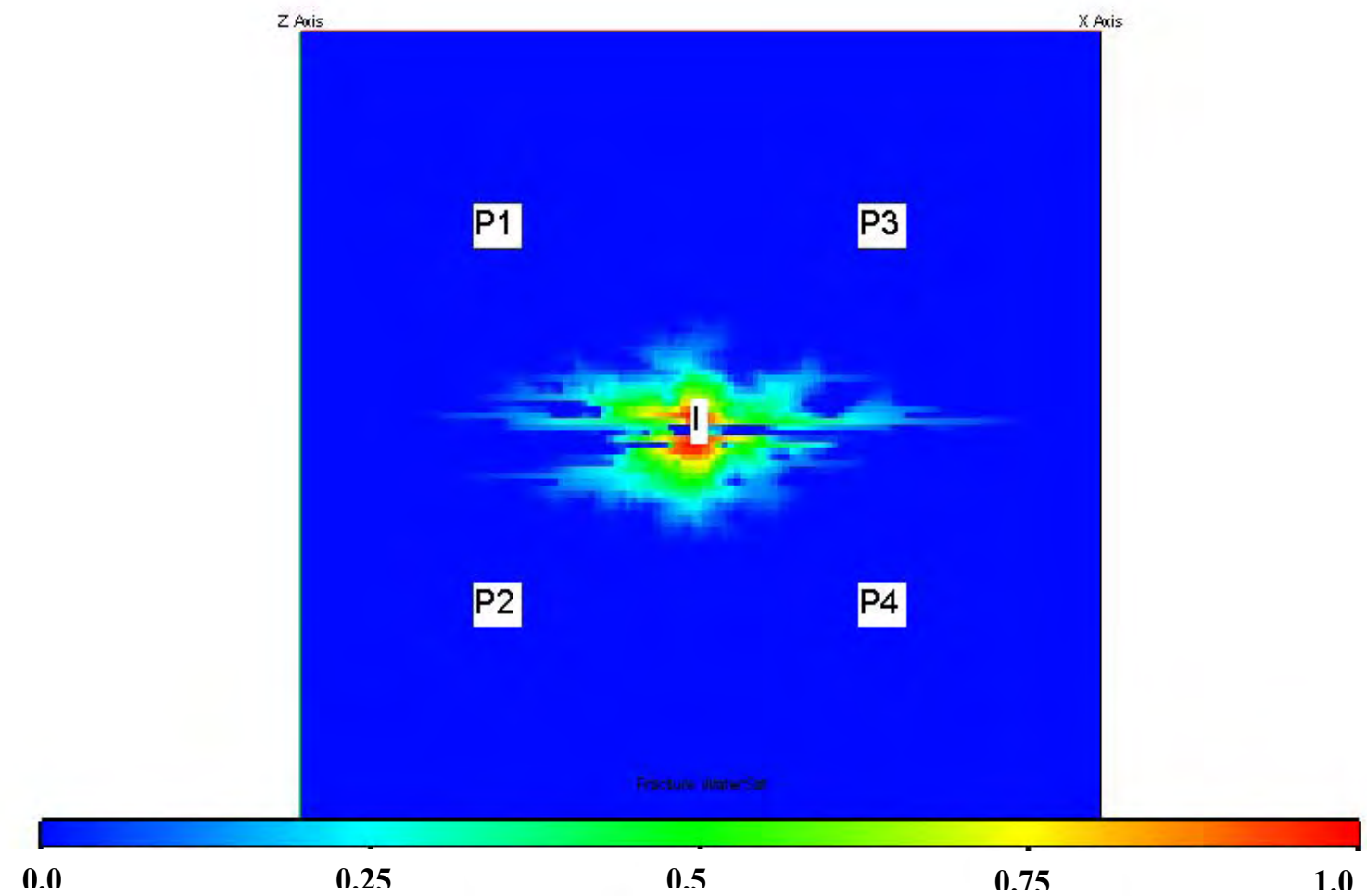

Fig. B.5d - Fracture water saturation at report step 5 (150 days) for test T2 final model combined data. 


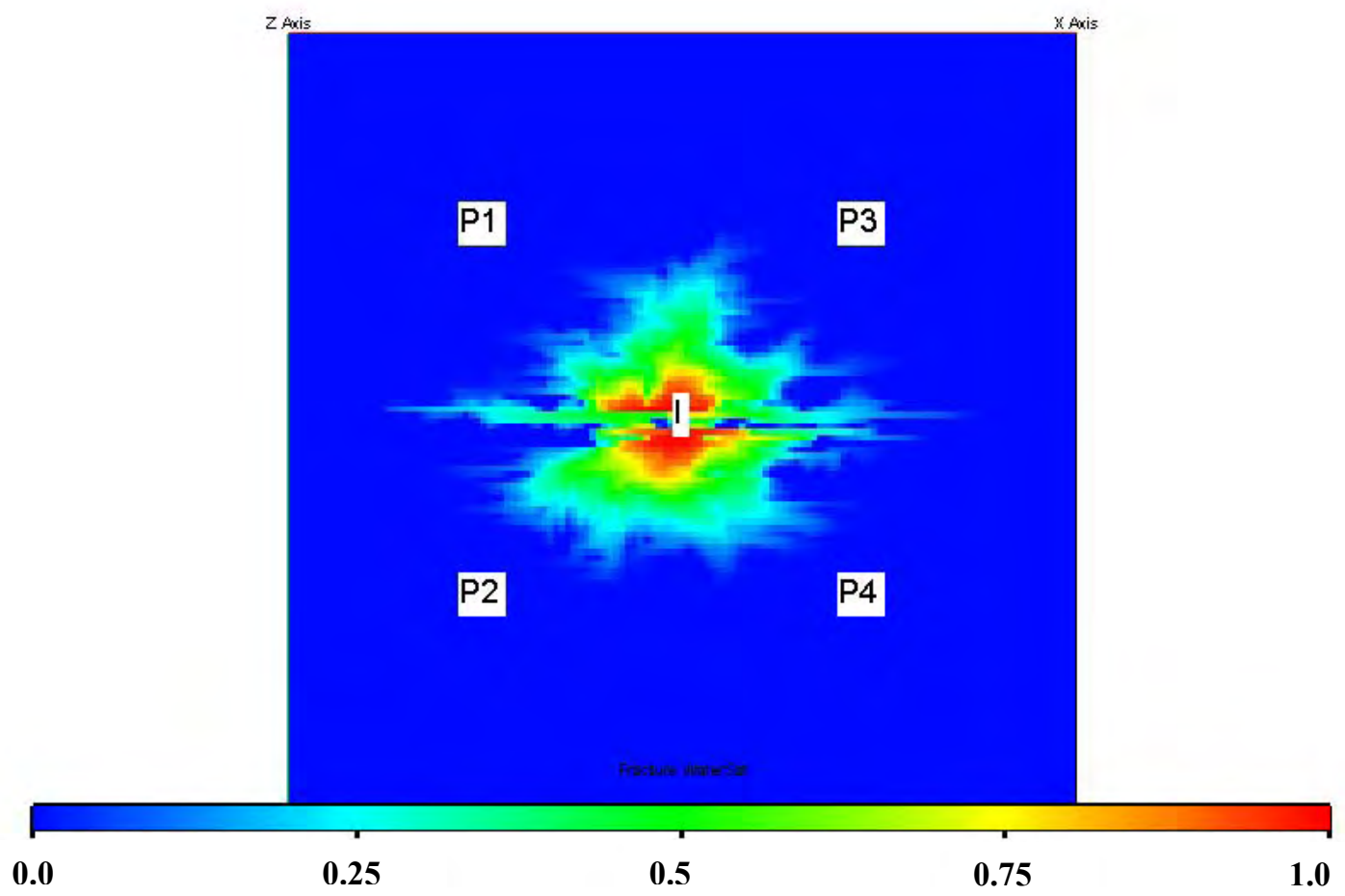

Fig. B.6a - Fracture water saturation at report step 10 (300 days) for test $\mathrm{T} 2$ base model

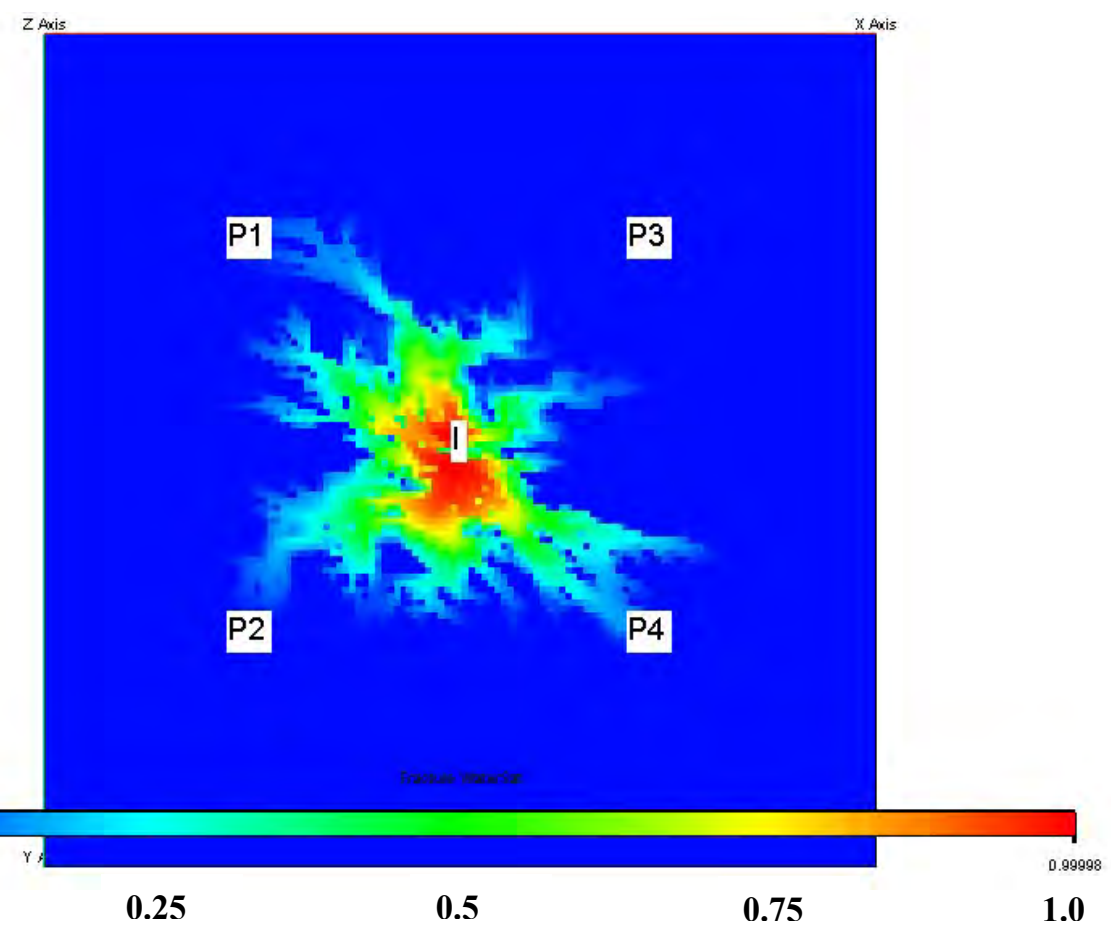

Fig. B.6b - Fracture water saturation at report step 10 (300 days) for test $\mathrm{T} 2$ initial estimate. 


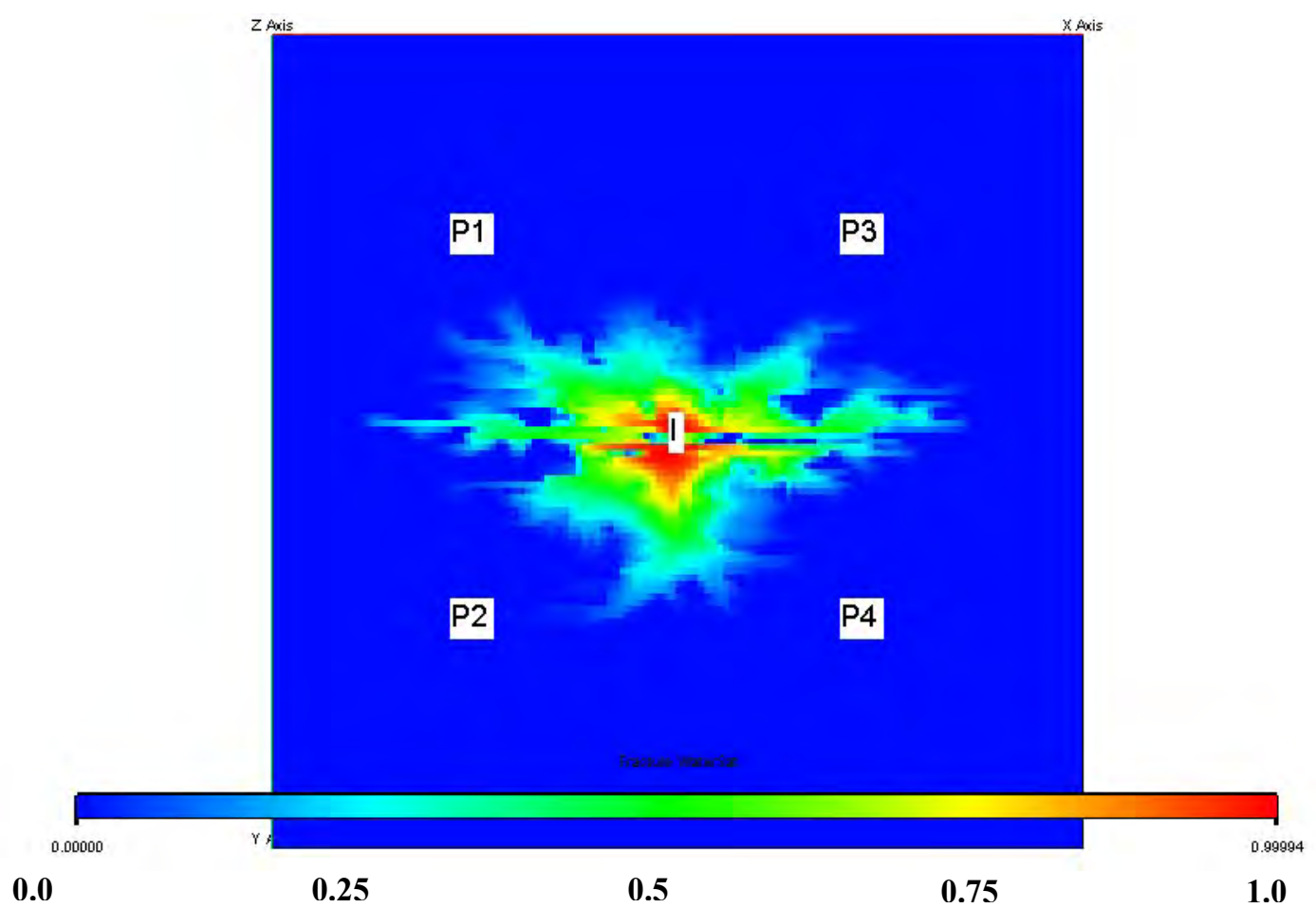

Fig. B 6c $\quad-\quad$ Fracture water saturation at report step 10 (300 days) for test T2 final model, production data only.

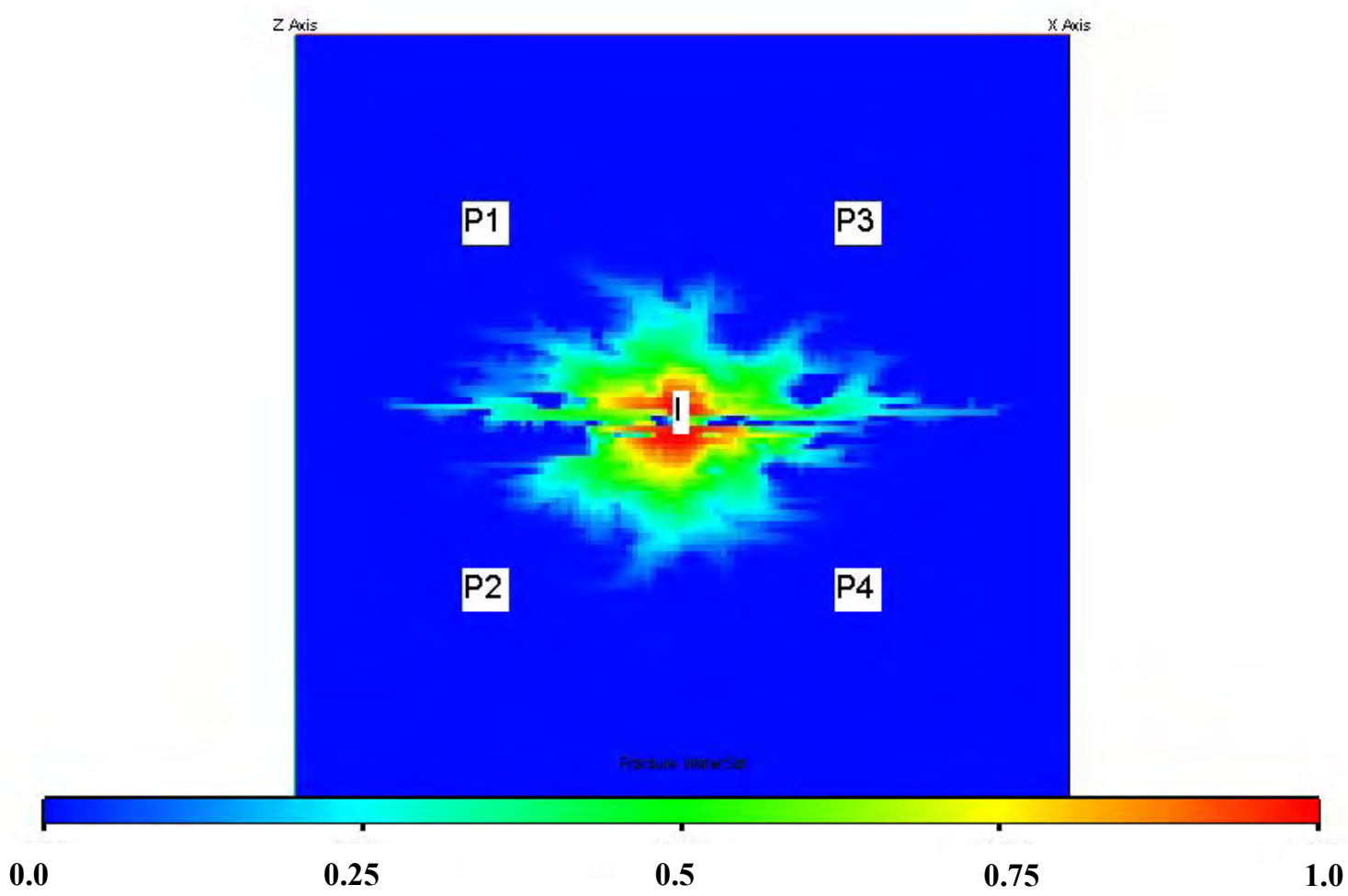

Fig. B.6d - Fracture water saturation at report step 10 (300 days) for test T2 final model, combined data. 


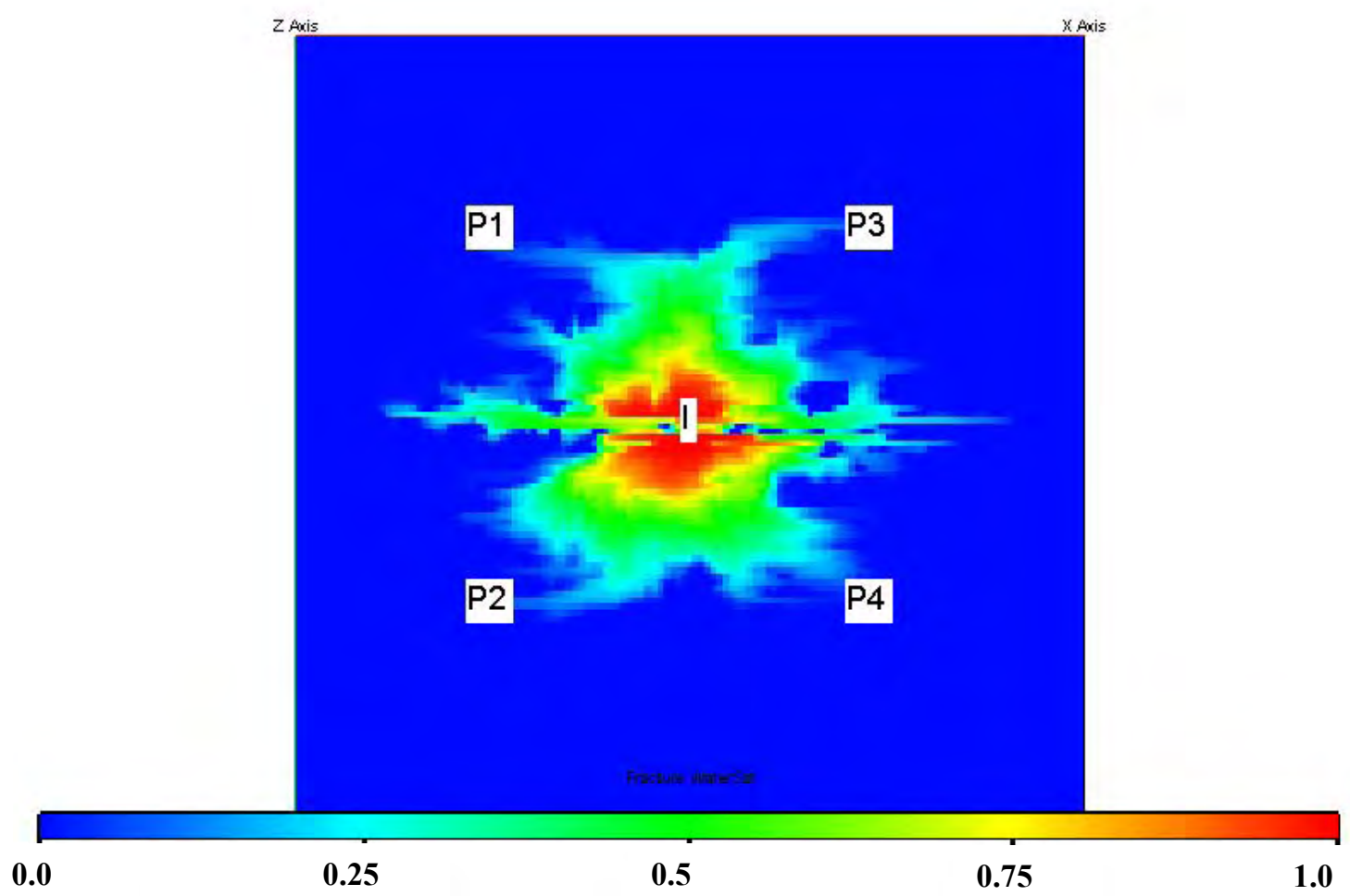

Fig. B.7a $\quad-\quad$ Fracture water saturation at report step 15 (450 days) for test T2 base model.

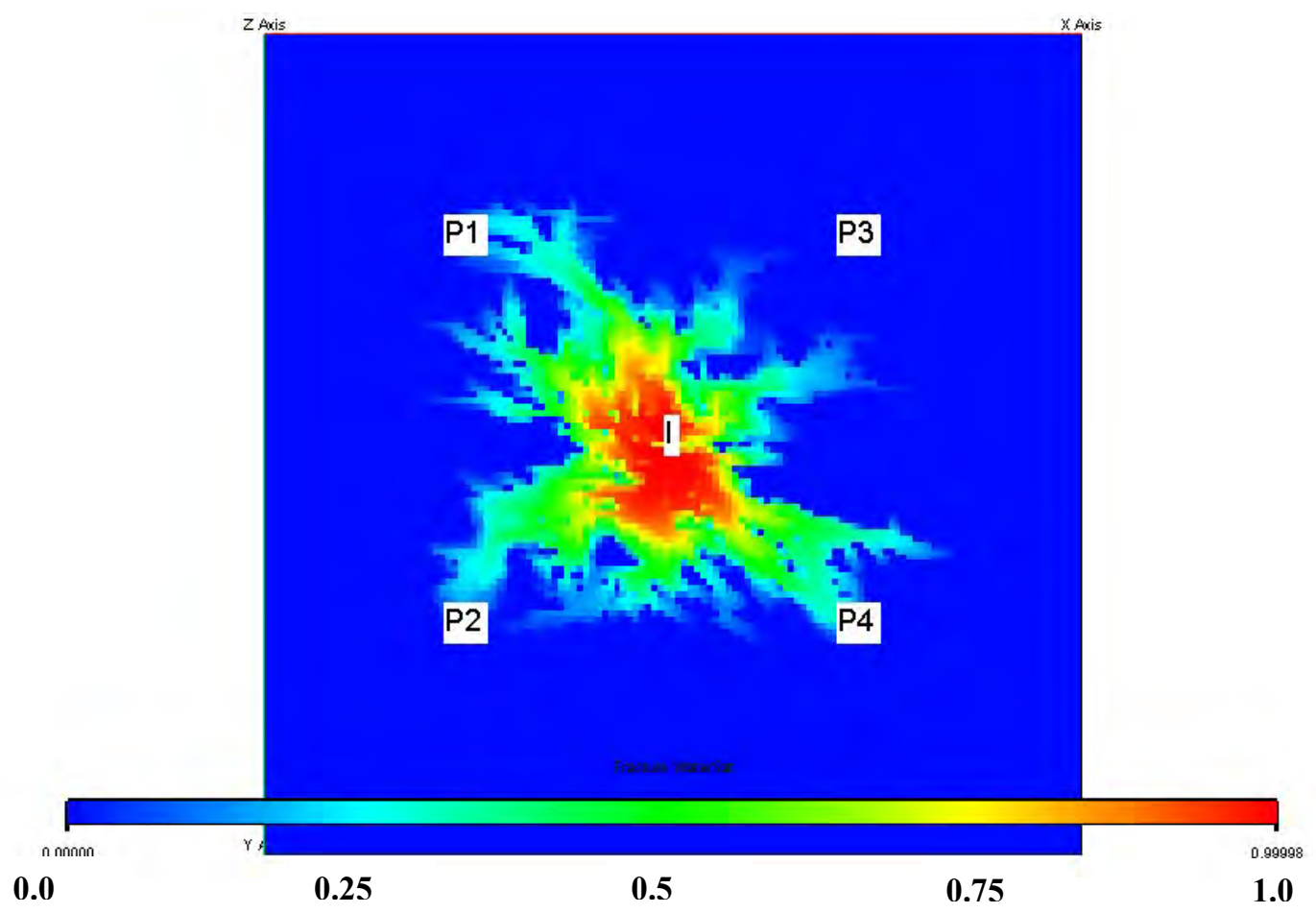

Fig. B 7b - Fracture water saturation at report step 15 (450 days) for test T2 initial estimate. 


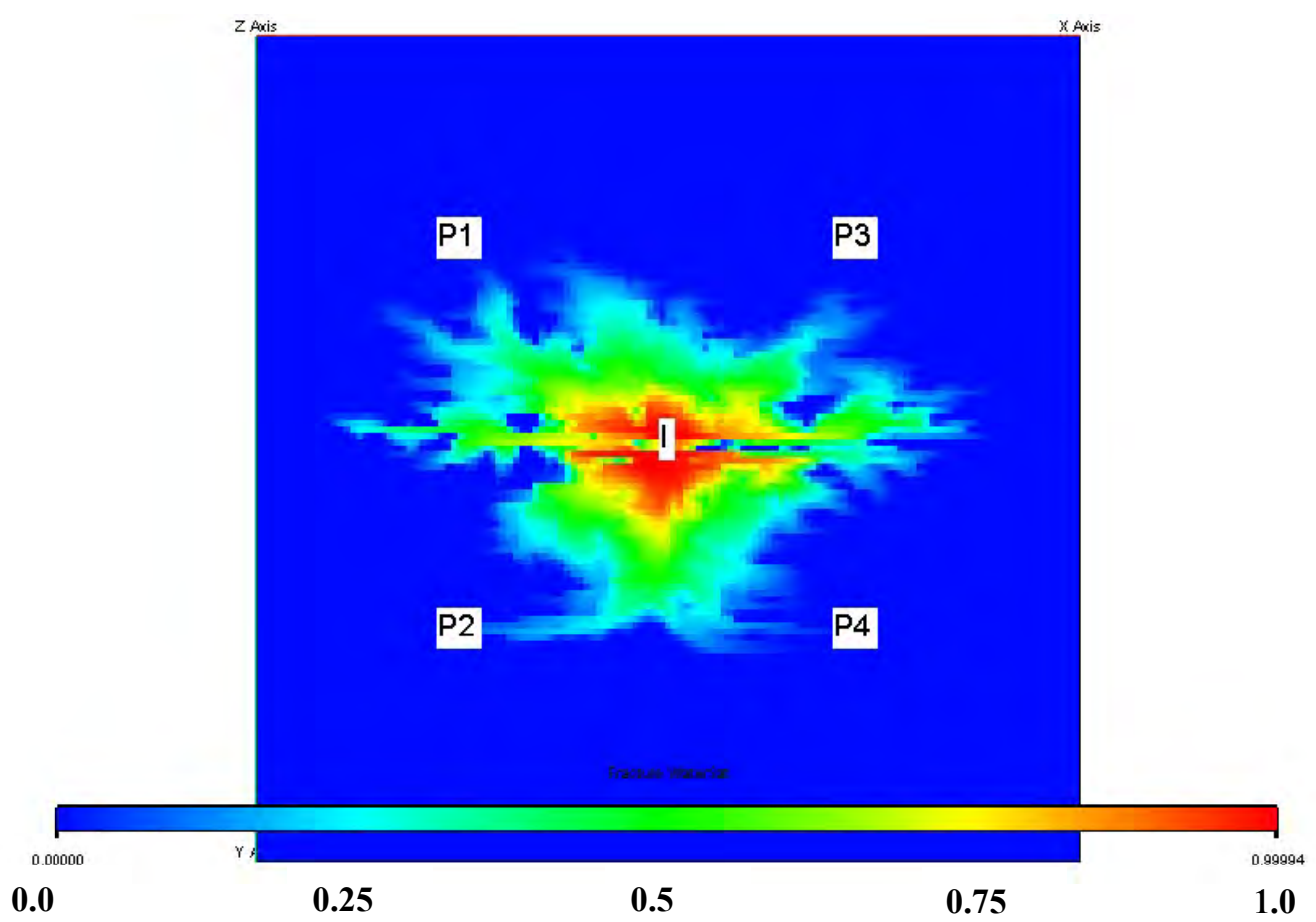

Fig. B.7c - Fracture water saturation at report step 15 (450 days) for test T2 final model, production data only.

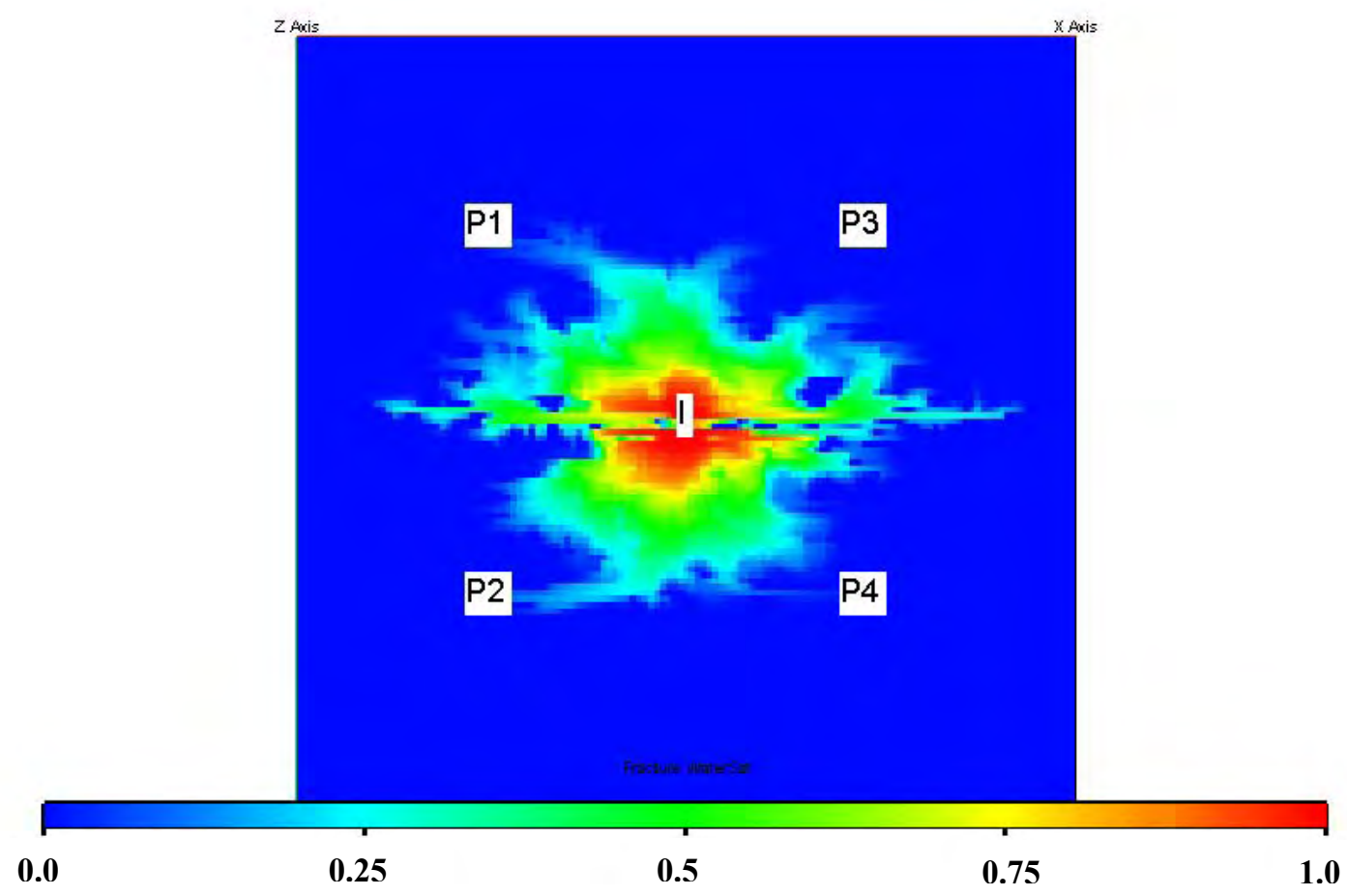

Fig. B 7d - Fracture water saturation at report step 15 (450 days) for test T2 final model, combined data. 


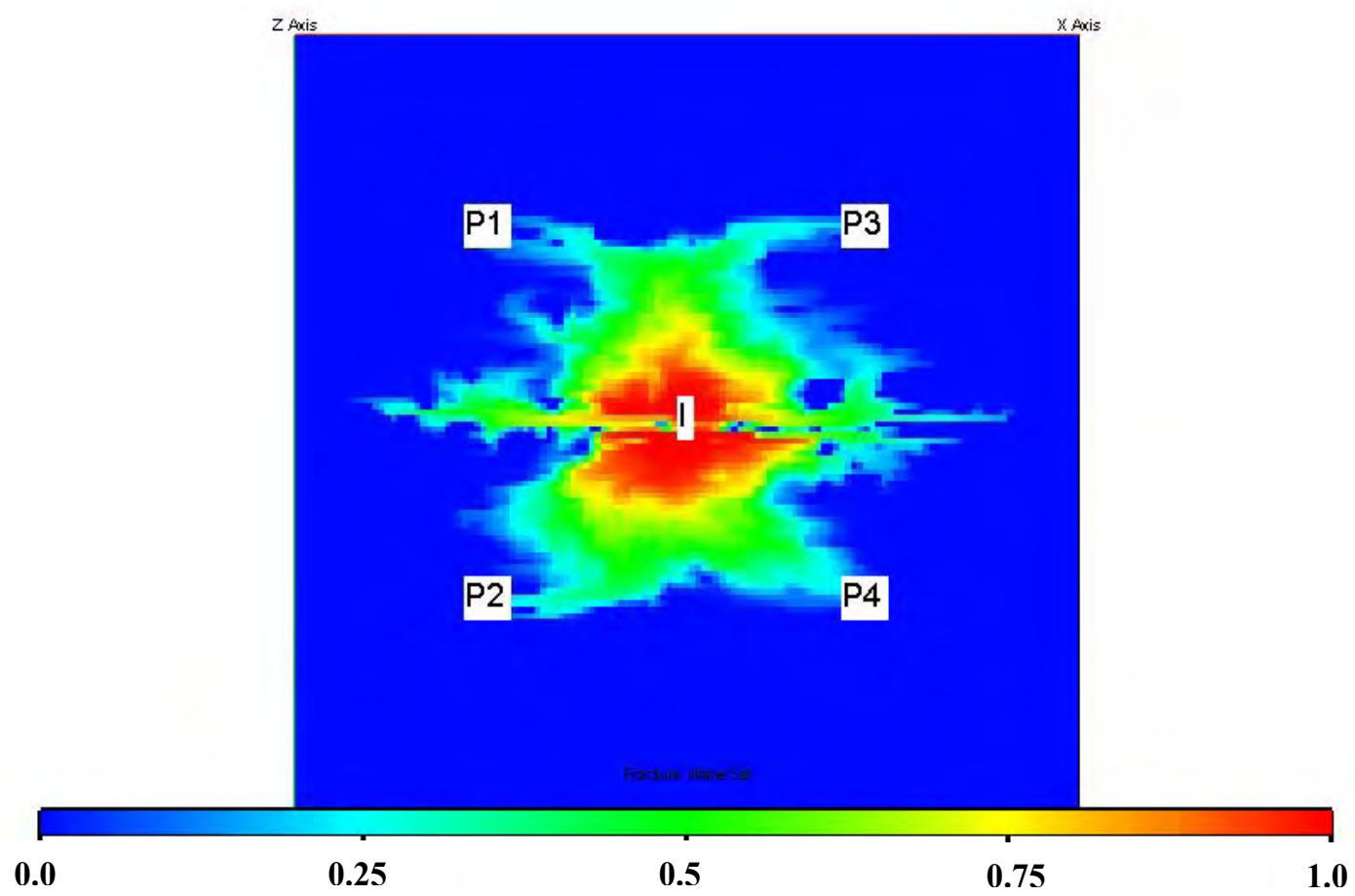

Fig. B.8a - Fracture water saturation at report step 20 (600 days) for test T2 base model.

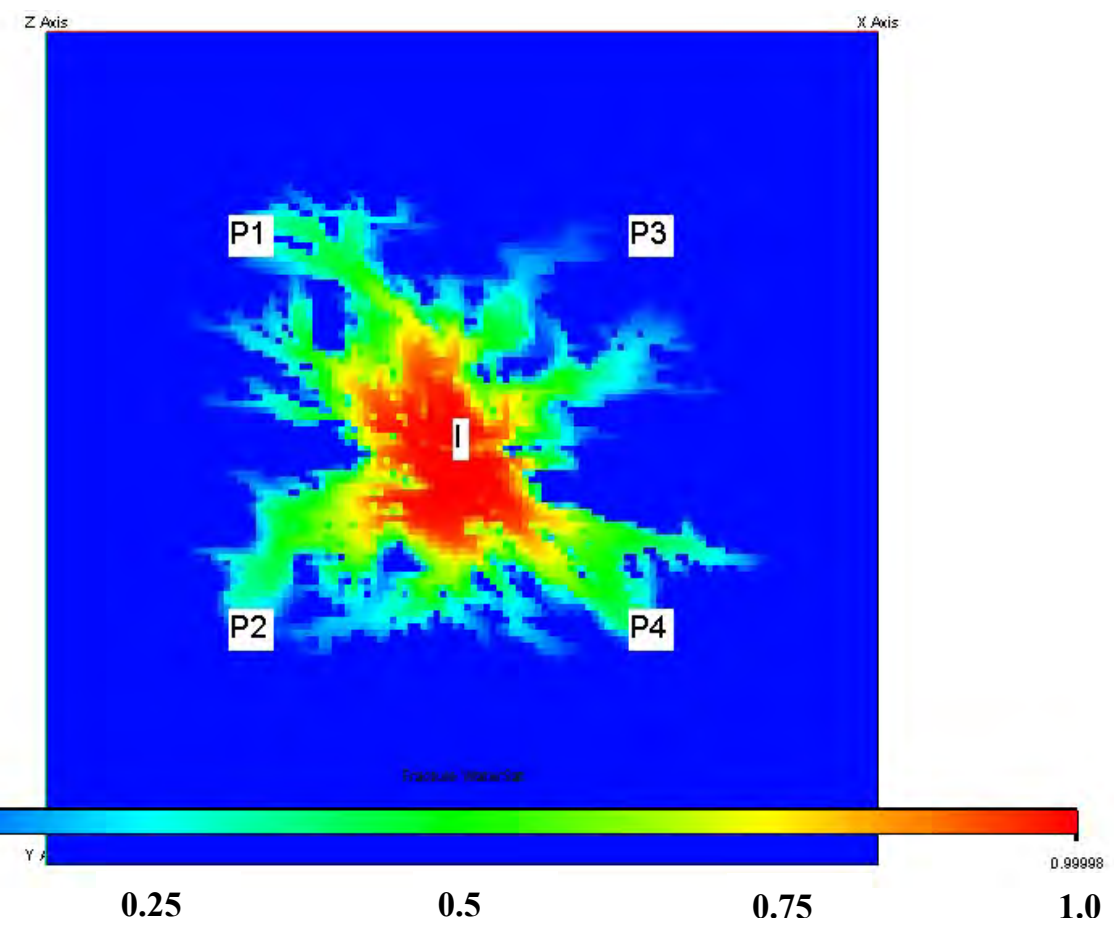

Fig. B.8b - Fracture water saturation at report step 20 (600 days) for test T2 initial estimate. 


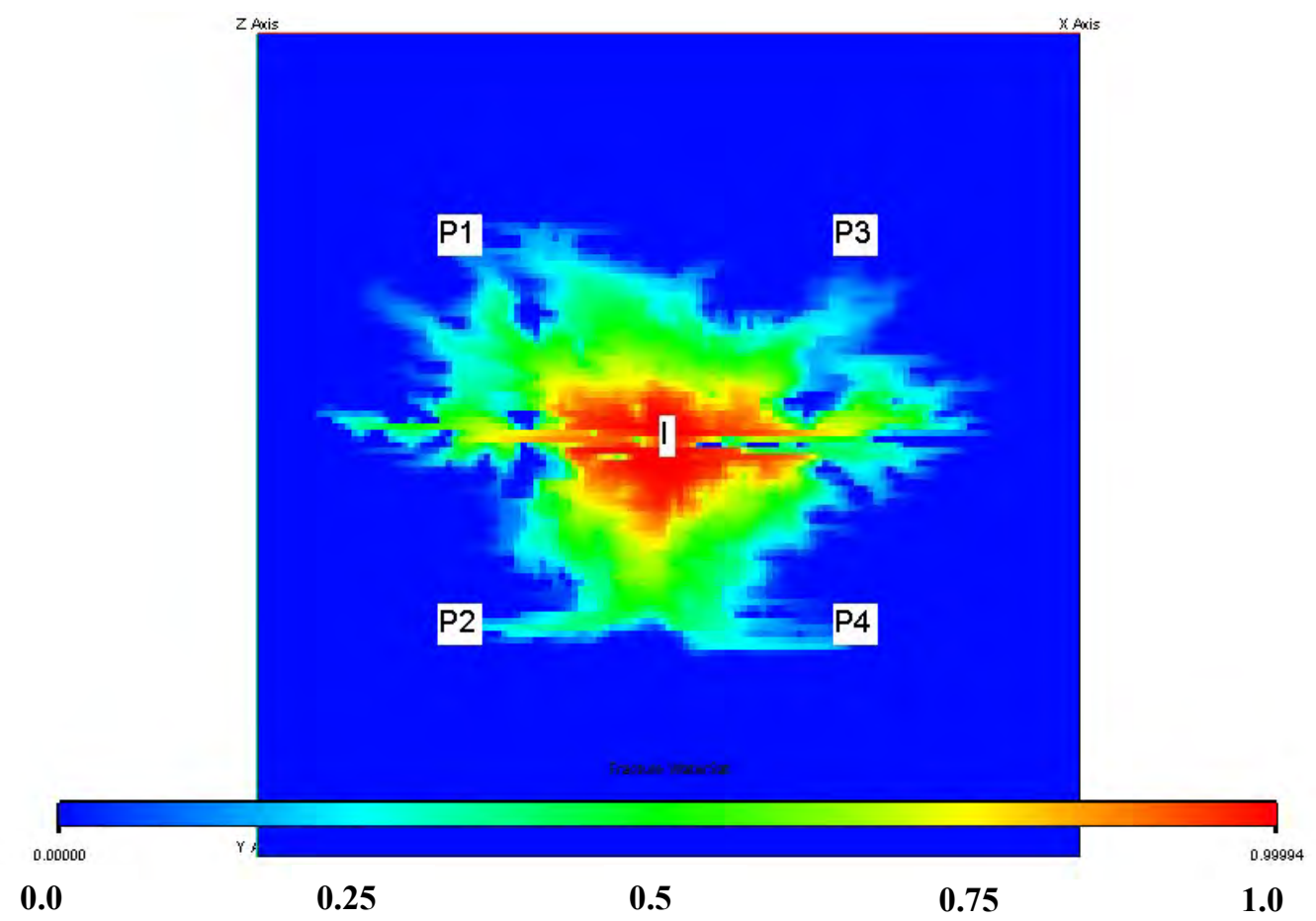

Fig. B.8c - Fracture water saturation at report step 20 (600 days) for test T2 final model, production data only.

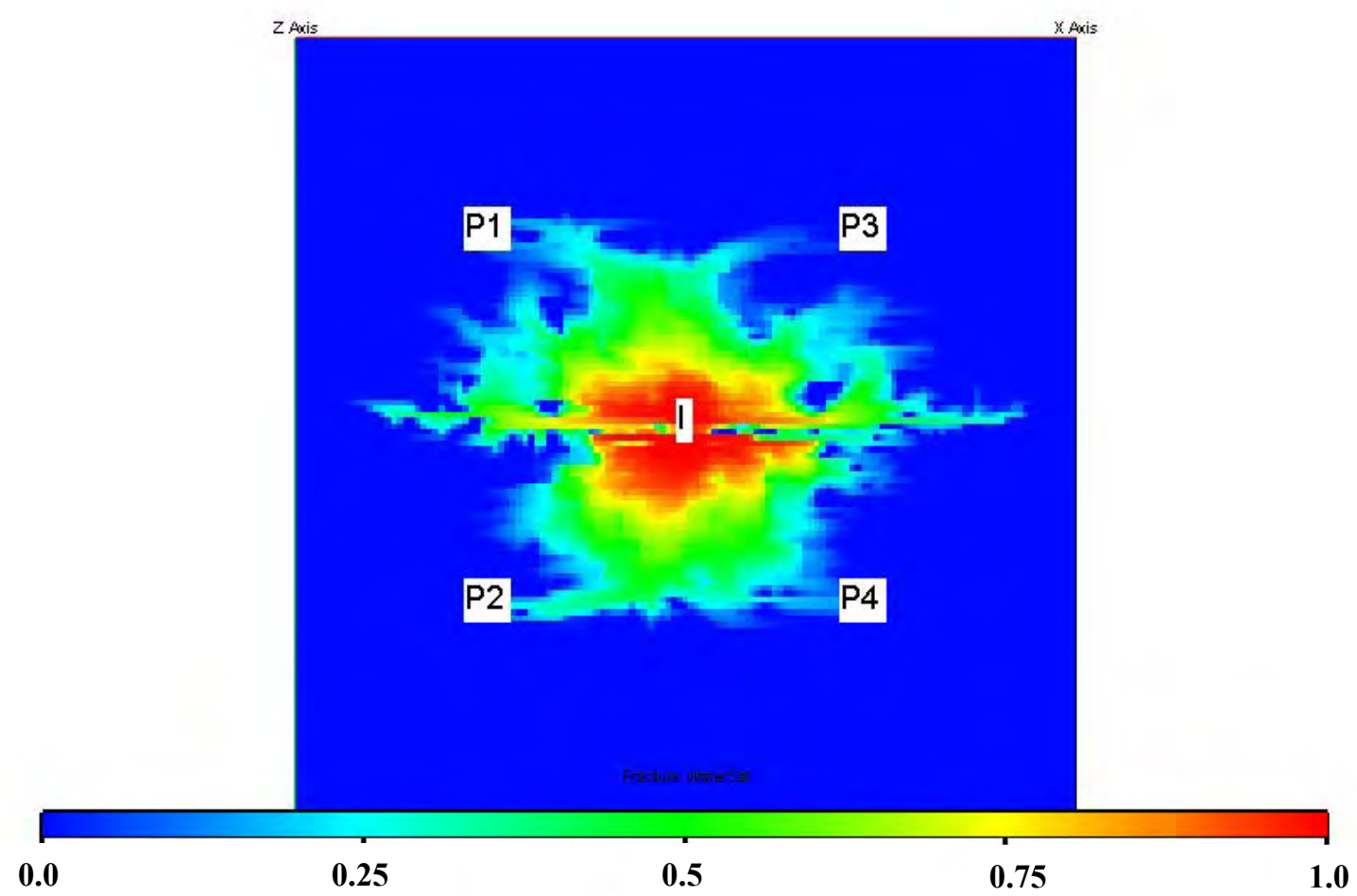

Fig. B.8d - Fracture water saturation at report step 20 (600 days)for test T2 final model, combined data. 
Matrix Water Saturation Maps (Base, Initial, Final)

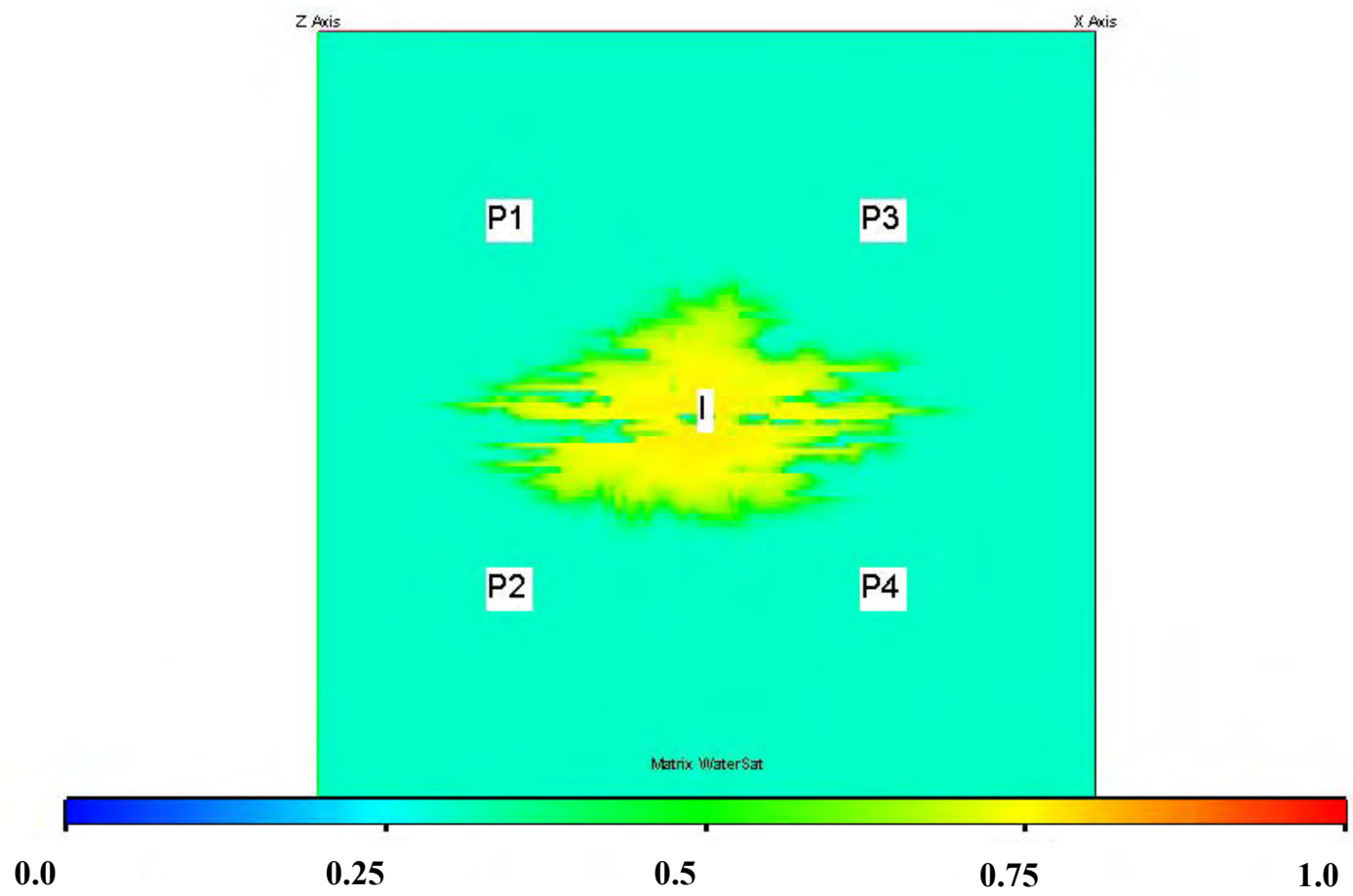

Fig. B 9a - Matrix water saturation at report step 5 (150 days) for test T2 base model.

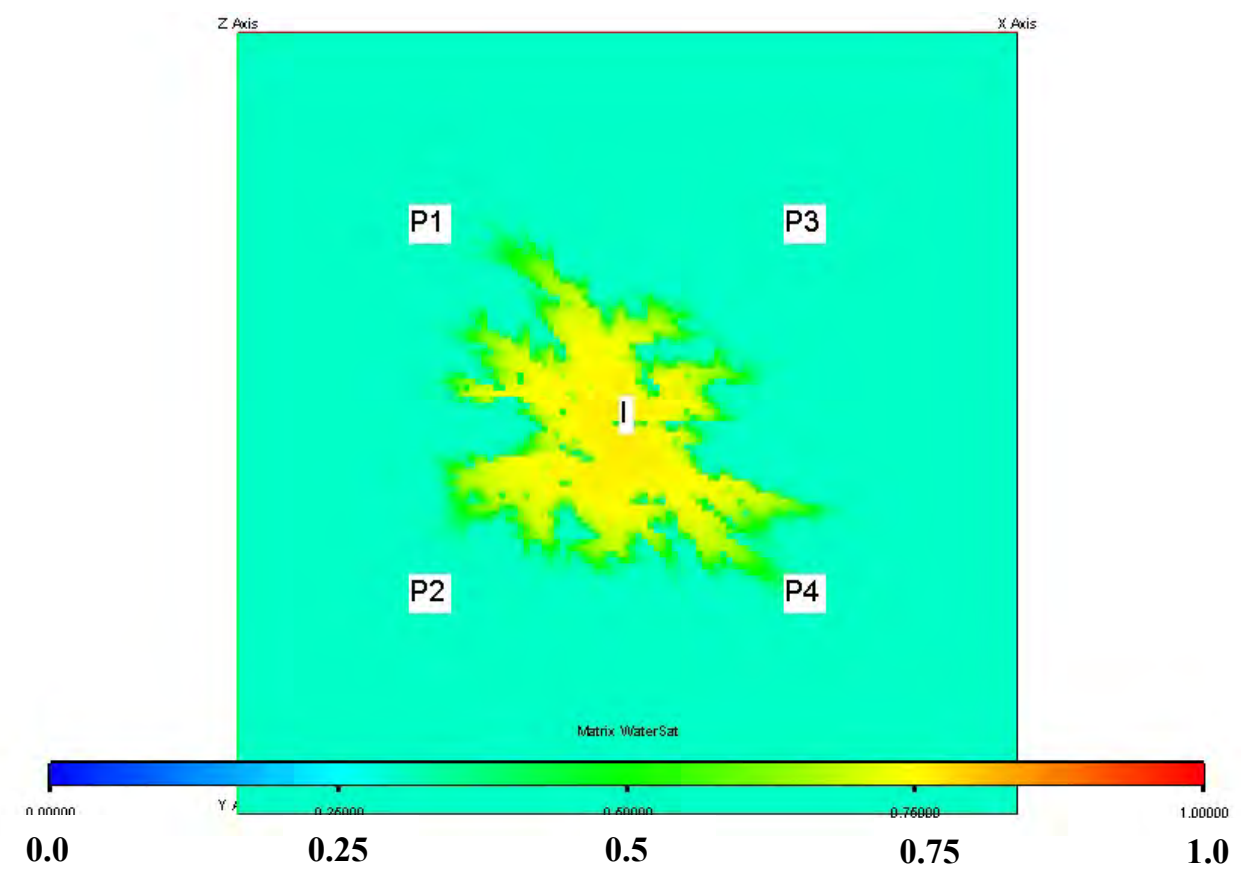

Fig. B 9b - Matrix water saturation at report step 5 (150 days)for test T2 initial estimate. 


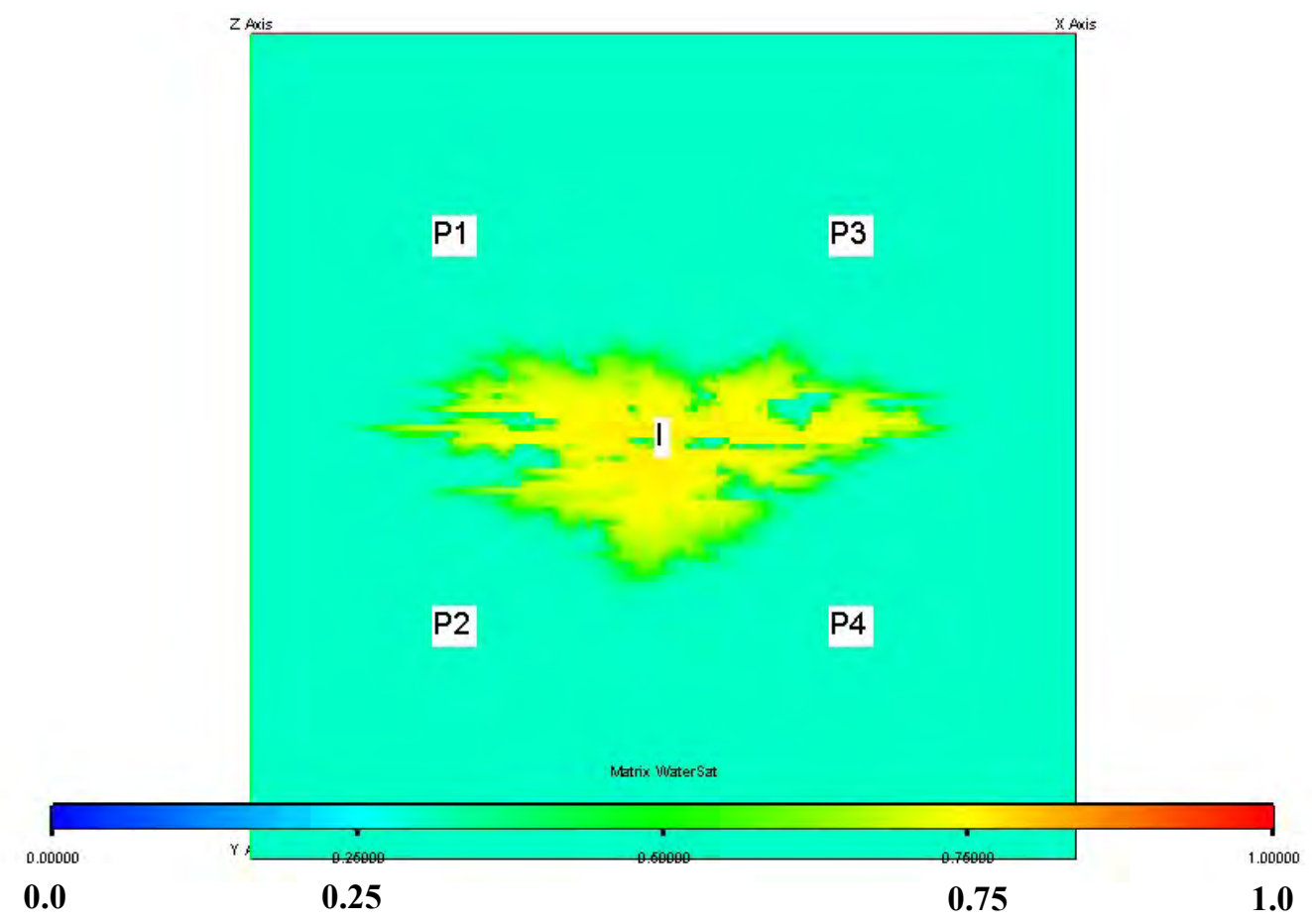

Fig. B.9c - Matrix water saturation at report step 5 (150 days) for test T2 final model, production data only.

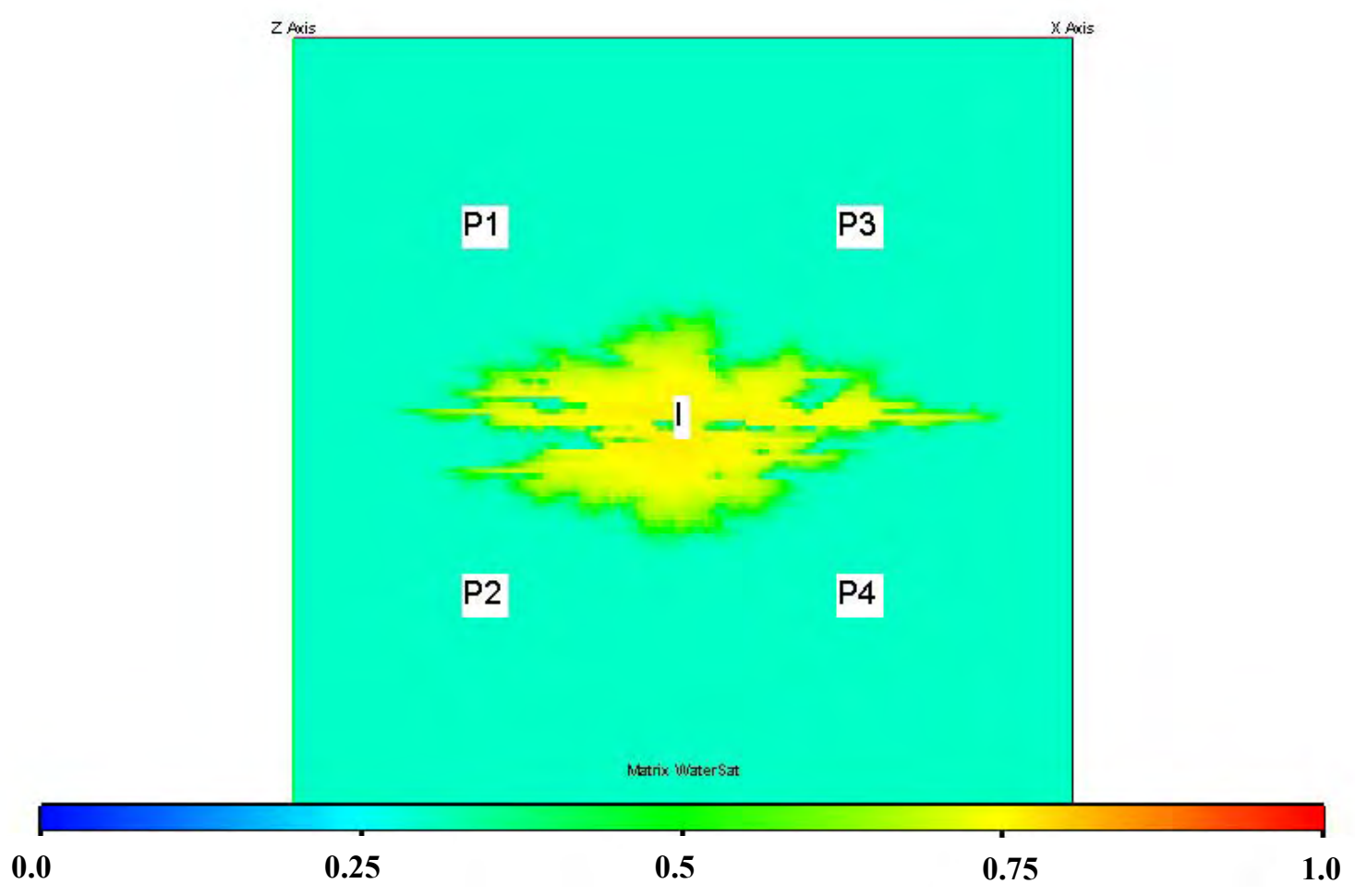

Fig. B.9d - Matrix water saturation at report step 5 (150 days) for test T2 final model, combined data. 


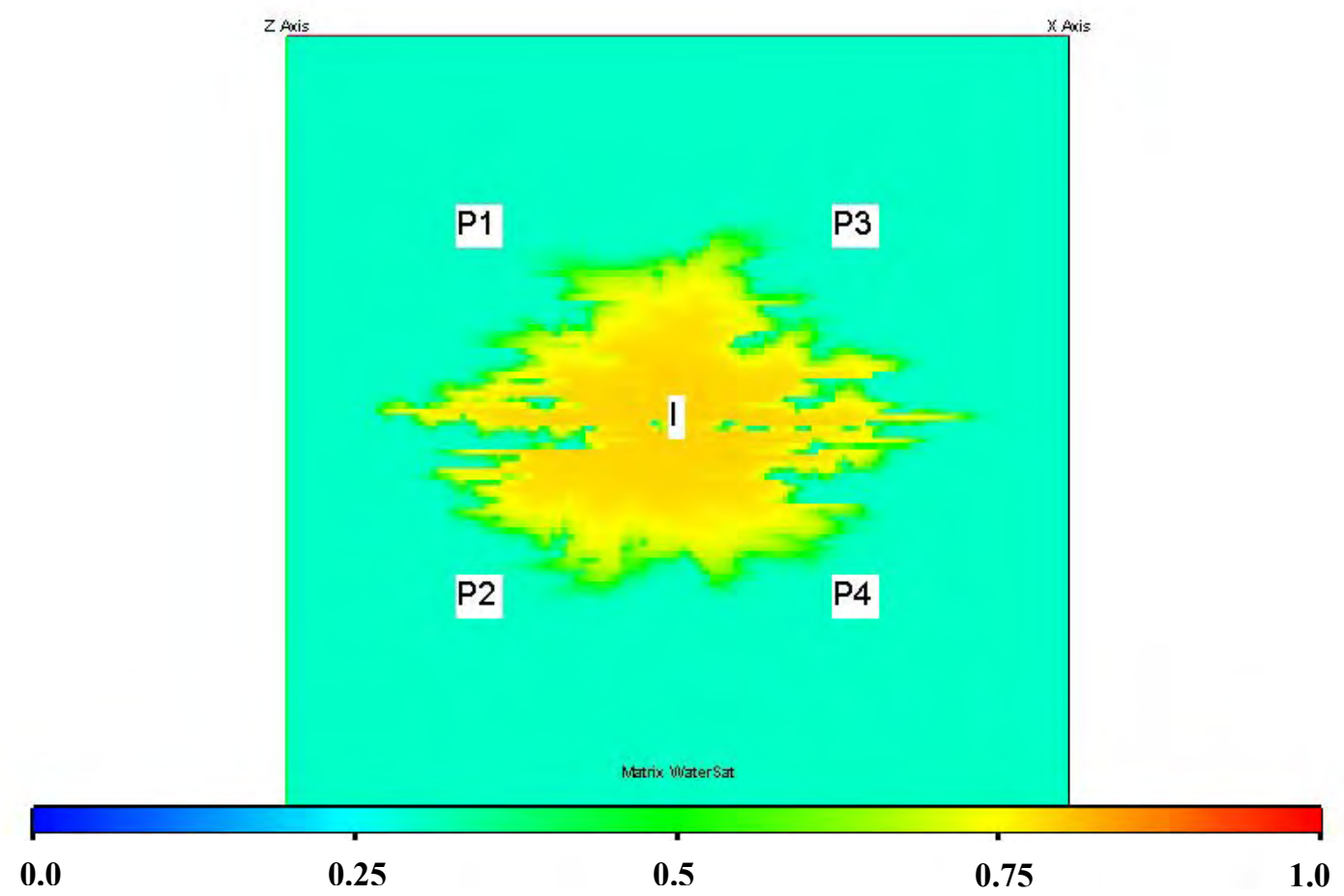

Fig. B.10a - Matrix water saturation at report step 10 (300 days) for test T2 base model.

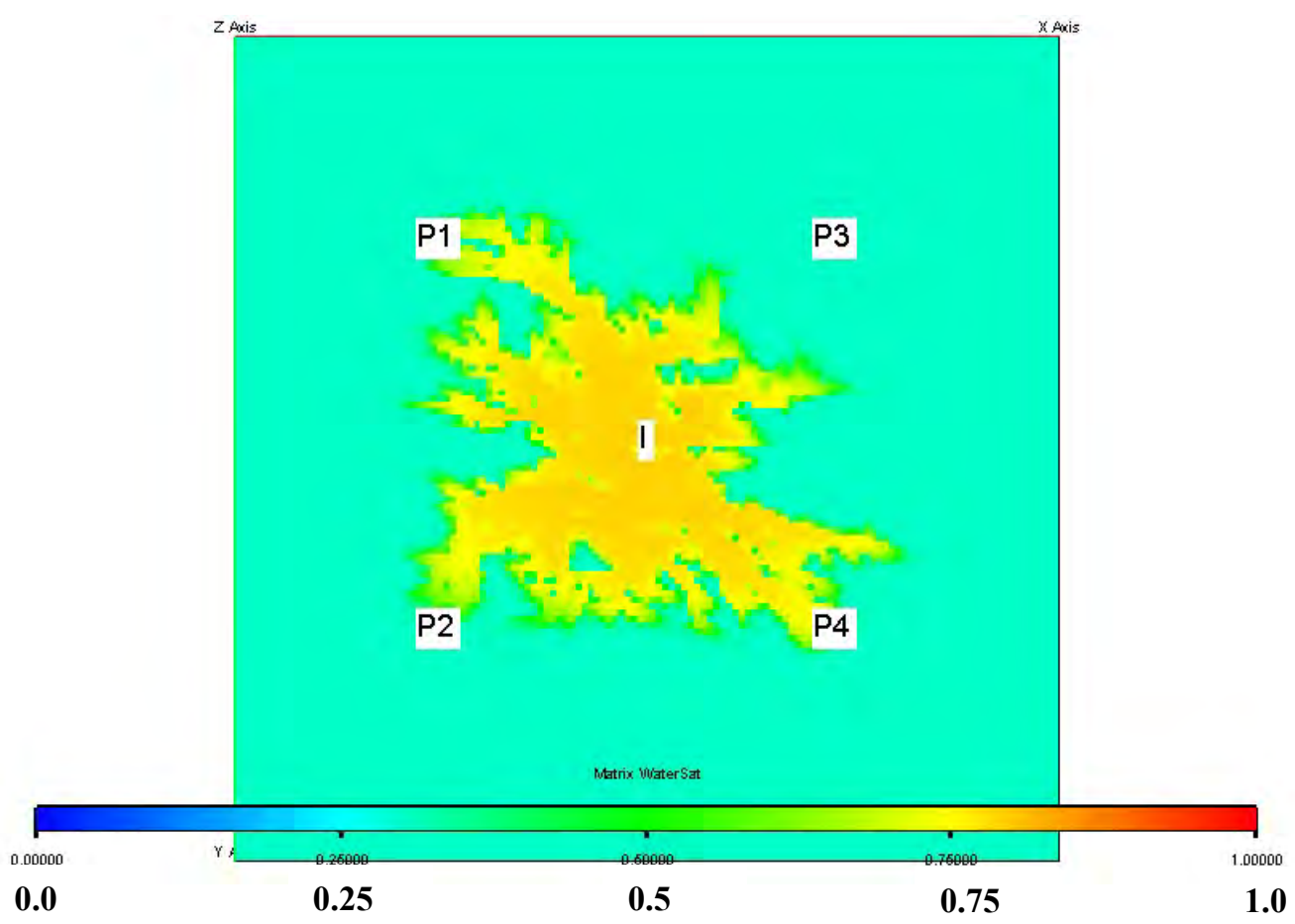

Fig. B.10b - Matrix water saturation at report step 10 (300 days) for test T2 initial estimate. 


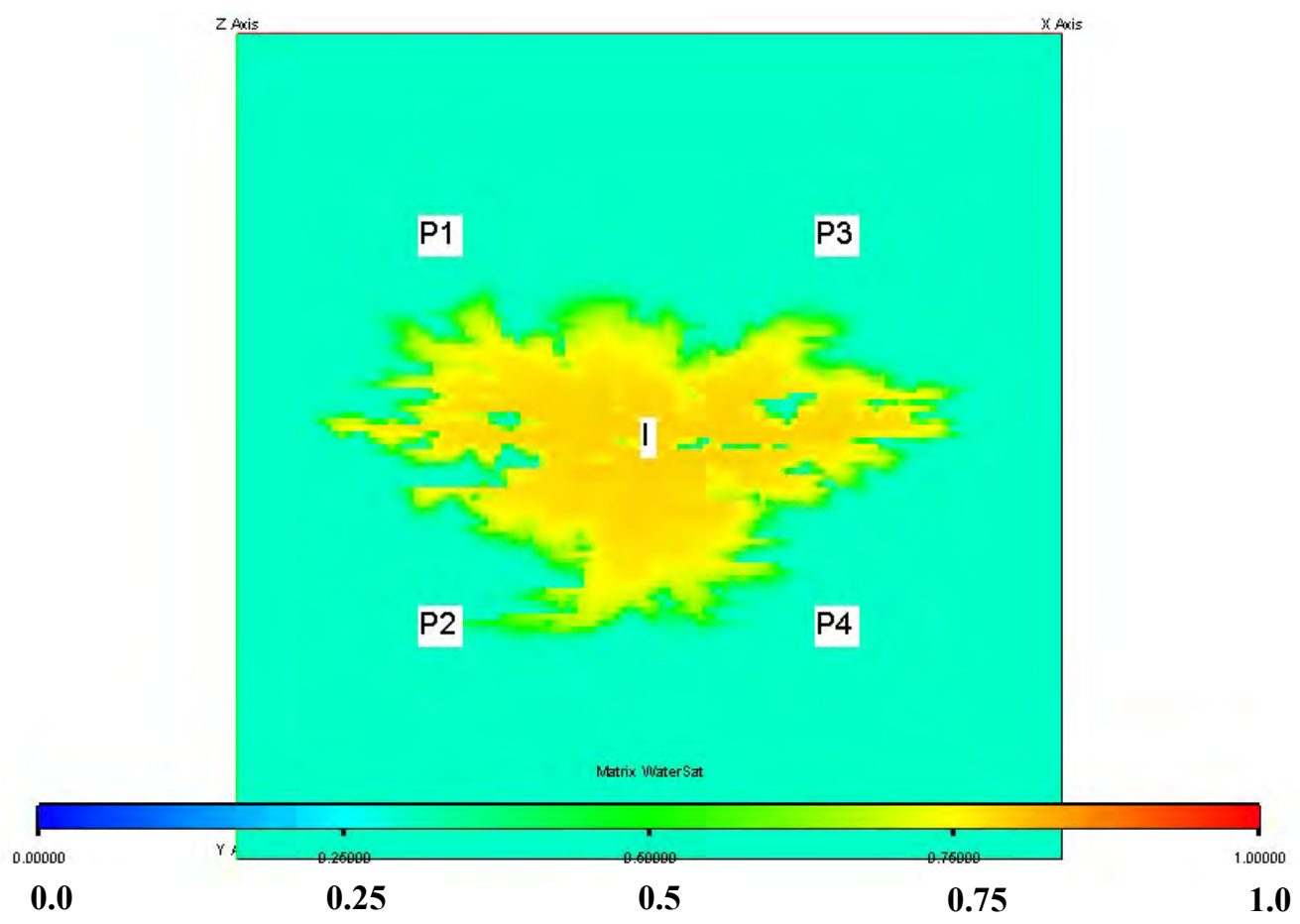

Fig. B.10c - Matrix water saturation at report step 10 (300 days) for test T2 final model, production data only.

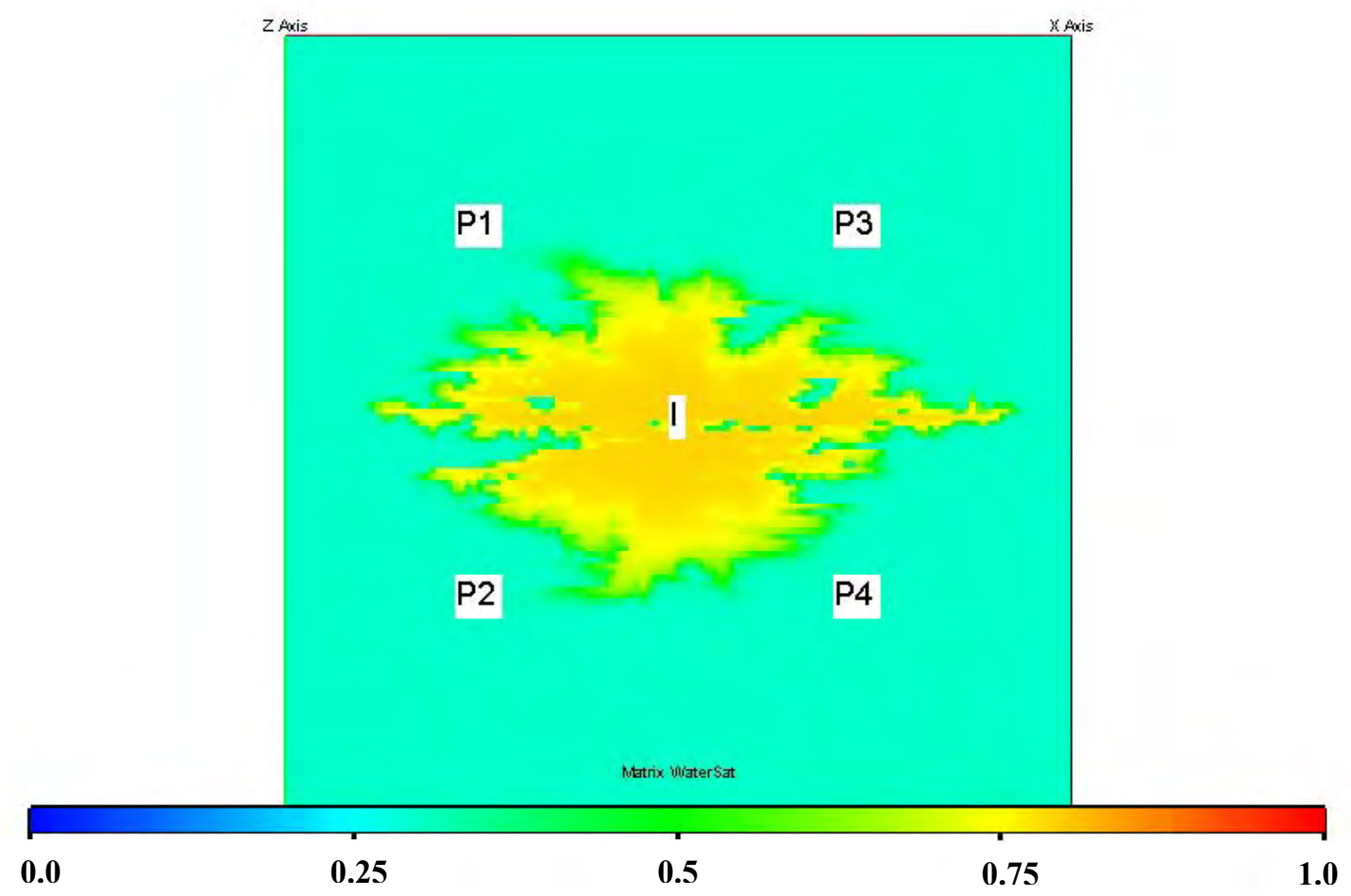

Fig. B.10d - Matrix water saturation at report step 10 (300 days) for test T2 final model, combined data. 


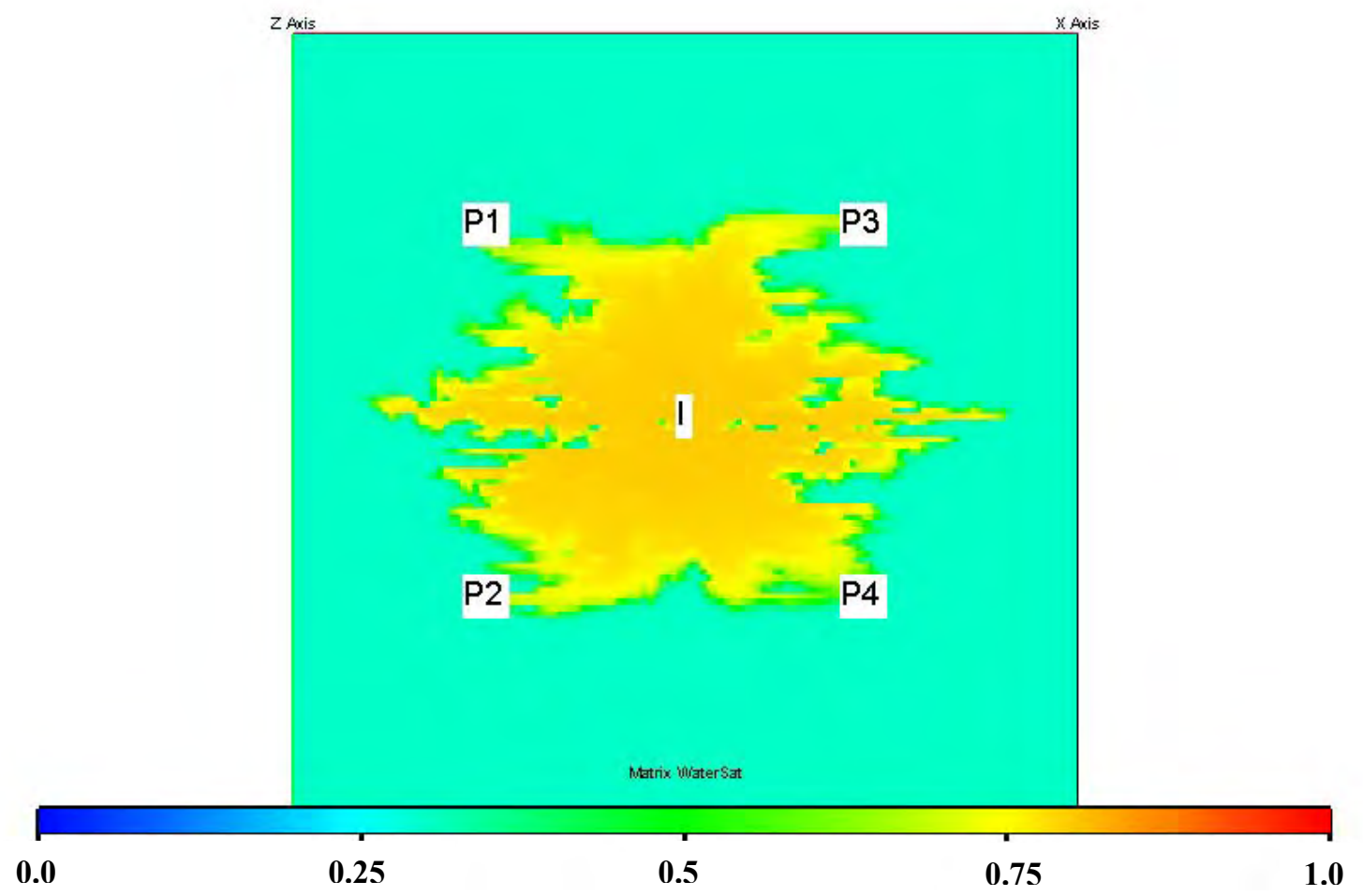

Fig. B.11a - Matrix water saturation at report step 15 (450 days) for test T2 base model.

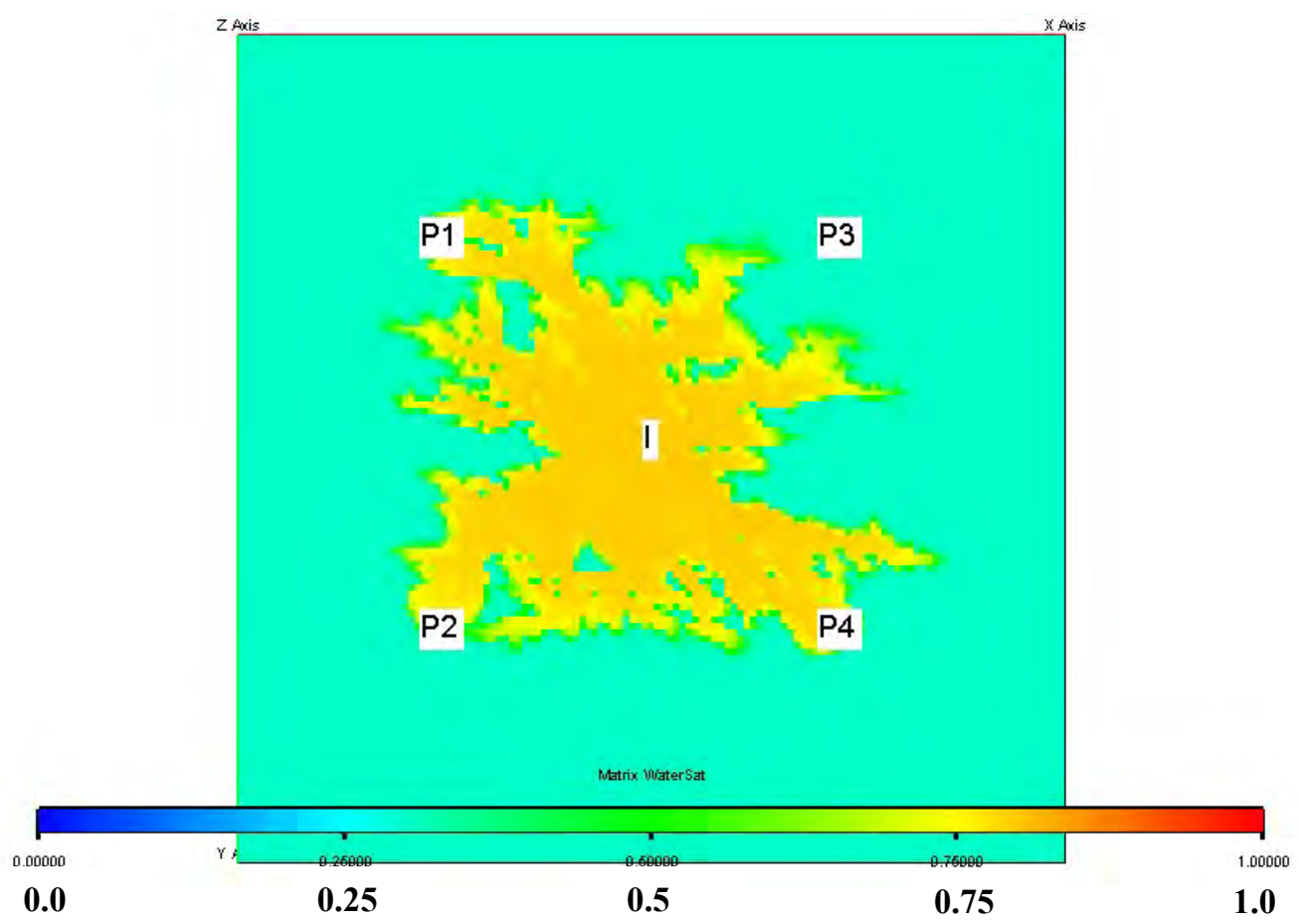

Fig. B.11b - Matrix water saturation at report step 15 (450 days) for test T2 initial estimate. 


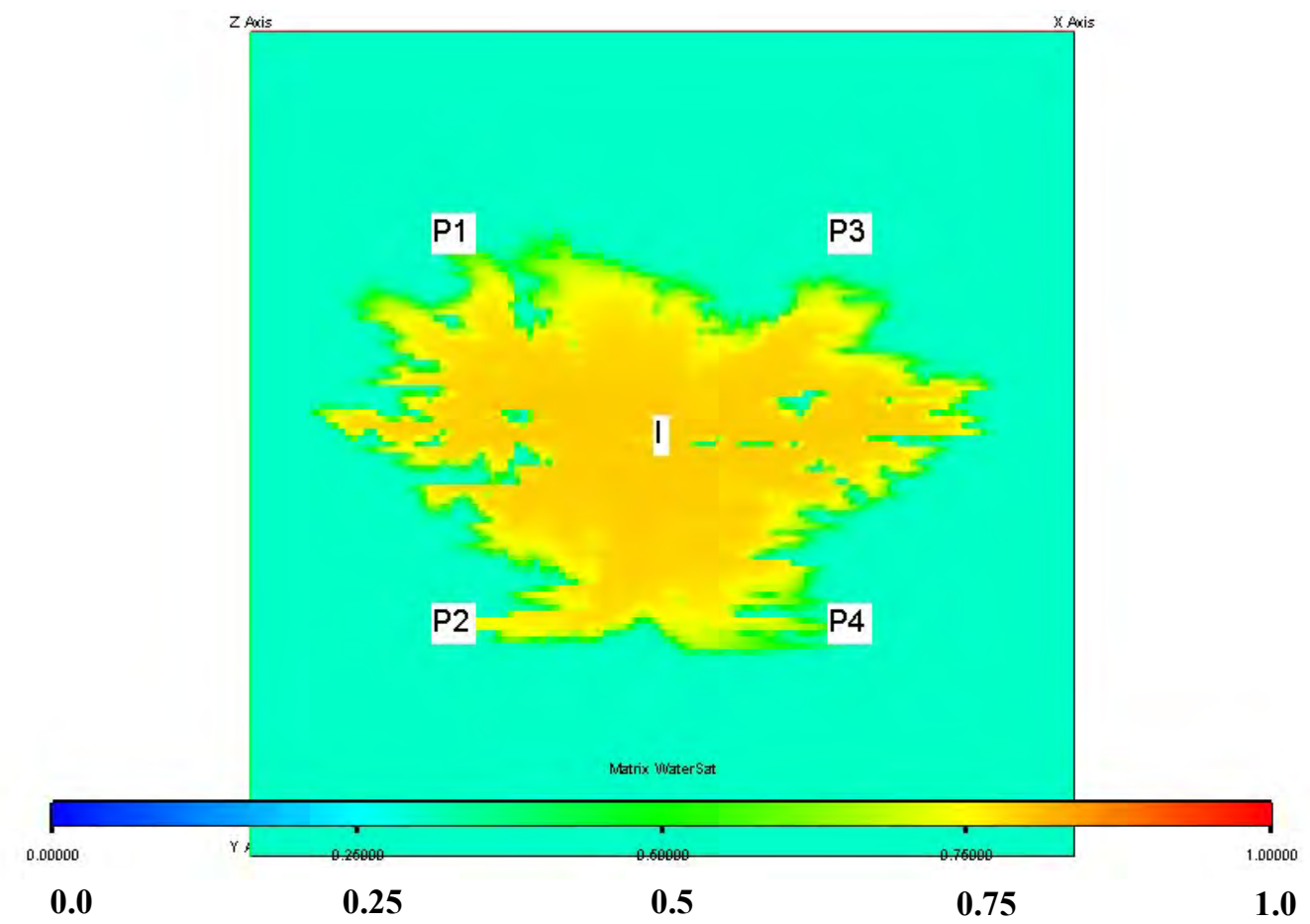

Fig. B.11c - Matrix water saturation at report step 15 (450 days) for test T2 final model, production data only.

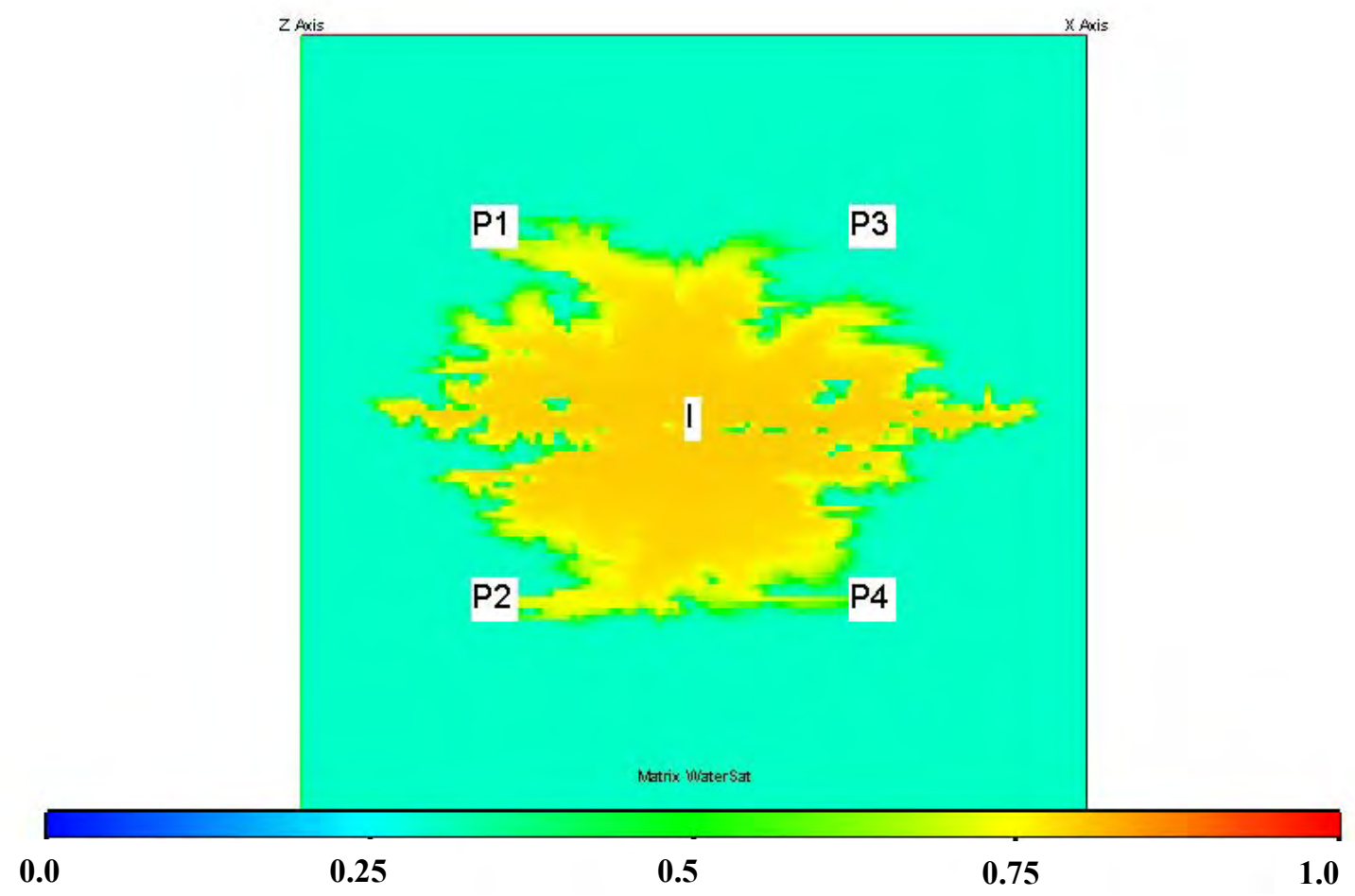

Fig. B.11d - Matrix water saturation at report step 15 (450 days) for test T2 final model, combined data. 


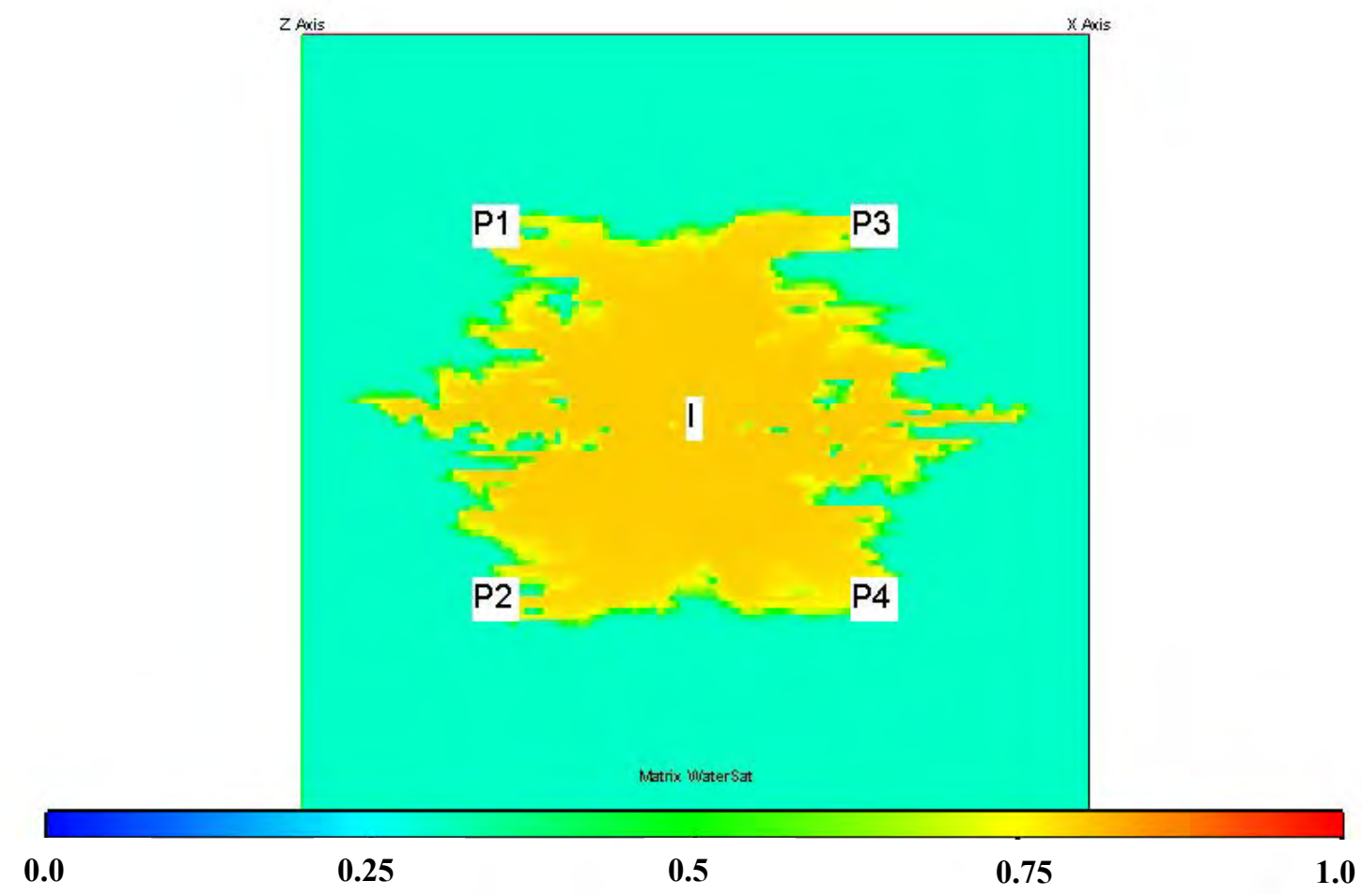

Fig. B.12a - Matrix water saturation at report step 20 (600 days) for test T2 base model.

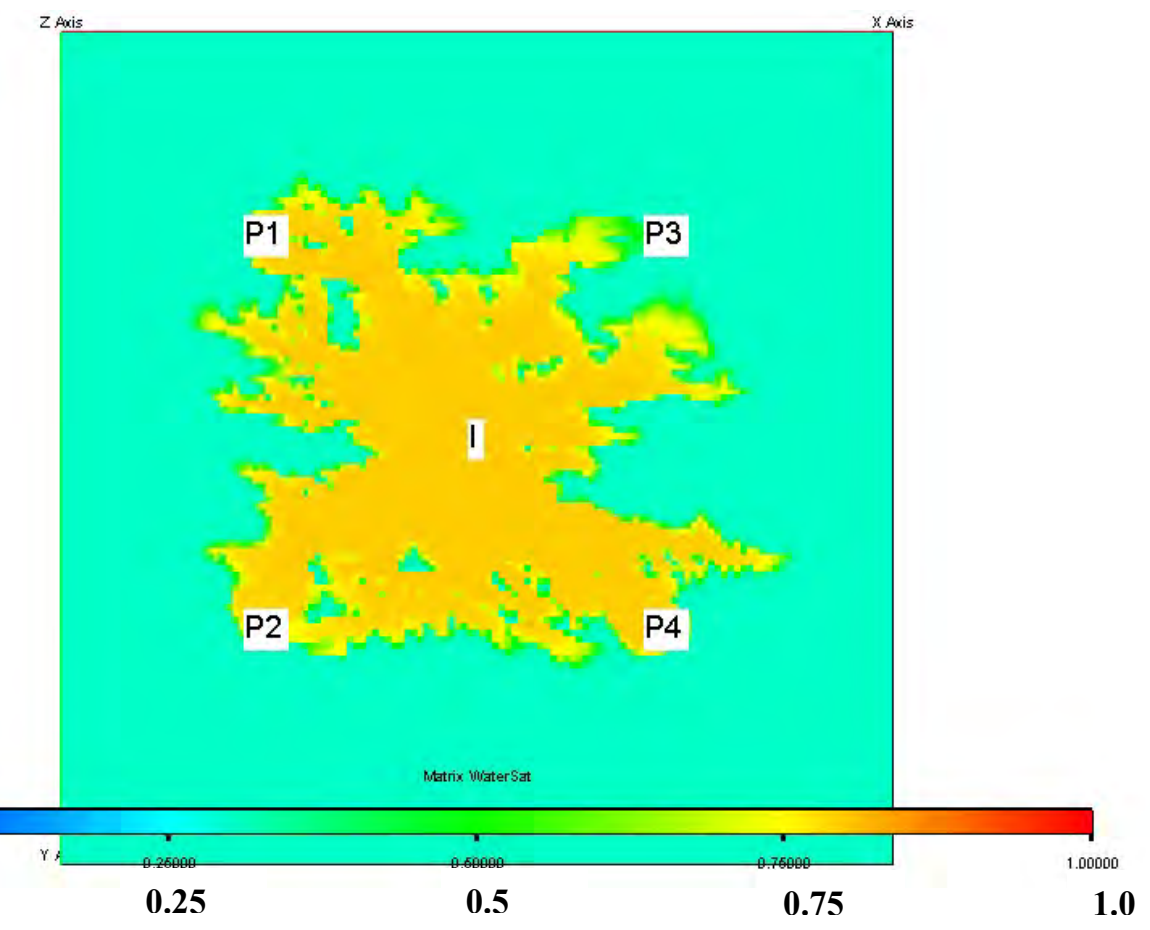

Fig. B.12b - Matrix water saturation at report step 20 (600 days) for test T2 initial estimate. 


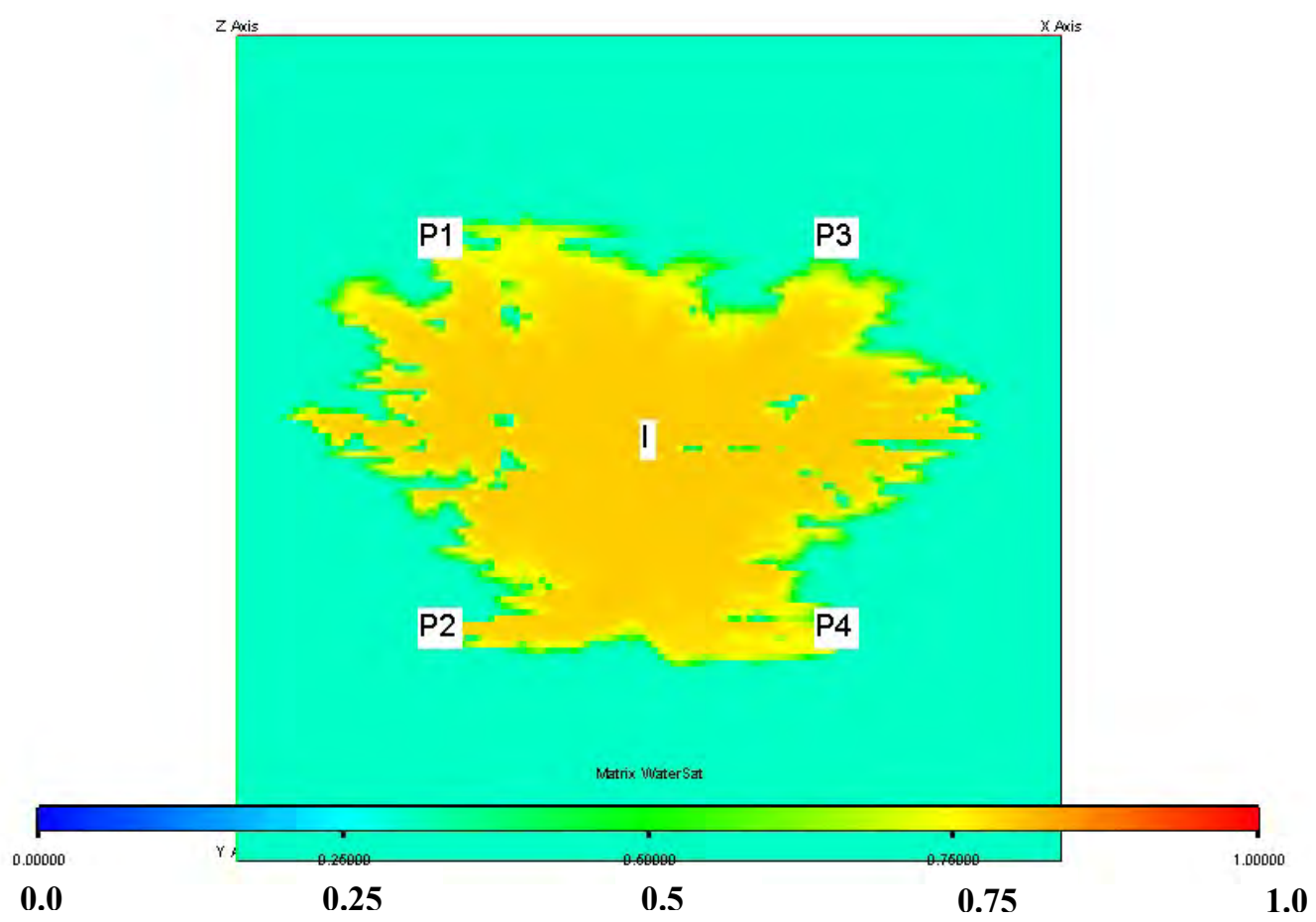

Fig. B 12c - Matrix water saturation at report step 20 (600 days) for test T2 final model, production data only.

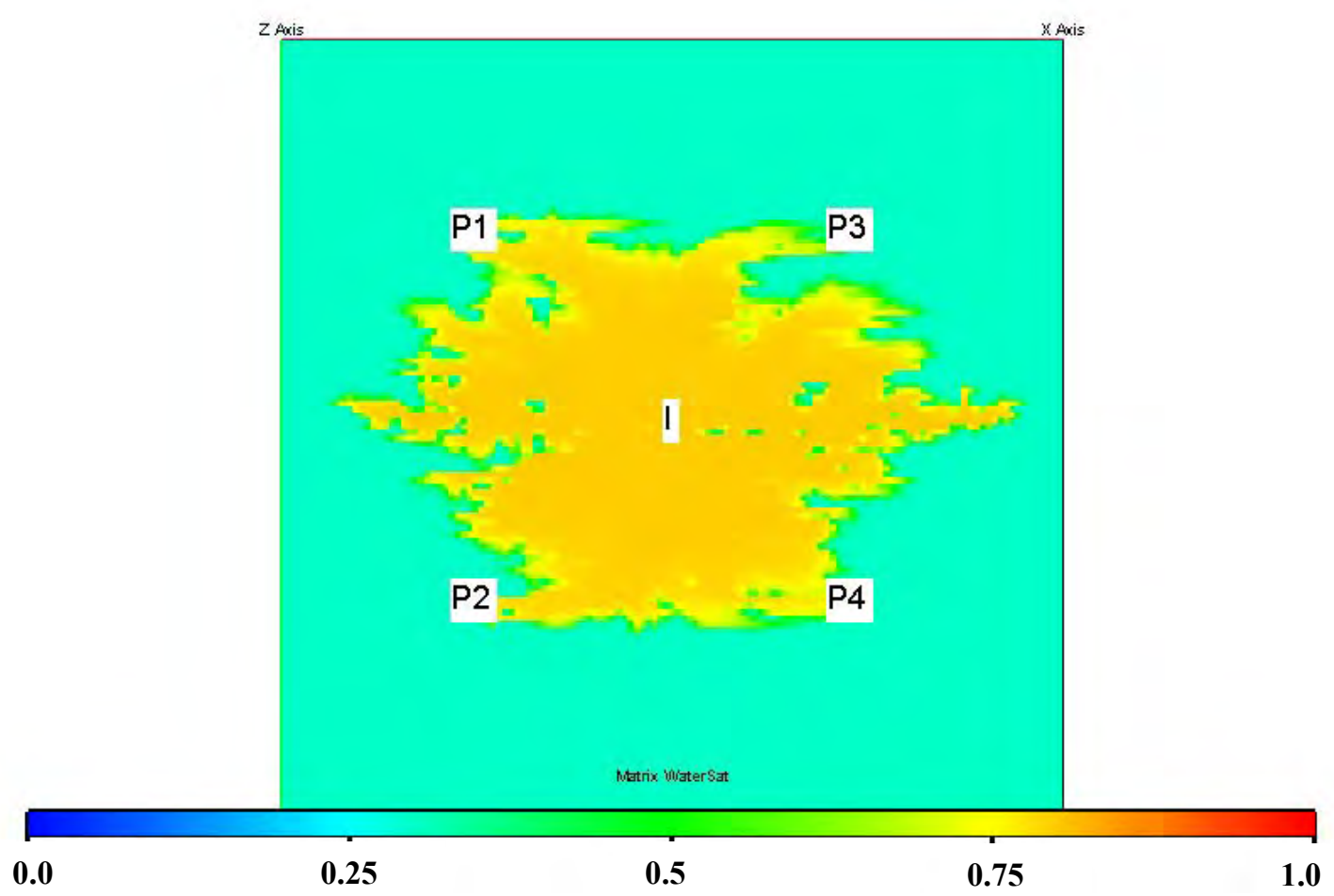

Fig. B.12d - Matrix water saturation at report step 20 (600 days) for test T2 final model, combined data. 


\section{Well Bottom Hole Pressure}

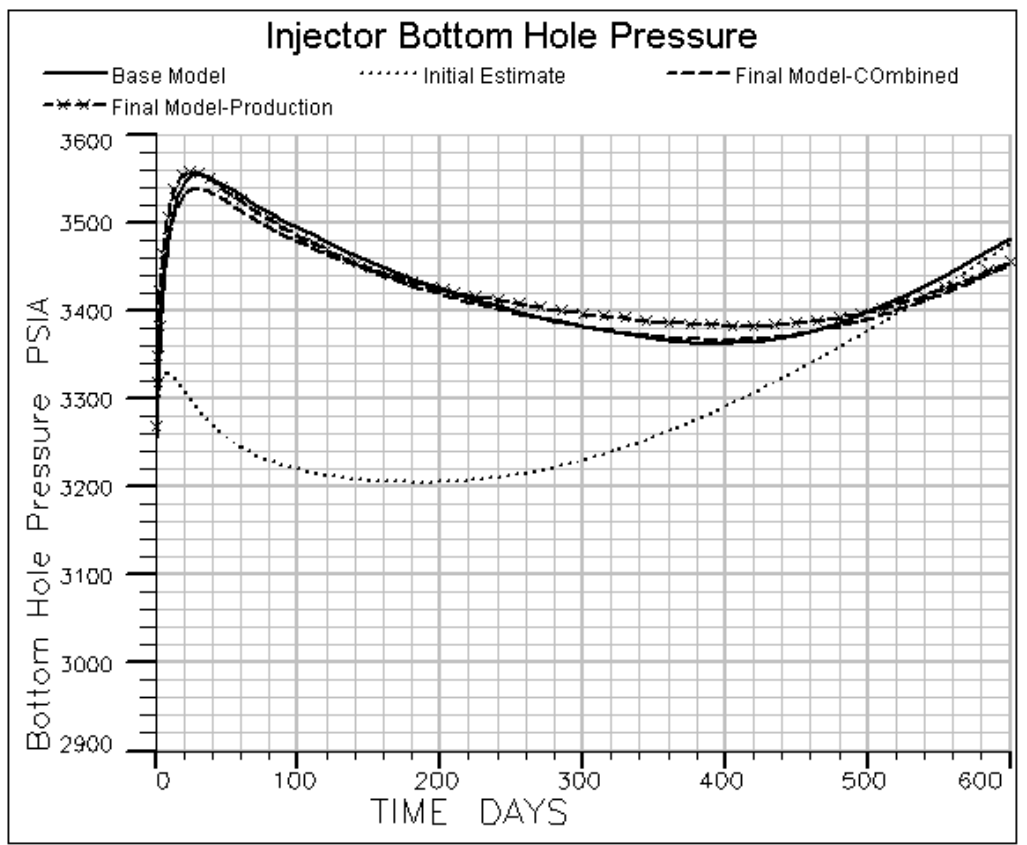

Fig. B.13a - Injector flowing bottom hole pressure for base model, initial estimate, and final model. Both production data only and combined data final models.

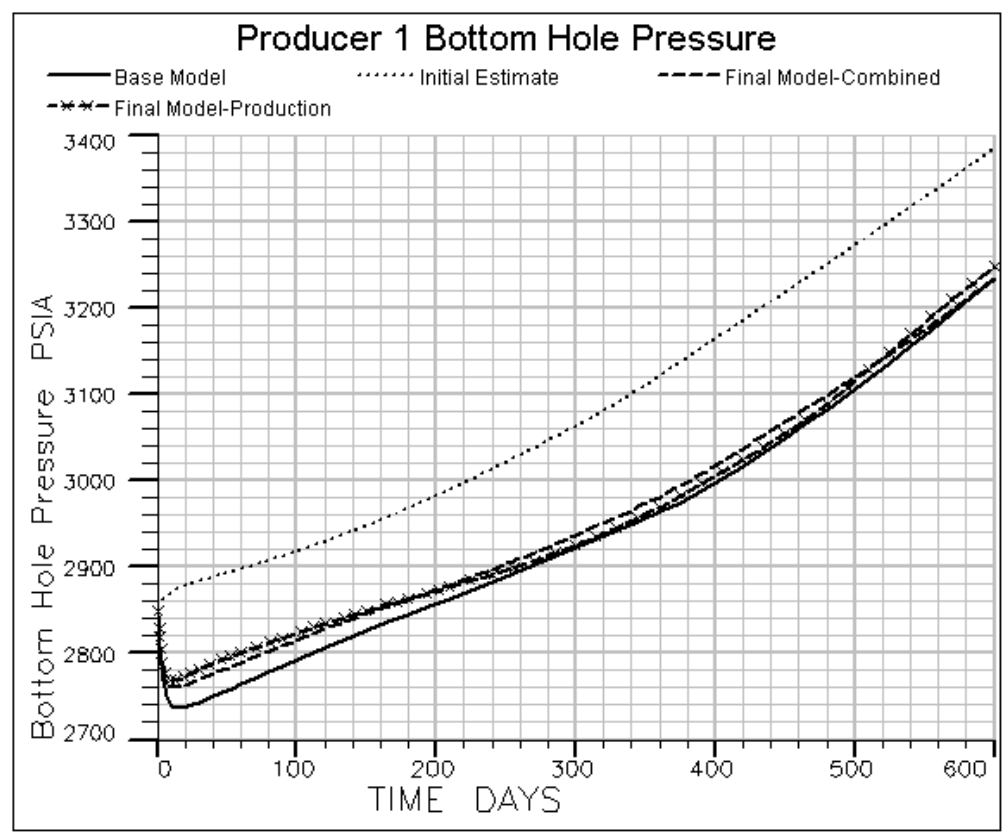

Fig. B.13b - Producer 1 flowing bottom hole pressure for base model, initial estimate, and final model. Both production data only and combined data final models. 


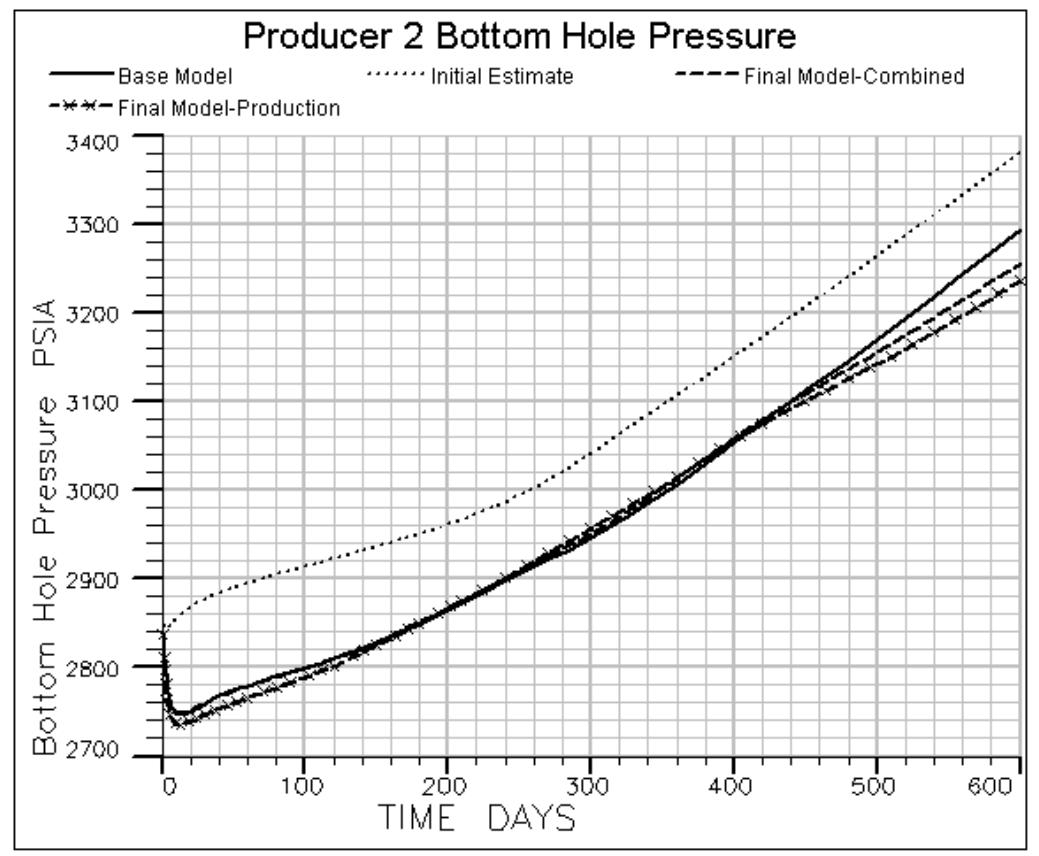

Fig. B.13c - Producer 2 flowing bottom hole pressure for base model, initial estimate, and final model. Both production data only and combined data final models.

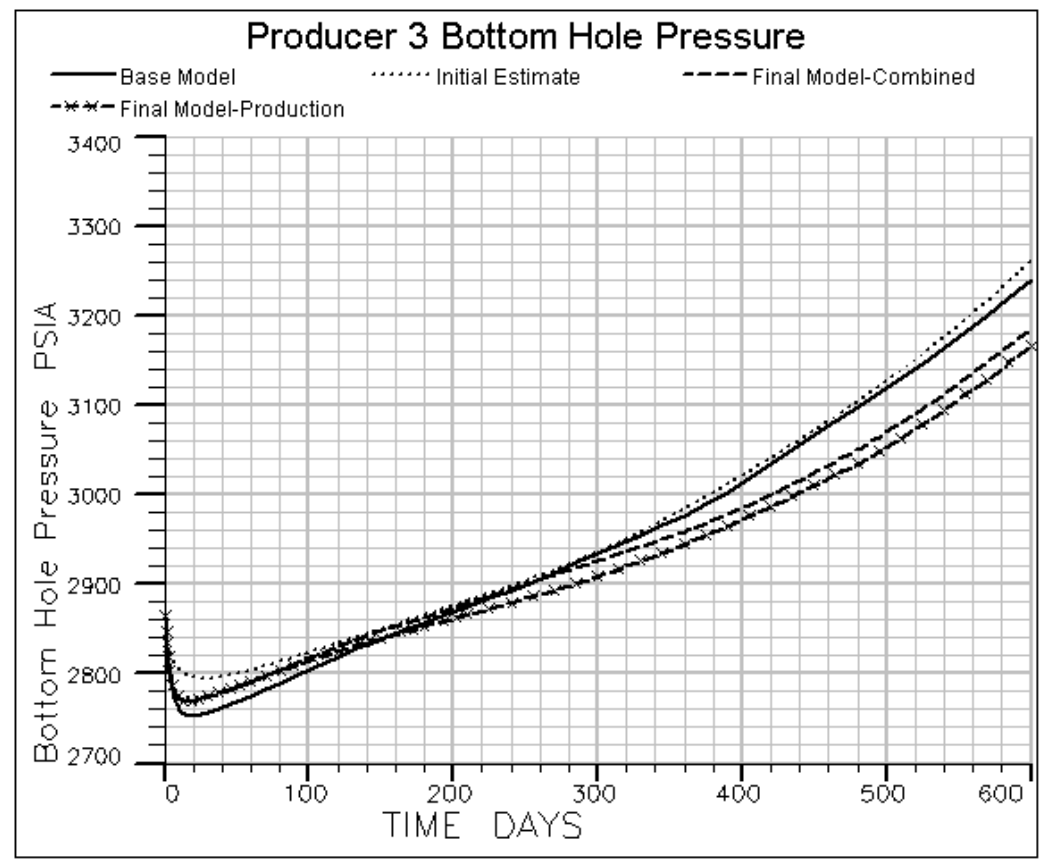

Fig. B.13d - Producer 3 flowing bottom hole pressure for base model, initial estimate, and final model. Both Production Data Only and Combined Data final models. 


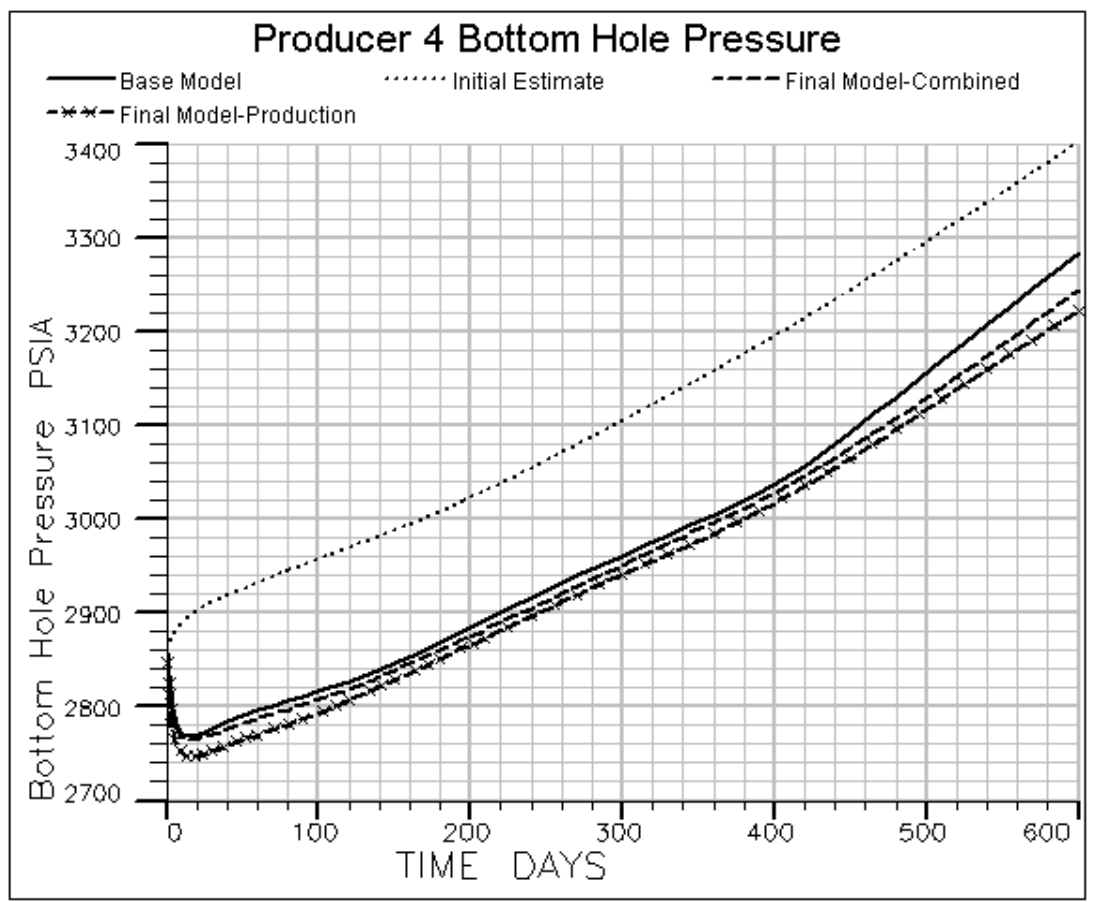

Fig. B.13e - Producer 4 flowing bottom hole pressure for base model, initial estimate, and final model. Both production data only and combined data final models. 


\section{Well Oil Production Rate}

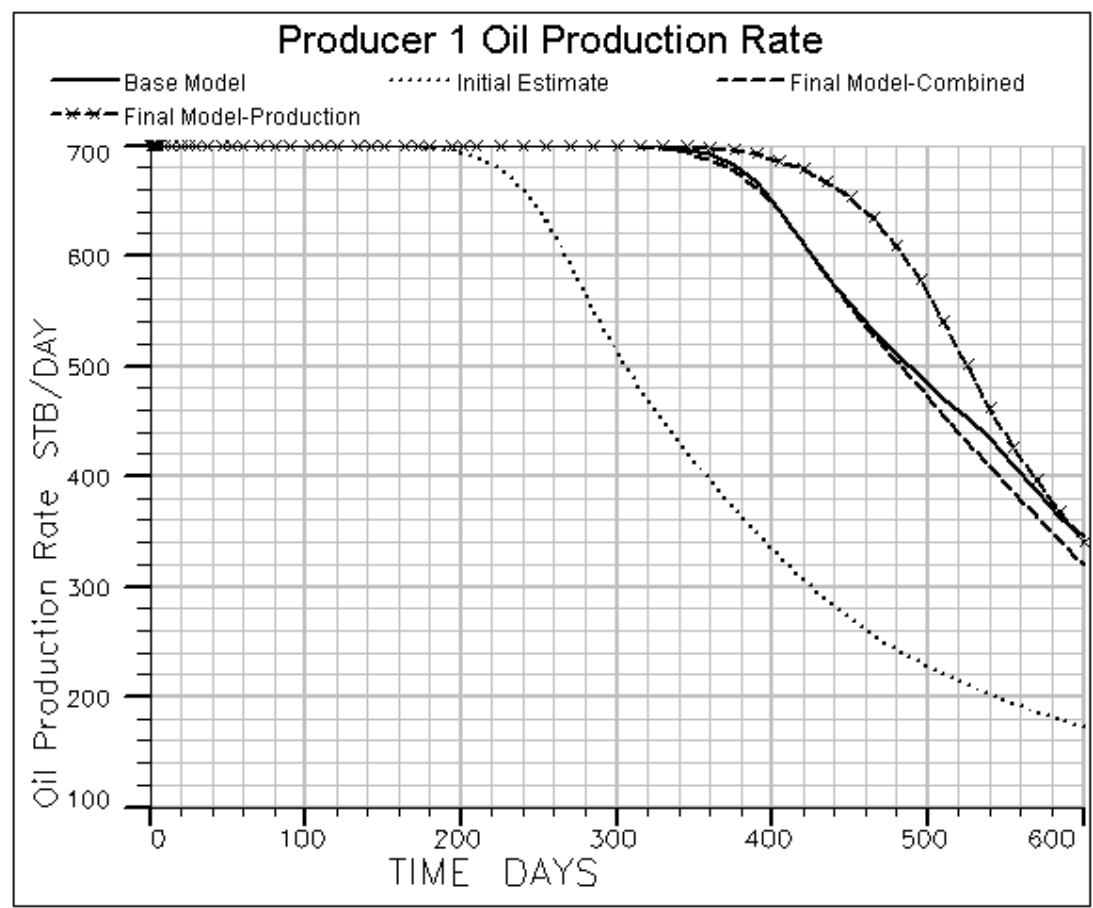

Fig. B.14a - Producer 1 oil production rate for base model, initial estimate, and final model. Both production data only and combined data final models.

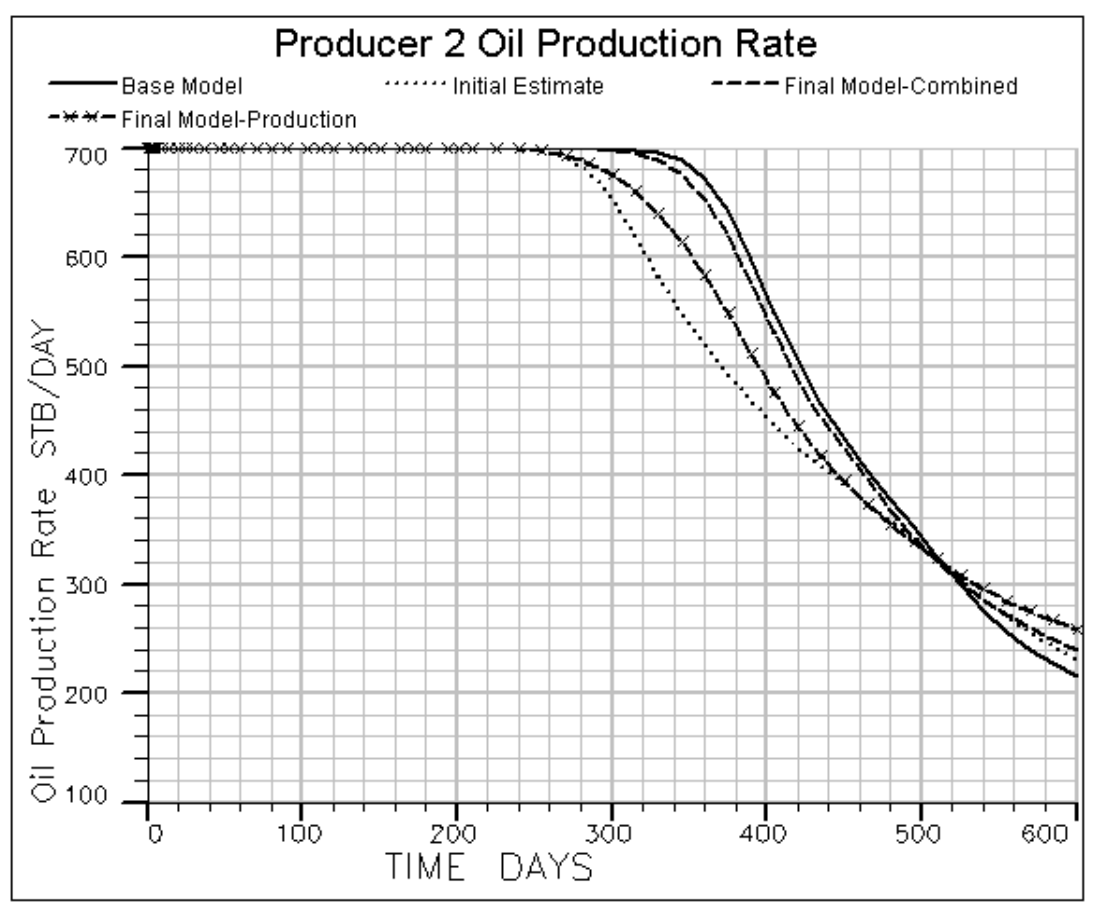

Fig. B.14b - Producer 2 oil production rate for base model, initial estimate, and final model. Both production data only and combined data final models. 


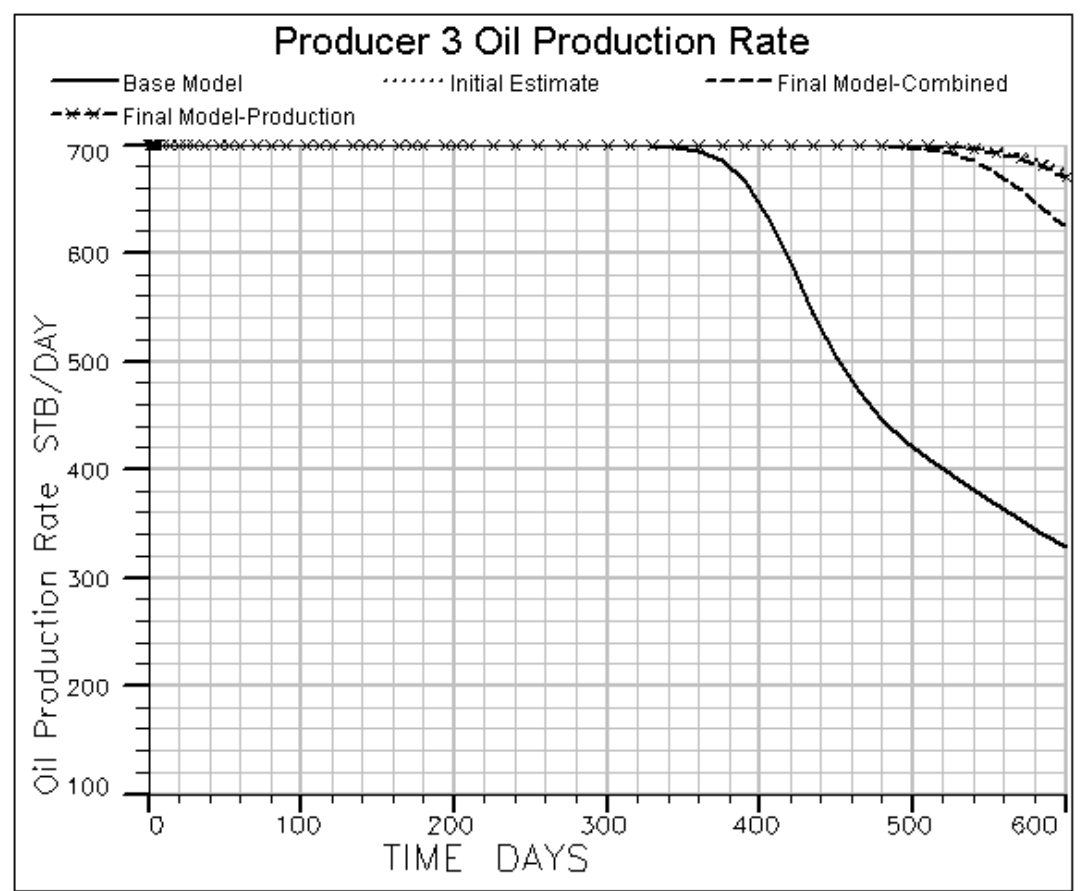

Fig. B.14c - Producer 3 oil production rate for base model, initial estimate, and final model. Both production data only and combined data final models.

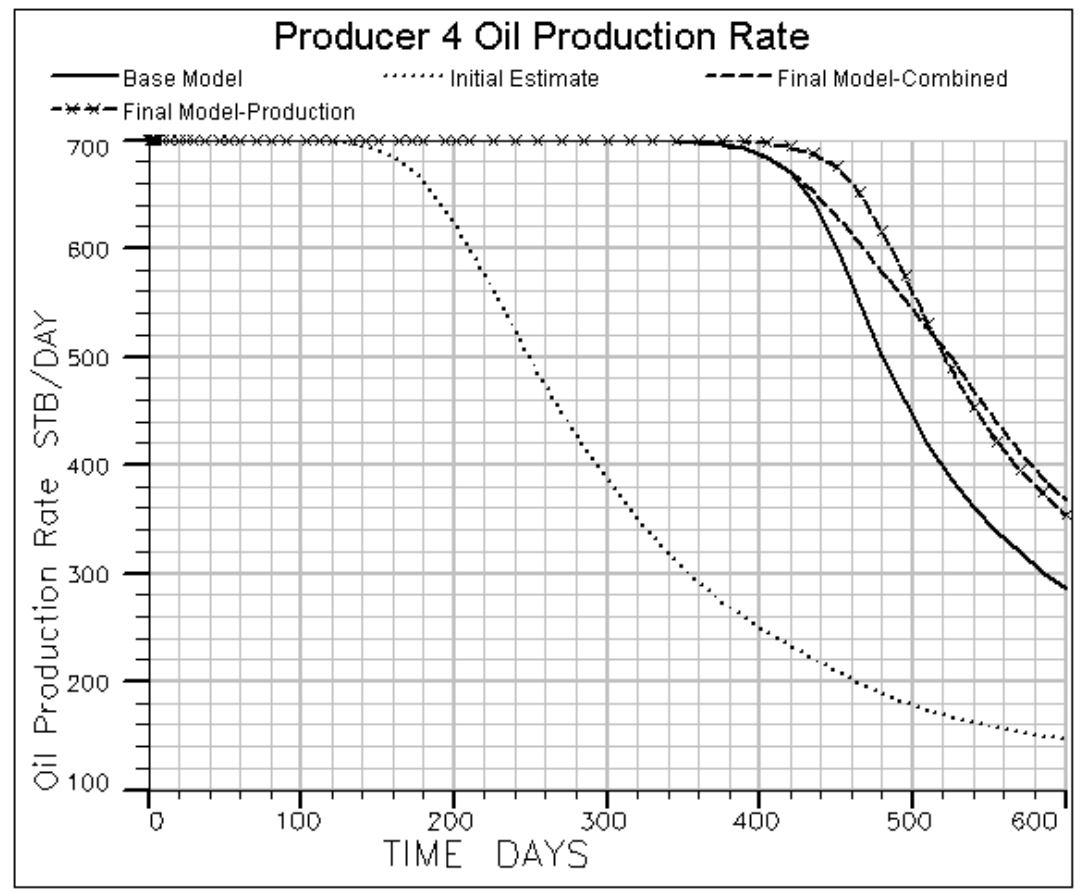

Fig. B.14d - Producer 4 oil production rate for base model, initial estimate, and final model. Both production data only and combined data final models. 
Sensitivity Coefficients (All Iterations)

Combined Data Test
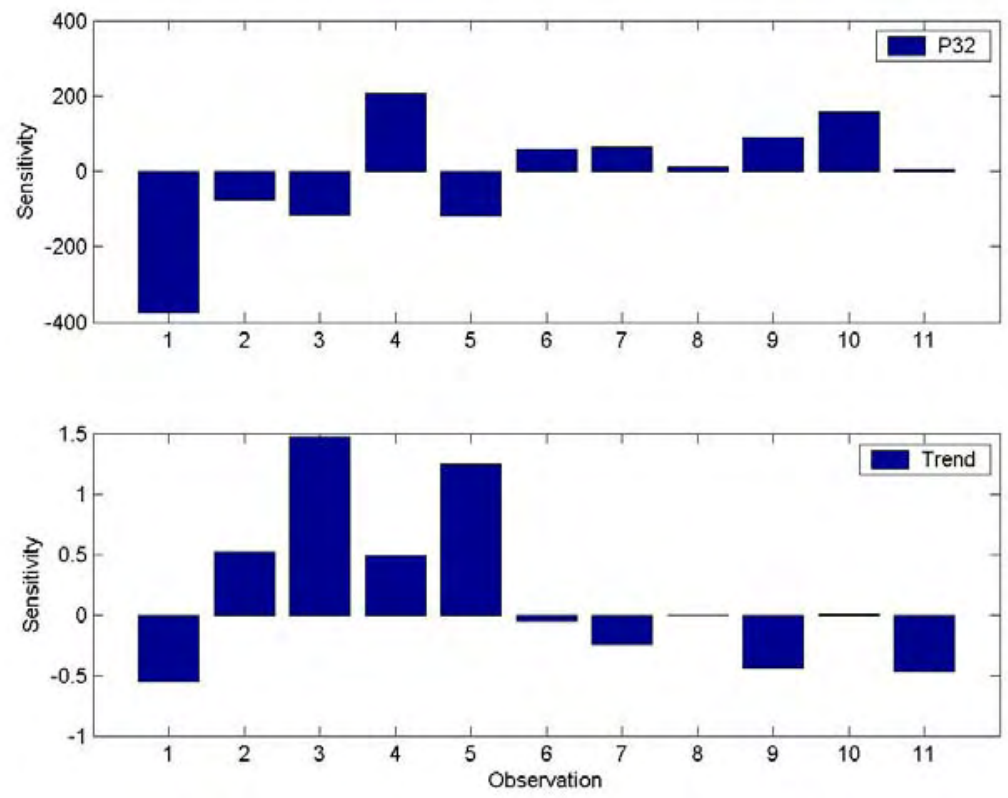

Fig. B.15a - Sensitivity coefficients for iteration 1 .
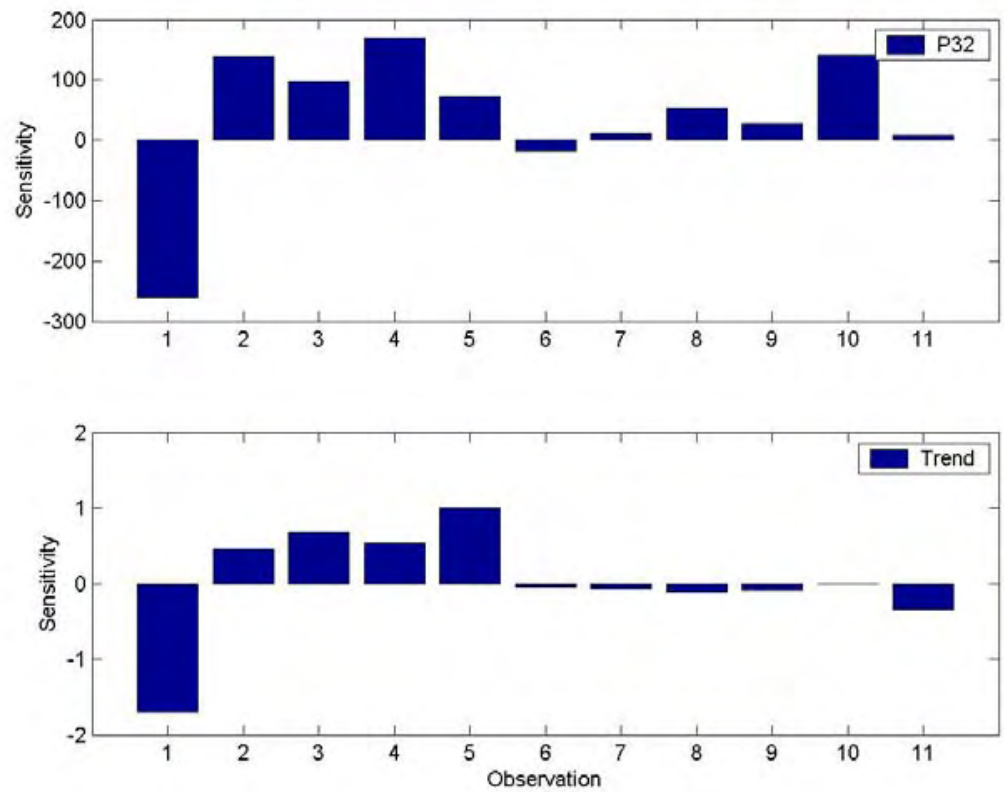

Fig. B.15b - Sensitivity coefficients for iteration 2 . 

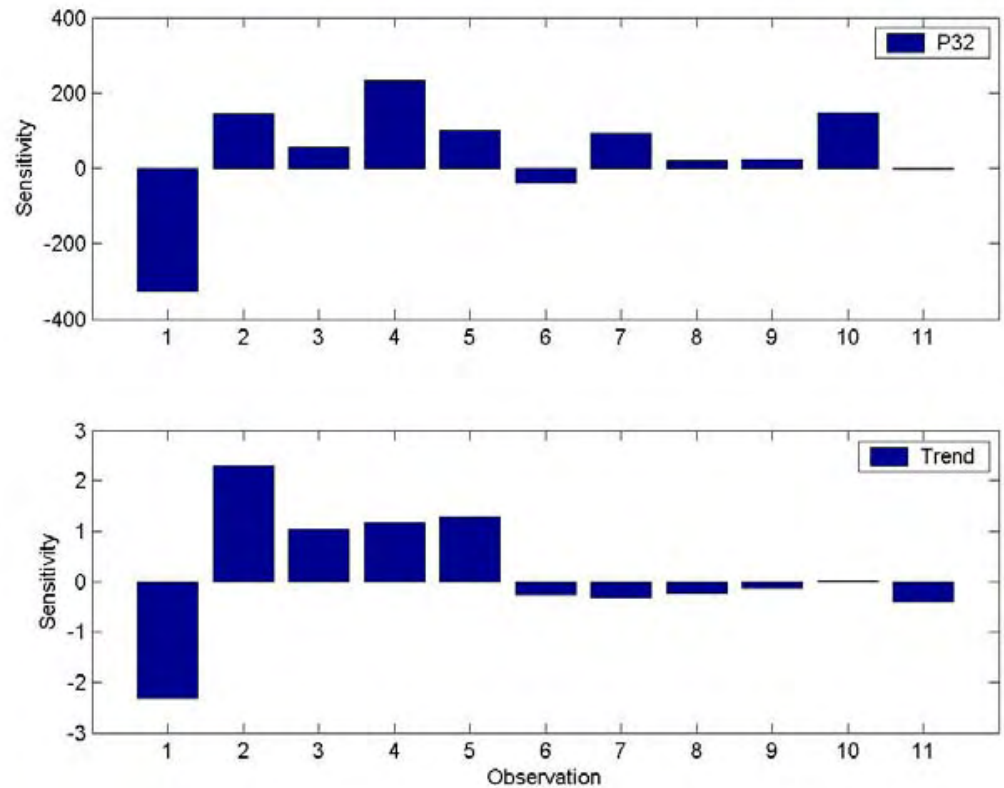

Fig. B.15c - Sensitivity coefficients for iteration 3 .
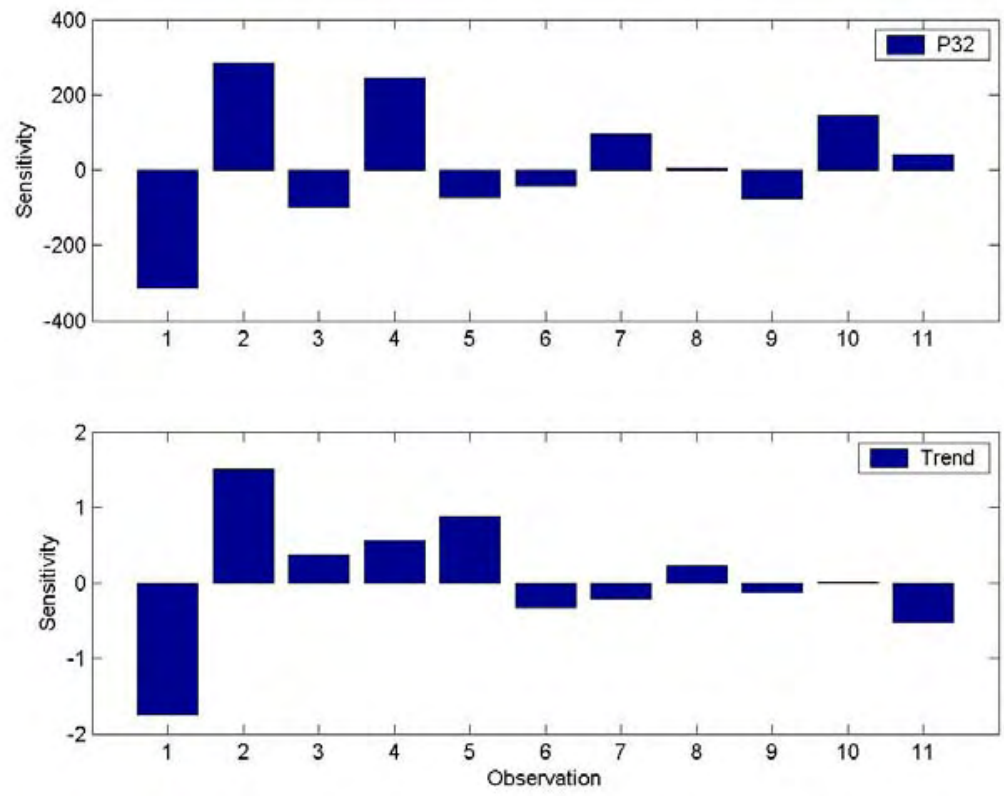

Fig. B.15d - Sensitivity coefficients for iteration 4 . 

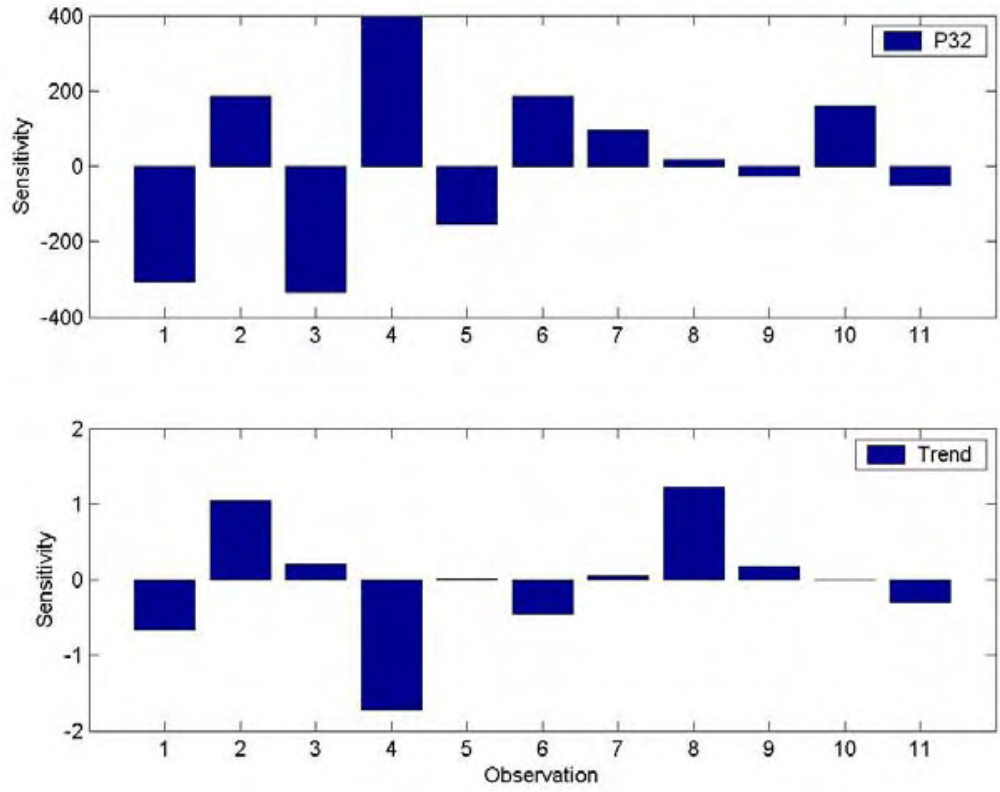

Fig. B.15e - Sensitivity coefficients for iteration 5 . 
Elastic Anisotropy versus Azimuth (Base, Initial, Final)

\section{Combined Data Test}

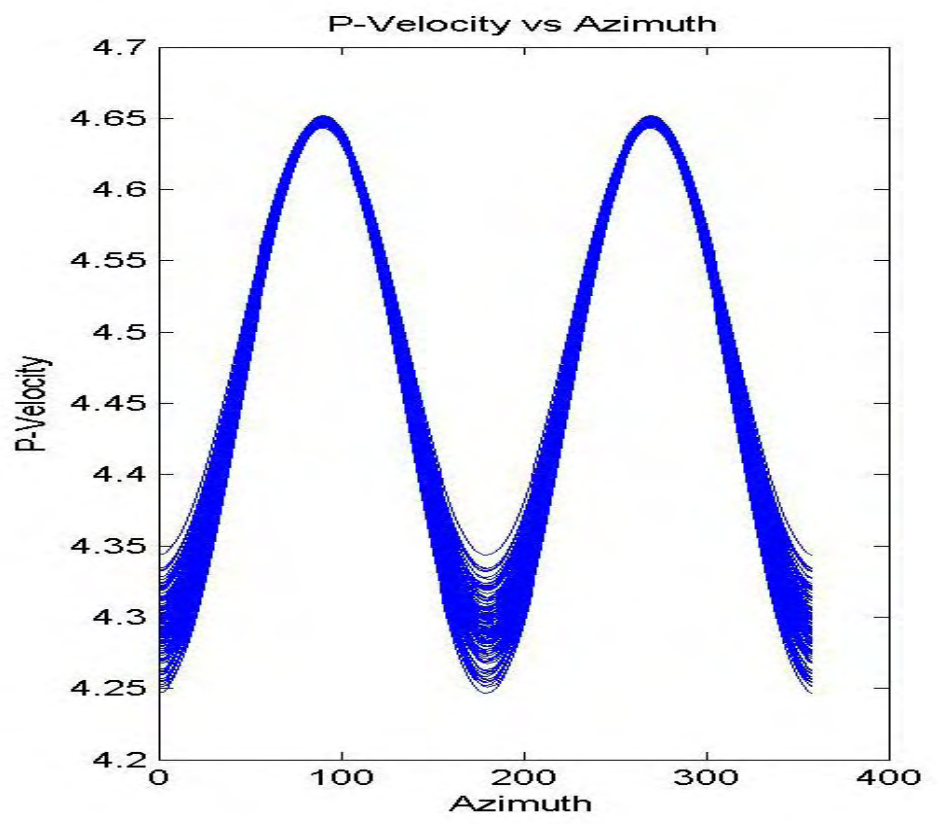

Fig. B.16a - Quasi-P wave velocity versus azimuth for base model.

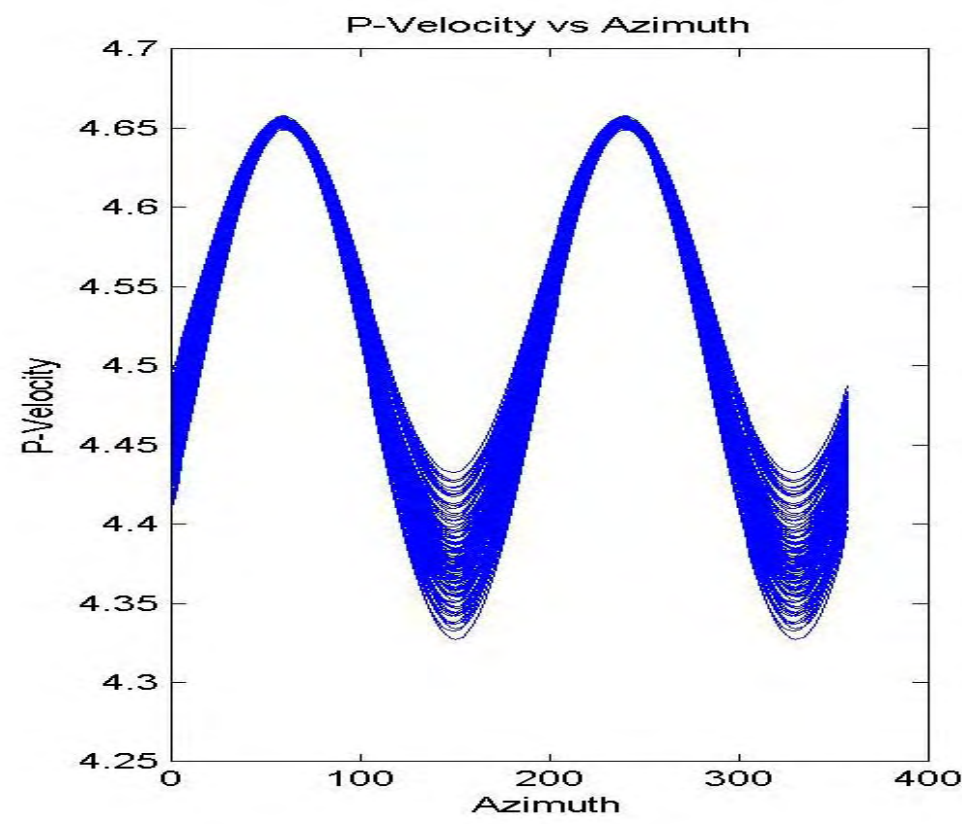

Fig. B.16b - Quasi-P wave velocity versus azimuth for initial estimate (M1). 
177

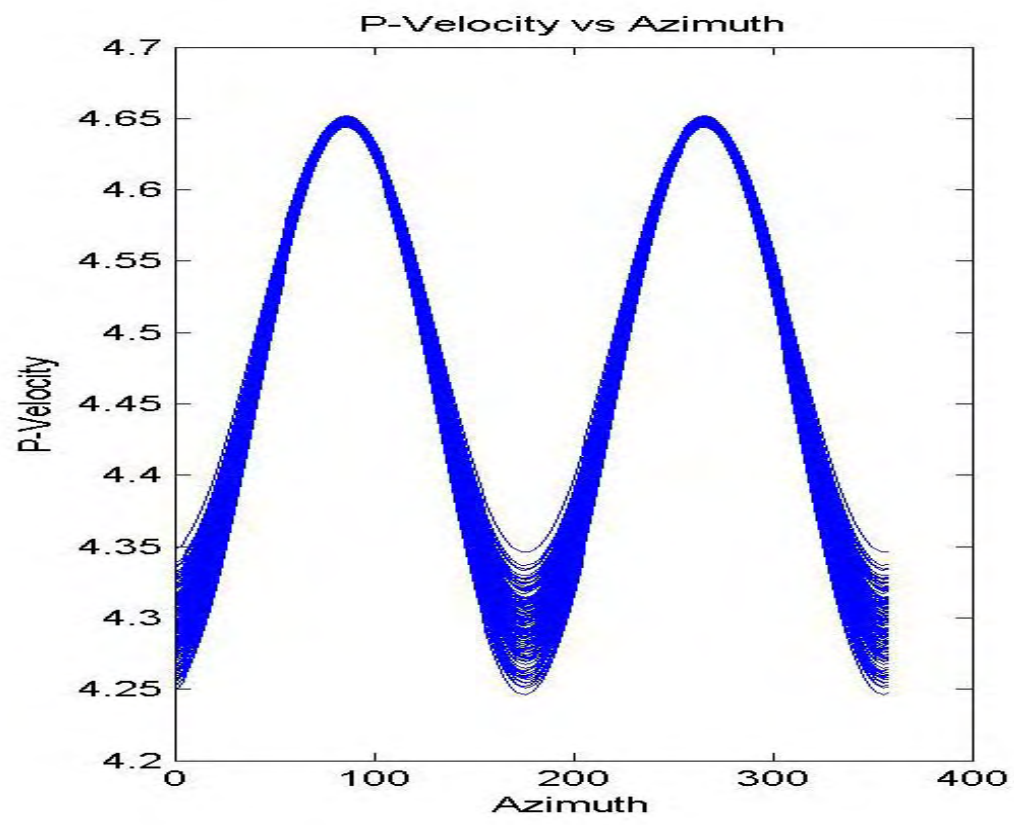

Fig. B.16c - Quasi-P wave velocity versus azimuth for final model (MB).

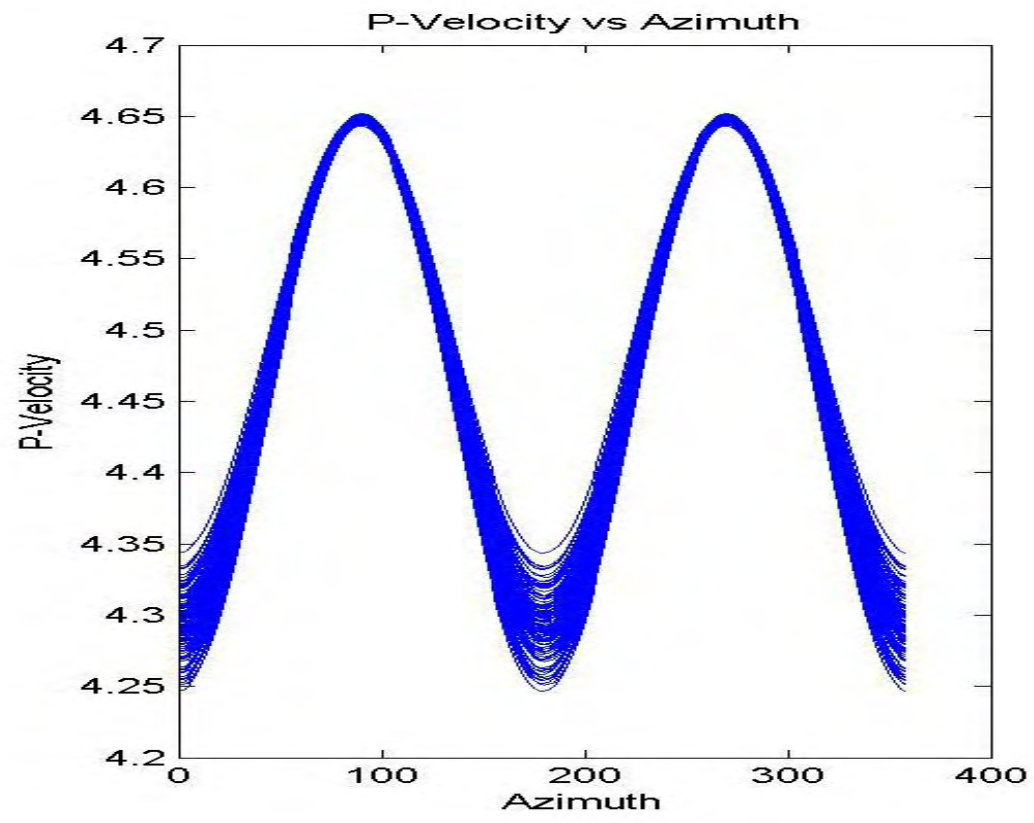

Fig. B.16d - Quasi-P wave velocity versus azimuth for base model for comparison with Fig. 16c. 

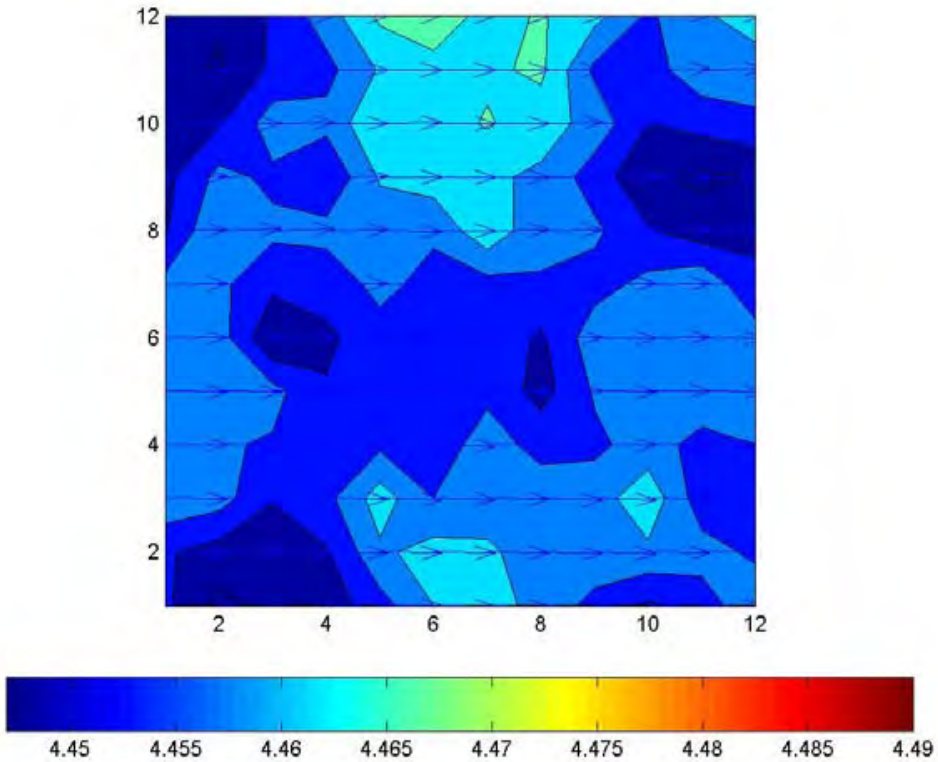

Fig. B.17a - Base model P velocity modulation amplitude and fast shear direction.
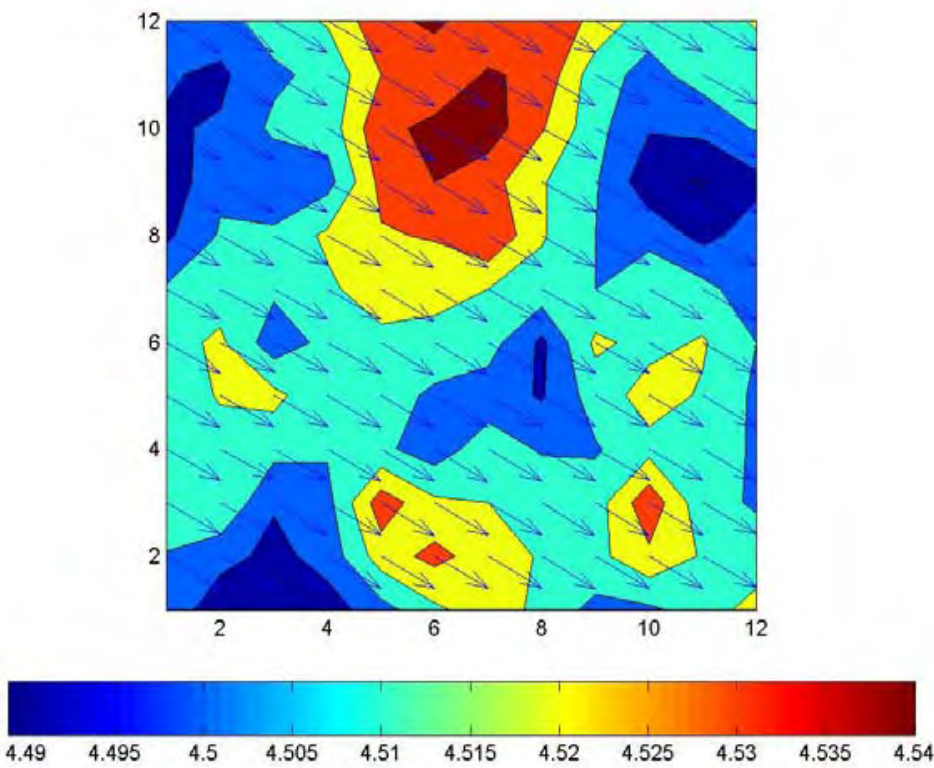

Fig. B.17b - Initial estimate $\mathrm{P}$ velocity modulation amplitude and fast shear direction. 

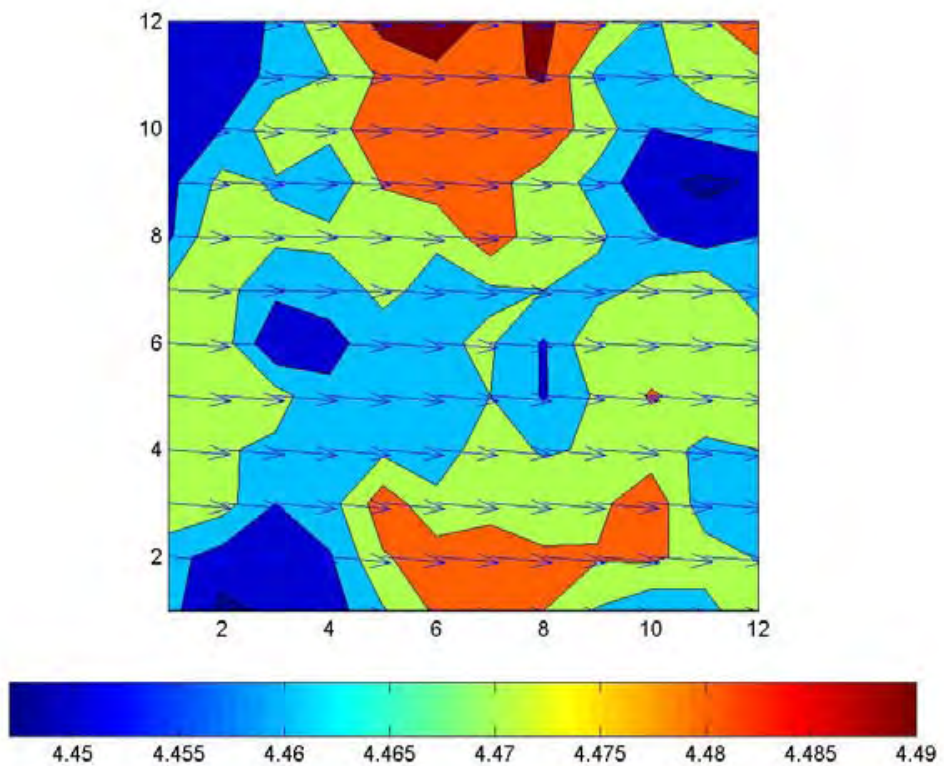

Fig. B.17c - Final P velocity modulation amplitude and fast shear direction.
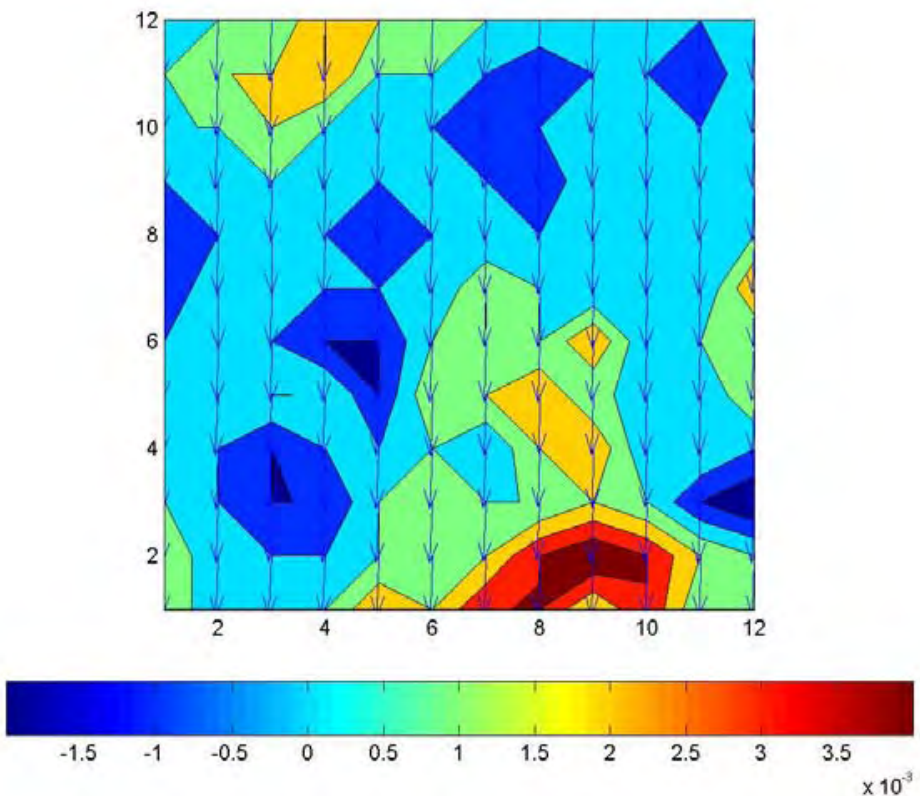

Fig. B.17d - P velocity modulation amplitude and fast shear direction difference between base and final modes. 


\section{APPENDIX C}

\section{SUMMARY OF TEST T3 RESULTS}

In this Appendix we provide plots and tables detailing optimization test T3. As this dissertation is based upon the application of the new optimization process to the test cases we provide this Appendix as a comprehensive documentation set for this test case. Selected portions of this information may be found in Chapter IV with a concise description of the optimization test.

\section{General Information}

- Fracture system properties.

- Petrophysical properties.

- Simulation parameters.

- Elastic properties.

\section{Summarized Test Results}

- Parameter error summary table and plot.

- Residual summary table and plot.

\section{Data for Each Iteration}

- Table of derivative perturbations.

- Table of prior model weights.

- Tables of raw parameter updates.

- Tables of raw residuals.

- Plots of sensitivity coefficients. 
For base case, initial estimate, and optimized model

- Plots of fracture distributions.

- Maps of fracture system water saturation report step 150 days, 300 days, 450 days, 600 days.

- Maps of matrix water saturation report step 150 days, 300 days, 450 days, 600 days.

- Bottom hole pressure plots.

- Oil production rate plots.

- Seismic P-wave velocity versus azimuth plots .

- Seismic anisotropy attribute maps. 
Table C.1 - Test T3 Base Model Fracture Distribution Parameters.

\begin{tabular}{|l|c|c|}
\hline \multicolumn{1}{|c|}{ Parameter } & Set 1 & Set 2 \\
\hline Trend (Geographical) & $\mathrm{N}[335,10]$ & $\mathrm{N}[45,10]$ \\
\hline P32 Intensity & 0.1 & $0.15 \mathrm{~m}$ \\
\hline Length & $\mathrm{LN}[50,3]$ & $\mathrm{LN}[50,3] \mathrm{m}$ \\
\hline Height & 60 & $60 \mathrm{~m}$ \\
\hline Termination & 50 & $50 \%$ \\
\hline Transmissivity & $\mathrm{LN}[8 \mathrm{e}-04,4.3 \mathrm{e}-05]$ & $\left.\mathrm{LN}[8 \mathrm{e}-04,4.3 \mathrm{e}-05] \quad \mathrm{m}^{2} / \mathrm{s}\right)$ \\
\hline
\end{tabular}

Table C.2 - Test T3 Reservoir Properties.

\begin{tabular}{|l|c|}
\hline Width & $2624 \mathrm{ft}$ \\
\hline Length & $2624 \mathrm{ft}$ \\
\hline Thickness & $100 \mathrm{ft}$ \\
\hline Matrix Porosity & 0.10 \\
\hline Matrix Permeability & $10 \mathrm{mD}$ \\
\hline Fracture Porosity & 0.015 \\
\hline
\end{tabular}

Table C.3 - Test T3 Flow Simulation Parameters.

\begin{tabular}{|l|c|}
\hline Sigma & 0.08 \\
\hline Report step & 30 days \\
\hline Number cells (i) & 131 \\
\hline Number cells (j) & 131 \\
\hline Cell size (i) & $20 \mathrm{ft}$ \\
\hline Cell size (j) & $20 \mathrm{ft}$ \\
\hline
\end{tabular}


Table C.4 - Test T3 Elastic Modeling Parameters.

\begin{tabular}{|l|c|}
\hline Matrix P-wave Velocity & $4670 \mathrm{ft} / \mathrm{sec}$ \\
\hline Matrix Shear-wave Velocity & $3060 \mathrm{ft} / \mathrm{sec}$ \\
\hline Matrix Density & $2510 \mathrm{~kg} / \mathrm{m}^{3}$ \\
\hline Overburden P wave Velocity & $4670 \mathrm{ft} / \mathrm{sec}$ \\
\hline Overburden S wave Velocity & $3060 \mathrm{ft} / \mathrm{sec}$ \\
\hline Overburden Density & $2510 \mathrm{~kg} / \mathrm{m}^{3}$ \\
\hline Incidence Angle & $30 \mathrm{degrees}$ \\
\hline
\end{tabular}

Table C.5 - Test T3 Derivative Perturbations.

\begin{tabular}{|l|r|r|r|r|r|r|}
\hline Iterations & \multicolumn{1}{|c|}{$\mathbf{1}$} & \multicolumn{1}{c|}{$\mathbf{2}$} & \multicolumn{1}{c|}{$\mathbf{3}$} & \multicolumn{1}{c|}{$\mathbf{4}$} & \multicolumn{1}{c|}{$\mathbf{5}$} & \multicolumn{1}{c|}{$\mathbf{6}$} \\
\hline Trend 1 (degrees) & -10 & -10 & -10 & -10 & -6 & -6 \\
\hline P32 1 (1/m) & -0.05 & -0.05 & -0.05 & -0.05 & -0.02 & 0.02 \\
\hline Trend 2 (degrees) & 10 & 10 & 10 & -10 & 6 & 6 \\
\hline P32 2 (1/m) & -0.05 & -0.05 & -0.05 & 0.05 & 0.02 & 0.02 \\
\hline
\end{tabular}

Table C.6 - Test T3 Prior Model Weights.

\begin{tabular}{|l|c|c|c|c|c|c|}
\hline Iterations & $\mathbf{1}$ & $\mathbf{2}$ & $\mathbf{3}$ & $\mathbf{4}$ & $\mathbf{5}$ & $\mathbf{6}$ \\
\hline Trend (1) & 4 & 4 & 0.5 & 0.5 & 1 & 0.5 \\
\hline P32 (1) & 4 & 4 & 4 & 4 & 2 & 2 \\
\hline Trend (2) & 4 & 4 & 4 & 1 & 0.5 & 2 \\
\hline P32 (2) & 4 & 4 & 100 & 2 & 2 & 4 \\
\hline
\end{tabular}


Table C.7 - Summary of absolute errors for fracture set trend and P32 intensity at each update step. Step 0 is the initial estimate.

\begin{tabular}{|r|r|r|r|r|}
\cline { 2 - 5 } \multicolumn{1}{c|}{} & \multicolumn{4}{|c|}{ Parameter Error } \\
\hline Iteration & Trend (1) & P32 (1) & Trend (2) & P32 (2) \\
\hline $\mathbf{0}$ & 20.0 & 0.100 & 20.0 & 0.050 \\
\hline $\mathbf{1}$ & 19.3 & 0.074 & 18.6 & 0.043 \\
\hline $\mathbf{2}$ & 16.4 & 0.050 & 15.6 & 0.056 \\
\hline $\mathbf{3}$ & 16.1 & 0.036 & 9.5 & 0.057 \\
\hline $\mathbf{4}$ & 8.2 & 0.024 & 5.5 & 0.035 \\
\hline $\mathbf{5}$ & 12.1 & 0.007 & 4.1 & 0.018 \\
\hline $\mathbf{6}$ & 1.8 & 0.003 & 0.6 & 0.005 \\
\hline
\end{tabular}

Table C.8 - Summary of unit normalized residual errors by observation type for fracture set trend and P32 intensity at each iteration. Iteration 0 is the initial estimate.

\begin{tabular}{|r|r|r|r|r|}
\cline { 2 - 5 } \multicolumn{1}{c|}{} & \multicolumn{4}{c|}{ Residuals } \\
\hline Iteration & \multicolumn{1}{c|}{ Total } & \multicolumn{1}{c|}{ BHP } & \multicolumn{1}{c|}{ OPR } & \multicolumn{1}{c|}{ SEIS } \\
\hline $\mathbf{0}$ & 18.80 & 8.31 & 3.93 & 16.40 \\
\hline $\mathbf{1}$ & 16.52 & 6.73 & 4.23 & 14.47 \\
\hline $\mathbf{2}$ & 11.83 & 3.93 & 3.29 & 10.68 \\
\hline $\mathbf{3}$ & 9.37 & 3.17 & 3.80 & 7.94 \\
\hline $\mathbf{4}$ & 5.08 & 1.34 & 1.18 & 4.74 \\
\hline $\mathbf{5}$ & 7.09 & 3.57 & 3.29 & 5.18 \\
\hline $\mathbf{6}$ & 4.52 & 3.26 & 3.06 & 0.77 \\
\hline
\end{tabular}




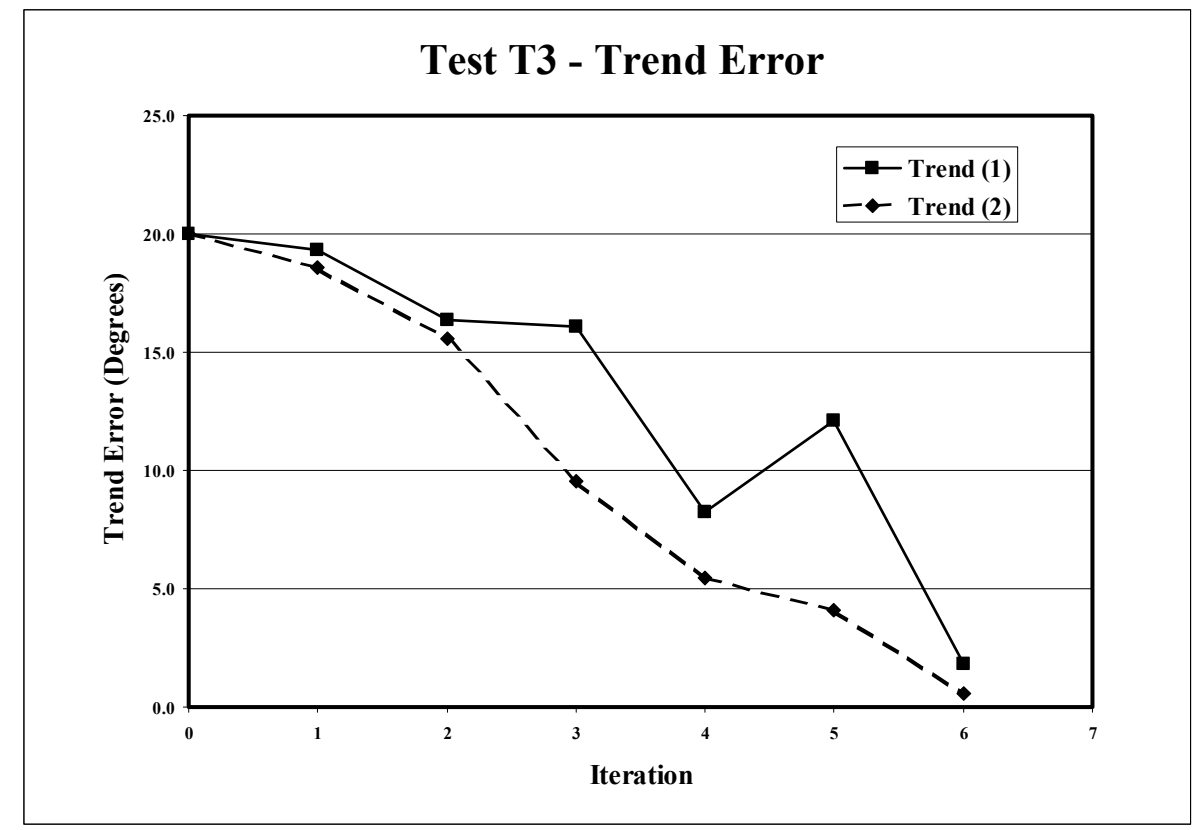

Fig. C.1 $\quad-\quad$ Graph of absolute error in fracture set trend versus iteration for test T3. Iteration 0 is the initial estimate.

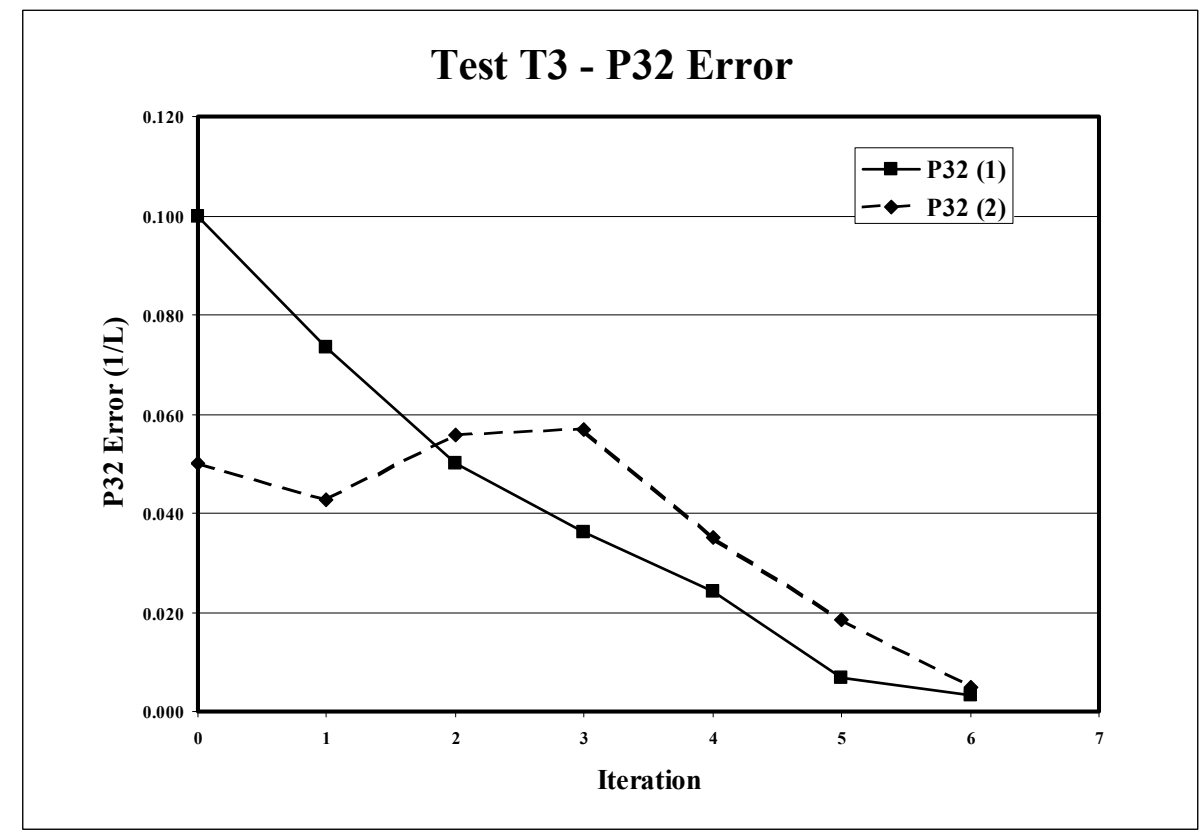

Fig. C.2 $\quad-\quad$ Graph of absolute error in fracture set P32 intensity versus iteration for test $\mathrm{T} 3$. Iteration 0 is the initial estimate. 


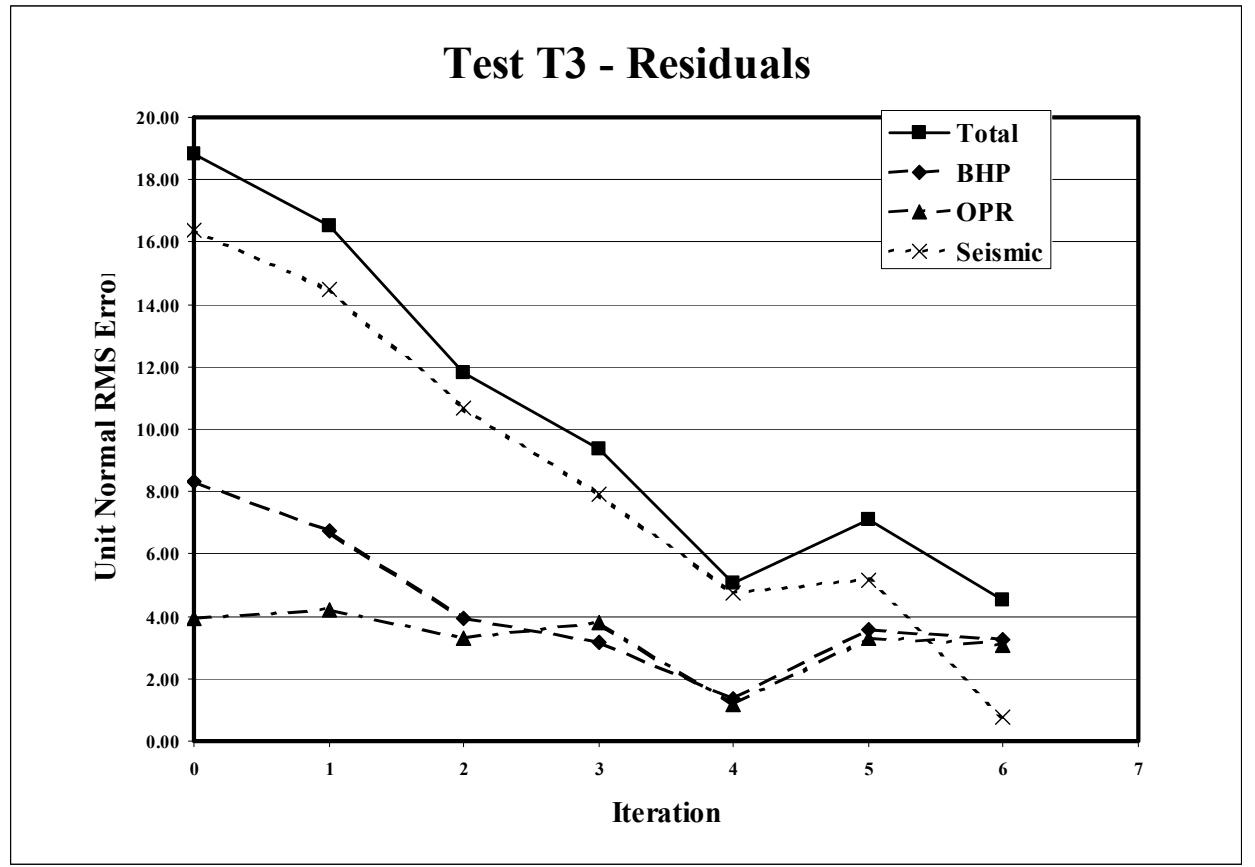

Fig. C.3 - Graph of unit normalized residual errors by observation type for fracture set trend and P32 intensity at each iteration. Iteration 0 is the initial estimate.

Table C.9a - Summary of iteration 1 Trend and P32 intensity updates and residual errors for 10 samples.

Step M1

\begin{tabular}{|r|r|r|r|r||r|r|r|r|}
\cline { 2 - 9 } \multicolumn{1}{c|}{} & \multicolumn{4}{|c||}{ Updates } & \multicolumn{4}{|c|}{ Residuals } \\
\hline SAMPLE & Trend (1) & P32 (1) & Trend (2) & P32 (2) & TOTAL & \multicolumn{1}{c|}{ BHP } & OPR & \multicolumn{1}{c|}{ SEIS } \\
\hline $\mathbf{1}$ & 84.6 & 0.172 & 112.9 & 0.111 & 18.92 & 8.40 & 3.90 & 16.40 \\
\hline $\mathbf{2}$ & 84.8 & 0.173 & 109.5 & 0.105 & 18.60 & 8.30 & 3.80 & 16.20 \\
\hline $\mathbf{3}$ & 84.7 & 0.168 & 115.7 & 0.117 & 18.90 & 8.30 & 3.90 & 16.50 \\
\hline $\mathbf{4}$ & 84.1 & 0.170 & 113.7 & 0.110 & 18.60 & 8.10 & 4.00 & 16.30 \\
\hline $\mathbf{5}$ & 84.4 & 0.173 & 112.9 & 0.109 & 18.70 & 8.20 & 3.70 & 16.40 \\
\hline $\mathbf{6}$ & 83.5 & 0.176 & 111.2 & 0.105 & 19.00 & 8.70 & 3.90 & 16.50 \\
\hline $\mathbf{7}$ & 83.8 & 0.178 & 107.0 & 0.099 & 18.70 & 8.10 & 4.20 & 16.40 \\
\hline $\mathbf{8}$ & 85.1 & 0.178 & 107.8 & 0.103 & 18.80 & 8.20 & 3.90 & 16.50 \\
\hline $\mathbf{9}$ & 83.3 & 0.176 & 111.9 & 0.106 & 18.90 & 8.50 & 3.80 & 16.40 \\
\hline $\mathbf{1 0}$ & 84.9 & 0.172 & 111.5 & 0.108 & 18.90 & 8.30 & 4.20 & 16.40 \\
\hline AVG & $\mathbf{8 4 . 3}$ & $\mathbf{0 . 1 7 4}$ & $\mathbf{1 1 1 . 4}$ & $\mathbf{0 . 1 0 7}$ & $\mathbf{1 8 . 8 0}$ & $\mathbf{8 . 3 1}$ & $\mathbf{3 . 9 3}$ & $\mathbf{1 6 . 4 0}$ \\
\hline
\end{tabular}


Table C.9b - Summary of iteration 2 Trend and P32 intensity updates and residual errors for 10 samples.

\begin{tabular}{|c|c|c|c|c|c|c|c|c|}
\hline & \multicolumn{4}{|c|}{ Step M2 } & \multirow{2}{*}{\multicolumn{4}{|c|}{ Posiduolo }} \\
\hline & \multicolumn{4}{|c|}{ Updates } & \multicolumn{2}{|c|}{ Residuals } & & \\
\hline SAMPLE & Trend (1) & P32 (1) & Trend (2) & P32 (2) & TOTAL & $\overline{\text { BHP }}$ & OPR & SEIS \\
\hline 1 & 81.1 & 0.146 & 114.2 & 0.097 & 16.30 & 6.40 & 4.20 & 14.40 \\
\hline 2 & 81.3 & 0.148 & 114.4 & 0.095 & 16.60 & 6.80 & 3.60 & 14.70 \\
\hline 3 & 81.0 & 0.148 & 114.3 & 0.096 & 16.50 & 6.70 & 4.30 & 14.50 \\
\hline 4 & 81.3 & 0.151 & 114.6 & 0.093 & 16.40 & 6.60 & 4.40 & 14.30 \\
\hline 5 & 81.1 & 0.152 & 113.8 & 0.095 & 16.50 & 6.80 & 4.30 & 14.40 \\
\hline 6 & 81.5 & 0.155 & 115.0 & 0.090 & 16.80 & 6.90 & 4.30 & 14.70 \\
\hline 7 & 81.3 & 0.150 & 114.0 & 0.096 & 16.50 & 6.90 & 4.50 & 14.30 \\
\hline 8 & 82.0 & 0.149 & 114.2 & 0.096 & 16.70 & 6.70 & 4.20 & 14.60 \\
\hline 9 & 81.6 & 0.150 & 114.5 & 0.093 & 16.50 & 6.90 & 4.40 & 14.40 \\
\hline 10 & 81.7 & 0.153 & 115.2 & 0.091 & 16.40 & 6.60 & 4.10 & 14.40 \\
\hline AVG & 81.4 & 0.150 & 114.4 & 0.094 & 16.52 & 6.73 & 4.23 & 14.47 \\
\hline
\end{tabular}

Table C.9c - $\quad$ Summary of iteration 3 Trend and P32 intensity updates and residual errors for 10 samples.

\begin{tabular}{|c|c|c|c|c|c|c|c|c|}
\hline & & & & ер M3 & & & & \\
\hline & & & & & RESID & RESID & RESID & RESID \\
\hline SAMPLE & T1 & I1 & $\overline{T 2}$ & $\overline{I 2}$ & TOTAL & $\overline{\text { BHP }}$ & $\overline{\text { OPR }}$ & SEIS \\
\hline 1 & 79.8 & 0.137 & 120.3 & 0.093 & 11.90 & 4.10 & 3.50 & 10.60 \\
\hline 2 & 83.4 & 0.137 & 120.3 & 0.093 & 12.00 & 4.06 & 3.36 & 10.80 \\
\hline 3 & 80.2 & 0.137 & 121.1 & 0.093 & 11.80 & 3.93 & 3.30 & 10.60 \\
\hline 4 & 81.8 & 0.136 & 120.9 & 0.093 & 11.60 & 3.80 & 2.90 & 10.60 \\
\hline 5 & 85.7 & 0.135 & 120.5 & 0.093 & 11.60 & 4.00 & 3.10 & 10.40 \\
\hline 6 & 80.5 & 0.134 & 120.2 & 0.093 & 11.90 & 4.10 & 3.40 & 10.70 \\
\hline 7 & 83.5 & 0.136 & 120.3 & 0.093 & 11.60 & 3.80 & 3.20 & 10.50 \\
\hline 8 & 76.8 & 0.137 & 119.8 & 0.093 & 11.90 & 3.70 & 3.50 & 10.80 \\
\hline 9 & 79.2 & 0.135 & 120.8 & 0.093 & 12.10 & 4.10 & 3.20 & 11.00 \\
\hline 10 & 79.8 & 0.137 & 120.6 & 0.093 & 11.90 & 3.70 & 3.40 & 10.80 \\
\hline AVG & 81.1 & 0.136 & 120.5 & 0.093 & 11.83 & 3.93 & 3.29 & 10.68 \\
\hline
\end{tabular}

Table C.9d - Summary of iteration 4 Trend and P32 intensity updates and residual errors for 10 samples.

Step M4

\begin{tabular}{|c|c|c|c|c|c|c|c|c|}
\hline & & & & & RESID & RESID & RESID & RESID \\
\hline SAMPLE & T1 & I1 & T2 & 12 & TOTAL & BHP & OPR & SEIS \\
\hline 1 & 79.8 & 0.137 & 120.3 & 0.093 & 11.90 & 4.10 & 3.50 & 10.60 \\
\hline 2 & 83.4 & 0.137 & 120.3 & 0.093 & 12.00 & 4.06 & 3.36 & 10.80 \\
\hline 3 & 80.2 & 0.137 & 121.1 & 0.093 & 11.80 & 3.93 & 3.30 & 10.60 \\
\hline 4 & 81.8 & 0.136 & 120.9 & 0.093 & 11.60 & 3.80 & 2.90 & 10.60 \\
\hline 5 & 85.7 & 0.135 & 120.5 & 0.093 & 11.60 & 4.00 & 3.10 & 10.40 \\
\hline 6 & 80.5 & 0.134 & 120.2 & 0.093 & 11.90 & 4.10 & 3.40 & 10.70 \\
\hline 7 & 83.5 & 0.136 & 120.3 & 0.093 & 11.60 & 3.80 & 3.20 & 10.50 \\
\hline 8 & 76.8 & 0.137 & 119.8 & 0.093 & 11.90 & 3.70 & 3.50 & 10.80 \\
\hline 9 & 79.2 & 0.135 & 120.8 & 0.093 & 12.10 & 4.10 & 3.20 & 11.00 \\
\hline 10 & 79.8 & 0.137 & 120.6 & 0.093 & 11.90 & 3.70 & 3.40 & 10.80 \\
\hline AVG & 81.1 & 0.136 & 120.5 & 0.093 & 11.83 & 3.93 & 3.29 & 10.68 \\
\hline
\end{tabular}


Table C.9e - Summary of iteration 5 Trend and P32 intensity updates and residual errors for 10 samples.

\begin{tabular}{|c|c|c|c|c|c|c|c|c|}
\hline \multicolumn{9}{|c|}{ Step M5 } \\
\hline & & & & & RESID & RESID & RESID & RESID \\
\hline SAMPLE & T1 & I1 & T2 & I2 & TOTAL & BHP & OPR & SEIS \\
\hline & 76.5 & 0.108 & 127.5 & 0.133 & 5.00 & 1.50 & 1.00 & 4.70 \\
\hline 2 & 77.9 & 0.103 & 120.6 & 0.128 & 5.20 & 1.30 & 1.40 & 4.80 \\
\hline$\overline{3}$ & 77.6 & 0.104 & 121.8 & 0.131 & 5.00 & 1.10 & 1.00 & 4.80 \\
\hline 4 & 78.7 & 0.101 & 117.6 & 0.120 & 5.30 & 1.80 & 1.30 & 4.80 \\
\hline$\overline{5}$ & 77.5 & 0.108 & 126.2 & 0.130 & 5.00 & 1.20 & 1.10 & 4.70 \\
\hline 6 & 76.2 & 0.110 & 130.5 & 0.136 & 5.30 & 1.10 & 1.60 & 4.90 \\
\hline 7 & 75.8 & 0.111 & 128.8 & 0.131 & 5.00 & 1.30 & 1.10 & 4.70 \\
\hline 8 & 76.9 & 0.108 & 130.1 & 0.138 & 4.90 & 1.40 & 0.90 & 4.60 \\
\hline$\overline{9}$ & 77.3 & 0.107 & 128.6 & 0.136 & 4.90 & 1.30 & 1.10 & 4.60 \\
\hline$\overline{10}$ & 76.9 & 0.108 & 127.6 & 0.133 & 5.20 & 1.40 & 1.30 & 4.80 \\
\hline$\overline{\mathrm{AVG}}$ & 77.1 & 0.107 & 125.9 & 0.132 & 5.08 & 1.34 & 1.18 & 4.74 \\
\hline
\end{tabular}

Table C.9f - Summary of iteration 6 Trend and P32 intensity updates and residual errors for 10 samples.

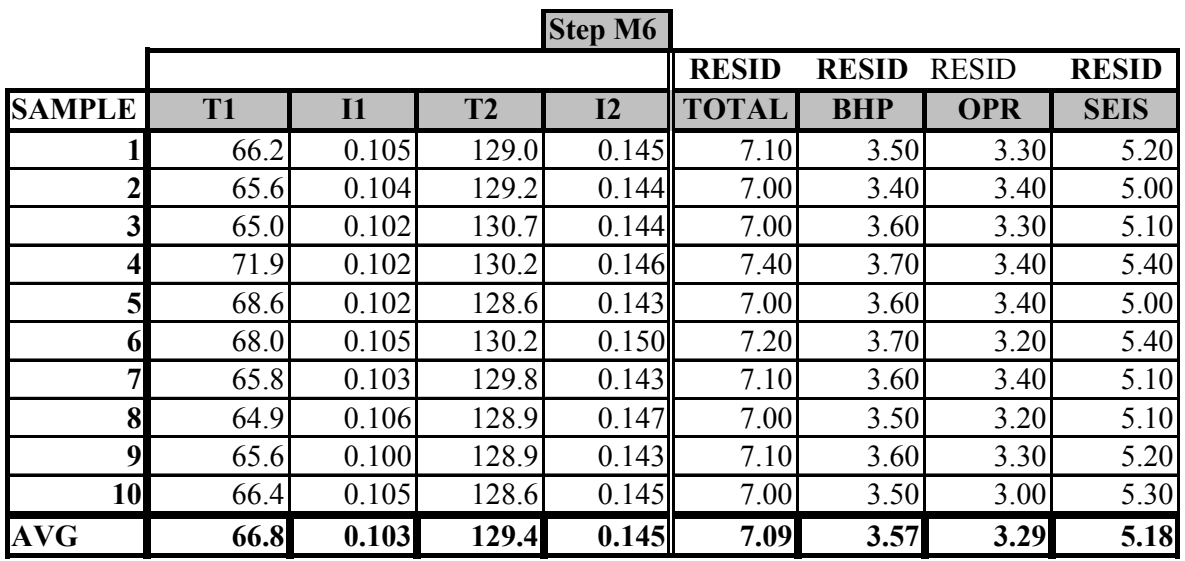


Sensitivity Coefficients (All Iterations)
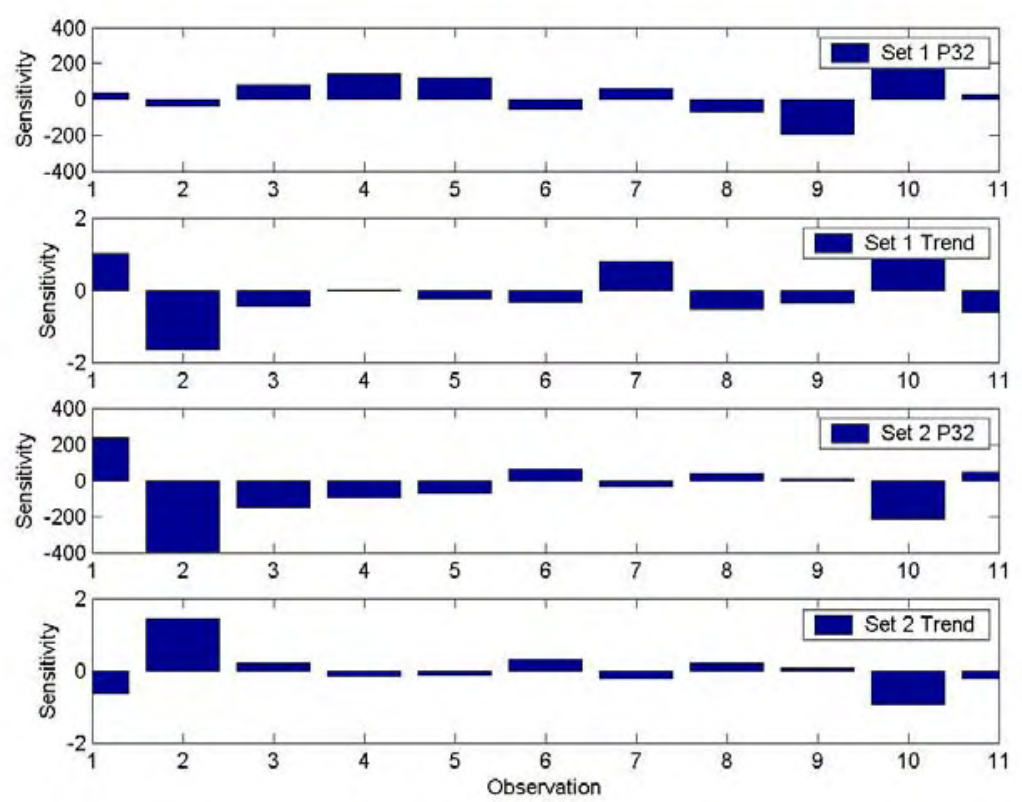

Fig. C.4a $\quad-\quad$ Sensitivity coefficients for iteration 1.
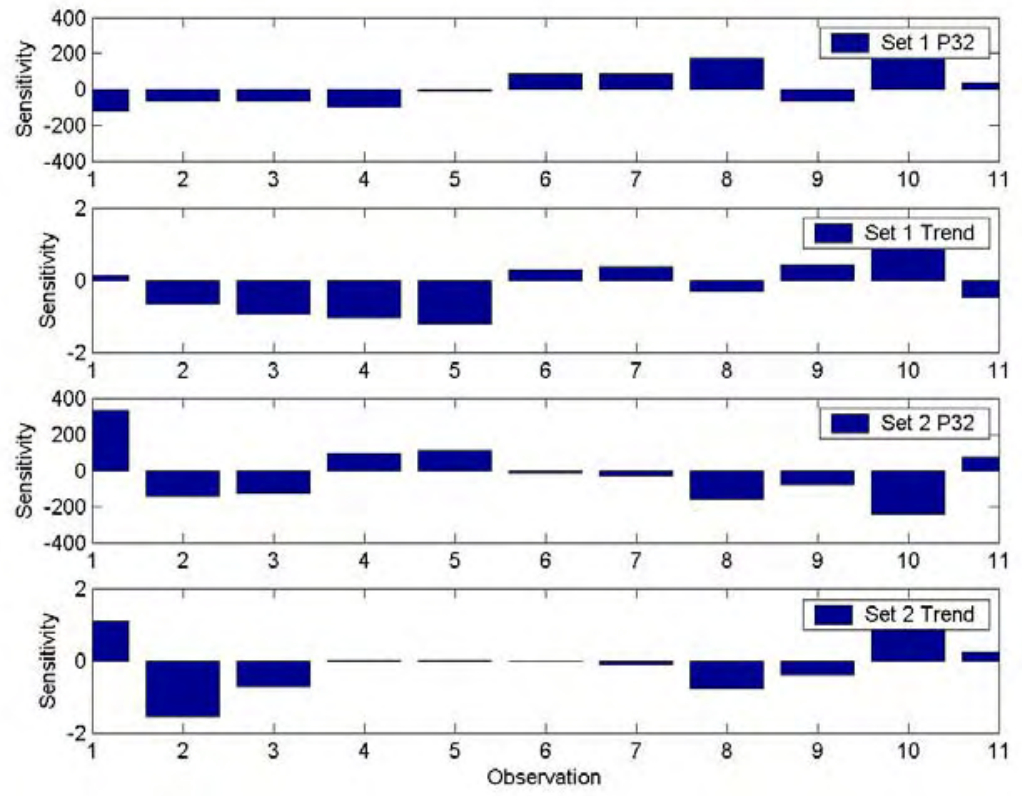

Fig. C.4b $\quad-\quad$ Sensitivity coefficients for iteration 2 . 

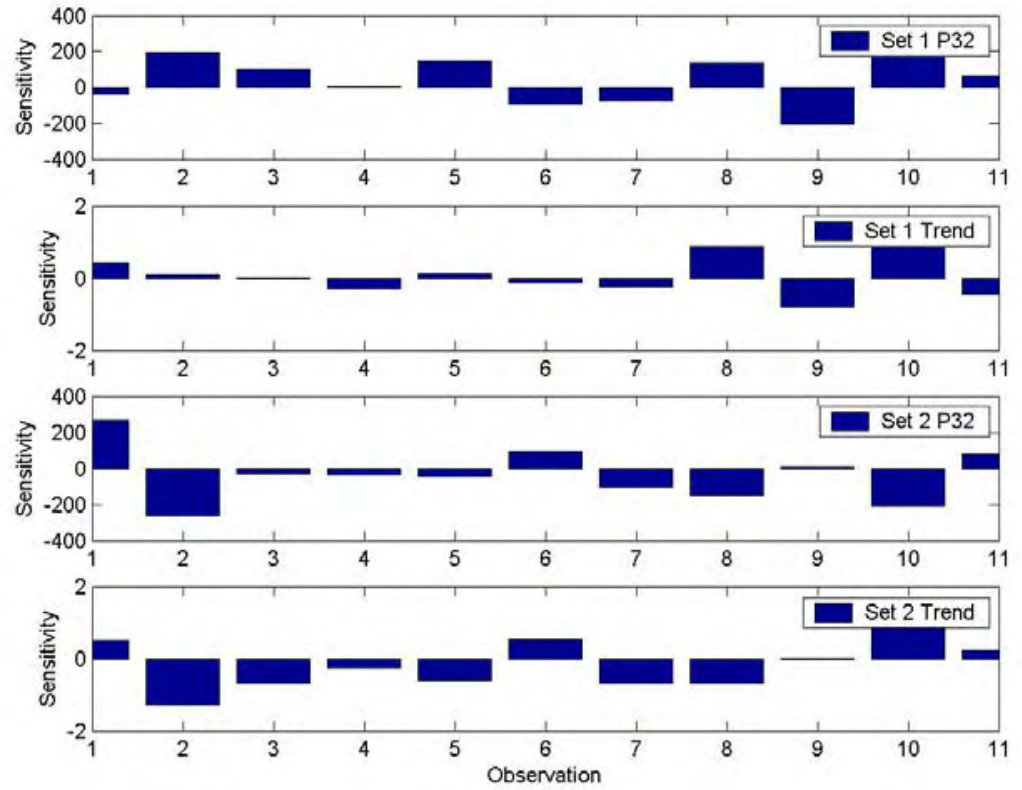

Fig. C.4c $\quad-\quad$ Sensitivity coefficients for iteration 3 .
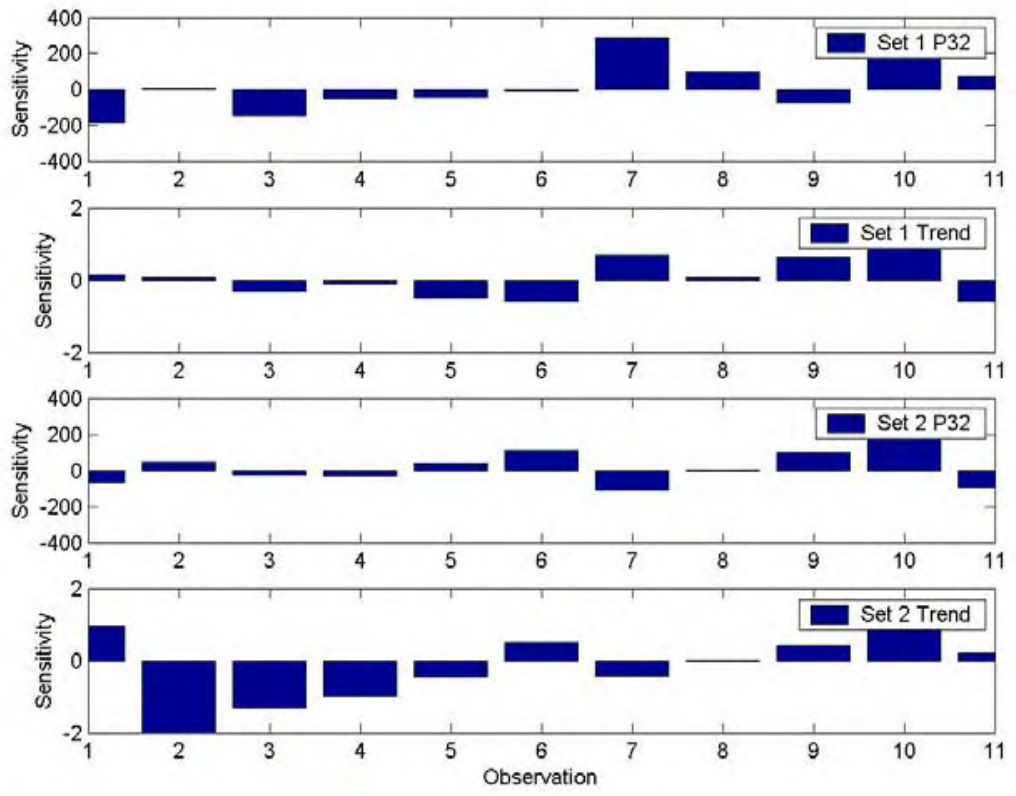

Fig. C.4d $\quad-\quad$ Sensitivity coefficients for iteration 4 . 

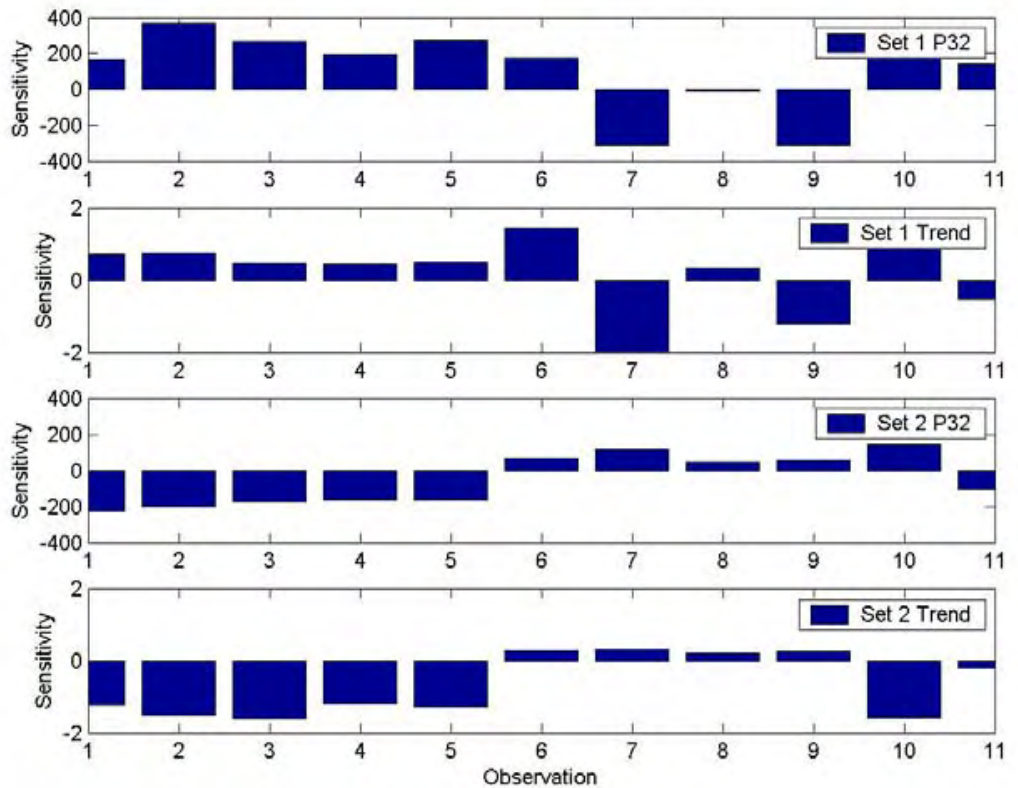

Fig. C.4e $\quad-\quad$ Sensitivity coefficients for iteration 5 .
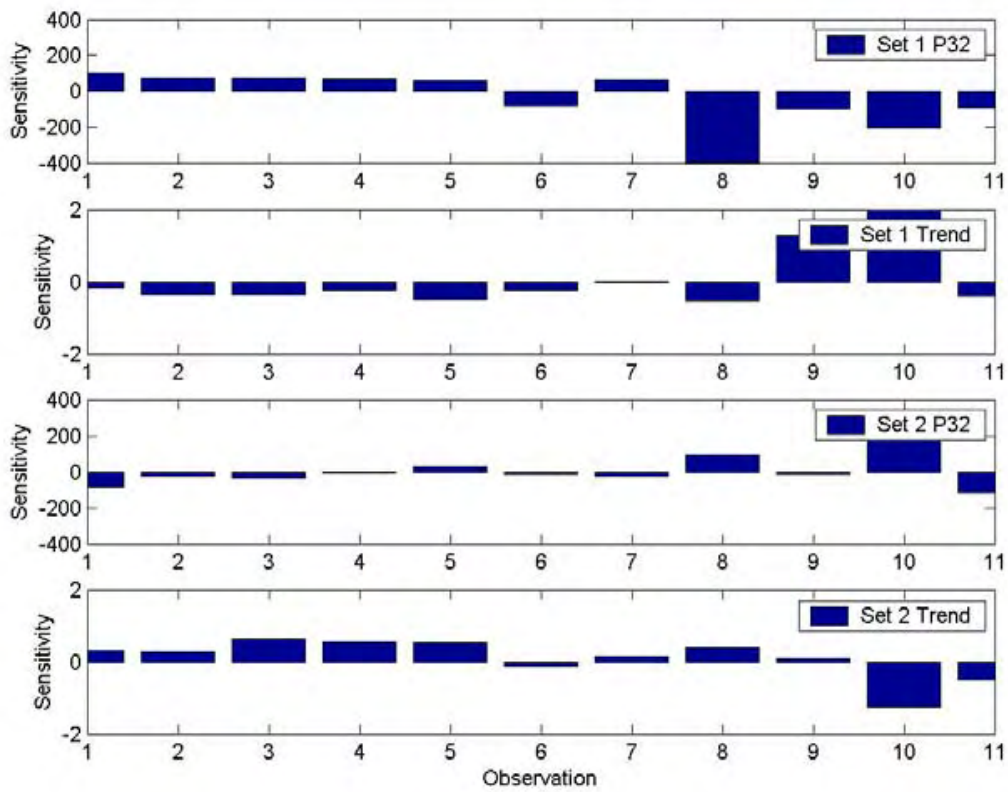

Fig. C.4f $\quad-\quad$ Sensitivity coefficients for iteration 6 . 
Fracture Distribution Maps, (Base, Initial, Final)

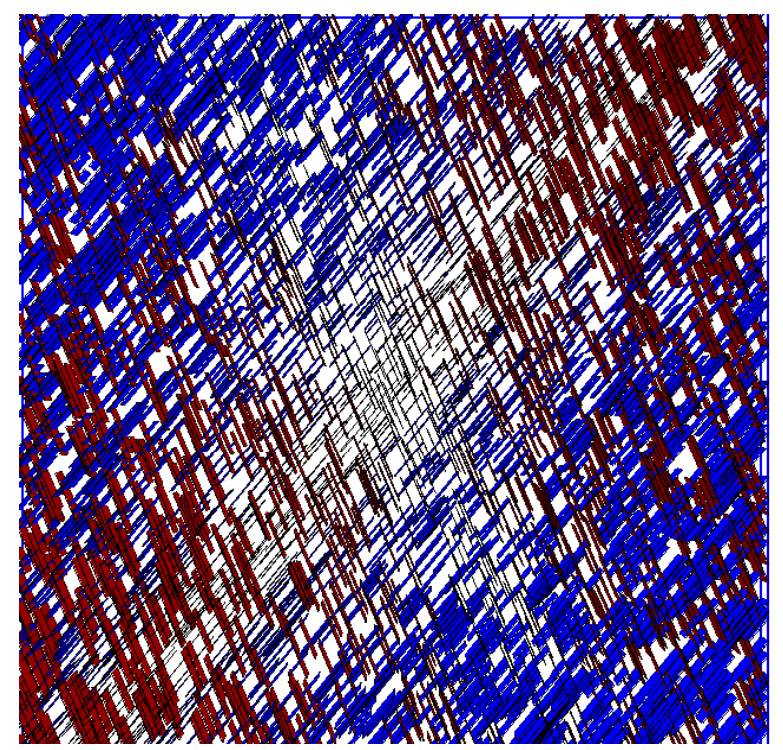

Fig. C.5a - Test T3 base model fracture distribution.

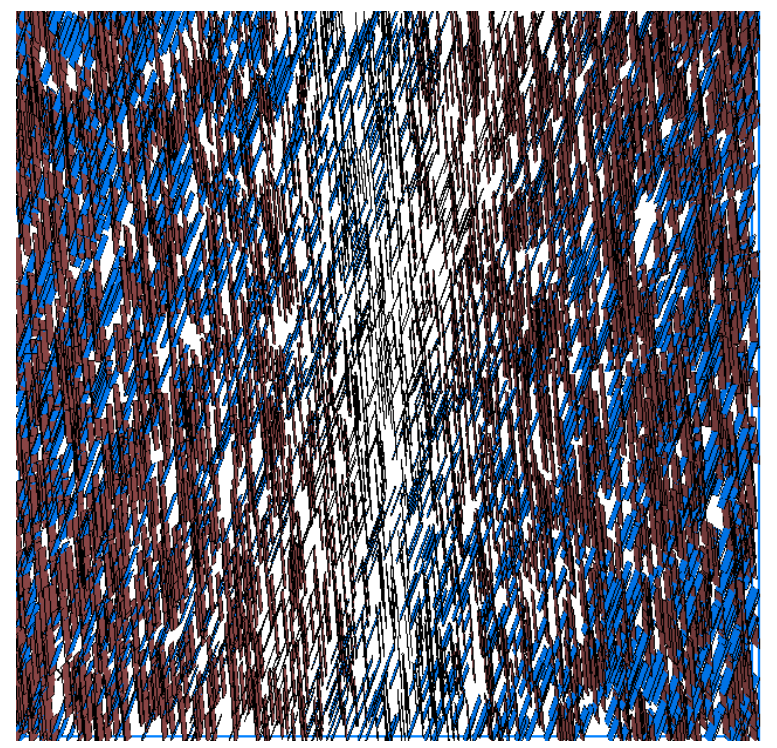

Fig. C.5b $\quad-\quad$ Test T3 initial estimate fracture distribution. 


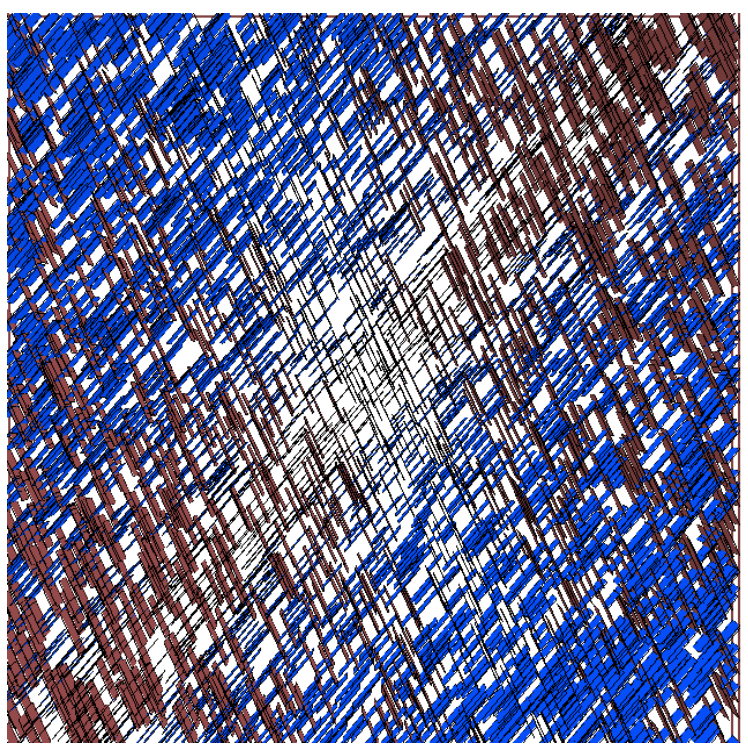

Fig.C.5c $\quad-\quad$ Test T3 final model fracture distribution.

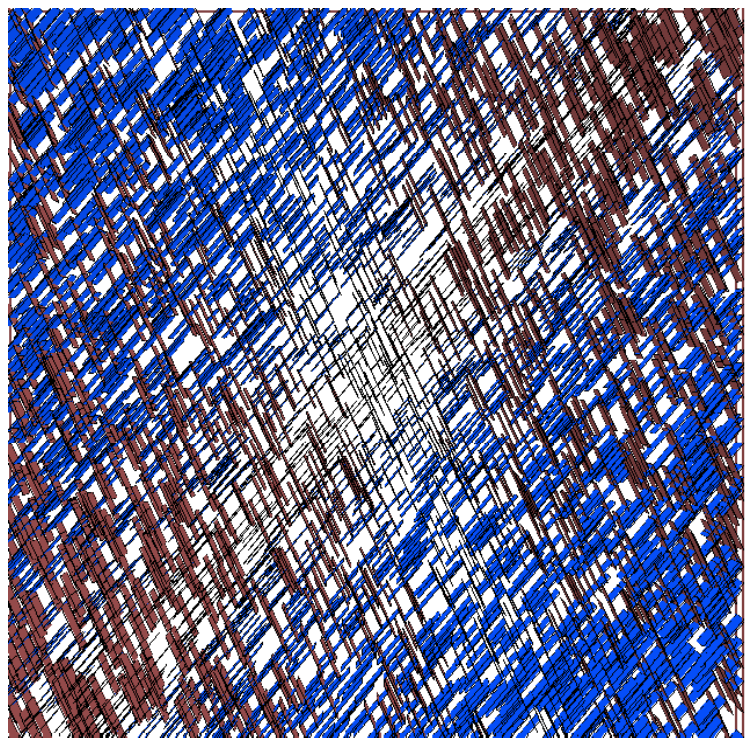

Fig. C.5d - Test T3 base model fracture distribution. 
Fracture Water Saturation Maps (Base, Initial, Final)

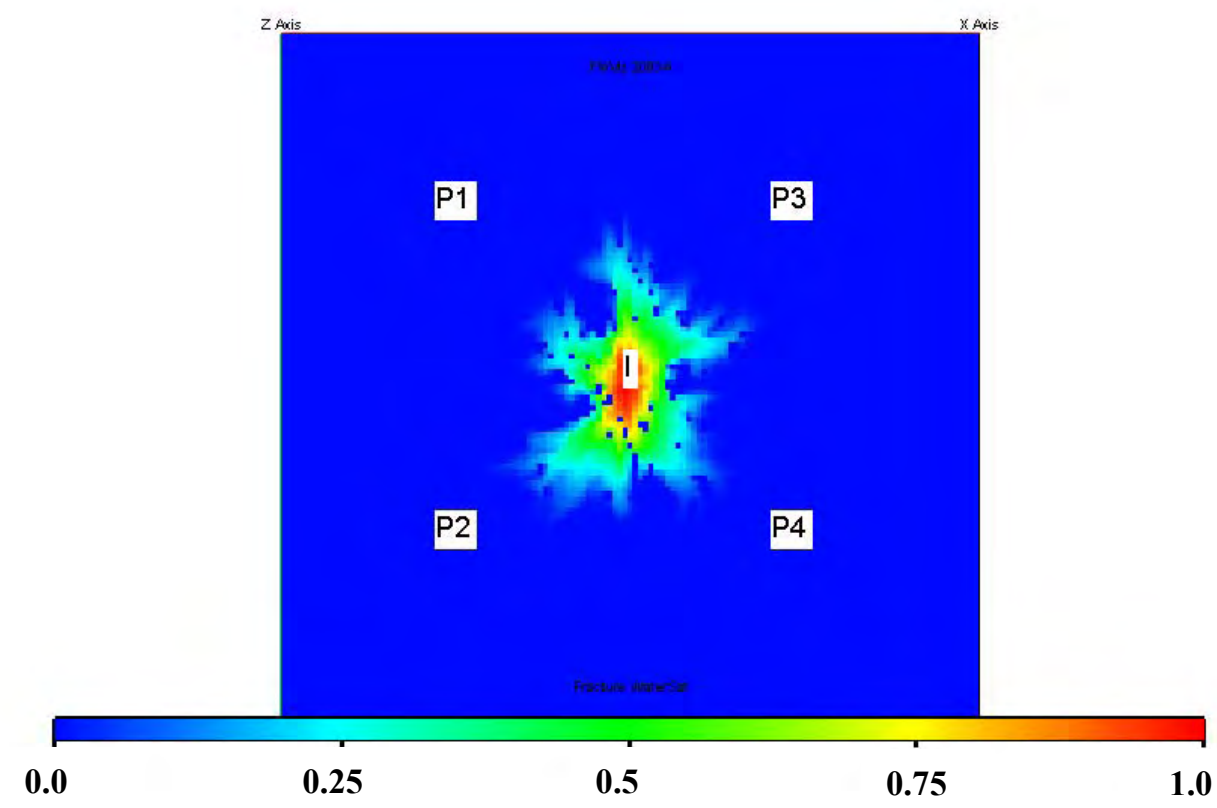

Fig. C.6a - Fracture water saturation at report step 5 (150 days) for test T3 base model.

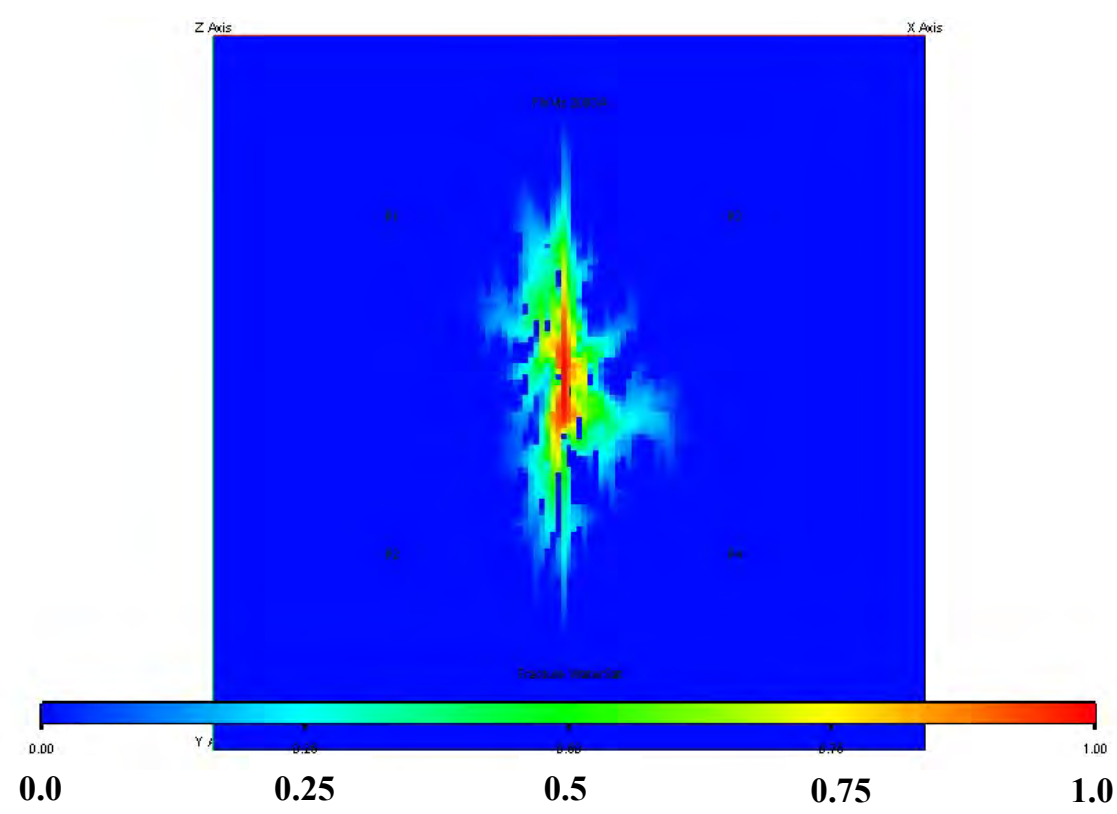

Fig. C.6b - Fracture water saturation at report step 5 (150 days) for test $\mathrm{T} 3$ initial estimate. 


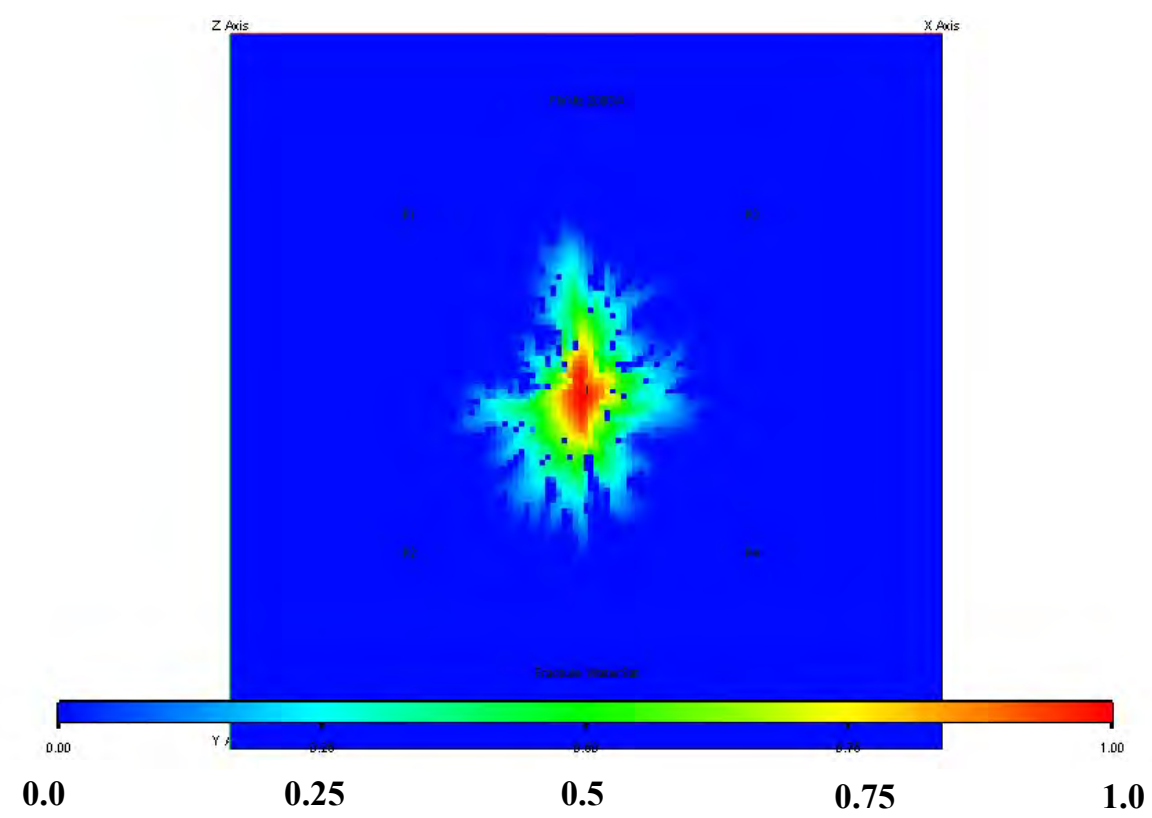

Fig. C.6c $\quad-\quad$ Fracture water saturation at report step 5 (150 days) for test T3 final model.

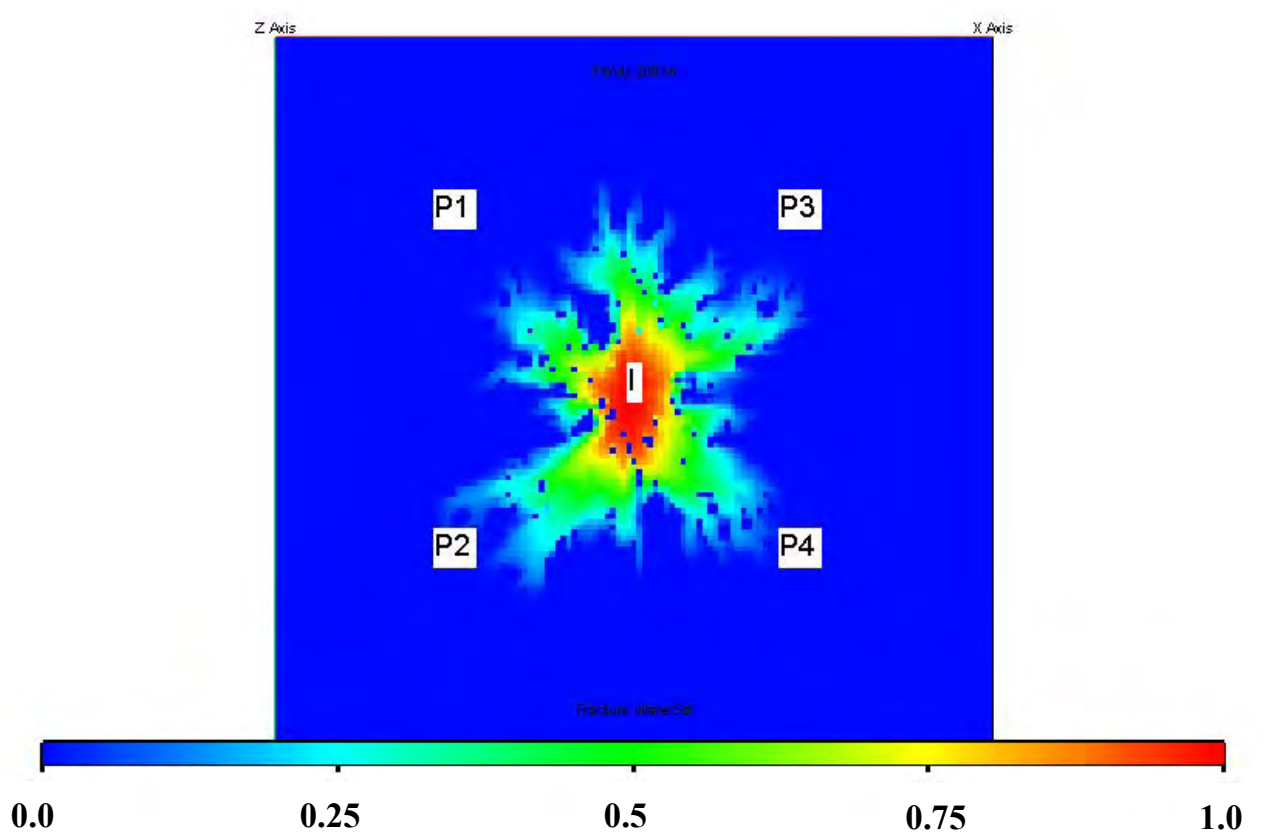

Fig. C.7a $\quad-\quad$ Fracture water saturation at report step $10(300$ days)for test T3 base model. 


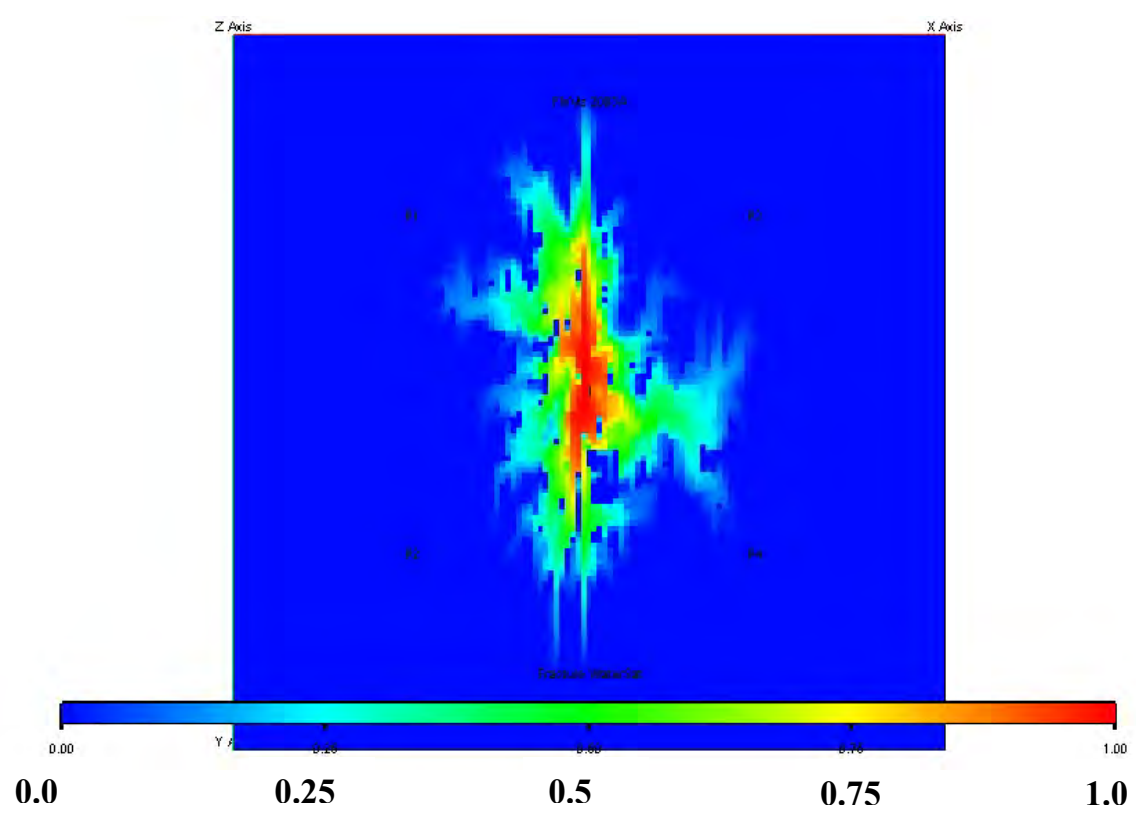

Fig. C.7b $\quad-\quad$ Fracture water saturation at report step 10 (300 days) for test T3 initial estimate.

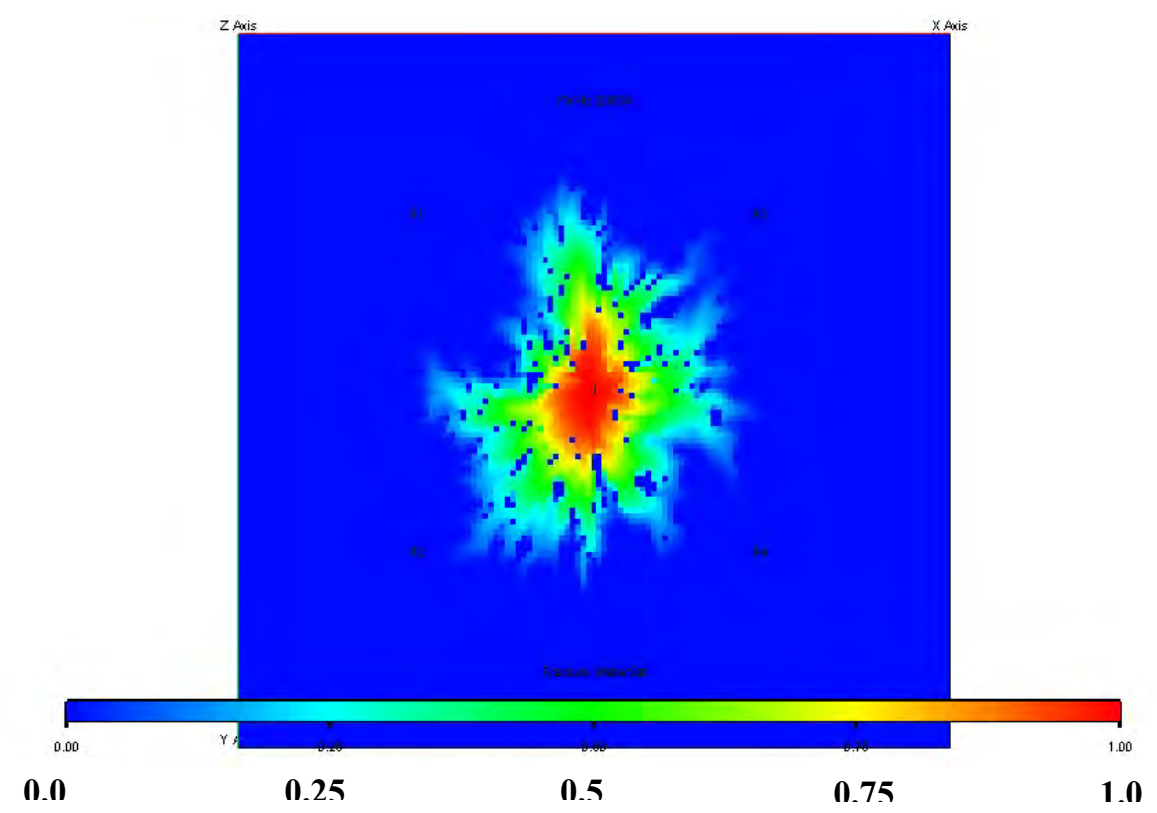

Fig. C.7c $\quad-\quad$ Fracture water saturation at report step 10 (300 days) for test T3 final model. 


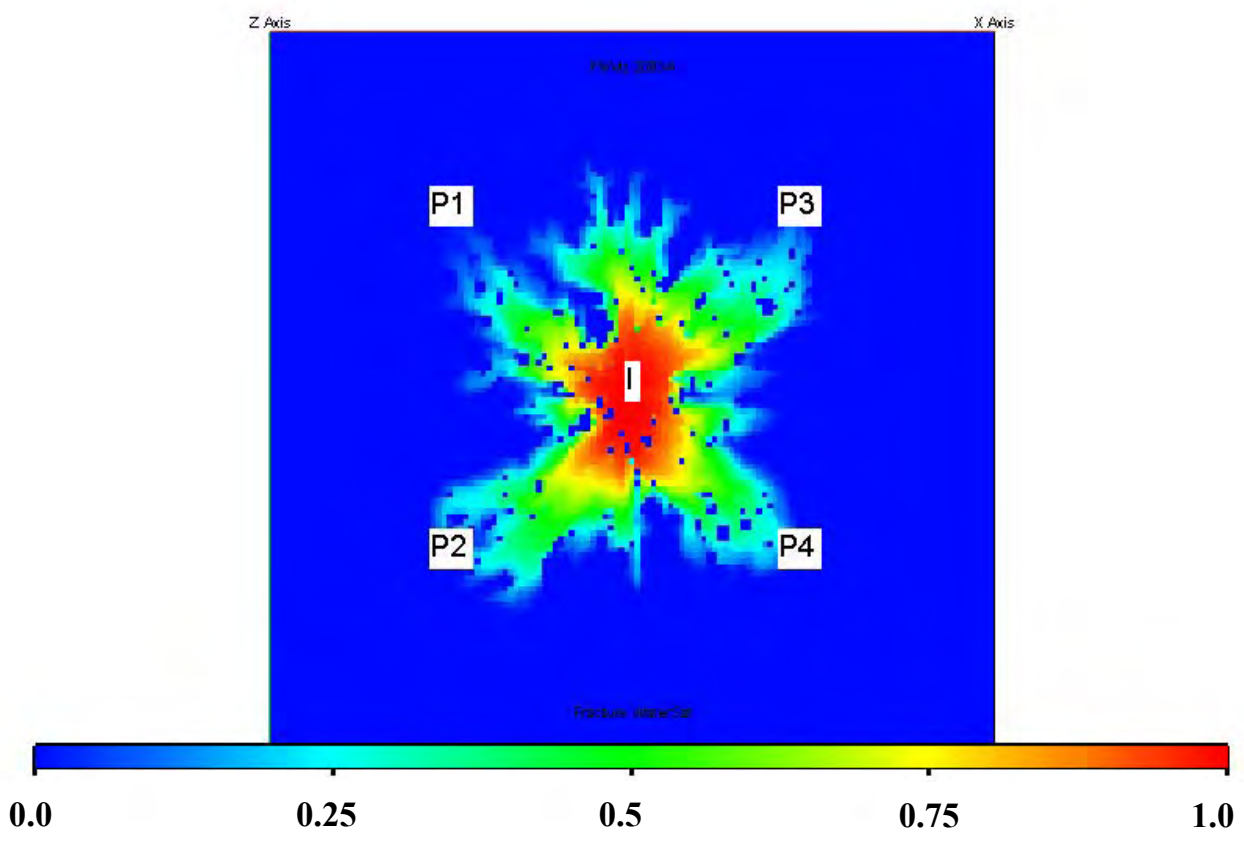

Fig. C.8a $\quad-\quad$ Fracture water saturation at report step 15 (450 days) for test $\mathrm{T} 3$ base model.

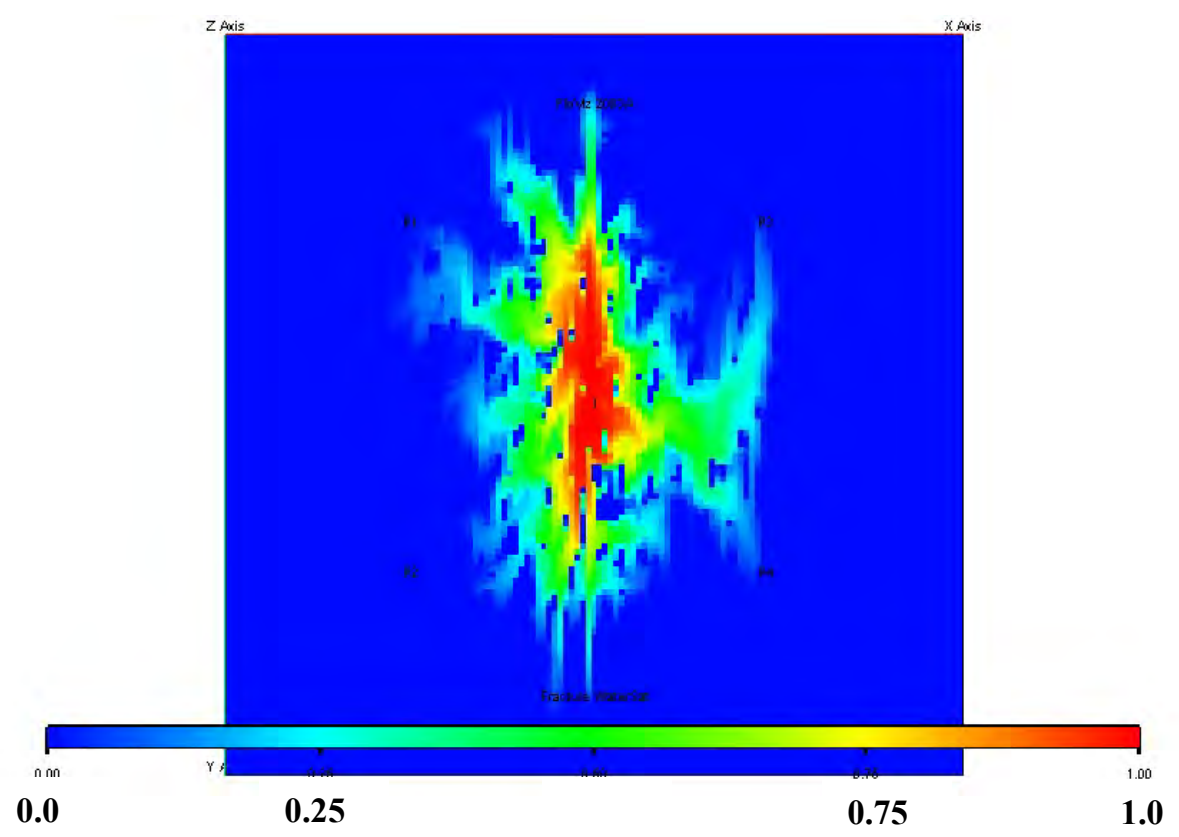

Fig. C.8b - Fracture water saturation at report step 15 (450 days) for test $\mathrm{T} 3$ initial estimate. 


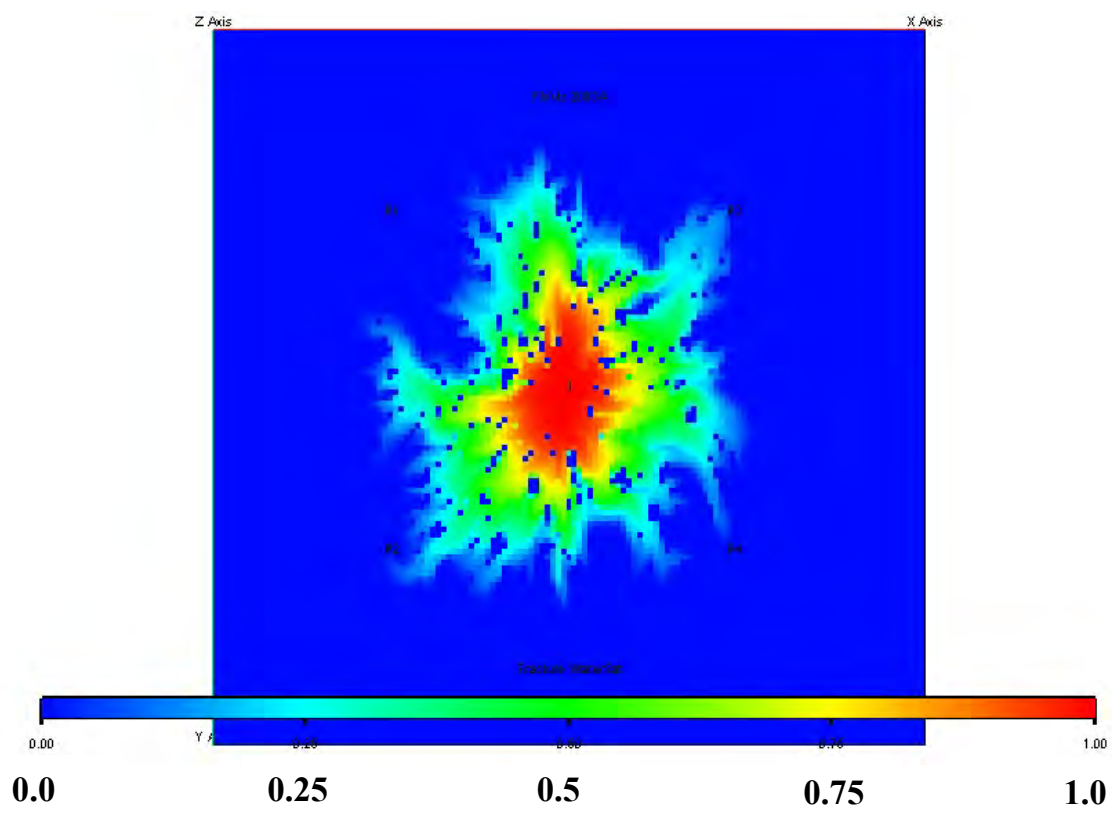

Fig. C.8c $\quad-\quad$ Fracture water saturation at report step 15 (450 days) for test T3 final model.

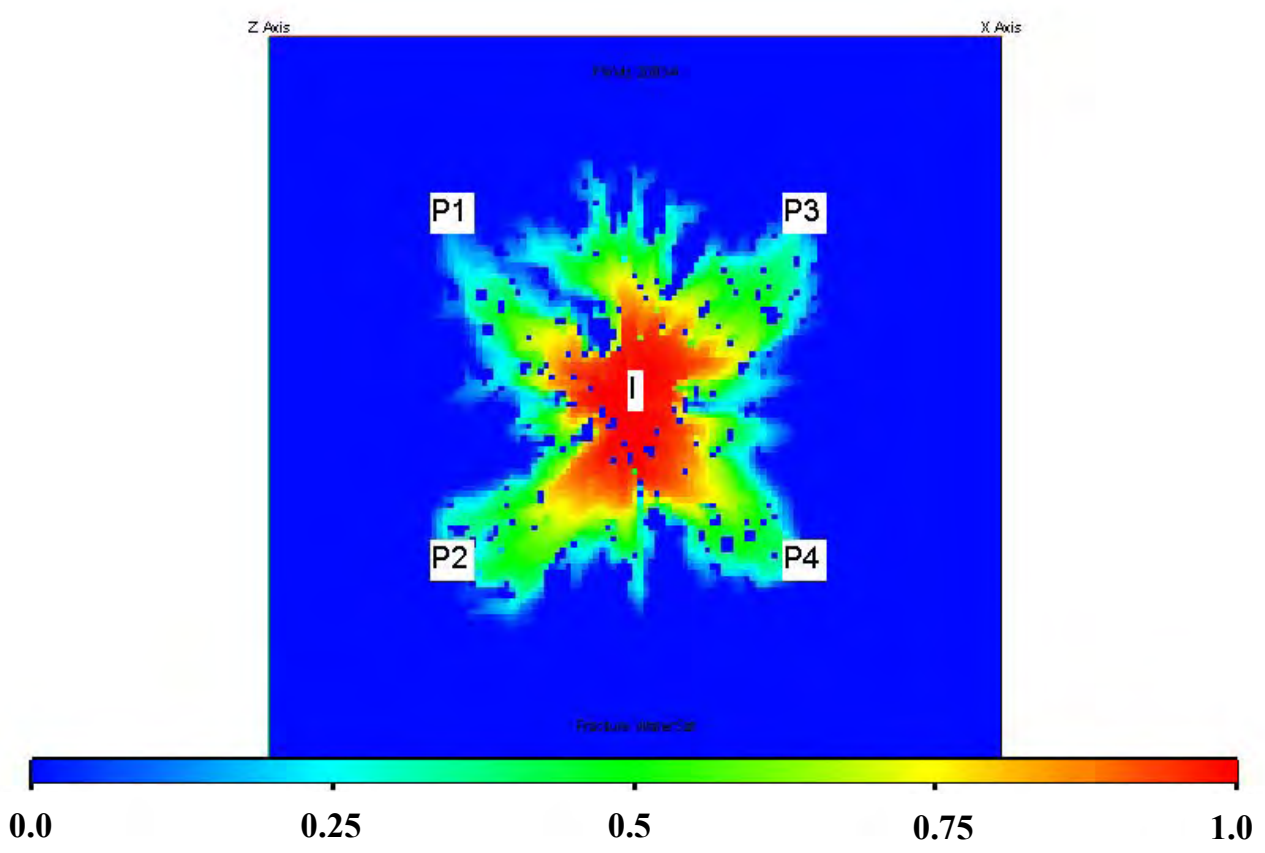

Fig. C.9a $\quad-\quad$ Fracture water saturation at report step 20 (600 days) for test T3 base model. 


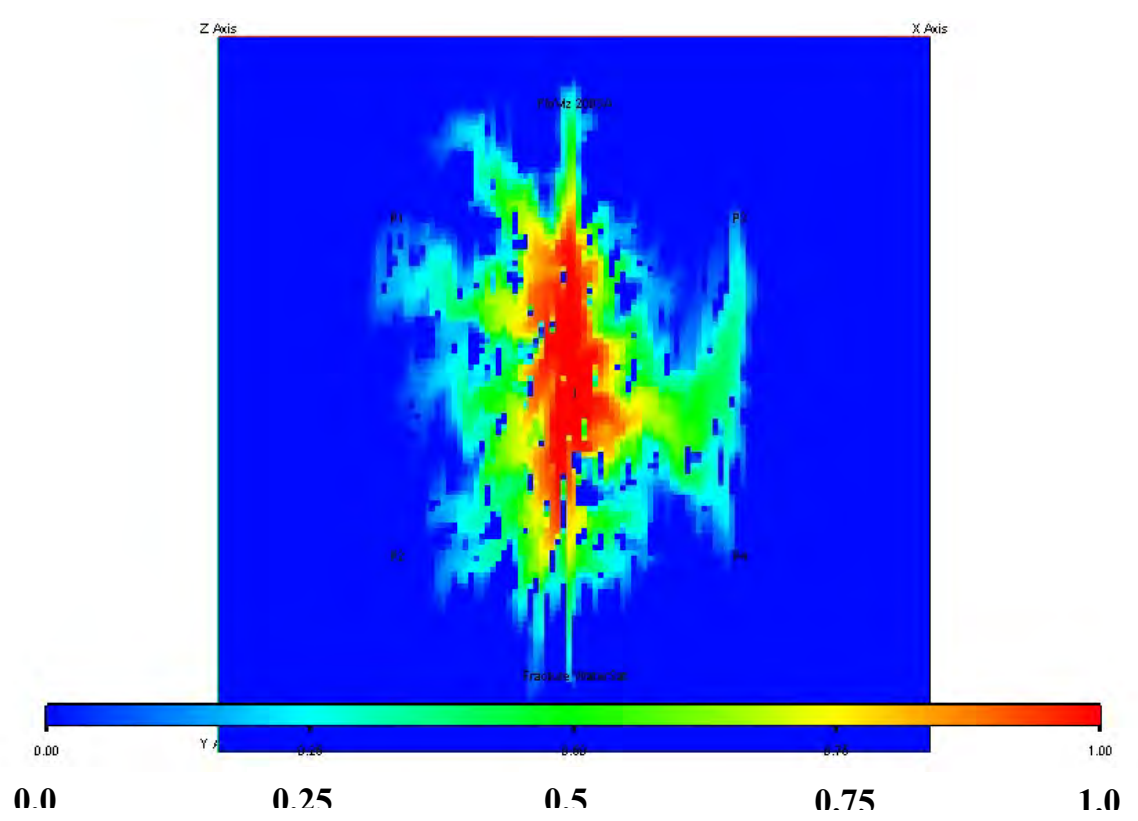

Fig. C.9b - Fracture water saturation at report step 20 (600 days) for test $\mathrm{T} 3$ initial estimate.

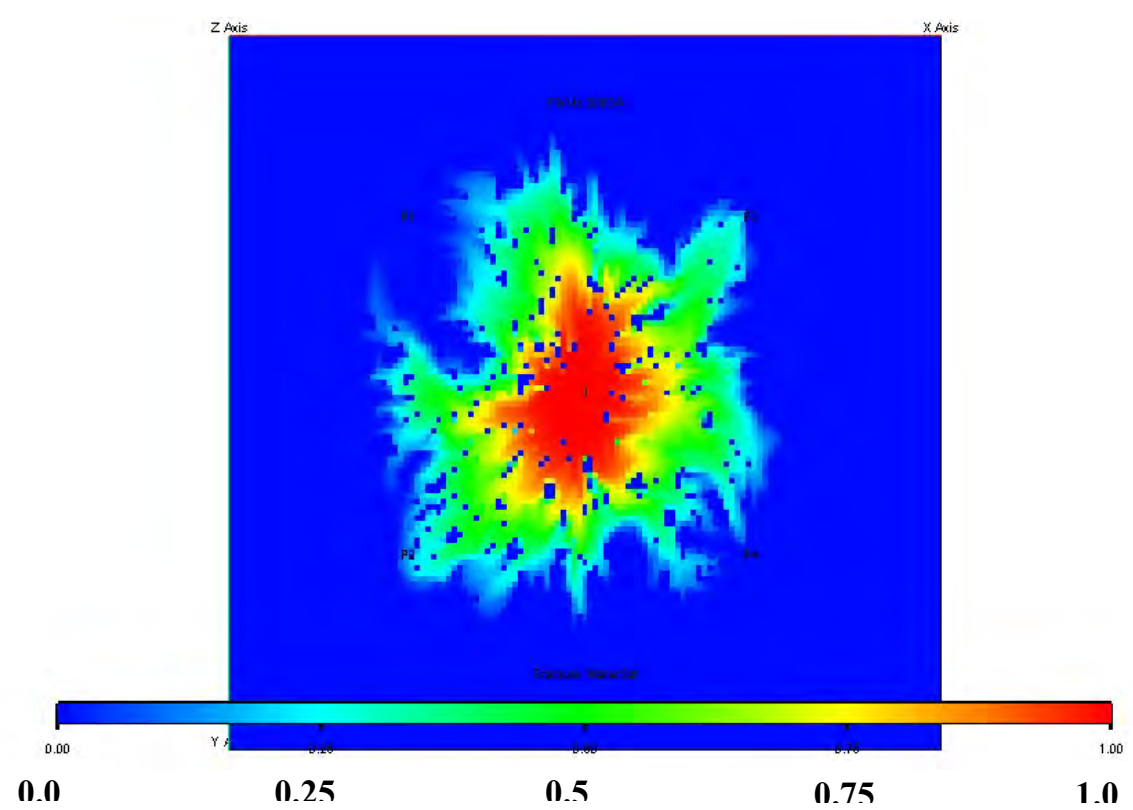

Fig. C.9c - Fracture water saturation at report step 20 (600 days) for test $\mathrm{T} 3$ final model. 
Matrix Water Saturation Maps (Base, Initial, Final)

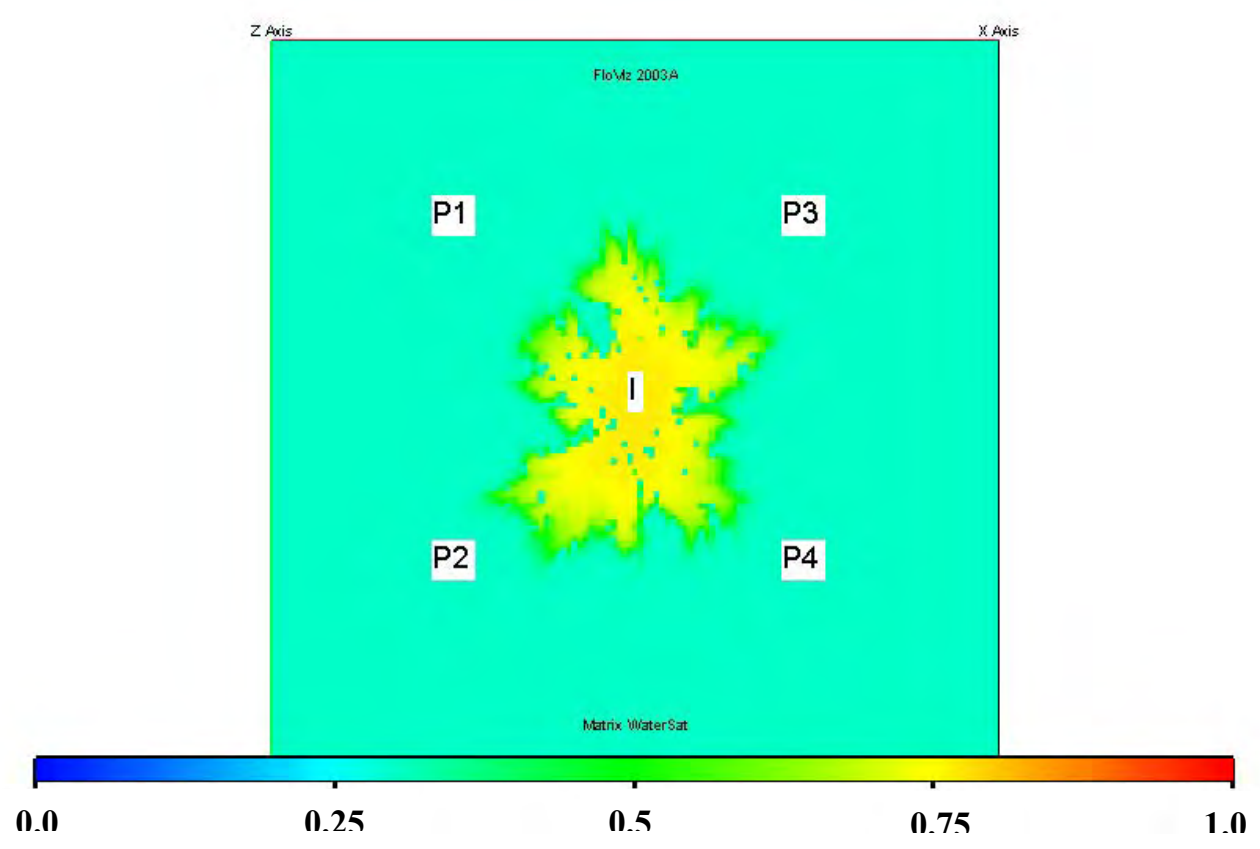

Fig. C.10a - Matrix water saturation at report step 5 (150 days) for test T3 base model.

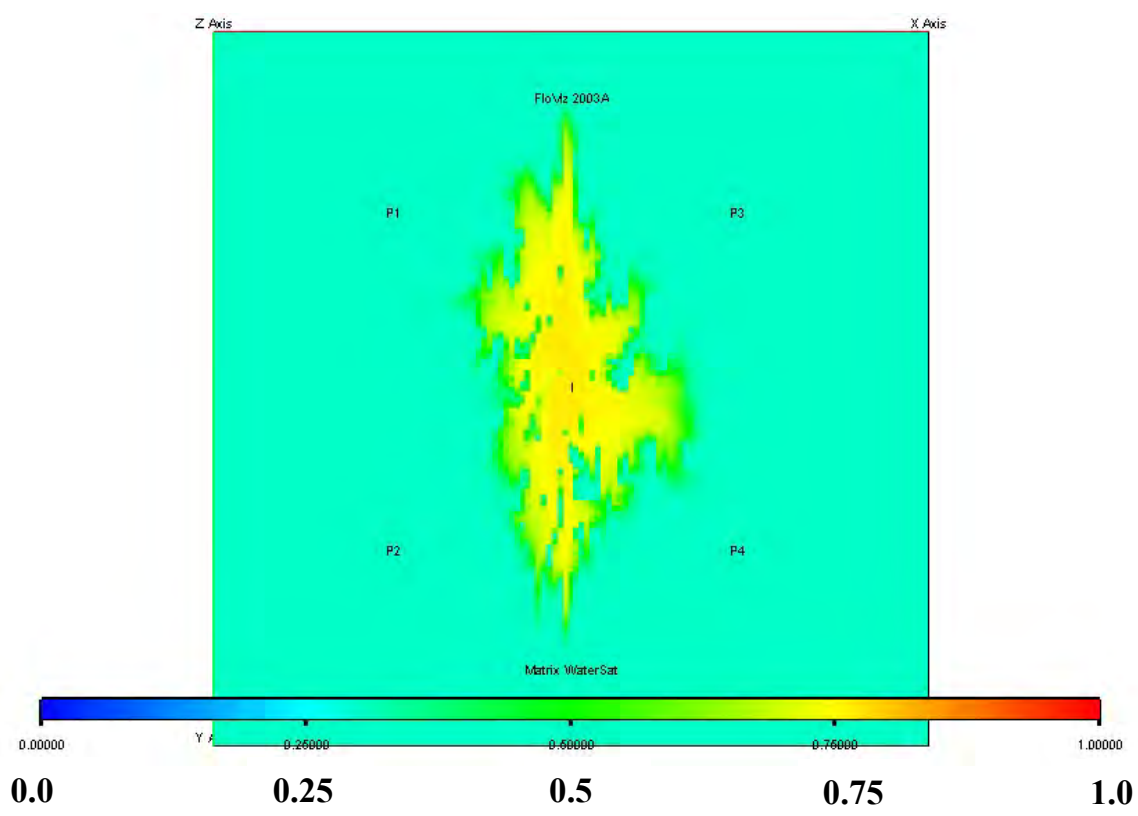

Fig. C.10b - Matrix water saturation at report step 5 (150 days) for test T3 initial estimate. 


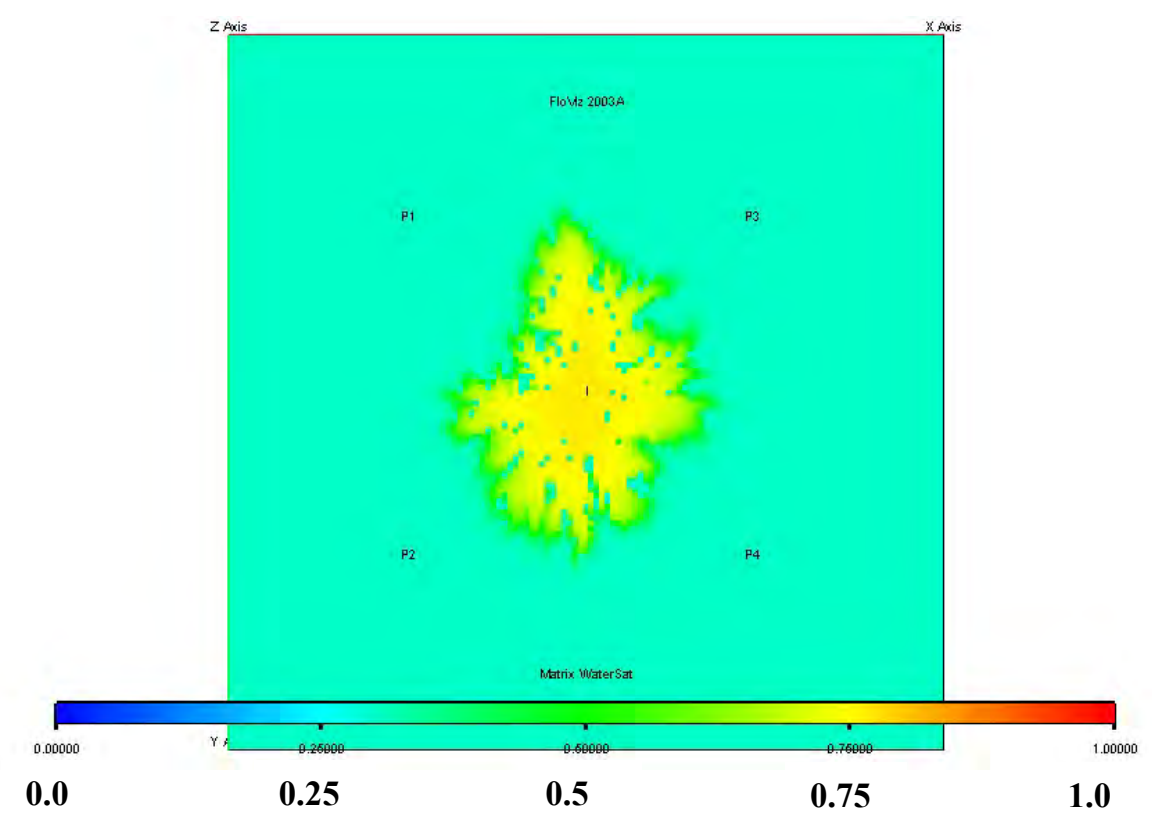

Fig. C.10c - Matrix water saturation at report step 5 (150 days) for test T3 final model.

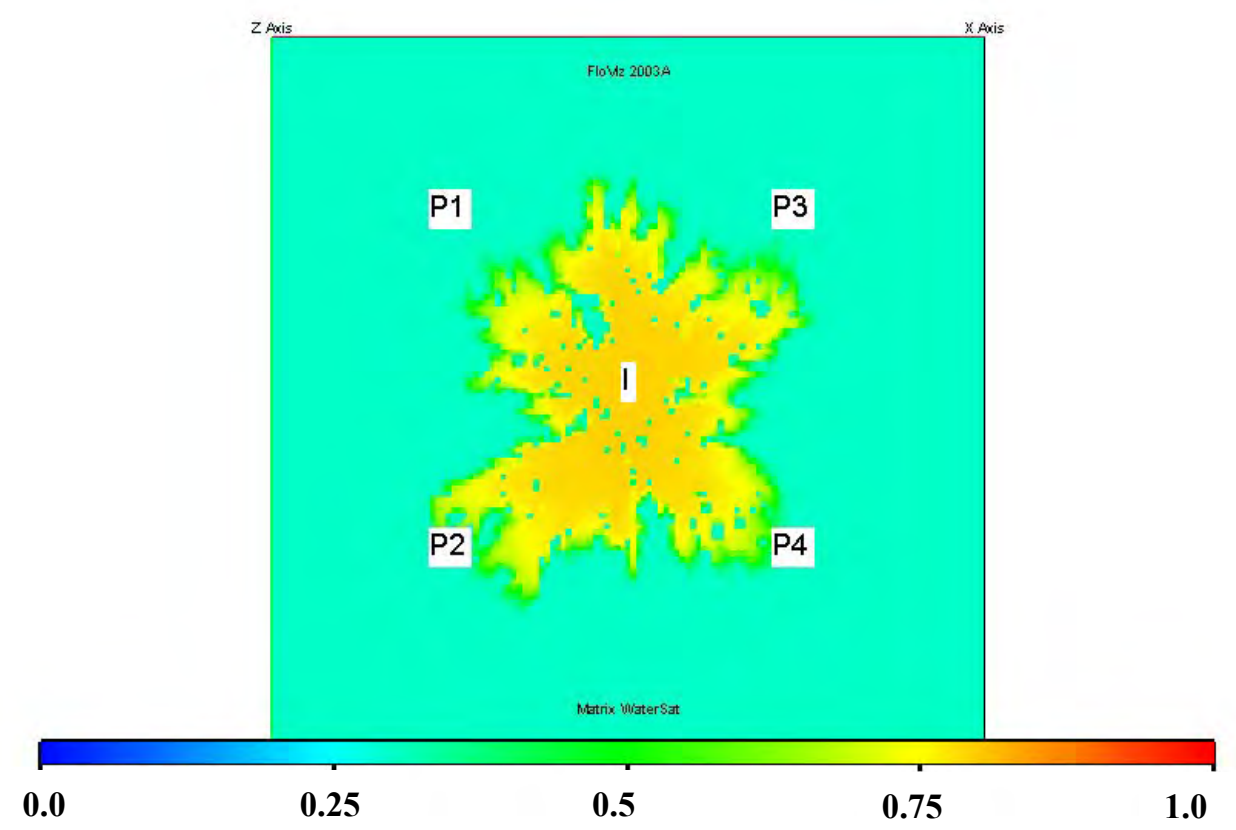

Fig. C.11a - Matrix water saturation at report step 10 (300 days) for test T3 base model. 


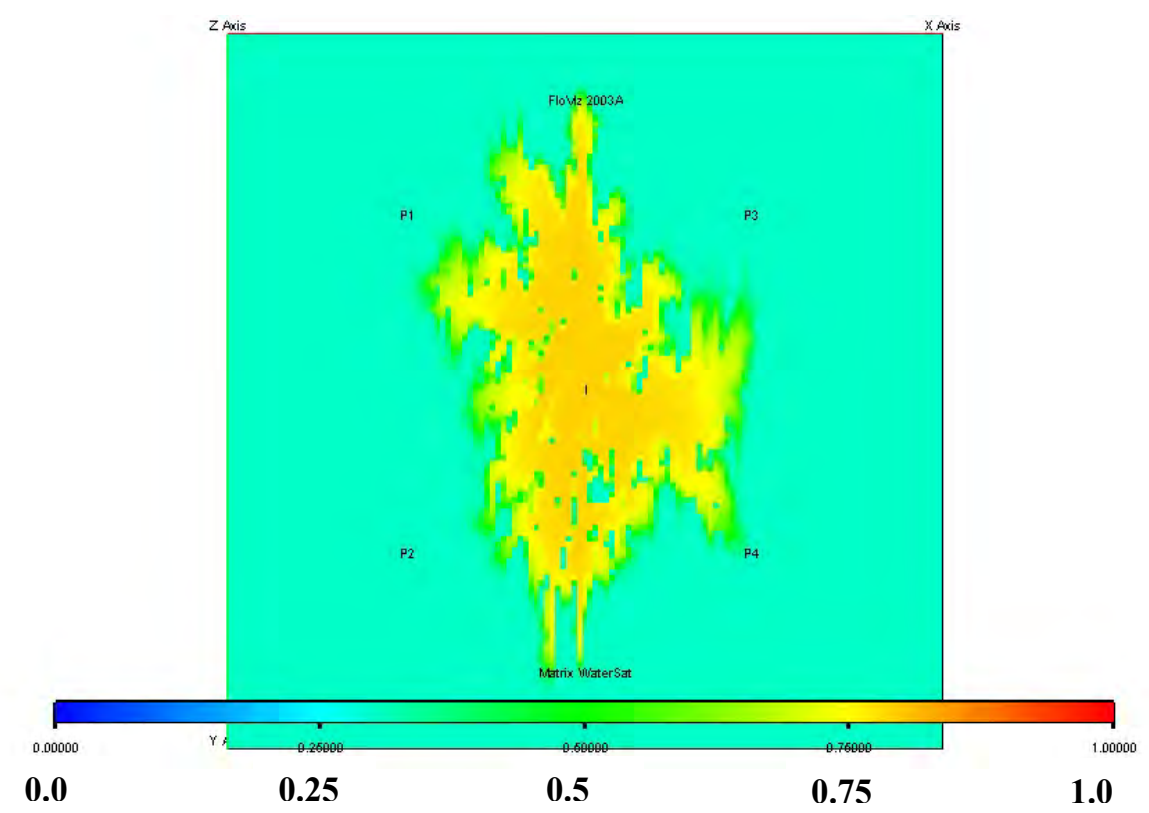

Fig. C.11b - Matrix water saturation at report step 10 (300 days) for test T3 initial estimate.

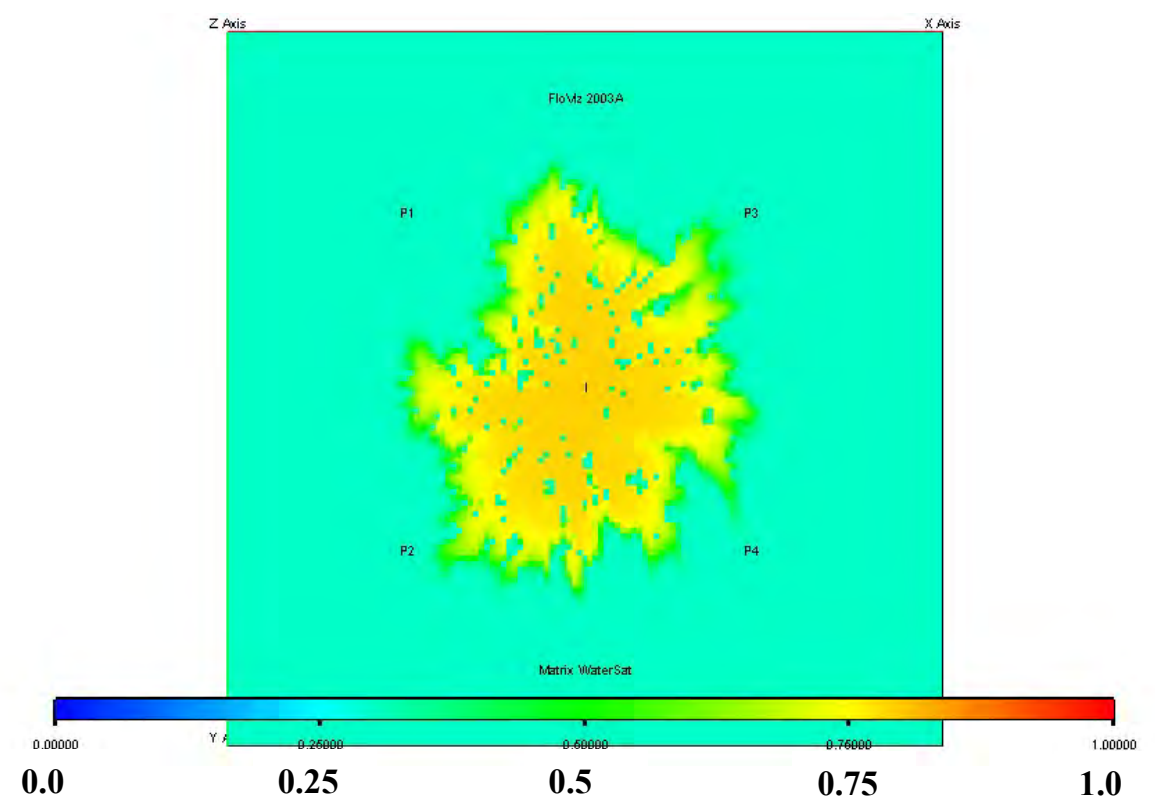

Fig. C.11c - Matrix water saturation at report step 10 (300 days) for test T3 final model. 


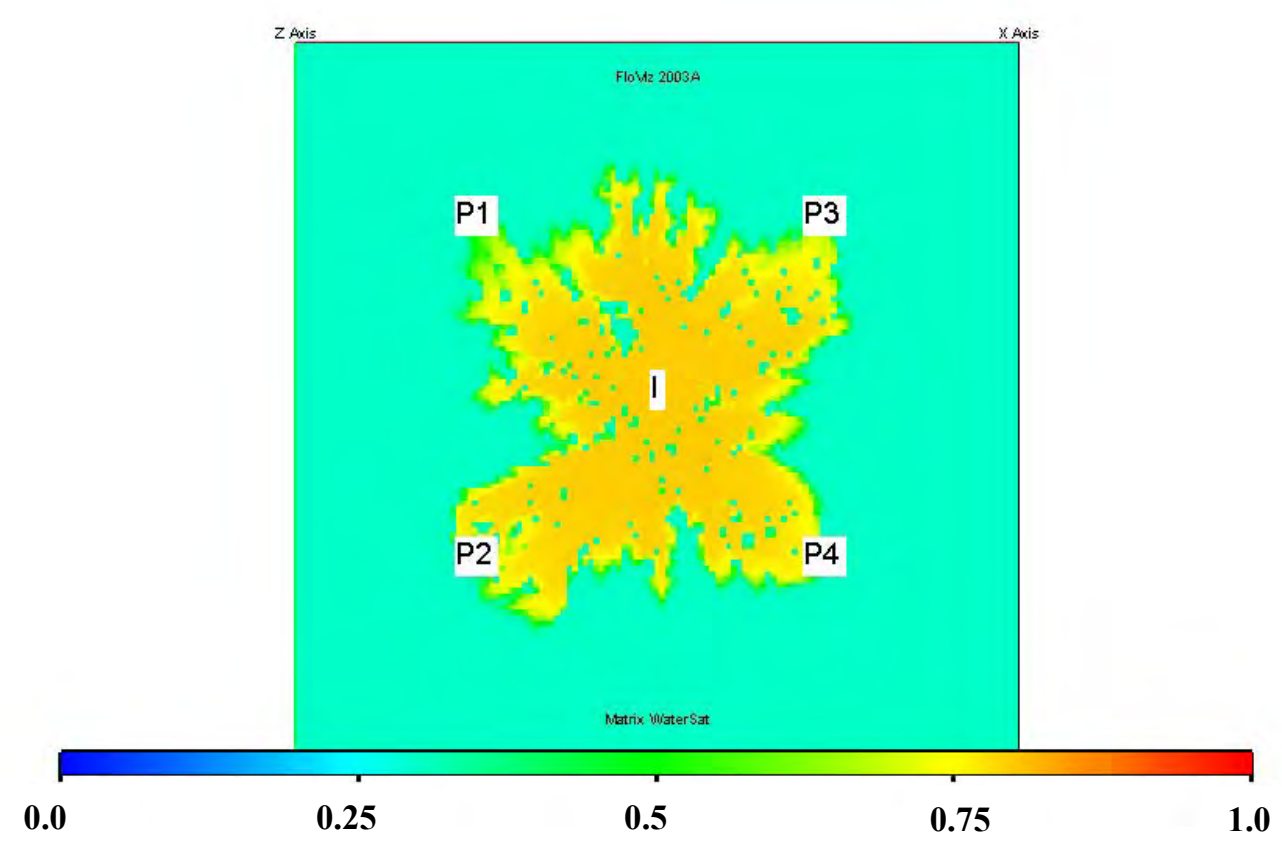

Fig. C.12a - Matrix water saturation at report step 15 (450 days) for test T3 base model.

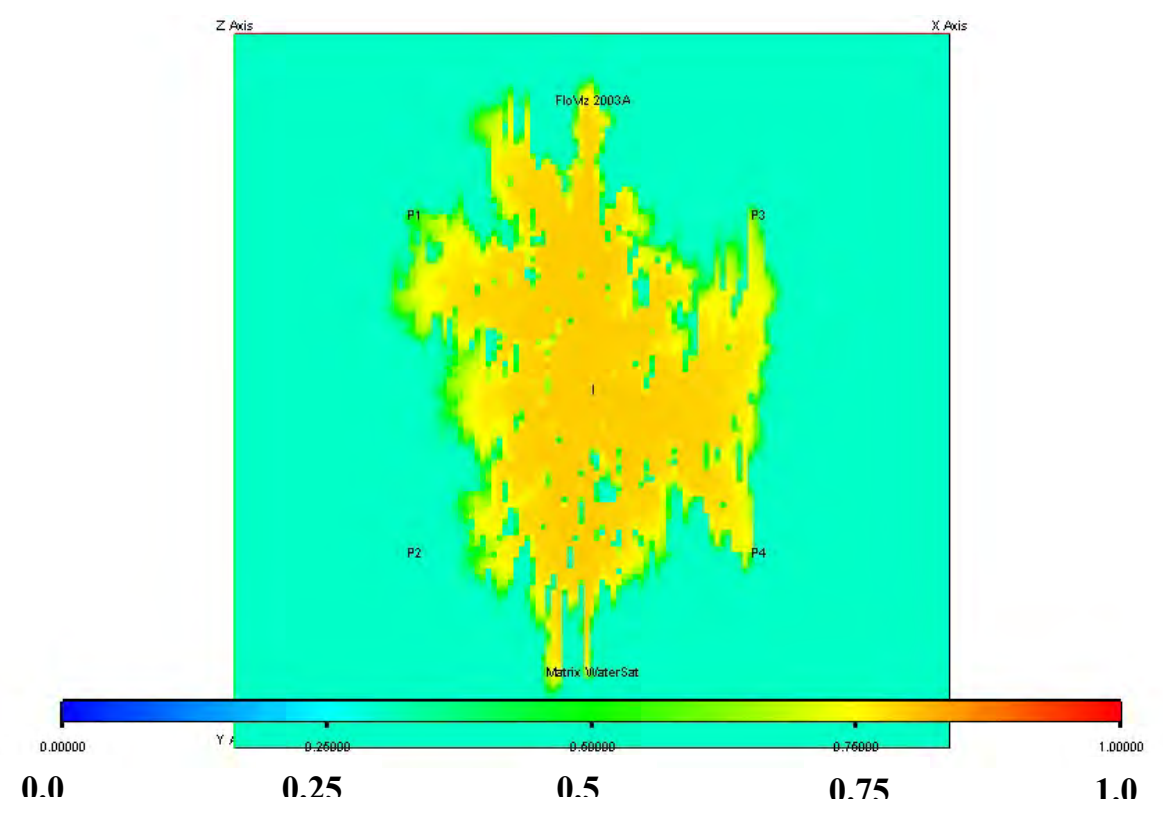

Fig. C.12b - Matrix water saturation at report step 15 (450 days) for test $\mathrm{T} 3$ initial estimate. 


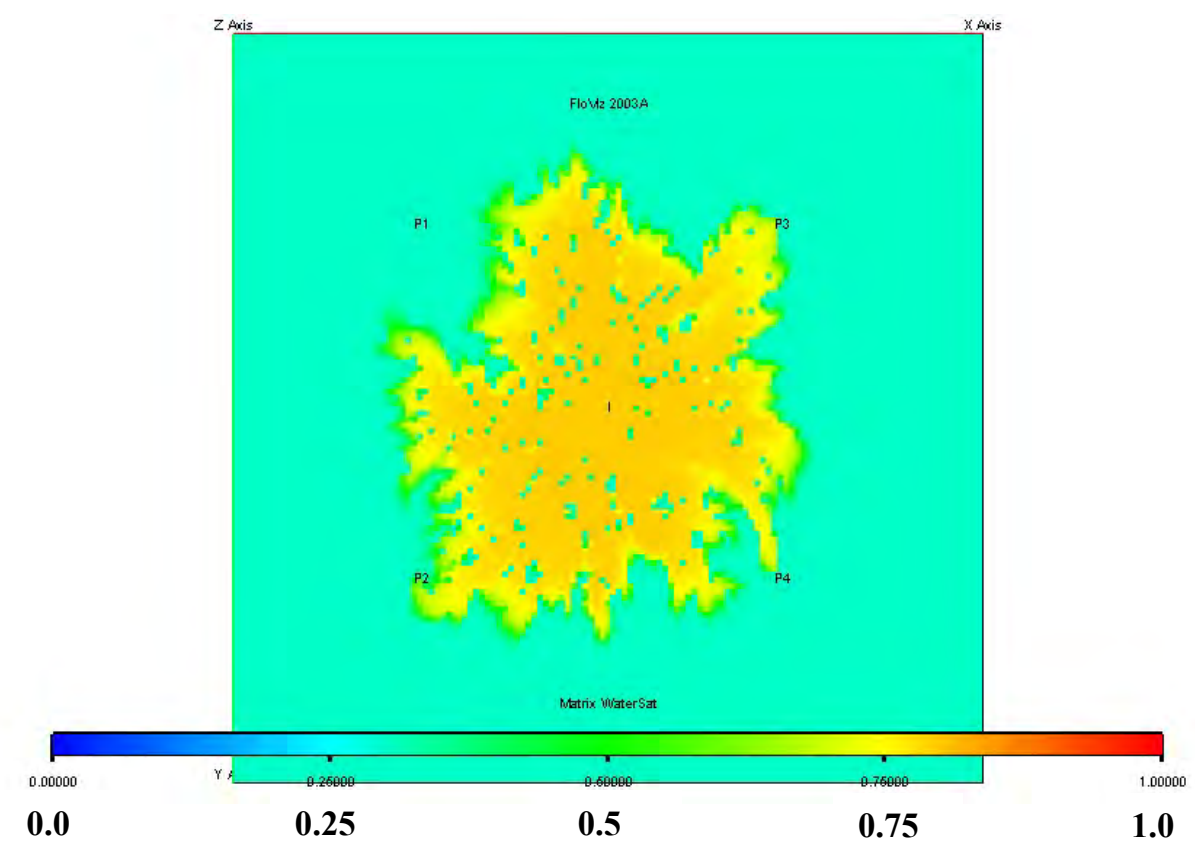

Fig. C.12c - Matrix water saturation at report step 15 (450 days) for test T3 final model.

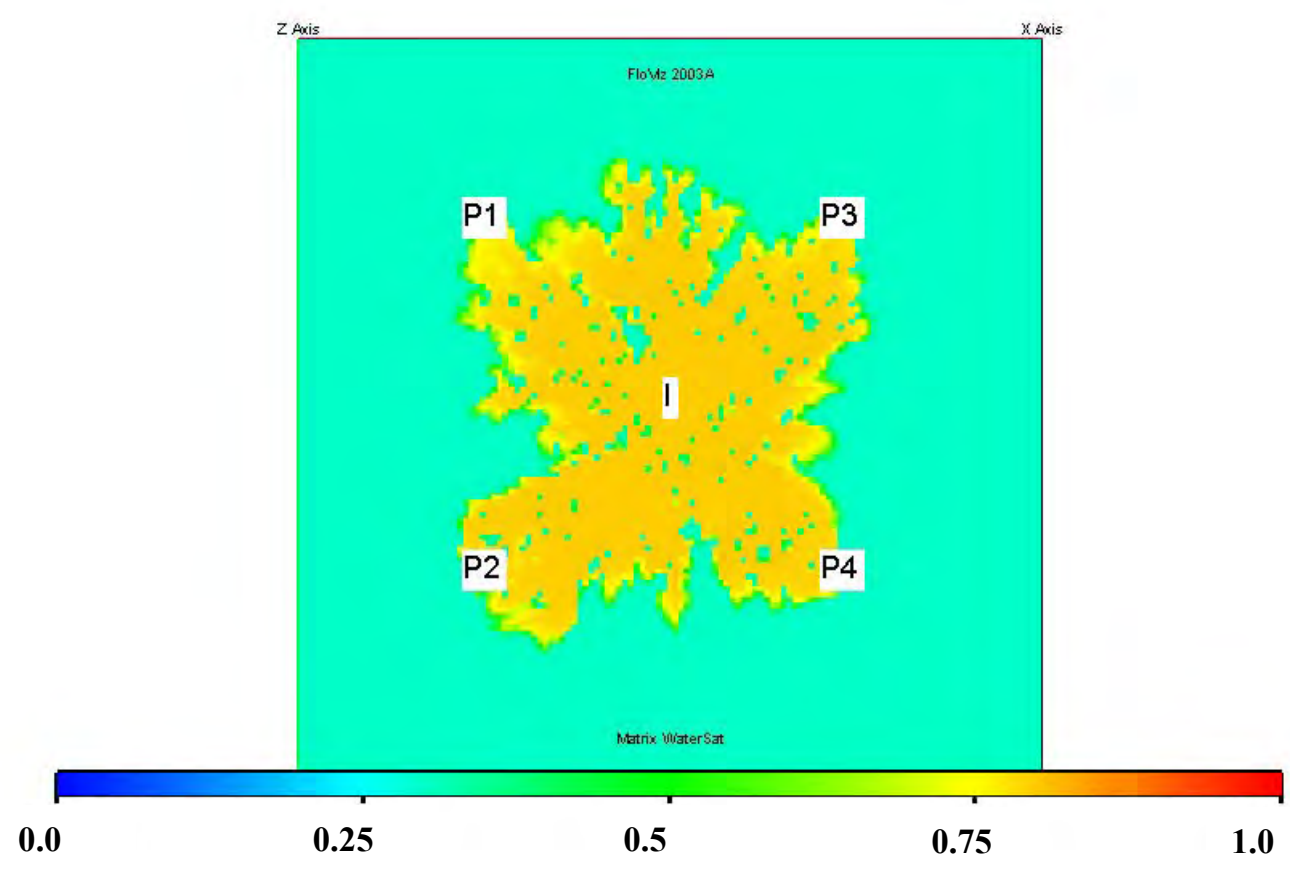

Fig. C.13a - Matrix water saturation at report step 20 (600 days) for test T3 base model. 


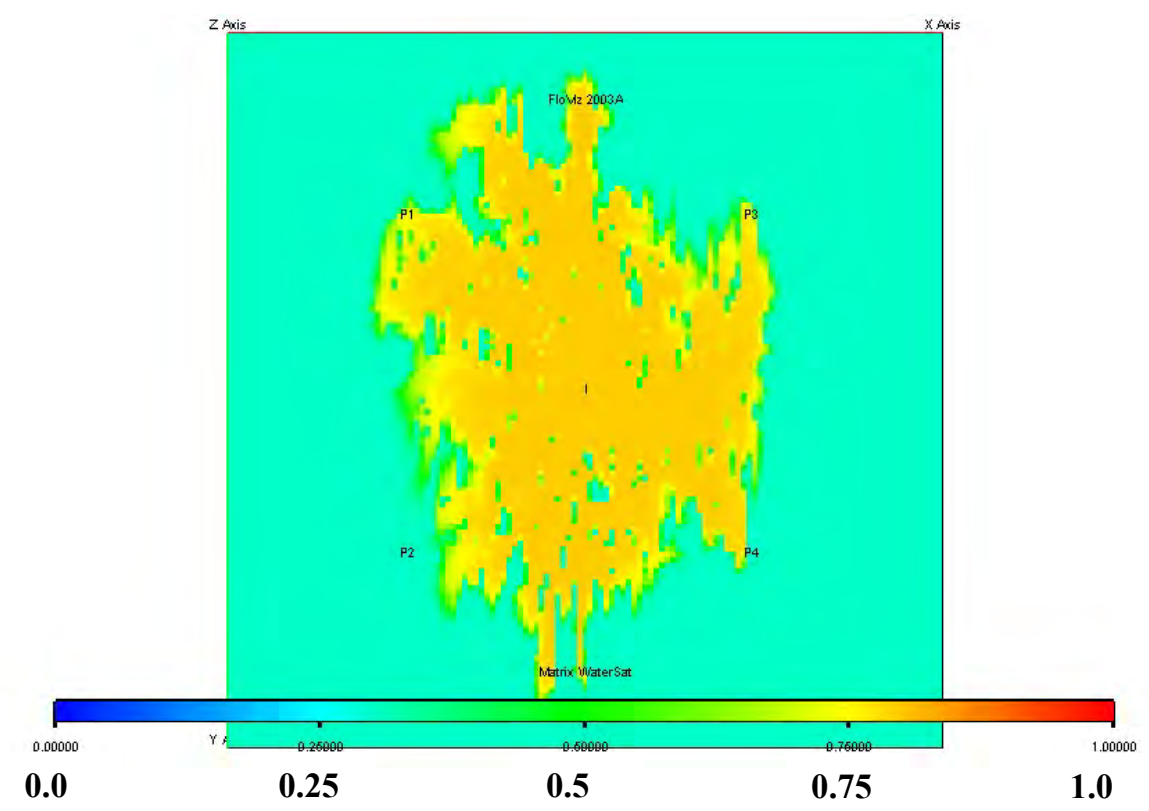

Fig. C.13b - Matrix water saturation at report step 20 (600 days) for test T3 initial estimate.

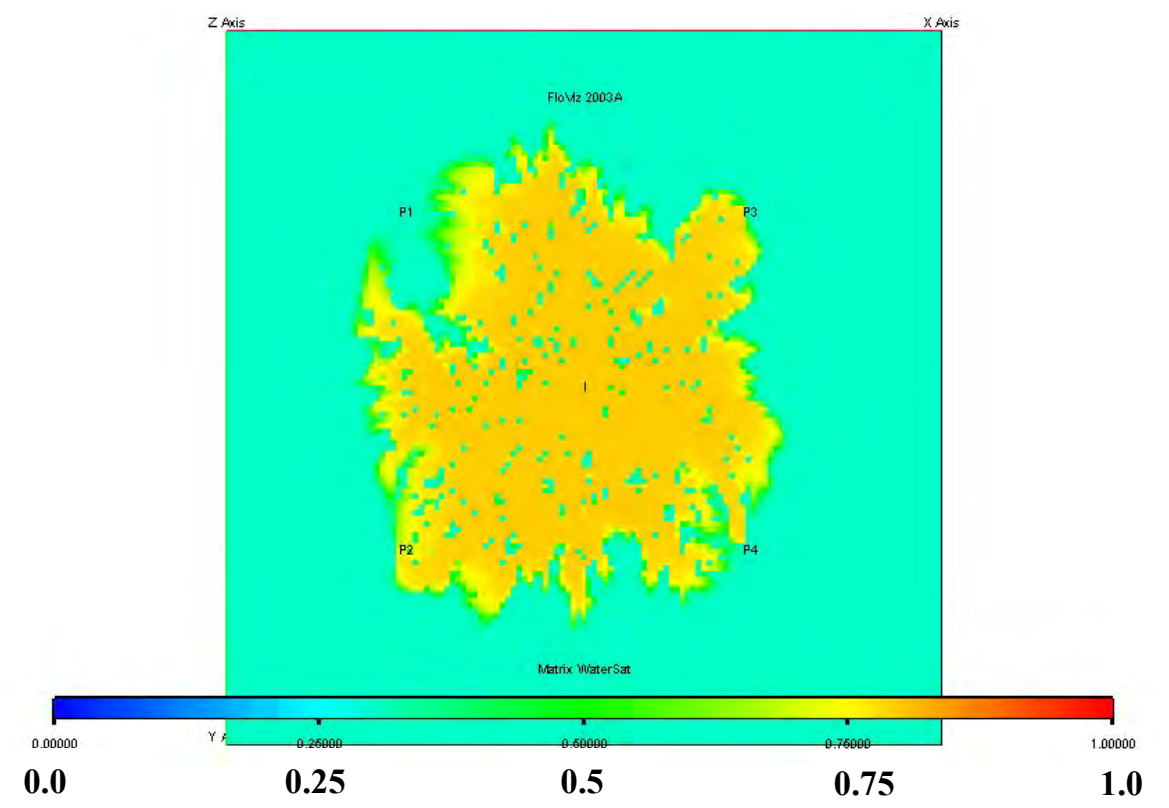

Fig. C.13c - Matrix water saturation at report step 20 (600 days) for test T3 final model. 


\section{Well Bottom Hole Pressure}

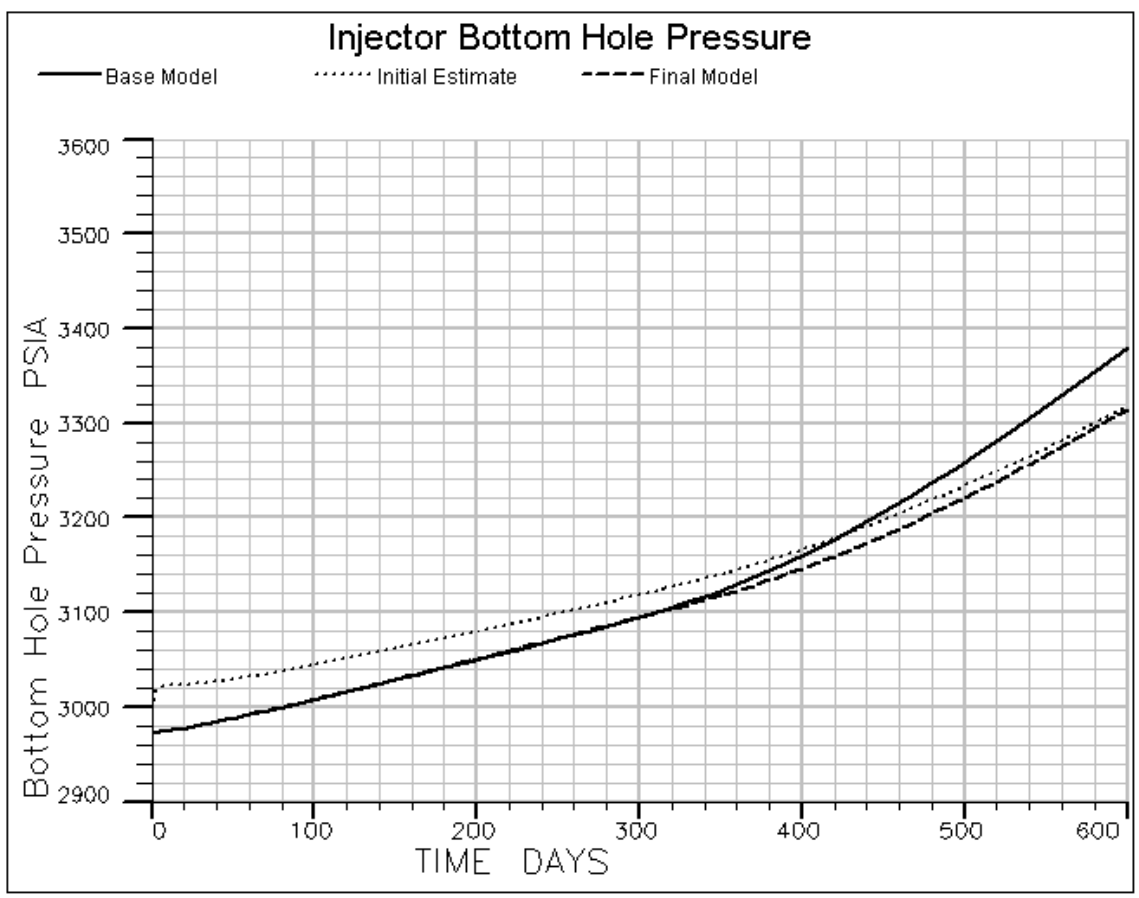

Fig. C.14a - Injector flowing bottom hole pressure for base model, initial estimate, and final model.

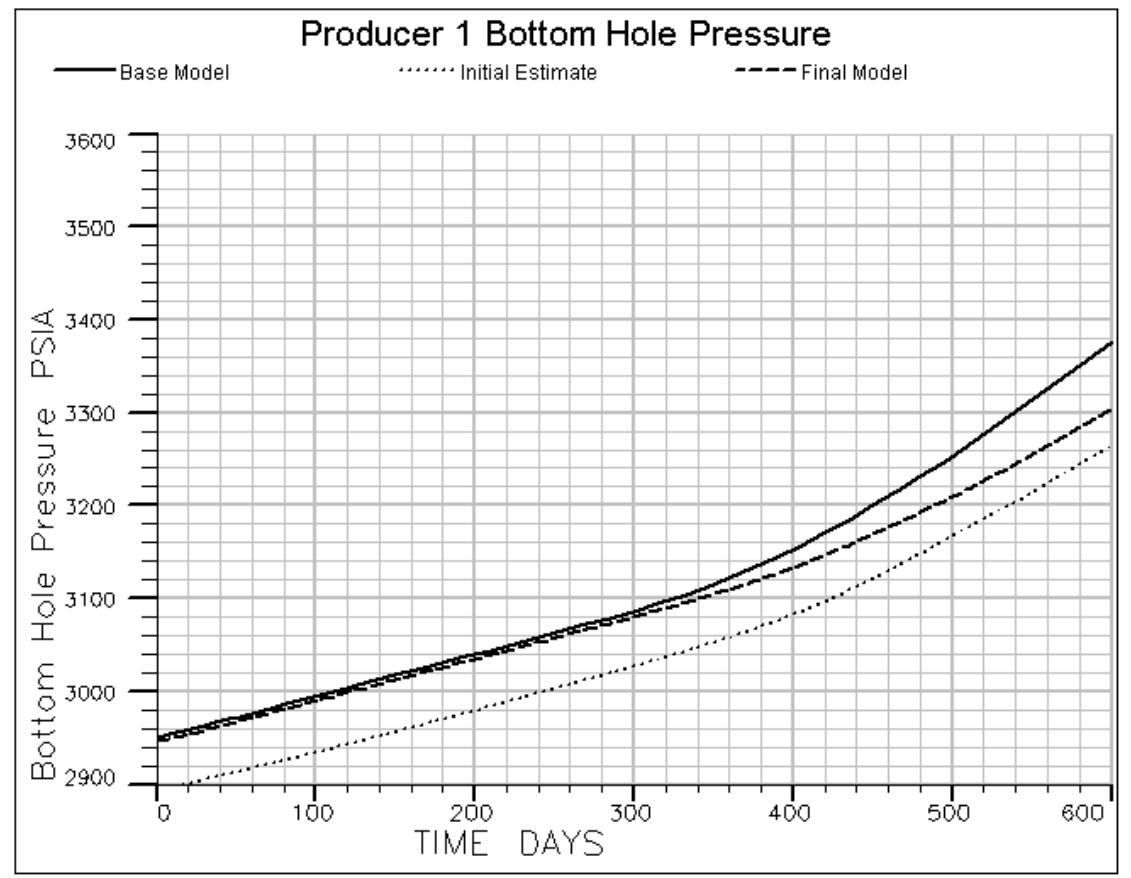

Fig. C.14b - Producer 1 flowing bottom hole pressure for base model, initial estimate, and final model. 


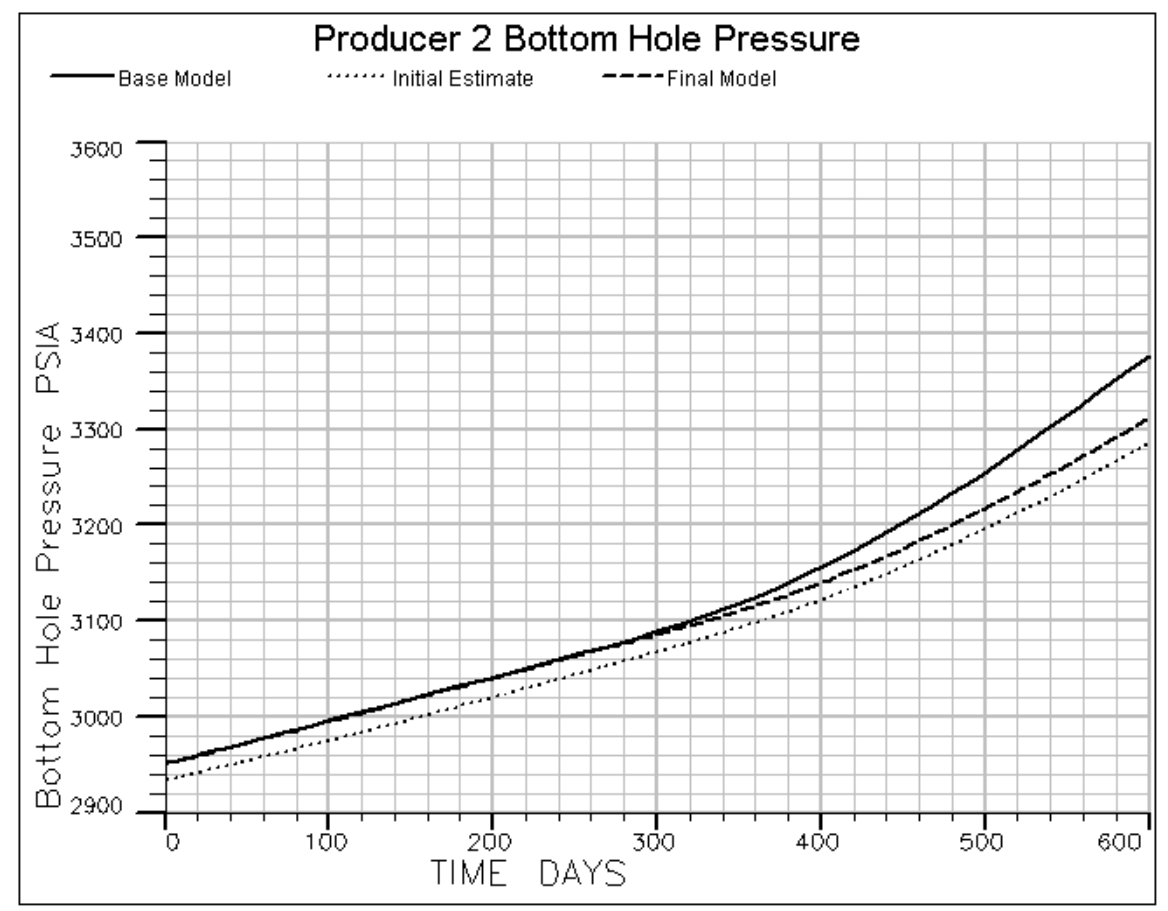

Fig. C.14c - Producer 2 flowing bottom hole pressure for base model, initial estimate, and final model.

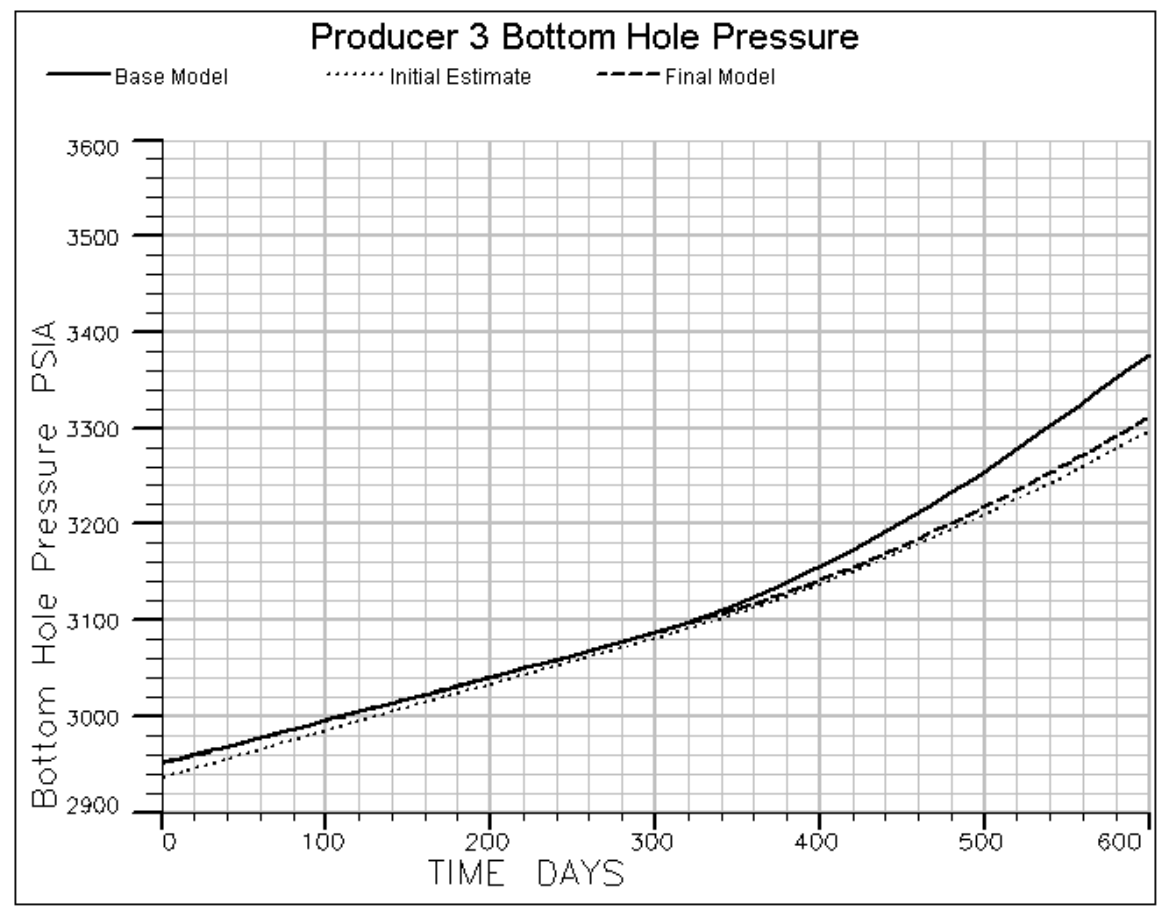

Fig. C.14d - Producer 3 flowing bottom hole pressure for base model, initial estimate, and final model. 


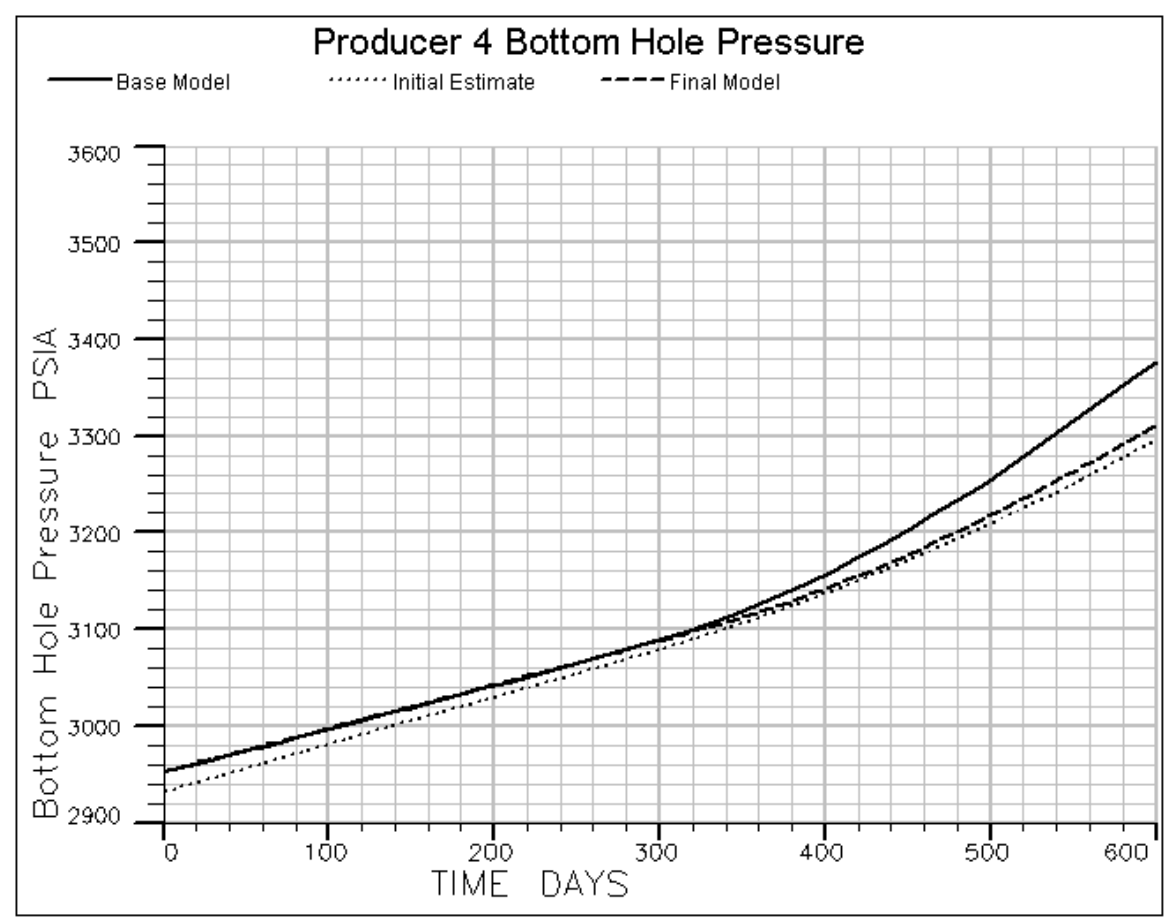

Fig. C.14e - Producer 4 flowing bottom hole pressure for base model, initial estimate, and final model. 


\section{Well Oil Production Rate}

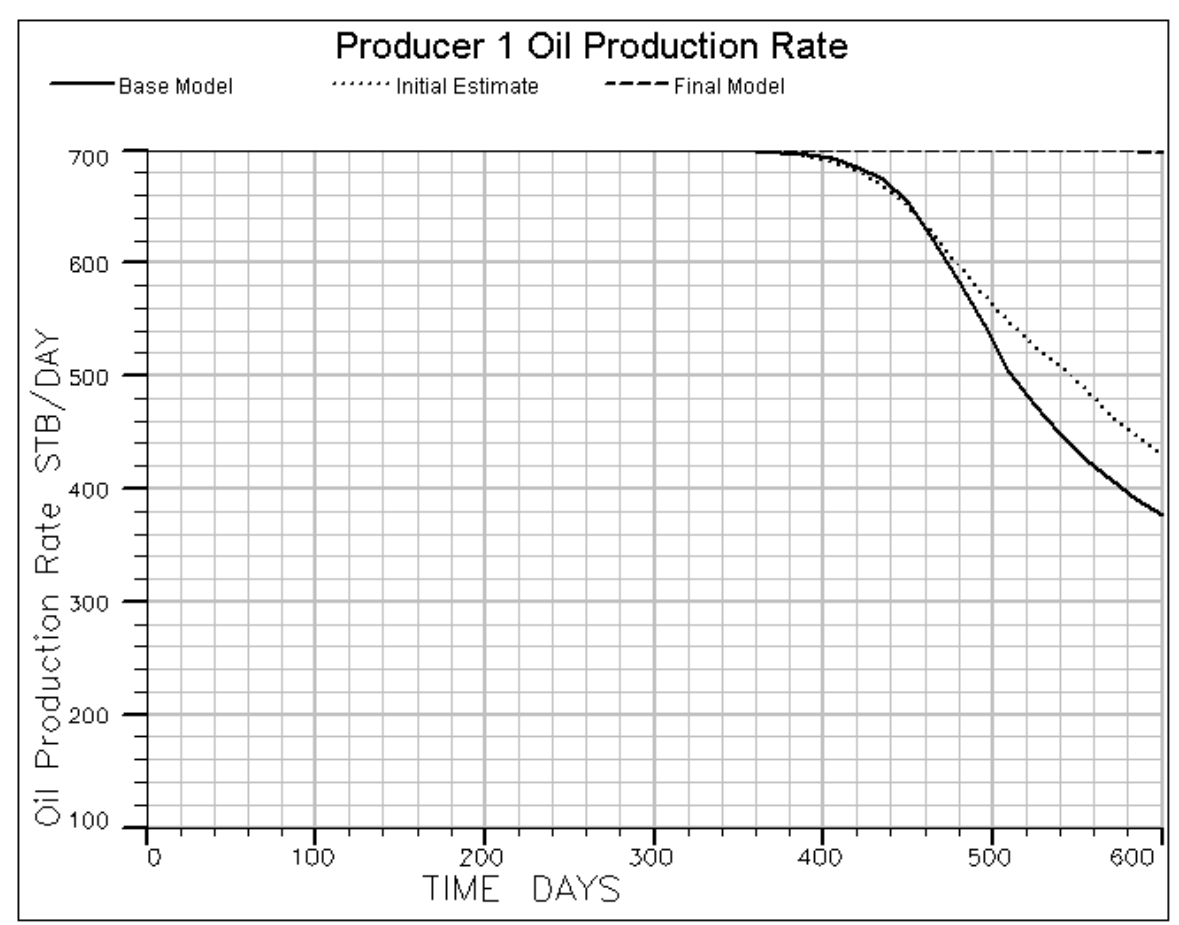

Fig. C.15a - Producer 1 oil production rate for base model, initial estimate, and final model.

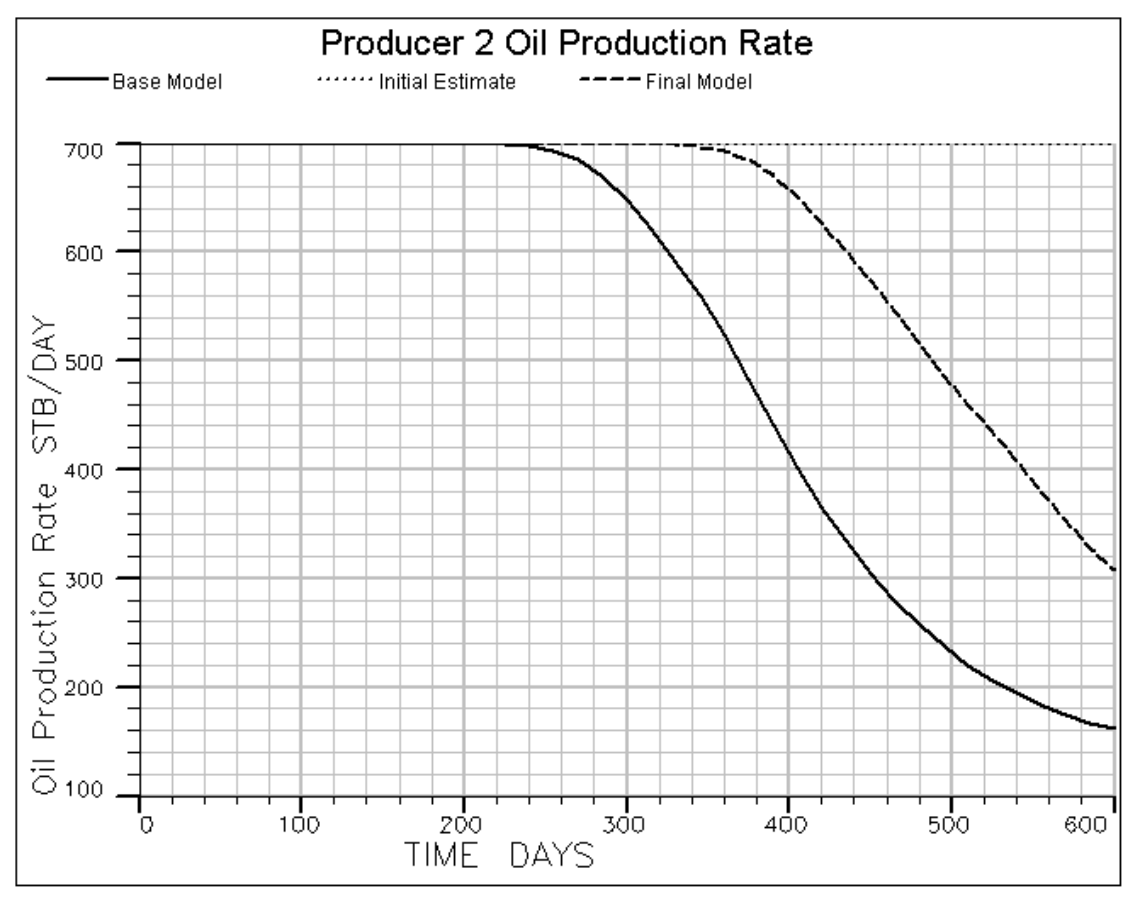

Fig. C.15b $\quad-\quad$ Producer 2 oil production rate for base model, initial estimate, and final model. 


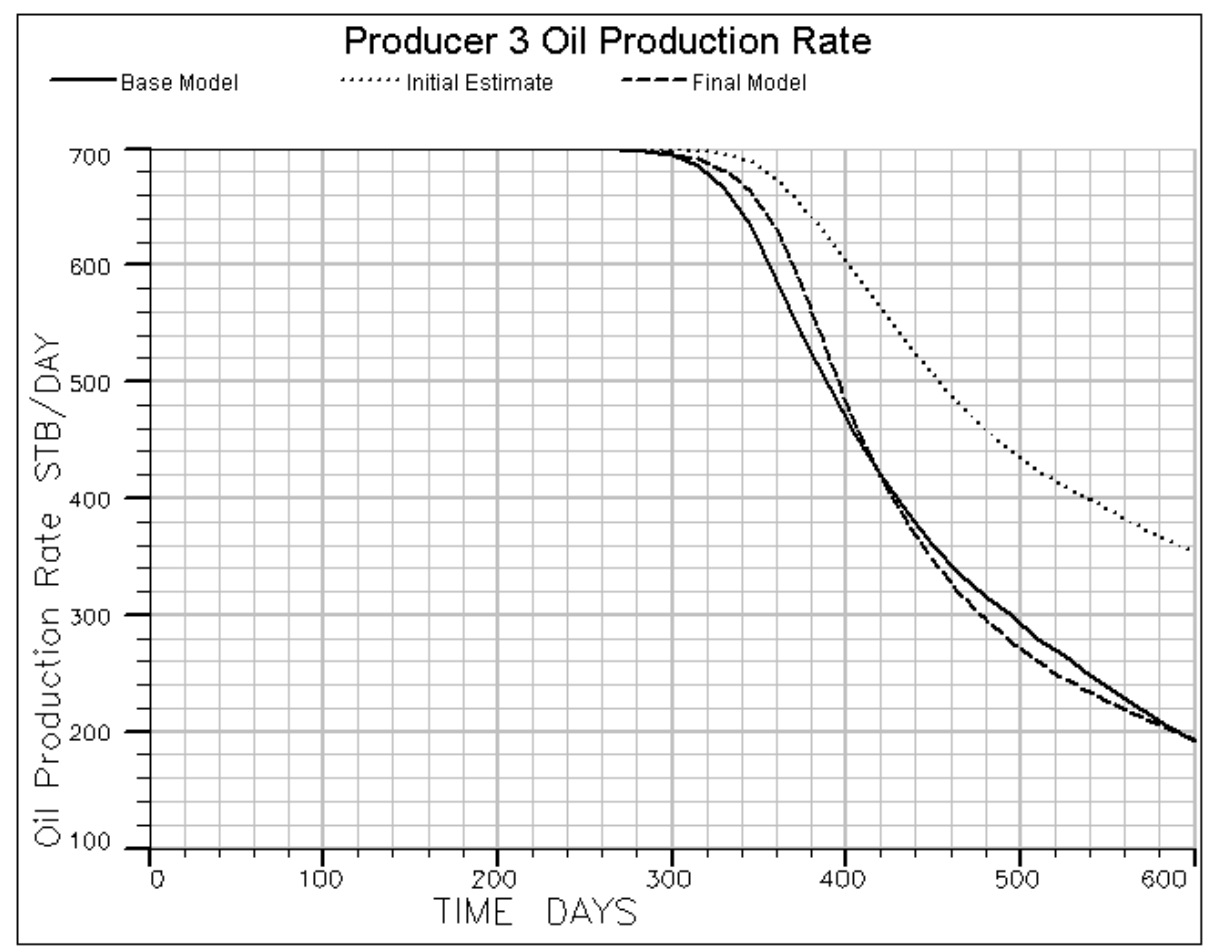

Fig. C.15c - Producer 3 oil production rate for base model, initial estimate, and final model.

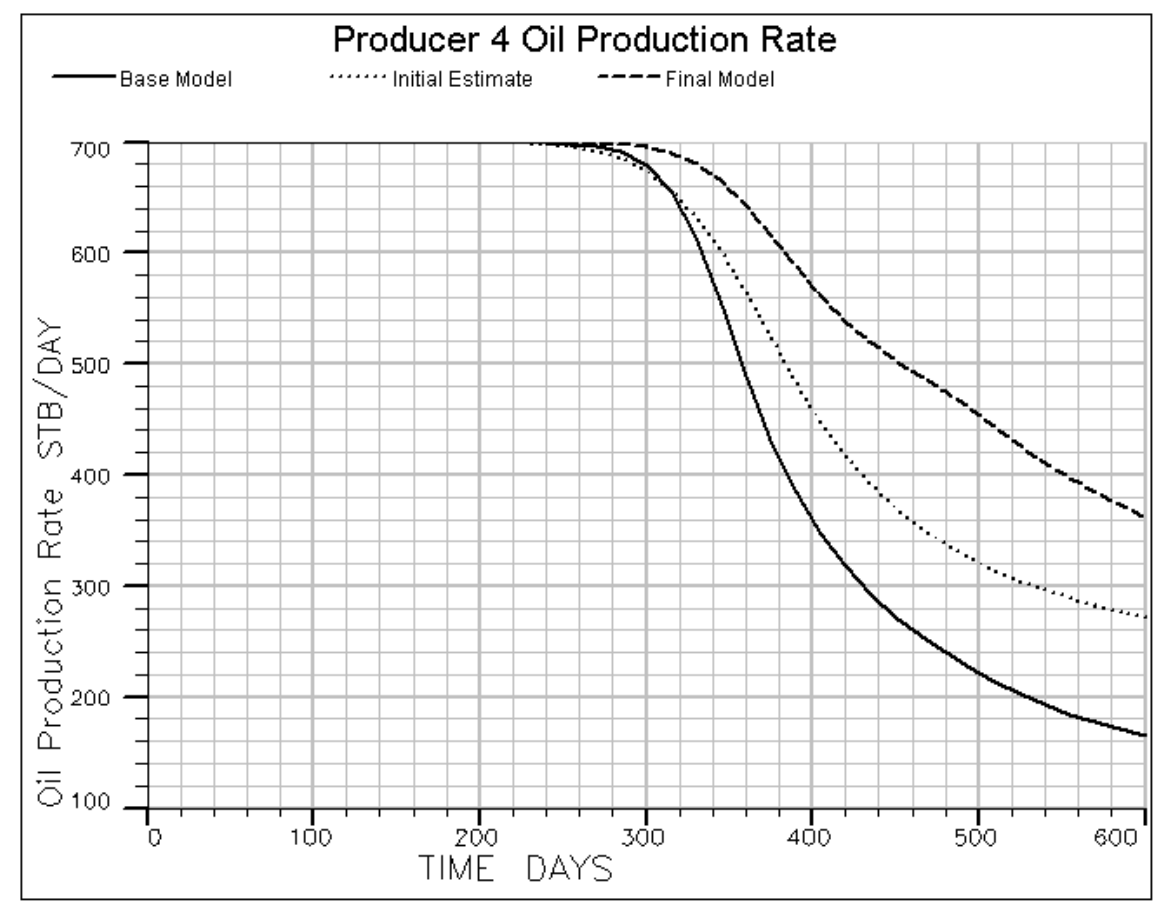

Fig. C.15d - Producer 4 oil production rate for base model, initial estimate, and final model. 
Elastic Anisotropy versus Azimuth (Base, Initial, Final)

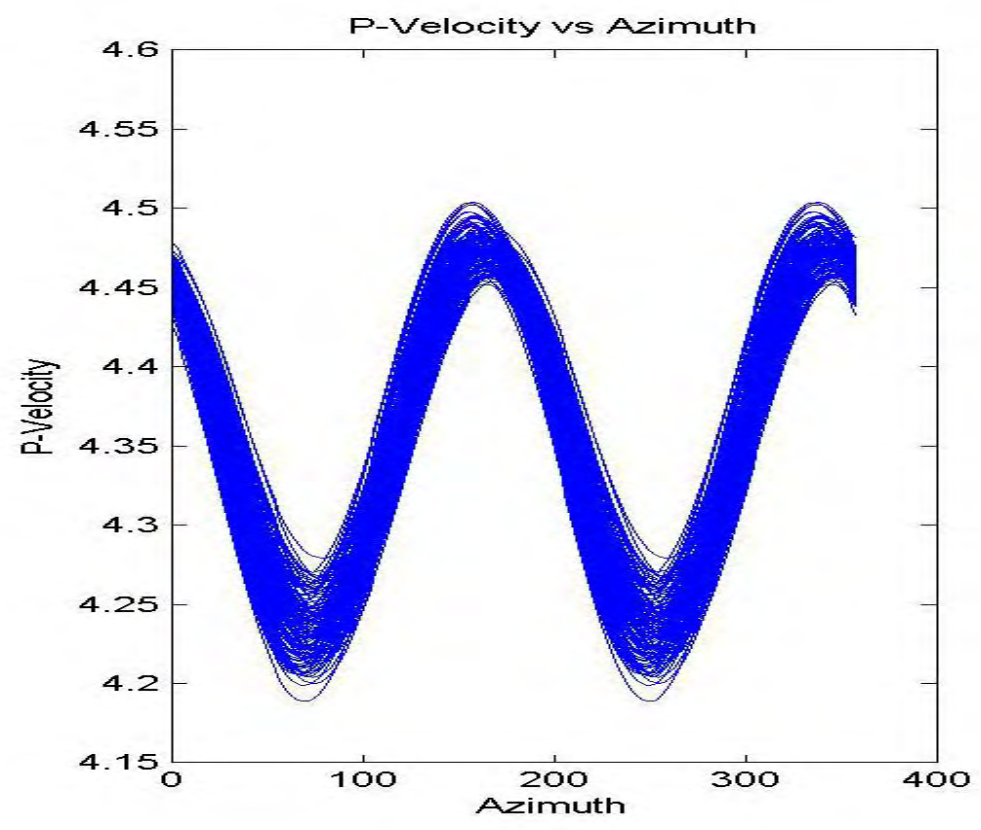

Fig. C.16a - Quasi-P wave velocity versus azimuth for base model.

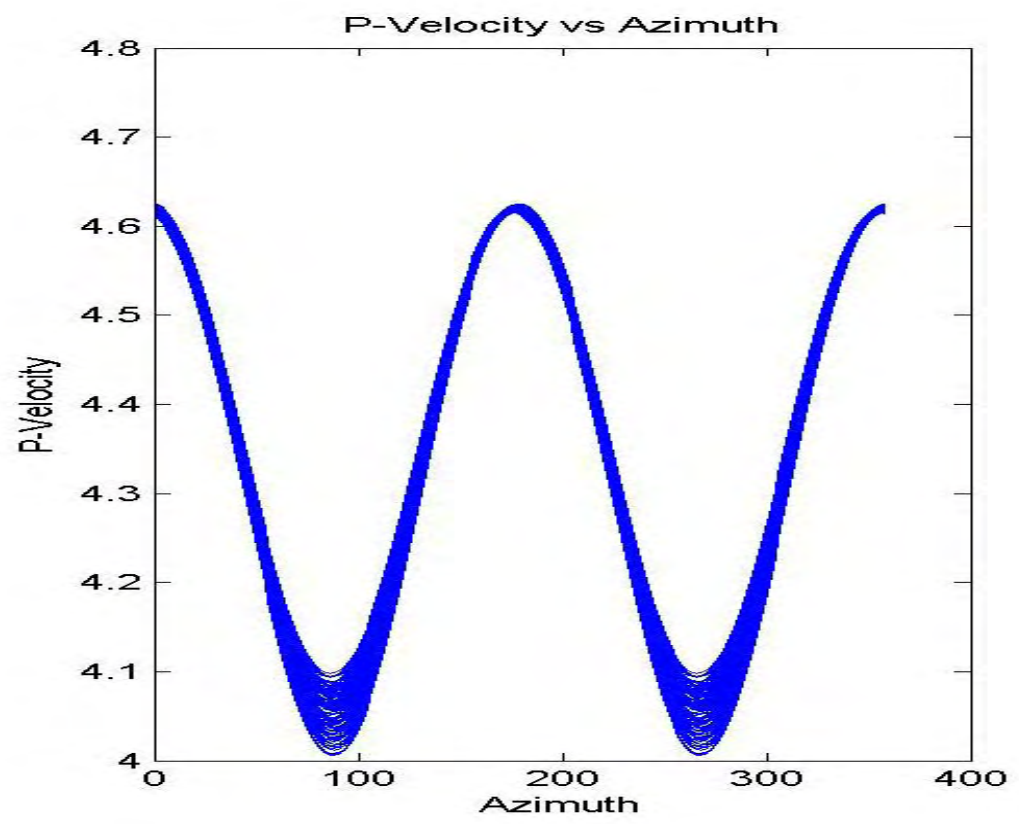

Fig. C.16b - Quasi-P wave velocity versus azimuth for initial estimate (M1). 


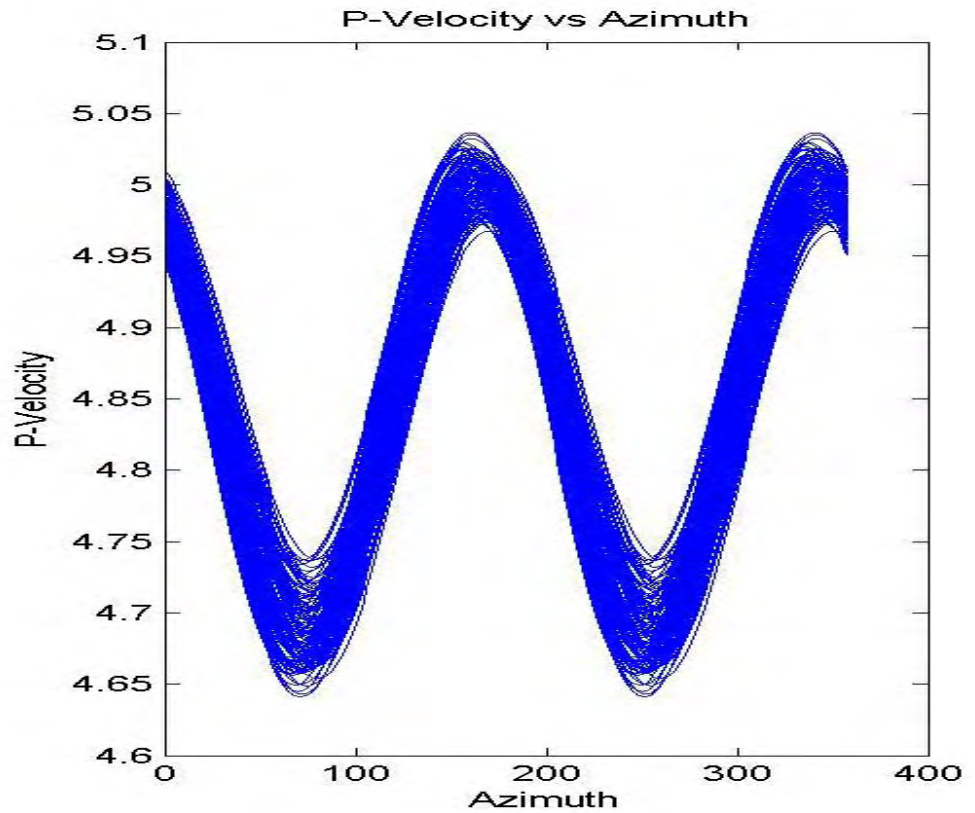

Fig. C.16c - Quasi-P wave velocity versus azimuth for final model (M6).

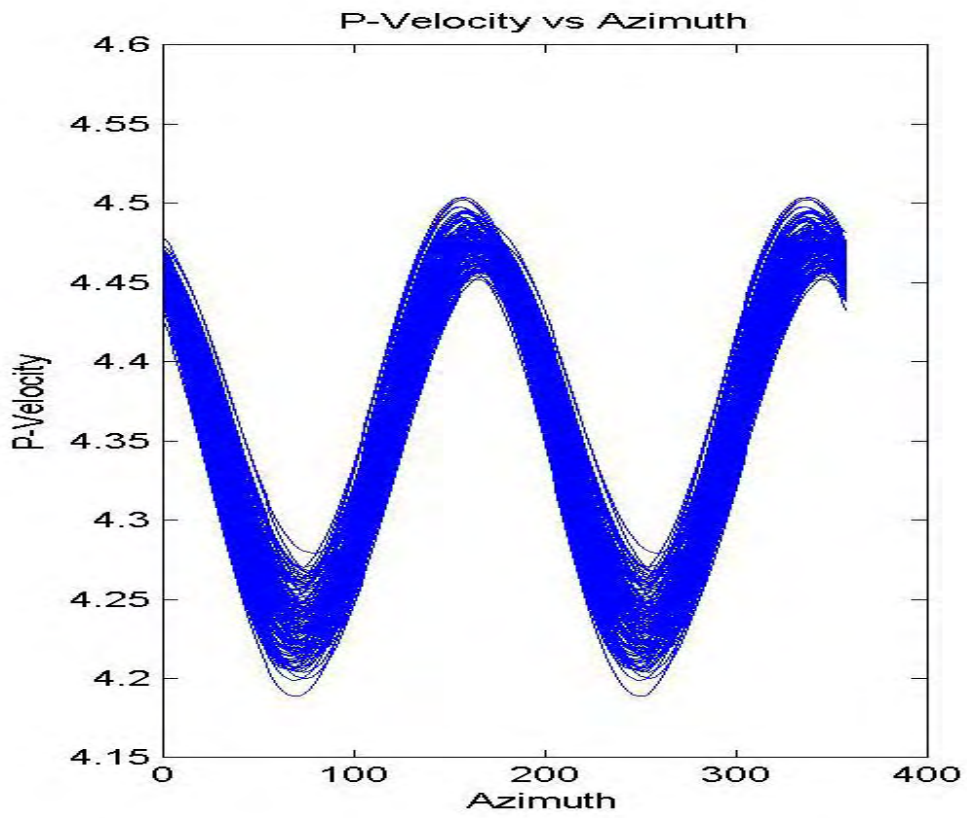

Fig. C.16d - Quasi-P wave velocity versus azimuth for base model for comparison with Fig. 16c. 

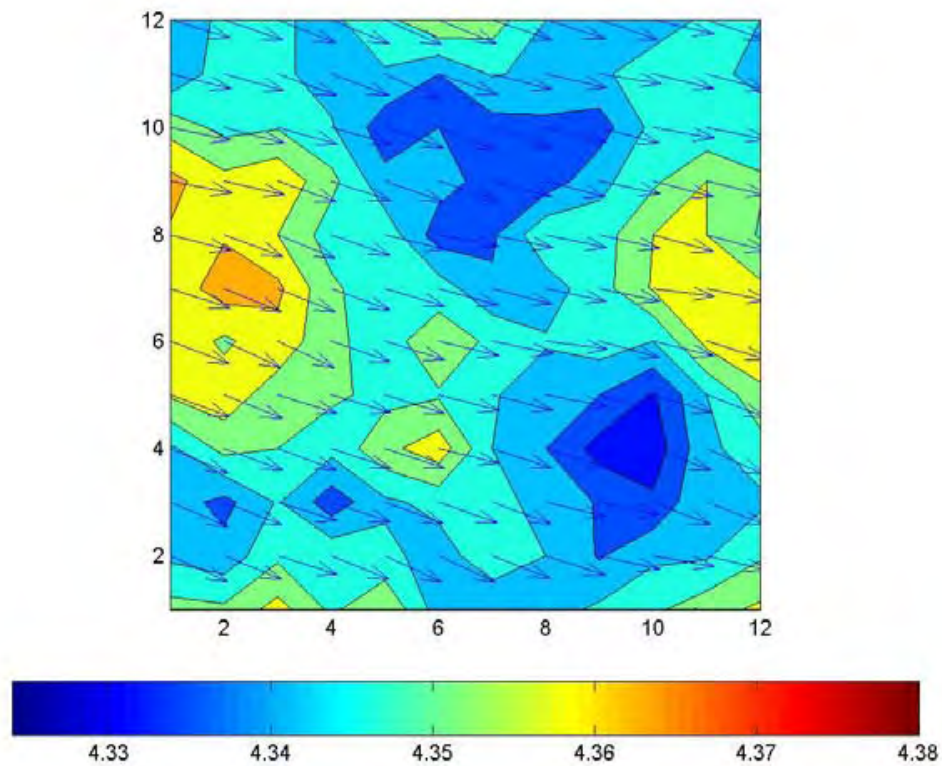

Fig. C.17a - Base model P velocity modulation amplitude and fast shear direction.
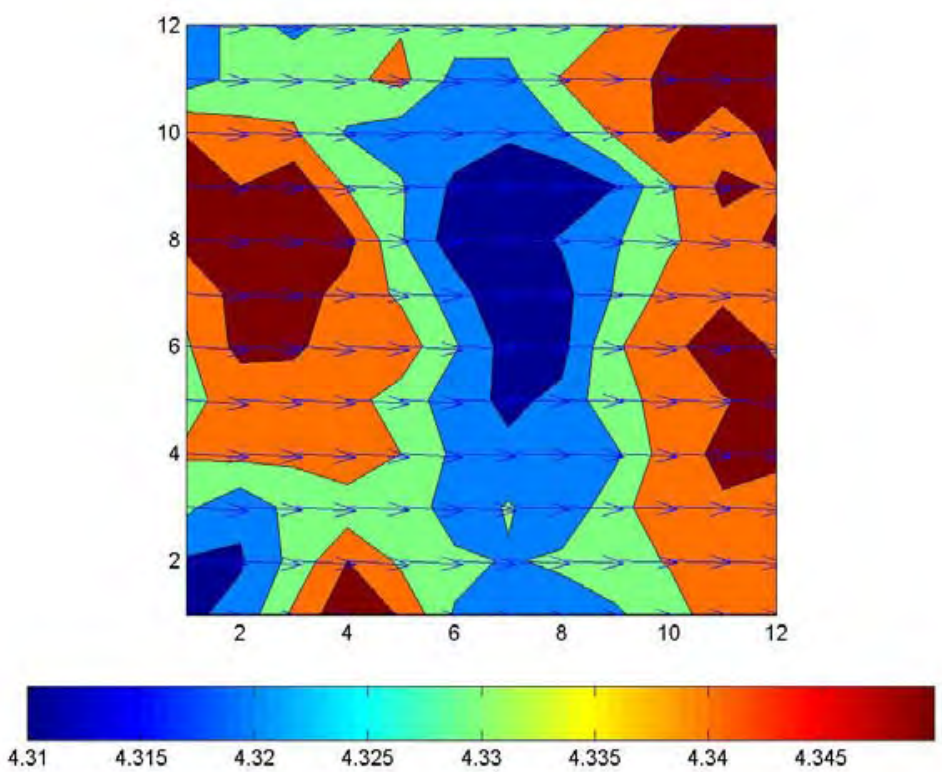

Fig. C.17b - Initial estimate $\mathrm{P}$ velocity modulation amplitude and fast shear direction. 

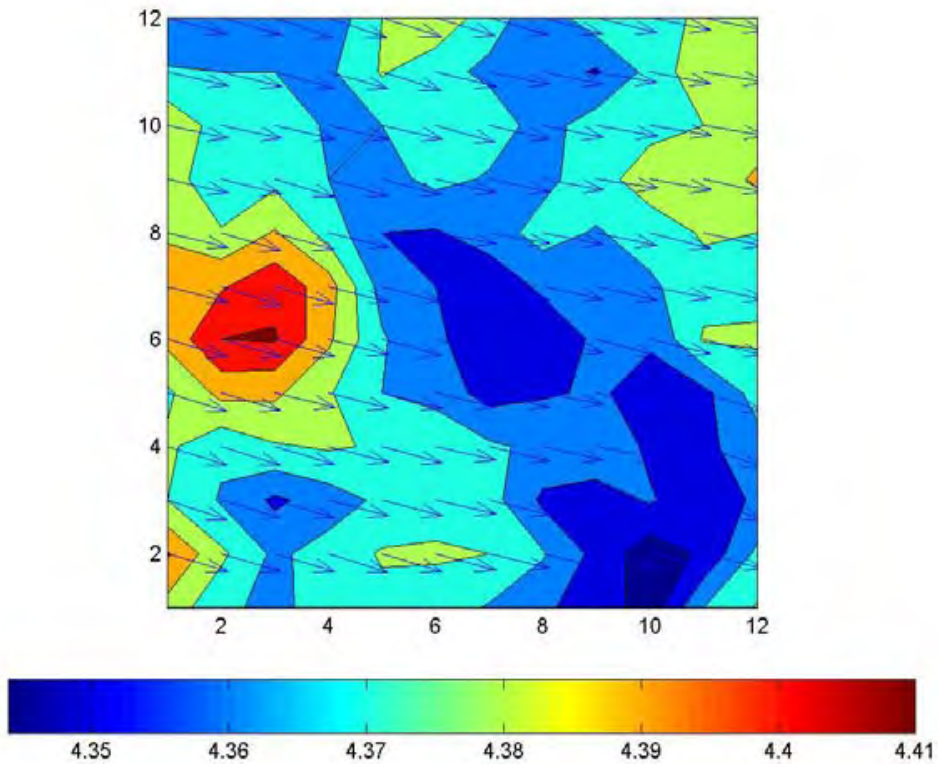

Fig. C.17c - Final $\mathrm{P}$ velocity modulation amplitude and fast shear direction.
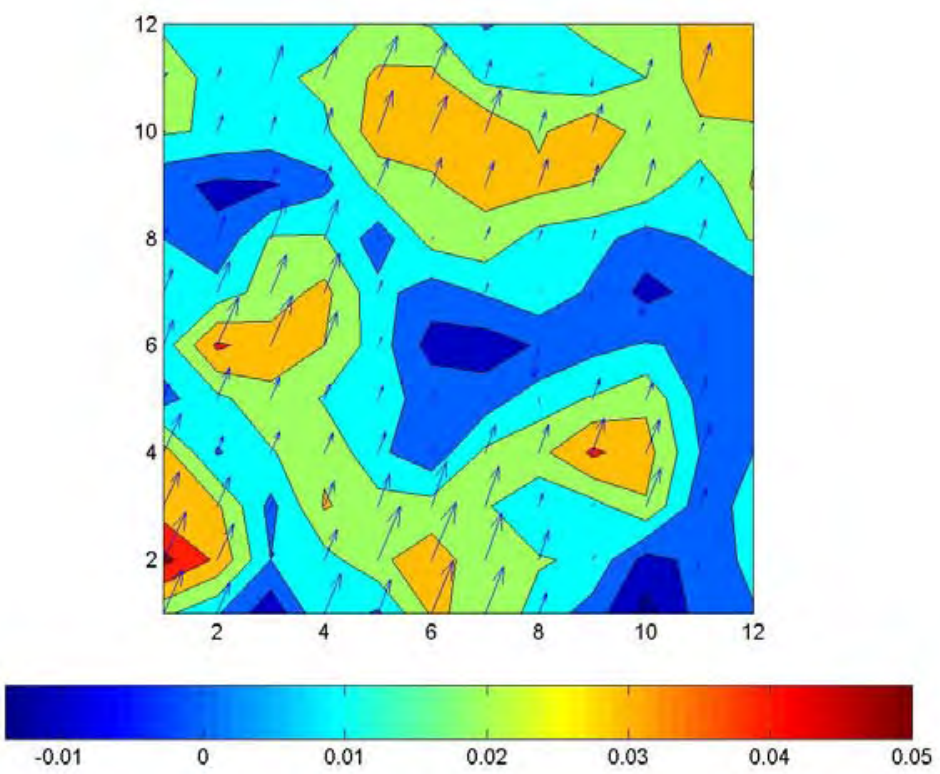

Fig. C.17d - P velocity modulation amplitude and fast shear direction difference between base and final modes. 


\section{Production Data Only Optimization}

Table C.10 - Test T3 production data only derivative perturbations.

\begin{tabular}{|l|r|r|r|r|r|}
\hline Iterations & \multicolumn{1}{c|}{$\mathbf{1}$} & \multicolumn{1}{c|}{$\mathbf{2}$} & \multicolumn{1}{c|}{$\mathbf{3}$} & \multicolumn{1}{c|}{$\mathbf{4}$} & \multicolumn{1}{c|}{$\mathbf{5}$} \\
\hline Trend 1 (degrees) & -10 & -10 & -10 & -10 & -10 \\
\hline P32 1 (1/m) & -0.05 & -0.05 & -0.05 & -0.05 & -0.05 \\
\hline Trend 2 (degrees) & -10 & 10 & 10 & 10 & 10 \\
\hline P32 2 (1/m) & -0.05 & 0.05 & 0.05 & 0.05 & 0.05 \\
\hline
\end{tabular}

Table C.11 - Test T3 production data only prior model weights.

\begin{tabular}{|l|c|c|c|c|c|}
\hline Iterations & $\mathbf{1}$ & $\mathbf{2}$ & $\mathbf{3}$ & $\mathbf{4}$ & $\mathbf{5}$ \\
\hline Trend (1) & .25 & .25 & .25 & .25 & .25 \\
\hline P32 (1) & .25 & .25 & .25 & .25 & .25 \\
\hline Trend (2) & .25 & .25 & .25 & .25 & .25 \\
\hline P32 (2) & .25 & .25 & .25 & .25 & .25 \\
\hline
\end{tabular}

Table C.12 - Summary of absolute errors for fracture set trend and P32 intensity at each update step using the production data only objective function. Step 0 is the initial estimate.

\begin{tabular}{|r|r|r|r|r|}
\cline { 2 - 5 } \multicolumn{1}{c|}{} & \multicolumn{4}{c|}{ Parameter Error } \\
\hline Iteration & Trend (1) & P32 (1) & Trend (2) & P32 (2) \\
\hline $\mathbf{0}$ & 20.0 & 0.100 & 20.0 & 0.050 \\
\hline $\mathbf{1}$ & 16.0 & 0.111 & 17.7 & 0.046 \\
\hline $\mathbf{2}$ & 15.2 & 0.116 & 12.8 & 0.065 \\
\hline $\mathbf{3}$ & 10.9 & 0.111 & 11.6 & 0.083 \\
\hline $\mathbf{4}$ & 12.2 & 0.118 & 16.8 & 0.068 \\
\hline $\mathbf{5}$ & 15.2 & 0.104 & 20.7 & 0.078 \\
\hline
\end{tabular}


Table C.13 - $\quad$ Summary of normalized residuals by observation type for fracture set trend and P32 intensity at each iteration using the production data only objective function. Iteration 0 is the initial estimate.

\begin{tabular}{|r|r|r|r|}
\cline { 2 - 4 } \multicolumn{1}{c|}{} & \multicolumn{3}{c|}{ Residuals } \\
\hline Iteration & \multicolumn{1}{c|}{ Total } & \multicolumn{1}{c|}{ BHP } & \multicolumn{1}{c|}{ OPR } \\
\hline $\mathbf{0}$ & 9.24 & 8.33 & 4.01 \\
\hline $\mathbf{1}$ & 5.27 & 3.55 & 3.89 \\
\hline $\mathbf{2}$ & 8.40 & 7.38 & 4.01 \\
\hline $\mathbf{3}$ & 3.46 & 2.76 & 2.06 \\
\hline $\mathbf{4}$ & 6.32 & 4.18 & 4.73 \\
\hline
\end{tabular}

Table C.14a - Summary of iteration 1 Trend and P32 intensity updates and residual errors for 10 samples.

\begin{tabular}{|c|c|c|c|c|c|c|c|}
\hline \multirow[b]{3}{*}{ SAMPLE } & \multicolumn{4}{|r|}{ Step M1 } & \multirow{2}{*}{\multicolumn{3}{|c|}{ Residuals }} \\
\hline & \multicolumn{4}{|c|}{ Updates } & & & \\
\hline & \begin{tabular}{|l|} 
Trend (1) \\
\end{tabular} & P32 (1) & Trend (2) & P32 (2) & TOTAL & BHP & OPR \\
\hline 1 & 81.3 & 0.212 & 110.8 & 0.103 & 9.40 & 8.53 & 3.94 \\
\hline 2 & 80.8 & 0.214 & 111.7 & 0.103 & 9.39 & 8.38 & 4.23 \\
\hline 3 & 80.5 & 0.210 & 113.1 & 0.104 & 9.36 & 8.42 & 4.10 \\
\hline 4 & 80.3 & 0.213 & 111.3 & 0.100 & 9.26 & 8.38 & 3.95 \\
\hline 5 & 81.3 & 0.209 & 113.2 & 0.106 & 8.92 & 8.03 & 3.89 \\
\hline 6 & 80.6 & 0.209 & 114.0 & 0.106 & 9.13 & 8.26 & 3.88 \\
\hline 7 & 81.5 & 0.212 & 110.8 & 0.103 & 9.52 & 8.55 & 4.18 \\
\hline 8 & 81.7 & 0.210 & 112.0 & 0.105 & 9.01 & 8.15 & 3.83 \\
\hline 9 & 80.6 & 0.209 & 112.3 & 0.103 & 9.37 & 8.36 & 4.24 \\
\hline 10 & 80.9 & 0.210 & 113.2 & 0.105 & 9.09 & 8.21 & 3.90 \\
\hline Avg & 81.0 & 0.211 & 112.3 & 0.104 & 9.24 & 8.33 & 4.01 \\
\hline
\end{tabular}


Table C.14b - $\quad$ Summary of iteration 1 Trend and P32 intensity updates and residual errors for 10 samples.

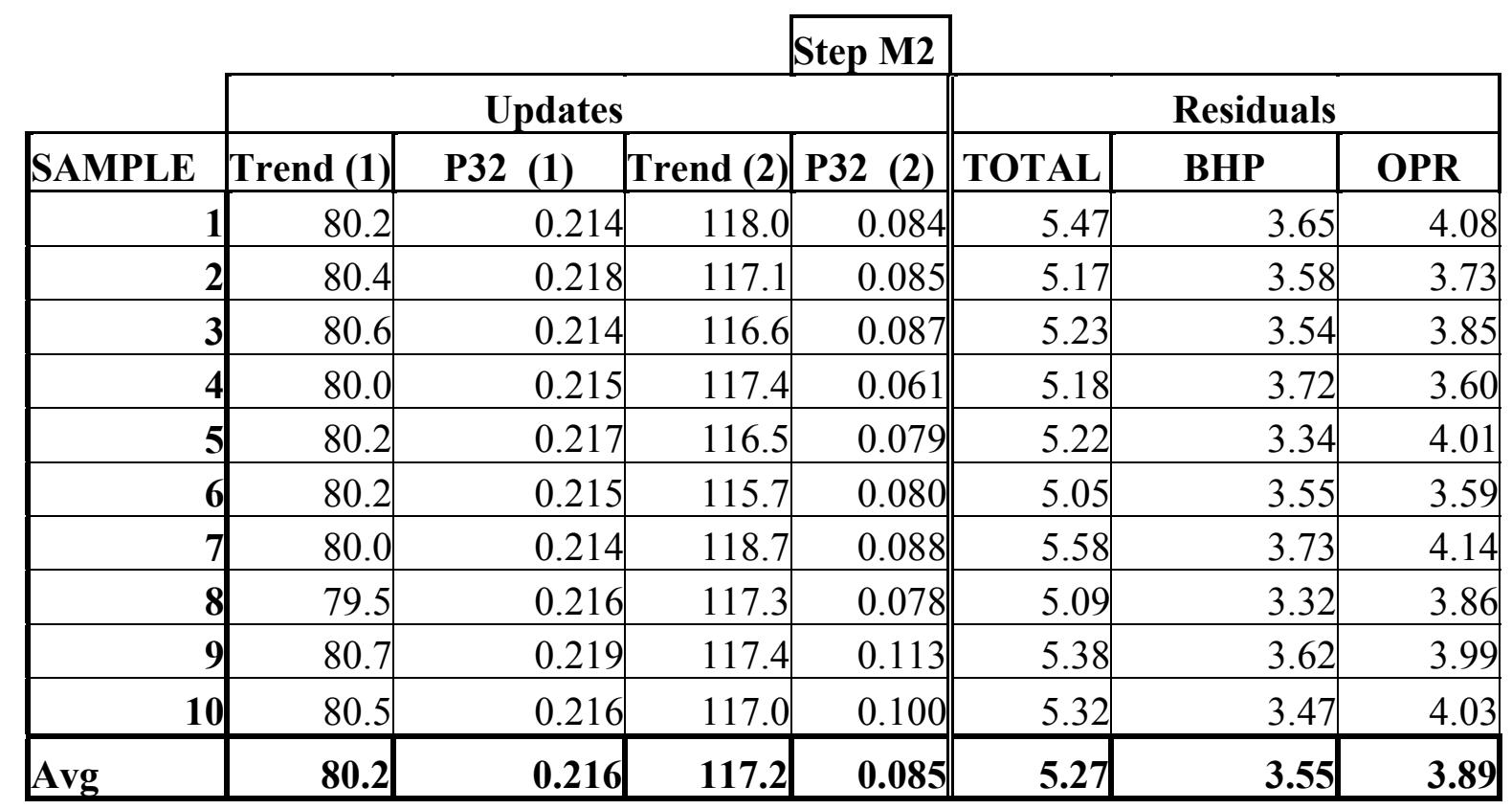

Table C.14c - Summary of iteration 1 Trend and P32 intensity updates and residual errors for 10 samples.

\begin{tabular}{|c|c|c|c|c|c|c|c|}
\hline \multirow[b]{3}{*}{ SAMPLE } & \multicolumn{7}{|c|}{ Step M3 } \\
\hline & \multicolumn{4}{|c|}{ Updates } & \multicolumn{3}{|c|}{ Residuals } \\
\hline & Trend (1) & P32 (1) & Trend (2) & P32 (2) & TOTAL & BHP & OPR \\
\hline 1 & 75.7 & 0.211 & 118.3 & 0.067 & 8.34 & 7.21 & 4.18 \\
\hline 2 & 76.2 & 0.212 & 119.5 & 0.064 & 8.46 & 7.52 & 3.87 \\
\hline 3 & 75.8 & 0.211 & 118.5 & 0.066 & 8.35 & 7.41 & 3.84 \\
\hline 4 & 74.8 & 0.213 & 118.6 & 0.060 & 8.59 & 7.61 & 3.98 \\
\hline 5 & 76.6 & 0.211 & 119.6 & 0.064 & 8.62 & 7.57 & 4.12 \\
\hline 6 & 75.7 & 0.210 & 117.2 & 0.071 & 8.41 & 7.34 & 4.11 \\
\hline 7 & 76.6 & 0.209 & 117.5 & 0.073 & 8.45 & 7.35 & 4.17 \\
\hline 8 & 76.4 & 0.212 & 119.0 & 0.066 & 8.44 & 7.50 & 3.86 \\
\hline 9 & 76.6 & 0.209 & 117.5 & 0.072 & 8.15 & 7.06 & 4.09 \\
\hline 10 & 74.9 & 0.212 & 118.4 & 0.063 & 8.25 & 7.27 & 3.89 \\
\hline Avg & 75.9 & 0.211 & 118.4 & 0.067 & 8.40 & 7.38 & 4.01 \\
\hline
\end{tabular}


Table C.14d - Summary of iteration 1 Trend and P32 intensity updates and residual errors for 10 samples.

\begin{tabular}{|c|c|c|c|c|c|c|c|}
\hline & & & & & & & \\
\hline & \multicolumn{7}{|c|}{ Step M4 } \\
\hline & \multicolumn{4}{|c|}{ Updates } & \multicolumn{3}{|c|}{ Residuals } \\
\hline SAMPLE & Trend (1) & P32 (1) & Trend (2) & P32 (2) & TOTAL & BHP & OPR \\
\hline 1 & 77.3 & 0.215 & 113.9 & 0.083 & 3.11 & 2.40 & 1.97 \\
\hline 2 & 77.7 & 0.218 & 112.3 & 0.089 & 3.44 & 2.64 & 2.20 \\
\hline 3 & 76.6 & 0.213 & 114.7 & 0.081 & 3.23 & 2.84 & 1.55 \\
\hline 4 & 76.7 & 0.225 & 114.9 & $0.061 \|$ & 3.27 & 2.38 & 2.24 \\
\hline 5 & 77.4 & 0.218 & 112.6 & 0.072 & 3.47 & 2.78 & 2.07 \\
\hline 6 & 77.7 & 0.219 & 113.9 & 0.076 & 3.41 & 2.57 & 2.23 \\
\hline 7 & 77.1 & 0.221 & 111.8 & 0.085 & 3.57 & 2.94 & 2.03 \\
\hline 8 & 77.1 & 0.210 & 112.6 & 0.101 & 3.57 & 2.83 & 2.17 \\
\hline 9 & 77.0 & 0.220 & 111.8 & 0.089 & 3.89 & 3.28 & 2.10 \\
\hline 10 & 77.0 & 0.222 & 113.5 & 0.081 & 3.61 & 2.96 & 2.06 \\
\hline Avg & 77.2 & 0.218 & 113.2 & 0.082 & 3.46 & 2.76 & 2.06 \\
\hline
\end{tabular}

Table C.14e - Summary of iteration 1 Trend and P32 intensity updates and residual errors for 10 samples.

\begin{tabular}{|c|c|c|c|c|c|c|c|}
\hline \multirow[b]{3}{*}{ SAMPLE } & \multicolumn{7}{|c|}{ Step M5 } \\
\hline & \multicolumn{4}{|c|}{ Updates } & \multicolumn{3}{|c|}{ Residuals } \\
\hline & Trend (1) & P32 (1) & Trend (2) & P32 (2) & \begin{tabular}{|l|} 
TOTAL \\
\end{tabular} & BHP & OPR \\
\hline 1 & 81.3 & 0.204 & 109.2 & 0.052 & 6.00 & 3.82 & 4.62 \\
\hline 2 & 82.0 & 0.202 & 109.4 & 0.040 & 6.34 & 4.13 & 4.82 \\
\hline 3 & 81.1 & 0.207 & 115.0 & 0.016 & 5.97 & 4.18 & 4.26 \\
\hline 4 & 80.7 & 0.202 & 107.9 & 0.092 & 6.21 & 3.75 & 4.94 \\
\hline 5 & 82.1 & 0.203 & 109.3 & 0.065 & 6.35 & 4.25 & 4.71 \\
\hline 6 & 81.5 & 0.202 & 106.8 & 0.122 & 6.36 & 3.98 & 4.96 \\
\hline 7 & 78.3 & 0.208 & 108.6 & -0.001 & 6.38 & 4.37 & 4.65 \\
\hline 8 & 77.2 & 0.208 & 105.6 & 0.171 & 6.41 & 4.22 & 4.82 \\
\hline 9 & 78.5 & 0.201 & 109.3 & 0.075 & 6.69 & 4.70 & 4.76 \\
\hline 10 & 79.6 & 0.201 & 112.1 & 0.087 & 6.46 & 4.37 & 4.76 \\
\hline Avg & 80.233 & 0.204 & 109.3 & 0.072 & 6.32 & 4.18 & 4.73 \\
\hline
\end{tabular}


Fracture Distribution Maps, (Base, Initial, Final) - Production Only Objective Function

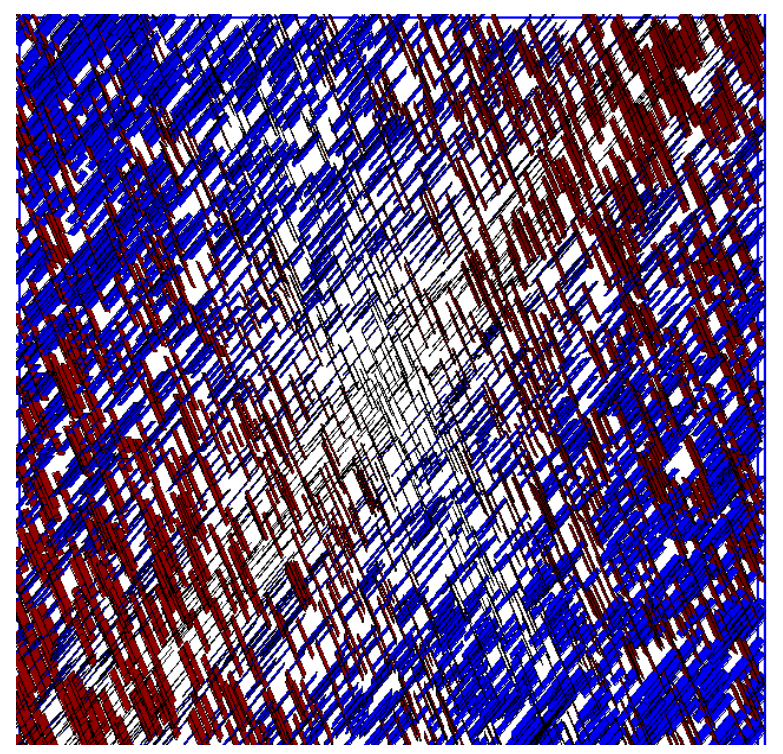

Fig. C.18a - Test T3 base model fracture distribution. Production only objective function.

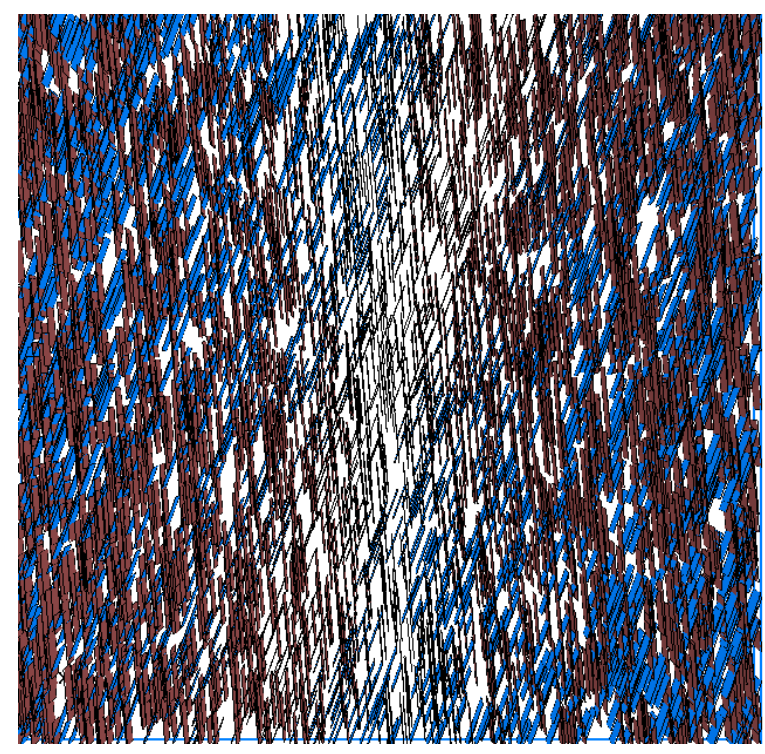

Fig. C.18b - Test T3 initial estimate fracture distribution. Production only objective function. 


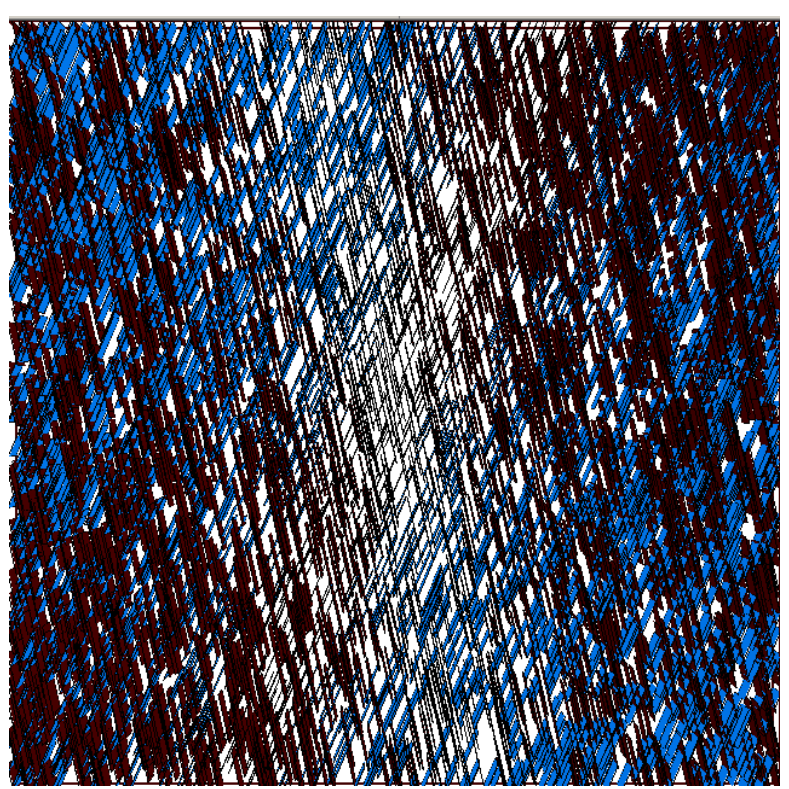

Fig.C.18c - Test T3 final model fracture distribution. Production only objective function. 


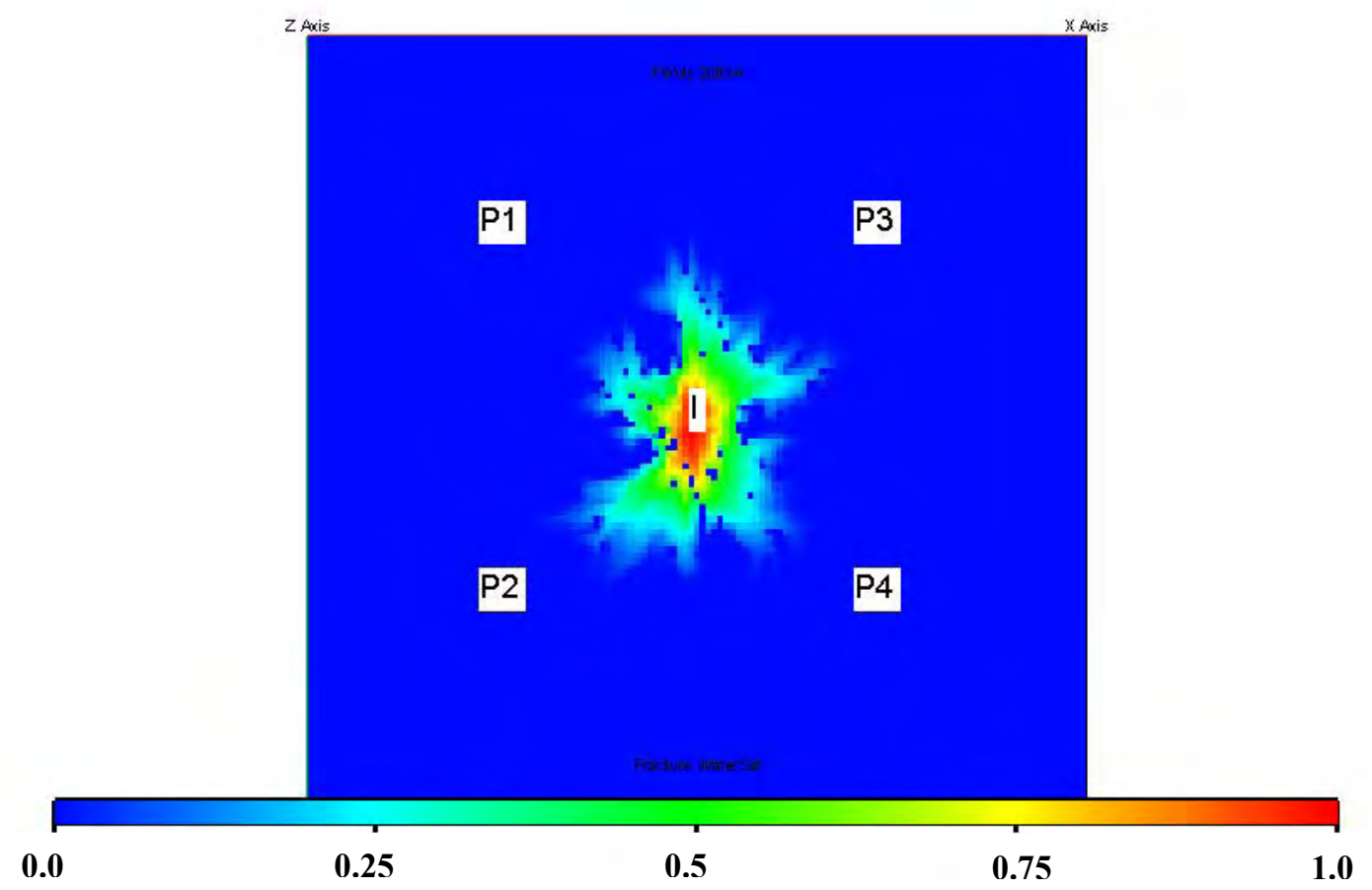

Fig. C.19a - Fracture water saturation at report step 5 (150 days) for test T3 base model. Production only objective function.

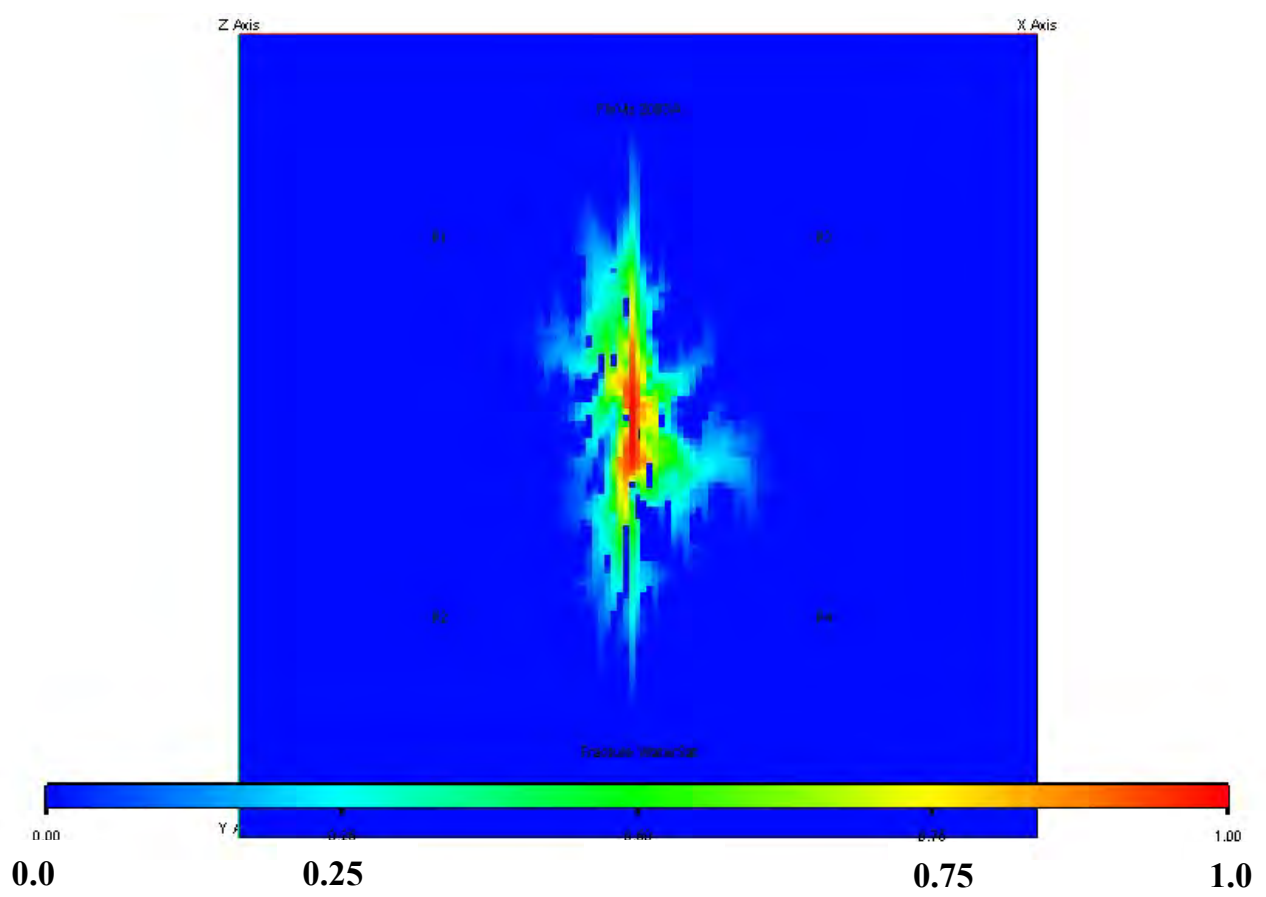

Fig. C.19b - Fracture water saturation at report step 5 (150 days) for test T3 initial estimate. Production only objective function. 


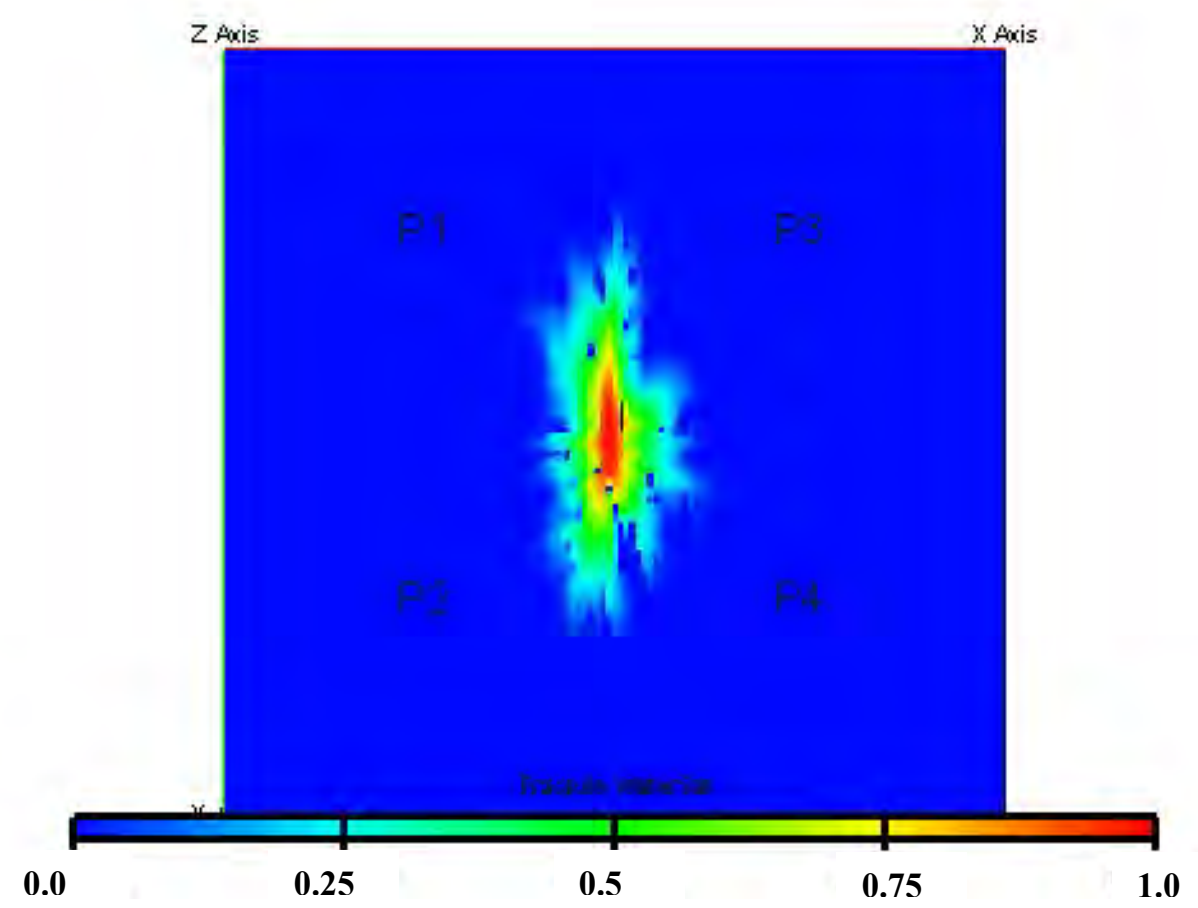

Fig. C.19c - Fracture water saturation at report step 5 (150 days) for test T3 final model. Production only objective function.

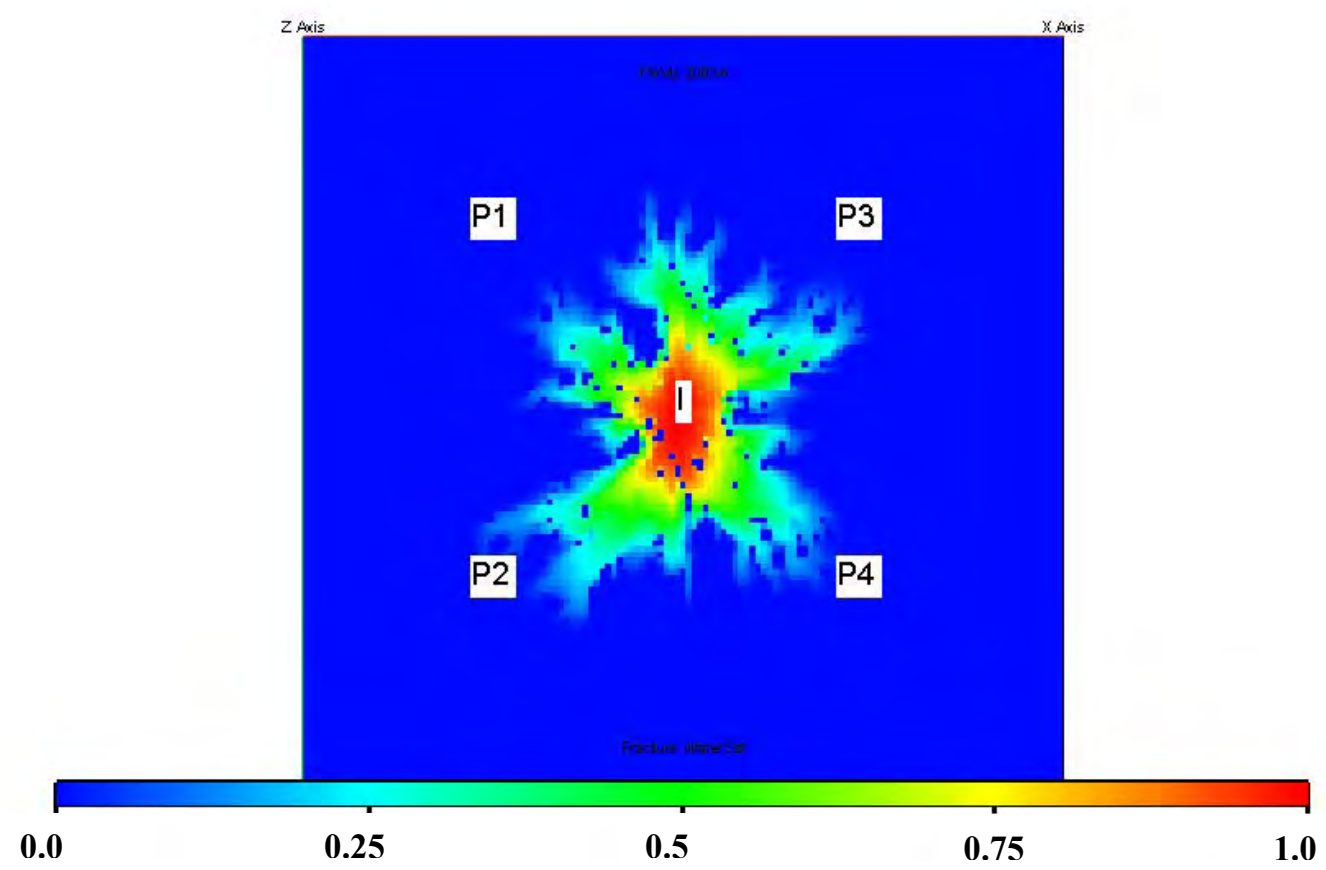

Fig. C.20a - Fracture water saturation at report step $10(300$ days)for test T3 base model. Production only objective function. 


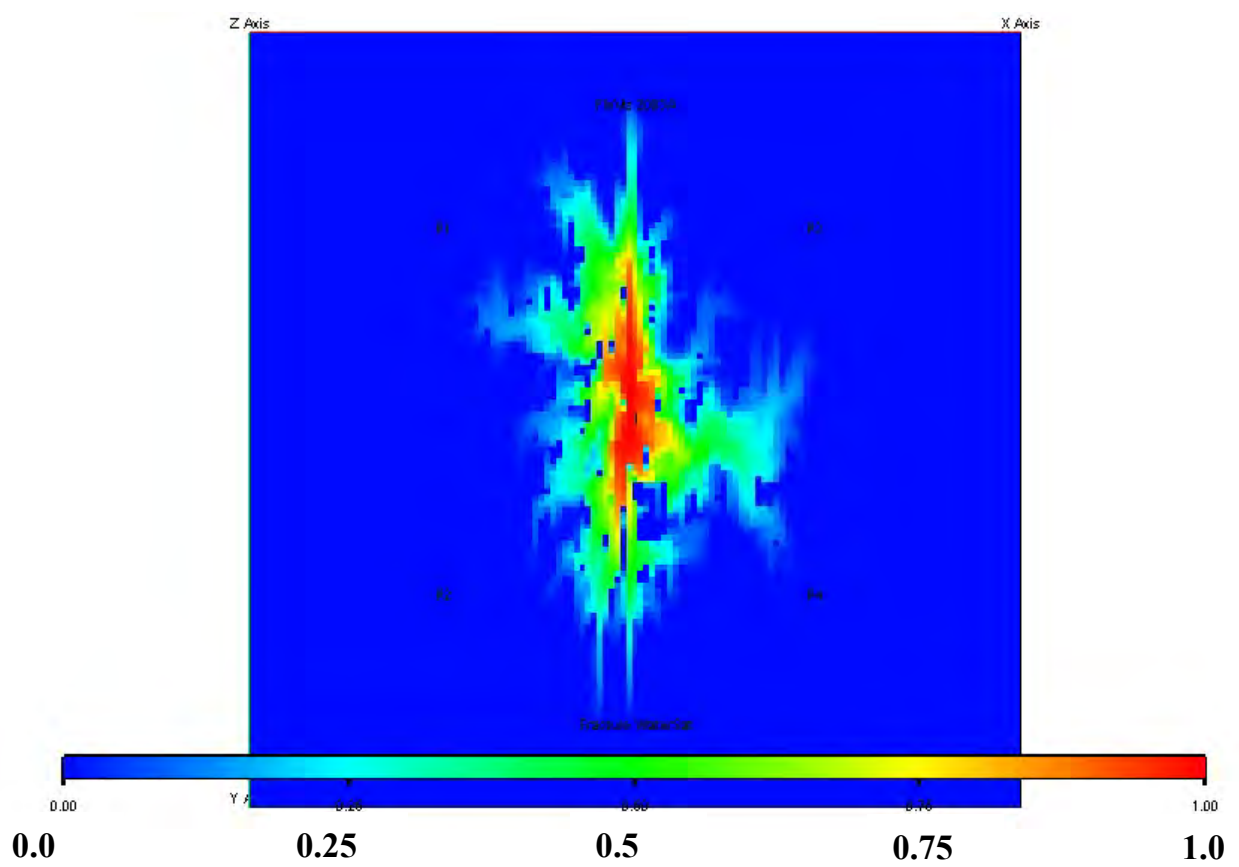

Fig. C.20b - Fracture water saturation at report step 10 (300 days) for test T3 initial estimate. Production only objective function.

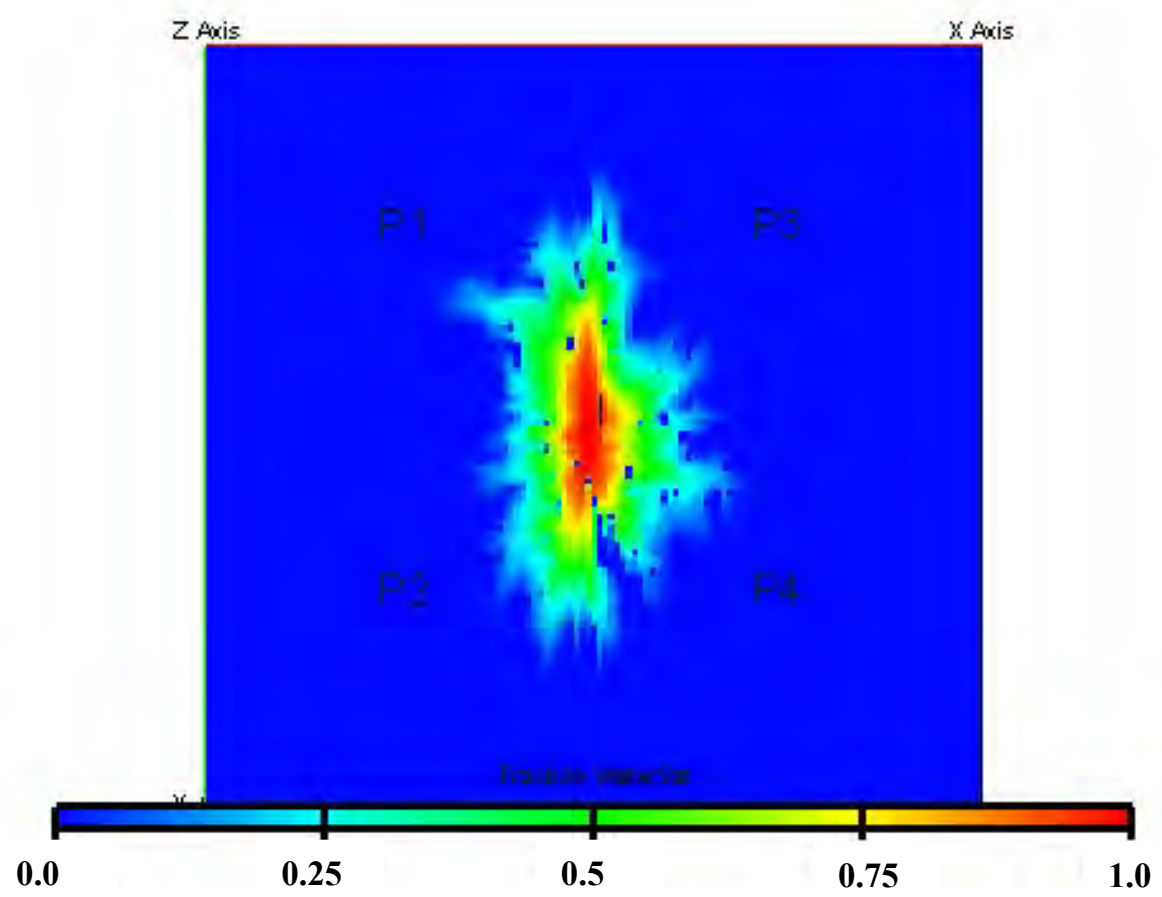

Fig. C.20c - Fracture water saturation at report step 10 (300 days) for test T3 final model. Production only objective function. 


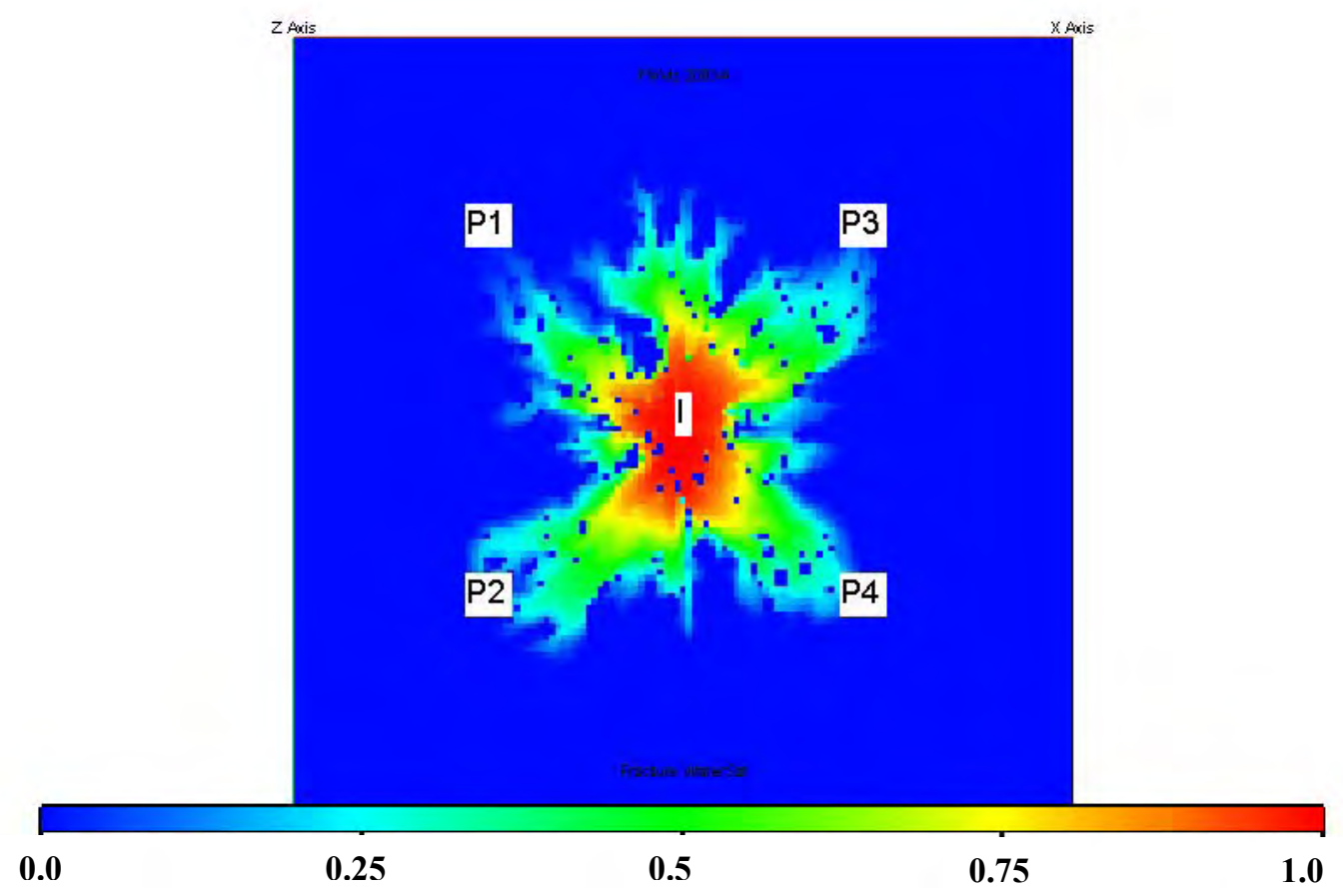

Fig. C.21a - Fracture water saturation at report step 15 (450 days) for test T3 base model. Production only objective function.

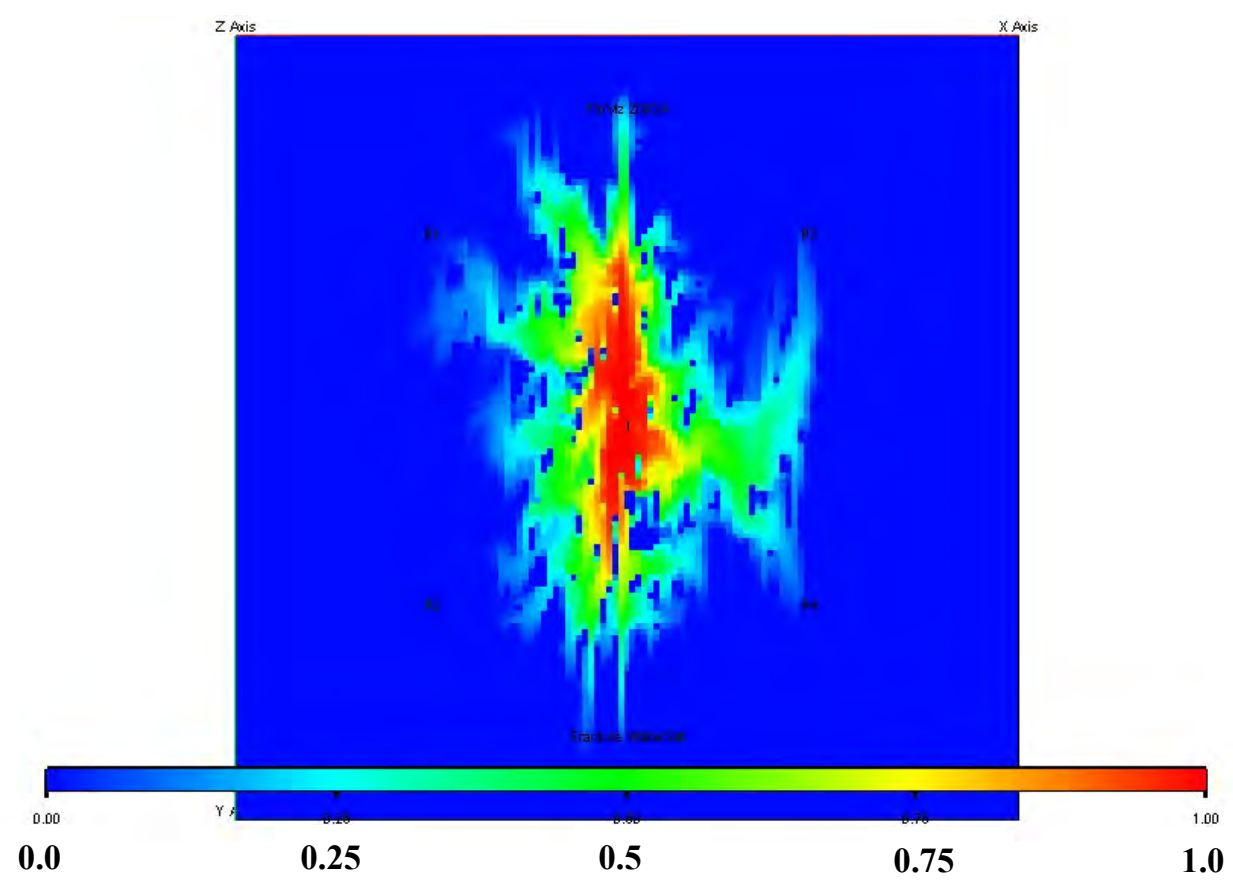

Fig. C.21b - Fracture water saturation at report step 15 (450 days) for test T3 initial estimate. Production only objective function. 


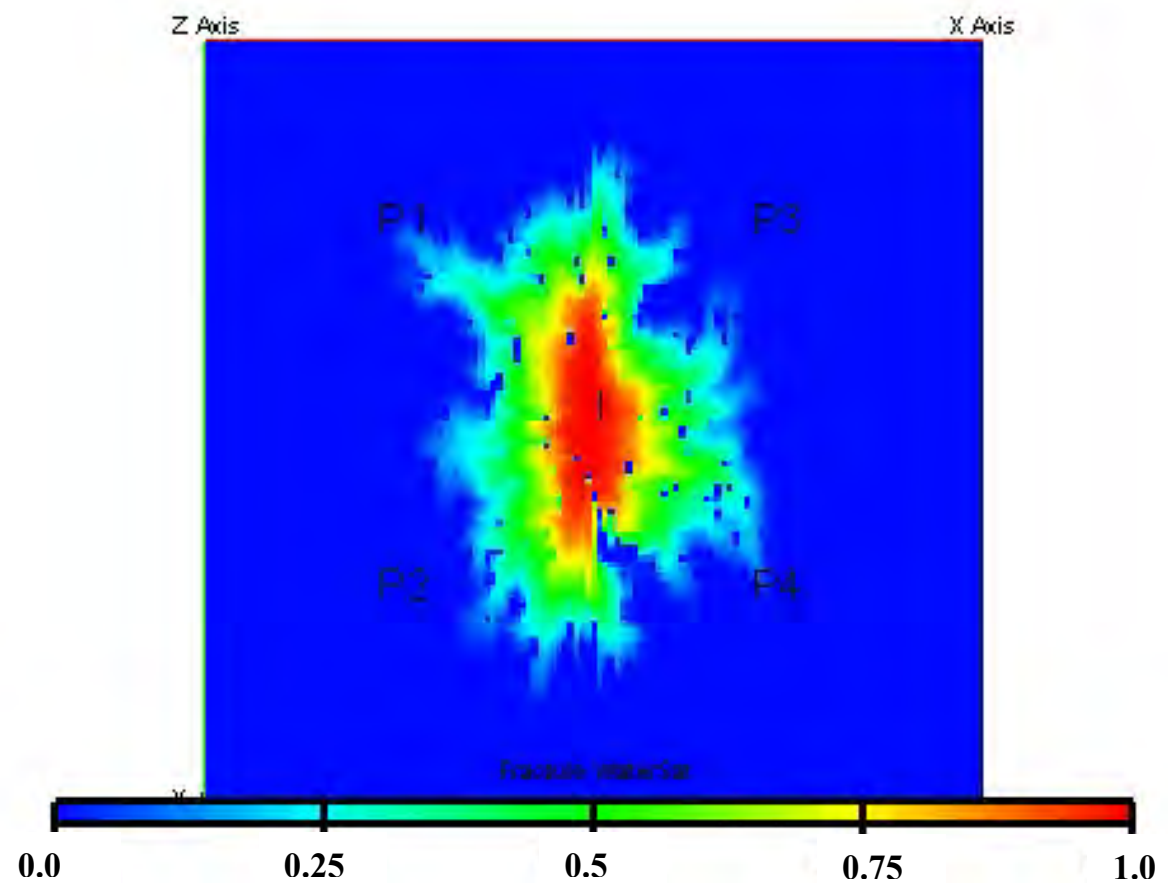

Fig. C.21c - Fracture water saturation at report step 15 (450 days) for test T3 final model. Production only objective function.

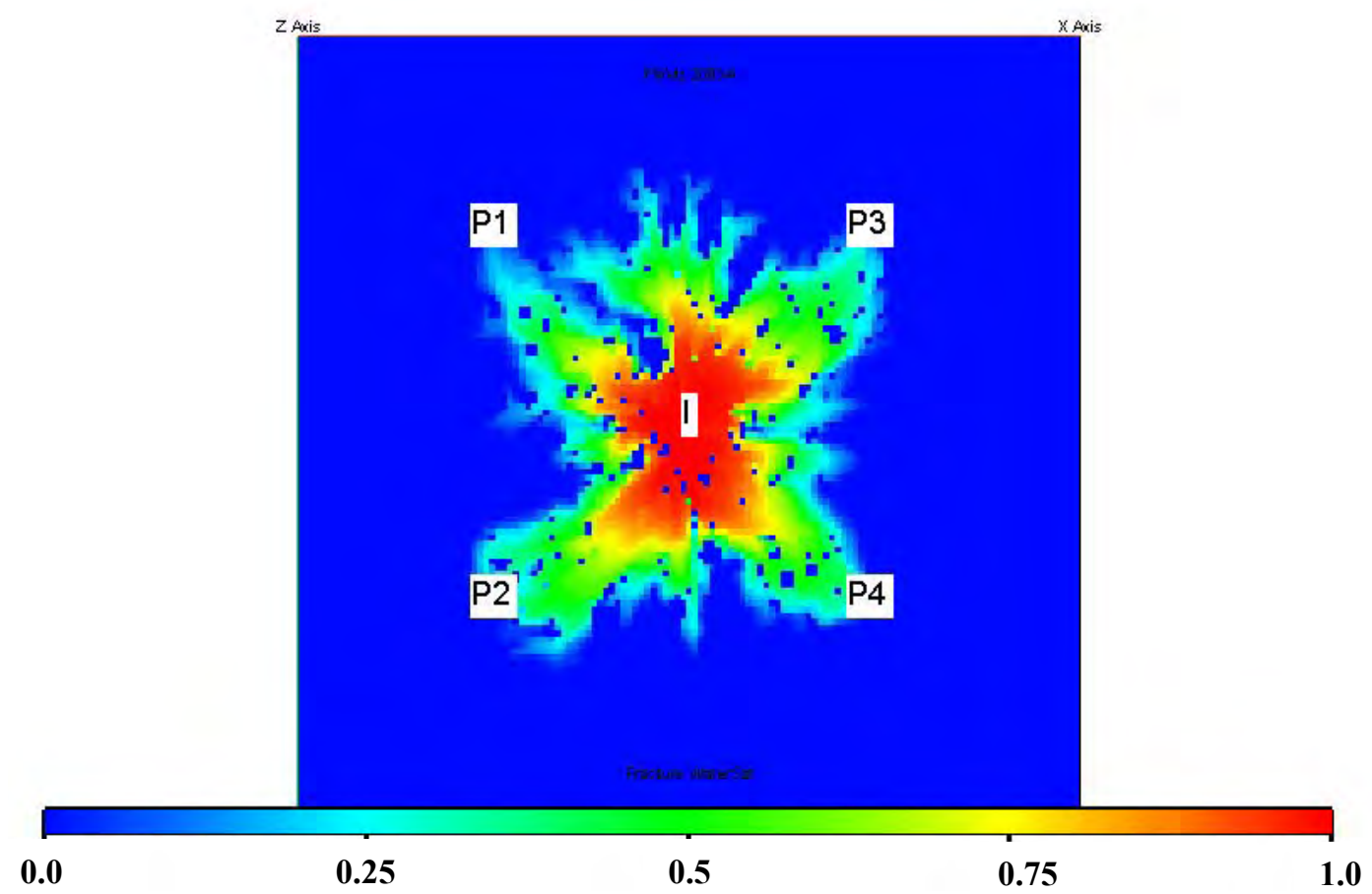

Fig. C.22a - Fracture water saturation at report step 20 (600 days) for test T3 base model. Production only objective function. 


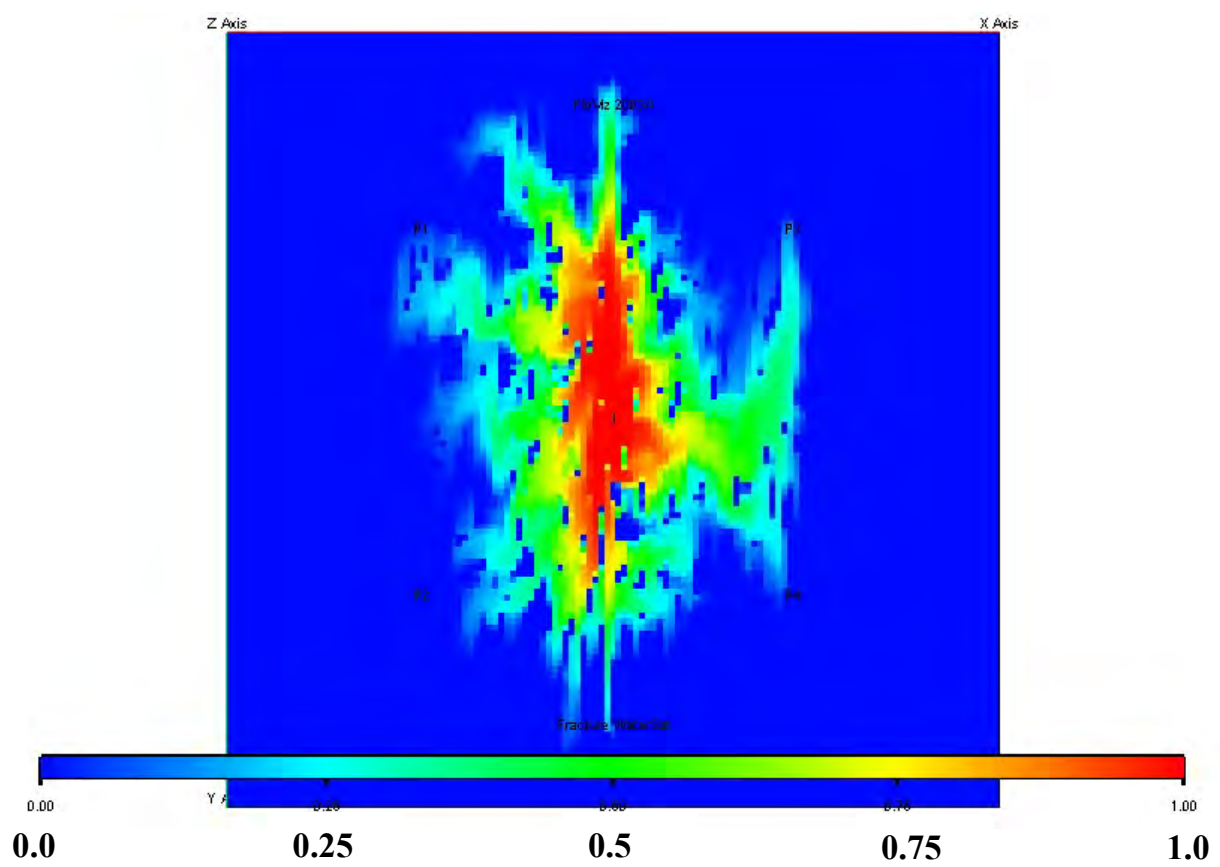

Fig. C.22b - Fracture water saturation at report step 20 (600 days) for test T3 initial estimate. Production only objective function.

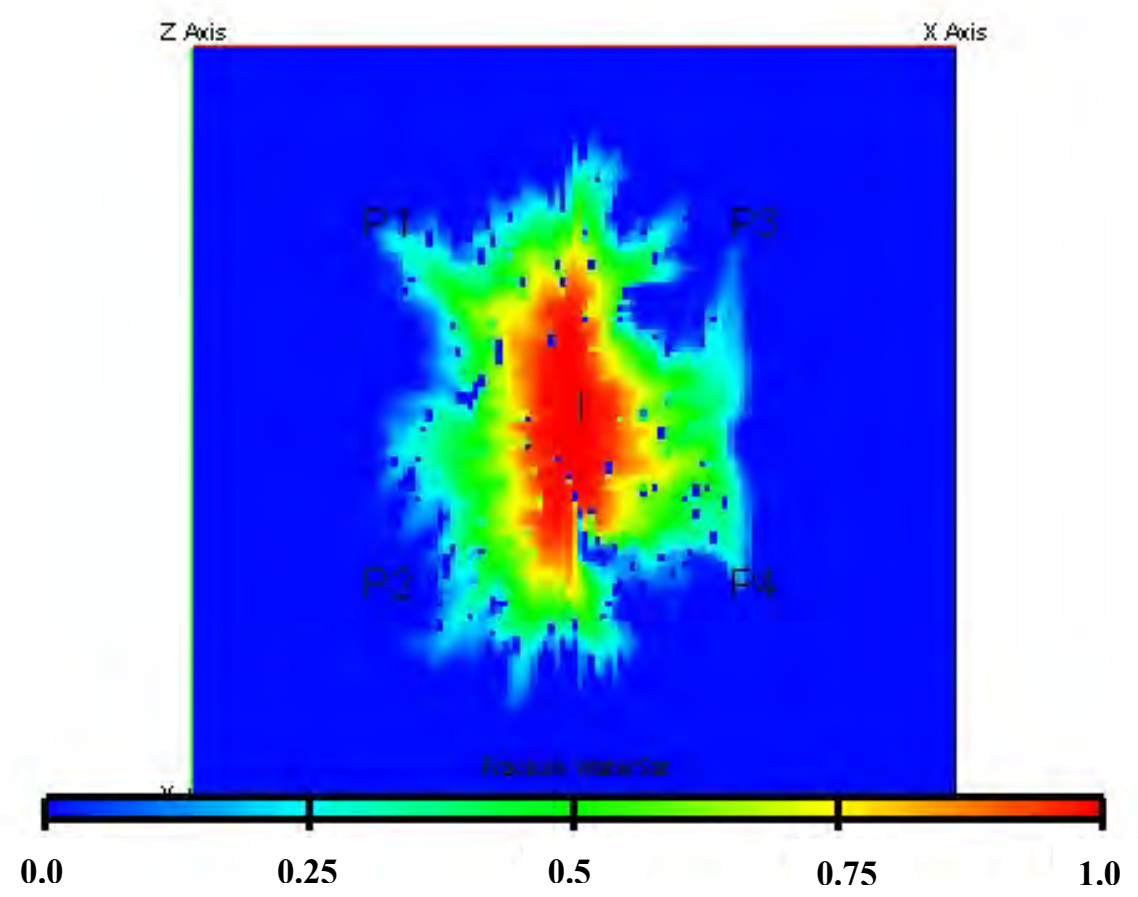

Fig. C.22c - Fracture water saturation at report step 20 (600 days) for test T3 final model. Production only objective function. 


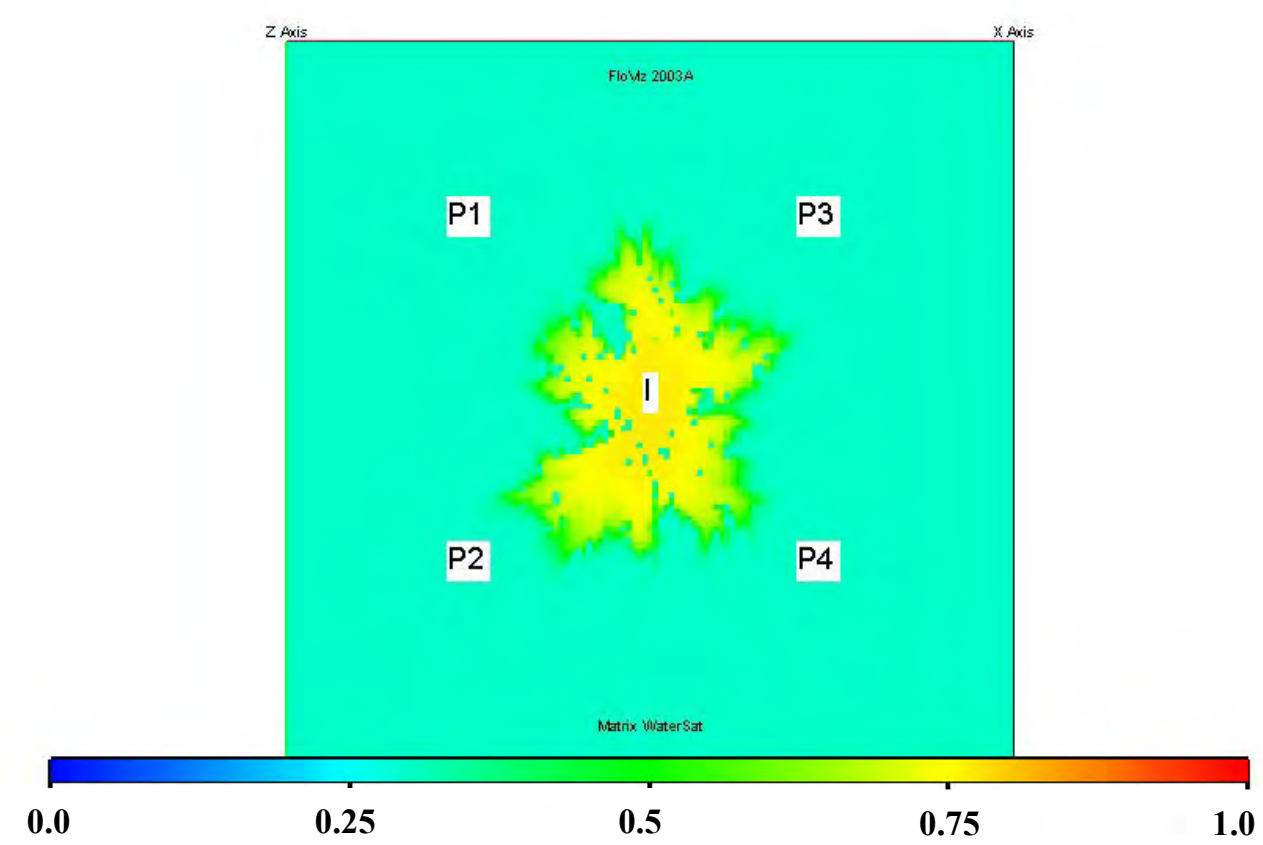

Fig. C.23 a - Matrix water saturation at report step 5 (150 days) for test T3 base model. Production only objective function.

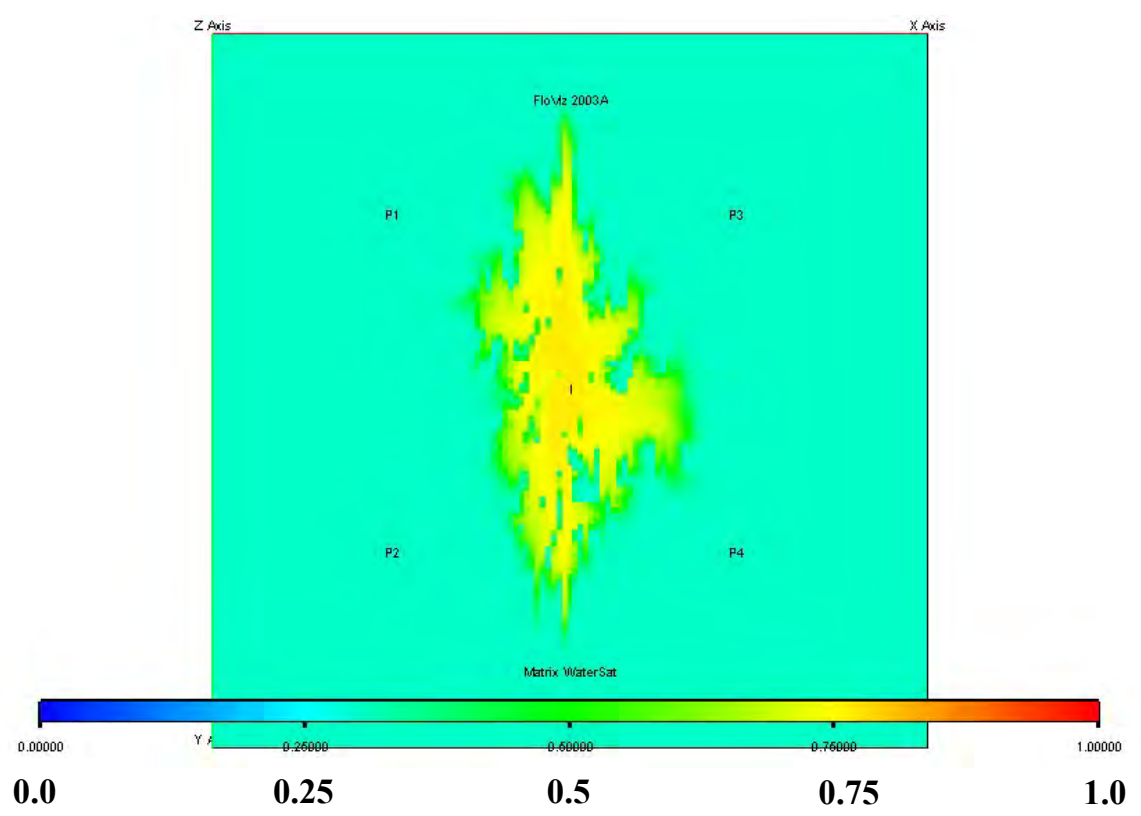

Fig. C.23b - Matrix water saturation at report step 5 (150 days) for test T3 initial estimate. Production only objective function. 

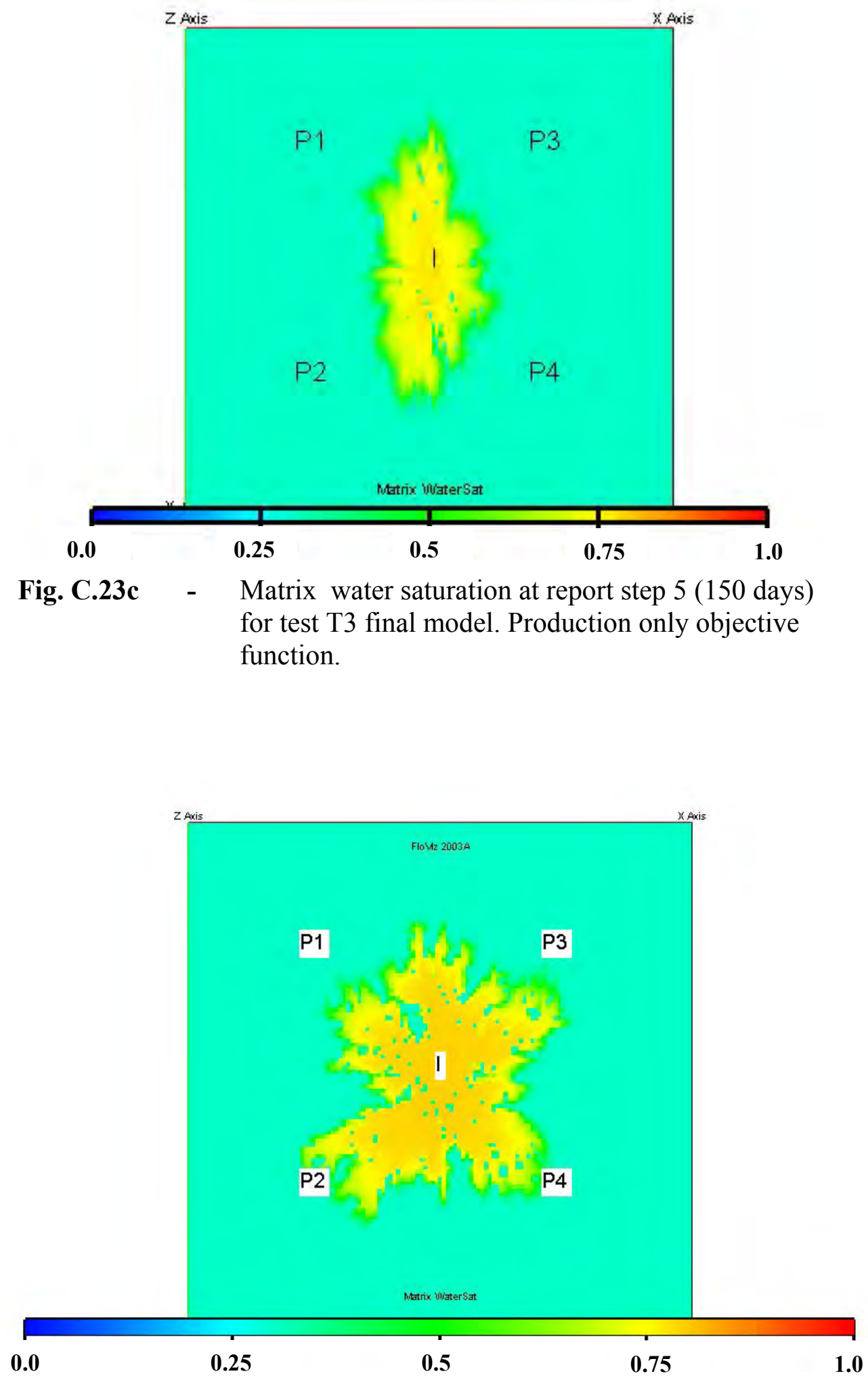

Fig. C.24 a $\quad-\quad$ Matrix water saturation at report step 10 (300 days) for test T3 base model. Production only objective function. 


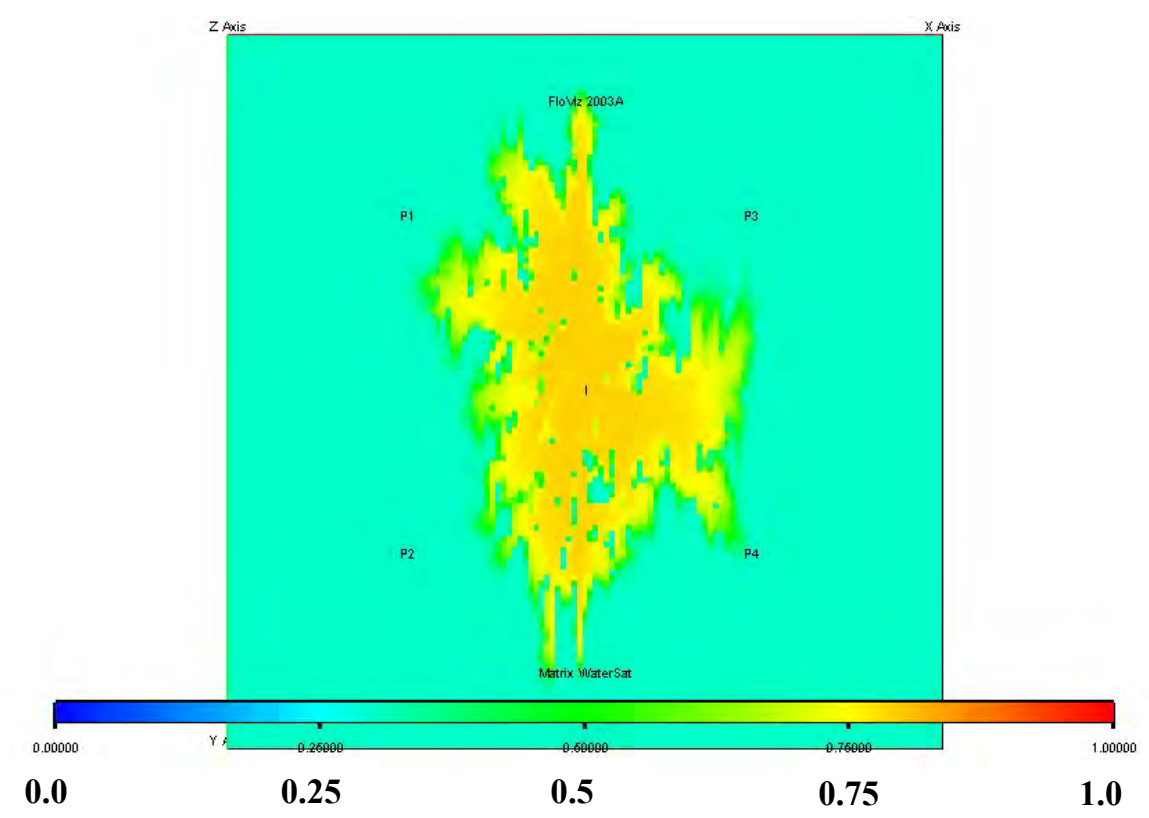

Fig. C.24b - Matrix water saturation at report step 10 (300 days) for test T3 initial estimate. Production only objective function.

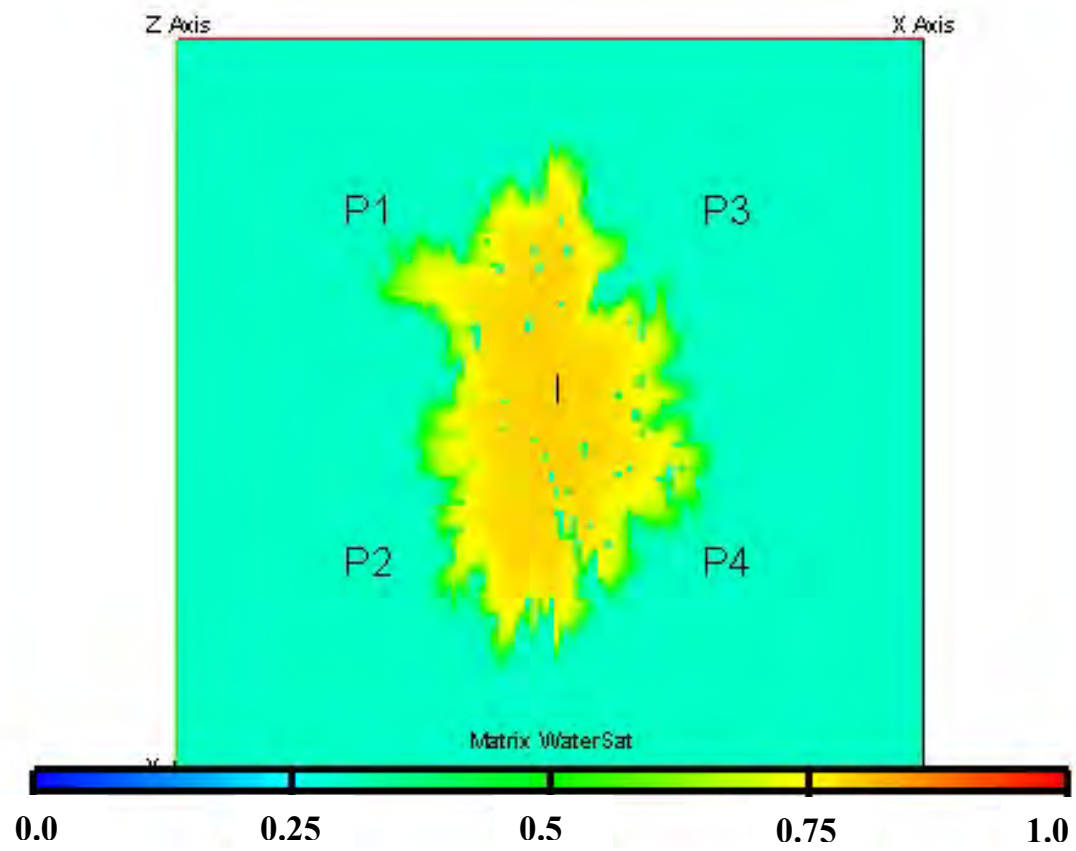

Fig. C.24c - Matrix water saturation at report step 10 (300 days) for test T3 final model. Production only objective function. 


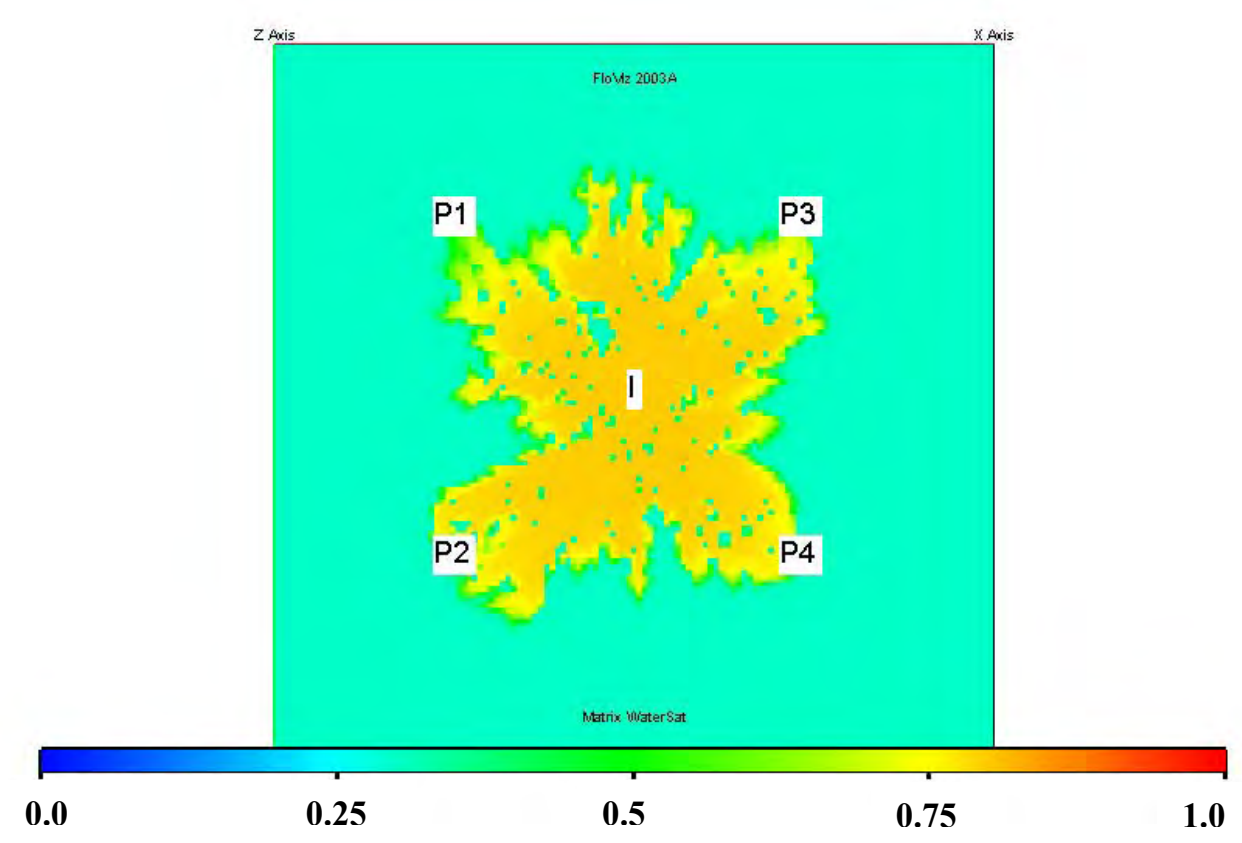

Fig. C.25 a $\quad-\quad$ Matrix water saturation at report step 15 (450 days) for test T3 base model. Production only objective function.

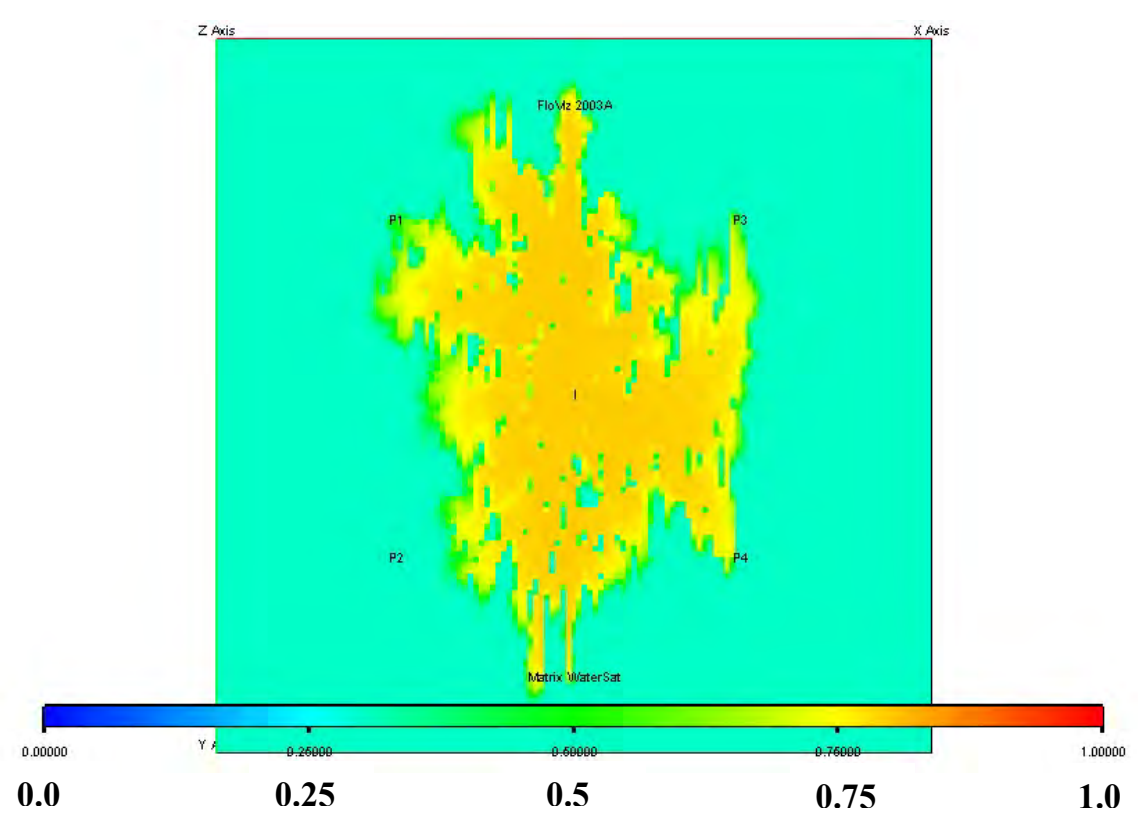

Fig. C.25b - Matrix water saturation at report step 15 (450 days) for test T3 initial estimate. Production only objective function. 


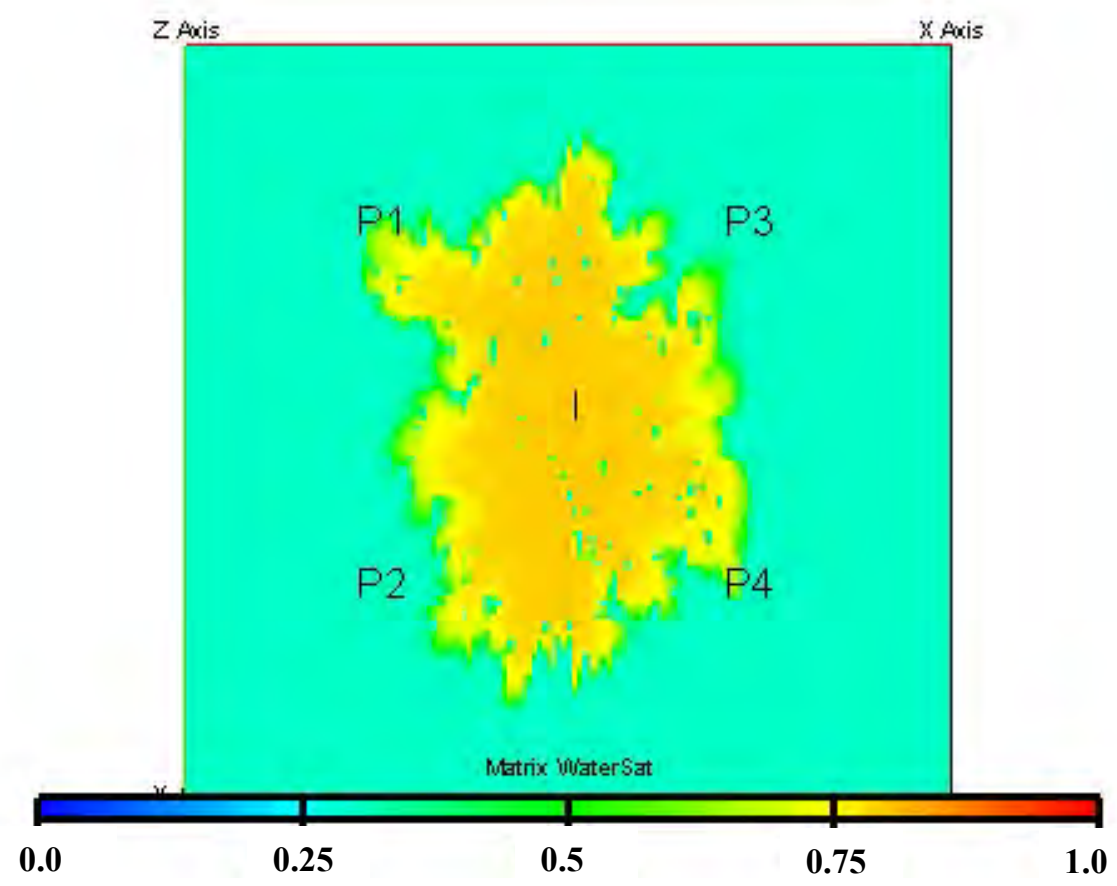

Fig. C.25c - Fracture Matrix water saturation at report step 15 (450 days) for test T3 final model. Production only objective function.

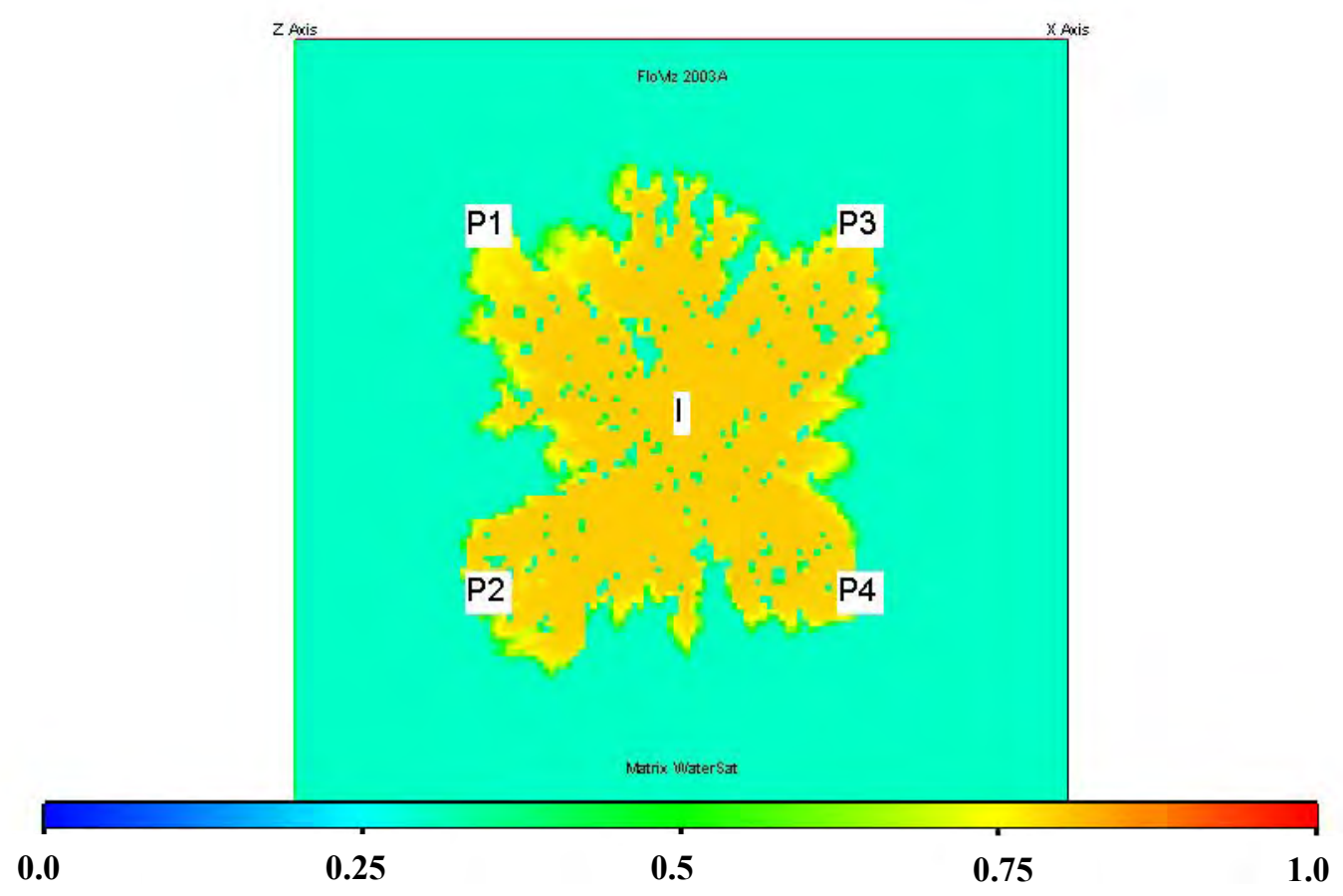

Fig. C.26 a - Matrix water saturation at report step 20 (600 days) for test T3 base model. . Production only objective function. 


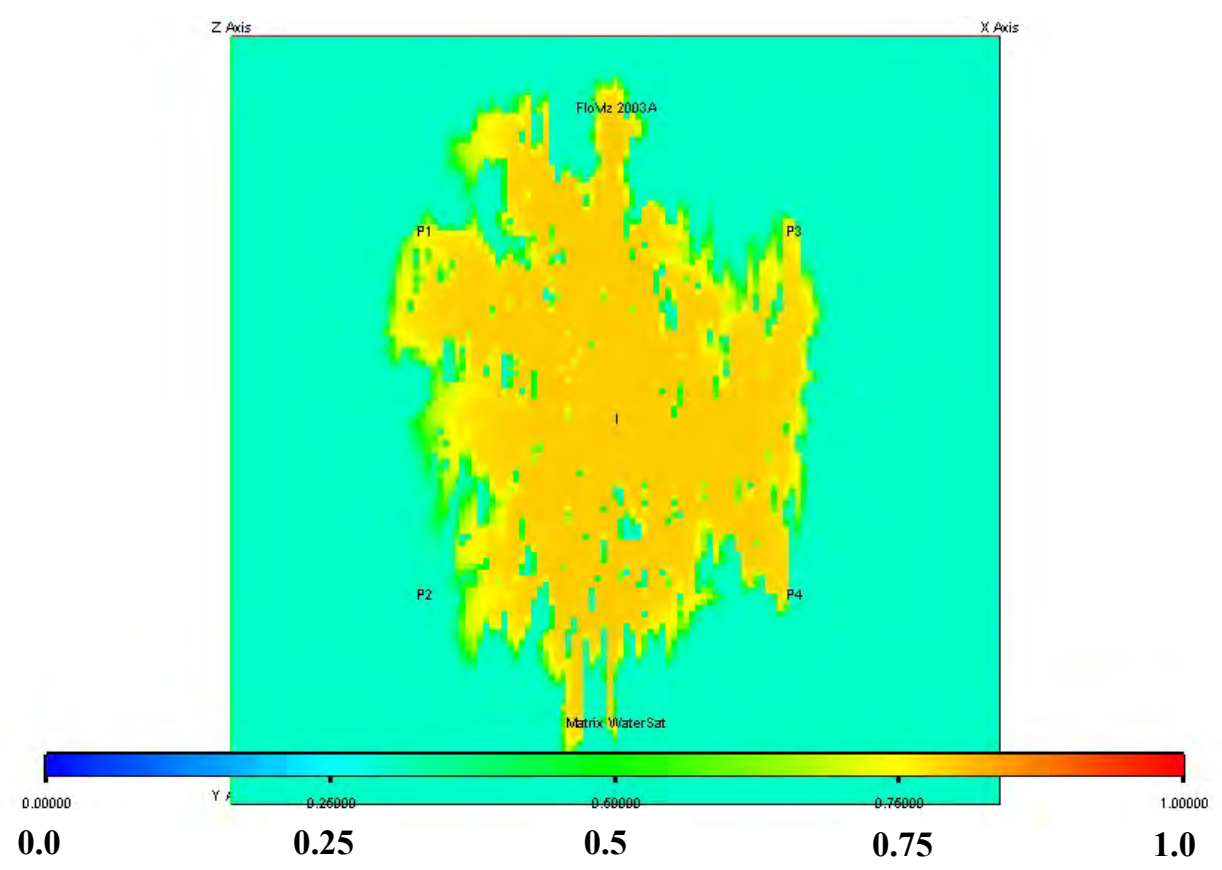

Fig. C.26b - Matrix water saturation at report step 20 (600 days) for test T3 initial estimate. Production only objective function.

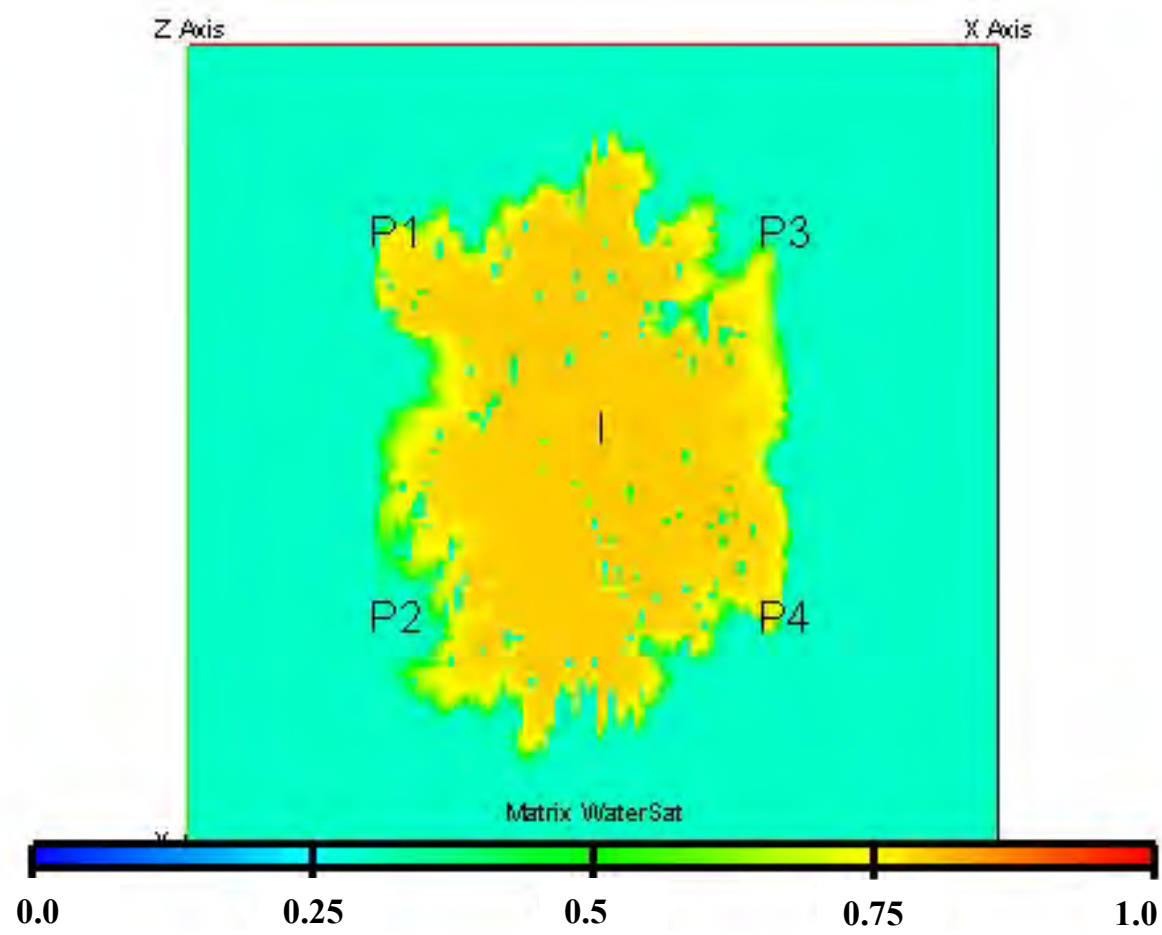

Fig. C.26c - Matrix water saturation at report step 20 (600 days) for test T3 final model. Production only objective function. 


\section{Bottom Hole Pressure - Production Data Only Objective Function}

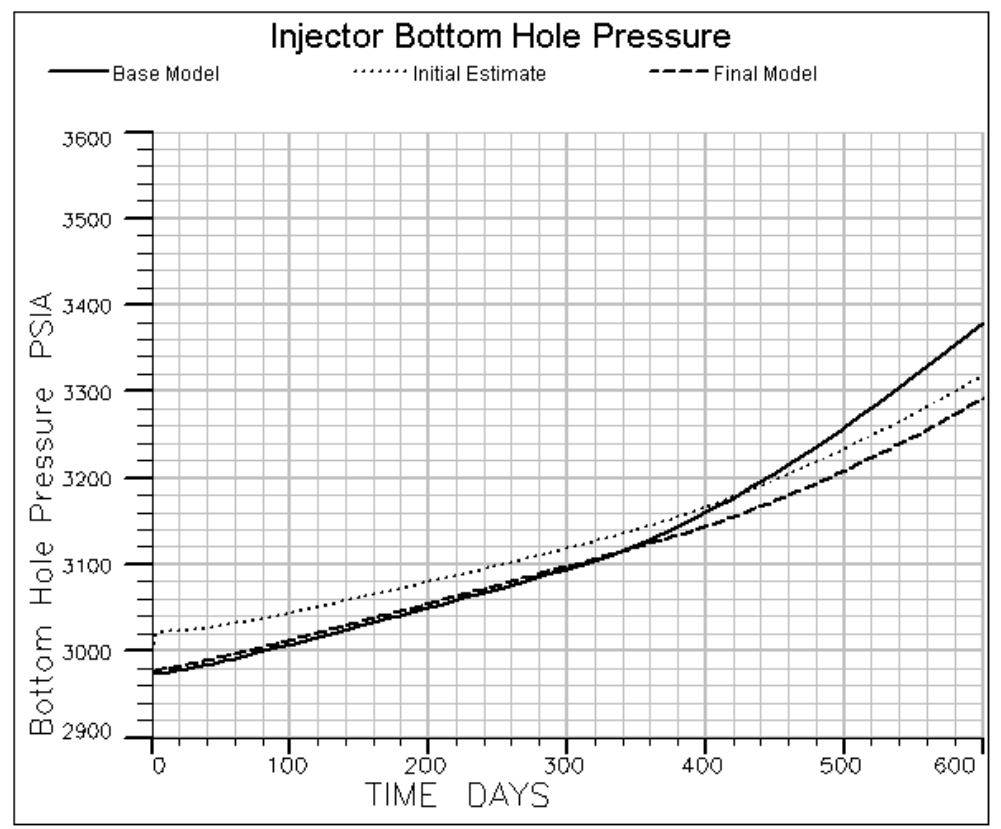

Fig. C.27a - Injector flowing bottom hole pressure for base model, initial estimate, and final model. Production only objective function.

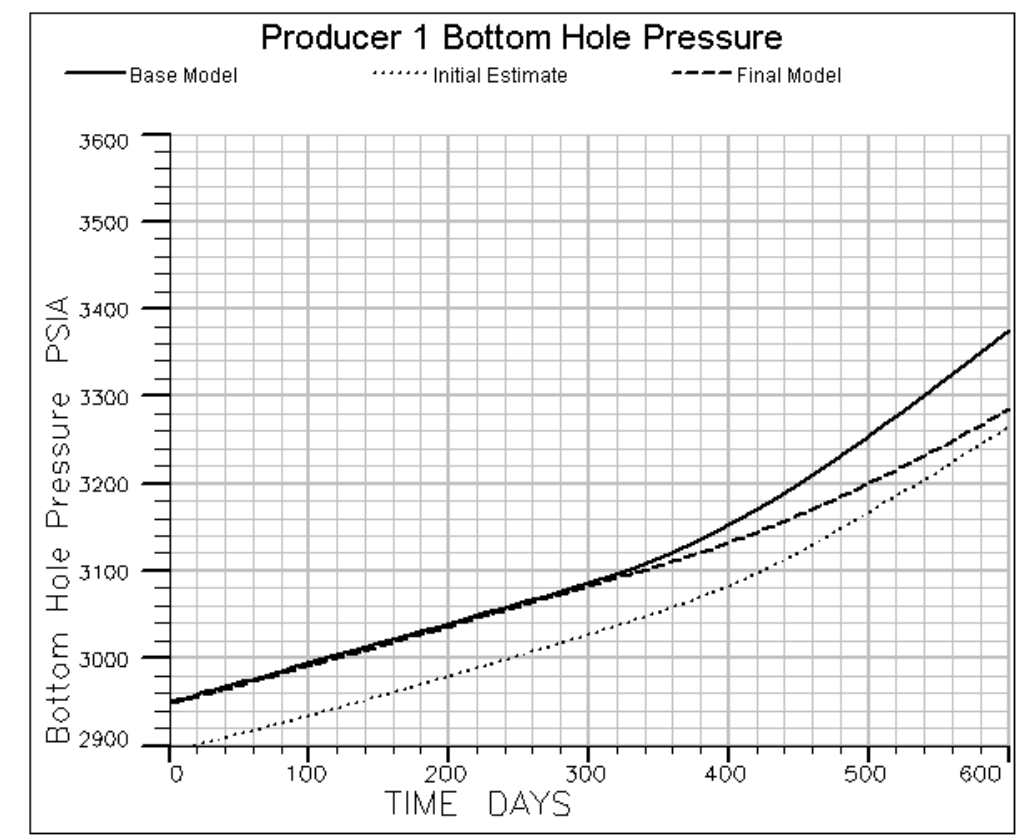

Fig. C.27b - Producer P1 flowing bottom hole pressure for base model, initial estimate, and final model. Production only objective function. 


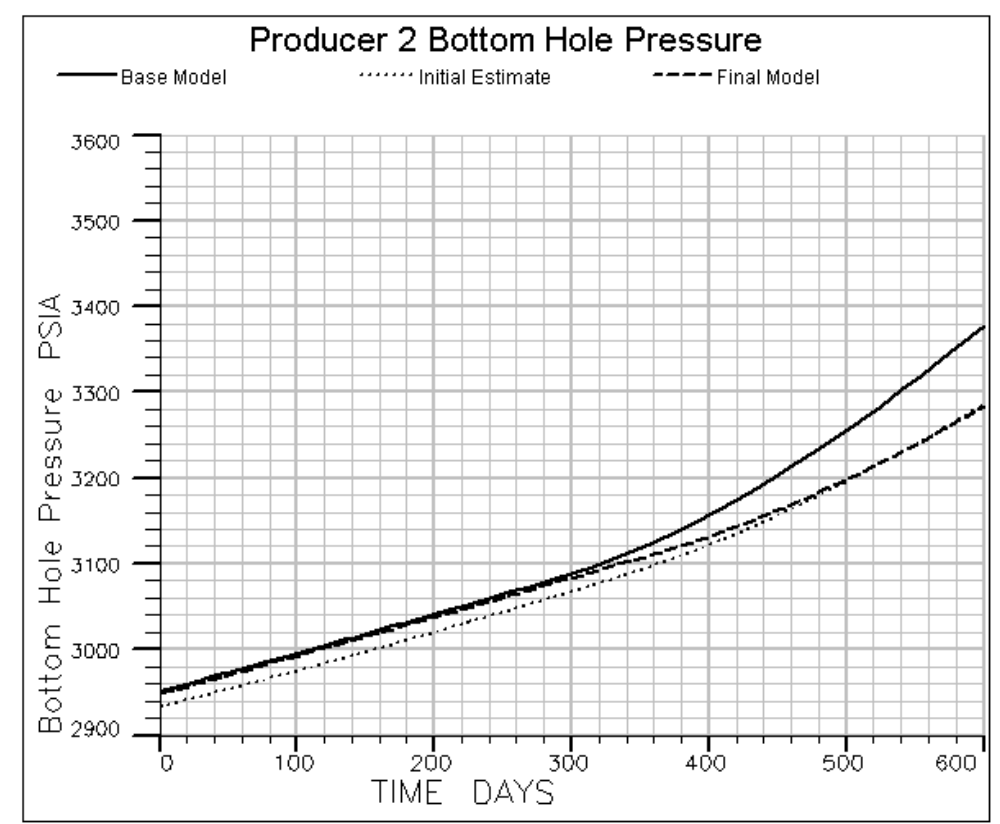

Fig. C.27c - Producer P2 flowing bottom hole pressure for base model, initial estimate, and final model. Production only objective function.

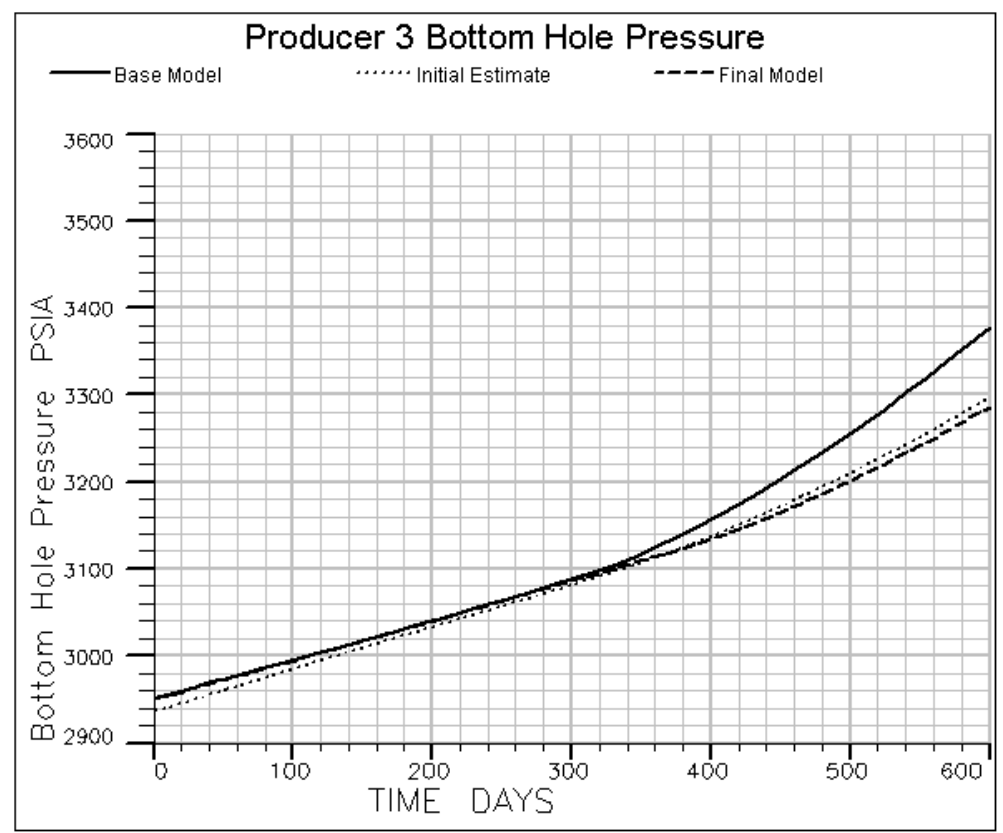

Fig. C.27d - Producer P3 flowing bottom hole pressure for base model, initial estimate, and final model. Production only objective function. 


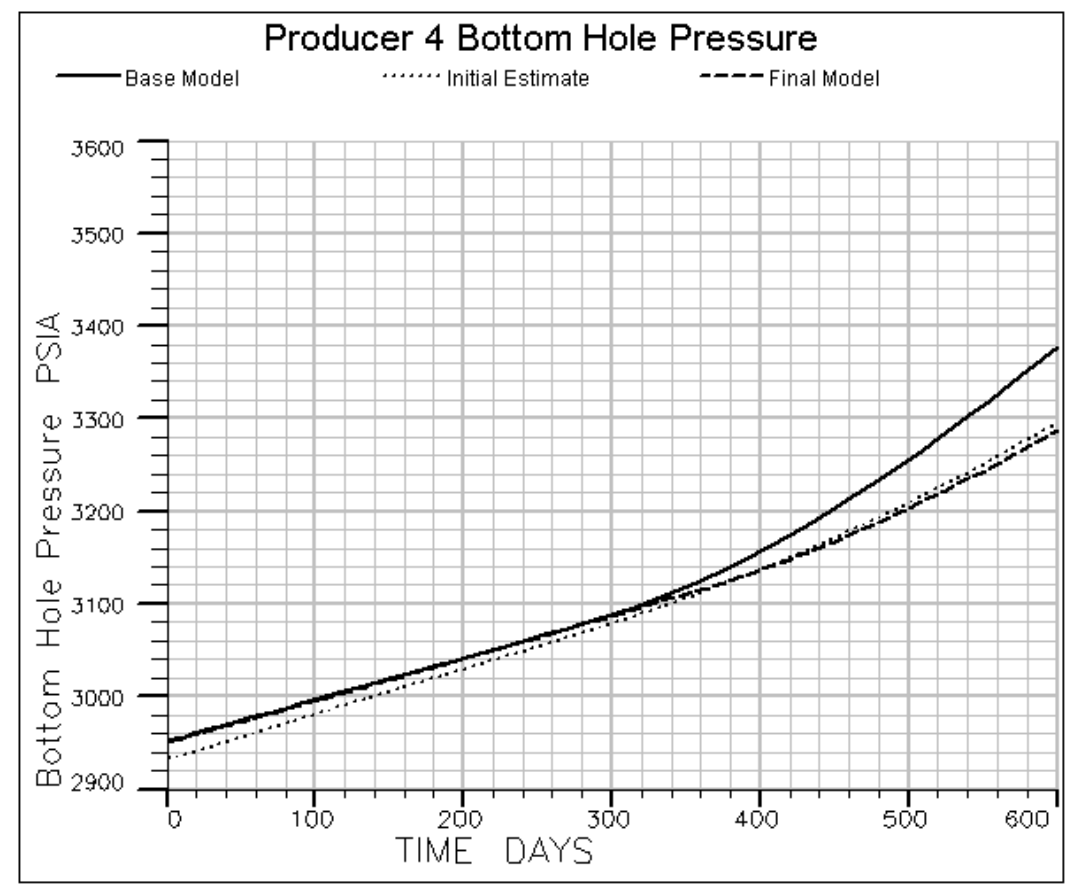

Fig. C.27e - Producer P4 flowing bottom hole pressure for base model, initial estimate, and final model. Production only objective function. 


\section{Oil Production Rate- Production Data Only Objective Function}

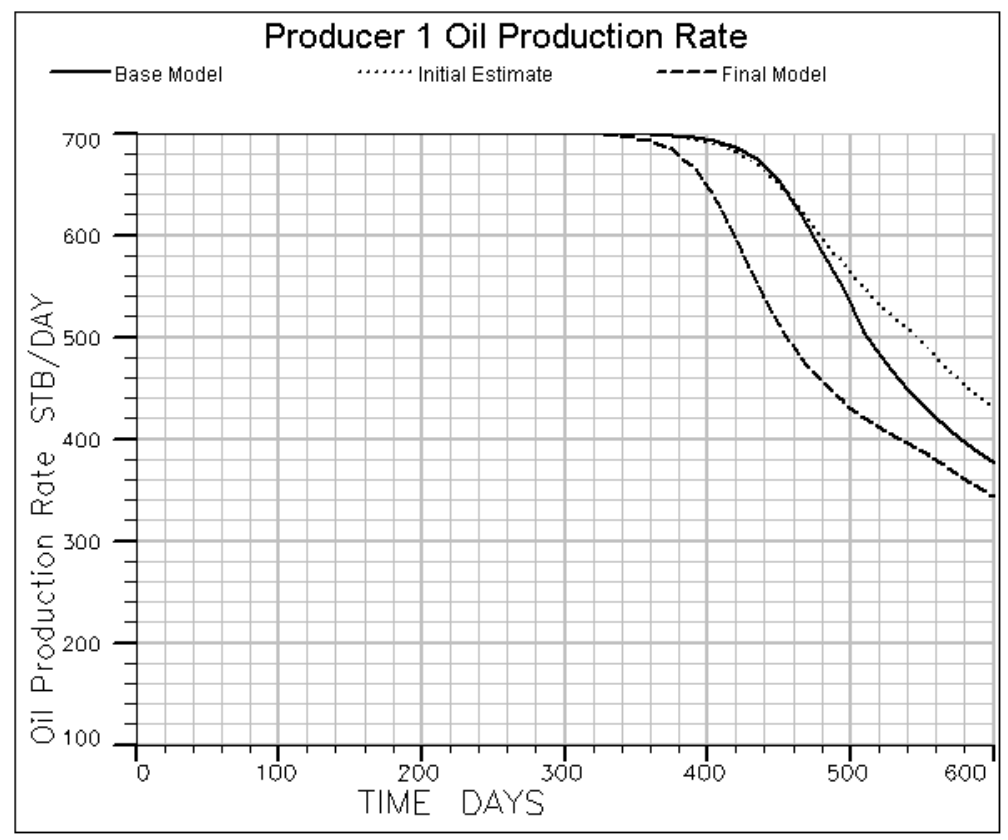

Fig. C.28a - Producer P1 oil production rate for base model, initial estimate, and final model. Production only objective function.

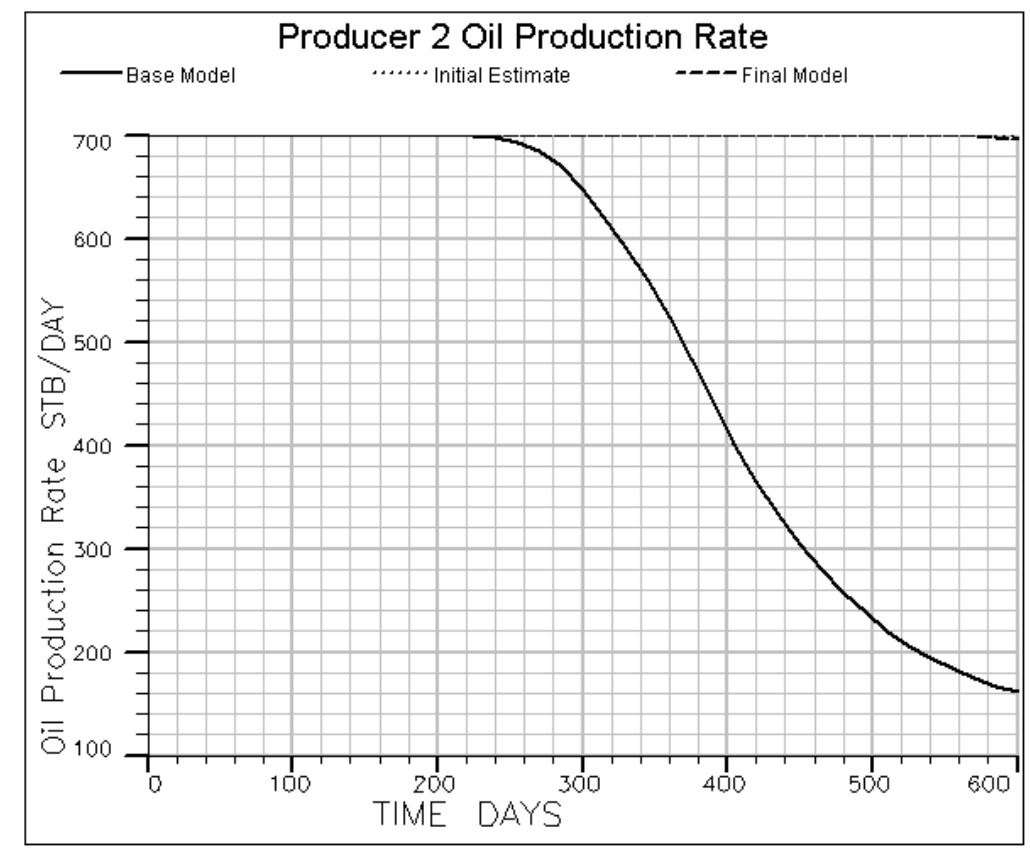

Fig. C.28b - Producer P2 oil production rate for base model, initial estimate, and final model. Production only objective function. 


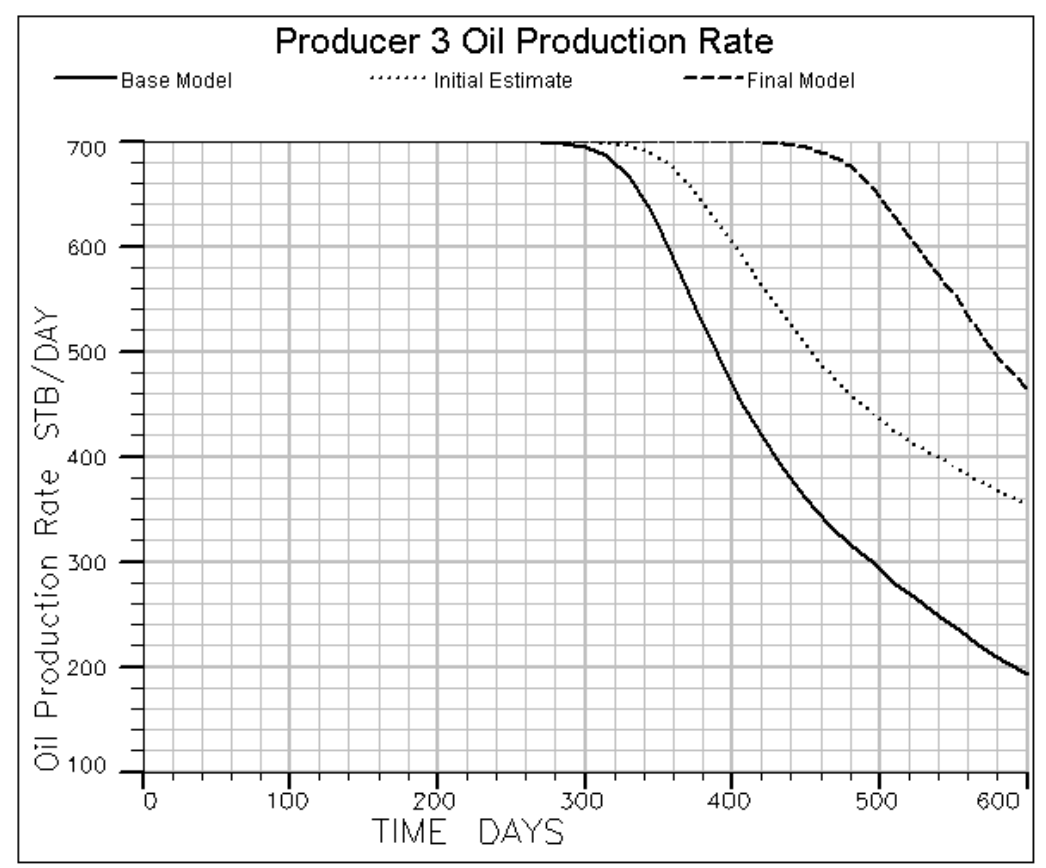

Fig. C.28c - Producer P3 oil production rate for base model, initial estimate, and final model. Production only objective function.

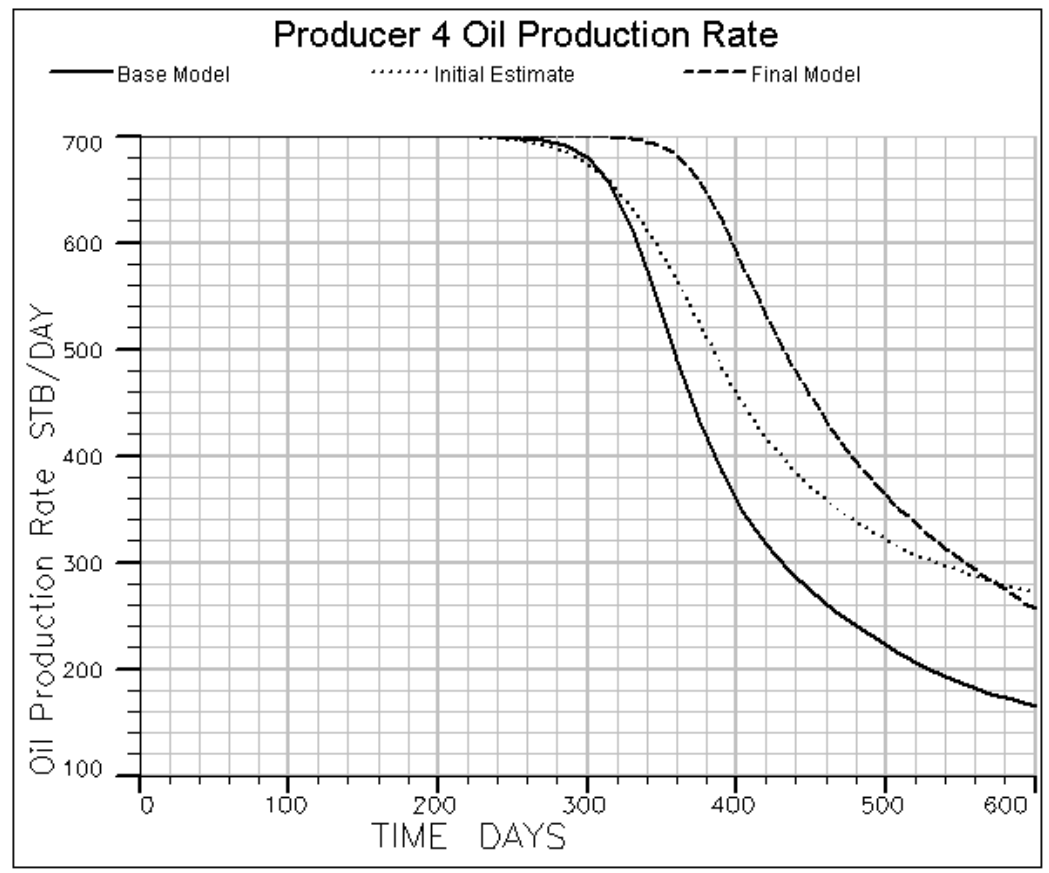

Fig. C.28d - Producer P4 oil production rate for base model, initial estimate, and final model. Production only objective function. 


\section{APPENDIX D}

\section{SUMMARY OF TEST T4 RESULTS}

In this Appendix we provide plots and tables detailing optimization test T4. As this dissertation is based upon the application of the new optimization process to the test cases we provide this Appendix as a comprehensive documentation set for this test case. Selected portions of this information may be found in Chapter IV with a concise description of the optimization test.

\section{General Information}

- Fracture system properties

- Petrophysical properties

- Simulation parameters

- Elastic properties

\section{Summarized Test Results}

- Parameter error summary table and plot

- Residual summary table and plot

\section{Data for Each Iteration}

- Table of derivative perturbations

- Table of prior model weights

- Tables of raw parameter updates

- Tables of raw residuals

- Plots of sensitivity coefficients 
For base case, initial estimate, and optimized model

- Plots of fracture distributions

- Maps of fracture system water saturation report step 150 days, 300 days, 450 days, 600 days

- Maps of matrix system water saturation report step 150 days, 300 days, 450 days, 600 days

- Bottom hole pressure plots

- Oil production rate plots

- Seismic P-wave velocity versus azimuth plots

- Seismic anisotropy attribute maps 
Table D.1 - Test T4 Base Model Fracture Distribution Parameters.

\begin{tabular}{|l|c|c|}
\hline \multicolumn{1}{|c|}{ Set } & Set 1 & Set 1 \\
\hline Trend (Geographical) & $\mathrm{N}[335,10]$ & $\mathrm{N}[45,10]$ \\
\hline P32 Intensity & 0.1 & $0.151 / \mathrm{m}$ \\
\hline Length & $\mathrm{LN}[50,3]$ & $\mathrm{LN}[50,3] \mathrm{m}$ \\
\hline Height & 60 & $60 \mathrm{~m}$ \\
\hline Termination & 50 & $50 \%$ \\
\hline Transmissivity & $\mathrm{LN}[8 \mathrm{e}-04,4.3 \mathrm{e}-05]$ & $\mathrm{LN}[8 \mathrm{e}-04,4.3 \mathrm{e}-05] \mathrm{m}^{2} / \mathrm{s}$ \\
\hline
\end{tabular}

Table D.2 - Test T4 Reservoir Properties.

\begin{tabular}{|l|c|}
\hline Width & $2624 \mathrm{ft}$ \\
\hline Length & $2624 \mathrm{ft}$ \\
\hline Thickness & $100 \mathrm{ft}$ \\
\hline Matrix Porosity & 0.13 \\
\hline Matrix Permeability & $0.1 \mathrm{mD}$ \\
\hline Fracture Porosity & 0.015 \\
\hline
\end{tabular}

Table D.3 - Test T4 Flow Simulation Parameters.

\begin{tabular}{|l|c|}
\hline Sigma & 0.08 \\
\hline Report step & 30 days \\
\hline Number cells (i) & 131 \\
\hline Number cells (j) & 131 \\
\hline Cell size (i) & $20 \mathrm{ft}$ \\
\hline Cell size (j) & $20 \mathrm{ft}$ \\
\hline
\end{tabular}

Table D.4 - Test T4 Elastic Modeling Parameters.

\begin{tabular}{|l|c|}
\hline Matrix P-wave Velocity & $5390 \mathrm{ft} / \mathrm{sec}$ \\
\hline Matrix Shear-wave Velocity & $2970 \mathrm{ft} / \mathrm{sec}$ \\
\hline Matrix Density & $2950 \mathrm{~kg} / \mathrm{m}^{3}$ \\
\hline Overburden P wave Velocity & $4670 \mathrm{ft} / \mathrm{sec}$ \\
\hline Overburden S wave Velocity & $3060 \mathrm{ft} / \mathrm{sec}$ \\
\hline Overburden Density & $2510 \mathrm{~kg} / \mathrm{m}^{3}$ \\
\hline Incidence Angle & $30 \mathrm{degrees}$ \\
\hline
\end{tabular}


Table D.5 - Test T4 Derivative Perturbations.

\begin{tabular}{|l|r|r|r|r|r|r|r|r|}
\hline Iterations & \multicolumn{1}{|c|}{$\mathbf{1}$} & \multicolumn{1}{l|}{$\mathbf{2}$} & \multicolumn{1}{l|}{$\mathbf{3}$} & \multicolumn{1}{c|}{$\mathbf{4}$} & \multicolumn{1}{c|}{$\mathbf{5}$} & \multicolumn{1}{c|}{$\mathbf{6}$} & \multicolumn{1}{c|}{$\mathbf{7}$} & \multicolumn{1}{c|}{$\mathbf{8}$} \\
\hline Trend 1 (degrees) & -10 & -10 & -15 & -5 & -5 & -5 & -5 & -5 \\
\hline P32 1 (1/m) & -0.05 & -0.1 & -0.05 & -0.05 & -0.02 & -0.02 & -0.02 & -0.02 \\
\hline Trend 2 (degrees) & 10 & 5 & 5 & 5 & 5 & 5 & 5 & 5 \\
\hline P32 2 (1/m) & 0.05 & 0.1 & 0.1 & 0.05 & 0.05 & 0.02 & 0.02 & 0.02 \\
\hline
\end{tabular}

Table D.6 - Test T4 Prior Model Weights.

\begin{tabular}{|l|c|c|c|c|c|c|c|c|}
\hline Iterations & $\mathbf{1}$ & $\mathbf{2}$ & $\mathbf{3}$ & $\mathbf{4}$ & $\mathbf{5}$ & $\mathbf{6}$ & $\mathbf{7}$ & $\mathbf{8}$ \\
\hline Trend (1) & 4 & 4 & 0.75 & 4 & 4 & 4 & 4 & 4 \\
\hline P32 (1) & 4 & 4 & 4 & 4 & 2 & 1 & 1 & 1 \\
\hline Trend (2) & 4 & 4 & 4 & 8 & 8 & 1 & 1 & 1 \\
\hline P32 (2) & 4 & 0.25 & 2 & 1 & 2 & 1 & 0.5 & 0.5 \\
\hline
\end{tabular}

Table D.7 - Summary of absolute errors for fracture set trend and P32 intensity at each update step. Step 0 is initial estimate.

\begin{tabular}{|r|r|r|r|r|}
\cline { 2 - 5 } \multicolumn{1}{c|}{} & \multicolumn{5}{|c|}{ Parameter Error } \\
\hline Iteration & Trend (1) & \multicolumn{1}{c|}{ P32 (1) } & Trend (2) & P32 (2) \\
\hline $\mathbf{0}$ & 20.0 & 0.100 & 20.0 & 0.050 \\
\hline $\mathbf{1}$ & 21.4 & 0.090 & 3.4 & 0.111 \\
\hline $\mathbf{2}$ & 22.0 & 0.061 & 2.5 & 0.080 \\
\hline $\mathbf{3}$ & 3.1 & 0.041 & 4.6 & 0.054 \\
\hline $\mathbf{4}$ & 0.4 & 0.029 & 5.4 & 0.043 \\
\hline $\mathbf{5}$ & 1.1 & 0.020 & 4.9 & 0.029 \\
\hline $\mathbf{6}$ & 1.5 & 0.001 & 0.6 & 0.019 \\
\hline $\mathbf{7}$ & 1.3 & 0.002 & 0.2 & 0.005 \\
\hline $\mathbf{8}$ & 0.7 & 0.001 & 0.8 & 0.001 \\
\hline
\end{tabular}


Table D.8 - Summary of unit normalized residual errors by observation type for fracture set trend and P32 intensity at each iteration. Iteration 0 is initial estimate.

\begin{tabular}{|r|r|r|r|r|}
\cline { 2 - 5 } \multicolumn{1}{c|}{} & \multicolumn{4}{c|}{ Residuals } \\
\hline Iteration & \multicolumn{1}{c|}{ Total } & \multicolumn{1}{c|}{ BHP } & \multicolumn{1}{c|}{ OPR } & \multicolumn{1}{c|}{ SEIS } \\
\hline $\mathbf{0}$ & 21.29 & 5.39 & 1.58 & 20.58 \\
\hline $\mathbf{1}$ & 14.72 & 5.45 & 2.06 & 13.52 \\
\hline $\mathbf{2}$ & 44.07 & 42.65 & 1.54 & 10.99 \\
\hline $\mathbf{3}$ & 10.51 & 2.34 & 8.97 & 4.96 \\
\hline $\mathbf{4}$ & 10.84 & 10.01 & 0.61 & 4.09 \\
\hline $\mathbf{5}$ & 6.95 & 6.13 & 0.90 & 3.14 \\
\hline $\mathbf{6}$ & 3.32 & 3.16 & 0.55 & 0.83 \\
\hline $\mathbf{7}$ & 8.13 & 8.07 & 0.55 & 0.79 \\
\hline
\end{tabular}

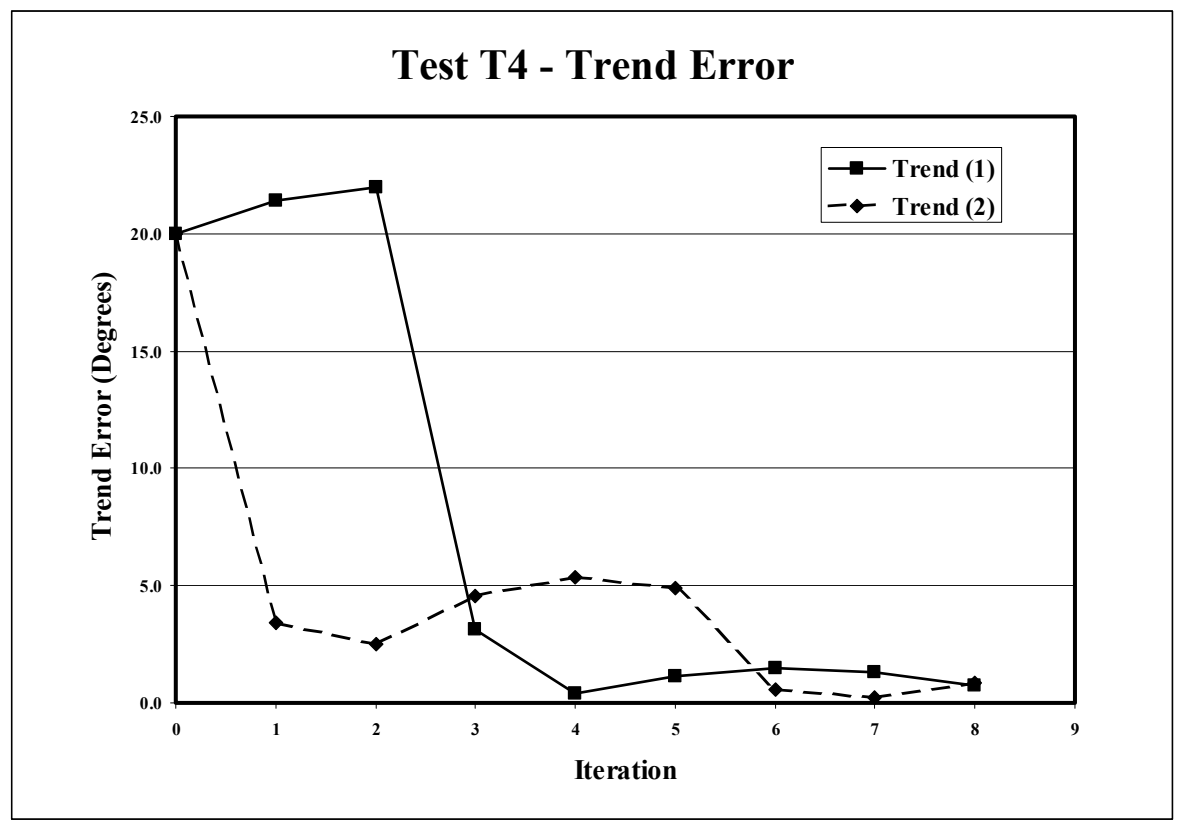

Fig. D.1 - Graph of absolute error in fracture set trend versus iteration for test $\mathrm{T} 4$. Iteration 0 is initial estimate. 


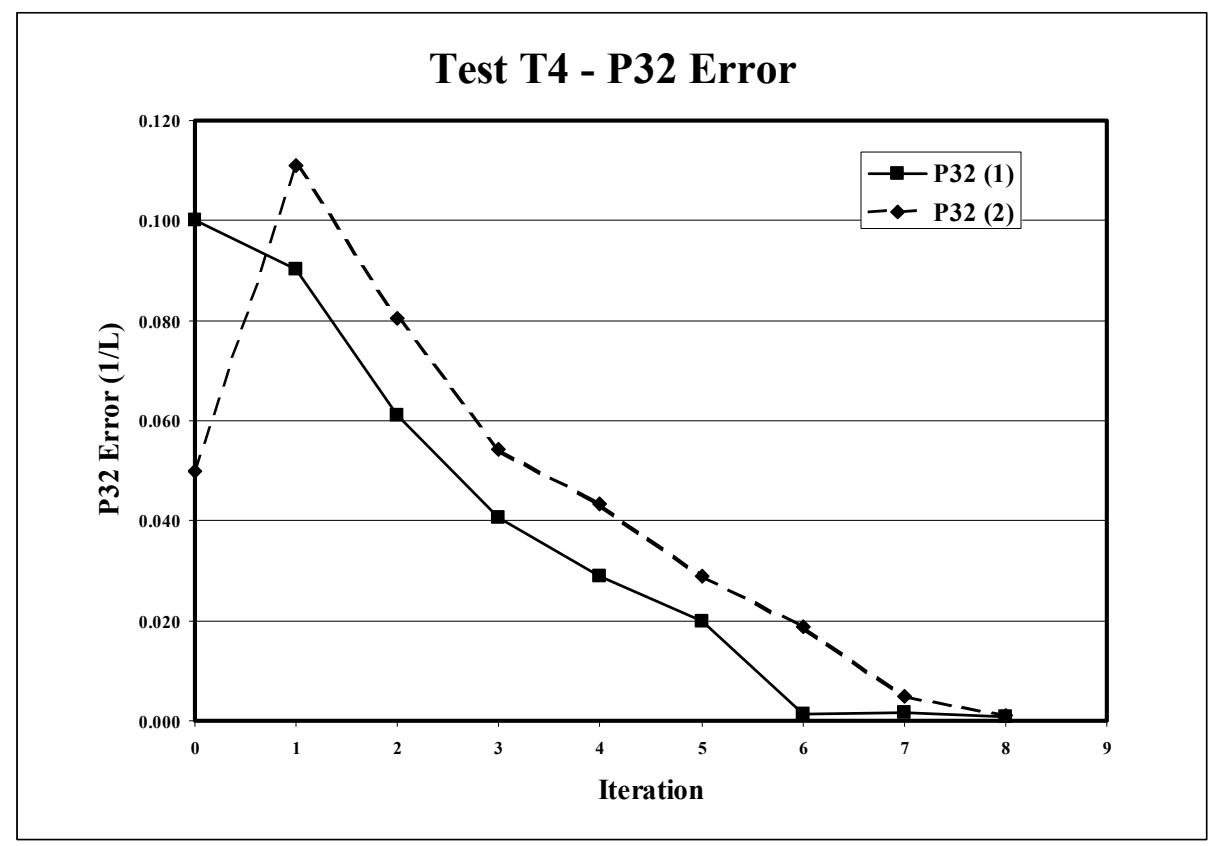

Fig. D.2 - Graph of absolute error in fracture set P32 intensity versus iteration for test T4. Iteration 0 is initial estimate.

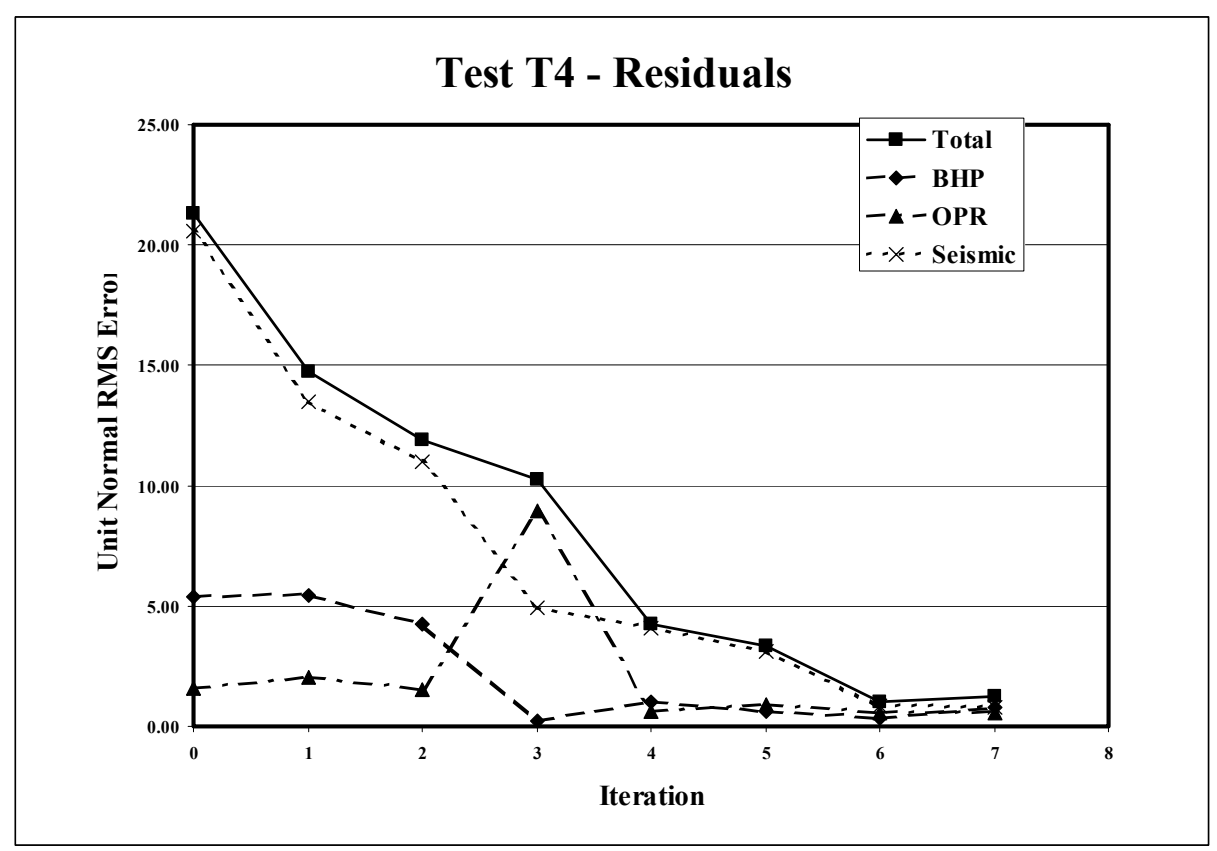

Fig. D.3 - Graph of unit normalized residual errors by observation type for fracture set trend and P32 intensity at each iteration. Iteration 0 is initial estimate. 
Table D.9a - Summary of iteration 1 Trend and P32 intensity updates and residual errors for 10 samples.

\begin{tabular}{|c|c|c|c|c|c|c|c|c|}
\hline & \multicolumn{4}{|c|}{ Step M1 } & \multirow{2}{*}{\multicolumn{4}{|c|}{ Residuals }} \\
\hline & \multicolumn{4}{|c|}{ Updates } & & & & \\
\hline SAMPLE & Trend (1) & P32 (1) & Trend (2) & P32 (2) & TOTAL & BHP & OPR & SEIS \\
\hline 1 & 86.4 & 0.196 & 128.4 & 0.032 & 21.30 & 5.50 & 1.50 & 20.60 \\
\hline 2 & 86.2 & 0.183 & 125.6 & 0.051 & 21.30 & 5.30 & 1.40 & 20.60 \\
\hline 3 & 85.5 & 0.183 & 124.0 & 0.049 & 21.40 & 5.30 & 2.00 & 20.70 \\
\hline 4 & 87.2 & 0.200 & 128.1 & 0.029 & 21.30 & 5.50 & 1.50 & 20.60 \\
\hline 5 & 85.7 & 0.176 & 121.6 & 0.058 & 21.20 & 5.60 & 1.40 & 20.40 \\
\hline 6 & 85.8 & 0.160 & 117.0 & 0.079 & 21.20 & 5.20 & 1.40 & 20.50 \\
\hline 7 & 86.2 & 0.208 & 133.3 & 0.010 & 21.40 & 5.50 & 1.60 & 20.70 \\
\hline 8 & 86.9 & 0.191 & 127.1 & 0.037 & 21.30 & 5.40 & 1.60 & 20.60 \\
\hline 9 & 87.9 & 0.190 & 125.8 & 0.040 & 21.30 & 5.20 & 1.80 & 20.60 \\
\hline 10 & 86.3 & 0.217 & 134.9 & 0.005 & 21.20 & 5.40 & 1.60 & 20.50 \\
\hline Average & 86.4 & 0.190 & 126.6 & 0.039 & 21.29 & 5.39 & 1.58 & 20.58 \\
\hline
\end{tabular}

Table D.9b - Summary of iteration 2 Trend and P32 intensity updates and residual errors for 10 samples.

\begin{tabular}{|c|c|c|c|c|c|c|c|c|}
\hline & \multicolumn{8}{|c|}{ Step M2 } \\
\hline & \multicolumn{4}{|c|}{ Updates } & \multicolumn{4}{|c|}{ Residuals } \\
\hline SAMPLE & Trend (1) & P32 (1) & Trend (2) & P32 (2) & TOTAL & BHP & OPR & SEIS \\
\hline 1 & 87.3 & 0.161 & 127.5 & 0.107 & 14.66 & 5.56 & 1.80 & 13.44 \\
\hline 2 & 87.1 & 0.162 & 127.6 & 0.062 & 14.94 & 5.52 & 2.24 & 13.70 \\
\hline 3 & 86.8 & 0.161 & 127.3 & 0.082 & 14.69 & 5.79 & 1.97 & 13.36 \\
\hline 4 & 87.0 & 0.161 & 127.4 & 0.054 & 14.73 & 5.21 & 1.69 & 13.67 \\
\hline 5 & 87.1 & 0.161 & 127.7 & 0.074 & 14.67 & 5.58 & 2.03 & 13.42 \\
\hline 6 & 86.8 & 0.162 & 127.4 & 0.048 & 14.62 & 5.33 & 2.17 & 13.44 \\
\hline 7 & 86.9 & 0.161 & 127.5 & 0.074 & 14.73 & 5.30 & 2.28 & 13.55 \\
\hline 8 & 86.9 & 0.161 & 127.6 & 0.052 & 14.85 & 5.51 & 2.22 & 13.61 \\
\hline 9 & 86.8 & 0.162 & 127.5 & 0.067 & 14.66 & 5.46 & 1.99 & 13.46 \\
\hline 10 & 86.9 & 0.161 & 127.4 & 0.075 & 14.69 & 5.31 & 2.22 & 13.51 \\
\hline Average & 87.0 & 0.161 & 127.5 & 0.070 & 14.72 & 5.45 & 2.06 & 13.52 \\
\hline
\end{tabular}


Table D.9c - $\quad$ Summary of iteration 3 Trend and P32 intensity updates and residual errors for 10 samples.

\begin{tabular}{|c|c|c|c|c|c|c|c|c|}
\hline & \multicolumn{8}{|c|}{ Step M3 } \\
\hline & \multicolumn{4}{|c|}{ Updates } & \multicolumn{4}{|c|}{ Residuals } \\
\hline SAMPLE & Trend (1) & P32 (1) & Trend (2) & P32 (2) & TOTAL & BHP & OPR & SEIS \\
\hline 1 & 67.5 & 0.144 & 125.9 & 0.088 & 44.05 & 42.65 & 1.64 & 10.88 \\
\hline 2 & 68.6 & 0.140 & 125.4 & 0.097 & 43.94 & 42.51 & 1.62 & 10.99 \\
\hline 3 & 67.8 & 0.141 & 125.5 & 0.094 & 44.16 & 42.72 & 1.72 & 11.05 \\
\hline 4 & 67.9 & 0.141 & 125.4 & 0.095 & 44.25 & 42.86 & 1.59 & 10.90 \\
\hline 5 & 69.9 & 0.139 & 125.3 & 0.098 & 43.82 & 42.40 & 1.56 & 10.95 \\
\hline 6 & 68.3 & 0.140 & 125.3 & 0.099 & 44.12 & 42.74 & 1.21 & 10.90 \\
\hline 7 & 67.8 & 0.140 & 125.4 & 0.097 & 44.17 & 42.76 & 1.47 & 10.96 \\
\hline 8 & 68.3 & 0.140 & 125.4 & 0.098 & 43.94 & 42.46 & 1.44 & 11.21 \\
\hline 9 & 67.5 & 0.142 & 125.5 & 0.094 & 44.15 & 42.71 & 1.66 & 11.03 \\
\hline 10 & 68.0 & 0.140 & 125.3 & 0.097 & 44.12 & 42.71 & 1.53 & 10.98 \\
\hline Average & 68.1 & 0.141 & 125.4 & 0.096 & 44.07 & 42.65 & 1.54 & 10.99 \\
\hline
\end{tabular}

Table D.9d - Summary of iteration 4 Trend and P32 intensity updates and residual errors for 10 samples.

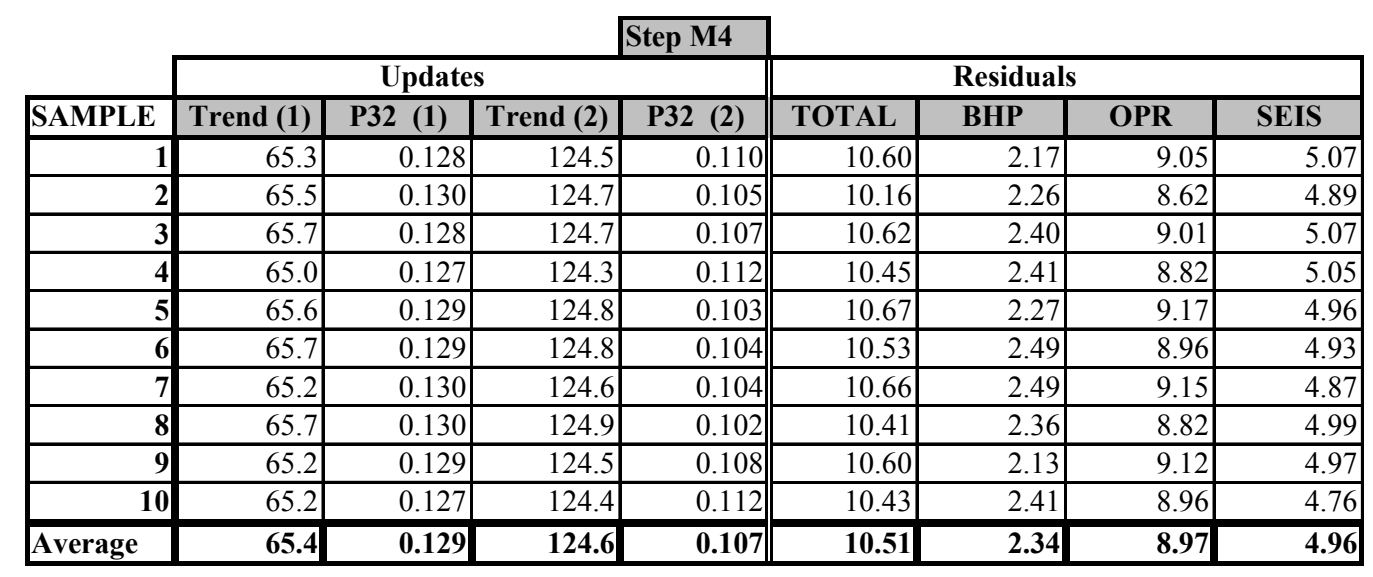


Table D.9e - $\quad$ Summary of iteration 5 Trend and P32 intensity updates and residual errors for 10 samples.

\begin{tabular}{|c|c|c|c|c|c|c|c|c|}
\hline & \multicolumn{8}{|c|}{ Step M5 } \\
\hline & \multicolumn{4}{|c|}{ Updates } & \multicolumn{4}{|c|}{ Residuals } \\
\hline SAMPLE & Trend (1) & P32 (1) & Trend (2) & P32 (2) & TOTAL & BHP & OPR & SEIS \\
\hline 1 & 66.0 & 0.120 & 125.0 & 0.120 & 10.78 & 10.00 & 0.81 & 3.95 \\
\hline 2 & 66.3 & 0.119 & 125.4 & 0.118 & 10.83 & 10.05 & 0.55 & 4.01 \\
\hline 3 & 66.1 & 0.120 & 125.1 & 0.121 & 10.87 & 10.02 & 0.50 & 4.20 \\
\hline 4 & 66.0 & 0.120 & 125.1 & 0.120 & 10.87 & 10.07 & 0.28 & 4.07 \\
\hline 5 & 66.5 & 0.119 & 125.5 & 0.119 & 10.87 & 10.04 & 0.70 & 4.11 \\
\hline 6 & 66.1 & 0.120 & 124.9 & 0.122 & 10.75 & 9.95 & 0.56 & 4.04 \\
\hline 7 & 66.2 & 0.120 & 125.1 & 0.123 & 10.95 & 10.14 & 0.81 & 4.07 \\
\hline 8 & 66.0 & 0.121 & 124.9 & 0.120 & 10.88 & 9.98 & 0.78 & 4.24 \\
\hline 9 & 66.0 & 0.120 & 124.7 & 0.126 & 10.74 & 9.89 & 0.52 & 4.15 \\
\hline 10 & 66.2 & 0.120 & 125.2 & 0.121 & 10.81 & 9.99 & 0.58 & 4.09 \\
\hline Average & 66.1 & 0.120 & 125.1 & 0.121 & 10.84 & 10.01 & 0.61 & 4.09 \\
\hline
\end{tabular}

Table D.9f - Summary of iteration 6 Trend and P32 intensity updates and residual errors for 10 samples.

\begin{tabular}{|c|c|c|c|c|c|c|c|c|}
\hline \multirow[b]{3}{*}{ SAMPLE } & \multicolumn{8}{|c|}{ Step M6 } \\
\hline & \multicolumn{4}{|c|}{ Updates } & \multicolumn{4}{|c|}{ Residuals } \\
\hline & Trend (1) & P32 (1) & Trend (2) & P32 (2) & TOTAL & BHP & $\overline{\text { OPR }}$ & SEIS \\
\hline 1 & 66.2 & 0.102 & 129.4 & 0.130 & 7.24 & 6.27 & 0.89 & 3.49 \\
\hline 2 & 66.4 & 0.104 & 129.1 & 0.130 & 7.16 & 6.35 & 1.18 & 3.09 \\
\hline 3 & 66.3 & 0.101 & 129.3 & 0.130 & 6.93 & 6.09 & 1.14 & 3.09 \\
\hline 4 & 66.5 & 0.103 & 130.3 & 0.135 & 6.64 & 5.77 & 0.55 & 3.23 \\
\hline 5 & 66.6 & 0.099 & 129.3 & 0.131 & 7.07 & 6.26 & 0.95 & 3.16 \\
\hline 6 & 66.9 & 0.101 & 129.2 & 0.132 & 6.99 & 6.28 & 0.85 & 2.95 \\
\hline 7 & 66.5 & 0.102 & 129.4 & 0.131 & 6.87 & 6.09 & 0.48 & 3.14 \\
\hline 8 & 66.3 & 0.103 & 129.9 & 0.131 & 6.81 & 6.02 & 1.17 & 2.95 \\
\hline 9 & 66.2 & 0.100 & 129.4 & 0.130 & 6.73 & 5.95 & 1.01 & 2.97 \\
\hline 10 & 67.1 & 0.100 & 128.8 & 0.133 & 7.05 & 6.18 & 0.79 & 3.29 \\
\hline Average & 66.5 & 0.101 & 129.4 & 0.131 & 6.95 & 6.13 & 0.90 & 3.14 \\
\hline
\end{tabular}


Table D.9g - Summary of iteration 7 Trend and P32 intensity updates and residual errors for 10 samples.

\begin{tabular}{|c|c|c|c|c|c|c|c|c|}
\hline & \multicolumn{8}{|c|}{ Step M7 } \\
\hline & \multicolumn{4}{|c|}{ Updates } & \multicolumn{4}{|c|}{ Residuals } \\
\hline SAMPLE & Trend (1) & P32 (1) & Trend (2) & P32 (2) & TOTAL & BHP & OPR & SEIS \\
\hline 1 & 66.1 & 0.101 & 129.6 & 0.149 & 3.44 & 3.19 & 0.64 & 1.10 \\
\hline 2 & 66.4 & 0.103 & 130.0 & 0.142 & 3.17 & 3.00 & 0.66 & 0.80 \\
\hline 3 & 66.3 & 0.103 & 129.4 & 0.151 & 3.48 & 3.37 & 0.37 & 0.83 \\
\hline 4 & 66.4 & 0.100 & 129.8 & 0.142 & 3.09 & 2.89 & 0.77 & 0.80 \\
\hline 5 & 66.3 & 0.102 & 130.0 & 0.143 & 3.25 & 3.15 & 0.39 & 0.72 \\
\hline 6 & 66.3 & 0.103 & 130.2 & 0.142 & 3.39 & 3.23 & 0.66 & 0.78 \\
\hline 7 & 66.5 & 0.101 & 129.7 & 0.143 & 3.23 & 3.06 & 0.47 & 0.93 \\
\hline 8 & 66.2 & 0.100 & 129.3 & 0.150 & 3.46 & 3.32 & 0.67 & 0.71 \\
\hline 9 & 66.4 & 0.104 & 129.8 & 0.148 & 3.47 & 3.33 & 0.36 & 0.89 \\
\hline 10 & 66.3 & 0.101 & 129.9 & 0.143 & 3.21 & 3.07 & 0.52 & 0.76 \\
\hline Average & 66.3 & 0.102 & 129.8 & 0.145 & 3.32 & 3.16 & 0.55 & 0.83 \\
\hline
\end{tabular}

Table D.9h - Summary of iteration 8 Trend and P32 intensity updates and residual errors for 10 samples.

\begin{tabular}{|c|c|c|c|c|c|c|c|c|}
\hline \multirow[b]{3}{*}{ SAMPLE } & \multicolumn{8}{|c|}{ Step M8 } \\
\hline & \multicolumn{4}{|c|}{ Updates } & \multicolumn{4}{|c|}{ Residuals } \\
\hline & Trend (1) & P32 (1) & Trend (2) & P32 (2) & TOTAL & BHP & OPR & SEIS \\
\hline 1 & 65.8 & 0.101 & 129.4 & 0.149 & 7.77 & 7.73 & 0.48 & 0.57 \\
\hline 2 & 65.7 & 0.103 & 130.7 & 0.147 & 8.24 & 8.17 & 0.67 & 0.82 \\
\hline 3 & 65.7 & 0.102 & 129.0 & 0.149 & 8.03 & 8.00 & 0.42 & 0.61 \\
\hline 4 & 65.7 & 0.101 & 128.9 & 0.153 & 8.01 & 7.95 & 0.44 & 0.84 \\
\hline 5 & 65.6 & 0.101 & 129.3 & 0.157 & 8.18 & 8.08 & 0.64 & 1.10 \\
\hline 6 & 65.7 & 0.100 & 127.9 & 0.153 & 8.34 & 8.27 & 0.66 & 0.80 \\
\hline 7 & 65.7 & 0.101 & 127.8 & 0.156 & 8.10 & 8.05 & 0.37 & 0.83 \\
\hline 8 & 65.8 & 0.099 & 130.1 & 0.148 & 8.34 & 8.27 & 0.77 & 0.80 \\
\hline 9 & 65.7 & 0.100 & 128.9 & 0.152 & 8.15 & 8.11 & 0.39 & 0.72 \\
\hline 10 & 65.8 & 0.101 & 129.7 & 0.147 & 8.11 & 8.04 & 0.66 & 0.78 \\
\hline Average & 65.7 & 0.101 & 129.2 & 0.151 & 8.13 & 8.07 & 0.55 & 0.79 \\
\hline
\end{tabular}




\section{Sensitivity Coefficients (All Iterations)}
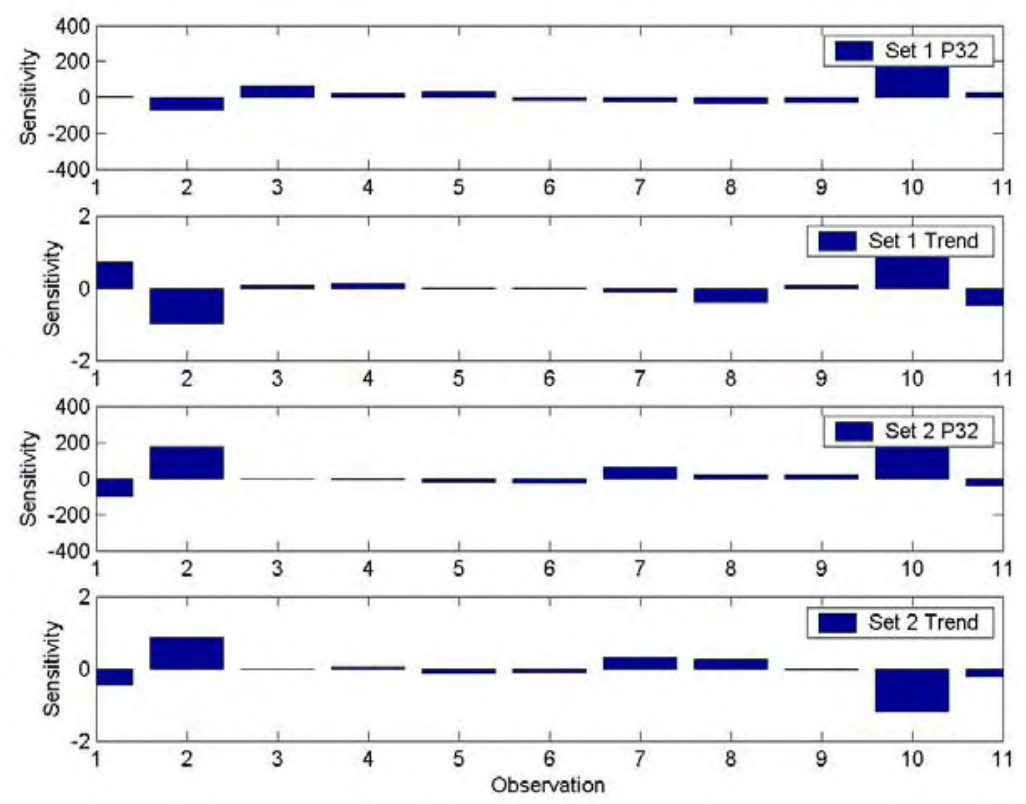

Fig. D.4a - Sensitivity coefficients for iteration 1 .
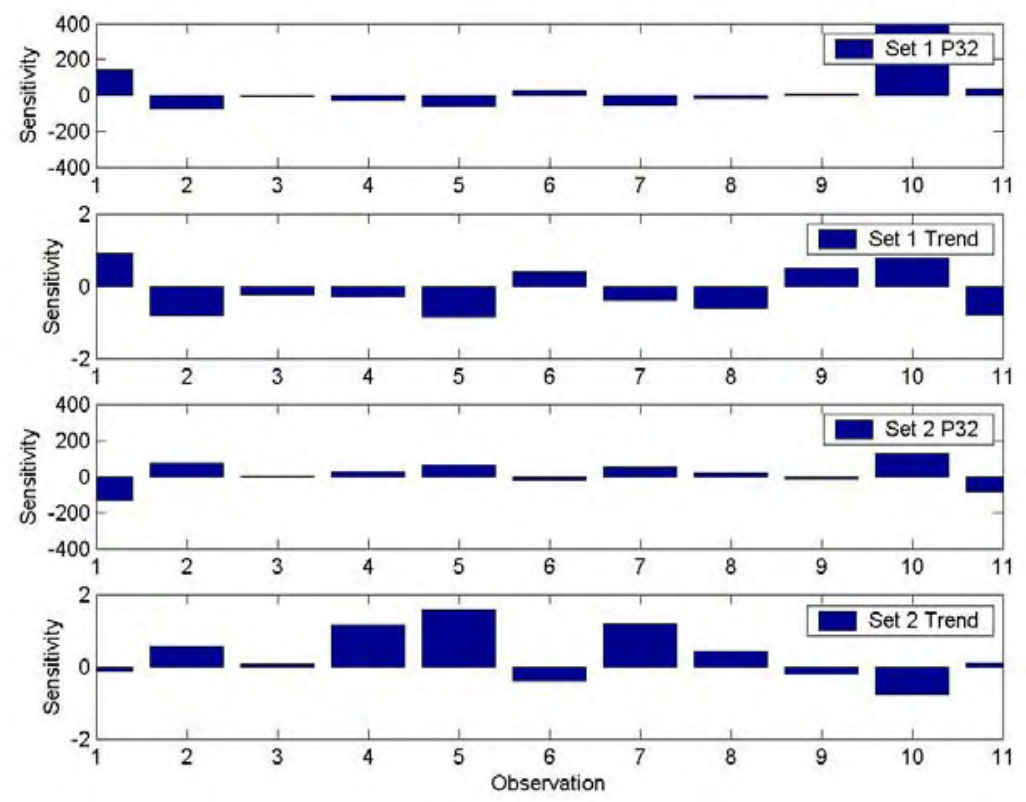

Fig. D.4b - Sensitivity coefficients for iteration 2 . 

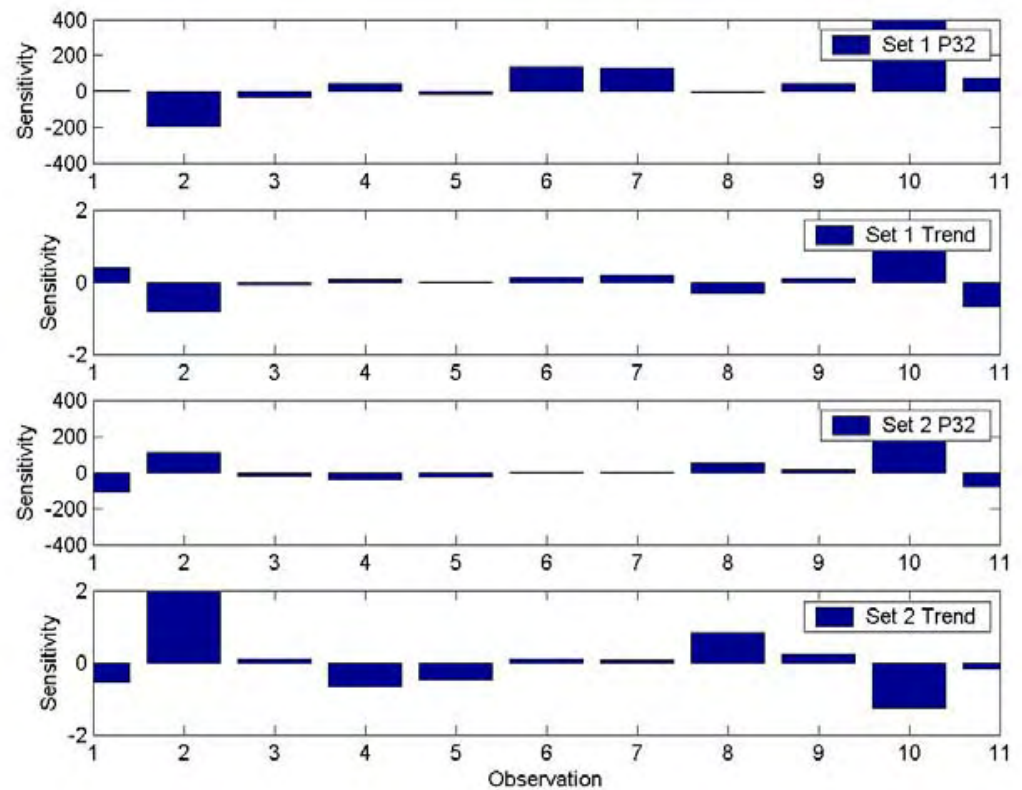

Fig. D.4c - Sensitivity coefficients for iteration 3 .
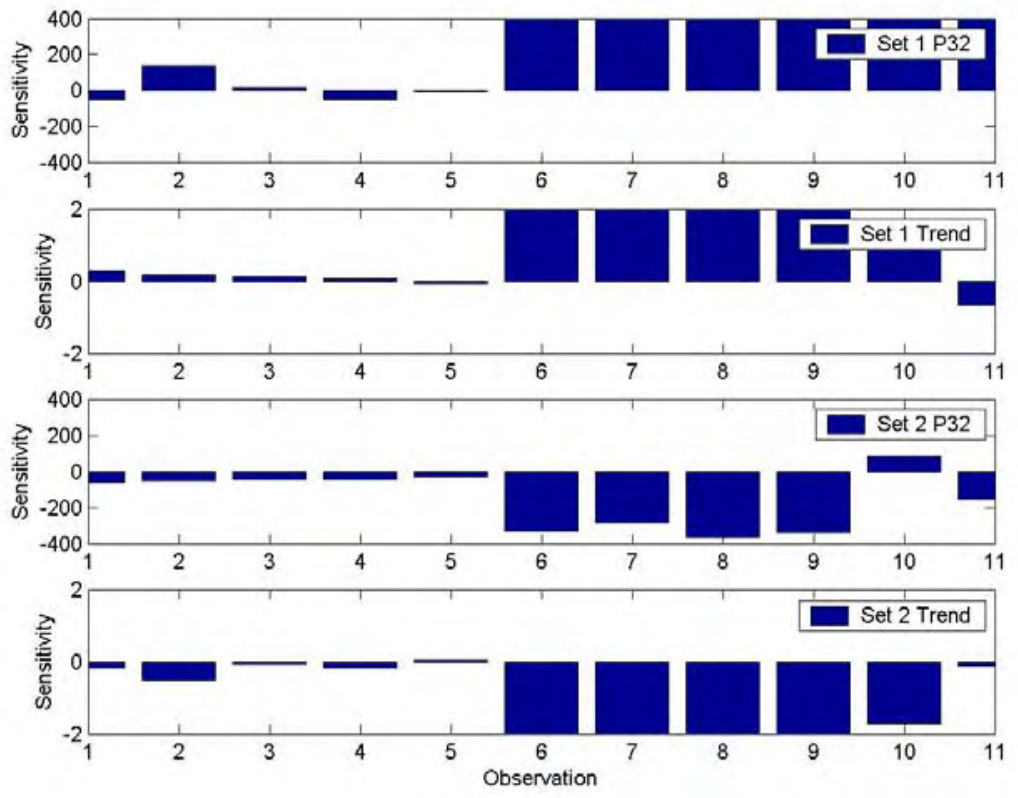

Fig. D.4d - Sensitivity coefficients for iteration 4 . 

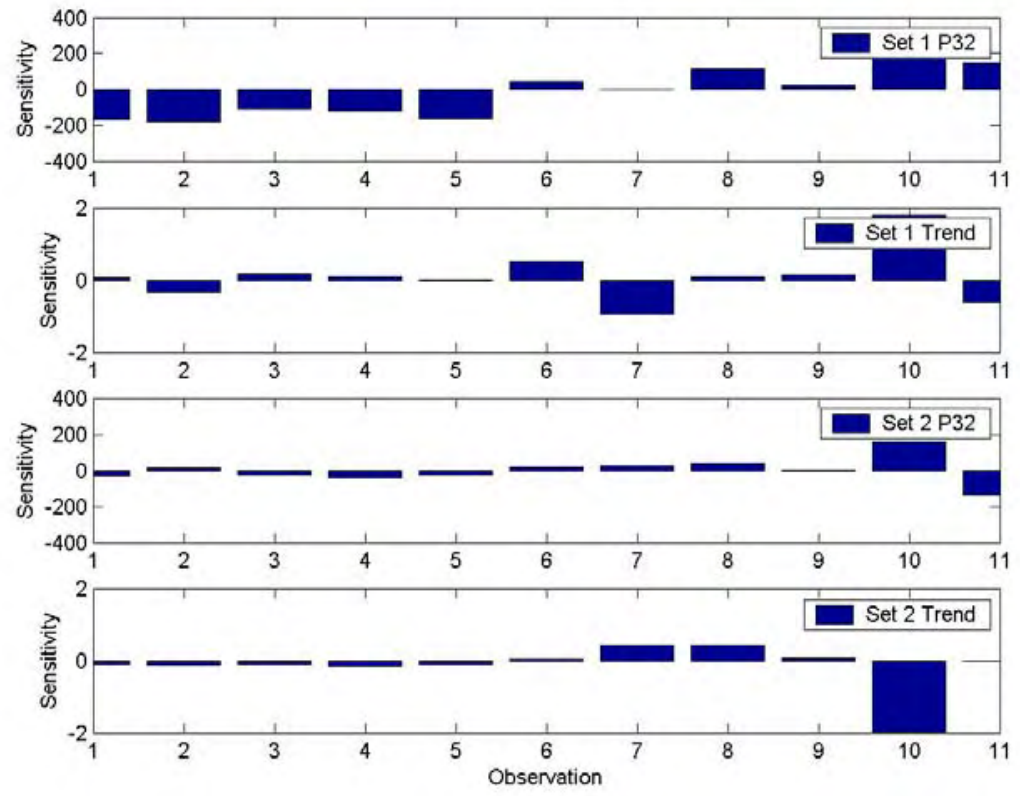

Fig. D.4e - Sensitivity coefficients for iteration 5 .
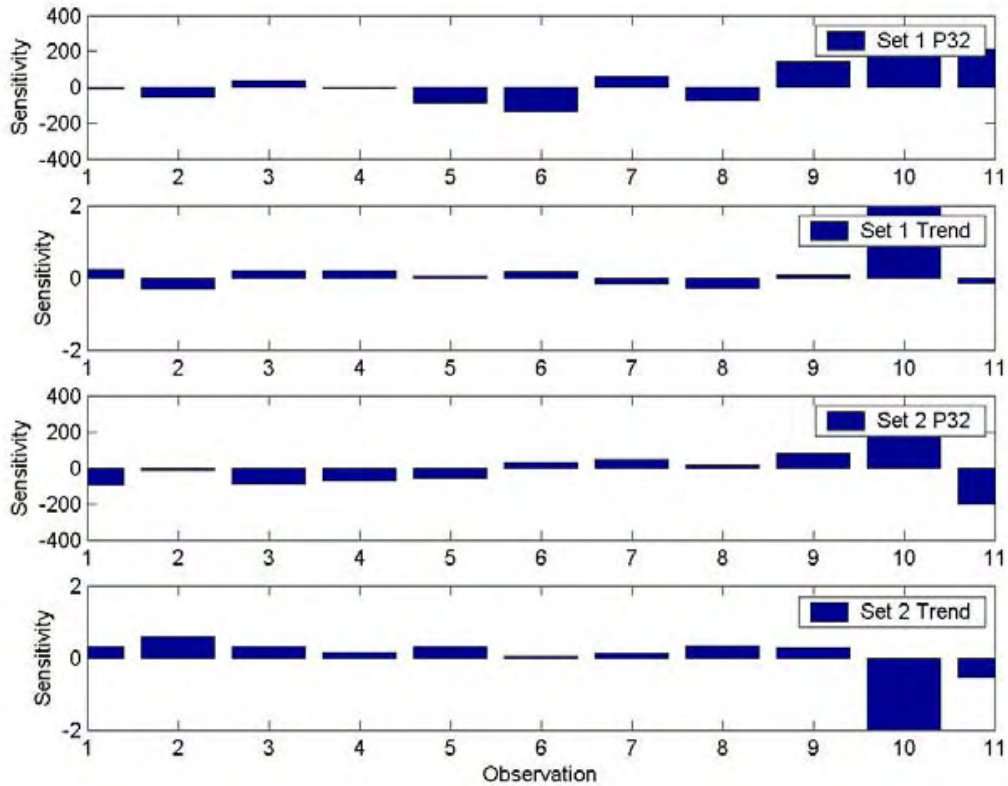

Fig. D.4f $\quad-\quad$ Sensitivity coefficients for iteration 6 . 

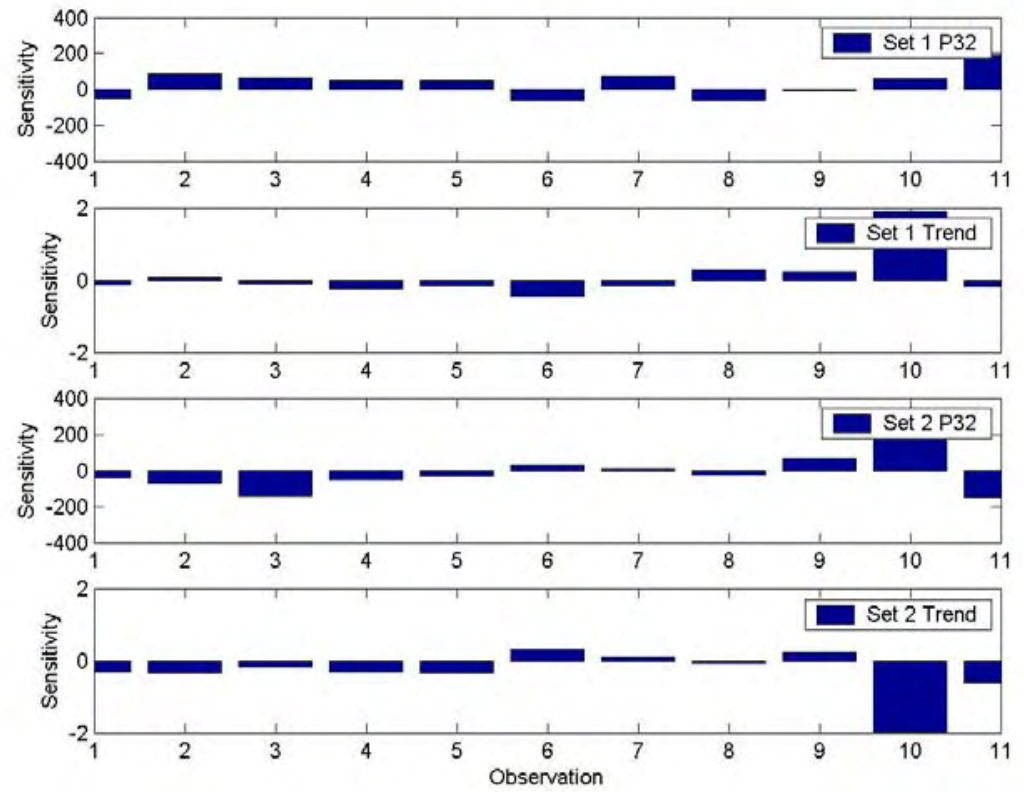

Fig. D.4g - Sensitivity coefficients for iteration 7.
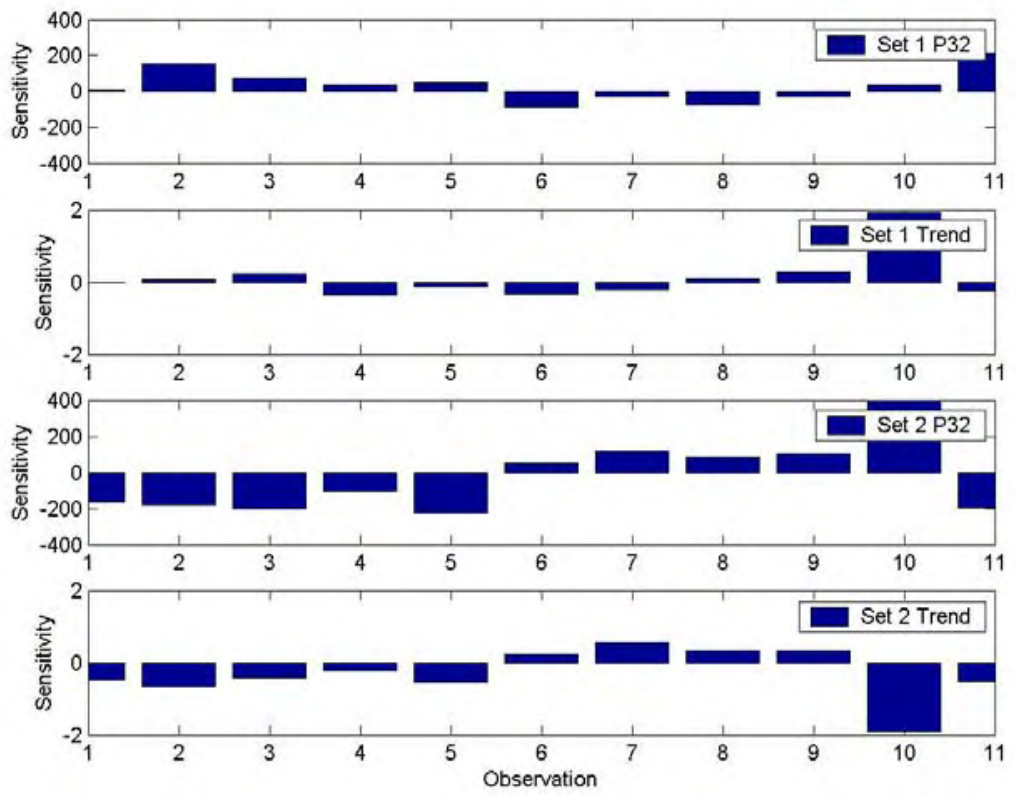

Fig. D.4h $\quad-\quad$ Sensitivity coefficients for iteration 8 . 
Fracture Distribution Maps (Base, Initial, Final

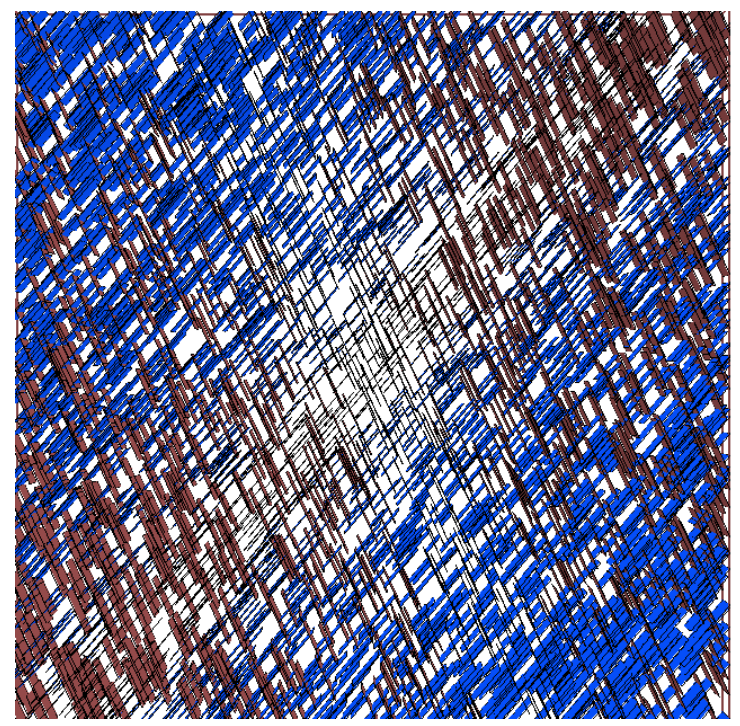

Fig. D 5a $\quad-\quad$ Test T4 base model fracture distribution.

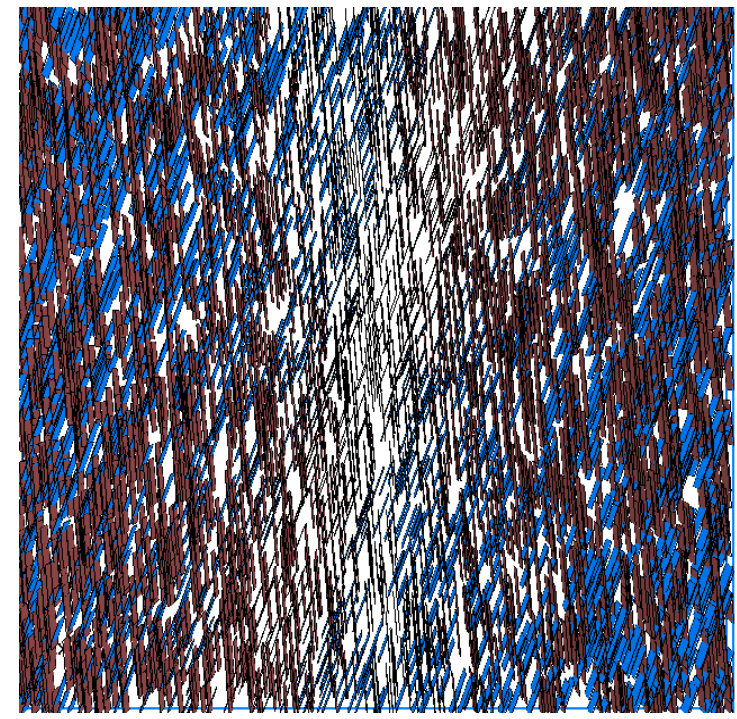

Fig. D 5b $\quad-\quad$ Test T4 initial estimate fracture distribution. 


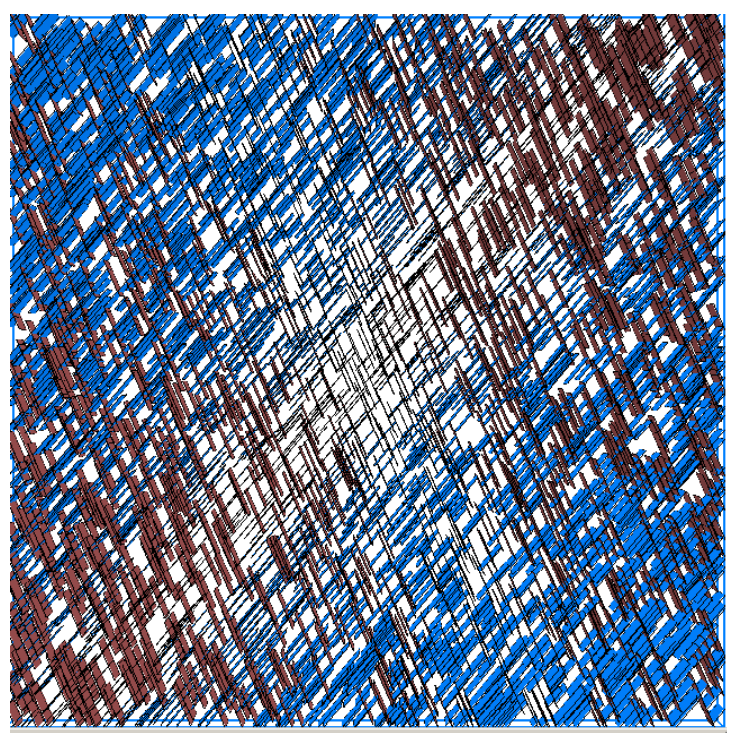

Fig. D 5c $\quad-\quad$ Test T4 final model fracture distribution.

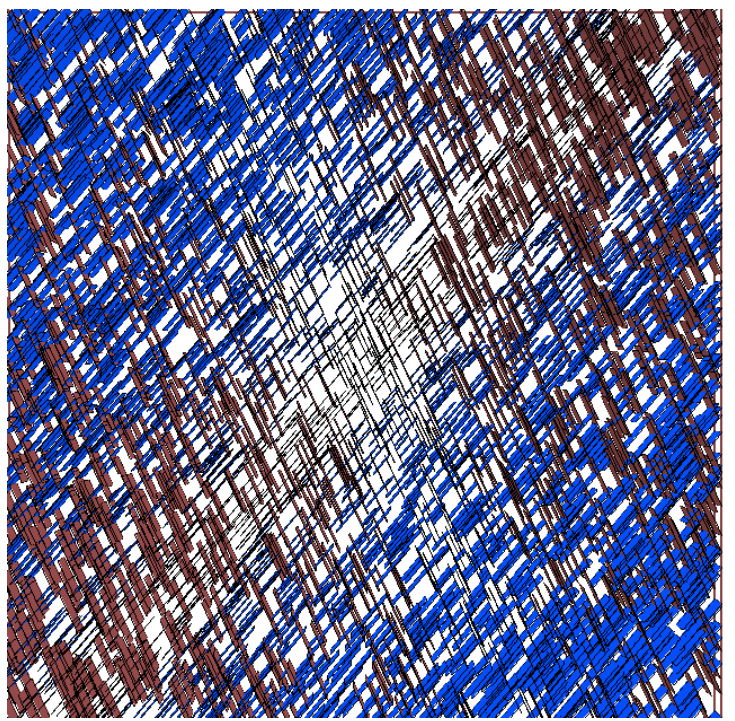

Fig. D 5d $\quad-\quad$ Test T4 base model fracture distribution. 


\section{Fracture Water Saturation Maps (Base, Initial, Final)}

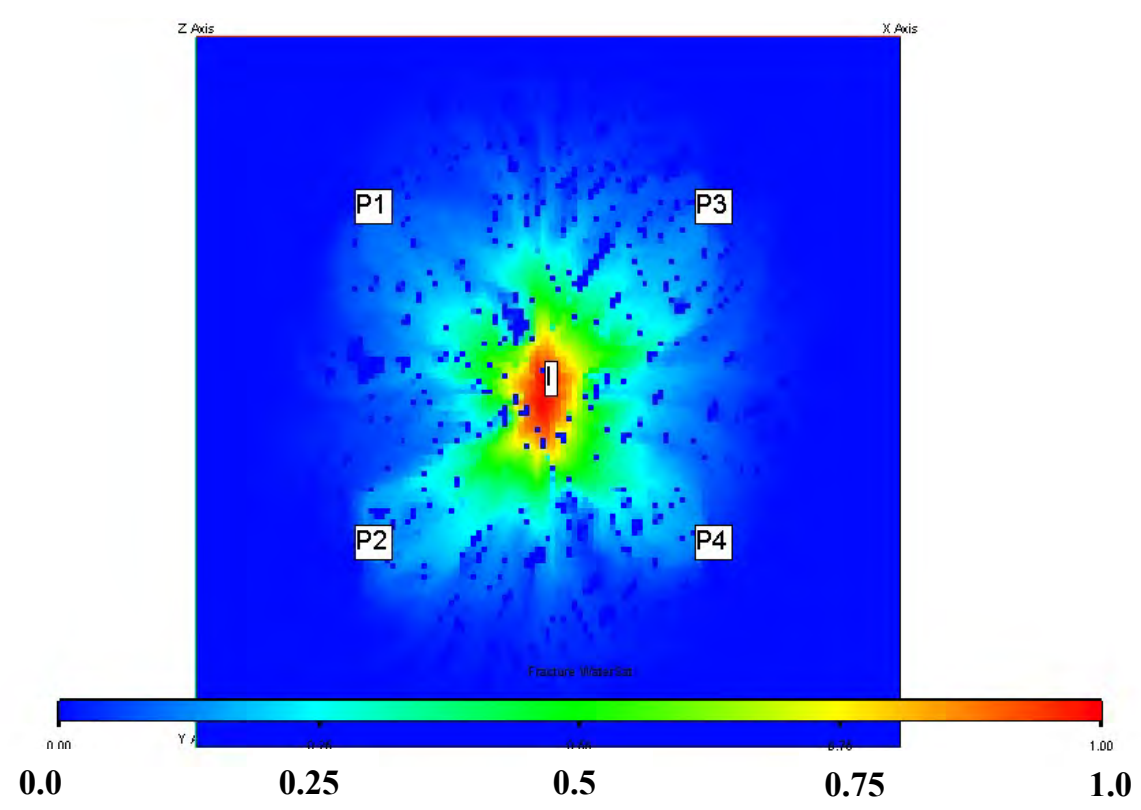

Fig. D.6a - Fracture water saturation at report step 5 (150 days) for test T4 base model.

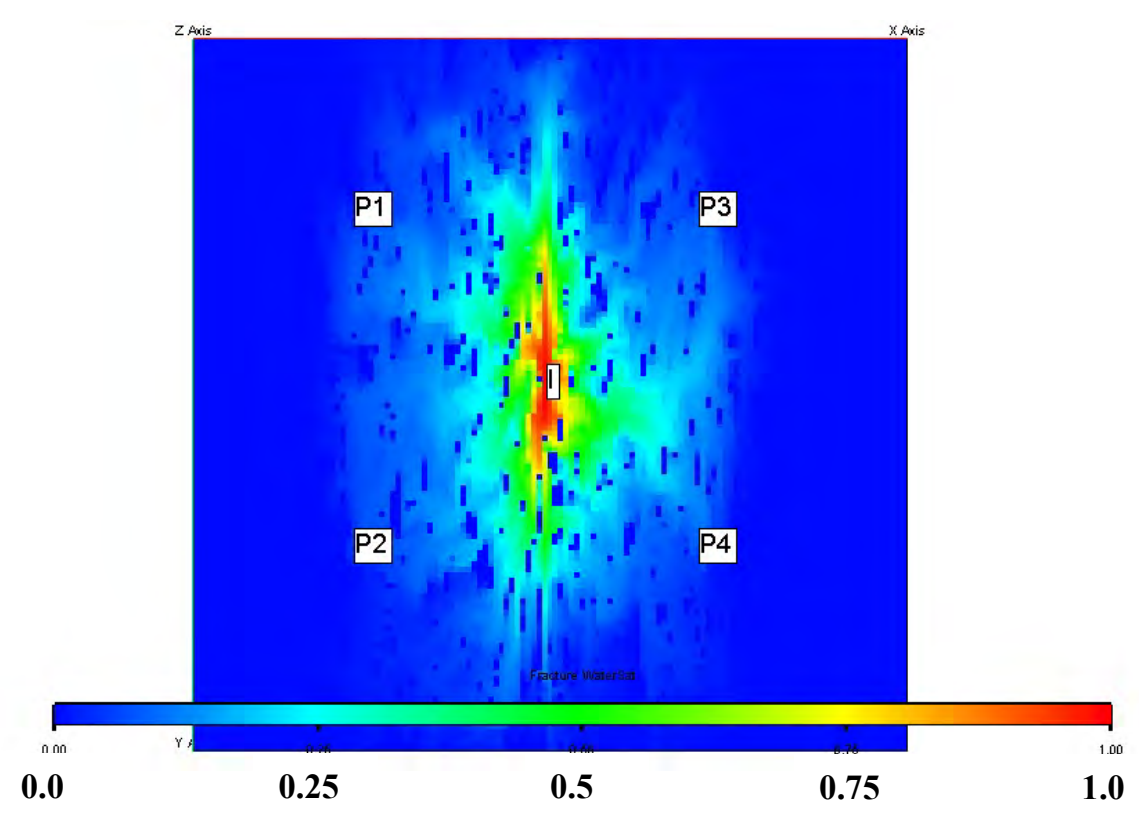

Fig. D.6b - Fracture water saturation at report step 5 (150 days) for test T4 initial estimate. 


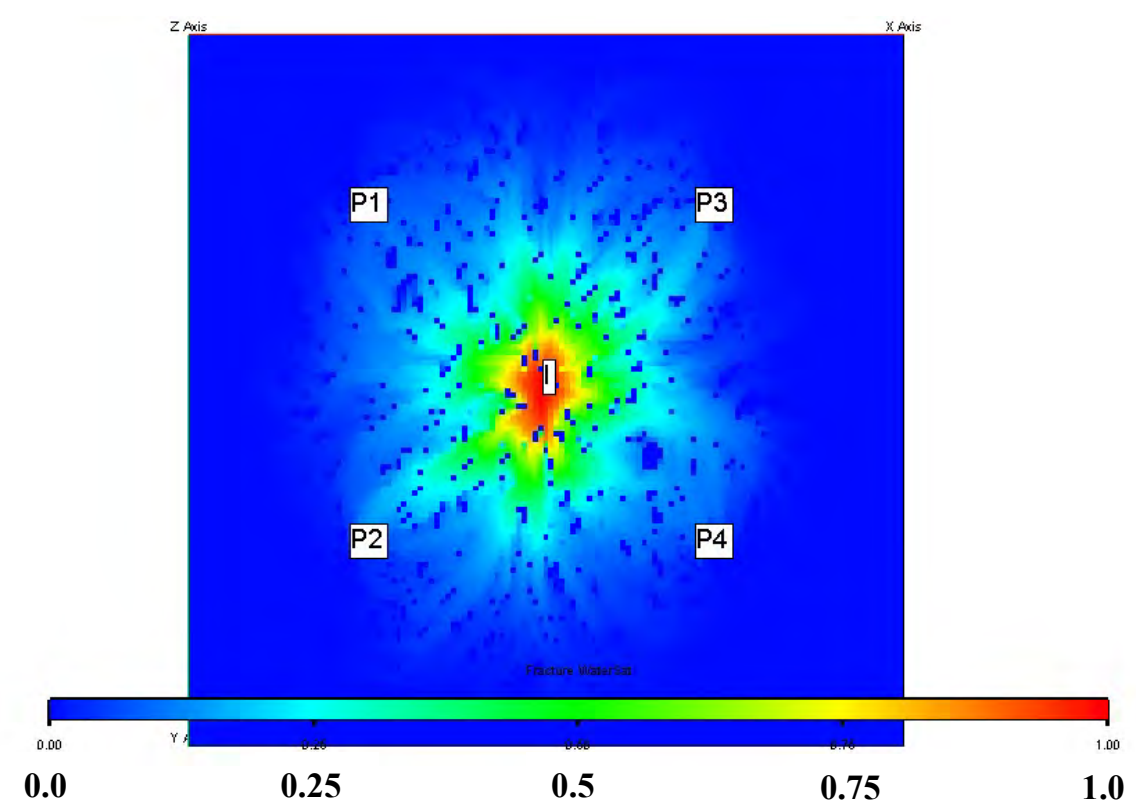

Fig. D.6c - Fracture water saturation at report step 5 (150 days) for test $\mathrm{T} 4$ final model.

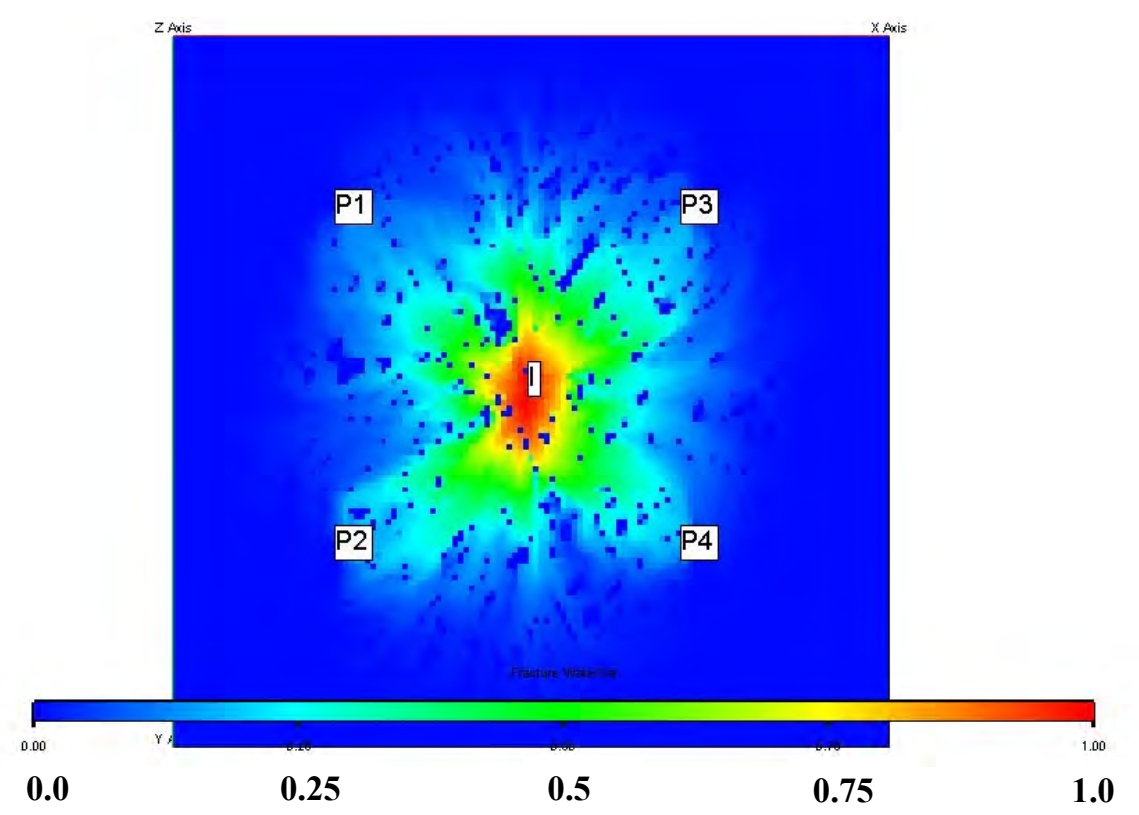

Fig. D.7a - Fracture water saturation at report step $10(300$ days) for test T4 base model. 


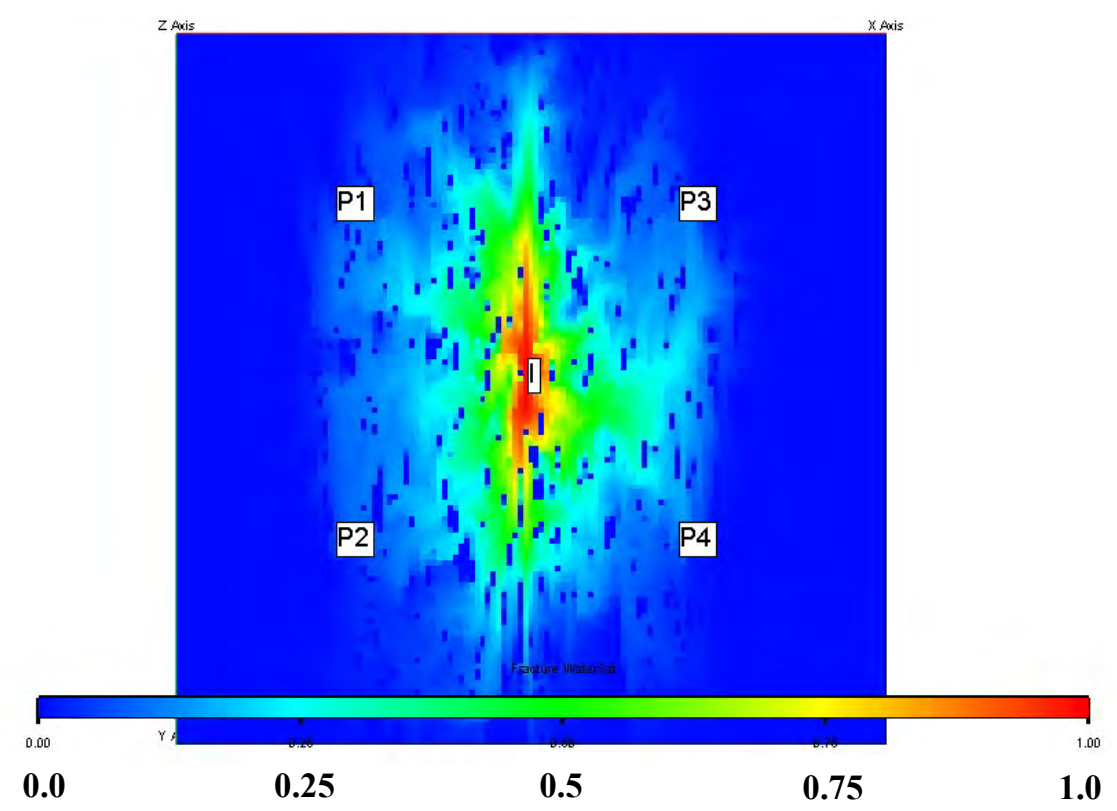

Fig. D.7b - Fracture water saturation at report step $10(300$ days) for test T4 initial estimate.

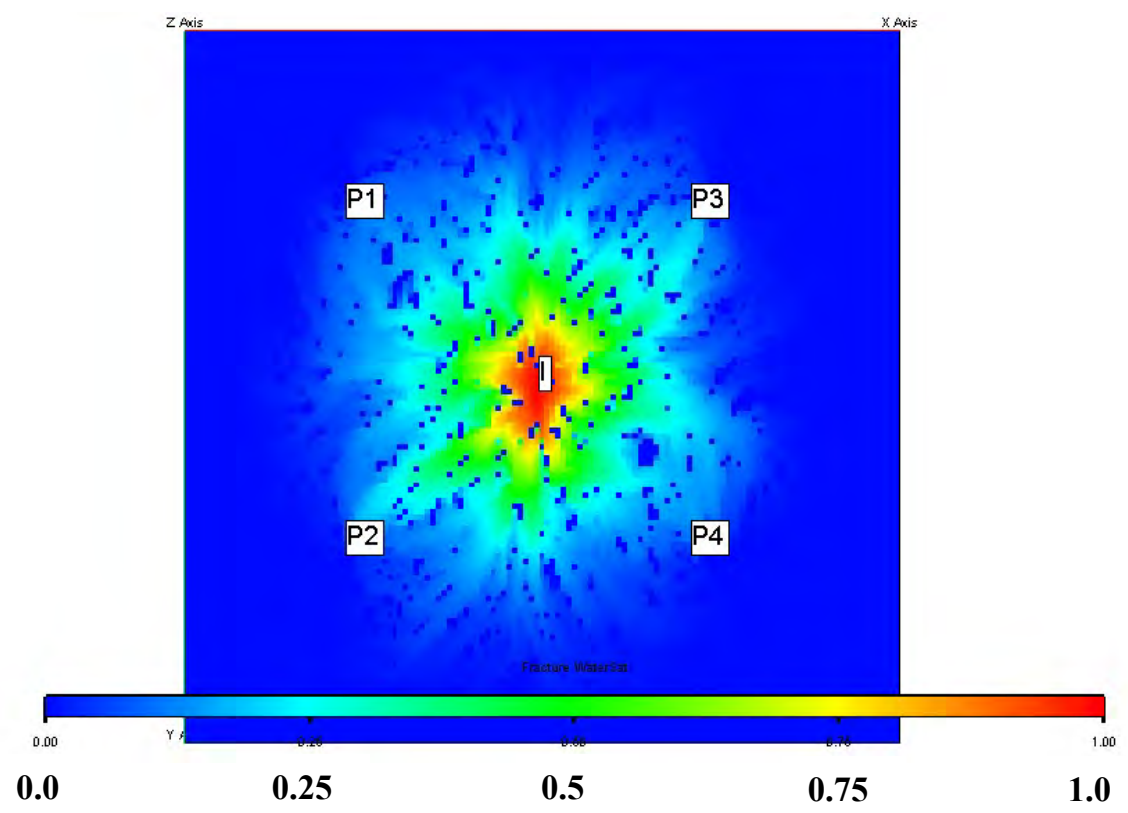

Fig. D.7c - Fracture water saturation at report step $10(300$ days) for test T4 final model. 


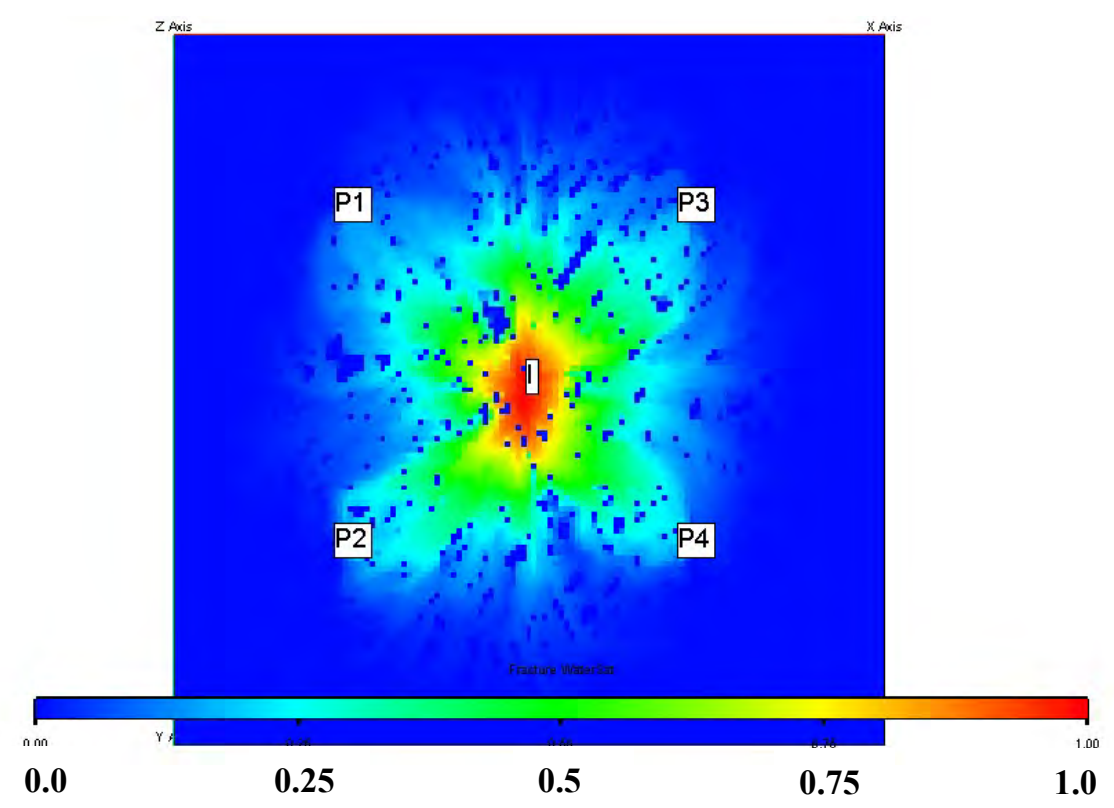

Fig. D.8a - Fracture water saturation at report step 15 (450 days) for test T4 base model.

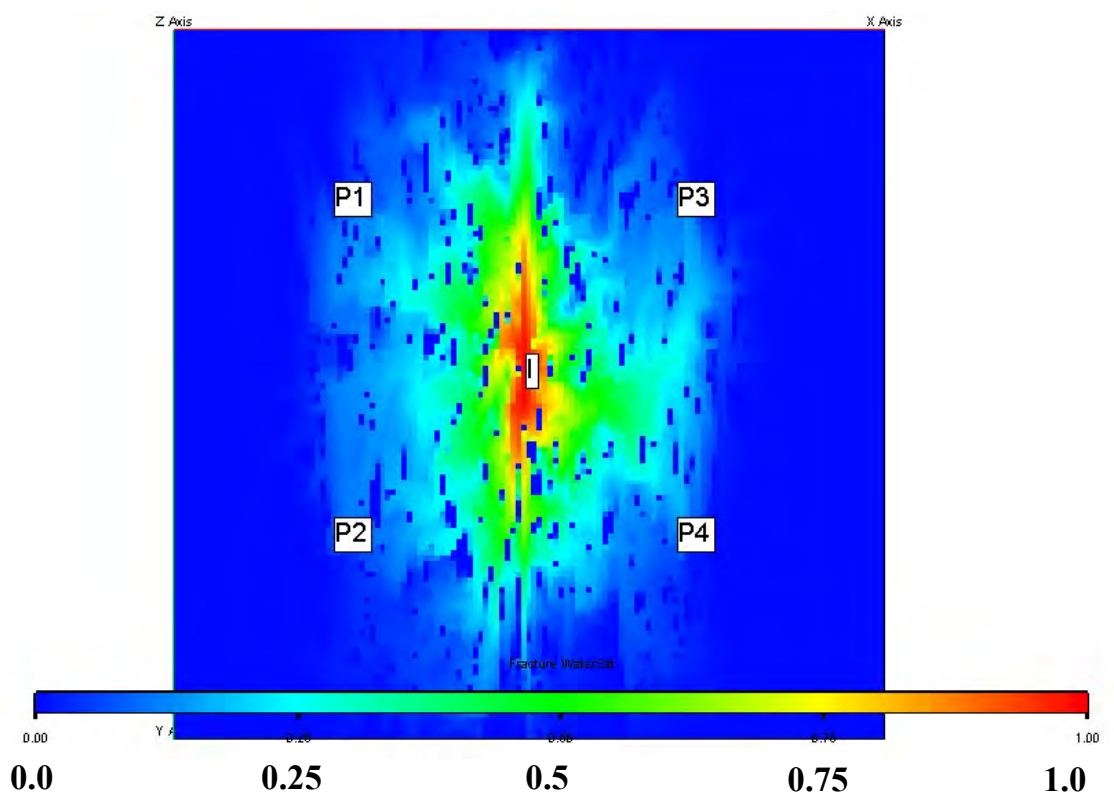

Fig. D.8b - Fracture water saturation at report step 15 (450 days) for test T4 initial estimate. 


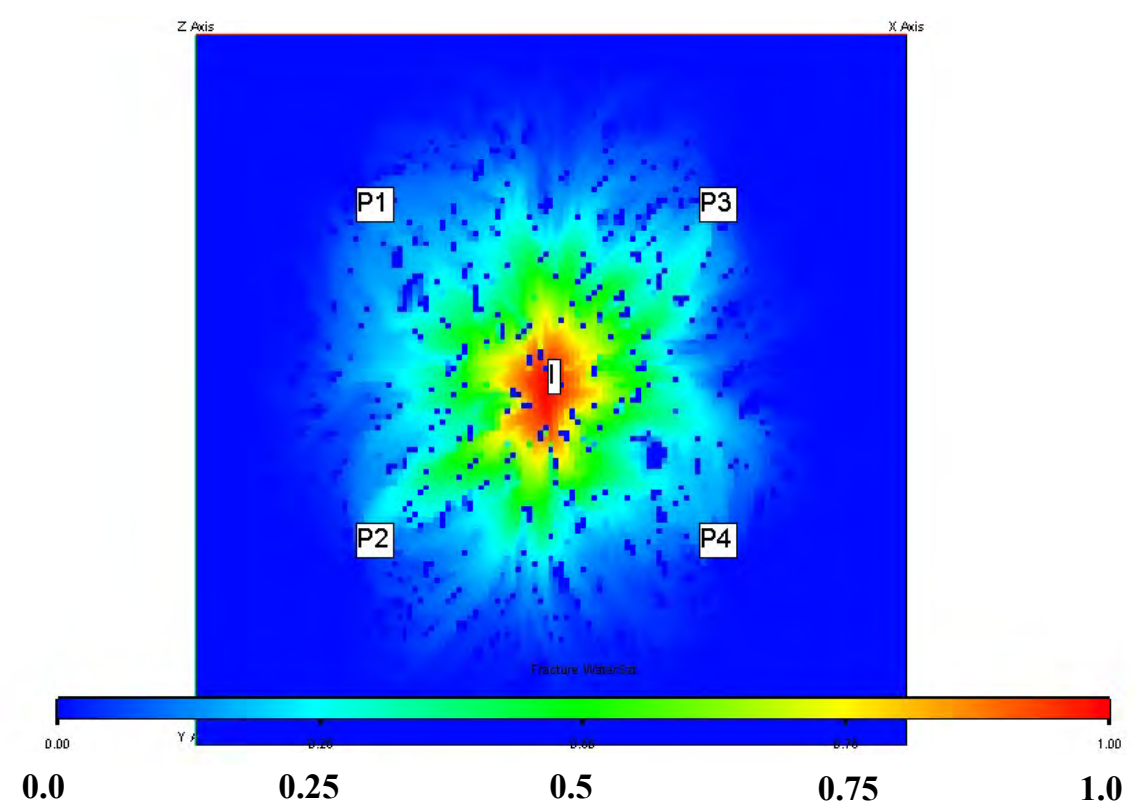

Fig. D.8c - Fracture water saturation at report step 15 (450 days) for test T4 final model.

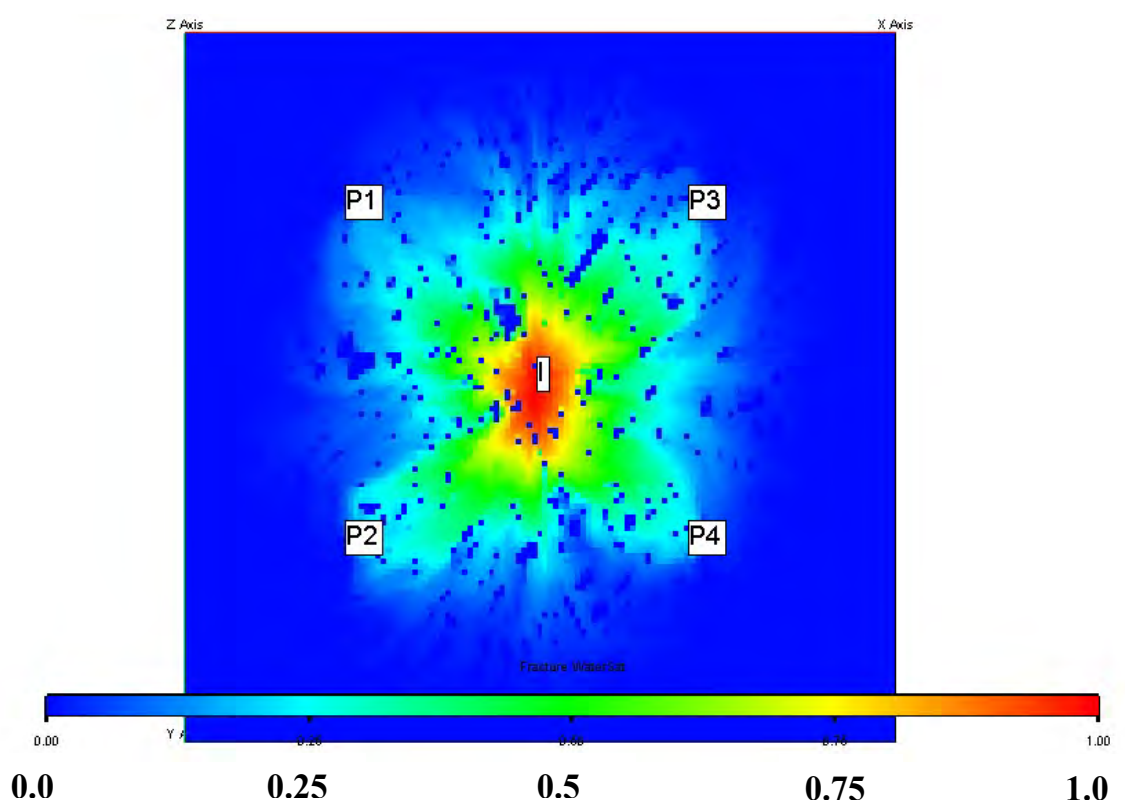

Fig. D.9a - Fracture water saturation at report step $20(600$ days) for test T4 base model 


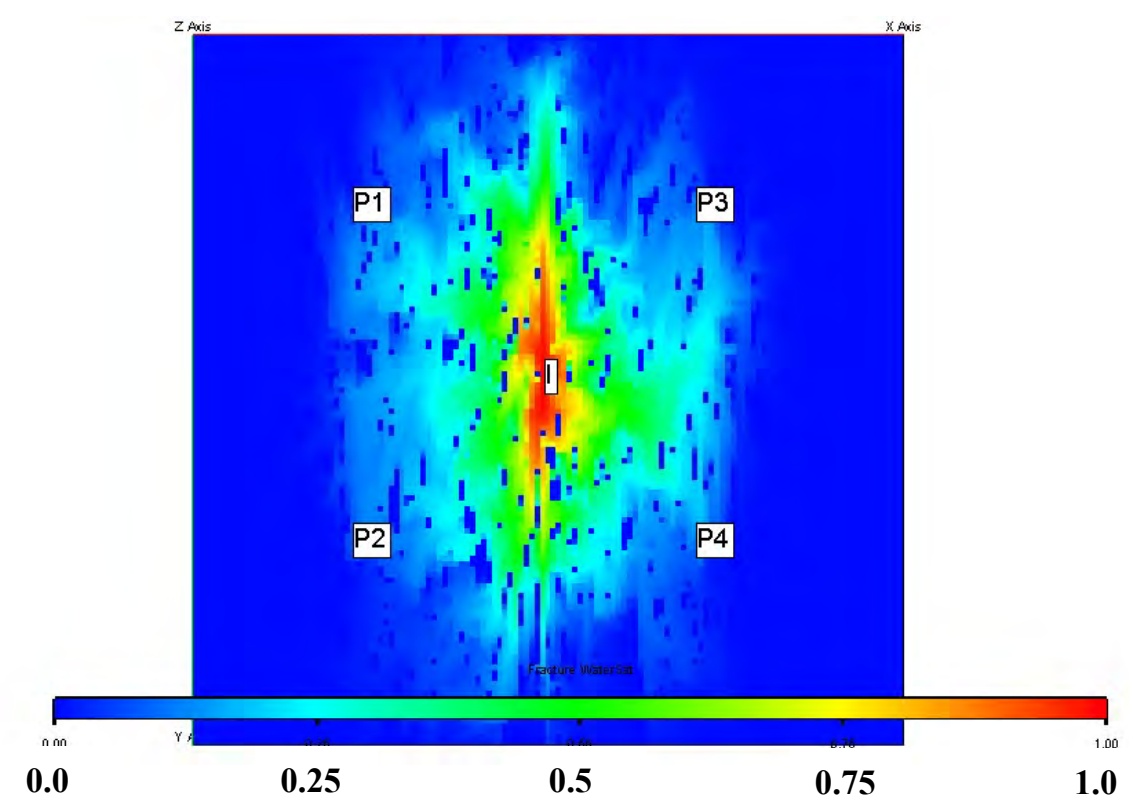

Fig. D.9b - Fracture water saturation at report step $20(600$ days) for test T4 initial estimate.

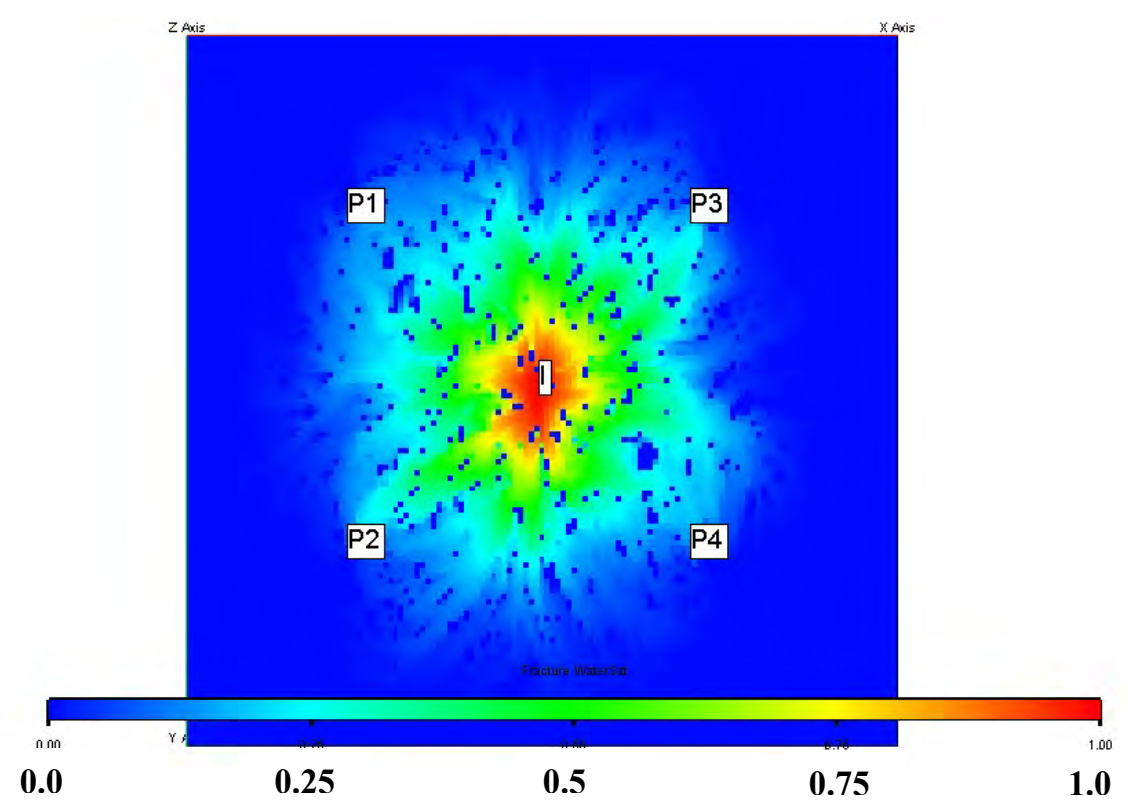

Fig. D.9c - Fracture water saturation at report step 20 (600 days) for test T4 final model. 
Matrix Water Saturation Maps (Base, Initial, Final)

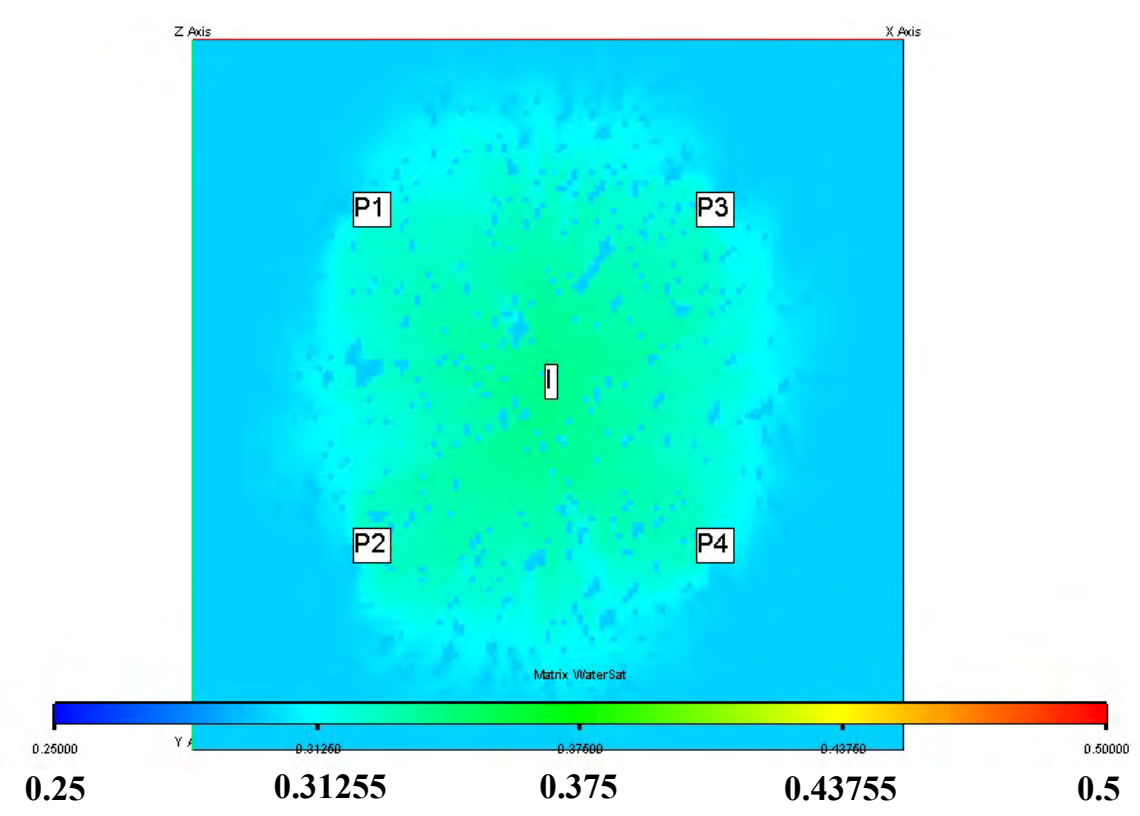

Fig. D.10a - Matrix water saturation at report step 5 (150 days) for test T4 base model.

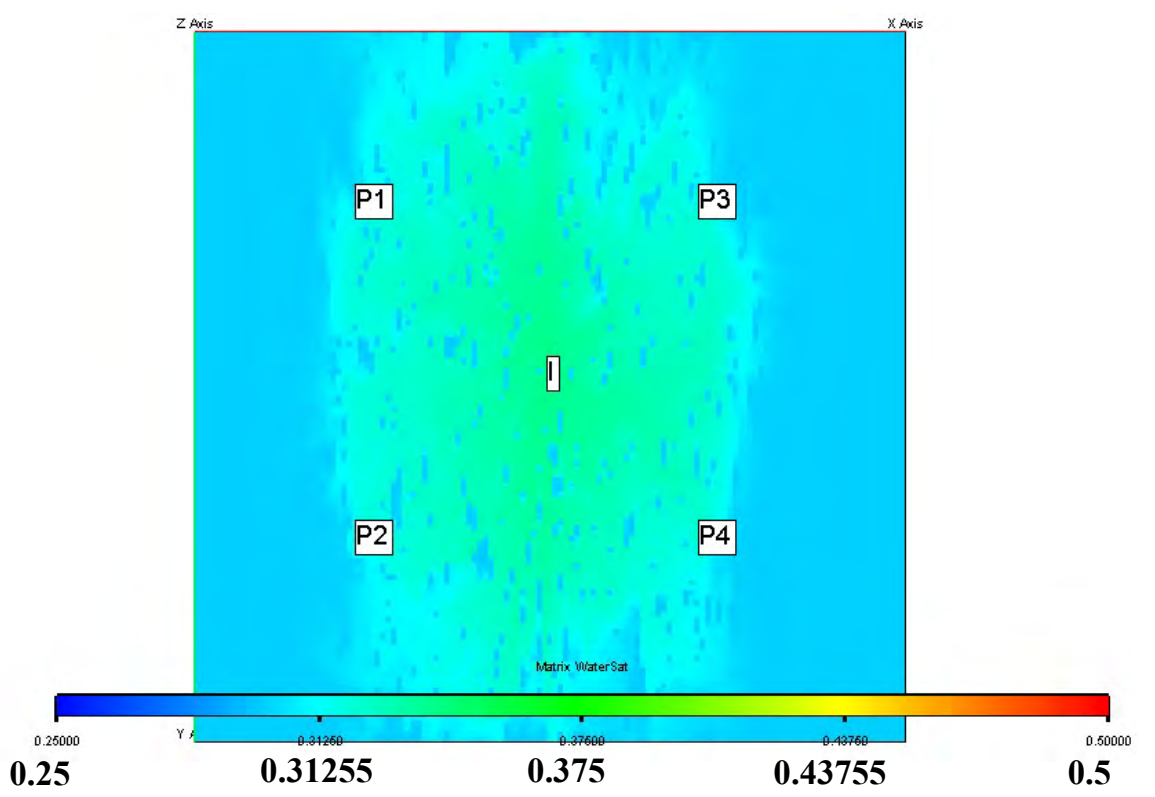

Fig. D.10b - Matrix water saturation at report step 5 (150 days) for test T4 initial estimate. 


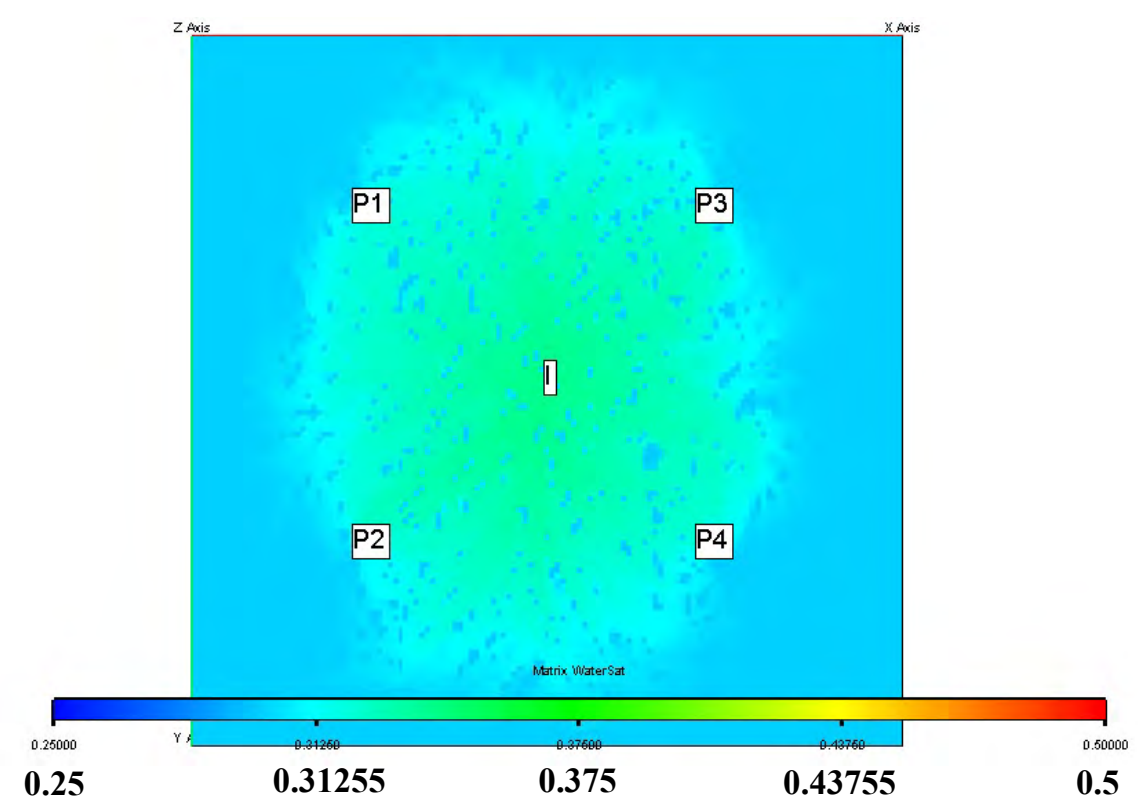

Fig. D.10c - Matrix water saturation at report step 5 (150 days) for test T4 final model.

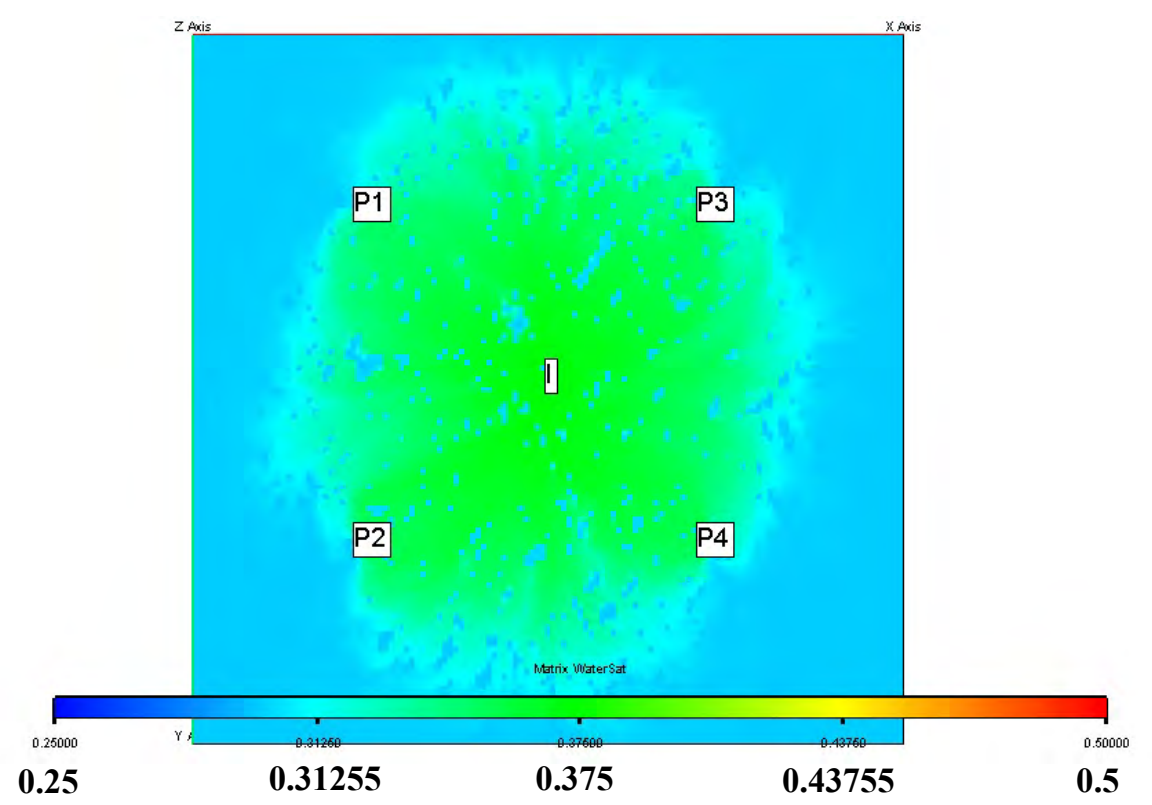

Fig. D.11a - Matrix water saturation at report step $10(300$ days) for test T4 base model. 


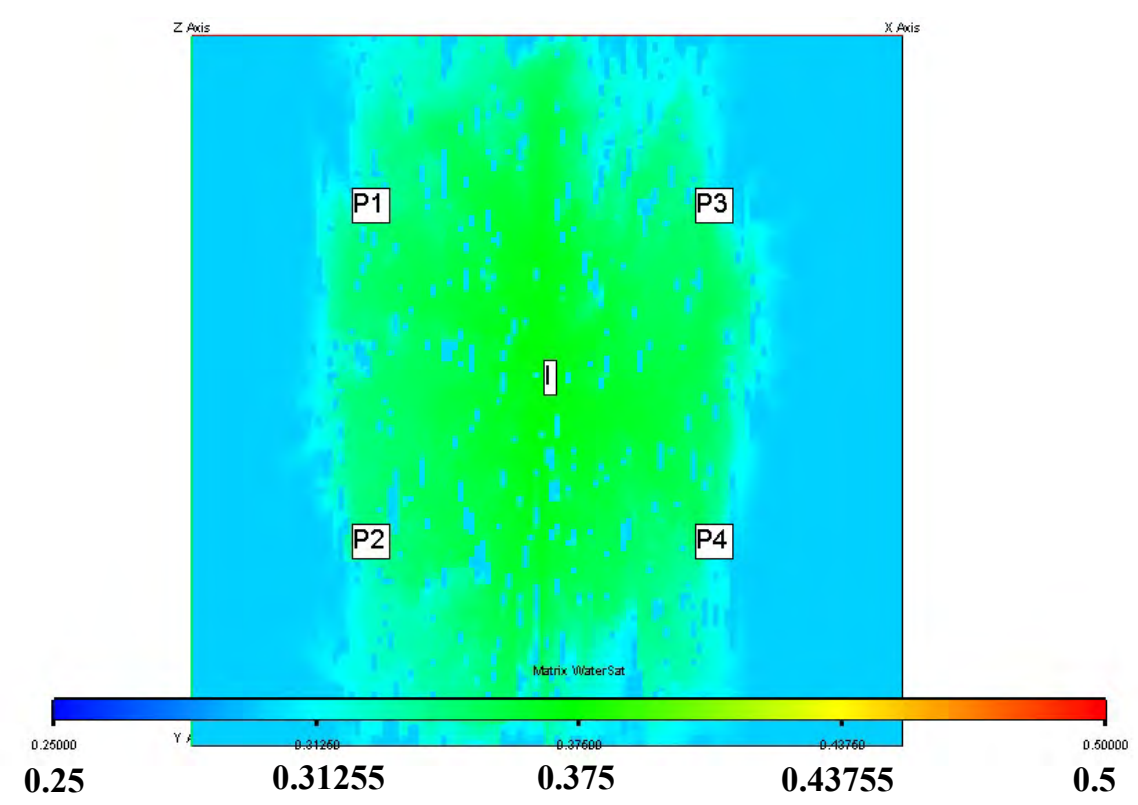

Fig. D.11b - Matrix water saturation at report step $10(300$ days) for test T4 initial estimate.

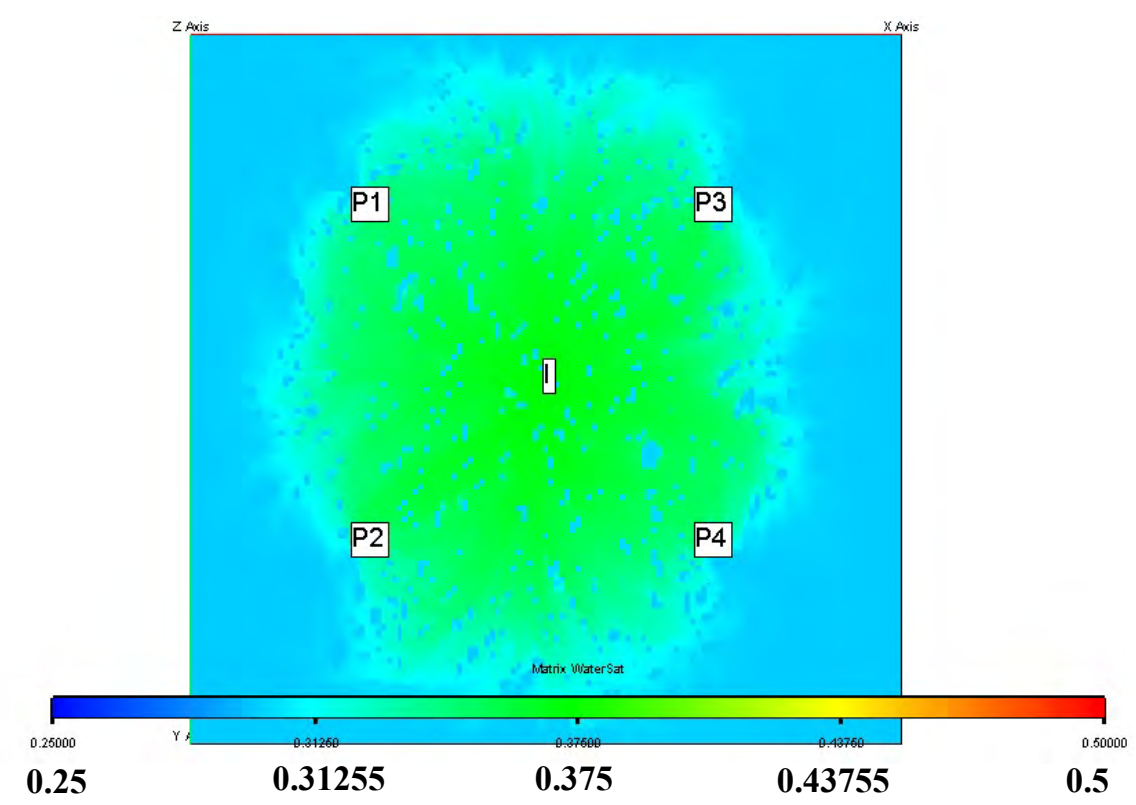

Fig. D.11.c - Matrix water saturation at report step $10(300$ days) for test $\mathrm{T} 4$ final model. 


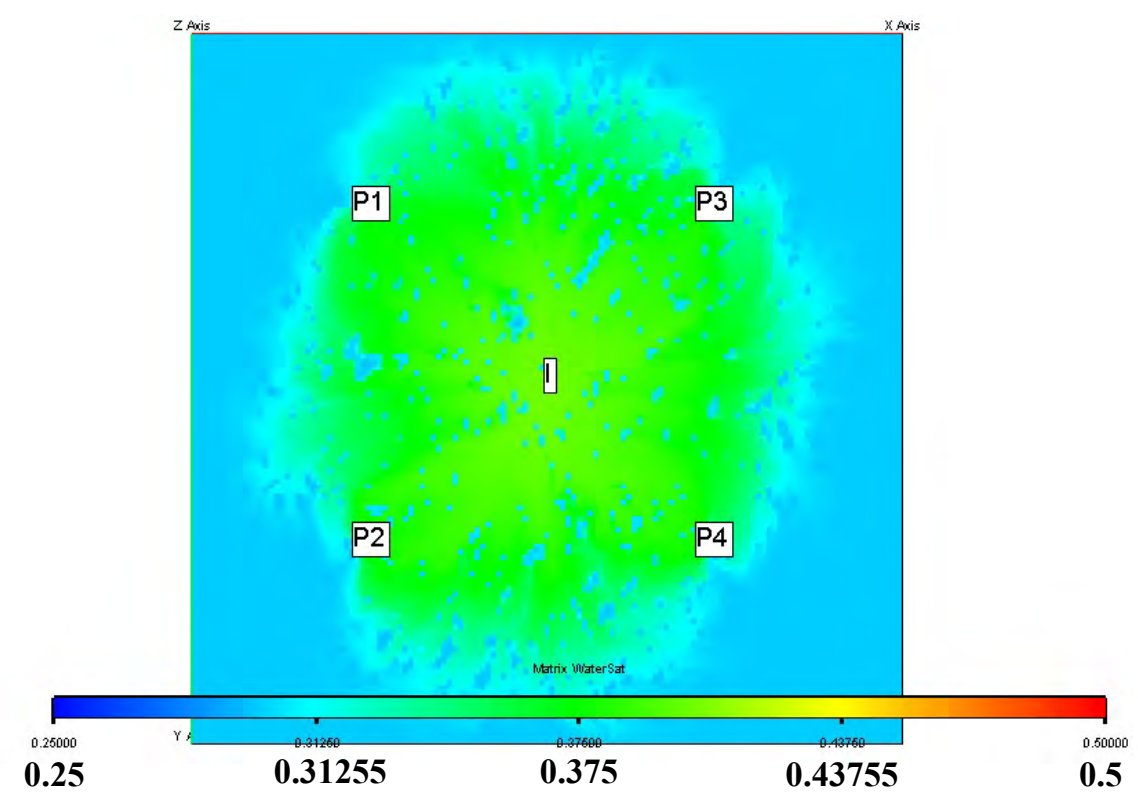

Fig. D.12a - Matrix water saturation at report step 15 (450 days) for test T4 base model.

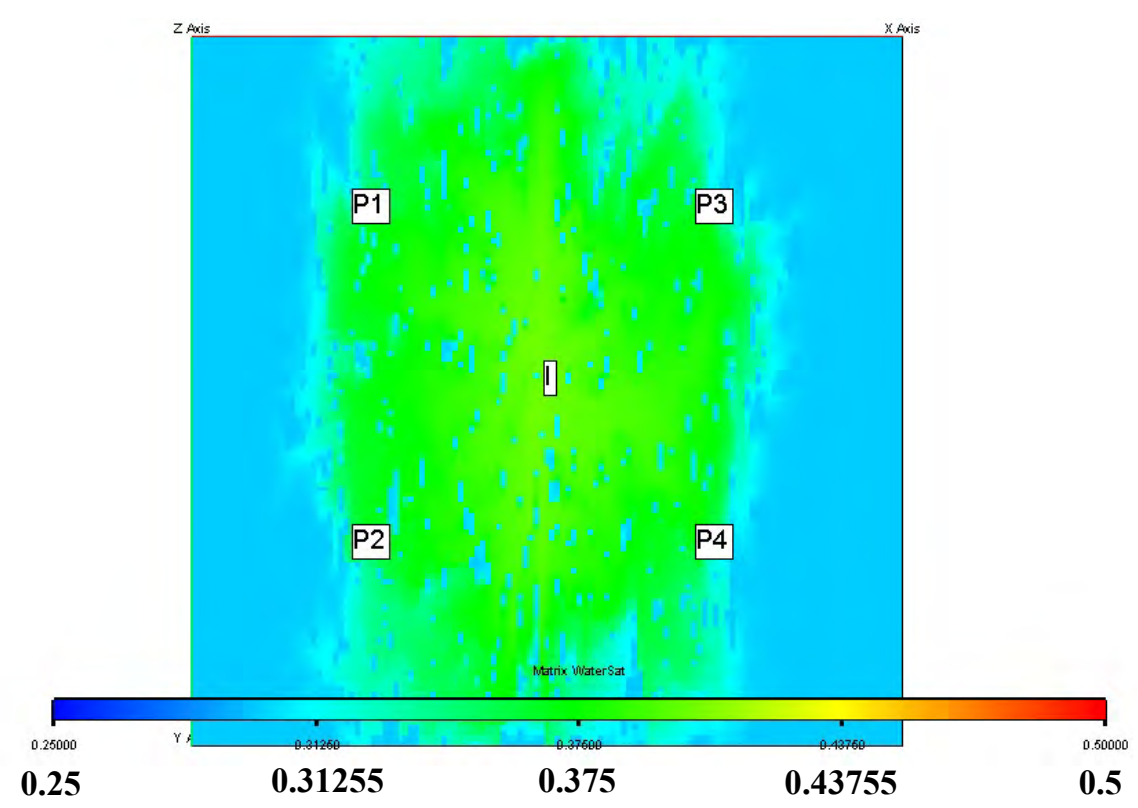

Fig. D.12b - Matrix water saturation at report step 15 (450 days) for test T4 initial estimate. 


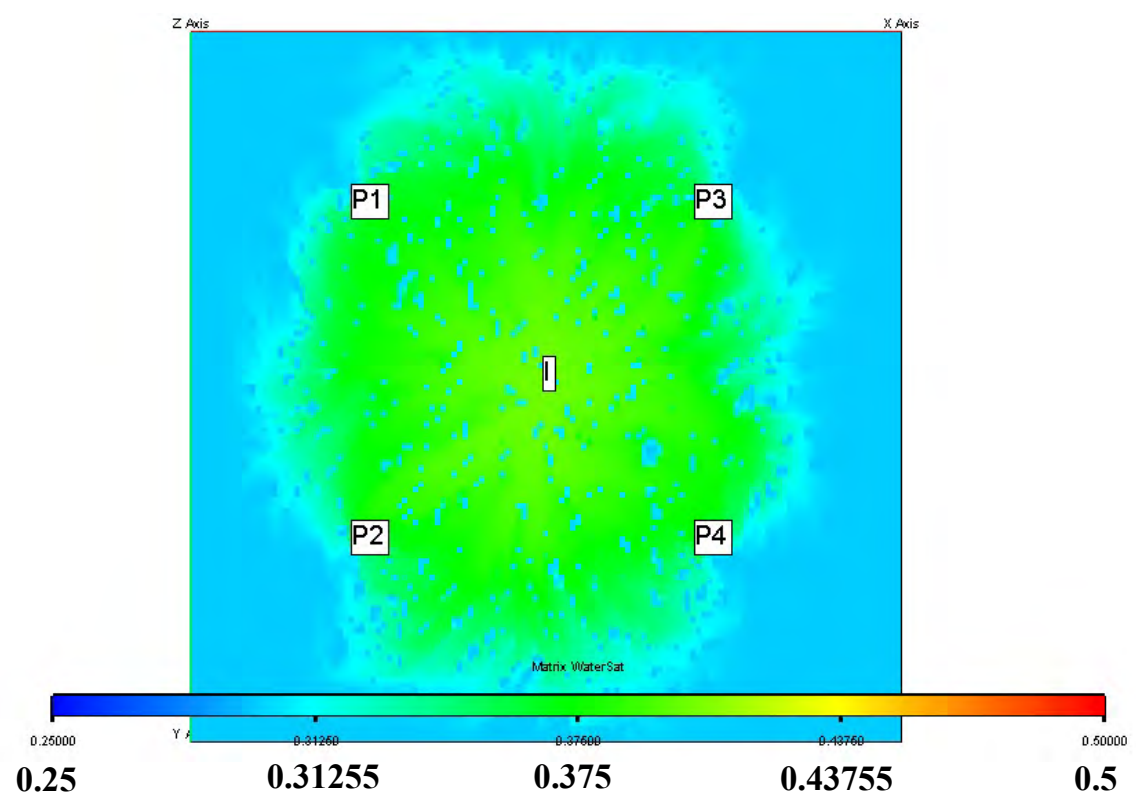

Fig. D.12c - Matrix water saturation at report step 15 (450 days) for test T4 final model.

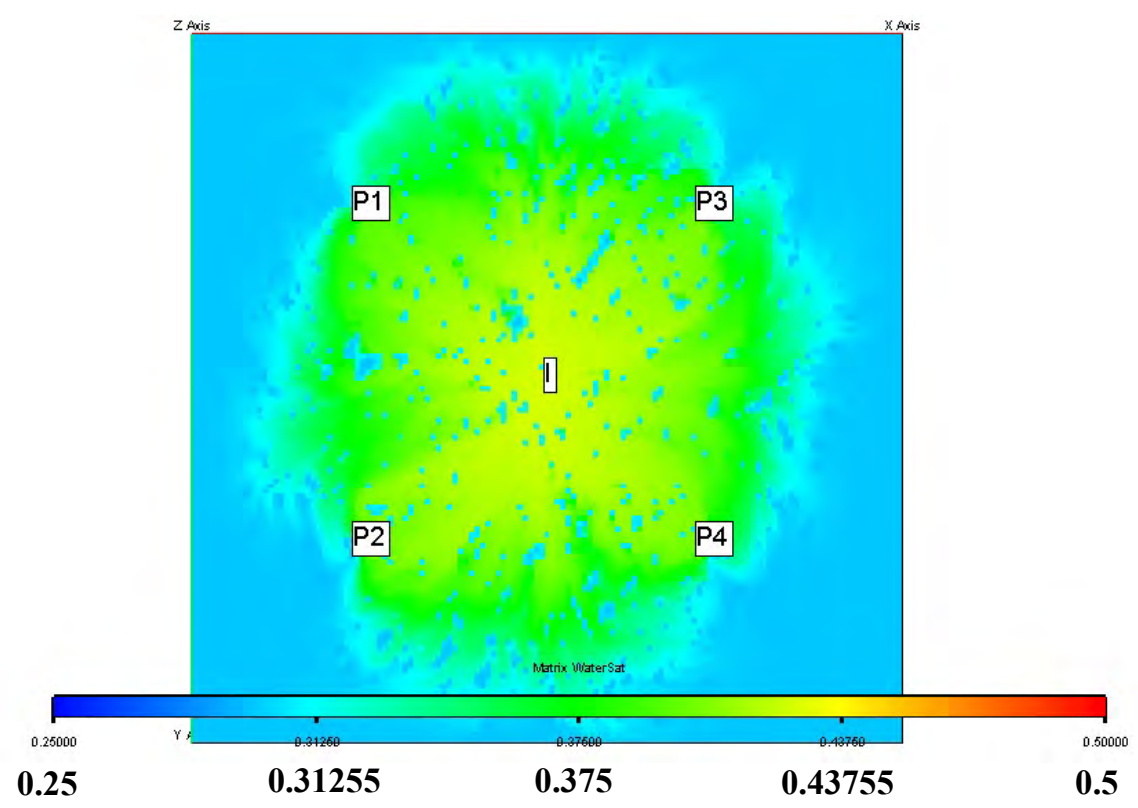

Fig. D.13a - Matrix water saturation at report step $20(600$ days) for test T4 base model. 


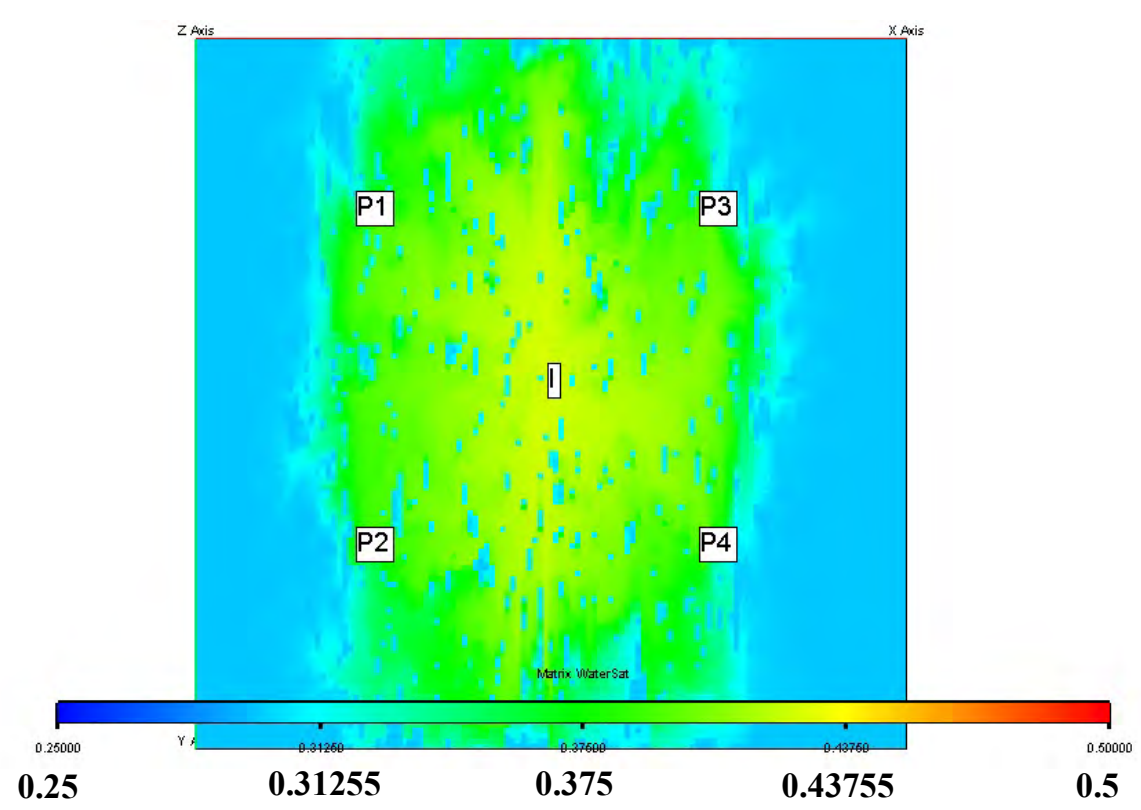

Fig. D.13b - Matrix water saturation at report step $20(600$ days) for test T4 initial estimate.

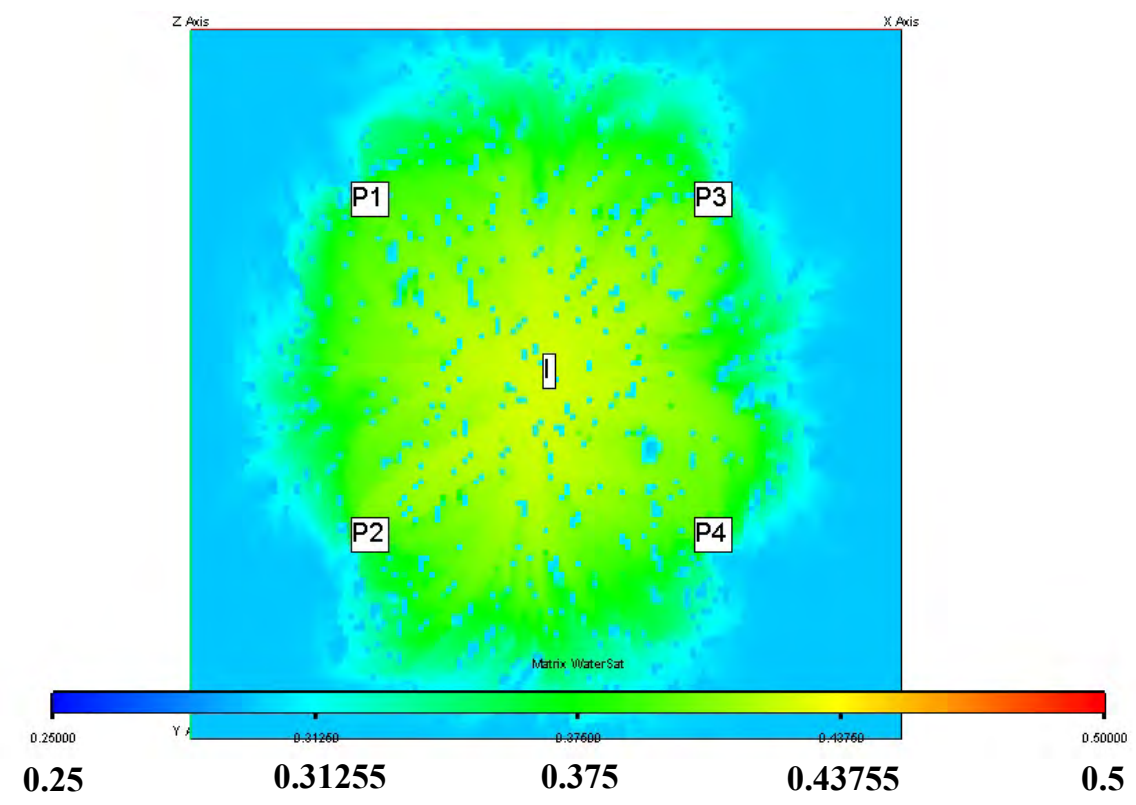

Fig. D.13c - Matrix water saturation at report step $20(600$ days) for test $\mathrm{T} 4$ final model. 


\section{Well Bottom Hole Pressure}

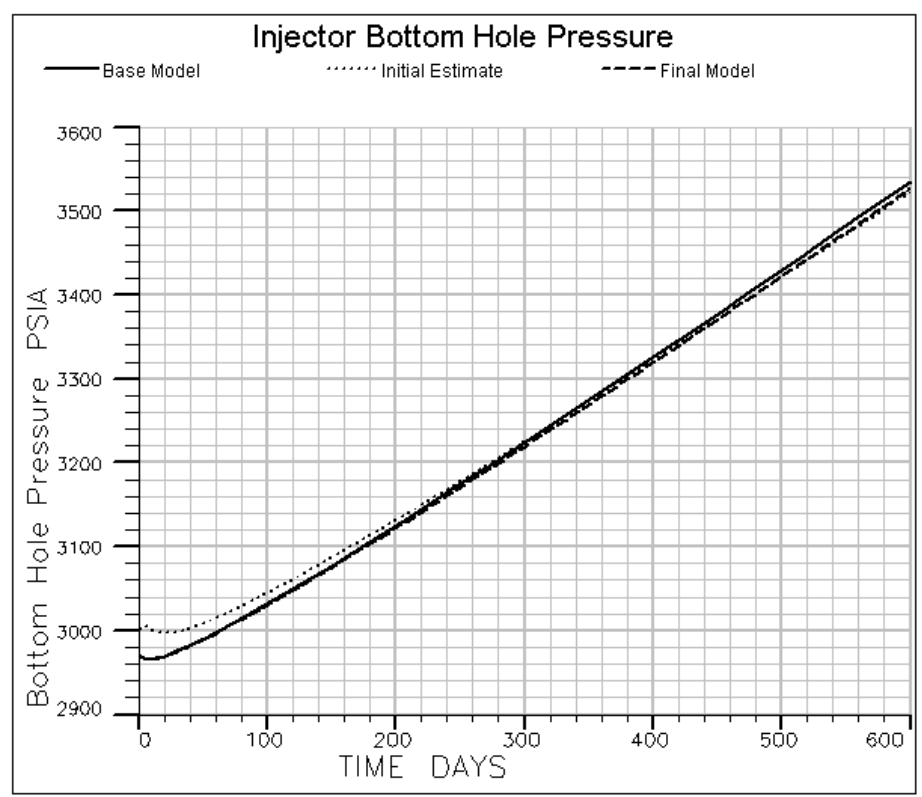

Fig. D.14a - Injector flowing bottom hole pressure for base model, initial estimate, and final model.

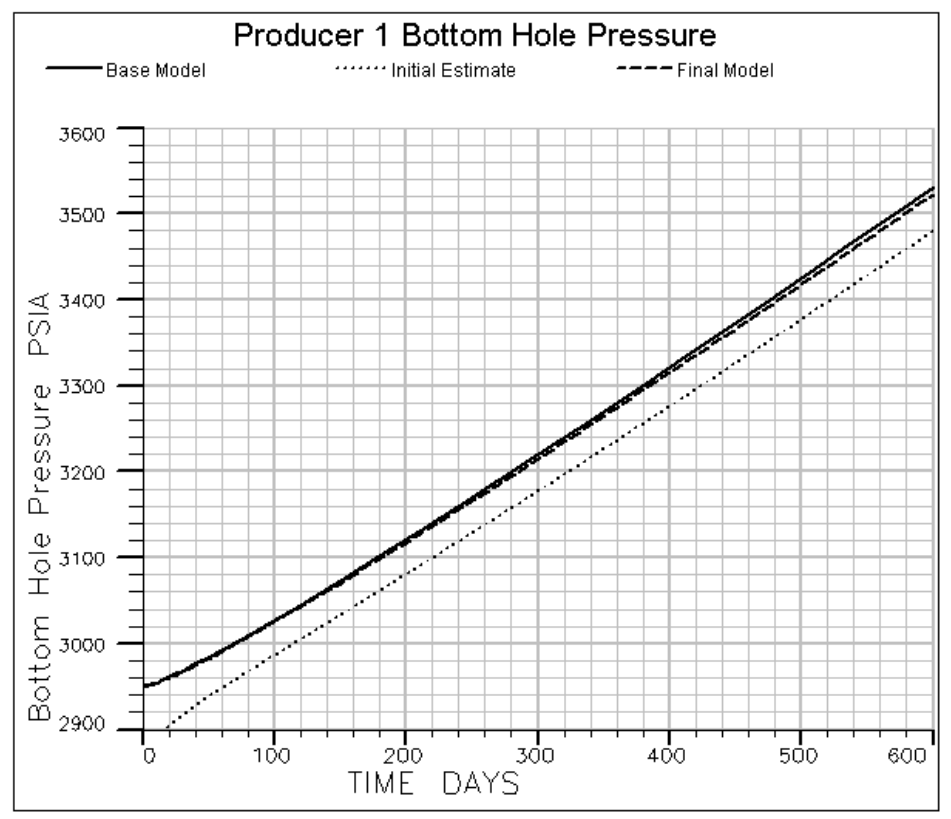

Fig. D.14b - Producer 1 flowing bottom hole pressure for base model, initial estimate, and final model. 


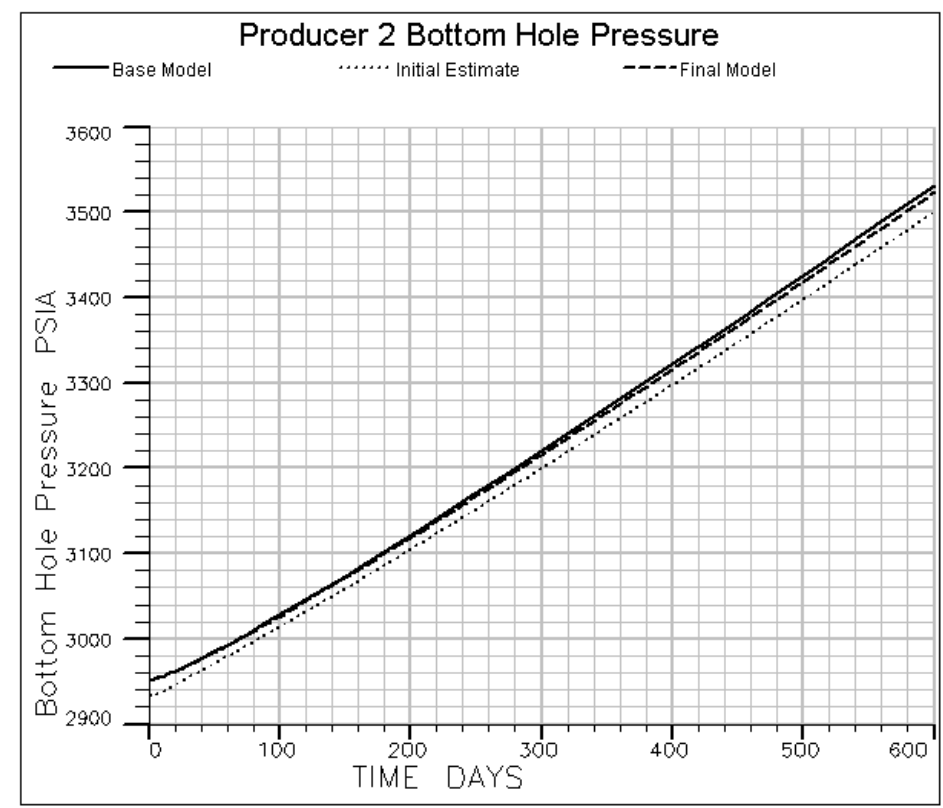

Fig. D.14c - Producer 2 flowing bottom hole pressure for base model, initial estimate, and final model.

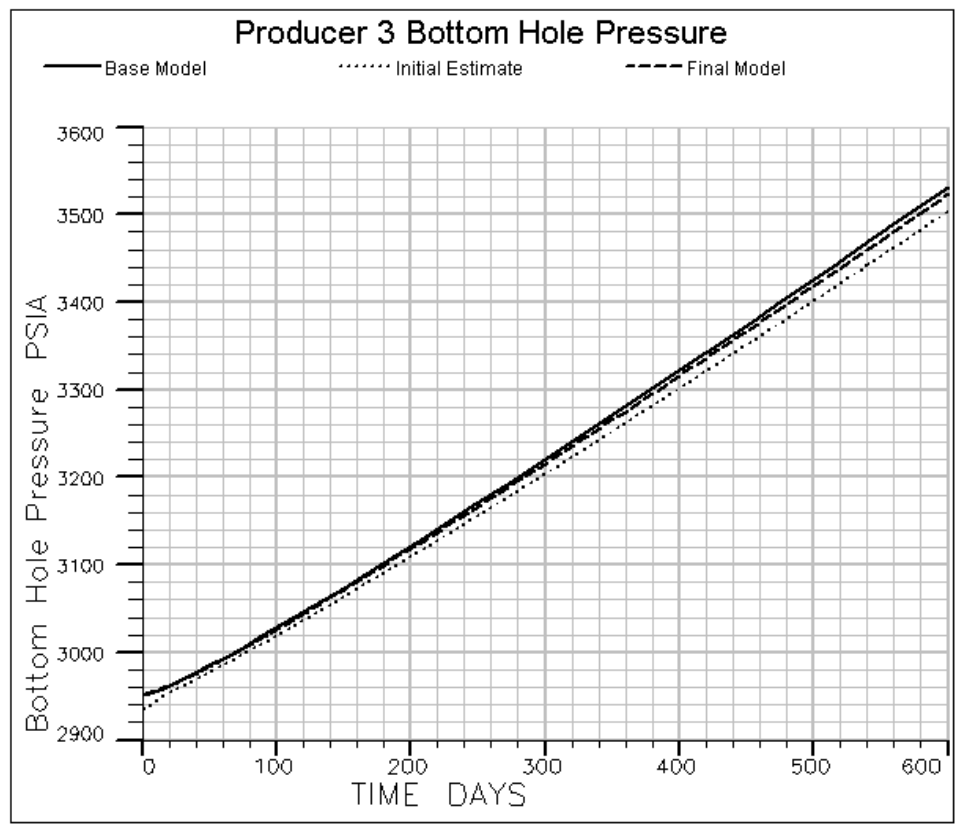

Fig. D.14d - Producer 3 flowing bottom hole pressure for base model, initial estimate, and final model. 


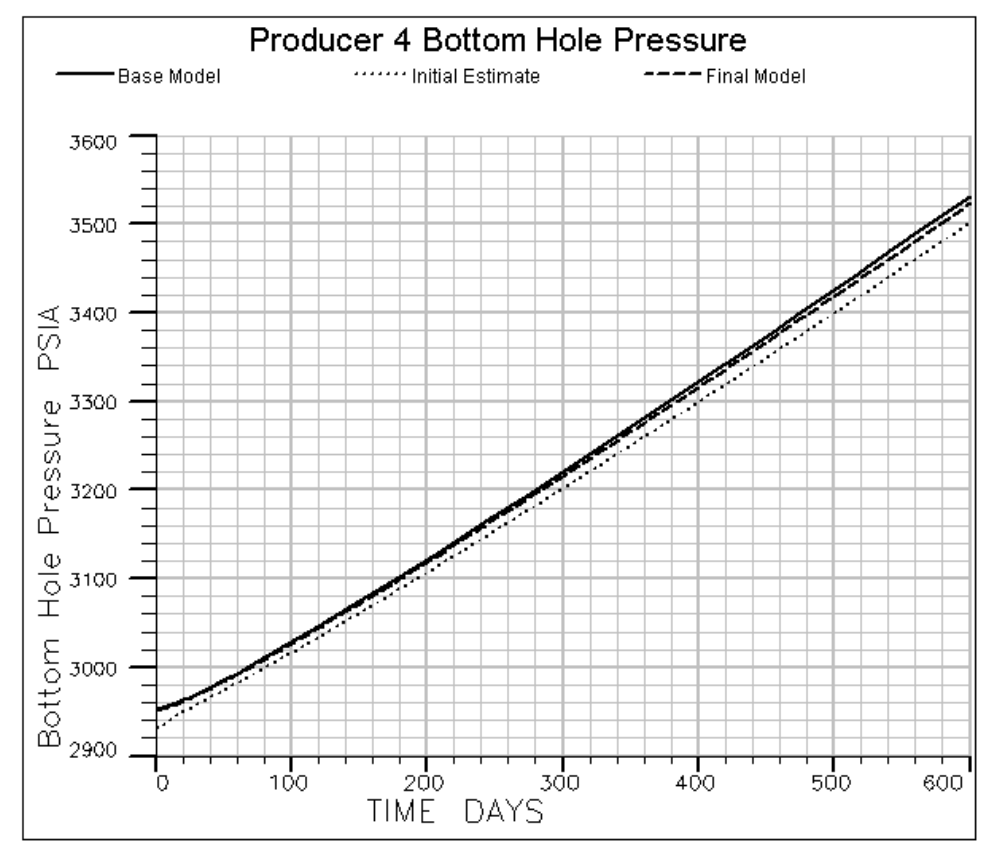

Fig. D.14e - Producer 4 flowing bottom hole pressure for base model, initial estimate, and final model. 


\section{Well Oil Production Rate}

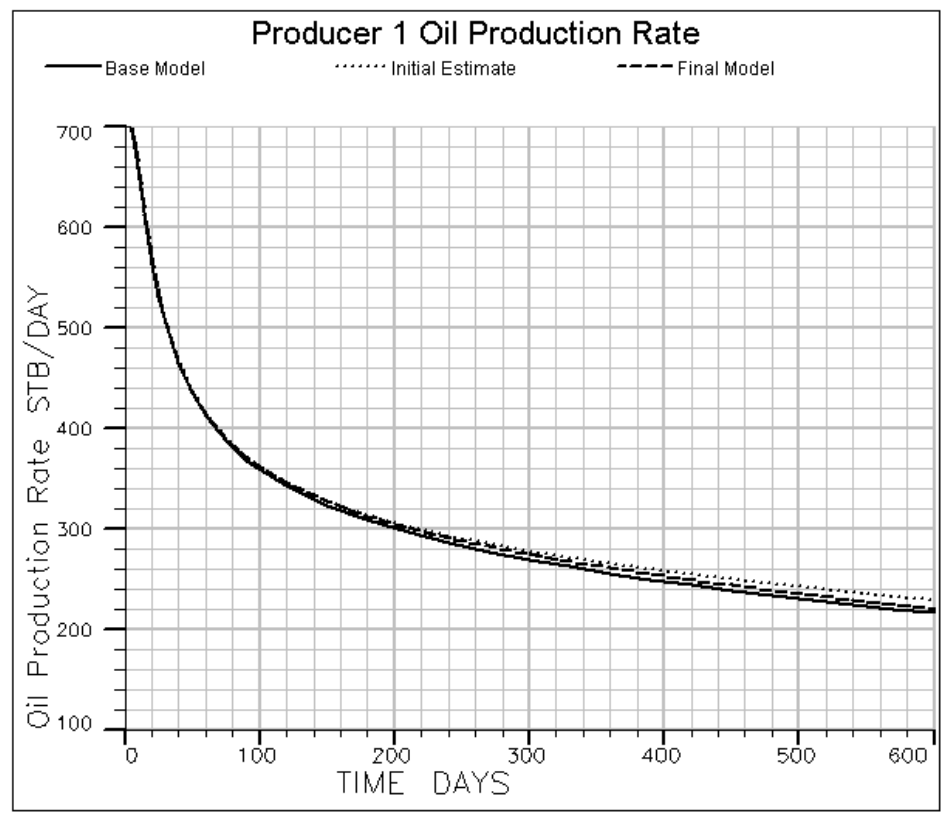

Fig. D. 15a - Producer 1 oil production rate for base model, initial estimate, and final model.

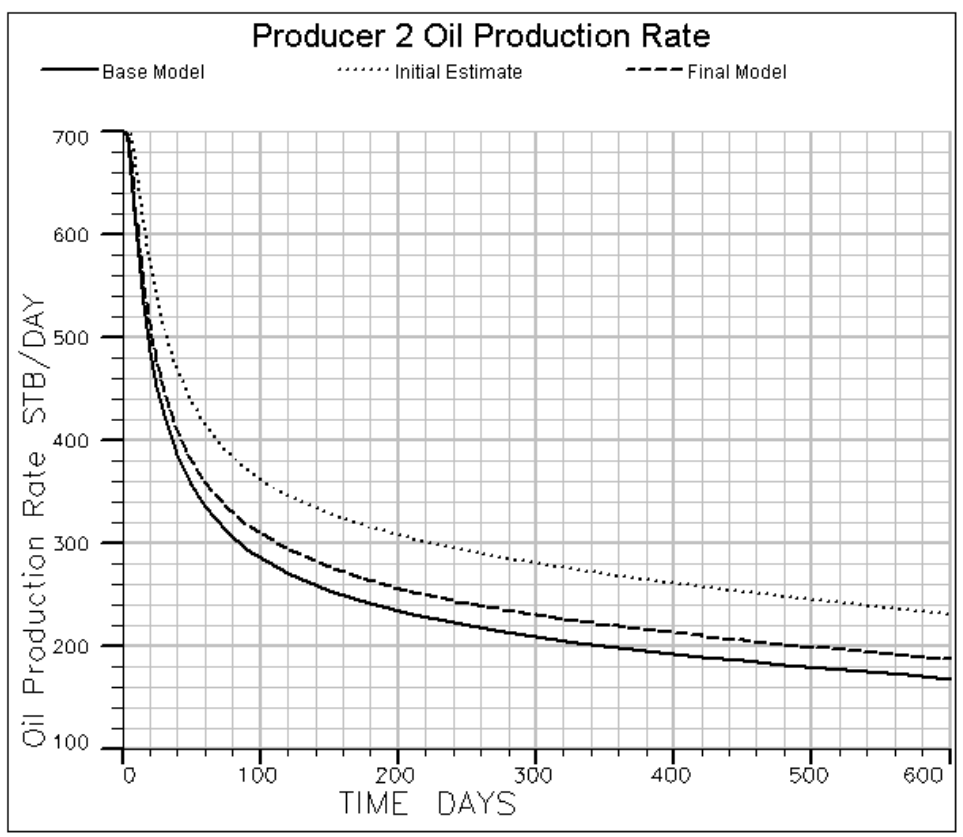

Fig. D.15b - Producer 2 oil production rate for base model, initial estimate, and final model. 


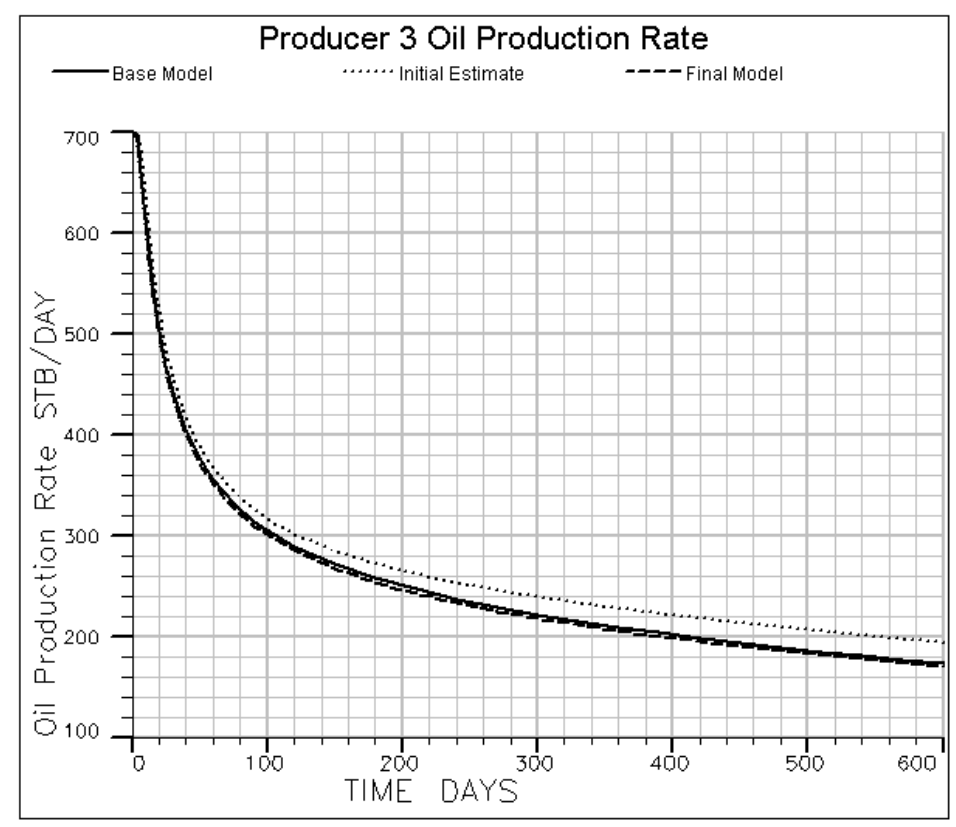

Fig. D.15c - Producer 3 oil production rate for base model, initial estimate, and final model.

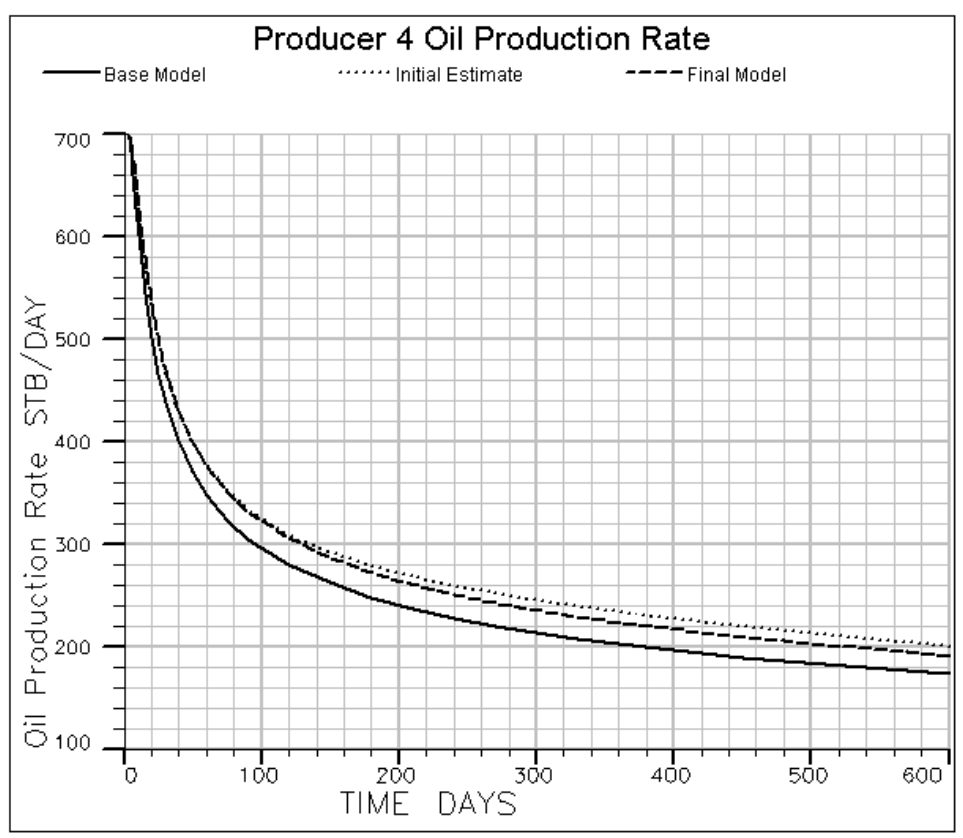

Fig. D.15d - Producer 4 oil production rate for base model, initial estimate, and final model. 
Elastic Anisotropy versus Azimuth (Base, Initial, Final)

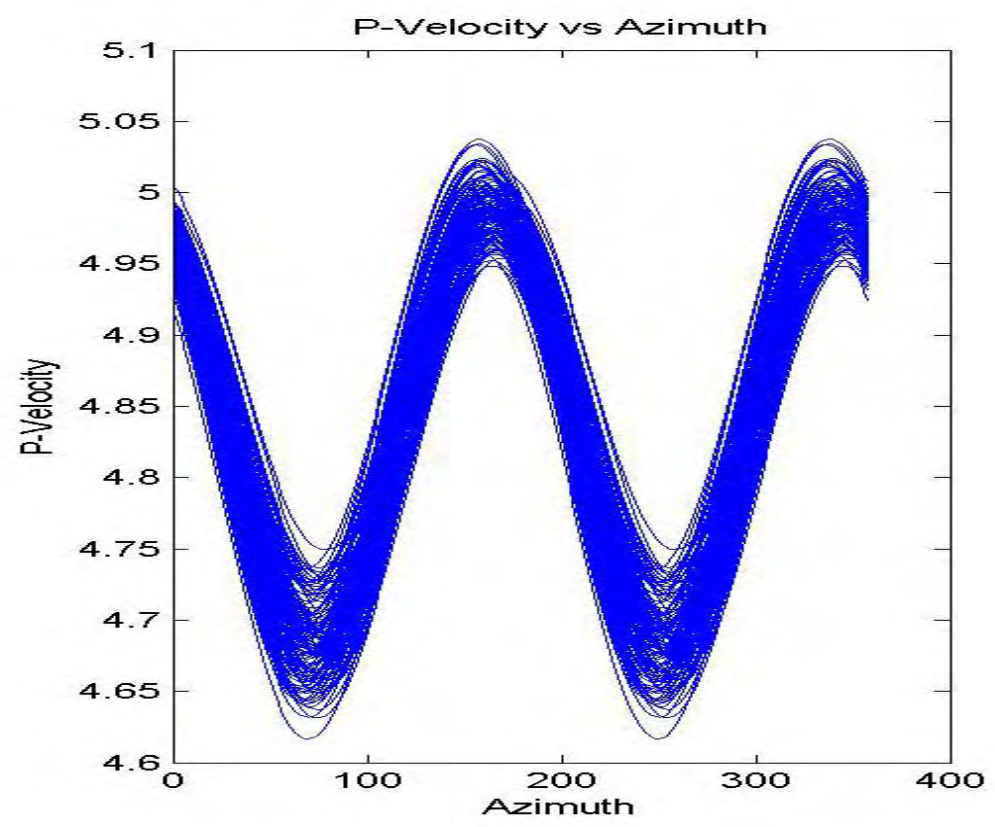

Fig. D.16a - Quasi-P wave velocity versus azimuth for base model.

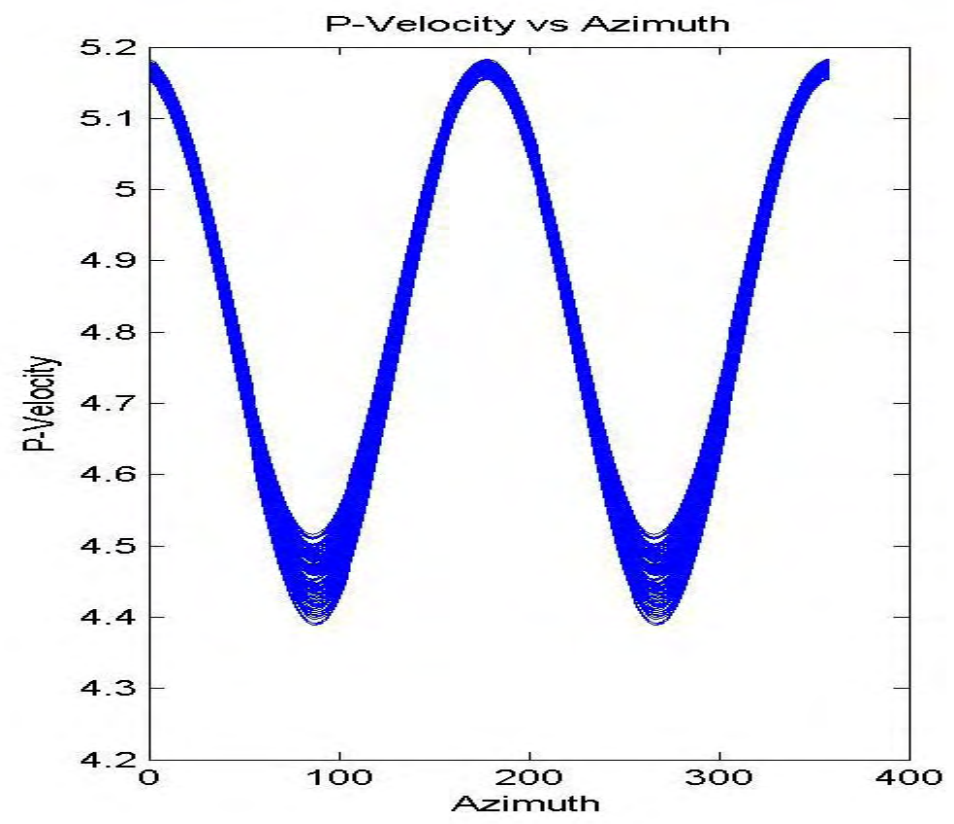

Fig. D.16b - Quasi-P wave velocity versus azimuth for initial estimate (M1). 


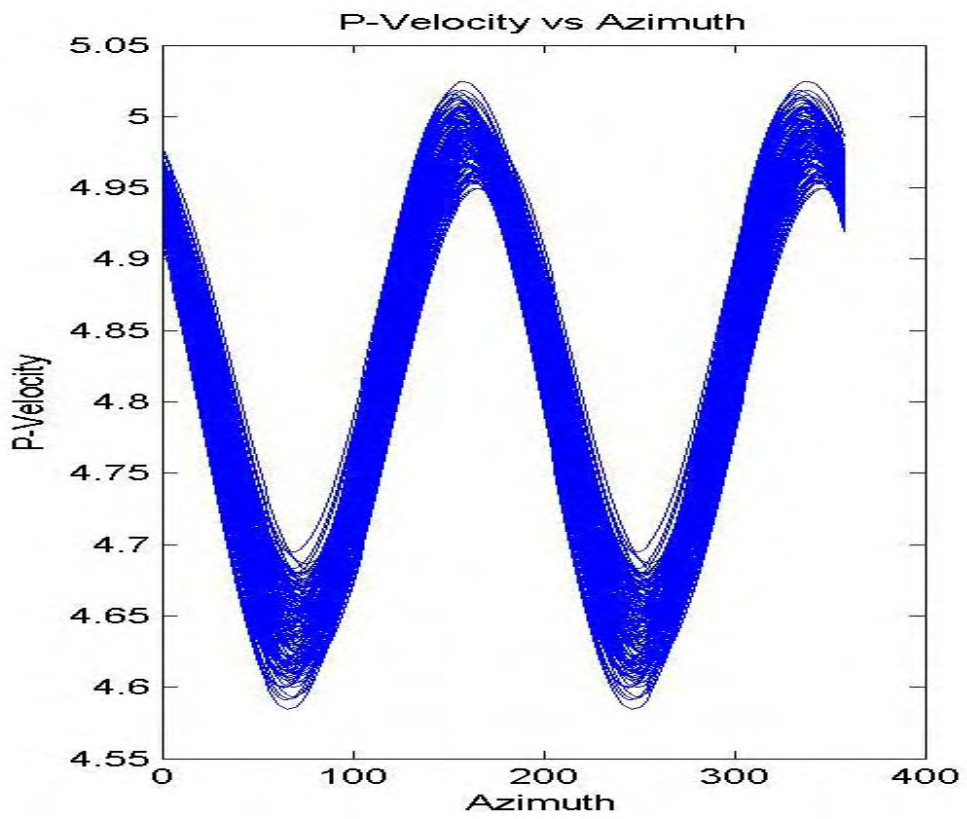

Fig. D.16c - Quasi-P wave velocity versus azimuth for final model (M8).

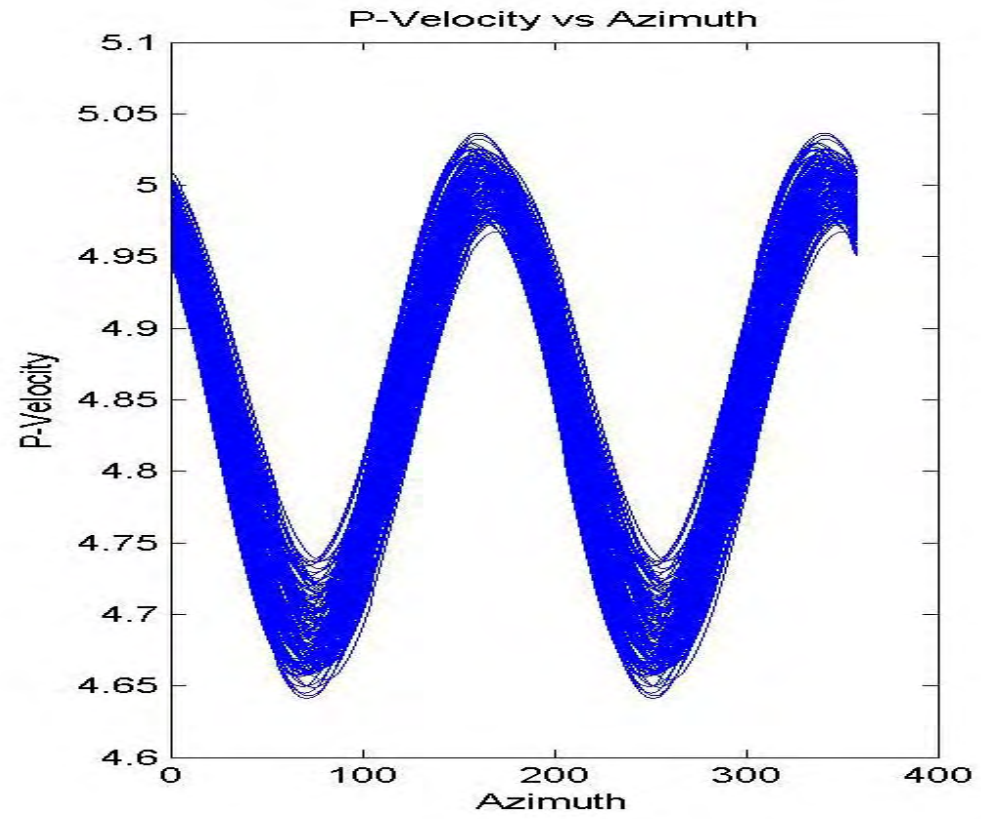

Fig. D.16d - Quasi-P wave velocity versus azimuth for base model for comparison with Fig. 16c. 

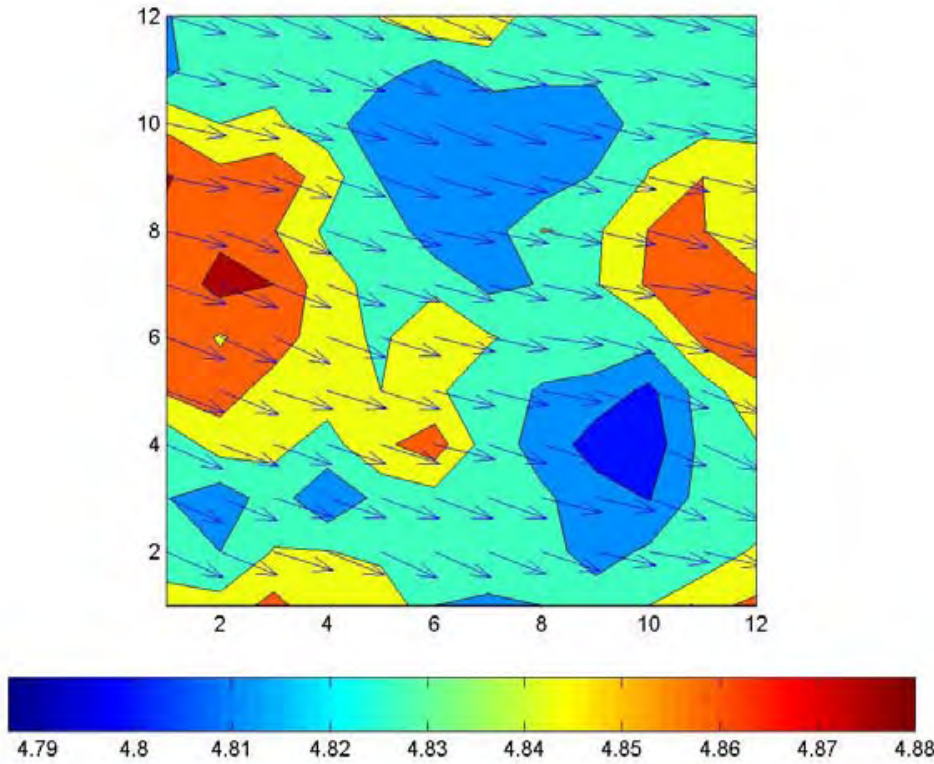

Fig. D.17a - Base model P velocity modulation amplitude and fast shear direction.
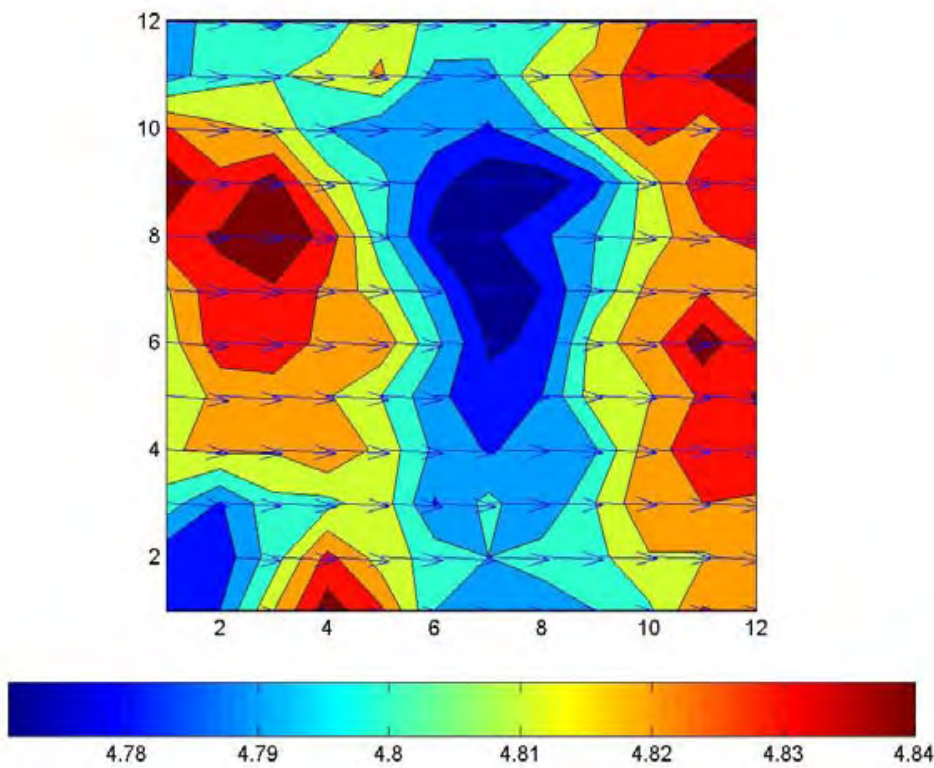

Fig. D.17b - Initial estimate $\mathrm{P}$ velocity modulation amplitude and fast shear direction. 

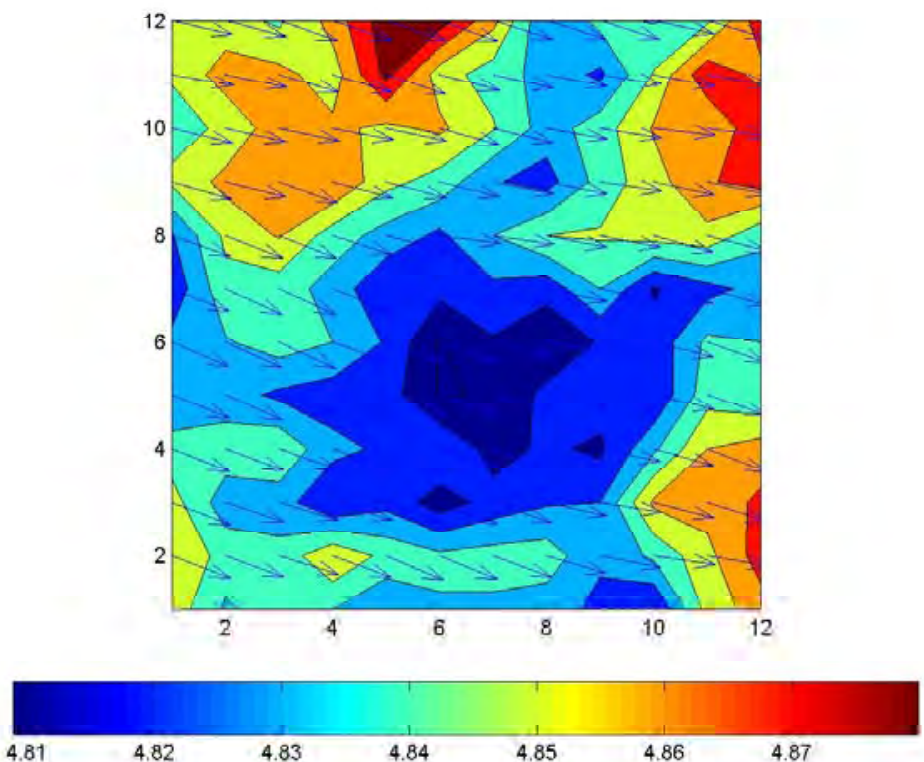

Fig. D.17c - Final $\mathrm{P}$ velocity modulation amplitude and fast shear direction.
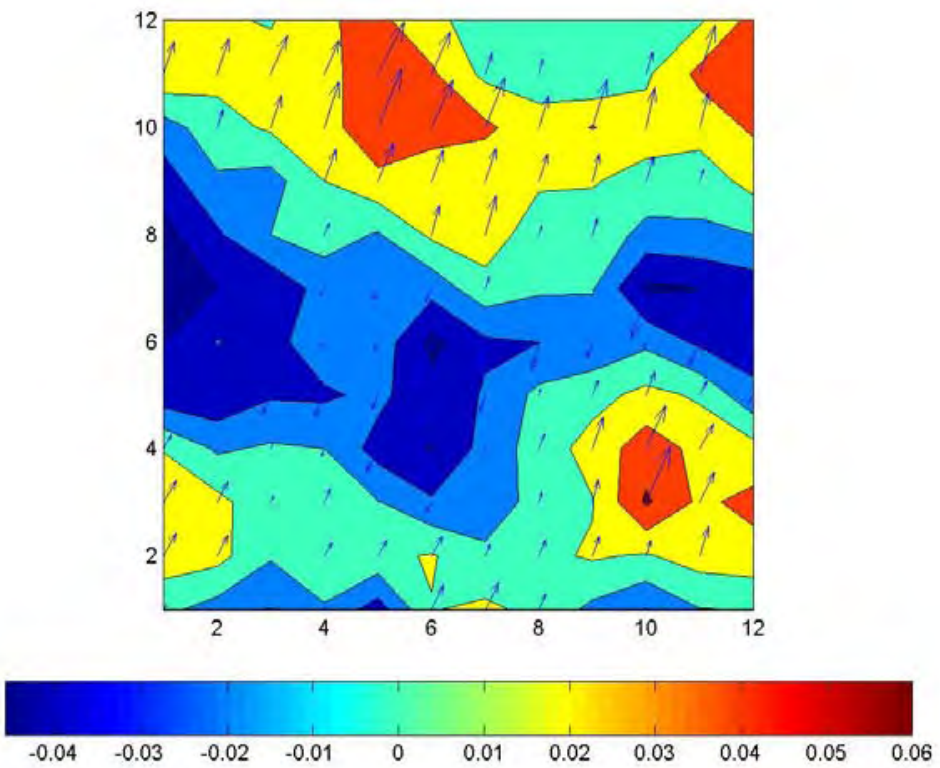

Fig. D.17d - P velocity modulation amplitude and fast shear direction difference between base and final modes. 


\section{APPENDIX E}

\section{SUMMARY OF SENSITIVITY TEST RESULTS}

In this Appendix we provide plots and tables detailing objective function sensitivity tests. Selected portions of this information may be found in Chapter $\mathrm{V}$ with a concise description of sensitivity testing program.

\section{General Information}

- Fracture system properties.

- Petrophysical properties.

- Simulation parameters.

- Elastic properties.

\section{Summarized Test Results}

- Parameter error summary table and plot.

- Residual summary table and plot.

\section{Data for Each Iteration}

- Table of derivative perturbations.

- Table of prior model weights.

- Tables of raw parameter updates.

- Tables of raw residuals.

- Plots of sensitivity coefficients.

For base case, initial estimate, and optimized model

- Plots of fracture distributions. 
- Maps of fracture system water saturation at report steps of 150 days, 300 days, 450 days, and 600 days.

- Maps of matrix water saturation at report step of 150 days, 300 days, 450 days, and 600 days.

- Bottom hole pressure plots.

- Oil production rate plots.

- Seismic P-wave velocity versus azimuth plots .

- Seismic anisotropy attribute maps. 
Objective Function Sensitivity

Fracture Set 2 Intensity and Trend

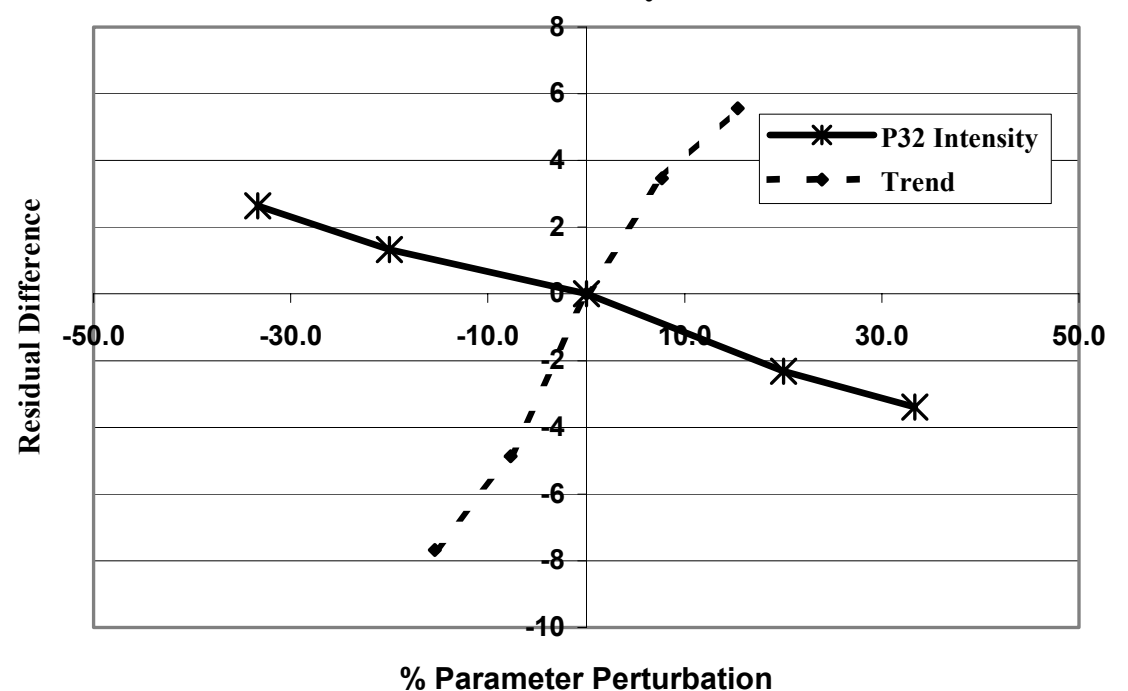

Fig. E.1 - Total unit residual error difference versus percent parameter perturbation, trend and P32 intensity.

Table E.1 - Component and total unit residual error versus P32 intensity.

\begin{tabular}{|r|r|r|r|r|}
\cline { 2 - 5 } \multicolumn{1}{c|}{} & \multicolumn{4}{c|}{ Unit Residual } \\
\hline $\begin{array}{c}\text { Parameter } \\
\text { Value }\end{array}$ & \multicolumn{1}{c|}{ TOTAL } & \multicolumn{1}{c|}{ BHP } & \multicolumn{1}{c|}{ OPR } & \multicolumn{1}{c|}{ SEIS } \\
\hline $\mathbf{0 . 1 0}$ & 21.22 & 9.26 & 4.61 & 18.52 \\
\hline $\mathbf{0 . 1 2}$ & 19.91 & 8.02 & 3.98 & 17.78 \\
\hline $\mathbf{0 . 1 5}$ & 18.58 & 8.04 & 3.50 & 16.38 \\
\hline $\mathbf{0 . 1 8}$ & 16.27 & 6.76 & 2.51 & 14.58 \\
\hline $\mathbf{0 . 2 0}$ & 15.19 & 6.64 & 2.39 & 13.45 \\
\hline
\end{tabular}

Table E.2 - Component and total unit residual error difference versus percent parameter perturbation, P32 intensity.

\begin{tabular}{|r|r|r|r|r|}
\cline { 2 - 5 } \multicolumn{1}{c|}{} & \multicolumn{4}{c|}{ Unit Residual Difference from Base } \\
\hline $\begin{array}{r}\text { \% Parameter } \\
\text { Perturbation }\end{array}$ & \multicolumn{1}{|c|}{ TOTAL } & \multicolumn{1}{c|}{ BHP } & \multicolumn{1}{c|}{ OPR } & \multicolumn{1}{c|}{ SEIS } \\
\hline $\mathbf{- 3 3 . 3}$ & 2.64 & 1.22 & 1.11 & 2.14 \\
\hline $\mathbf{- 2 0 . 0}$ & 1.33 & -0.03 & 0.48 & 1.40 \\
\hline $\mathbf{0 . 0}$ & 0.00 & 0.00 & 0.00 & 0.00 \\
\hline $\mathbf{2 0 . 0}$ & -2.32 & -1.28 & -0.99 & -1.80 \\
\hline $\mathbf{3 3 . 3}$ & -3.39 & -1.41 & -1.10 & -2.93 \\
\hline
\end{tabular}




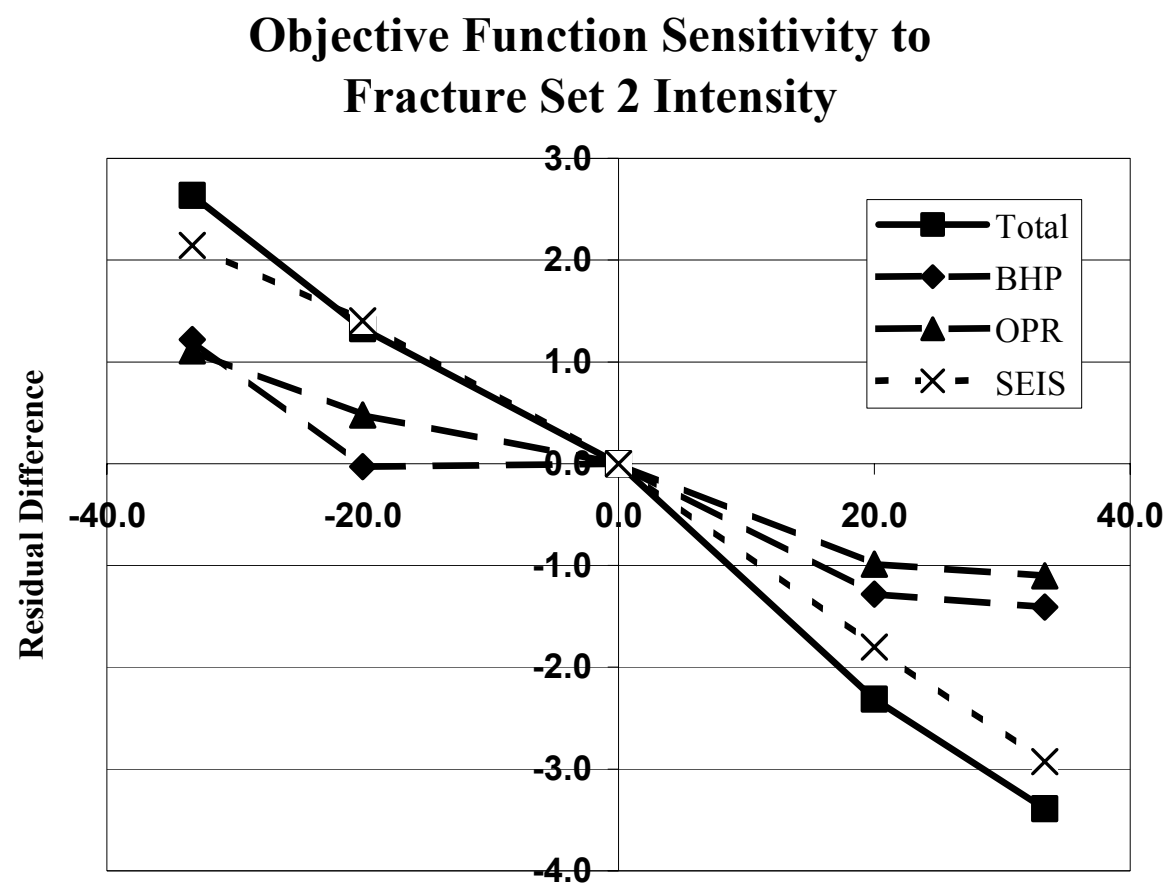

Parameter Perturbation

Fig. E.2 - Component and total unit residual error difference versus percent parameter perturbation, P32 intensity.

Table E.3 - Component and total unit residual error versus trend.

\begin{tabular}{|r|r|r|r|r|}
\cline { 2 - 5 } \multicolumn{1}{c|}{} & \multicolumn{4}{c|}{ Unit Residual } \\
\hline $\begin{array}{c}\text { Parameter } \\
\text { Value }\end{array}$ & \multicolumn{1}{c|}{ TOTAL } & \multicolumn{1}{c|}{ BHP } & \multicolumn{1}{c|}{ OPR } & \multicolumn{1}{c|}{ SEIS } \\
\hline $\mathbf{1 1 0 . 0}$ & 11.08 & 6.08 & 2.03 & 9.04 \\
\hline $\mathbf{1 2 0 . 0}$ & 13.90 & 6.13 & 1.03 & 12.43 \\
\hline $\mathbf{1 3 0 . 0}$ & 18.76 & 8.33 & 3.97 & 16.33 \\
\hline $\mathbf{1 4 0 . 0}$ & 22.23 & 8.02 & 2.60 & 20.57 \\
\hline $\mathbf{1 5 0 . 0}$ & 24.33 & 6.63 & 1.40 & 23.37 \\
\hline
\end{tabular}


Table E.4 - Component and total unit residual error difference versus percent parameter perturbation, trend.

\begin{tabular}{|r|r|r|r|r|}
\cline { 2 - 5 } \multicolumn{1}{c|}{} & \multicolumn{4}{c|}{ Unit Residual Difference from Base } \\
\hline $\begin{array}{r}\text { \% Parameter } \\
\text { Perturbation }\end{array}$ & \multicolumn{1}{c|}{ TOTAL } & \multicolumn{1}{c|}{ BHP } & \multicolumn{1}{c|}{ OPR } & \multicolumn{1}{c|}{ SEIS } \\
\hline $\mathbf{- 1 5 . 4}$ & -7.68 & -2.25 & -1.95 & -7.30 \\
\hline $\mathbf{- 7 . 7}$ & -4.87 & -2.20 & -2.95 & -3.91 \\
\hline $\mathbf{0 . 0}$ & 0.00 & 0.00 & 0.00 & 0.00 \\
\hline $\mathbf{7 . 7}$ & 3.47 & -0.31 & -1.38 & 4.24 \\
\hline $\mathbf{1 5 . 4}$ & 5.57 & -1.69 & -2.58 & 7.03 \\
\hline
\end{tabular}

\section{Objective Function Sensitivity to}

Fracture Set 2 Trend

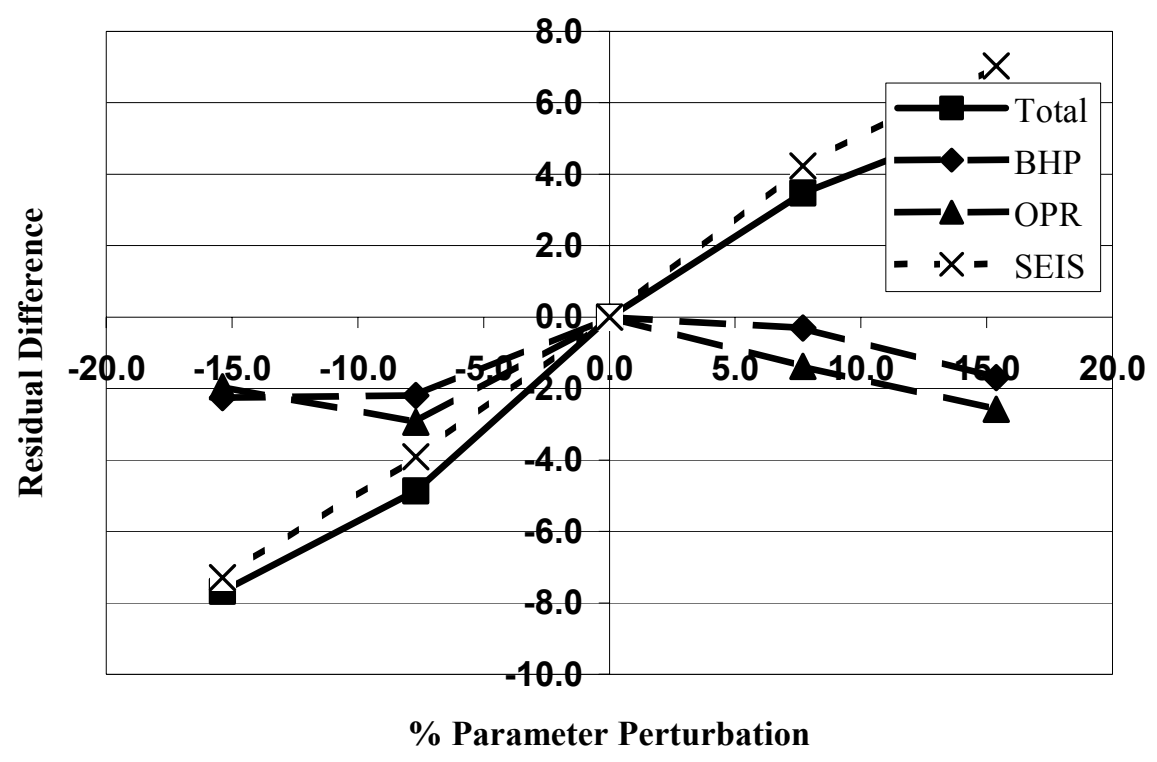

Fig. E.3 - Component and total unit residual error difference versus percent parameter perturbation, trend. 
Table E.5 - Component and total unit residual error versus hydraulic REV.

\begin{tabular}{|r|r|r|r|r|}
\cline { 2 - 5 } \multicolumn{1}{c|}{} & \multicolumn{4}{c|}{ Unit Residual } \\
\hline $\begin{array}{c}\text { Parameter } \\
\text { Value }\end{array}$ & \multicolumn{1}{c|}{ TOTAL } & \multicolumn{1}{c|}{ BHP } & \multicolumn{1}{c|}{ OPR } & \multicolumn{1}{c|}{ SEIS } \\
\hline 3.0 & 18.76 & 8.33 & 3.97 & 16.33 \\
\hline 10.0 & 17.82 & 6.71 & 2.03 & 16.38 \\
\hline 20.0 & 17.84 & 6.66 & 1.96 & 16.43 \\
\hline 30.0 & 17.82 & 6.77 & 1.83 & 16.38 \\
\hline 40.0 & 17.87 & 6.75 & 2.06 & 16.42 \\
\hline 50.0 & 17.81 & 6.70 & 1.90 & 16.39 \\
\hline
\end{tabular}

Table E.6 - Component and total unit residual error difference versus percent parameter perturbation, hydraulic REV.

\begin{tabular}{|r|r|r|r|r|}
\cline { 2 - 5 } \multicolumn{1}{c|}{} & \multicolumn{4}{c|}{ Unit Residual Difference from Base } \\
\hline $\begin{array}{r}\text { \% Parameter } \\
\text { Perturbation }\end{array}$ & \multicolumn{1}{c|}{ TOTAL } & \multicolumn{1}{c|}{ BHP } & \multicolumn{1}{c|}{ OPR } & \multicolumn{1}{c|}{ SEIS } \\
\hline 0.0 & 0.00 & 0.00 & 0.00 & 0.00 \\
\hline 233.3 & -0.94 & -1.62 & -1.94 & 0.05 \\
\hline 566.7 & -0.93 & -1.66 & -2.01 & 0.09 \\
\hline 900.0 & -0.95 & -1.56 & -2.14 & 0.04 \\
\hline 1233.3 & -0.89 & -1.58 & -1.91 & 0.08 \\
\hline 1566.7 & -0.96 & -1.63 & -2.08 & 0.05 \\
\hline
\end{tabular}




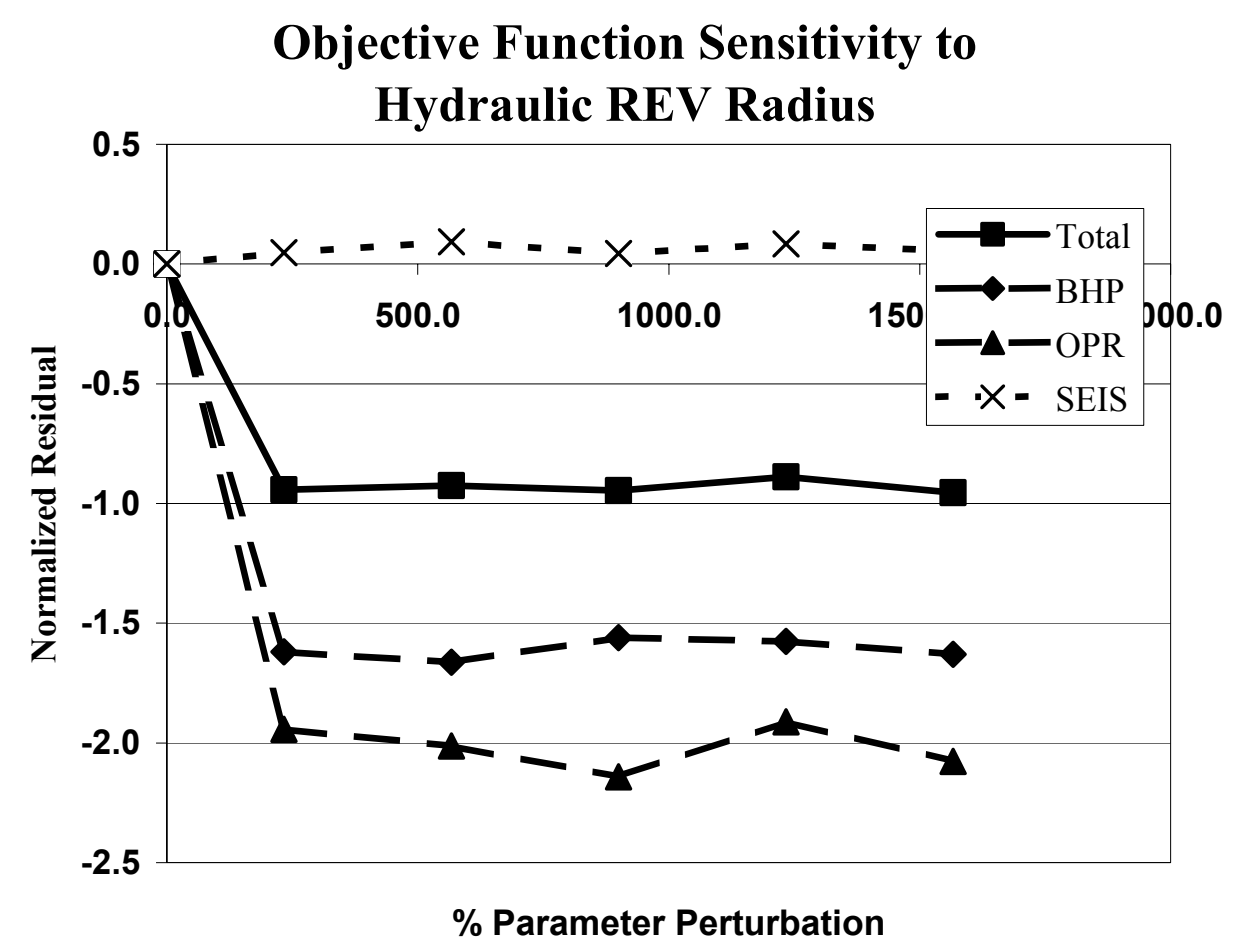

Fig. E.4 - Component and total unit residual error difference versus percent parameter perturbation, hydraulic $\mathrm{REV}$ radius.

Table E.7 - Component and total unit residual error versus sigma.

\begin{tabular}{|r|r|r|r|r|}
\cline { 2 - 5 } \multicolumn{1}{c|}{} & \multicolumn{4}{c|}{ Unit Residual } \\
\hline $\begin{array}{c}\text { Parameter } \\
\text { Value }\end{array}$ & \multicolumn{1}{c|}{ TOTAL } & \multicolumn{1}{c|}{ BHP } & \multicolumn{1}{c|}{ OPR } & \multicolumn{1}{c|}{ SEIS } \\
\hline $\mathbf{0 . 0 1}$ & 22.46 & 14.20 & 5.82 & 16.40 \\
\hline $\mathbf{0 . 0 8}$ & 18.76 & 8.33 & 3.97 & 16.33 \\
\hline $\mathbf{0 . 1 5}$ & 18.85 & 8.31 & 4.20 & 16.39 \\
\hline $\mathbf{0 . 2 5}$ & 19.01 & 8.59 & 4.42 & 16.36 \\
\hline $\mathbf{0 . 5}$ & 19.01 & 8.59 & 4.42 & 16.36 \\
\hline
\end{tabular}


Table E.8 - Component and total unit residual error difference versus percent parameter perturbation, sigma.

\begin{tabular}{|rr|r|r|r|}
\cline { 2 - 5 } \multicolumn{1}{c|}{} & \multicolumn{4}{c|}{ Unit Residual Difference from Base } \\
\hline $\begin{array}{r}\text { \% Parameter } \\
\text { Perturbation }\end{array}$ & \multicolumn{1}{c|}{ TOTAL } & \multicolumn{1}{c|}{ BHP } & \multicolumn{1}{c|}{ OPR } & \multicolumn{1}{c|}{ SEIS } \\
\hline $\mathbf{- 8 7 . 5}$ & 3.70 & 5.88 & 1.85 & 0.07 \\
\hline $\mathbf{0 . 0}$ & 0.00 & 0.00 & 0.00 & 0.00 \\
\hline $\mathbf{8 7 . 5}$ & 0.09 & -0.02 & 0.23 & 0.06 \\
\hline $\mathbf{2 1 2 . 5}$ & 0.25 & 0.27 & 0.45 & 0.03 \\
\hline $\mathbf{5 2 5 . 0}$ & 0.25 & 0.27 & 0.45 & 0.03 \\
\hline
\end{tabular}

\section{Objective Function Sensitivity to Dual Porosity Shape Factor}

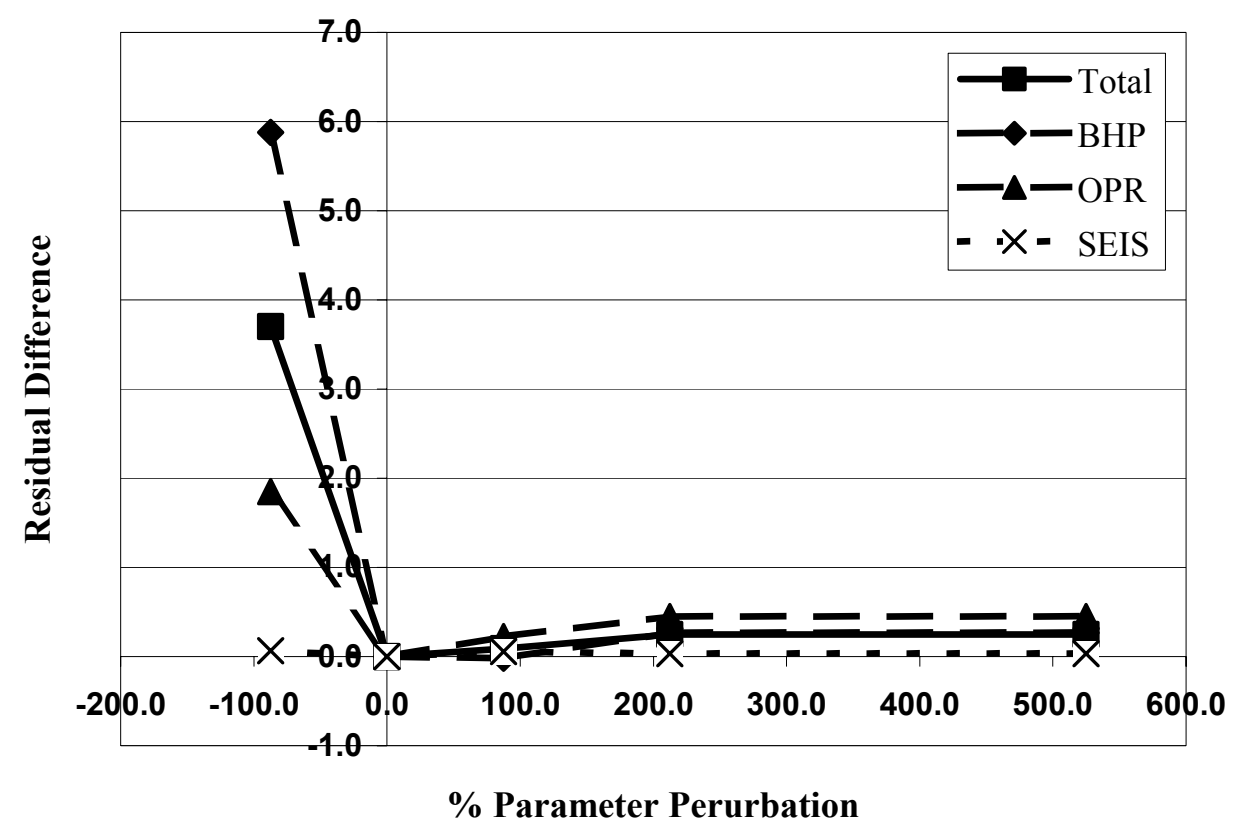

Fig. E.5 - Component and total unit residual error difference versus percent parameter perturbation, sigma. 
Table E.9 - Component and total unit residual error versus matrix permeability.

\begin{tabular}{|r|r|r|r|r|}
\cline { 2 - 5 } \multicolumn{1}{c|}{} & \multicolumn{4}{c|}{ Unit Residual } \\
\hline $\begin{array}{r}\text { Parameter } \\
\text { Value }\end{array}$ & \multicolumn{1}{c|}{ TOTAL } & \multicolumn{1}{c|}{ BHP } & \multicolumn{1}{c|}{ OPR } & \multicolumn{1}{c|}{ SEIS } \\
\hline $\mathbf{0 . 1}$ & 50.43 & 45.24 & 15.12 & 16.38 \\
\hline $\mathbf{1 . 0}$ & 23.49 & 15.65 & 6.23 & 16.37 \\
\hline $\mathbf{1 0 . 0}$ & 18.83 & 8.35 & 4.01 & 16.39 \\
\hline $\mathbf{5 0 . 0}$ & 19.18 & 8.74 & 4.58 & 16.44 \\
\hline $\mathbf{1 0 0 . 0}$ & 19.33 & 8.92 & 4.89 & 16.44 \\
\hline
\end{tabular}

Table E.10 - Component and total unit residual error difference versus percent parameter perturbation, matrix permeability.

\begin{tabular}{|r|r|r|r|r|}
\cline { 2 - 5 } \multicolumn{1}{c|}{} & \multicolumn{4}{c|}{ Unit Residual Difference from Base } \\
\hline $\begin{array}{r}\text { \% Parameter } \\
\text { Perturbation }\end{array}$ & \multicolumn{1}{c|}{ TOTAL } & \multicolumn{1}{c|}{ BHP } & \multicolumn{1}{c|}{ OPR } & \multicolumn{1}{c|}{ SEIS } \\
\hline $\mathbf{- 9 9 . 0}$ & 31.60 & 36.89 & 11.11 & -0.01 \\
\hline $\mathbf{9 0 . 0}$ & 4.66 & 7.30 & 2.22 & -0.02 \\
\hline $\mathbf{0 . 0}$ & 0.00 & 0.00 & 0.00 & 0.00 \\
\hline $\mathbf{4 0 0 . 0}$ & 0.35 & 0.39 & 0.57 & 0.05 \\
\hline $\mathbf{9 0 0 . 0}$ & 0.50 & 0.57 & 0.88 & 0.05 \\
\hline
\end{tabular}




\section{Objective Function Sensitivity to} Matrix Permeability

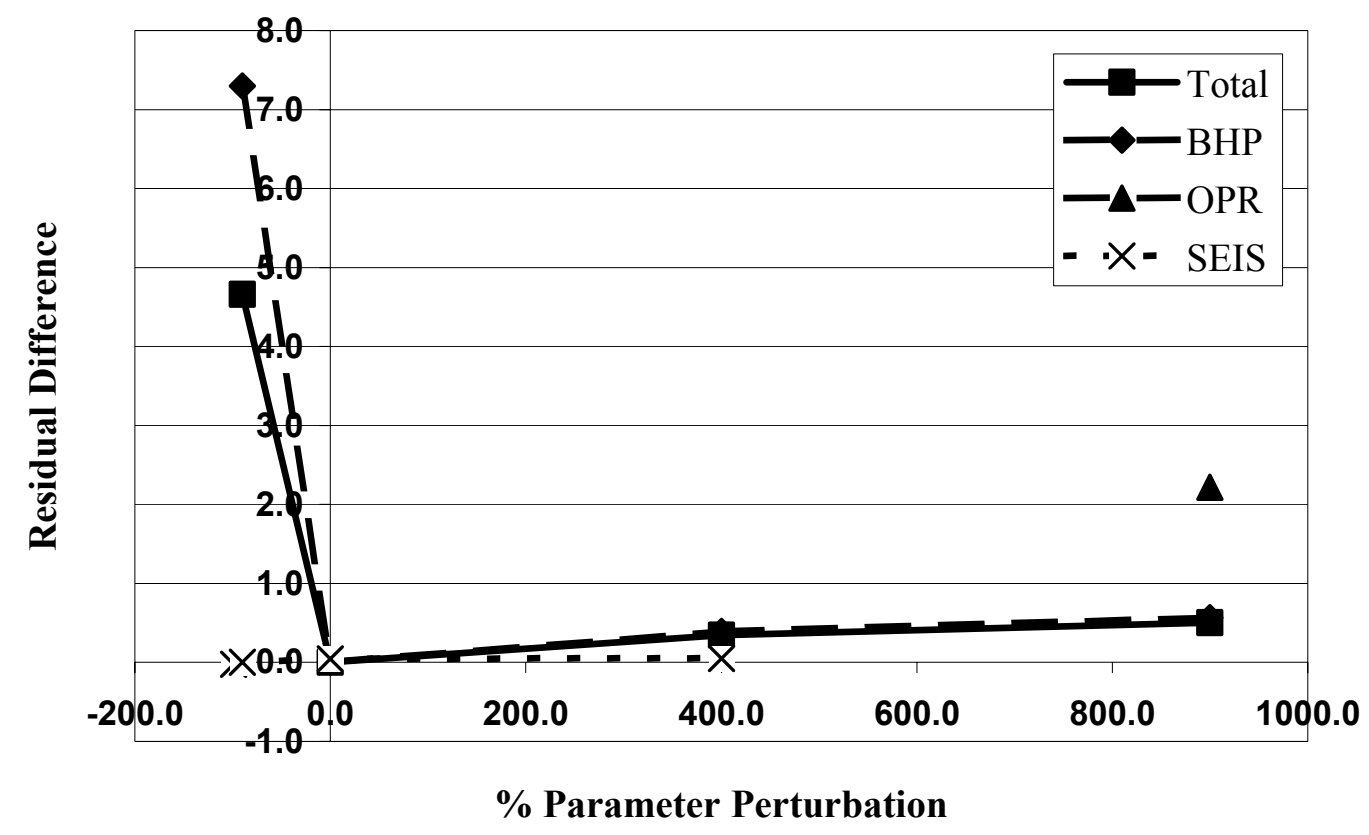

Fig. E.6 - Component and total unit residual error difference versus percent parameter perturbation, matrix permeability.

Table E.11 - Component and total unit residual error versus elastic REV radius.

\begin{tabular}{|r|r|r|r|r|}
\cline { 2 - 5 } \multicolumn{1}{c|}{} & \multicolumn{4}{c|}{ Unit Residual } \\
\hline $\begin{array}{r}\text { Parameter } \\
\text { Value }\end{array}$ & \multicolumn{1}{c|}{ TOTAL } & \multicolumn{1}{c|}{ BHP } & \multicolumn{1}{c|}{ OPR } & \multicolumn{1}{c|}{ SEIS } \\
\hline $\mathbf{2 0 . 0}$ & 17.38 & 8.45 & 3.93 & 14.67 \\
\hline $\mathbf{4 0 . 0}$ & 18.40 & 8.34 & 4.01 & 15.90 \\
\hline $\mathbf{6 0 . 0}$ & 18.76 & 8.39 & 4.00 & 16.30 \\
\hline $\mathbf{8 0 . 0}$ & 18.78 & 8.30 & 4.01 & 16.36 \\
\hline $\mathbf{1 0 0 . 0}$ & 18.77 & 8.35 & 3.89 & 16.36 \\
\hline $\mathbf{1 2 0 . 0}$ & 18.83 & 8.38 & 3.90 & 16.41 \\
\hline $\mathbf{1 4 0 . 0}$ & 18.98 & 8.34 & 4.13 & 16.54 \\
\hline $\mathbf{1 6 0 . 0}$ & 19.05 & 8.34 & 3.96 & 16.65 \\
\hline $\mathbf{1 8 0 . 0}$ & 19.14 & 8.35 & 3.98 & 16.75 \\
\hline $\mathbf{2 0 0 . 0}$ & 19.29 & 8.41 & 3.94 & 16.91 \\
\hline
\end{tabular}


Table E.12 - Component and total unit residual error difference versus percent parameter perturbation, elastic REV radius.

\begin{tabular}{|r|r|r|r|r|}
\cline { 2 - 5 } \multicolumn{1}{c|}{} & \multicolumn{4}{c|}{ Unit Residual Difference from Base } \\
\hline $\begin{array}{r}\text { \% Parameter } \\
\text { Perturbation }\end{array}$ & \multicolumn{1}{c|}{ TOTAL } & \multicolumn{1}{c|}{ BHP } & \multicolumn{1}{c|}{ OPR } & \multicolumn{1}{c|}{ SEIS } \\
\hline $\mathbf{- 8 0 . 0}$ & -1.39 & 0.10 & 0.04 & -1.68 \\
\hline $\mathbf{- 6 0 . 0}$ & -0.37 & -0.01 & 0.12 & -0.46 \\
\hline $\mathbf{- 4 0 . 0}$ & -0.01 & 0.04 & 0.12 & -0.06 \\
\hline $\mathbf{- 2 0 . 0}$ & 0.01 & -0.05 & 0.12 & 0.01 \\
\hline $\mathbf{0 . 0}$ & 0.00 & 0.00 & 0.00 & 0.00 \\
\hline $\mathbf{2 0 . 0}$ & 0.06 & 0.03 & 0.01 & 0.05 \\
\hline $\mathbf{4 0 . 0}$ & 0.20 & -0.01 & 0.24 & 0.18 \\
\hline $\mathbf{6 0 . 0}$ & 0.27 & -0.01 & 0.07 & 0.30 \\
\hline $\mathbf{8 0 . 0}$ & 0.37 & 0.00 & 0.10 & 0.40 \\
\hline $\mathbf{1 0 0 . 0}$ & 0.52 & 0.06 & 0.05 & 0.55 \\
\hline
\end{tabular}

\section{Objective Function Sensitivity to Elastic REV Radius}

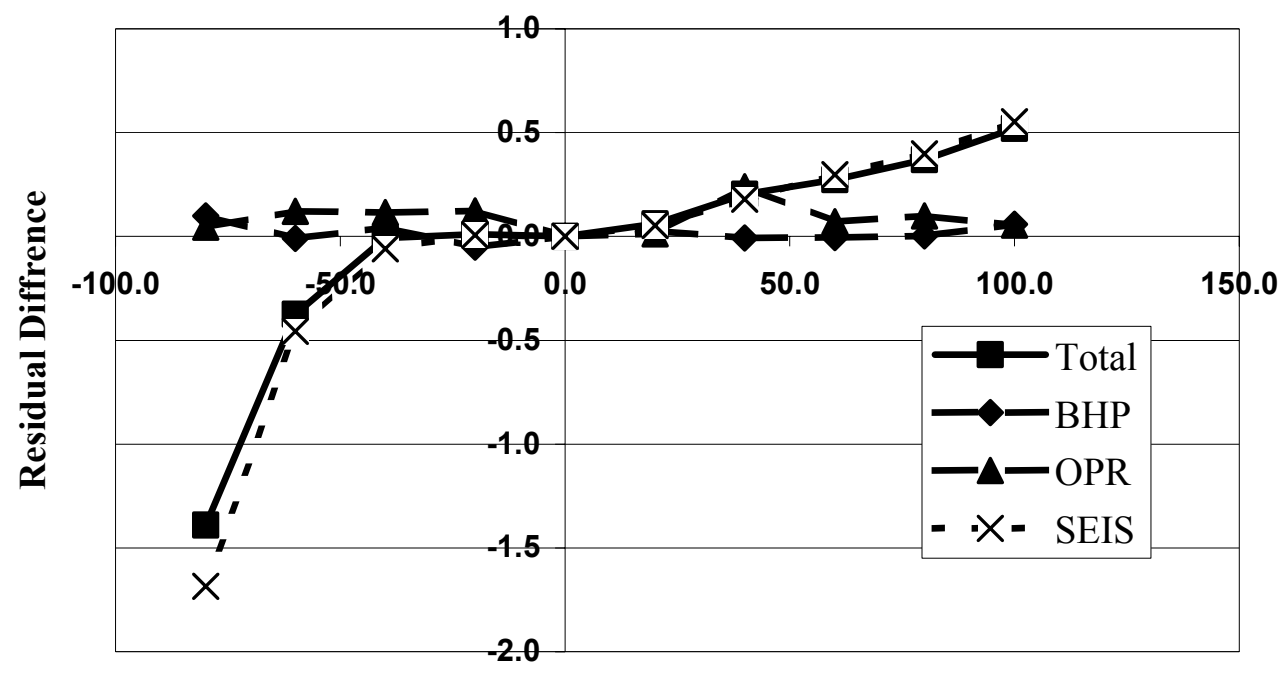

\% Parameter Perturbation

Fig. E.7 - Component and total unit residual error difference versus percent parameter perturbation, elastic REV radius. 
Table E.13 - Component and total unit residual error versus fracture compliance.

\begin{tabular}{|r|r|r|r|r|}
\cline { 2 - 5 } \multicolumn{1}{c|}{} & \multicolumn{4}{c|}{ Unit Residual } \\
\hline $\begin{array}{c}\text { Parameter } \\
\text { Value }\end{array}$ & \multicolumn{1}{c|}{ TOTAL } & \multicolumn{1}{c|}{ BHP } & \multicolumn{1}{c|}{ OPR } & \multicolumn{1}{c|}{ SEIS } \\
\hline $\mathbf{1 . 0 E}-11$ & 25.23 & 8.30 & 3.96 & 23.50 \\
\hline $\mathbf{1 . 5 E}-11$ & 23.33 & 8.37 & 3.93 & 21.42 \\
\hline $\mathbf{2 . 0 E}-11$ & 21.70 & 8.38 & 3.93 & 19.62 \\
\hline $\mathbf{2 . 5 E}-11$ & 20.14 & 8.36 & 4.00 & 17.88 \\
\hline $\mathbf{3 . 0 E}-11$ & 18.87 & 8.41 & 4.03 & 16.40 \\
\hline $\mathbf{3 . 5 E}-11$ & 17.61 & 8.30 & 3.93 & 15.03 \\
\hline $\mathbf{4 . 0 E}-11$ & 16.58 & 8.33 & 4.03 & 13.75 \\
\hline $\mathbf{4 . 5 E}-11$ & 15.66 & 8.27 & 3.97 & 12.69 \\
\hline $\mathbf{5 . 0 E}-11$ & 14.90 & 8.45 & 3.91 & 11.63 \\
\hline
\end{tabular}

Table E.14 - Component and total unit residual error difference versus percent parameter perturbation, fracture compliance.

\begin{tabular}{|r|r|r|r|r|}
\cline { 2 - 5 } \multicolumn{1}{c|}{} & \multicolumn{4}{c|}{ Unit Residual Difference from Base } \\
\hline $\begin{array}{r}\text { \% Parameter } \\
\text { Perturbation }\end{array}$ & \multicolumn{1}{c|}{ TOTAL } & \multicolumn{1}{c|}{ BHP } & \multicolumn{1}{c|}{ OPR } & \multicolumn{1}{c|}{ SEIS } \\
\hline $\mathbf{- 6 6 . 7}$ & 6.37 & -0.11 & -0.07 & 7.10 \\
\hline $\mathbf{- 5 0 . 0}$ & 4.46 & -0.03 & -0.10 & 5.01 \\
\hline $\mathbf{- 3 3 . 3}$ & 2.83 & -0.03 & -0.10 & 3.22 \\
\hline $\mathbf{- 1 6 . 7}$ & 1.27 & -0.04 & -0.03 & 1.48 \\
\hline $\mathbf{0 . 0}$ & 0.00 & 0.00 & 0.00 & 0.00 \\
\hline $\mathbf{1 6 . 7}$ & -1.25 & -0.10 & -0.10 & -1.38 \\
\hline $\mathbf{3 3 . 3}$ & -2.29 & -0.08 & 0.00 & -2.65 \\
\hline $\mathbf{5 0 . 0}$ & -3.21 & -0.14 & -0.05 & -3.71 \\
\hline $\mathbf{6 6 . 7}$ & -3.97 & 0.05 & -0.12 & -4.77 \\
\hline
\end{tabular}




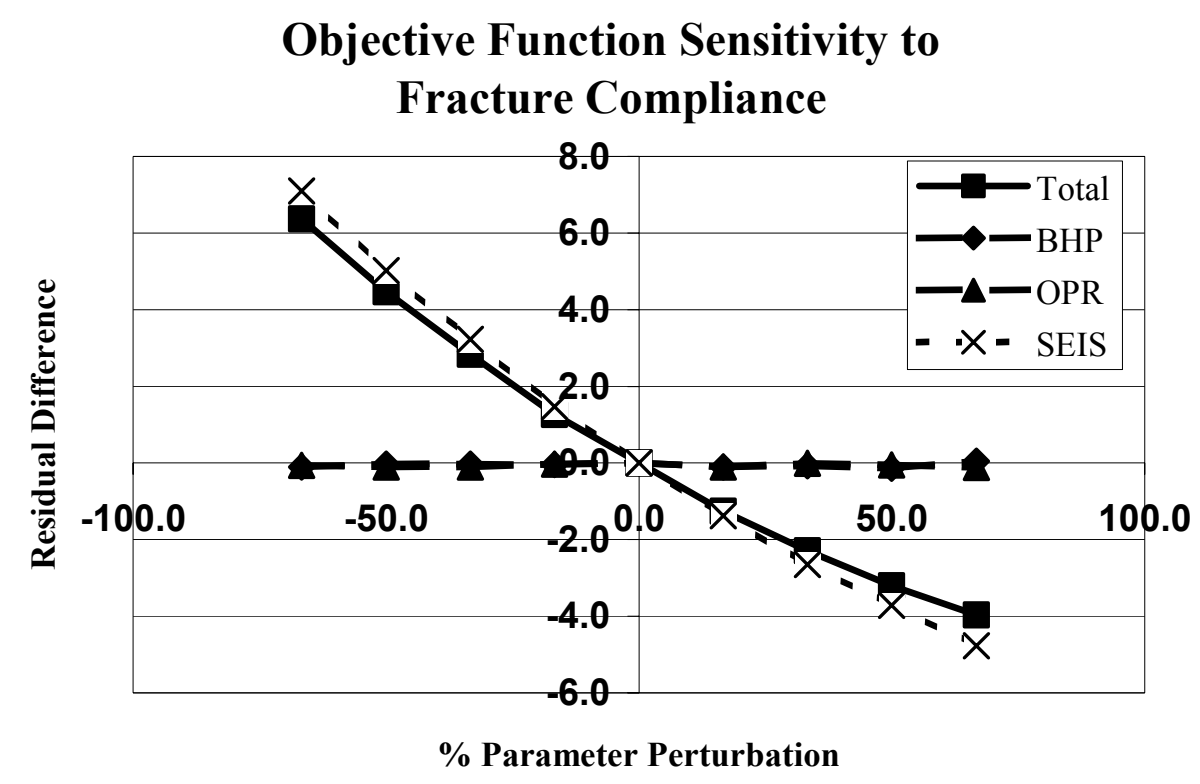

Fig. E.8 - Component and total unit residual error difference versus percent parameter perturbation, fracture compliance.

Table E.15 - Component and total unit residual error versus incidence angle.

\begin{tabular}{|r|r|r|r|r|}
\cline { 2 - 5 } \multicolumn{1}{c|}{} & \multicolumn{4}{c|}{ Unit Residual } \\
\hline Parameter Value & \multicolumn{1}{c|}{ TOTAL } & \multicolumn{1}{c|}{ BHP } & \multicolumn{1}{c|}{ OPR } & \multicolumn{1}{c|}{ SEIS } \\
\hline $\mathbf{1 6 . 0}$ & 16.26 & 8.34 & 3.90 & 13.40 \\
\hline $\mathbf{2 0 . 0}$ & 16.89 & 8.37 & 3.91 & 14.14 \\
\hline $\mathbf{2 6 . 0}$ & 16.89 & 8.37 & 3.91 & 14.14 \\
\hline $\mathbf{3 0 . 0}$ & 18.77 & 8.35 & 3.93 & 16.34 \\
\hline $\mathbf{3 6 . 0}$ & 20.22 & 8.33 & 3.96 & 17.99 \\
\hline
\end{tabular}

Table E.16 - Component and total unit residual error difference versus percent parameter perturbation, incidence angle.

\begin{tabular}{|r|r|r|r|r|}
\cline { 2 - 5 } \multicolumn{1}{c|}{} & \multicolumn{4}{c|}{ Unit Residual Difference from Base } \\
\hline $\begin{array}{r}\text { \% Parameter } \\
\text { Perturbation }\end{array}$ & \multicolumn{1}{c|}{ TOTAL } & \multicolumn{1}{c|}{ BHP } & \multicolumn{1}{c|}{ OPR } & \multicolumn{1}{c|}{ SEIS } \\
\hline $\mathbf{- 4 6 . 7}$ & -2.51 & -0.02 & -0.02 & -2.94 \\
\hline $\mathbf{- 3 3 . 3}$ & -1.88 & 0.01 & -0.02 & -2.20 \\
\hline $\mathbf{- 1 3 . 3}$ & -1.88 & 0.01 & -0.02 & -2.20 \\
\hline $\mathbf{0 . 0}$ & 0.00 & 0.00 & 0.00 & 0.00 \\
\hline $\mathbf{2 0 . 0}$ & 1.45 & -0.02 & 0.03 & 1.65 \\
\hline
\end{tabular}




\section{Objective Function Sensitivity to} Seismic Incidence Angle

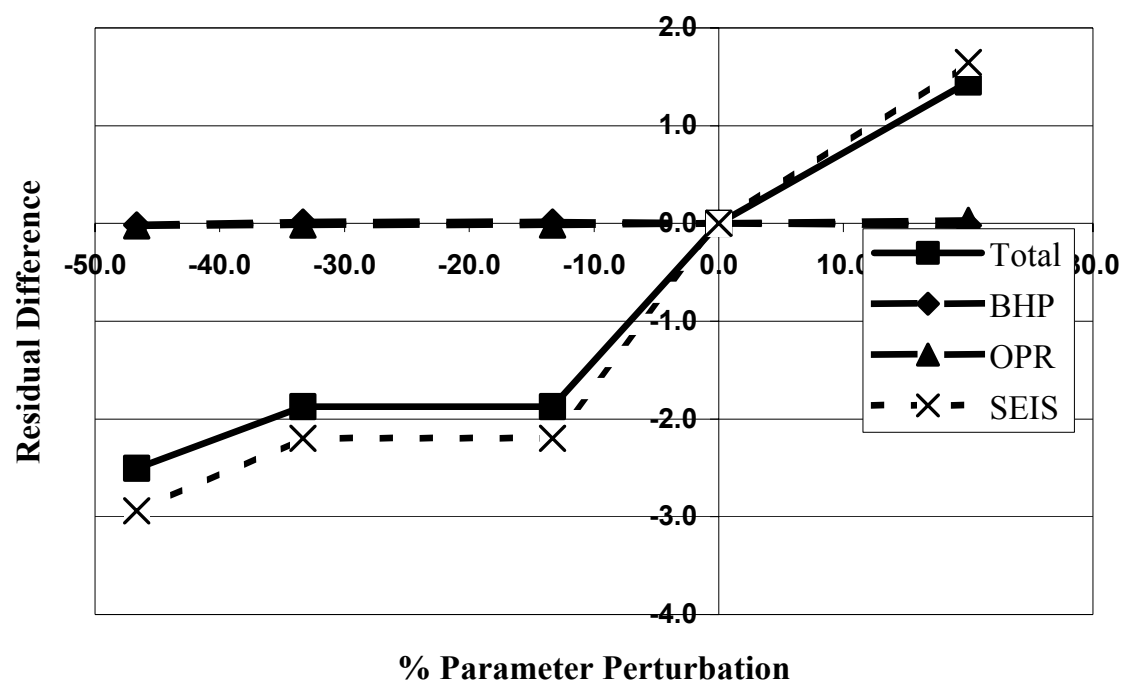

Fig. E.9 - Component and total unit residual error difference versus percent parameter perturbation, incidence angle.

Table E.17 - Component and total unit residual error versus reservoir thickness.

\begin{tabular}{|r|r|r|r|r|}
\cline { 2 - 5 } \multicolumn{1}{c|}{} & \multicolumn{4}{c|}{ Unit Residual } \\
\hline $\begin{array}{c}\text { Parameter } \\
\text { Value }\end{array}$ & \multicolumn{1}{c|}{ TOTAL } & \multicolumn{1}{c|}{ BHP } & \multicolumn{1}{c|}{ OPR } & \multicolumn{1}{c|}{ SEIS } \\
\hline $\mathbf{2 0 . 0}$ & 46.69 & 42.90 & 8.39 & 16.40 \\
\hline $\mathbf{3 0 . 0}$ & 18.76 & 8.33 & 3.97 & 16.33 \\
\hline $\mathbf{4 0 . 0}$ & 19.34 & 9.69 & 3.49 & 16.37 \\
\hline $\mathbf{5 0 . 0}$ & 22.21 & 14.54 & 3.53 & 16.41 \\
\hline $\mathbf{6 0 . 0}$ & 24.37 & 17.67 & 3.55 & 16.40 \\
\hline $\mathbf{7 0 . 0}$ & 26.21 & 20.14 & 3.58 & 16.39 \\
\hline $\mathbf{8 0 . 0}$ & 27.51 & 21.77 & 3.56 & 16.44 \\
\hline
\end{tabular}


Table E.18 - Component and total unit residual error difference versus percent parameter perturbation, reservoir thickness.

\begin{tabular}{|rr|r|r|r|}
\cline { 2 - 5 } \multicolumn{1}{c|}{} & \multicolumn{4}{c|}{ Unit Residual Difference from Base } \\
\hline $\begin{array}{r}\text { \% Parameter } \\
\text { Perturbation }\end{array}$ & \multicolumn{1}{c|}{ TOTAL } & \multicolumn{1}{c|}{ BHP } & \multicolumn{1}{c|}{ OPR } & \multicolumn{1}{c|}{ SEIS } \\
\hline $\mathbf{- 3 3 . 3}$ & 27.93 & 34.57 & 4.42 & 0.07 \\
\hline $\mathbf{0 . 0}$ & 0.00 & 0.00 & 0.00 & 0.00 \\
\hline $\mathbf{3 3 . 3}$ & 0.58 & 1.37 & -0.48 & 0.04 \\
\hline $\mathbf{6 6 . 7}$ & 3.45 & 6.22 & -0.44 & 0.08 \\
\hline $\mathbf{1 0 0 . 0}$ & 5.61 & 9.34 & -0.43 & 0.07 \\
\hline $\mathbf{1 3 3 . 3}$ & 7.45 & 11.82 & -0.40 & 0.05 \\
\hline $\mathbf{1 6 6 . 7}$ & 8.75 & 13.45 & -0.41 & 0.11 \\
\hline
\end{tabular}

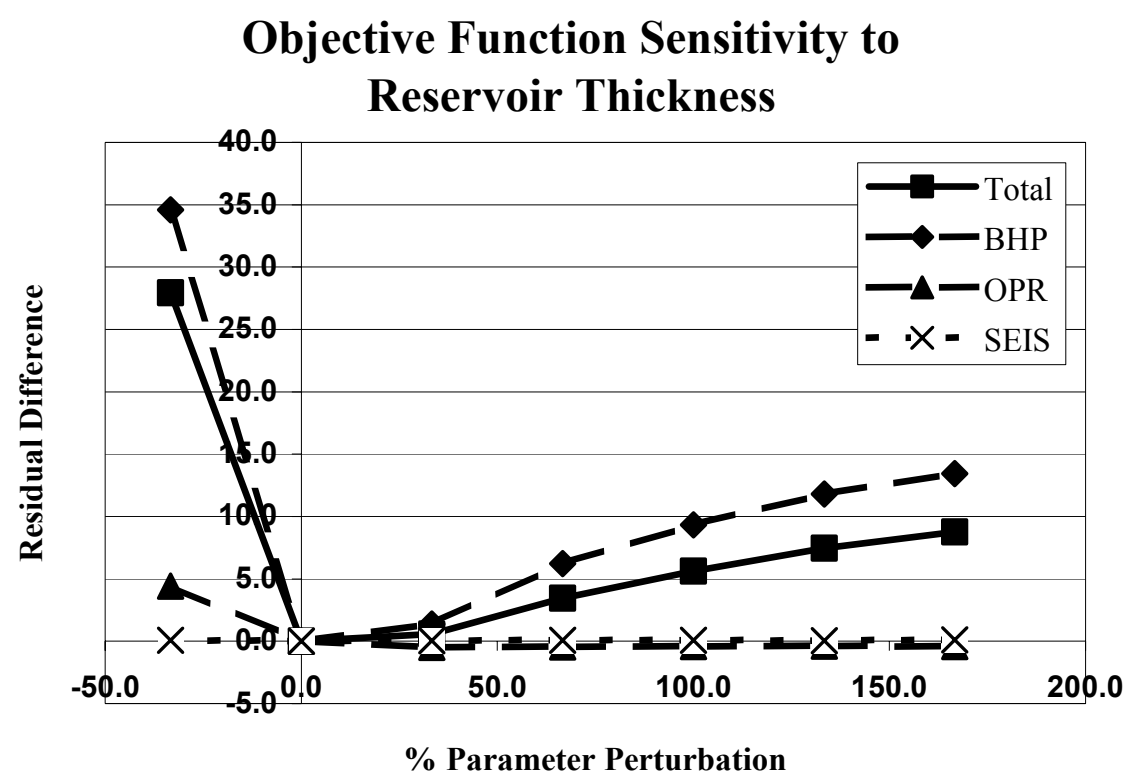

Fig. E.10 - Component and total unit residual error difference versus percent parameter perturbation, reservoir thickness. 
Objective Function Sensitivity

Elastic Parameters and Fracture Set 2 Intensity

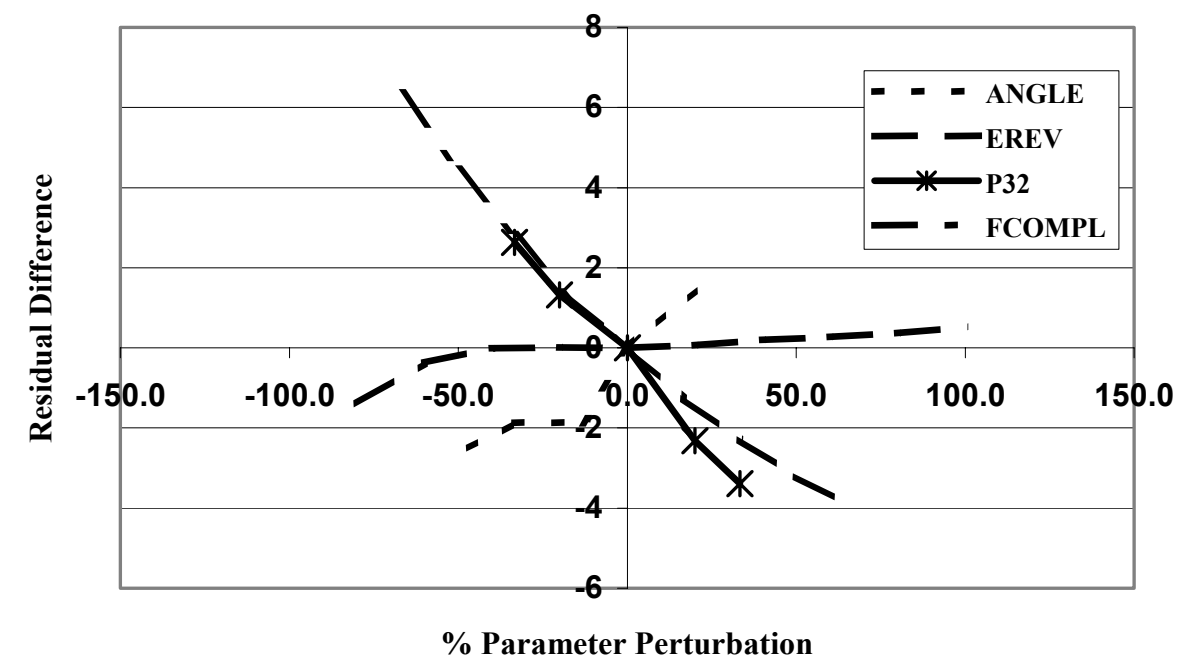

Fig. E.11 - Component and total unit residual error difference versus percent parameter perturbation, P32 intensity and elastic parameters.

Objective Function Sensitivity

Elastic Parameters and Fracture Set 2 Trend

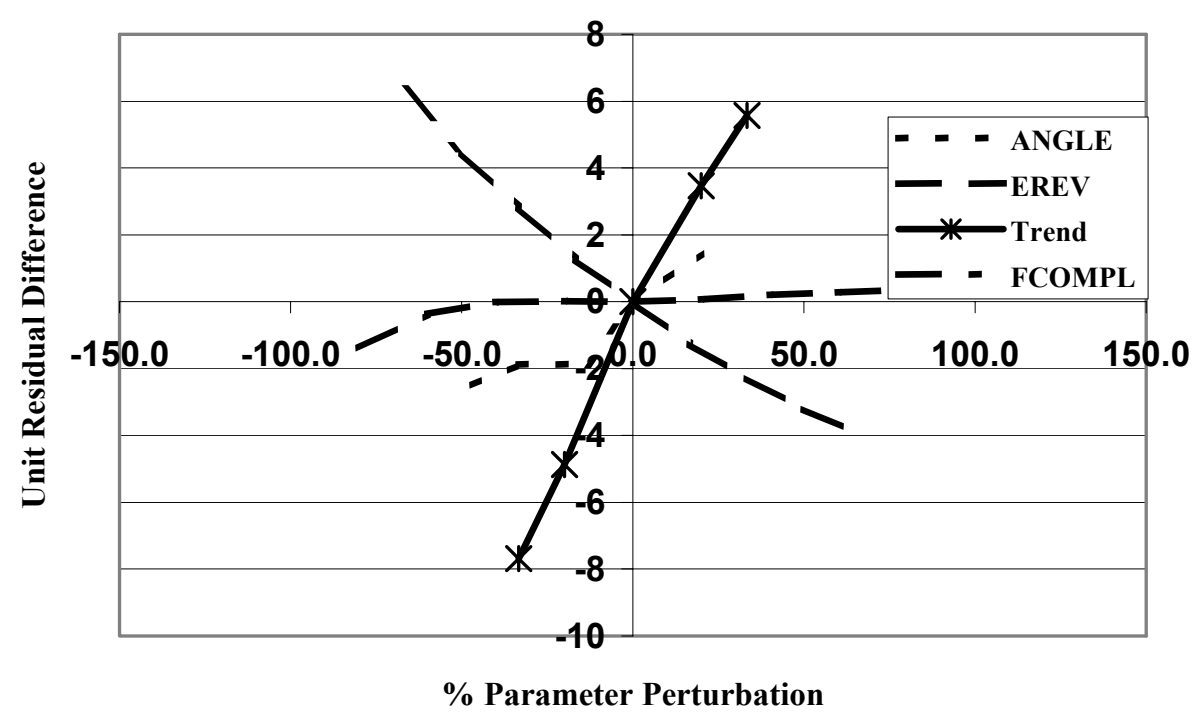

Fig. E.12 - Component and total unit residual error difference versus percent parameter perturbation, trend and elastic parameters. 


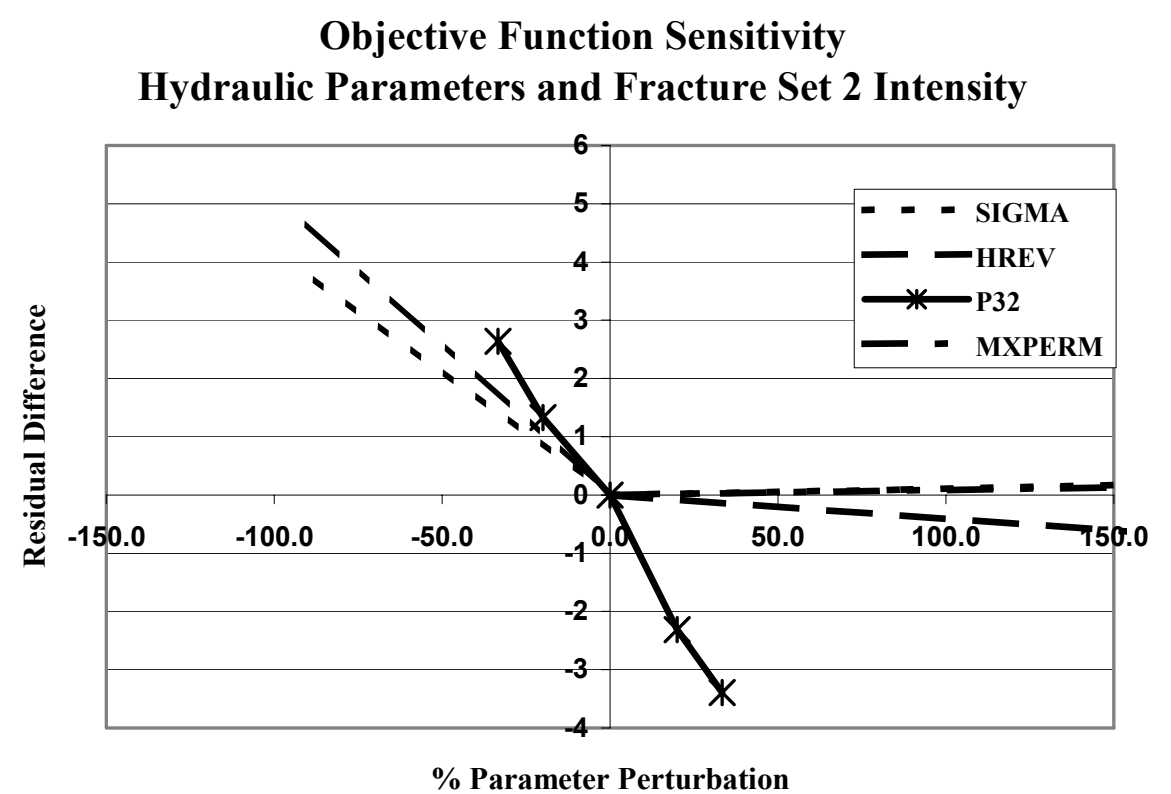

Fig. E.13 - Component and total unit residual error difference versus percent parameter perturbation, P32 intensity and hydraulic parameters.

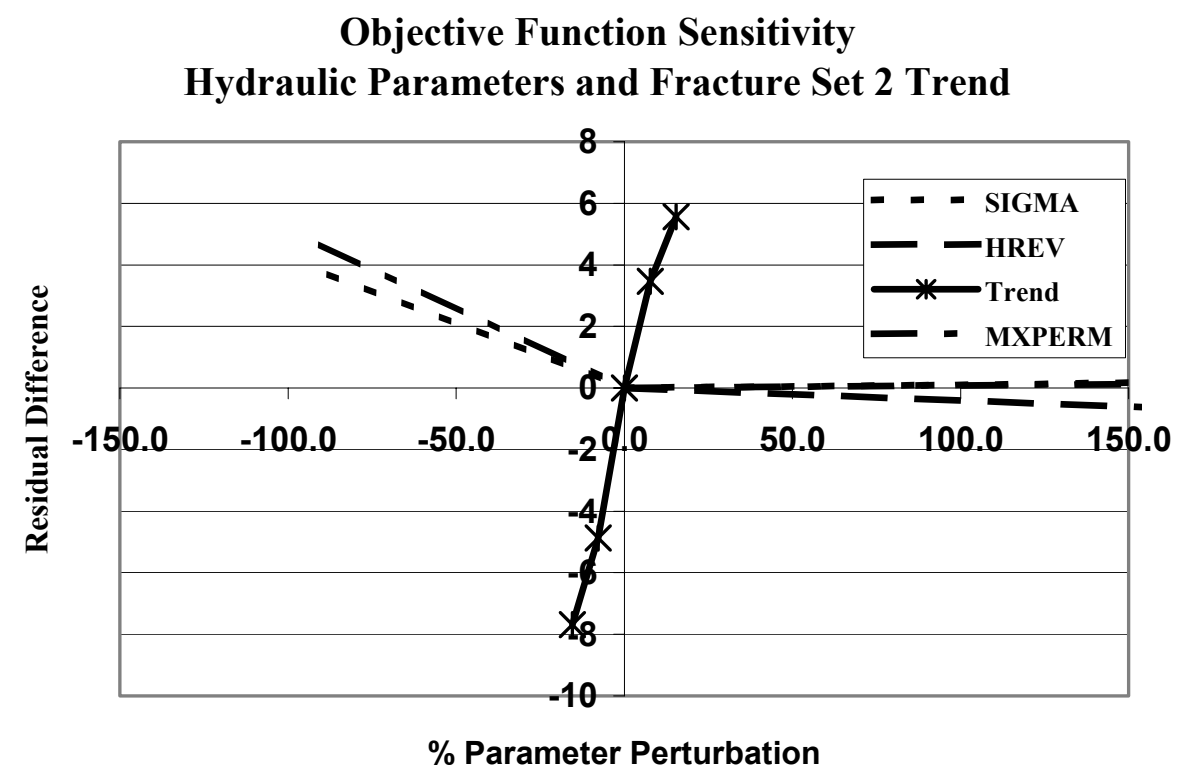

Fig. E.14 - Component and total unit residual error difference versus percent parameter perturbation, trend and hydraulic parameters. 


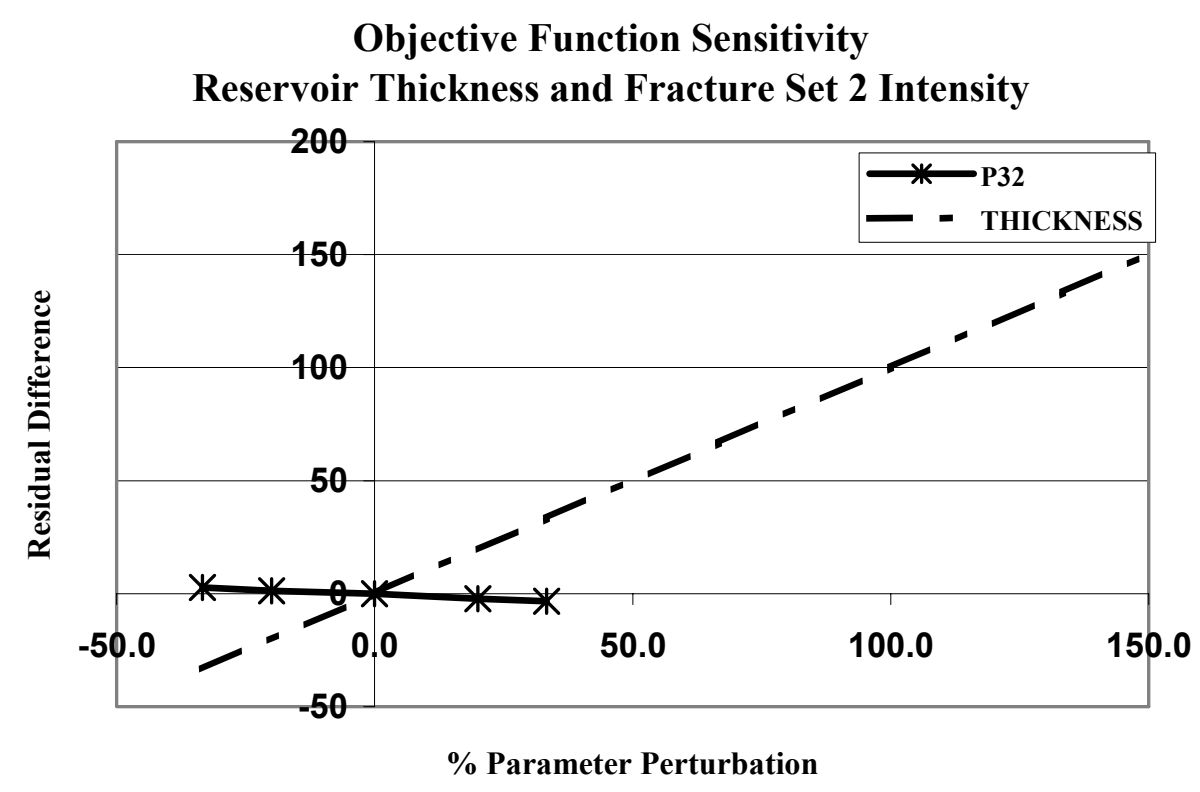

Fig. E.15 - Component and total unit residual error difference versus percent parameter perturbation, P32 intensity and reservoir thickness.

Objective Function Sensitivity Reservoir Thickness and Fracture Set 2 Trend

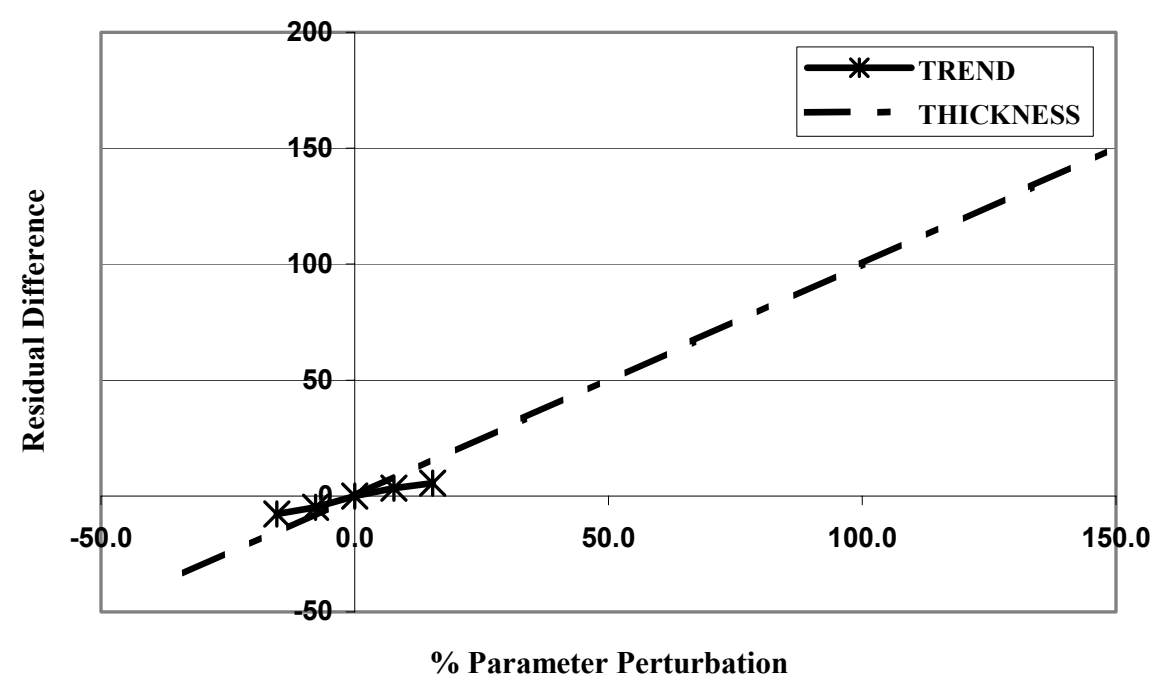

Fig. E.16 - Component and total unit residual error difference versus percent parameter perturbation, trend and reservoir thickness. 


\section{APPENDIX F}

\section{MatLab Script}

STIFF

(Effective Elastic Media Modeling) 


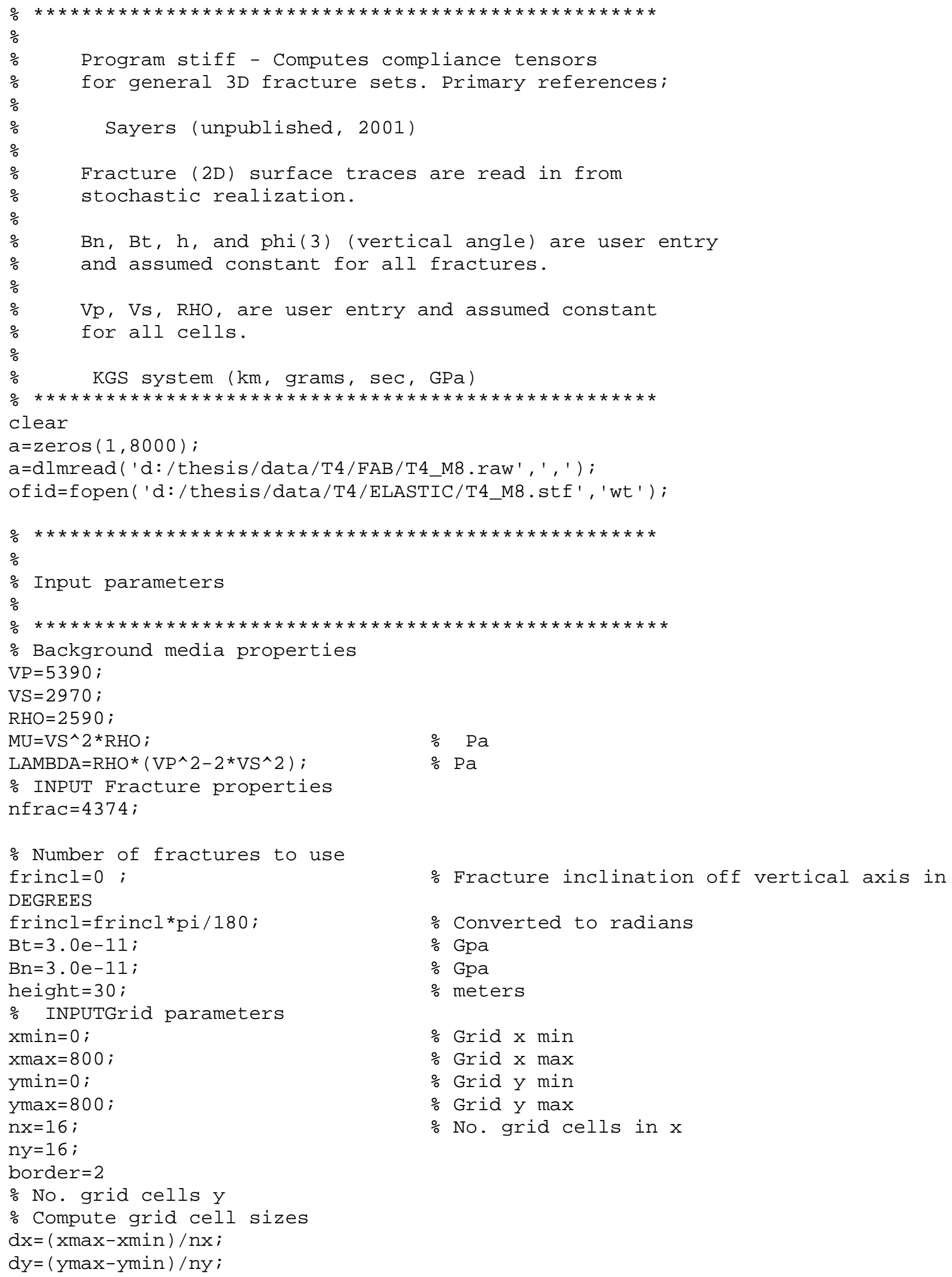




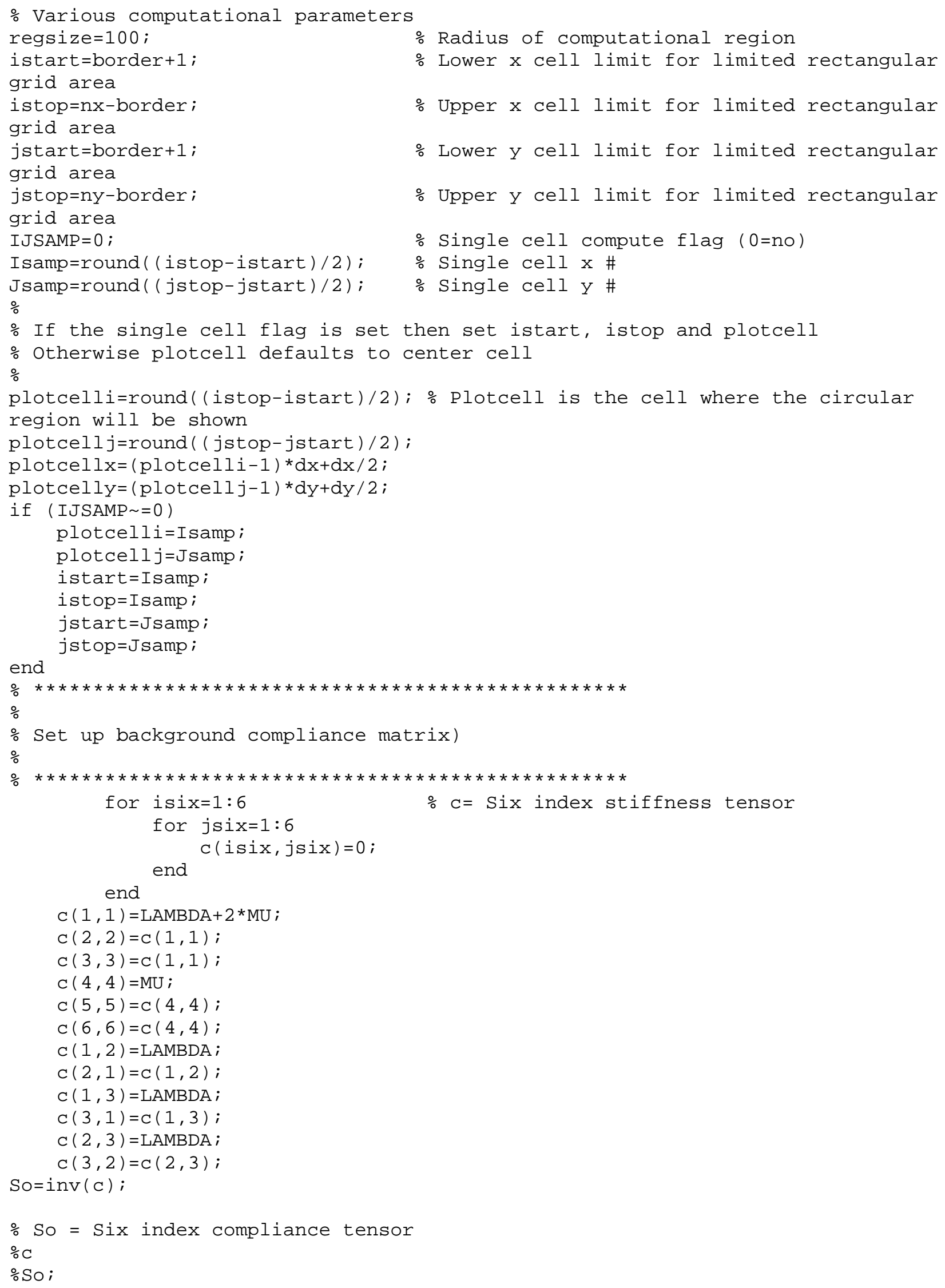




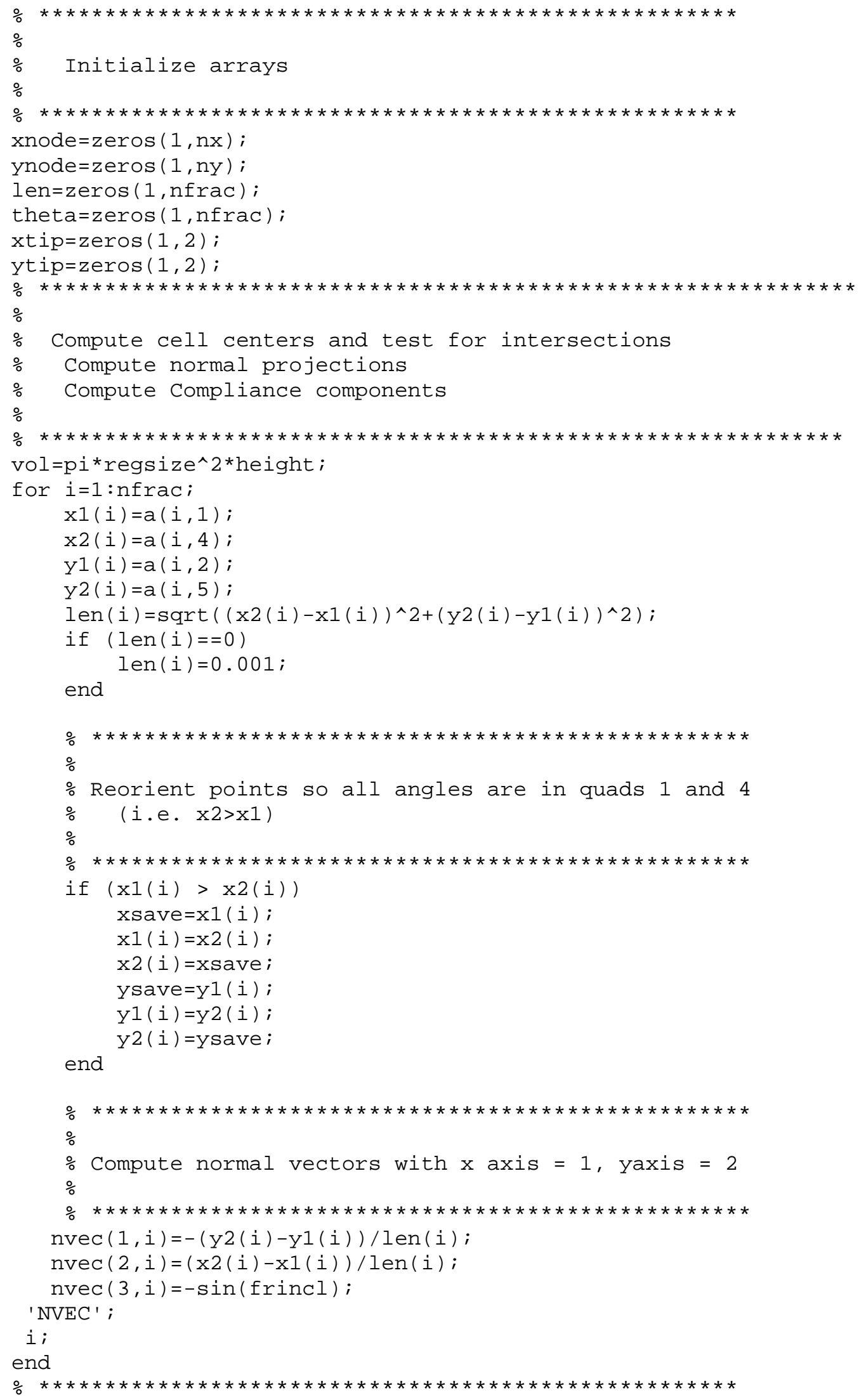




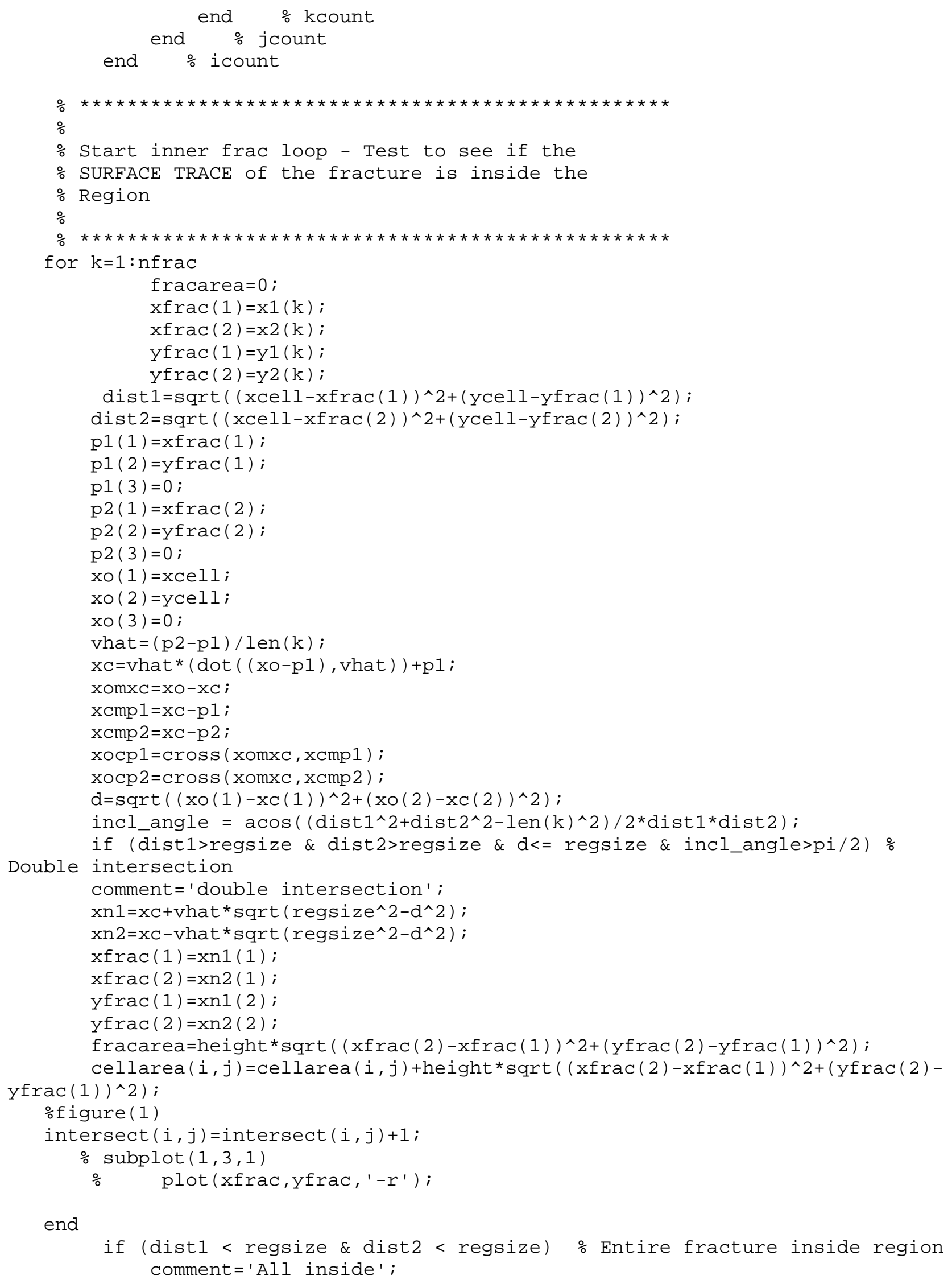




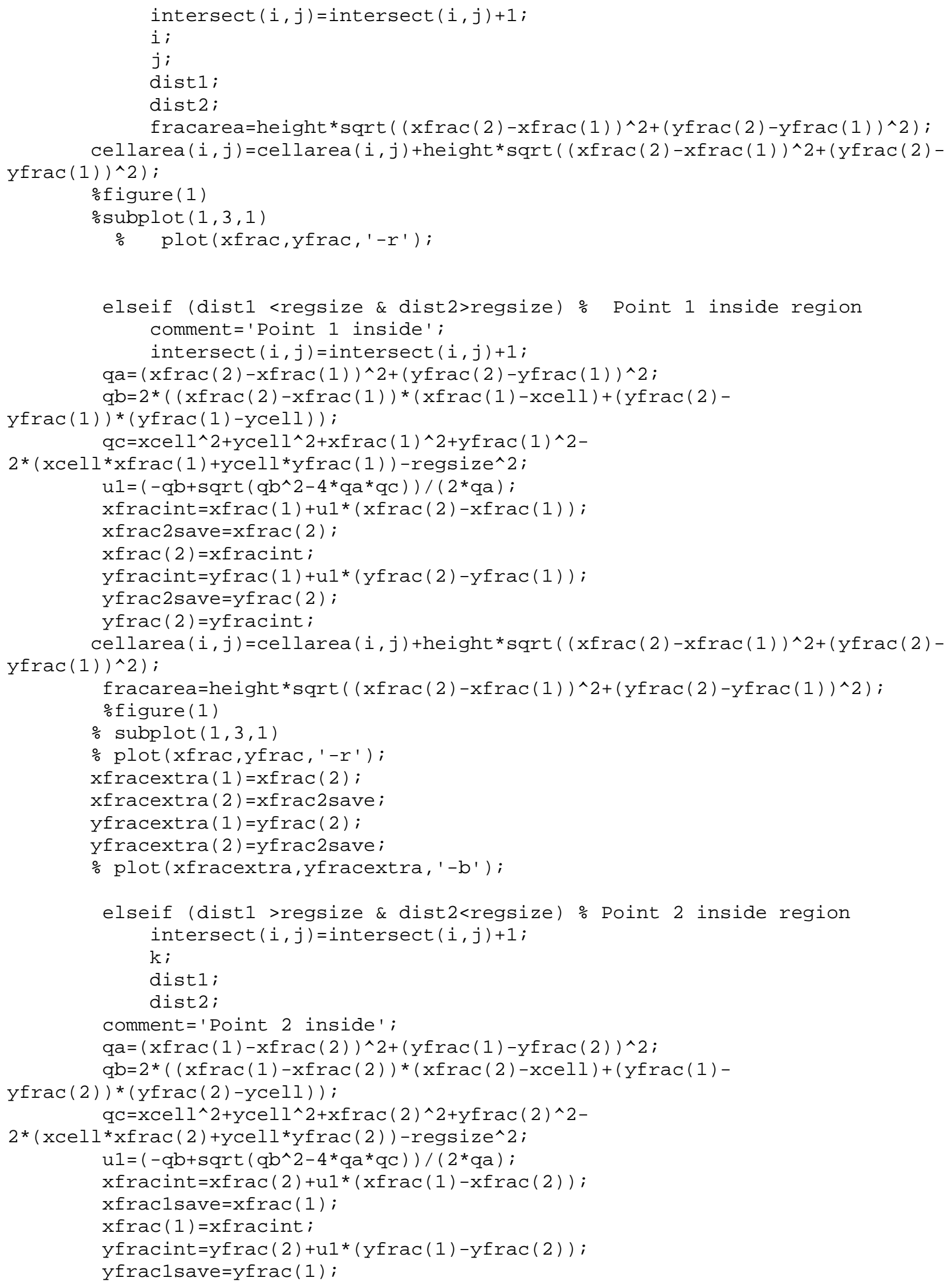




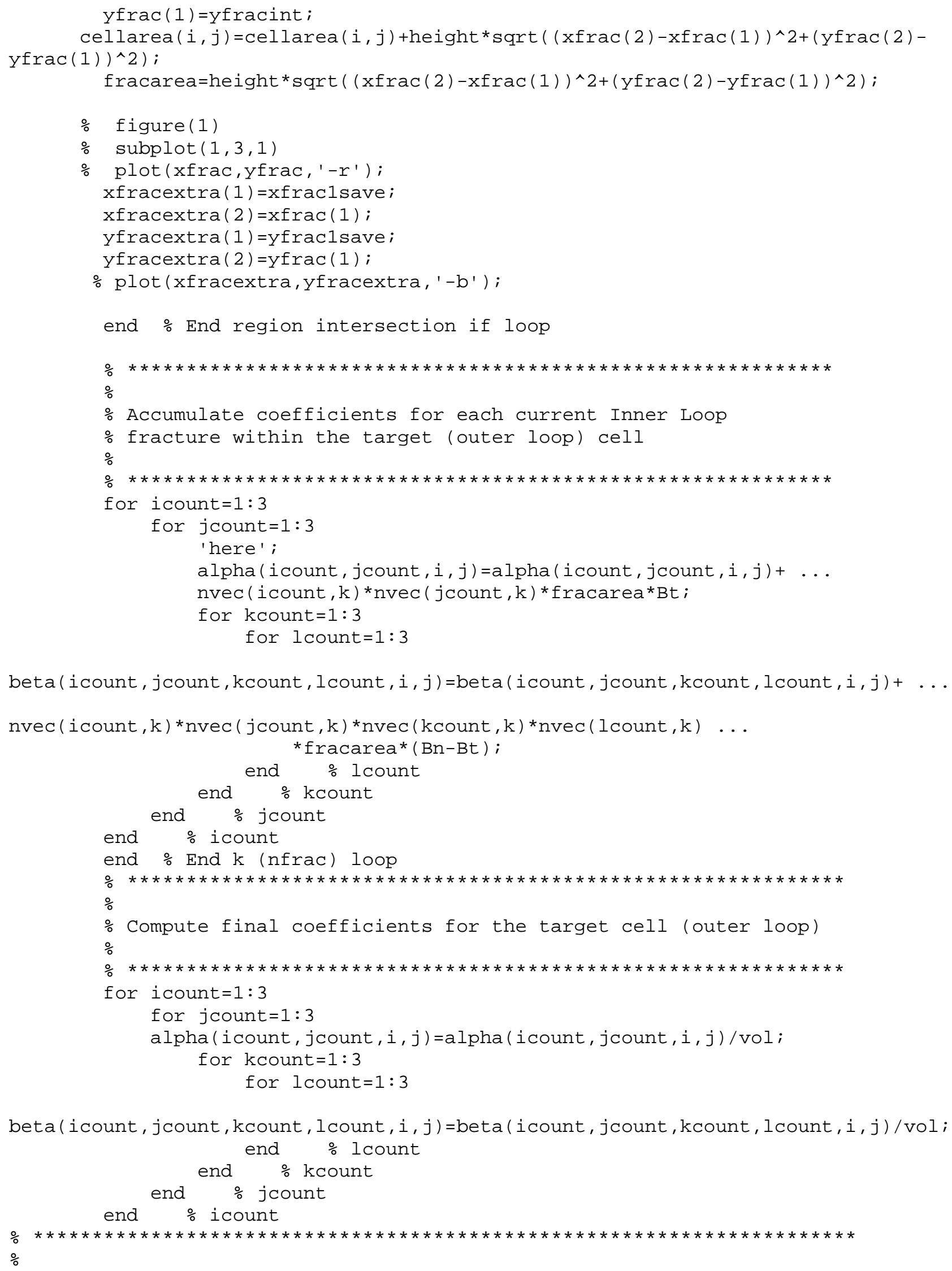




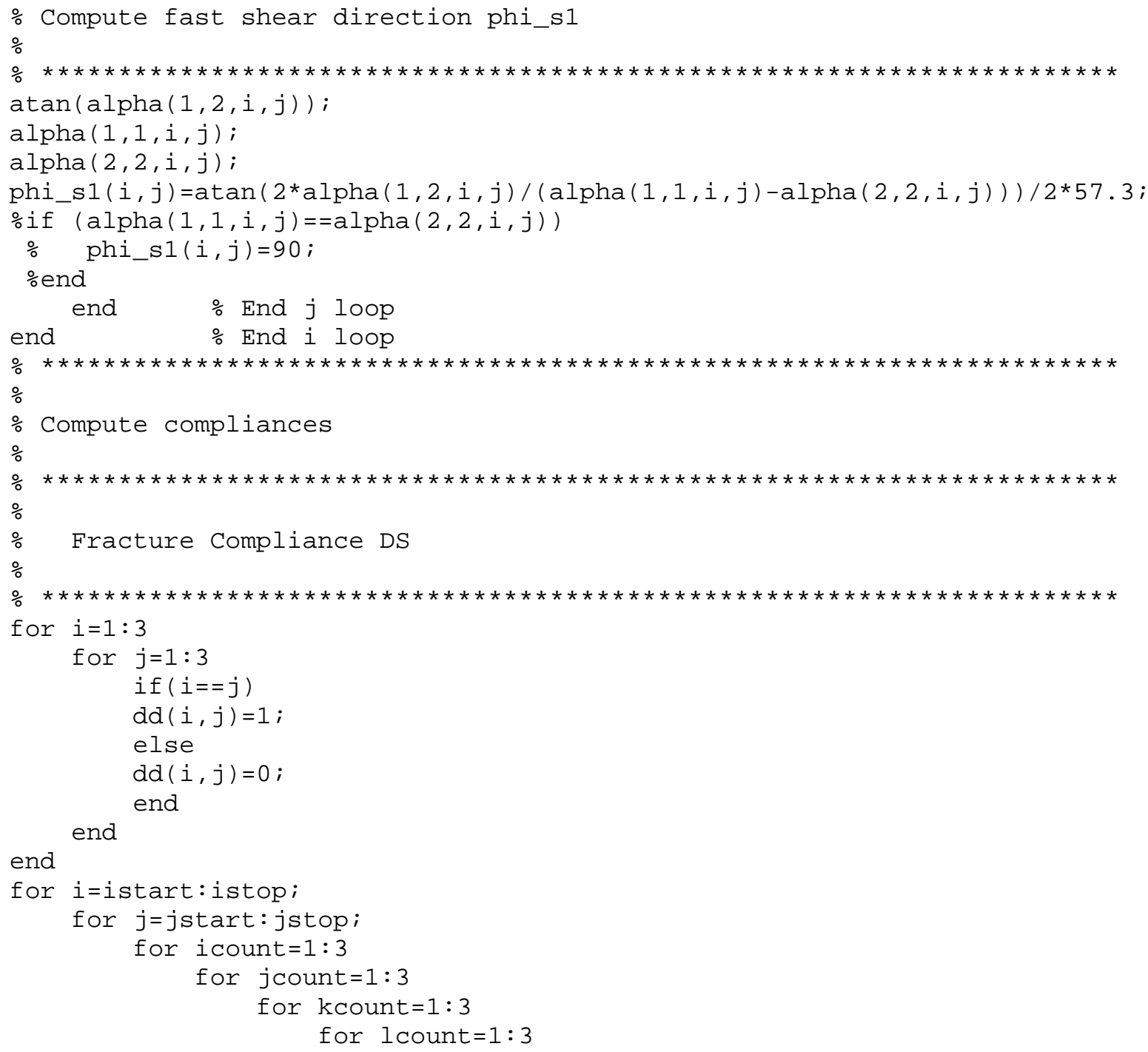

DS (icount, jcount, kcount, lcount, $i, j)=0.25 *$ (dd (icount, kcount)*alpha (jcount, lcount, $i, j) \ldots$

$+d d(i c o u n t, l$ count $) * a l p h a(j c o u n t, k c o u n t, i, j)+d d(j \operatorname{count}, k$ count $)$ *..

alpha (icount, lcount, $i, j)+d d(j c o u n t, ~ l c o u n t) * a l p h a(i c o u n t, k c o u n t, i, j)) \ldots$ +beta (icount, jcount, kcount, lcount, $i, j$ ) ; end end

end

end

- Transform to 6 index compliance tensor DS2

$\operatorname{DS} 2(1,1, i, j)=\operatorname{DS}(1,1,1,1, i, j)$;

$\operatorname{DS} 2(2,2, i, j)=\operatorname{DS}(2,2,2,2, i, j)$;

$\operatorname{DS} 2(3,3, i, j)=\operatorname{DS}(3,3,3,3, i, j)$;

$\operatorname{DS} 2(1,2, i, j)=\operatorname{DS}(1,1,2,2, i, j)$;

$\operatorname{DS} 2(1,3, i, j)=\operatorname{DS}(1,1,3,3, i, j)$;

$\operatorname{DS} 2(1,4, i, j)=2 \star \operatorname{DS}(1,1,2,3, i, j)$;

$\operatorname{DS} 2(1,5, i, j)=2 * \operatorname{DS}(1,1,1,3, i, j)$;

$\operatorname{DS} 2(1,6, i, j)=2 * \operatorname{DS}(1,1,1,2, i, j)$; 


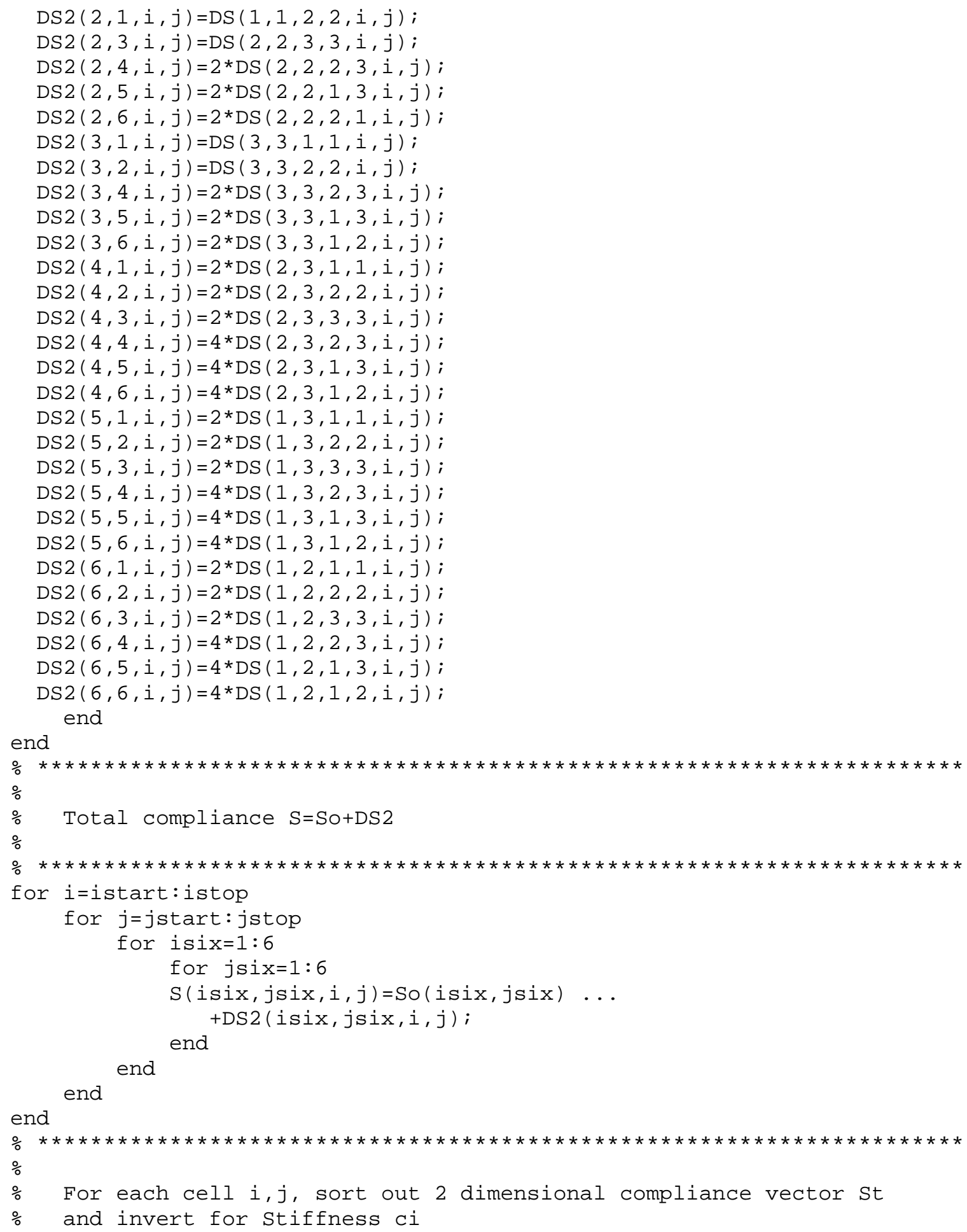

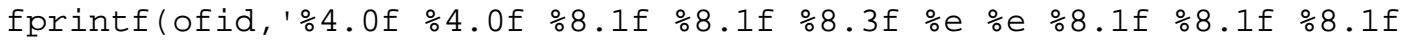
ㅇ. $1 f \backslash n^{\prime},[n x-2 *$ border, ny-2*border, VP, VS, RHO/1000, Bt, Bn, xmin, xmax, ymin, ymax ] ) ; for $i=i s t a r t:$ istop

for j=jstart: jstop for isix $=1: 6$ 


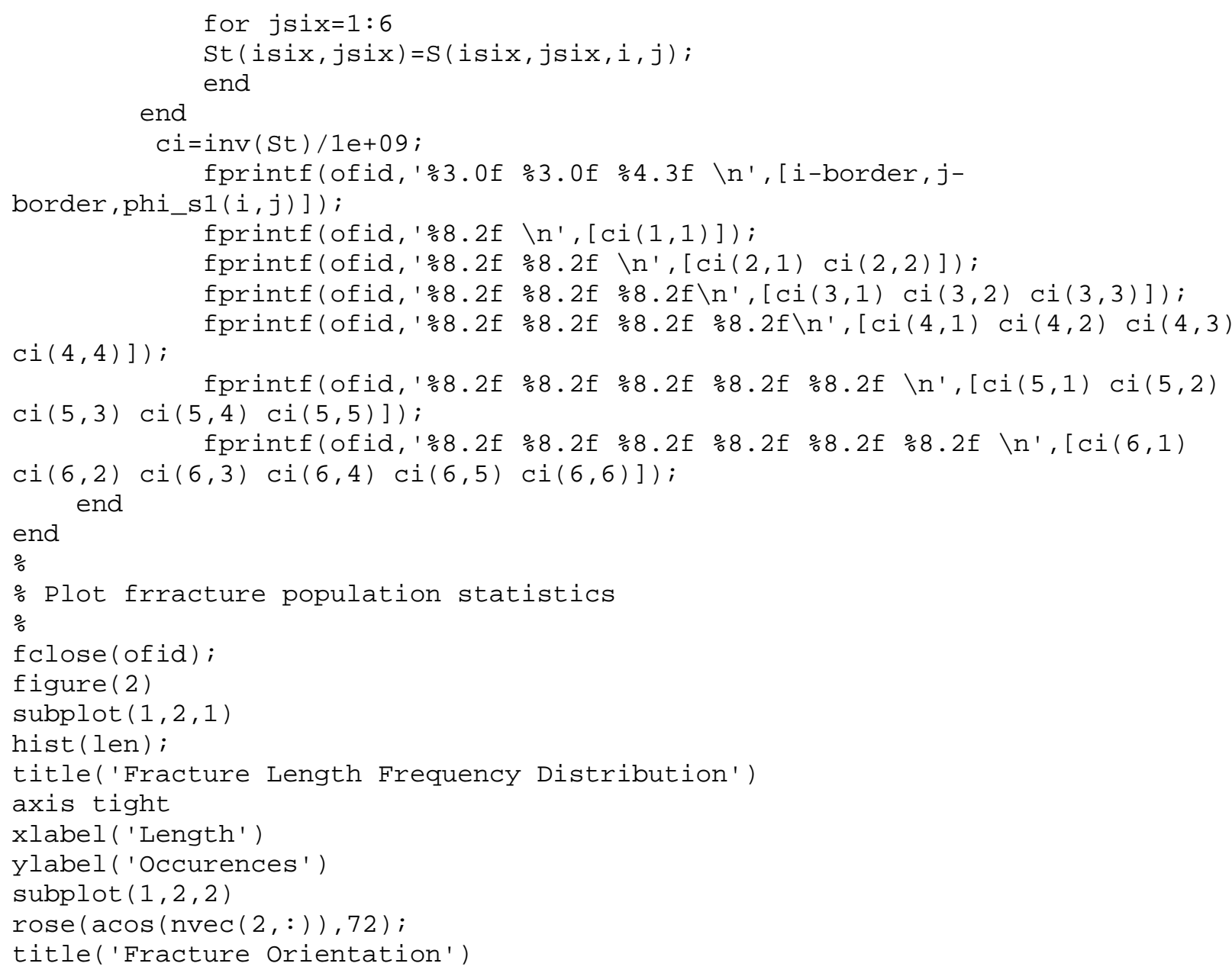


APPENDIX G

MatLab Script

CREATE_ATTR

(Seismic Attribute Calculation) 


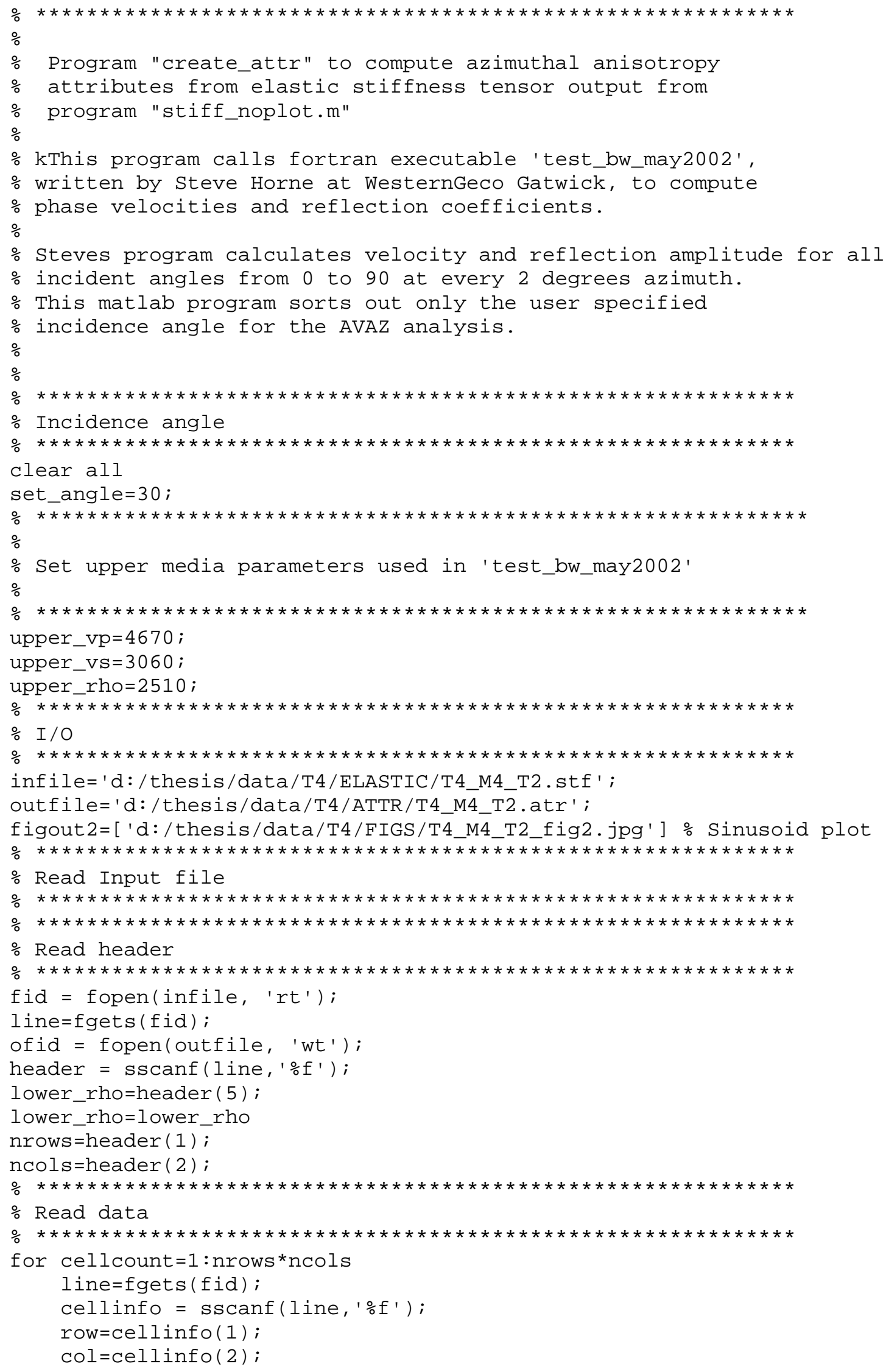




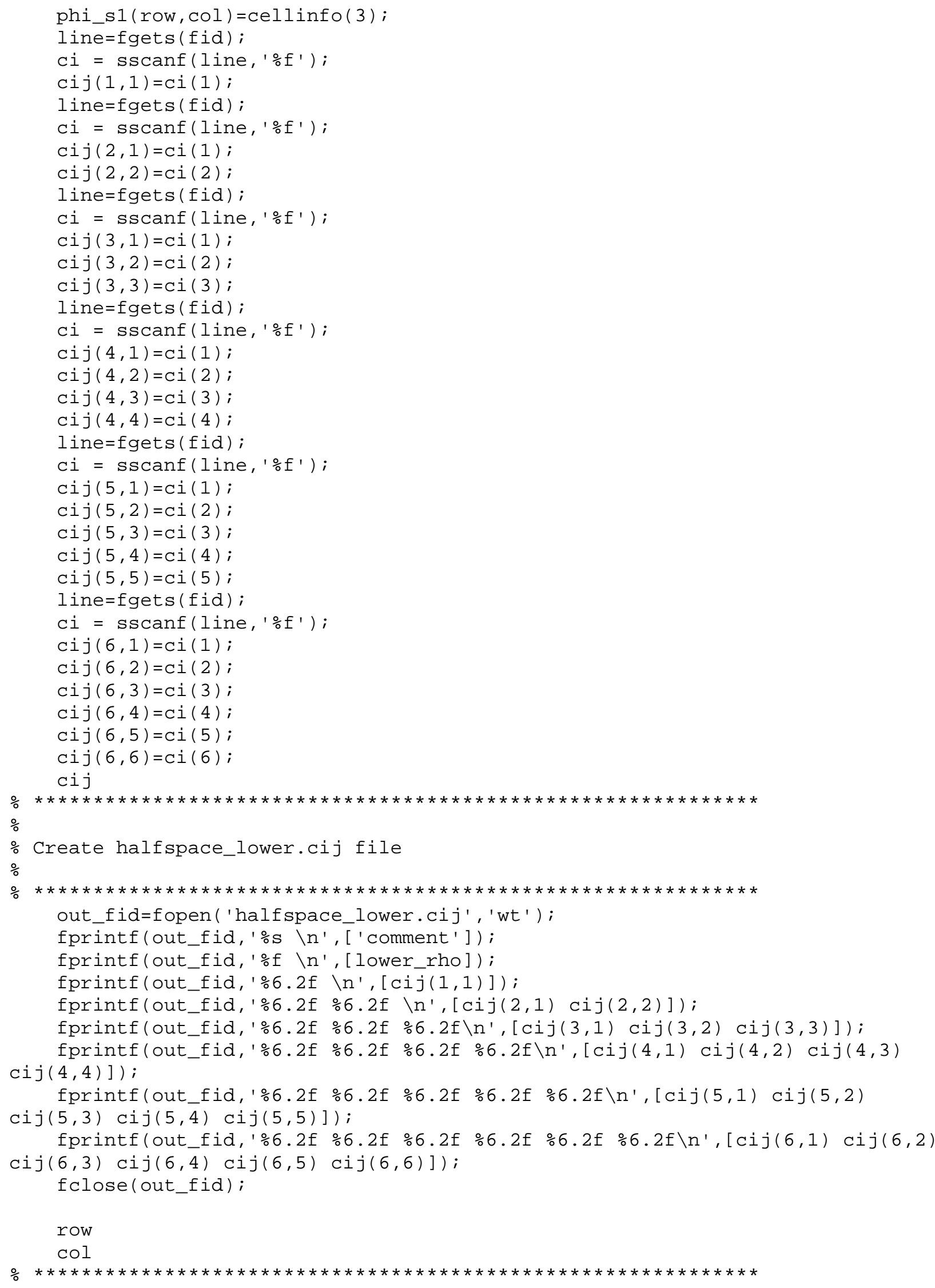




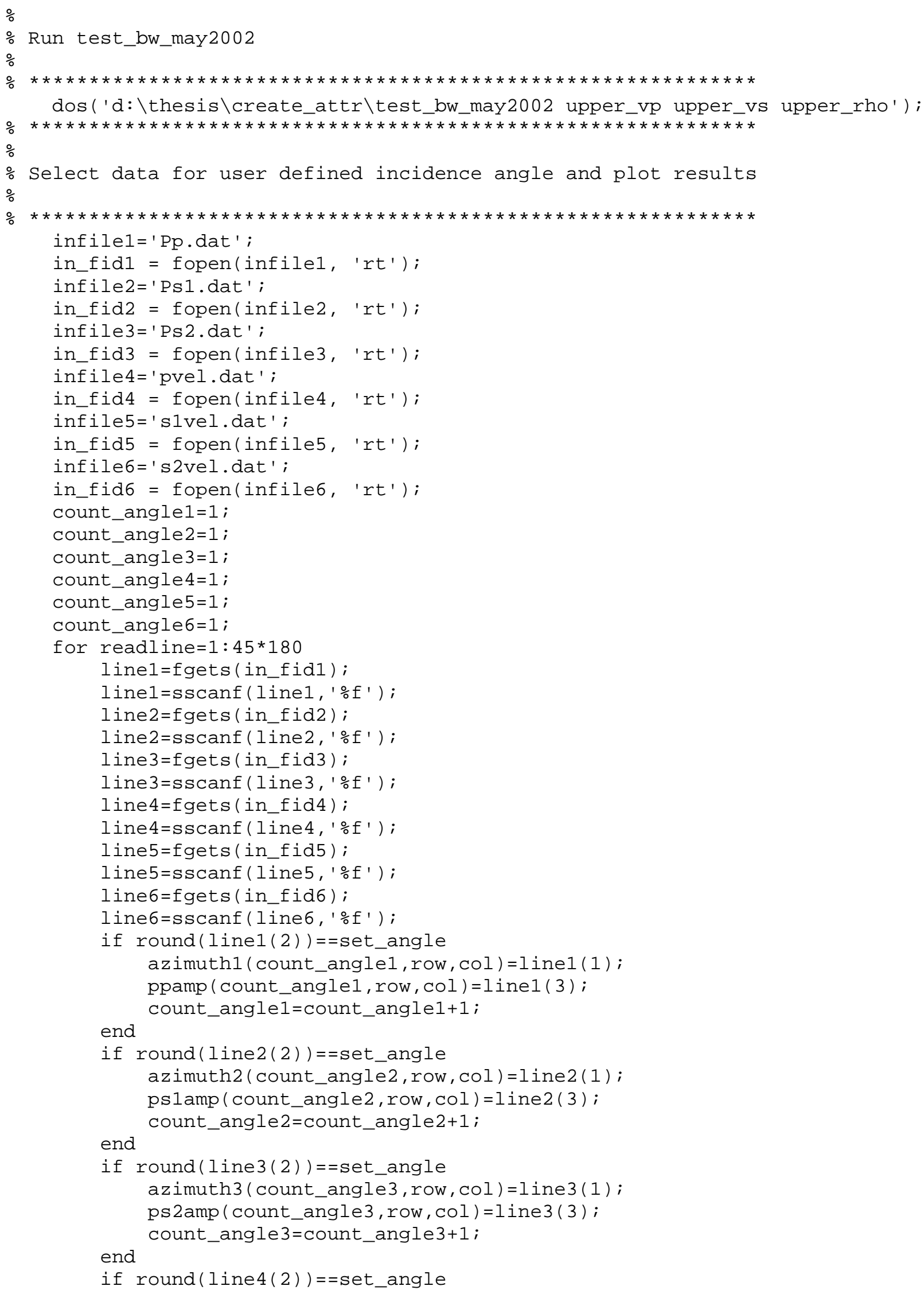




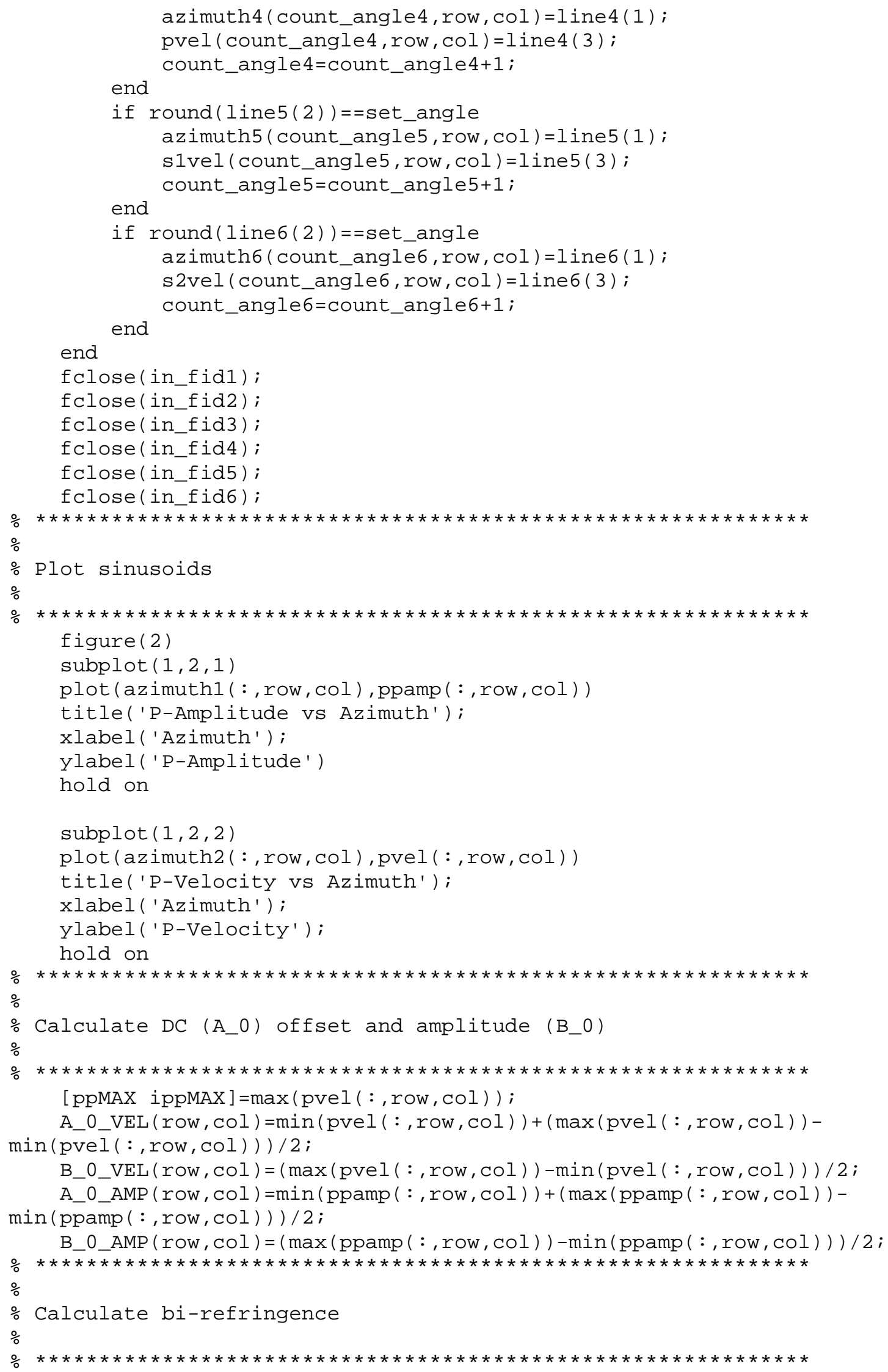




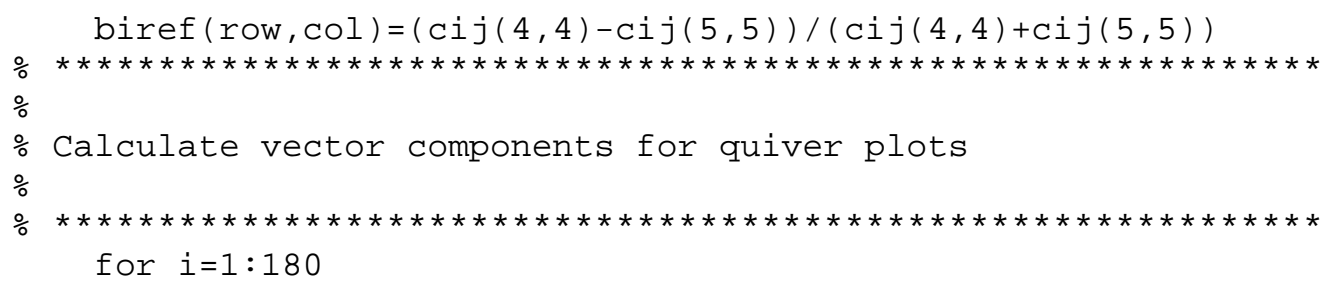

pvel_0 $(i, r o w, c o l)=A \_0 \_V E L(r o w, c o l)+B \_0 \_V E L(r o w, c o l) * \cos (2 *($ a zimuth2 $(i$, row, col $) / 5$ 7.3-phi_s1 (row, col)/57.3));

pamp_0 $(i, r o w$, col $)=$ A_0_AMP $($ row , col $)+$ B_0_AMP $($ row, col $){ }^{*}$ cos $(2 *($ a zimuth2 (i, row , col $) / 5$

7.3 -phi_s1 (row, col)/57.3)); end

end

saveas (gcf, figout2)

for $i=1:$ nrows

for $j=1:$ ncols

$U(i, j)=B \_0 \_V E L(i, j) * \cos \left(\operatorname{phi} \_s 1(i, j) / 57.3\right)$;

$V(i, j)=B \_0 \_V E L(i, j) * \sin (\operatorname{phi} \operatorname{si}(i, j) / 57.3)$; end

end

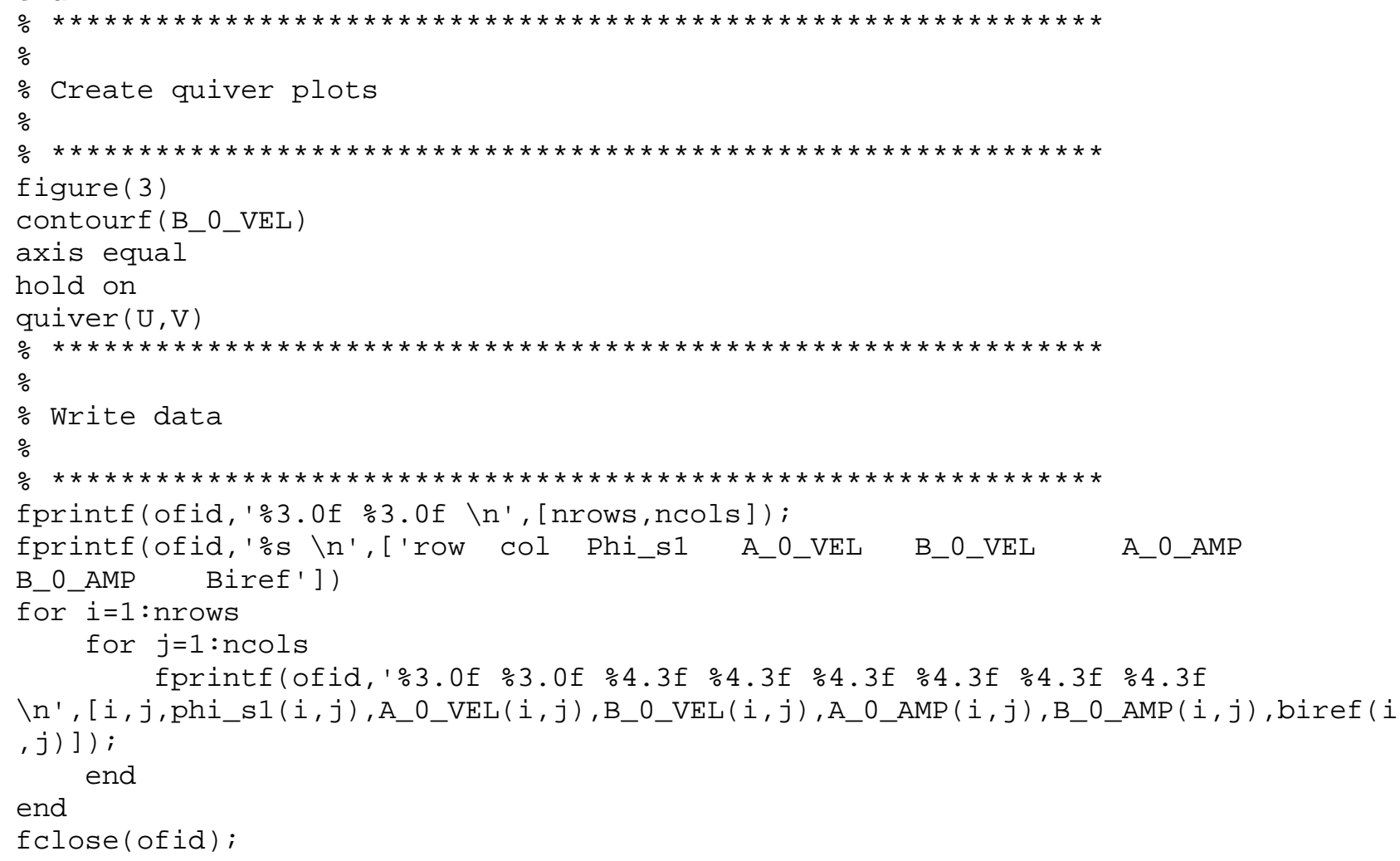




\section{APPENDIX H}

\section{MatLab Script}

\section{SENS}

(Sensitivity Coefficients and Parameter Updates) 


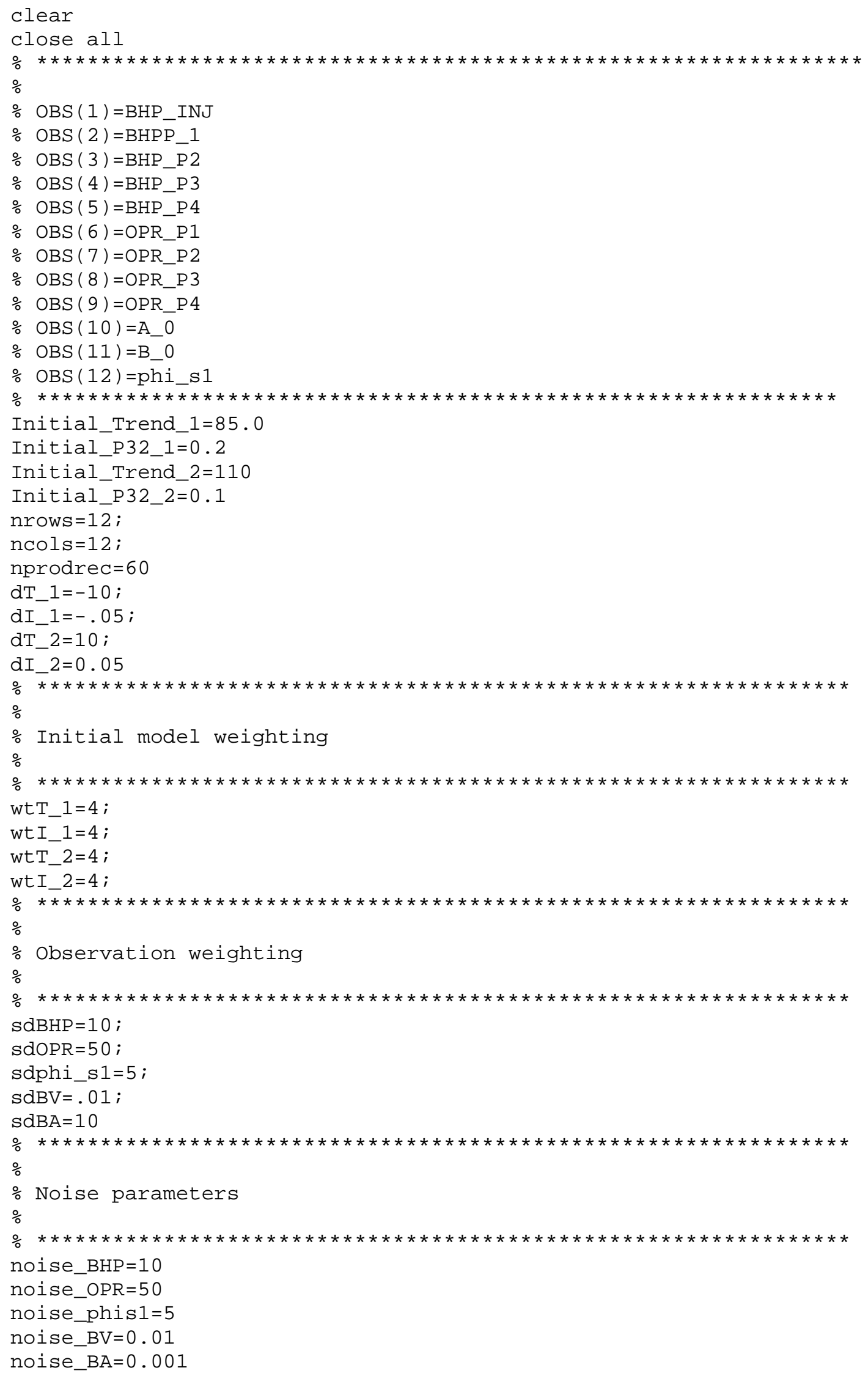




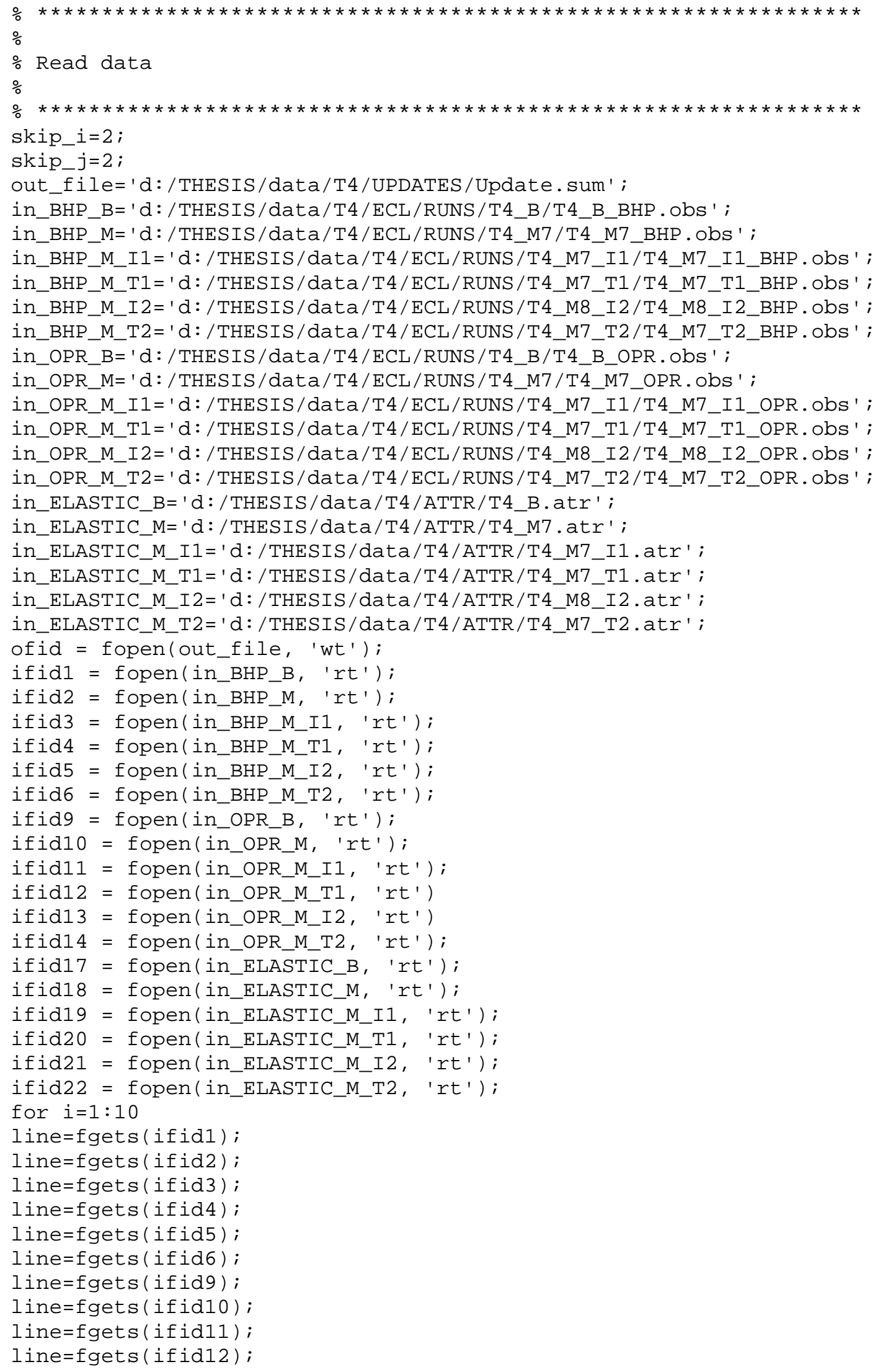




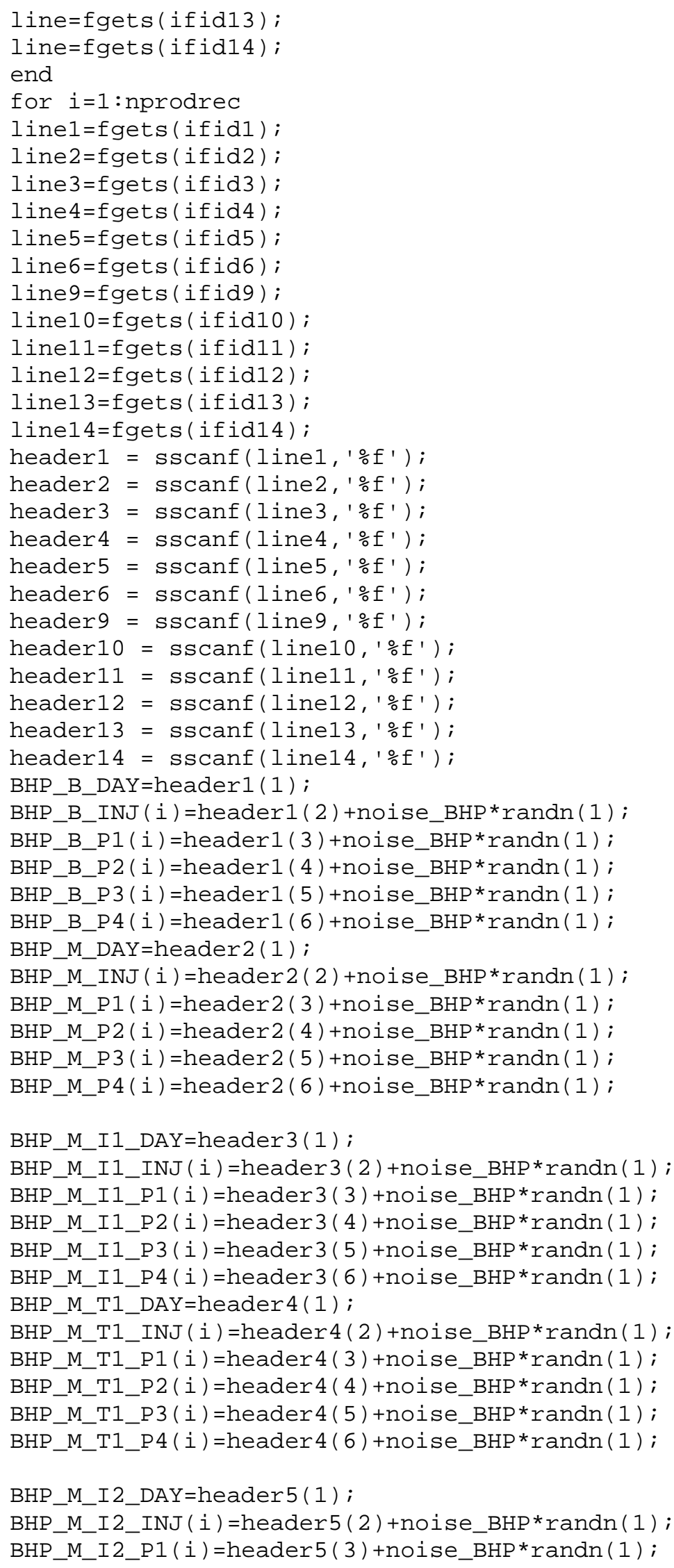




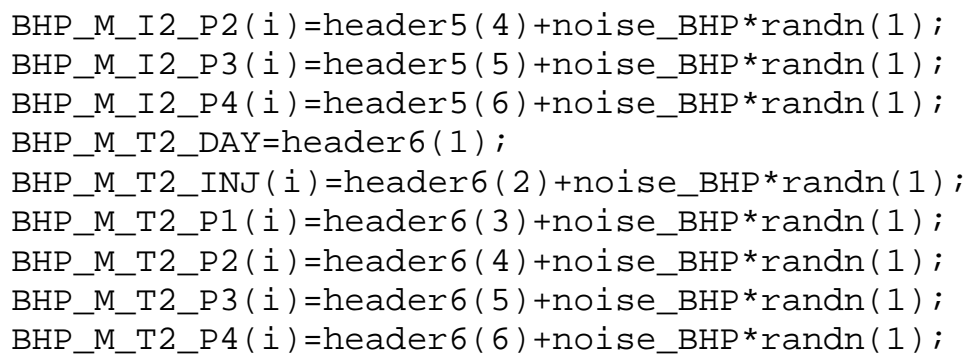

OPR_B_DAY (i) =header9 (1);

OPR_B_P1 (i) =header9 (2) +noise_OPR*randn (1) ;

OPR_B_P2 (i) =header9 (3) +noise_OPR*randn (1) ;

OPR_B_P3 (i) =header9 (4) +noise_OPR $*$ randn (1);

OPR_B_P4 (i) =header9 (5) +noise_OPR*randn (1) ;

OPR_M_DAY $(i)=$ header10 (1);

OPR_M_P1 (i) =header10 (2) +noise_OPR*randn (1);

OPR_M_P2 (i) =header10 (3) +noise_OPR*randn (1);

OPR_M_P3 (i) =header10 (4) +noise_OPR*randn (1) ;

OPR_M_P4 (i) =header10 (5) +noise_OPR* randn (1) ;

OPR_M_I1_DAY (i) =header11 (1) ;

OPR_M_I1_P1 (i) =header11 (2) +noise_OPR*randn (1) ;

OPR_M_I1_P2 (i) =header11 (3) +noise_OPR*randn (1) ;

OPR_M_I1_P3 (i) =header11 (4) +noise_OPR* randn (1) ;

OPR_M_I1_P4 (i) =header11 (5) +noise_OPR*randn (1) ;

OPR_M_T1_DAY $(i)=$ header12 (1);

OPR_M_T1_P1 (i) =header12 (2) +noise_OPR*randn (1) ;

OPR_M_T1_P2 (i) =header12 (3) +noise_OPR* randn (1) ;

OPR_M_T1_P3 (i) =header12 (4) +noise_OPR*randn (1) ;

OPR_M_T1_P4 (i) =header12 (5) +noise_OPR*randn (1) ;

OPR_M_I2_DAY (i) =header13 (1);

OPR_M_I2_P1 (i) =header13 (2) +noise_OPR*randn (1) ;

OPR_M_I2_P2 (i) =header13 (3) +noise_OPR* randn (1) ;

OPR_M_I2_P3 (i) =header13 (4) +noise_OPR* randn (1) ;

OPR_M_I2_P4 (i) =header13 (5) +noise_OPR*randn (1) ;

OPR_M_T2_DAY $(i)=$ header13 (1);

OPR_M_T2_P1 (i) =header13 (2) +noise_OPR* randn (1) ;

OPR_M_T2_P2 (i) =header13 (3) +noise_OPR*randn (1) ;

OPR_M_T2_P3 (i) =header13 (4) +noise_OPR*randn (1) ;

OPR_M_T2_P4 (i) =header13 (5) +noise_OPR* randn (1) ;

end

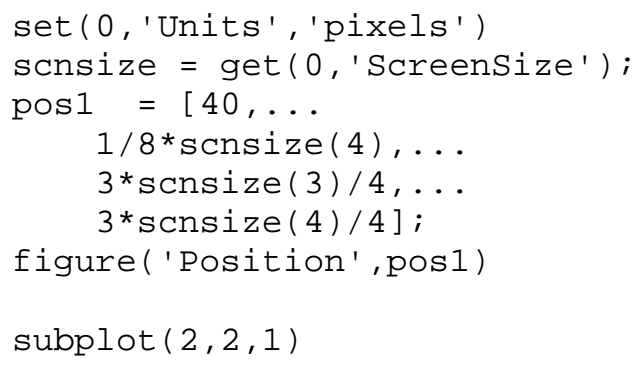




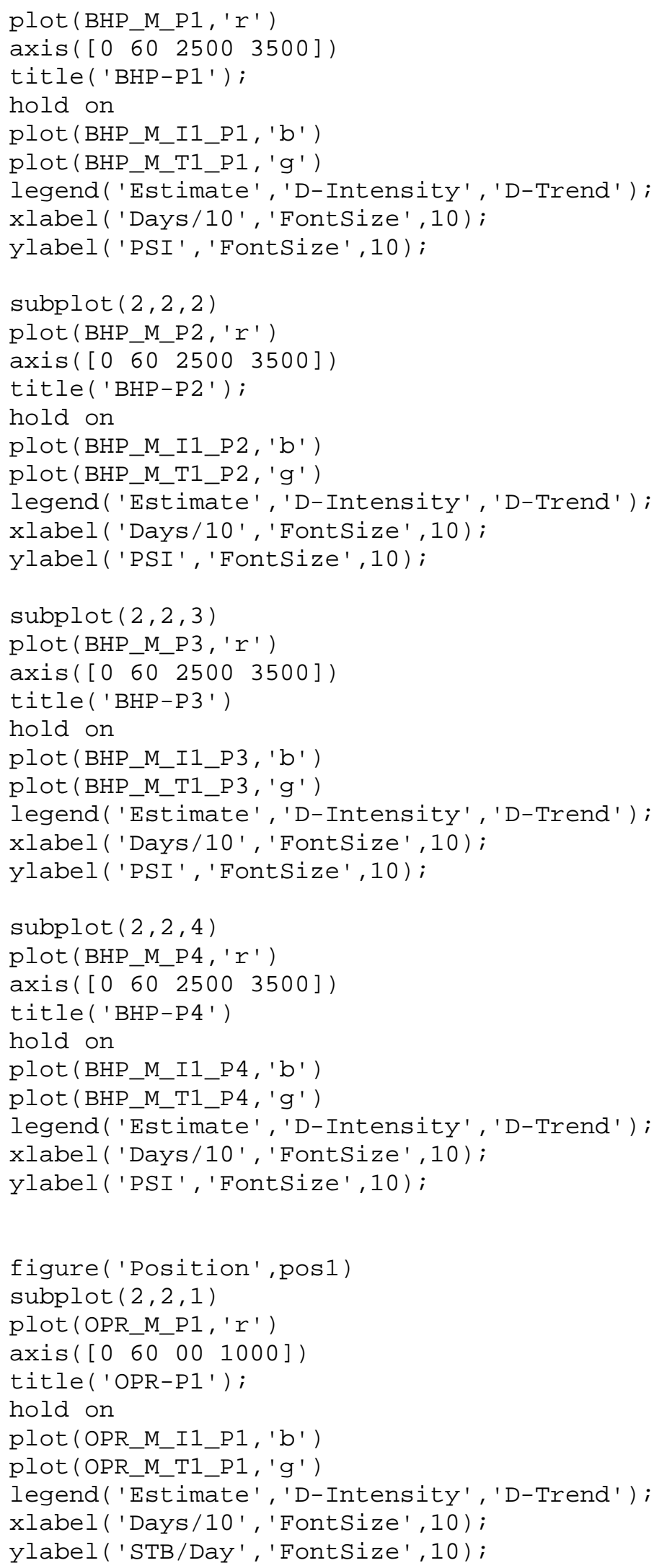




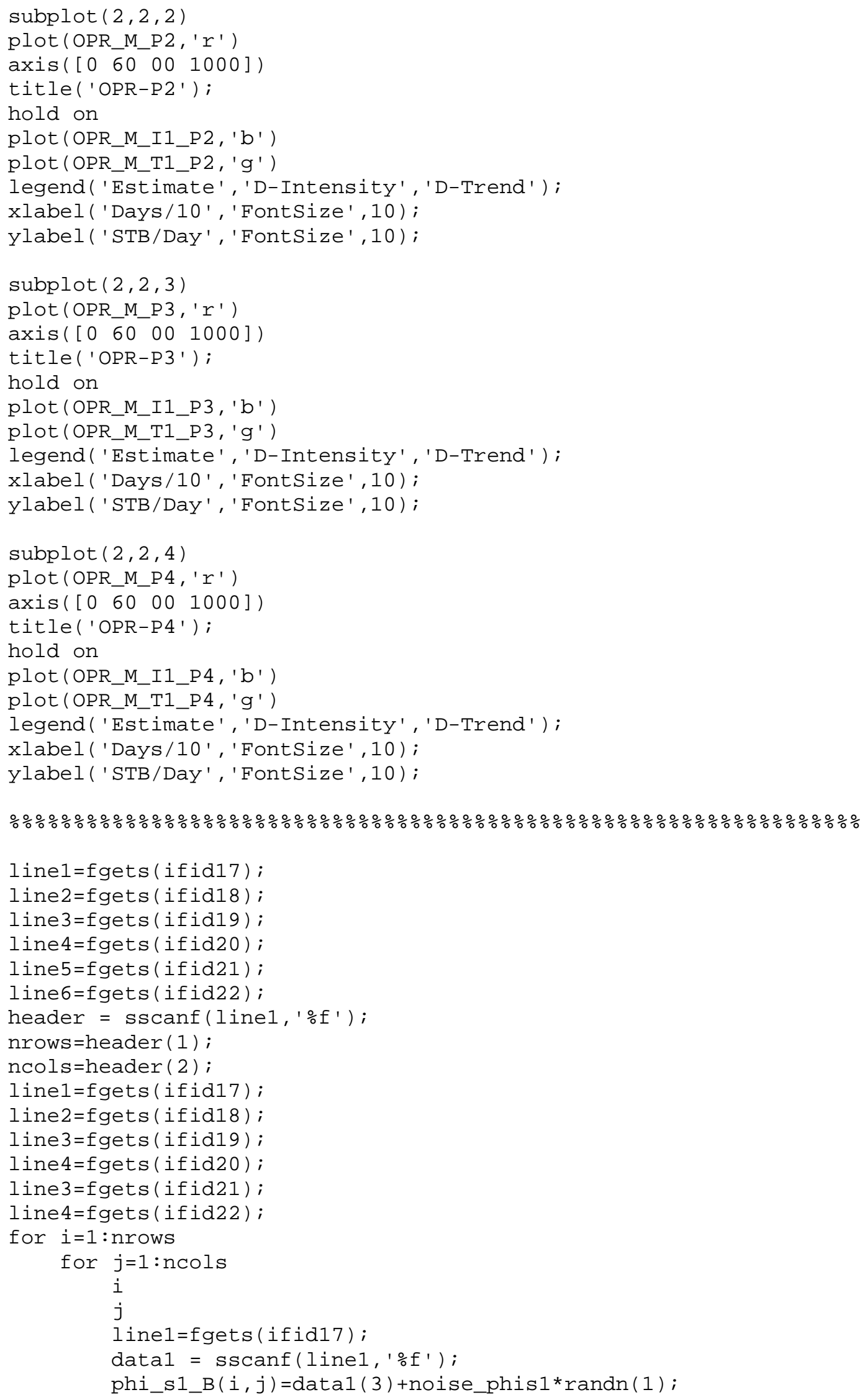




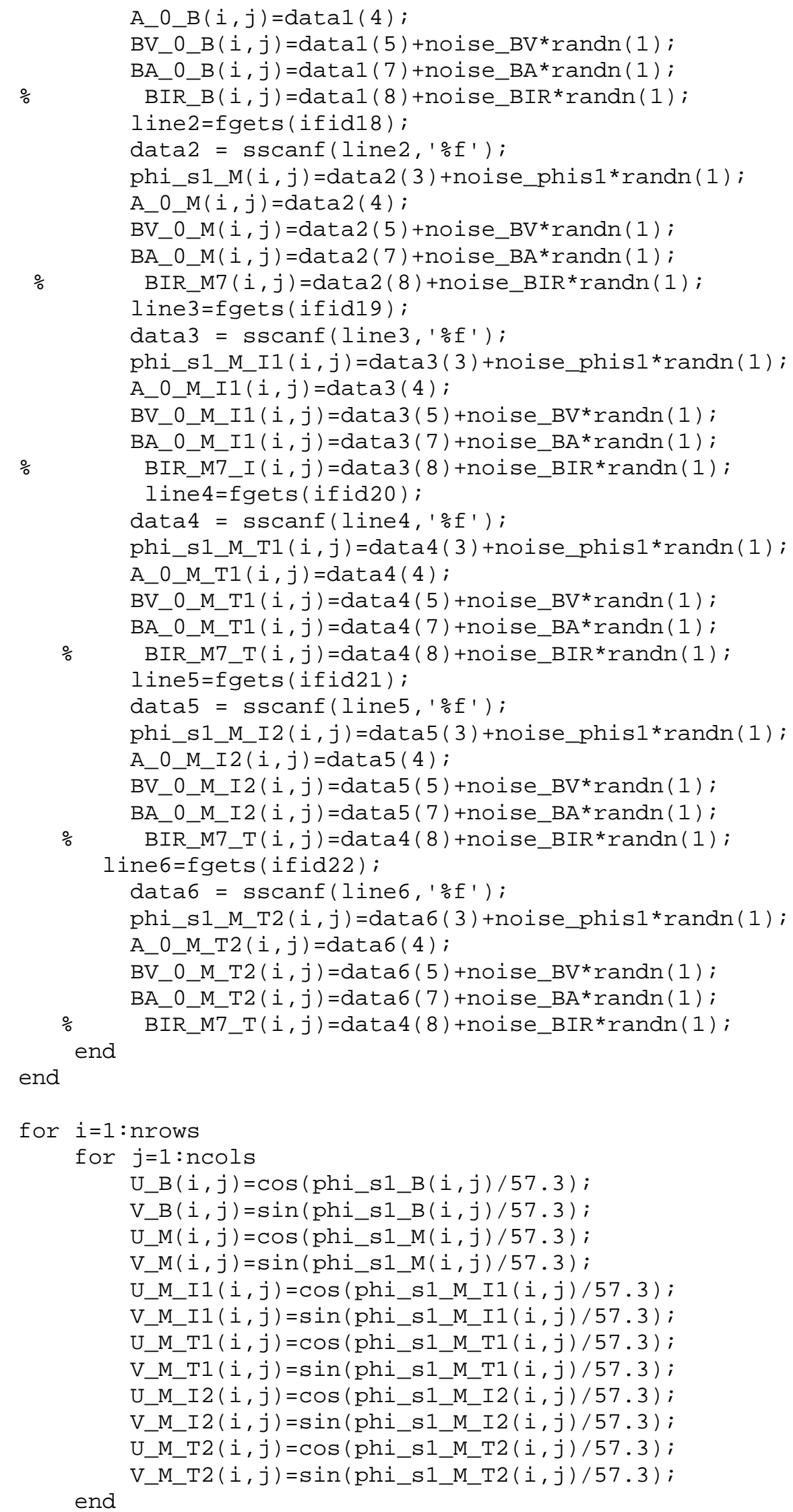




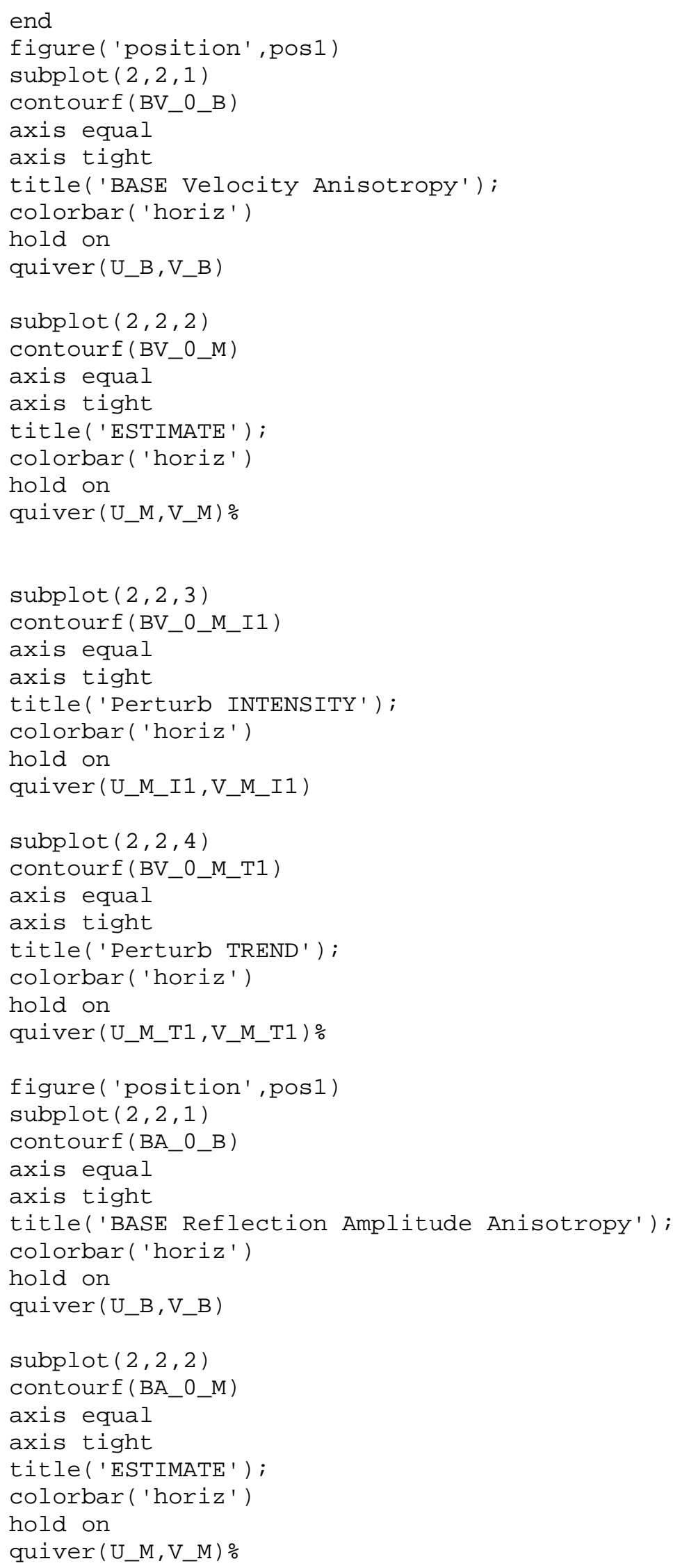




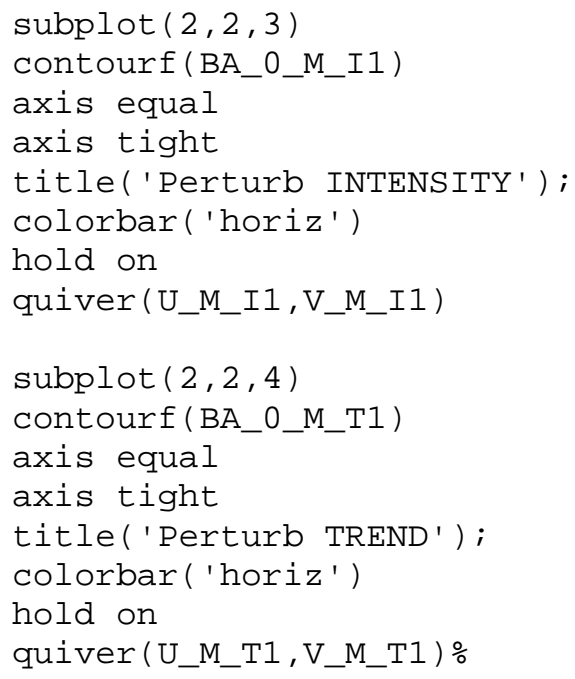




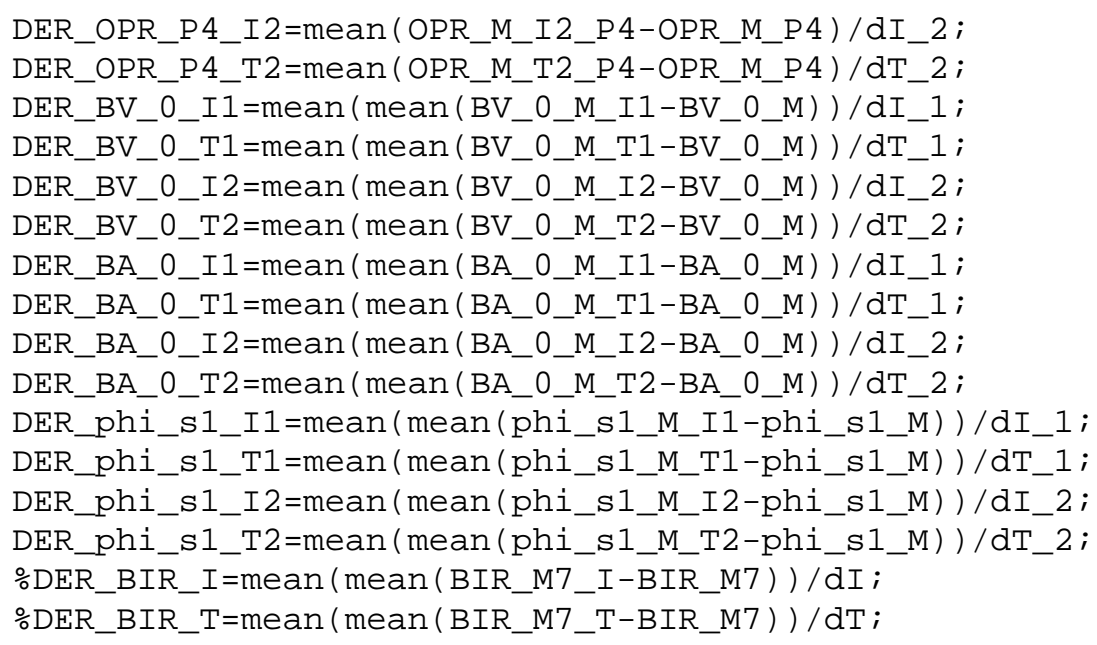




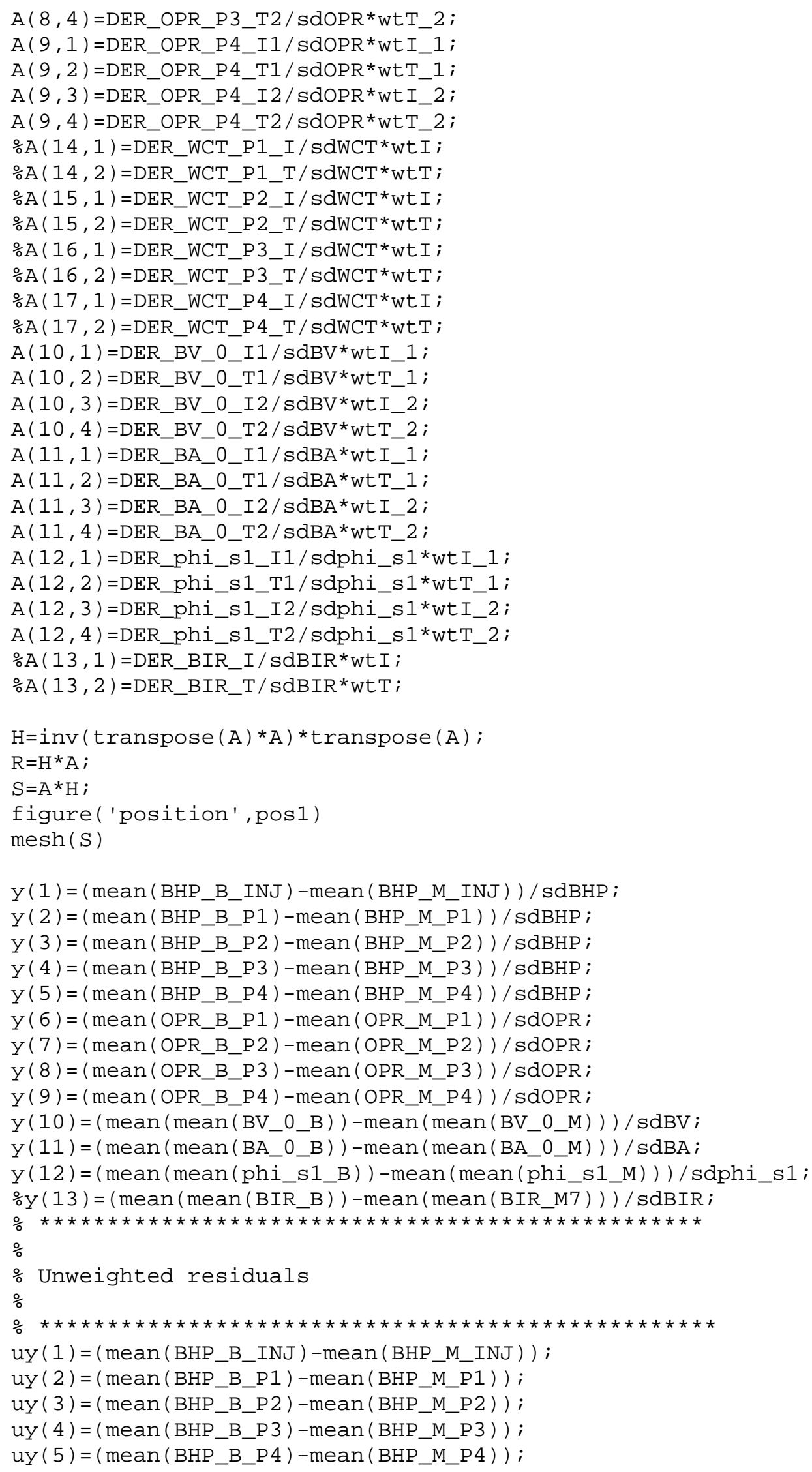




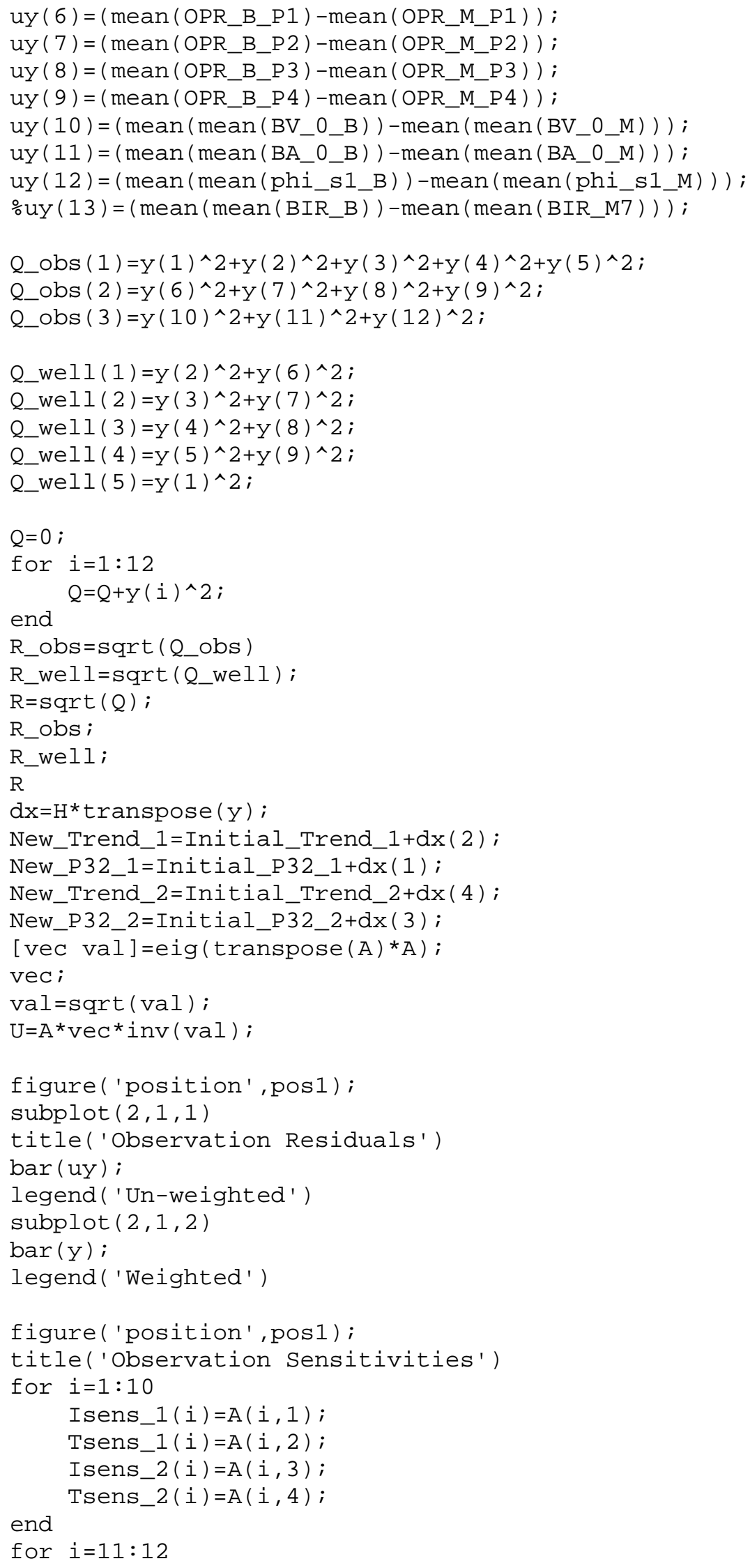




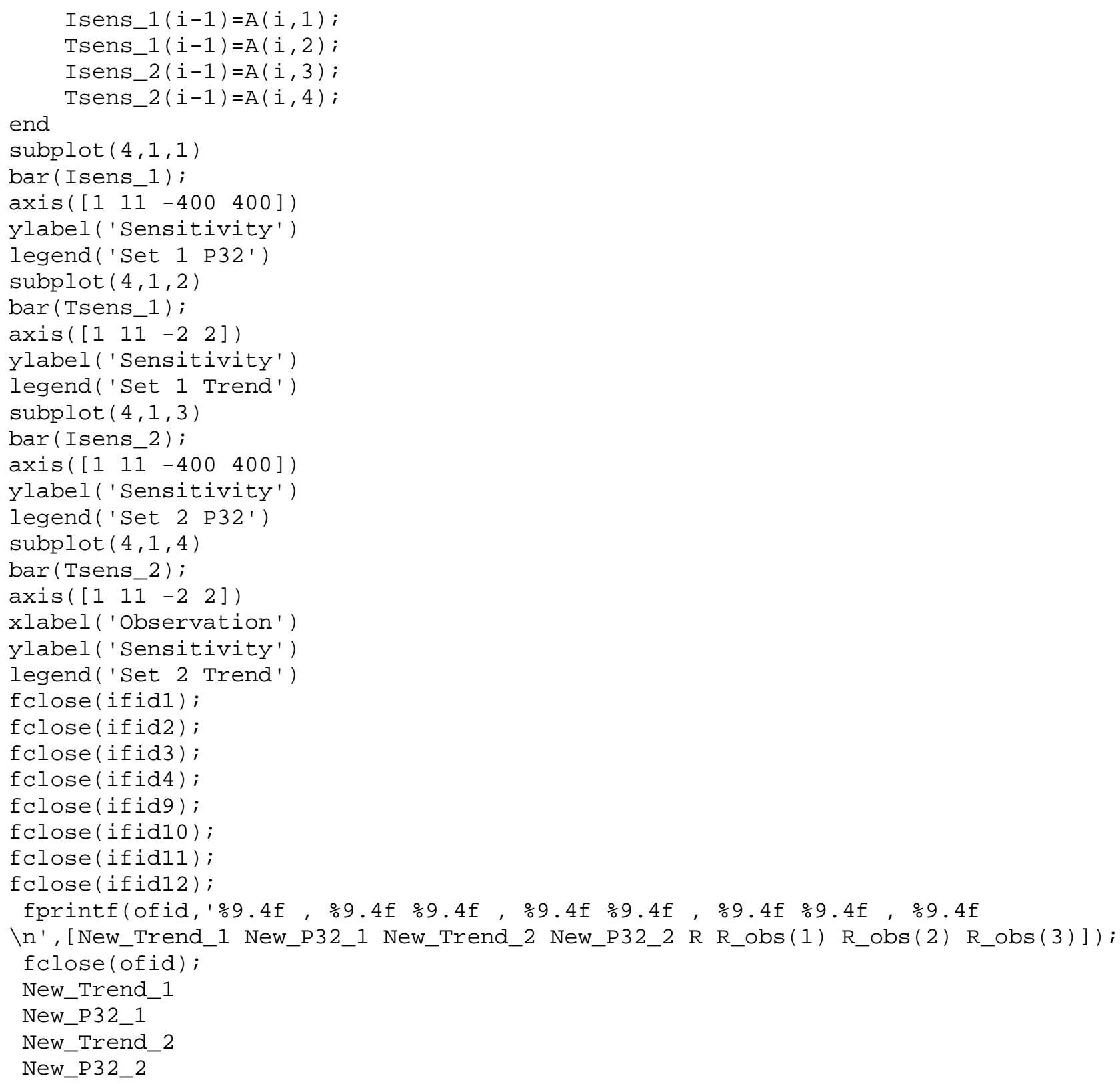




\section{APPENDIX I}

Sample Eclipse Data File 


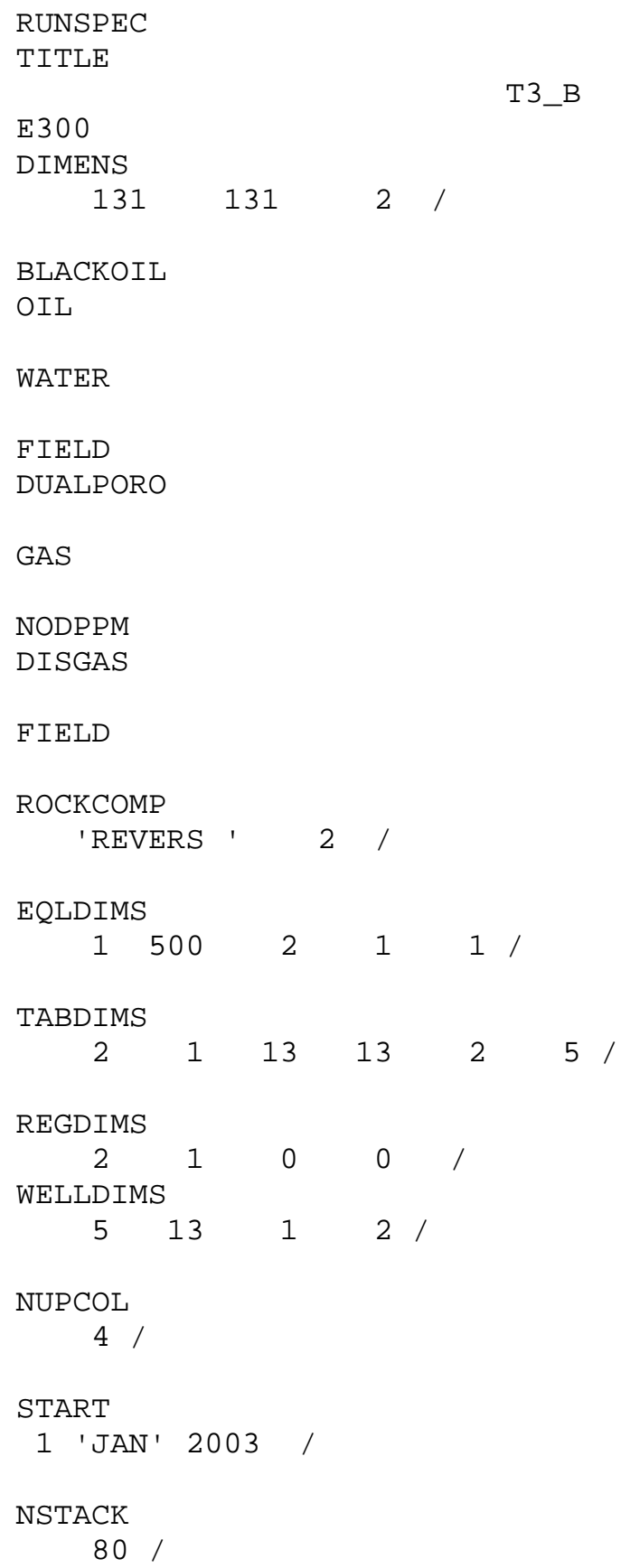




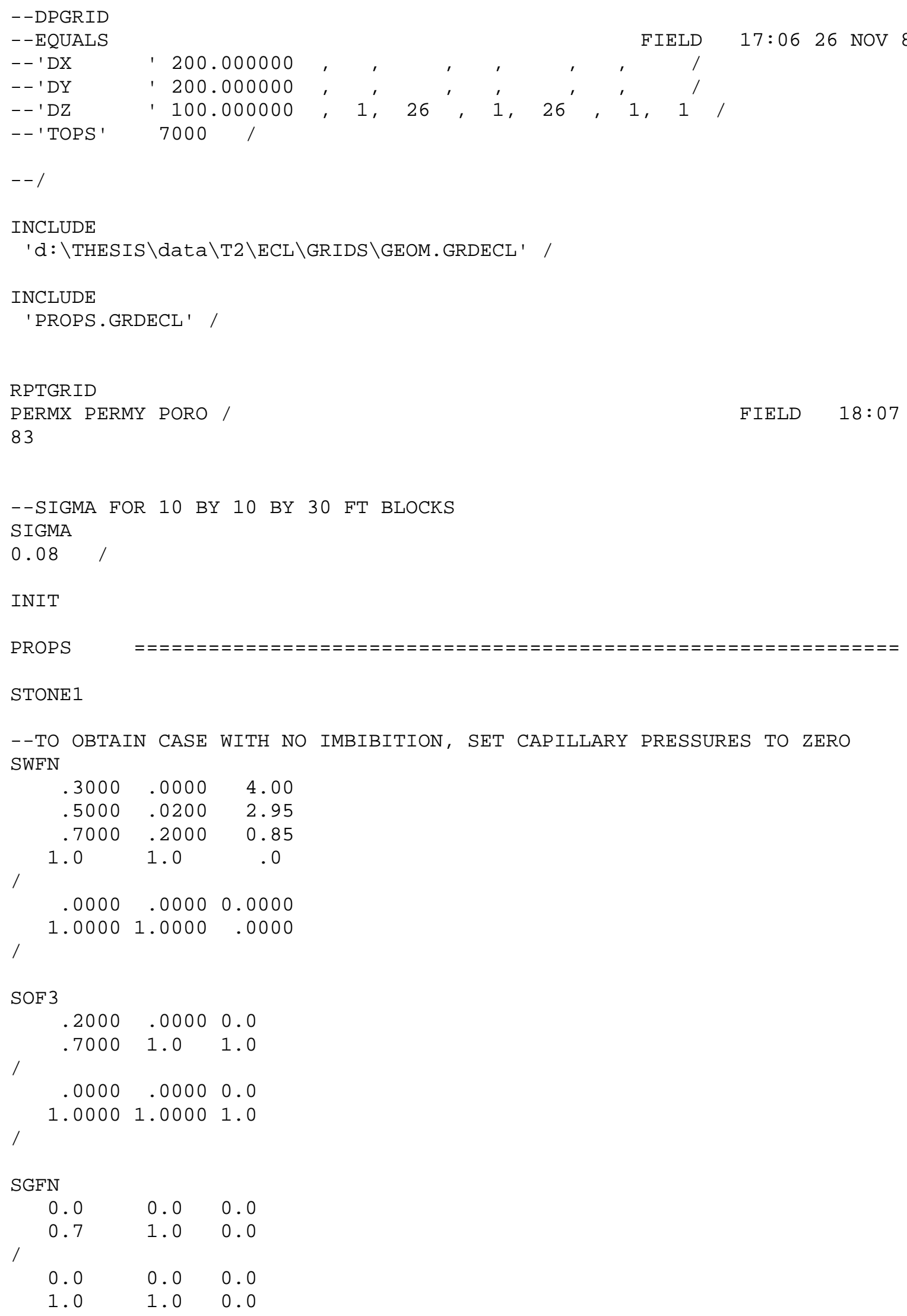




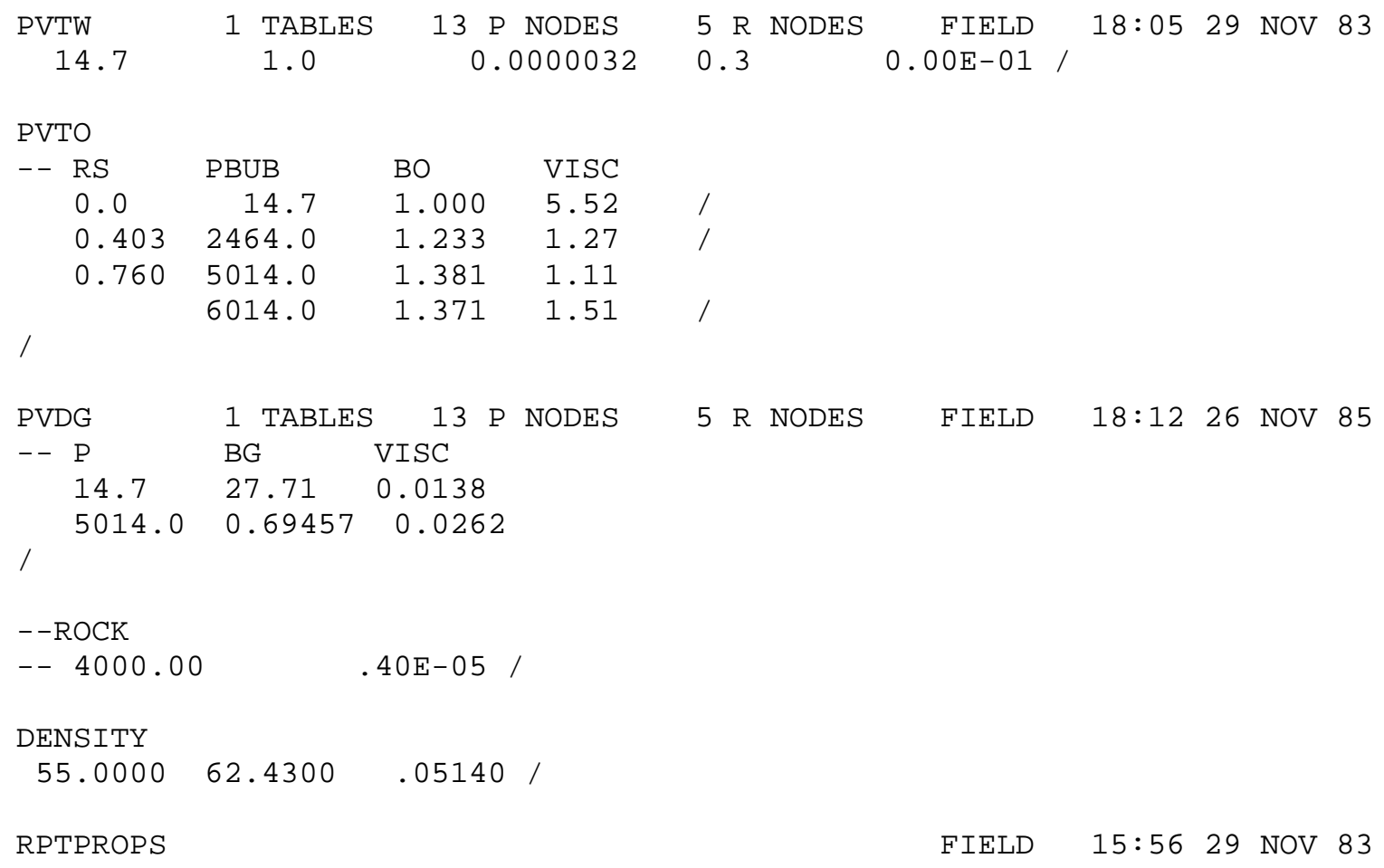




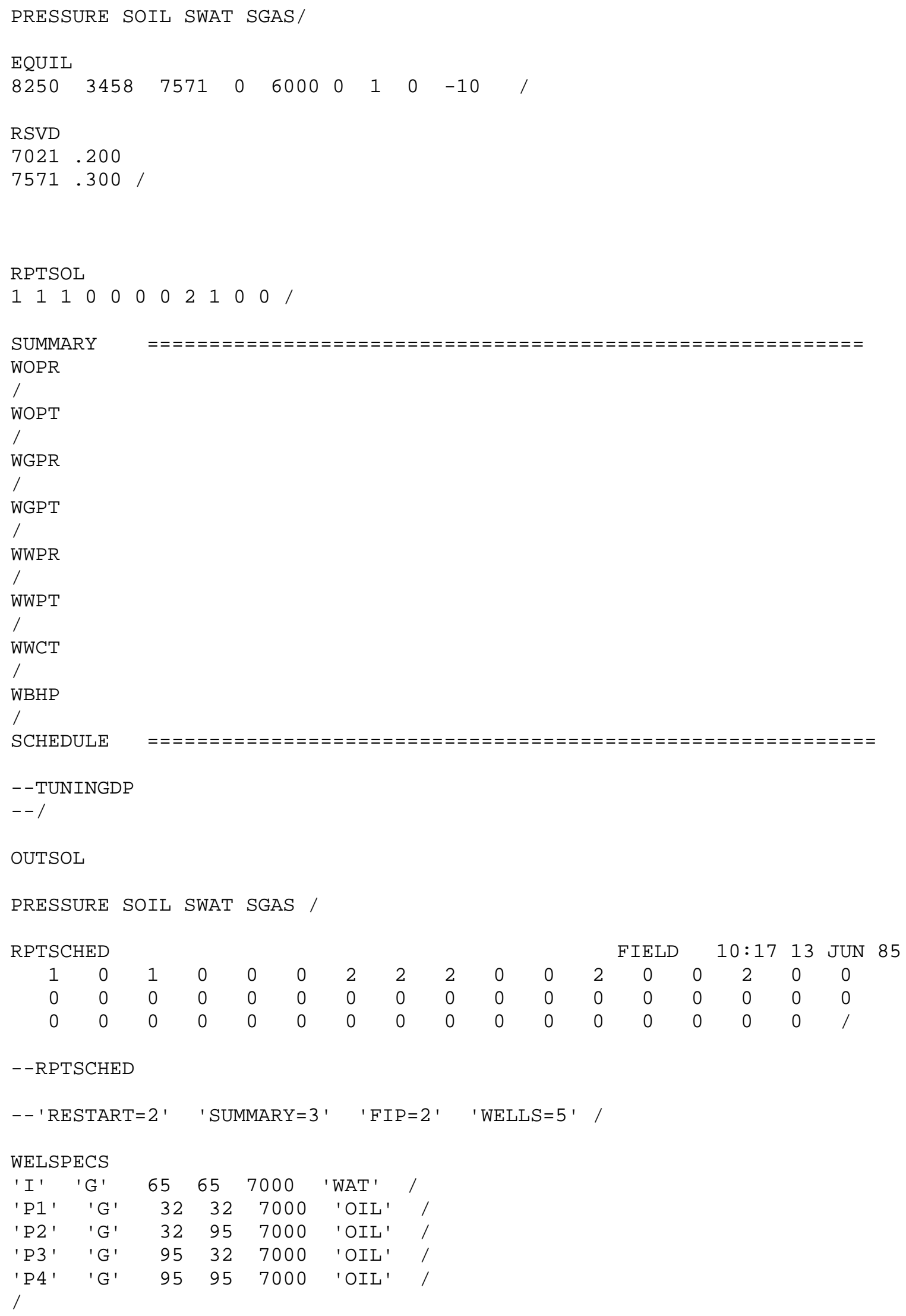




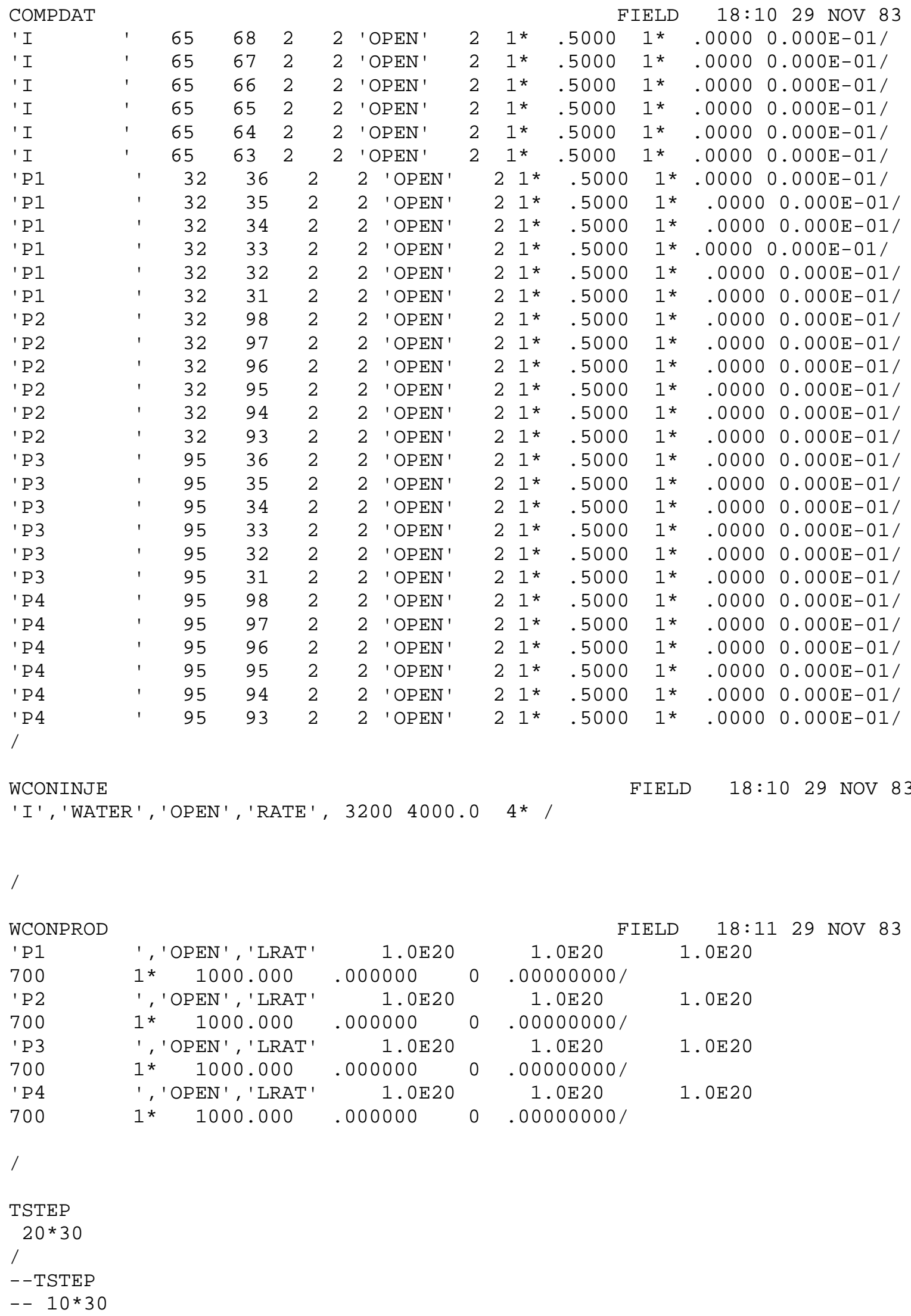


$--/$

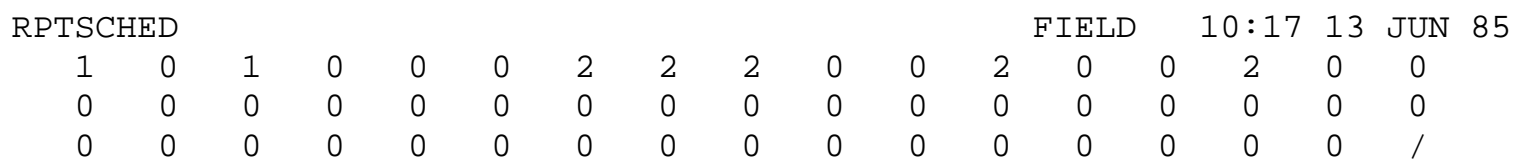

END 


\section{VITA}

Name:

Place of Birth:

Parents:

Permanent Address:

Educations:

Professional Affiliations:

Professional Experience:
Robert A. Will

Toledo, Ohio

Harold L. and Rachel L. Will

12867 Kingsbridge Lane

Houston, TX 77077

B.A. Geology (May 1977)

Hope College

Holland, Michigan

M.S., Geophysics (May 1983)

New Mexico Institute of Mining and Technology,

Socorro, New Mexico

Ph.D., Petroleum Engineering (May 2004)

Texas A\&M University

College Station, Texas

Society of Petroleum Engineers.

Society of Exploration Geophysicists.

Western Geophysical (1981-2000)

Schlumberger, (2000 - present). 